Louisiana State University

LSU Digital Commons

1969

\title{
Fluid Dynamics and Flow Patterns in Stirred Tanks With a Turbine Impeller.
}

Abel Desouza

Louisiana State University and Agricultural \& Mechanical College

Follow this and additional works at: https://digitalcommons.Isu.edu/gradschool_disstheses

\section{Recommended Citation}

Desouza, Abel, "Fluid Dynamics and Flow Patterns in Stirred Tanks With a Turbine Impeller." (1969). LSU Historical Dissertations and Theses. 1650.

https://digitalcommons.Isu.edu/gradschool_disstheses/1650

This Dissertation is brought to you for free and open access by the Graduate School at LSU Digital Commons. It has been accepted for inclusion in LSU Historical Dissertations and Theses by an authorized administrator of LSU Digital Commons. For more information, please contact gradetd@lsu.edu. 
This dissertation has been microfilmed exactly as received

$70-9050$

DeSOUZA, Abel, 1936-

FLUID DYNAMICS AND FLOW PATTERNS IN STIRRED TANKS WITH A TURBINE IMPELLER.

The Louisiana State University and Agricultural and Mechanical College, Ph.D., 1969

Engineering, chemical

University Microfilms, Inc., Ann Arbor, Michigan 


\title{
FLUID DYNAMICS AND FLOW PATTERNS IN STIRRED
}

TANKS WITH A TURBINE IMPELLER

\author{
A Dissertation \\ Submitted to the Graduate Faculty of the \\ Louisiana State University and \\ Agricultural and Mechanical College \\ in partial fulfillment of the \\ requirements for the degree of \\ Doctor of Philosophy
}

in

The Department of Chemical Engineering

by

Abel DeSouza

B.S., University of Bombay, 1959

M.S., Loufsiana State University, 1966

August, 1969 


\section{ACKNOWLEDGEMENTS}

This work was performed under the direction of $\mathrm{Dr}$. Ralph W. Pike. His encouragement, counsel and guidance is gratefully acknowledged.

The author would like to express his thanks to the Chemical Engineering Department for financial support during his years in Graduate School and the LSU Computer Research Center for unlimited use of their facilities. The author would also like to thank Dr. P. M. Arnold, Vice-President of Research and Development, The Phillips Petroleum Company, for his keen interest and encouragement in this project, and the following companies who have supported this project: The Dow Chemical Eompany, Esso Research and Engineering Company, and The Phillips Petroleum Company.

The publication and typing costs of this dissertation was partially provided by the Charles E. Coates Memorial Fund of the LSU Foundation, donated by George H. Coates.

Special thanks are due to L. E. Veilleux for help in building the necessary apparatus of this project; to Miss Suzanne Hackler for typing the first drafts, Miss Madeline Jewe11 and Miss Debbie Kleinpeter for typing the manuscript and to my many friends whose advice and help is greatly appreciated. 
LIST OF COMPUTER LISTINGS

$x x i 1$

ABSTRACT

xxili

CHAPTER

2. Statement of the Problem 4

3. Literature Review 7

3:1. The Turbine Impeller 9

3.2. Power Consumption in Stirred Tanks 11

3.3. Flow Patterns with Turbine Impellers 12

Comparison of Turbine Impellers by 13

Nagata and Associates

Measurement of Velocity Profiles

.Near the Impeller by Sachs

Nielson's Tangential Jet Mode1

Velocity Profdles in Baffled and

Unbaffled Tanks by Aiba

Measurement of Flow Profiles at the 31

Impeller by Cutter

Potential Flow Solution by Larson 32

Flow Pattern Studies by Schumm 35 
Velocity Measurements in the Region of the Impeller by Cooper

Flow Patterns in Newtonian and Non-

41. Newtonian Fluids by Metzner

Turbulence Measurement in Stirred : Tanks by Bowers and Mujumdar

Summary

3.4. Pumping Capacities of Turbine Impellers

3.5. Velocity Measurements in Stirred Tanks

4. Conclusions

1. Development of a Mathematical Model for the Stirred Tank

1.1. Region I - Nefghborhood of the

Impe11er

The Tangential Jet

Equations of Continuity and Momentum for 66 the Tangential Jet

Solutions of the Tangential Jet Equations 69

Results of Tangential Jet Solution $\quad 70$

1.2. Regions II, III, and IV 71

1.3. Region V 75

1.4, Region VI 77

2. Development of Computer Program to Draw the 78 Flow Pattern 
2.1. Equations for Streamlines in 79 Region I

2.2. Region II, III, IV 82

2.3. Mechanics of Plotting Streamlines 85: in Regions I, II, III and IV

$\begin{array}{ll}\text { Region I } & 86\end{array}$

$\begin{array}{ll}\text { Region II } & 87\end{array}$

Scale Used for Plotting 88

Region III and IV 91

2.4. Region V 92

3, Theoretical Flow Patterns 95

3.1. Extent of Region IV 97

3.2. Boundary Between Regions III and IV 101

3.3. Locating the Origin of the Circular 101 Jet

3.4. Desirable Values of Boundary 104 Parameters

4. Summary and Conclusions 106

III EXPERIMENTAL APPARATUS AND OPERATING PROCEDURE 108

3.1. Mixing Vessel 108

3.2. Impeller and Impeller Drive Assembly 112

3,3. Velocity Measuring Probe and Probe 116 Assembly

3.4. Measurement of Velocities 119

3,5. Manometers 120

3.6. Measurement of the Impeller Speed 124

3.7. Problems Encountered 124

3.8. Summary 125 
4.1. Veloctty Profiles in the Region

of the Impeller

Analysis of Velocity Profiles 128

Radius of Source Parameter a 130

Effect of Impeller Depth, Radial 137

Angle, and Radial Distance on

Tangential Jet Parameters

Effect of Impeller Speed on

Tangential Jet Parameter

Effect of High Impeller Speed on

Velocity Profiles Measurements

Effect of Tank Diameter

Correlation of Tangential Jet

Parameters

4.2. Effect of Constant Jet Width and' Radius of Source on the Analysis

Width of the Tangential Jet

Jet Displacement Parameter

4.3. Velocity. Profile Measurementsin

the. Rest of the Tank

Velocity Measurements in the Rest of the Tank

Experillental Velocity Profiles

Theoretical Velocity Profiles

176

4.4. Detailed Analyses of a Velocity

Profile in the Neighborhood of

. the Impeller

Radial Velocity Profile Analysis

4.5. Interpretation of Manometer D 
4.6. Accuracy of the Three Dimensional 200 Pitot Tube as a Measuring Device : ' $1 . . . . "$. in Turbulent Flow Fields

4.7. Comparison with Cooper's Data 204

$\begin{array}{ll}\text { Jet Width } \sigma & 207\end{array}$

Radius of Source a $\quad 210$

Dimensionless Pumping Capacity $\mathrm{N}_{Q} \quad 215$.

Pumping Capacity Q 215

Volumetric Flow Parameter A 220

Results of Correlating Tangential 223

Jet Parameters

4.8. Analysis of Cooper's Data at Varying 227 Radial Distance and Varying Impeller Impeller Blade Width

Analysis at Varying Radial Distance 228

Analysis at Varying Blade Widths 230

4.9. Analysis of Nielson's and Cutter's 235 Data

Analysis of Nielson's Data $236^{\circ}$

Analysis of Cutter's Data 241

4.10. Summary of Velocity Profiles Obtained 247 by the Light Streak Method

4.11. Extent of the Tangential Jet 249

Jet Width 250

4.12. Summary 251

V 5๖l. Three-Dimensional Velocity Probe 255

vii 
5.2. Flow Model for the Stirred

Tank

Tangential Jet
Potential Flow
Recommendation

\section{APPENDIX}

A

TANGENTIAL OR RING JET

Derivation of Differential Equation

Momentum of Tangential Jet

B

SIMILARITY SOLUTION FOR A TANGENTIAL JET

Properties of the Tangential Jet

NON-LINEAR LEAST SQUARE FIT OF EXPERIMENTAL

297 VELOCITY PROFILE

D TWO DIMENSIONAL POTENTIAL FLOW IN $x-z$

304 PLANE

E.

DESCRIPTION OF PROGRAM PLOTER USED FOR PLOTER USED FOR PLOTTING STREAMLINES

Golden Section Search

Evaluation of Velocity profiles

Sample Printout from PLOTER

LISTING AND DESCRIPTION OF COMPUTER PROGRAMS 
PAGE

$\begin{array}{ll}\text { Program COOPER and NEILCUT } & 346\end{array}$

$\begin{array}{ll}\text { Program GRAPH } & 346\end{array}$

G SUMMARY OF TANGENTIAL JET ANALYSIS 448

Summary of Cooper's Data $\quad 449$

Sunmary of Nielson's and Cutter's Data 450

H DETAILED ANALYSIS OF A VELOCITY PROFILE 497

IN THE NETGHBORHOOD OF THE IMPELLER

508

FROM PROGRAM TANKANL

VITA

514 
TABLE

NUMBER

PAGE

CHAPTER I

I-1 Classification of Stirred Tanks According to Functions Performed in the Tank

I-2 Summary of Neilson's Investigation of

Turbine Impe1lers

I-3 Comparisson of Impellers and Conditions Under Which Flow Patterns Were Examined. Al1 Measurements reported are in inches.

\section{CHAPTER II}

II-1 List and Definition of Symbols Used in Figure II-6

II-2 Operating Conditions and Pertinent data on the Stirred Tank Whose Theoretical Profiles , :: are drawn in Figures II-5 to II-9

\section{CHAPTER III}

\section{CHAPTER IV}

IV-1 Conditions Under Which Velocity Profiles Were Measured

IV-2 Effect of Velocity Factor on Least Square

Fit of a Velocity Profile for 3.0 Inch

Diameter Impeller at $333.3 \mathrm{RPM}$ in a 12.25

Inch Diameter Tank

IV.3 Effect of Impeller Depth, Radial Angle, and

Radial Distance on Tangential Jet Parameters at Impeller Speed of 500 RPM

IV-4 Effect of Radial Distance on Tangential Jet Parameters for a $3.0 \mathrm{In}$. Impeller at $243 \mathrm{RPM}$ and Impeller Depth of $3.0 \mathrm{in}$.

IV-5 Average value of Jet Width Parameter $\sigma$ for

a 3.0 Inch Diameter Impeller 
TABLE

NUMBER

IV $\rightarrow 6$ Average Value of Volumetric Flow

Parameter A, For a $3.0 \mathrm{In}$. Diameter

Impeller

IV-7 Average Value of Radius of Source a

146

for a 3.0 Inch Diameter. Impeller in a

12.5 Inch Diameter Tank

IV-8 Summary of Results of Tangential Jet

Parameters for a 3.0 Inch Diameter Impeller

151

in a 12.25 Inch Diameter Tank

IV-9 Effect of Constant $\sigma$ and, $a$, on the Süm of

Squares SS, and Correlation Coefficient for $\bar{q}$ and $\bar{v}_{r}$ at Impeller Speed of 243 RPM

IV-10 Effect of Constant $\sigma$ and, $a$, on the Volumetric Flow Parameter $A$ and Jet Displacement Parameter $z_{0}$ at Impeller speed of $243 \mathrm{RPM}$

IV-11 Effect of Constant $\sigma$ and a on Volumetric Flow at Impeller Periphery and at Radial Distance $r$ for a Constant Impeller Speed of 243 RPM

IV-12 Effect of Constant $\sigma$ and $a$ on the Average Values of the Volumetric Flow Parameter A

IV-13 Theoretical Velocity Profiles Calculated by

181 Program VELPRO for Regions II and III Above the Impeller

IV-14 Theoretical Velocity Profile Calculated by Program VELPRO for Regions II and III Below Impeller. Shows Effect of Considering a Boundary Layer in the Theoretical Model

IV-15 Theoretical Velocity Profiles in Region V Obtained from the Circular Jet

IV-16 Typical Results of Velocity Profile Analysis in : the Impeller From Program FLOWANL and Illustrated for Experimental Data From Run Number 29

IV-17 Typical Results of Radial Velocity Profile Analysis. Illustrated for Experimental Data From Run Number 29 
TABLE

NUMBER

PAGE

IV-18 Results of Program YAWANL Which Treats the Impeller Region As a Three-Dimensional Flow Field, and Illustrated for Experimental Data from Run Number 29

IV-19 Result of Analysis by Subroutine YAW that Treats 199 the Region of the Impeller as a Region of High Shear, for Experimental Data of Run Number 29

IV-20 Summary of Tangential Jet Analysis for. Data 205 Obtained with a Directional Pitot Tube

IV-21 Average Values of Jet Width $\sigma$, Radius of 208 Source a, Dimensionless Pumping Capacity $\mathrm{N}_{Q}$ and Weighted Average Yaw Angle $\theta_{\mathrm{y}}$ Extracted for Data Sets of Table IV-20

IV-22 Predicted and Experimenta11y Determined Values of $a$, The Radius of Source

IV-23 Eddy Viscosity E. Calculated From Predicted Values of $\sigma, A$ and $a$

IV-24 Analysis of Cooper's Data With Varying Radial Distance, Showing Effect of $\sigma$

IV-25 Analysis of Cooper's Data at Varying Blade Width and RPM

IV-26 Effect of Constant $\sigma$ Equal to 12.621 on Varying Blade Width at 100 RPM and Impeller Diameter of 4.0 Inch

IV-27 Effect of $\bar{\theta}_{y}$ on the Analysis of Nielson's

IV-28 Effect of Keeping $\sigma$ a Constant Value of 12.621 on Nielson's Data. $\bar{\theta}_{y}=65^{\circ}$

IV-29 Analysis of Nielson's Data Resulting From an Optimum Selection of $\bar{\theta}_{y}$

IV-30 Analysis of Cutter's Data at 200 RPM for a 4.0 Inch Impeller Showing Effect of Varying $\bar{\theta}_{\mathrm{y}}$ 
TABLE

NUMBER

PAGE

IV-31 Analysis of Cutter's Data at 200 RPM

for a 4.0 Inch Impeller, with $\sigma$

Constant and Varying $\bar{\theta}_{y}$

IV-32 Results of Analysis of Cutter's Data

at $400 \mathrm{RPM}$, for a 4.0 Inch Impel1er,

$\sigma$ Constant and $\bar{\theta}_{y}=65^{\circ}$

IV-33 Results of Analysis of Cutter's Data at

246

600 RPM, for a 4.0 Inch Impeller, $\sigma$

Constant and $\bar{\theta}_{y}=65^{\circ}$

IV-34 Summary of Results for the Tangential Jet

by the Light Streak Method

CHAPTER V

\section{APPENDIX G}

G-1 List of Abbreviations Used for Column

Headings in Tables of Appendix G

G-2 Summary of Velocity Profile Analysis at .

243 RPM, in a 12.25 Inch Diameter Tank

G-3 Summary of Velocity Profile Analysis at

455

243 RPM $\sigma$ and a Constant, in a 12.25 Inch

Diameter Tank

G-4 Summary of Velocity Profile Analysis at

250 RPM in a 12.25 Inch Diameter Tank

G-5 Summary of Velocity Profile Analysis at

458

250 RPM $\sigma$ and a Constant

G-6 Summary of Velocity Profile Analysis at

333.3 RPM in a 12.25 Inch Diameter Tank

G-7 Summary of Velocity Profile Analysis at

460

333.3 RPM $\sigma$ and a Constant

G-8 Summary of Velocity Profile Analysis at

461

400 RPM in a 12.25 Inch Diameter Tank

G-9 Summary of Velocity Profile Analysis at 400 RPM $\sigma$ and a Constant 
G-10 Summary of Velocity Profiled.Analysis at 500 RPM in a 12.25 Inch Diameter Tank

G-11 Summary of Velocity Profile Analysis

465 at $500 \mathrm{RPM}, \sigma$ and a Constant

G-12 Summary of the Velocity Profile Analysis at 550 RPM in a 12.25 Inch Diameter Tank

G-13 Summary of Velocity Profile Analysis at $550 \mathrm{RPM}, \sigma$ and a Constant

G-14 Summary of Velocity Profile Analysis at 333.3 RPM in a 11.5 Inch Diameter Tank

G-15 Summary of Velocity Profile Analysis at 333.3 RPM, $\sigma$ and a Constant in a 11.5 Inch Diameter Tank

G-16 Analysis of Cooper's Data in Water for a 471 3.0 Inch Diameter Impeller

G-17 Analysis of Cooper's Data for a 3.0 Inch Diameter Impeller in Water, $\sigma$ Constant 4.0 Inch Diameter Impelier

G-19 Analysis of Cooper's Data for a 4.0 Inch Diameter Impeller in Water, $\sigma$ Constant

G-20 Analysis of Cooper's Data in Water for a 5.0 Inch Diameter Impeller

475

G-21 Analysis of Cooper's Data for a 5.0 Inch Diameter Impeller in Water, $\sigma$ Constant

G-22 Analysis of Cooper's Data in Water for a 6.0 Inch Diameter Impeller

G-23 Analysis of Cooper's Data for a 6.0 Diameter Impeller in Water, $\sigma$ Constant 4.0 Diameter Impeller 
G-26 Analysis of Cooper's Data in Air for a 5.0 Inch Diameter Impeller

G-27 Analysis of Cooper's Data for a 5.0

482

Diameter Impeller in Air, $\sigma$ Constant

G-28 Analysis of Cooper's Data in Air for a

483 6.0 Inch Diameter Impeller

G-29 Analysis of Cooper's Data for a 6.0 Inch Diameter Impeller in Air, $\sigma$ Constant

484

G-30 Analysis of Cooper's Data for a 4.0 Inch

485 Impeller at 280 RMP and Varying Radial Distance

G-31 Analysis of Cooper's Data for a 4.0 Inch Impeller at 280 RPM and Varying Distance, $\sigma$ Constant and a Evaluated from Equation IV-38

G-32 Analysis of Cooper?'s Data for a 4.0 Inch Impeller at 100 RPM with Varying Blade Widths

G-33 Analysis of Cooper's Data for a 4.0 Inch Impeller at 200 RPM with Varying Blade Widths

G-34 Analysis of Nielson's Data at Varying 489 Impeller Diameter, Impeller Speed. Profile 5 is Measured in Corn Syrup andra was Calculated From $\theta_{y}$

G-35 Analysis of Nielson's Data At Varying Impeller Diameter Impeller Speed. Profile 5 is Measured Corn Syrup, a Was Calculated from $\bar{\theta}_{\mathrm{y}}$ and $\sigma$ is Constant

G-36 Analysis of Cutter's Data with a 4.0 Inch Diameter Impeller at Varying Radial Distance $r$. Impeller Speed is $200 \mathrm{RPM}$

G-37 Analysis of Cutter's Data with a 4.0 Inch Diameter Impellez at Varying Radial Distance $r$. Impeller Speed is 200 RPM and $\sigma$ is Constant 
TABLE

NUMBER

G-38 Analysis of Cutter's Data at 400 RPM Impeller Diameter $=4.0$ Inch and Varying Radial Distance $r$

G-39 Analysis of Cutter's Data at 400 RPM, 4.0

484 Inch Diameter Impeller, with Varying Radial Distance and $\sigma$ Constant

G-40 Analysis of Cutter's Data at 600 RPM, 4.0 Inch Diameter Impeller, with Varying Radial Distance

G-41 Analysis of Cutter's Data at 600 RPM, 4.0 496 Inch Diameter Impeller, with Varying Radial Distance and $\sigma$ Constant

APPENDIX H

H-1 Raw Data for Run No. $29 \quad 498$

H-2 Analysis of Velocity Profile $\vec{q}$, for Run 500 No. 29

H-3 Radial Velocity Profile Analysis for Run No. 29

H-4 Results of Analysing Manometer D for

507 Run No. 29

APPENDIX $J$

J-1 List of Abbreviations Used for Column Headings

J-2 Raw Data Converted to Pressure Drops Across Manometers $B$ and $D$ for Port 1

J-3 Results of Velocity Profile Analysis for Port 1

J-4 Results of Velocity Profile Analysis for

J-5 Results of Velocity Profile Analy.sis for Port 3 


\section{LIST OF FIGURES}

FIGURE

NUMBER

PAGE

CHAPTER I

I-1 Flow Patterns in a Baffled Tank with a Turbine

5 Impeller after Rushton

I-2 Types of Turbine Impellers

10

I-3 Schematic Representation of Velocity Profiles

15 in Baffled Tanks, after Nagata

I-4 Schematic Diagram of Vortex Formation in

18 Baffled Tanks from Nagata

I-5 Typical Streamlines in a Baffled Tank, From Nagata

I-6 Tangential Jet

I-7 Nielson's Comparison of Experimental and Theoretical Shear Stress at $r$ is equal to 4.0 inches

I-8 Anamolous Flow Pattern Observed by Schumm With Type B Turbine, with the Turbine Located Close to Tank Bottom

I-9 Schematic Diagram for Cooper's Model of Flow Through the Impeller

\section{CHAPTER II}

II-1 Typical Computer Drawn Solution of Flow Patterns Showing the Regions into which the Tank is Divided

II-2 The Tangential Jet

II-3 Potential Flow Solution of Equation D-9 74

II-4 The Circular Jet 76

II-5 Tangential Jet Model in Region $I \quad 83$ 
FIGURE

NUMBER

PAGE

II-6 Dimensionless Ratios Used for Obtainding

the Theoretical Streamlines by Program

PLOTER. Note all Symbols and Mathematical

Operations are in FØRTRAN

II-7 Effect of Varying the Boundary Between

98

Regions IV and $V$ in a Horizontal Plane on

the Theoretical Flow Patterns

II-8 Effect of Varying the Boundary Between Regions

99

IV and $V$ in a Cylinderical Plane on the Theo-

retical Flow Patterns

II-9 Effect of Varying the Boundary Between

102

Regions III and IV on the Theoretical

Flow Patterns

II-10 Effect of Varying the Boundary Between

103

Regions II and III on the Theoretical

Flow Patterns

II-11 Effect of Varying the Origin, $K$ of the

105

Circular Jet on the Theoretical Flow

Patterns

CHAPTER III

III-1 Details of Stirred Tank and Probe

109

Ass embly

III-2 Plexiglass Tank

111

III-3 - Overa11 Views of the Experimental Apparatus

113

III-4 Close-up View Showing Tank Drive, Vernier

114

Stand, Strobotac and Tank

III-5 Close-up Showing Impeller and Probe Tip

III-6 Details of Three Dimensional Pitot Tube

III-7 Manometer Connections to Three Dimensional

118

Pitot Tubes

III-8 Pressure Measurement of Pressure Taps $P_{4}$ and

$P_{5}$ in High Shear Flows 
FIGURE

NUMBER

PAGE

CHAPTER IV

IV-1 Angle Profile for Run Number 29,

134

Illustrating the Definition of

Velocity Factor

IV-2 Correlation for 3 in. Impeller in

153

12.25 in. Diameter Tank

IV-3

Theoretical Streamlines for a 3.0 in.

164

Impeller at 500 RPM Values of Parameters

are $=11.192, A=28.24, a=0.1147, h=$

6.0. Depth of Water 12.0 in, and Tank

Diameter $=11.5$ in. Each Pair of Streamlines

Enclose $5 \mathrm{ft}^{3} / \mathrm{min}$ of Water. This Theoretical

Profile Illustrates a Boundary Layer at the

Tank Bottom? having a width of $10 \%$ of the Fluid

Depth.

IV-4 Resolution of Velocity into its Components

167

When the Three Dimensional Probe is in a

Horizontal Position

IV-5 Manufacturers Calibration Chart for

168

Three Dimensional Pitot Tube

Experimental Velocity Profiles of $\overrightarrow{v_{r}}$
for Ports 1,2 , and 3

171

172

Ports 1, 2, and 3

IV-8 Experimental Velocity Profile of $\vec{v}_{z}$

173

IV-9 Comparison Between Experimental and

Theoretical Velocity Profiles for $\overline{\mathbf{v}}_{\boldsymbol{r}}$ for Ports 1, 2, and 3 
FIGURE

NUMBER

PAGE

IV-10 Comparison Between Experimental and Theoretical Velocity Profiles for Ports 1, 2, and 3

IV-11 Velocity Profile $\vec{q}$ for Run No. 29 190

IV-12 Resolution of Velocity $\vec{\nabla}$ into its 194

Components, when Probe is Placed in the Region of the Impeller

IV-13 Correlation for Jet Width Parameter $\sigma$

209

IV-14 Correlation for Radius of Source a With $\mathrm{D} / \mathrm{T}$

IV-15 Correlation of Radius of Source a With $(T-D) / T$

IV-16 Correlation for Dimensionless Pumping Capacity $\mathrm{N}_{\mathrm{Q}}$

IV-17 Velocity Profile $\bar{v}_{\mathrm{r}}$ for Run Number 29

IV-18 Correlation for Impeller Pumping

219

Capacity $Q$

IV-19 Correlation for Volumetric Flow

221

Parameter A

IV-20 Correlation of $A$ versus $N D^{3} /\left((D / 2)^{2}-a^{2}\right)^{\frac{1}{4}}$

222

IV-21 Comparison between Calculated and Experimentally Determined Values of $Q$ the Impeller Discharge

IV-22 Comparison Between Calculated and Experimentally Determined Values of Eddy Viscosity

IV-23 Effect of Blade Width on the Impeller Discharge $Q$ for a 4.0 in. Impeller 
APPENDIX A

A-1 The Tangential Jet

APPENDIX :C

C-1. Pattern Search: in Two Dimensipipn 300

C-2 Flow Diagram for Pattern Search Subroutine 303

APPENDIX E

E-1 Flow Diagram for Main Program PLOTER 312

E-2 Flow Diagram for Subroutine STREAM 316

$\begin{array}{lll}\text { E-3 Flow Diagram for Subroutine GOLD } & 318\end{array}$

E-4 Illustration of Golden Section Search 319 Procedure

APPENDIX F

F-1 Flow Diagram for Program FLOWANL 349

F-2 Flow Diagram for Program YAW $\quad 350$

F-3 Flow Diagram for Subroutine AVG 351

F-4 Flow Diagram for Program VELPRO 353.

F-5 Flow Diagram for Program TANKANL and YAWANL 354 


\section{LIST OF COMPUTER LISTINGS}

\section{LISTING:}

NUMBER

E-1 FORTRAN List of Program PLOTER, With

Supporting Subroutines STREAM, GOLD and SPRINT, and Sample Output

F-1 FORTRAN 1isting Program FLOWANL with Supporting Subroutines YAW, AVG, PROC, BOUNDS, OMEGA and PATERN. This listing also Gives Raw Data Which is the Input to the Program and a Detailed : Sample Output for Run Number 29

F-2 FORTRAN 1isting for program VELPRO, Supporting Substances are POLY and CURVEF. Also Given are the Necessary Input Data And Sample Output

F-3 FORTRAN Listing for Program TANKANL with Supporting Subroutines OMEGA. Also Gien are the Raw Data and a Sample Output for Port 1

F-4 FORTRAN Listing for Program YAWANL With Supporting Subroutine OMEGA and I1lustrated With Sample Output for Experimental Data from Run Number 29

F-5 FORTRAN Listing for Program COOPER with 407 Supporting Subroutines PATERN, PROC, BOUNDS, AVG and OMEGA. Also Given is Cooper's Velocity Profile Data

F-6 FORTRAN Listing for Program NIELCUT With Supporting Subroutines, PATERN, BOUNDS and OMEGA. Also Given are Velocity Profile Data of Nielson and Cutter

F-7 FORTRAN Listing For GRAPH with Supporting Subroutine SOLVE and Sample Input. Typical Output from Program is also given

F-8 FORTRAN Listing for Main Program RESULT With Supporting Subroutine VARI and Sample Output 


\section{ABSTRACT}

Velocity measurements were made.in a stirred tank with a turbine impeller. Two sizes of vertical cylinderical tanks were used. (11.5 and 12.25 inches in diameter) and a simgle 3.0 inch diameter six flat-blade turbine impeller. The velocity measurements were made with a three dimensional pitot tube probe. A tangential jet.. model was found to adequately describe the flow in the region of the impeller. The velocity profile in the region of the impeller exhibits an angle profile which is not predicted by the tangential jet mode1. The model is, however, relatively insensitive to the angle profile and a weighted average angle was found to give satisfactory resuits.

The significant velocity in the neighborhood of the impeller centerline was shown to be $\vec{q}$ the resultant of $\bar{v}_{r}$ and $\bar{v}_{\theta}$. It was also shown that the three dimensional pitot tube is not sensitive enough to measure velocities below $0.5 \cdot \mathrm{ft} / \mathrm{sec}$, the lower limit of probe response. $\vec{q}$ was found to 'fall räpidly: bejöond $\frac{1}{2} \bar{q}_{\text {max }}$ ', and this point was close to the limit of the probe response. However the tangential jet model shows that the half width of the jet is more than twice the limit of probe response.

The tangential jet model. was successfully used to predict velocity profiles of the impeller streams reported in the 1iterature. 
The results of the combined data includes measurements made in air and. water and spans varying. impeller diameter, tank diameter and impeller speeds. For geometrically similar impellers it was found that the jet width was independent of the physical properties of the fluid, impeller diameter and tank.diameter.

The tangential jet model is a three parameter model and correlations are presented for these parameters. The impeller discharge. $Q$ was found to be $a$ function of $\mathrm{ND}^{3}$ and the dimensionless pumping capacity. $Q / \mathrm{ND}^{3}$ was found to be a constant with a value of $0.73 \pm 0.28$ for the combined data in the literature and in this study.

In the region away from the jet flow, from the impeller it was shown that a three dimensional, low velocity, flow field exists. At 500 RPM for the 3.0 inch diameter impeller the resultant velocity was found to vary from 30 to $70 \mathrm{ft} / \mathrm{min}$. The center of circulation is not a true stagnation point but appears to be so for streamlines plotted in the $r-z$ plane. The flow at this point is predominantly tangentia1.

Two-dimensional potential flow was used to model this flow away from the..impeller region, and the tank was divided into five regions. The intersection of these regions were adjusted to have smooth streamlines that clopsed and thus satisfied continuity. Due to the three-dimensional nature of the actual flow it was found that this.model was not satisfacory to predict the velocities in these regions. A Calcomp plotter was used to draw streamlines from the mode1. It was demonstrated that this is a useful tool for 
drawing flow patterns.

. It was observed that the branching flow form the impeller was found to be restricted to the periphery of the tank.in a narrow. region about a baffle width thick. The $10 \%$ baffle is thus not only correctly placed but its width effectively controls the flow in the tank. 
CHAPTER I

INTRODUCTION AND BACKGROUND

\section{Introduction}

The stirred tank is extensively used in fluid processing plants because of its simple mechanical construction and reliability. In addition it performs a variety of functions in liquids whose viscosity ranges from water to corn syrup or molasses. This range of viscosity is roughly $1: 1,000,000$ centipoises.

In Table 1 the use of a stirred tank, is divided into five broad classifications. These classifications may be further subdivided. For example, solid suspension might be easy or difficult depending on whether the solid material is a finely divided precipitate or a heavy mineral of varying particle size. Similarly, emulsification might be stable (permanent) or unstable as in the case of liquid-liquid extraction.

In the stirred tank these functions are performed with an impeller. The rotating impeller in typical industrial applications causes turbulent flow. For a particlar fluid and impeller the degree of turbulence and flow is in general a function of impeller diameter, tank diameter, and impeller speed. A wide variety of impellers are available. All these impellers can be 
Table I-1: Classification of Stirred Tanks According to Functions Performed in the Tank.'(23)

Physical Criteria

Suspension

Dispersion

Emuls ification

Blending

Pumping
Application Class

Liquid-Solid

Liquid-Gas

Immiscible Liquids

Miscible Liquids

Fluid Motion
Mass Transfer Criteria

Dissolving

Absorption

Extraction

Reaction

Heat Transfer 
classified into three types depending on the three types of flow patterns observed in stirred tanks. (27) These patterns are observed in fully baffled tanks and are,

1. Axial flow; typical impeller that causes this type of flow is the three-blade marine propeller.

2. Radial flow; typical impeller is the flat blade turbine. 3. Mixed flow, which can be obtained by a pitch blade turbine. Presently the design of stirred tanks, to meet the process conditions of Table I-1 is still an art in spite of the voluminous body of literature on the subject. This is because the mixing flow pattern required for a variety of processes are not only different, but mixing in the stirred tank is poorly understood. For example, suspension of solids and blending of miscible liquids required relatively larger bulk fluid motion rather than regions of intense turbulence. On the other hand, dispersion of gases in 1iquids and emulsification requires regions of intense turbulence and shear and relative less bulk fluid motion in the tank. In general each process situation demands its own optimum flow pattern. Thus what is good mixing in a stirred tank reduces to arriving at the right design so as to obtain the desired flow patterns.

The present approach used in the design of stirred tanks is through scale-up, using the principles of dynamic similarity. This requires previous knowledge either from an existing plant, or through experimental data obtained from careful laboratory measurements. It is thus desirable to make a thorough study of the flow patterns in a stirred tank to permit design of such units without the need of specific experimental data; which data in many cases is 
usually expensive and difficult to obtain.

The purpose of this research work is to characterize the flow regions in a stirred tank and to arrive at a model which will describe the flow patterns generated in the vessel. As previously mentioned there are three basic types of flow patterns, and it was decided to study the radial flow pattern as generated by a turbine impeller. The turbine was selected as the impeller since it is more often used in industrial applications. Also an extension of this work to the other two types of flow patterns should not be overly difficult. The turbine used in this work was the Mixing Equipment Company's, 6-flat blade, turbine impeller since this unit is most frequently used in process applications, and considerable data is available in the literature for comparison.

2. Statement of the Problem

In unbaffled tanks, a turbine-impeller (or any other symmetrically located impeller) produces mere swirl in liquids of relatively low viscosity such as water. The introduction of baffles produces the characteristic radial flow pattern. The fluid emerges as a radial jet with a tangential component which is significant near the impeller, but decreasing as it approaches the wa11. The jet as it emerges from the impeller has a high velocity and hence entrains a considerable amount of fluid which tends to diminish its velocity as it approaches the wall. At the wall the jet divides into two streams one above the impeller and one below. These streams loop and return to the impeller. The loops form acenter of circulation one above and one below the impeller as is seen in Figure I-1. 


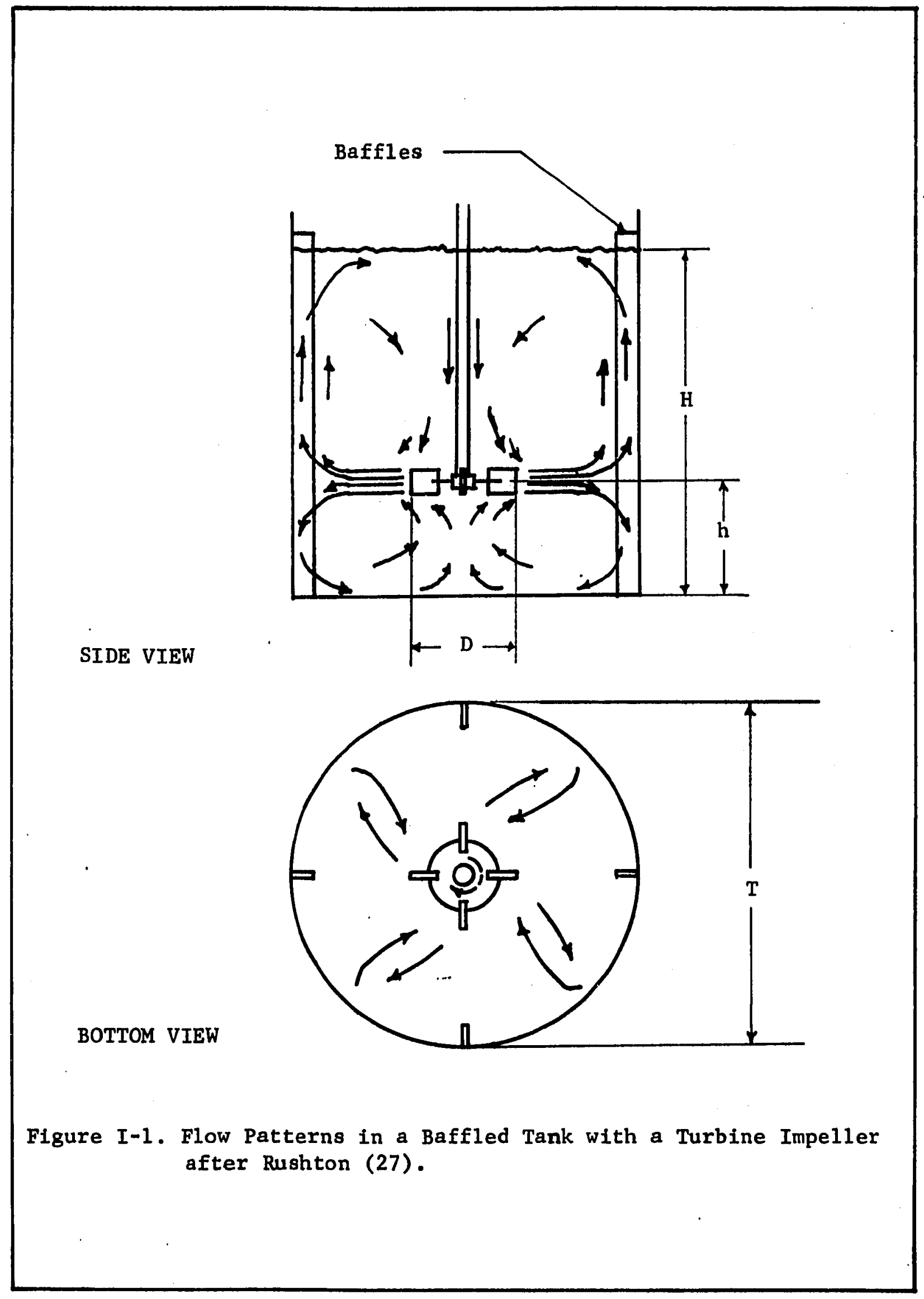


The flow in a stirred tank is not a simple phenomena, and it is decided to restrict this work to a Newtonian fluid. Specifically distilled water was selected as the fluid, since it is easily handled and has a low viscosity typical of process fluids commonly used in stirred tanks. Furthermore, since stirred tanks are most frequently used in fully turbulent flow, this work will be restricted to a tank Reynolds number $\mathrm{N}_{\mathrm{Re}}=\mathrm{oND}^{2} / \mu$ greater than 10,000 .

After surveying the literature, a summary of which will be presented in the next section, it was apparent that the flow in stirred tank was far from simple. There are approximately two regions of flow in the tank. One is the flow near the impeller, and this has received considerable attention. The other region is the rest of the tank. After considerable thought it was decided to divide the tank into several additional regions and model each separately.

The objectives of this research can now be clearly defined as:

1. To study the flow patterns in a tank stirred with a standard turbine impeller and to arrive at a suitable mathematical model to describe the flow in the tank.

2. To experimentally measure the actual velocities in the vessel with a three-dimensional pitot tube.

3. To obtain streamlines of the flow in the tank so as to have a visual picture of the flow, and to compare the experimental and theoretical velocity profiles in various regions of the tank for a 3.0 inch diameter impeller.

4. To correlate the parameters in the flow model so that quantities such as pumping capacity and eddy viscosity 
in the region of the impeller can be predicted.

5. Compare the experimental and theoretical results of this work with that reported in the literature.

\section{Literature Review}

This section will be concerned with a review of the literature pertinent to flow patterns in a stirred tank. The stirred tank as a mixing device has been described in a considerable body of literature because of its extensive application, and the ingenuity of various investigators who have tried to describe and analyse the phenomena. Frequently the term mixing and stirred tank are synonymous as demonstrated by large sections devoted to the stirred tank in three books $(12,33,34)$ published on mixing. There have been three dissertations and numerous articles on flow patterns. It is our opinion that the last word has not been said on the fluid dynamics of the stirred tank.

The description of the dynamics of the stirred tank has been approached by two methods.

1. The direct approach is used in which flow patterns and pumping capacities of impellers have been measured. Typical papers describing this approach are by Nagata $(18,19,20)$ and Cooper (8).

2. Stirred Tanks are frequently used as perfect mixers or devices that will smooth out instantaneously any inhomogenuity in fluid property present in the tank. Actual stirred tanks seldom conform to this ideal but behave as perfect mixers in the region near the impeller 
where high shear and turbulence exist and have very little mixing taking place in the rest of the tank. To obtain an idea of the extent of perfect mixing two methods of approach are used.

(i) Terminal blending time, or the time required to smooth out an impulse change in fluid property, for example, the concentration of a chemical species. An excellent review of work in this area is presented by Grey (40).

(ii) In the alternate approach, the tank is divided into four arbitrary elements or regions. These are plug flow, perfectly mixed, short circuit and dead water. The various fraction of the fluid residing in these regions is determined by measuring the residence time distribution. The residence time distribution is the response obtained from injection of pulse of a tracer material. A summary of the various 1 inear models that can be built through combinations of the above four elements is given by Camps (7) and Uh1 (34). The fact that only linear combination of elements is used restricts this method to linear systems. For example, in the study of chemical reactions in stirred tanks only first order reactions can be considered by this method. For second and higher order reactions additional information such as the flow pattern is needed.

Terminal blending time and residence time distributions, a1though useful in specific instances, give no insight into the 
fundamental phenomena which is responsible for the observed behavior of the system. An accurate model of the fluid dynamics could be used to obtain these functions, if desired through theoretical analysis and without the need for additional experimental data.

In presenting a review of the literature it was found convenient to divide the material under various subheadings such as Power Consumption, Flow Patterns, etc. This approach was considered better than reviewing each individual work in its entirety, as it prevented undue repetition and put each topic in proper perspective. The reader will therefore find references to the same worker in one or more subheadings.

3.1. The Turbine Impe1ler

In the study of turbine impellers a variety of designs have been proposed and tested. Not all of these designs are of industrial importance. However the literature abounds with studies on different kinds of turbine impellers. In order to form a uniform basis for comparison and reference, five different basic designs of turbine impellers are shown in Figure I-2.

Type A is the flat blade, disk type turbine. Type B is the full flat blade turbine, and Type $\mathrm{C}$ is the curve blade turbine. Types $B$ and $C$ can have an additional parameter by raising the blades a distance $h_{1}$, above the plane of the impeller. This is shown by dotted 1 ines for Type $B$ in Figure I-2. Type $D$ is the hammer headed turbine and Type $\mathrm{E}$ is the full blade turbine with a disk. Type A with six blades is the most frequently used turbine and is considered a standard design. 


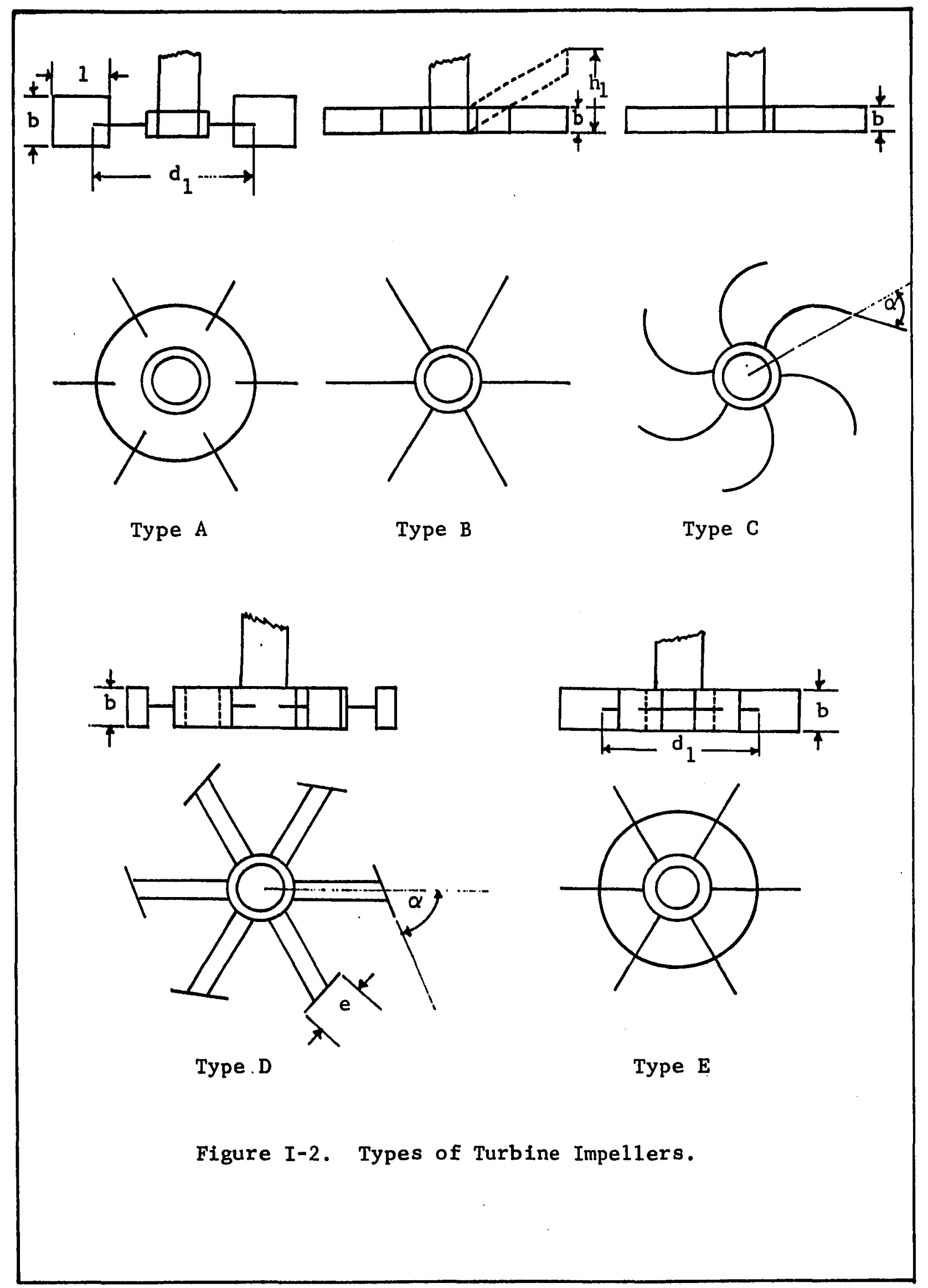


The parameters that can be varied are the breadth of the blade b, blade length 1 , disk diameter $d_{1}$, height of blade above impeller plane $h_{1}$, and in case of types $C$ and $D$ the angle $\alpha$. In addition, the number of blades on the impeller can also be varied.

\subsection{Power Consumption in Stirred Tanks}

Power consumption was the first variable to be studied and reliable correlations of power input has been know for more than a decade. The most recent work is that of Bates and associates (3)

The correlation of power consumption using dimensional analysis is we11 known (26). For geometrically similar systems in baffled tanks (i.e. absence of swirl), dimensional analysis gives the following relationship,

$$
\mathrm{N}_{\mathrm{p}}=k\left(\mathrm{~N}_{\mathrm{Re}}\right)^{\mathrm{a}}
$$

where $N_{P}=\frac{\mathrm{Pg}_{c}}{\rho N^{3} D^{5}}$, is the Power Number and $N_{R e}=\frac{\rho N^{2}}{\mu}$, is the Reynolds Number. As pointed out by Bates, when comparing or using power correlations of the form of equation I-1 care should be taken to ascertain if the systems considered are geometrically similar. This is frequently not the case, and is the reason why data reported in the literature often cannot be compared.

For the Type A turbine (see Figure 1-2) used in this work, the log-log plot of $\mathrm{N}_{\mathrm{p}}$ versus $\mathrm{N}_{\mathrm{Re}}$ as reported by Bates is not in agreement with the earlier and well known work of Rushton (26). The curves have the same general shape, and they reach a constant value of Power Number for Reynolds Number greater than 10,000. This limiting value of $\mathrm{N}_{\mathrm{p}}$ is reported by Rushton to be 6.3 while that 
reported by Bates is 5.0. The higher value of Rushton is said to be due to frictional errors. Lower values of $\mathrm{N}_{\mathrm{p}}$ have been reported by other workers (35). Cutter (9) reported a 1 imiting value of $\mathrm{N}_{\mathrm{p}}$ to be 5.95. For a comprehensive review of power consumption the reader is referred to Grey (34). Bates (3) also claims that $\mathrm{N}_{\mathrm{p}}$ is affected somewhat by number of baffles, baffle width and height of impeller above the bottom of the tank. These should therefore have some effect on the flow patterns and thus the power consumption. 3.3. Flow Patterns with Turbine Impellers

The word flow pattern by itself can and has been misconstrued. It can refer to:

1. Streamlines: These streamlines may be qualitative studies as in the work of Metzner (16) and Schumm (32), or the actual drawing of streamlines in a particular region as done by Nagata (19).

2. Velocity profiles: The velocity profile is the distribution of mean velocity across any plane. These planes are typically a cylinder surrounding the impeller or horizontal planes perpendicular to the axis of the impe11er.

In addition flow patterns involve other factors or aspects. These are:

1. Whether the tank is baffled or unbaffled.

2. Kind of measuring device used. As there are a variety of devices employed, these will be reviewed separately in Section $3-5$.

3. Frequently a theoretical analysis for the flow in a region 
or the entire tank is attempted, and these will also be reviewed.

Comparison of Turbine Impellers by Nagata and Associates $(18,19)$

Any reference to flow patterns in stirred tanks is not complete without reviewing the extensive work of Nagata and associates. Their work is presented in three papers, and the object is to compare the performance of different types of impellers in unbaffled and baffled tanks. In order to make this comparison possible, the impeller diameter to tank diameter ( $D / T$ ratio) and the Reynolds number was kept approximately constant. In all but a few cases $\mathrm{D} / \mathrm{T}=0.513$

On the basis of kinds of impellers employed, in their first paper thirty impellers were investigated in unbaffled tanks. The impellers included types A, B, C and D of Figure I-2, All the impellers had eight or more blades. On the basis of the number of blades, there were eight different kinds of impellers. The thirty impellers were obtained by varying $b, d_{1}$ and 1 , the impeller parameter parameters shown in Figure I-2. $ّ$ th the second paper (19) the number of impellers was reduced to six. These six impellers were run in baffled tanks. In the third and last paper (20) the number of impellers were reduced to three, types $A, B$ and $C$. All impellers had eight blades. The purpose of this study was to investigate the laminar and transitional regions in unbaffled tanks. Five runs were made using each impeller at approximately the same $\mathrm{N}_{\mathrm{Re}}$. The range of $\mathrm{N}_{\mathrm{Re}}$ studied was approximately 6.7 to $10^{5}$. 
In all the work done by Nagata the impeller was centeraily located. By centrally located it is meant that the impeller is placed at half the depth of the fluid in the tank. At high $\mathrm{N}_{\mathrm{Re}}$ a specially designed pitot tube was used to measure velocities in the tank. At low $\mathrm{N}_{\mathrm{Re}}$, velocity measurements were made using the photographic method of Sachs (28)

Nagata presents his results as velocity profiles across a number of planes in the tank for one impeller, a sixteen blade type B impeller. It is implied that profiles for other impellers investigated are similar in form. For the purpose of quantative comparison Nagata defines a coefficient of discharge $\mathrm{N}_{\mathrm{Q}_{1}}=\mathrm{Q}_{1} / \mathrm{ND}^{3}$ and a coefficient of circulation $N_{Q_{2}}=\frac{Q_{2}}{N_{3}}$. The quantity $Q_{1}$ is defined as the volumetric flow discharged by the impeller. To evaluate $Q_{1}$ consider Figure $I-3$, in which is shown a schematic velocity distribution in the upper quadrant of the tank. The dotted 1 ines $B^{\prime} B^{\prime \prime}$ and $C^{\prime} C^{\prime \prime}$ are the streamlines that pass through the corners of the impeller, and thus enclosed the fluid discharged through the impeller. Nagata defines $Q_{1}$ to be this quantity fluid that is enclosed by the streamline $B^{\prime} B^{\prime \prime}$ and $C^{\prime} C{ }^{\prime}$. In the tank the re will be some flow in the vicinity of the impeller blades. Hence the quantity $Q_{1}$, as defined by Nagata, will be lower than the value obtained if the discharge from the impeller is considered to be the flow across a cylinderical plane of diameter $D$, the impeller diameter. The latter definition of $Q_{1}$ is more often used.

The flow rate, $Q_{2}$, is obtained by integrating $\bar{v}_{z}$ in the plane that passes through the center of circulation and is from the 


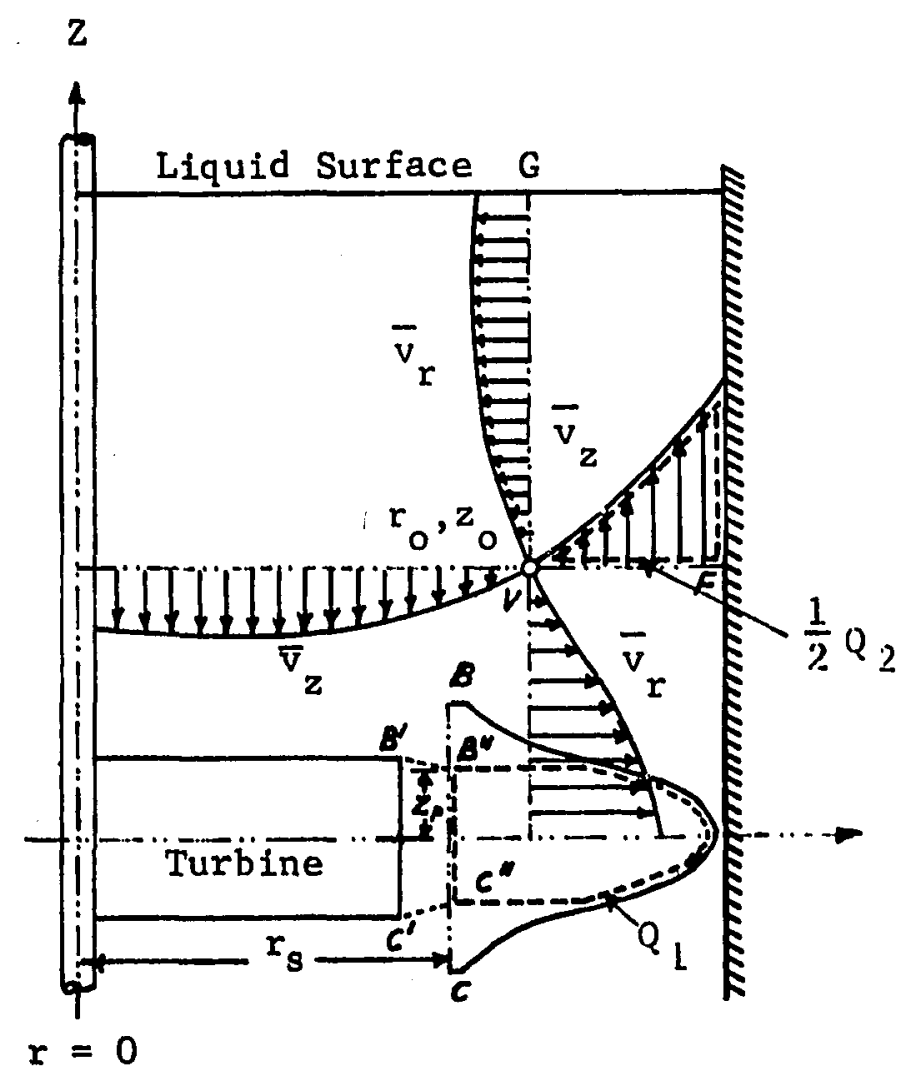

Figure I-3. Schematic Representation of Velocity Profiles in Baffled Tanks, after Nagata (19). 
point $V$ to $F$ in Figure $I-3$. Thus,

$$
\begin{aligned}
& Q_{1}=4 \pi r \int_{0}^{z} p v_{\dot{r}} d z \\
& Q_{2}=4 \pi \int_{r_{0}}^{T / 2} v_{z} d x
\end{aligned}
$$

In equation $I-2, z_{p}$ is the height at which the streamline B ' $C$ ' intersects the cylinderical plane $B C$ of radius $r_{s}$. In equation I-2 and I-3 it is assumed that the velocity profiles $\bar{v}_{z}$ and $\bar{v}_{r}$ are independent of $\theta$, i.e. axial symmetry exists.

In unbaffled tanks Nagata (18) observed that the flow.can be considered as two zones. An inner zone of radius $r_{c}$ in which the tangential velocity $\bar{v}_{\theta}$ is approximately proportional to the radius $r$, and an outer zone in which the flow is quasi-potential flow. The radius $r_{c}$ is about $80 \%$ of the impeller radius. There is a small circulatory flow which is caused by a small radial component, emerging from the impeller. Nagata called this a secondary circulation since the bulk of the flow is due to the tangential velocity $\overline{\mathbf{v}}_{\dot{\theta}}$

The secondary circulation is caused by the centrifugal forces due to the rotating impeller. At low $_{\mathrm{Re}}$ the radial velocity profile at the impeller has a broad width. This width narrows considerably as $\mathrm{N}_{\mathrm{Re}}$ increases. Nagata observed that the secondary circulation reaches a maximum in the transistion flow region being small in laminar or fully turbulent flow 
In baffled tanks, the introduction of baffle plates was found to increase the width of the discharge from the impeller and also the velocity. The secondary circulation becomes the main flow and is well defined. The value of $\mathrm{N}_{\mathrm{Q}_{1}}$ was found to range from 0.23 to 0.59 for most impellers in unbaffled tanks. In baffled tanks $\mathrm{N}_{\mathrm{Q}_{1}}$ ranged from 0.78 to 1.34 indicating an increase in the discharge flow from the impeller by a factor of 2-4 on introducing baffles into the tank.

In baffled tanks Nagata (19) observed that the flow pattern repeats itself on an average, changing continuously with time in a complicated manner. These conclusions were based on observing the flow in five radial planes in a tank with four baffles as shown in Figure I-4(a). Progressing from Sections I to IV of Figure I-4(a), it was seen that the upper and lower centers of circulation in Section I were located very close to the impeller as shown in Figure I-4(d). In section II the center of circulation moved further apart as shown in Figure I-4(e). In sections III and IV the center of circulation becomes unstable and sometimes breaks into two eddies, as shown in Figure $I-4(f)$. It was also observed that the baffles give rise to the eddy A shown in Figure I-4(b). The eddy $A$ was found to break down into two eddies $B$ and $C$, the eddy $\mathrm{C}$ being weak is easily dissipated. The eddy $\mathrm{B}$ is much stronger and is reinforced by similar eddies from other baffles and appears as a hollow vortex on the surface of the tank that rotates slowly in the same direction as the impeller. This surface vortex often sucks air into the system. Frequently it was found that all the eddies A, B and C completely disappear. 


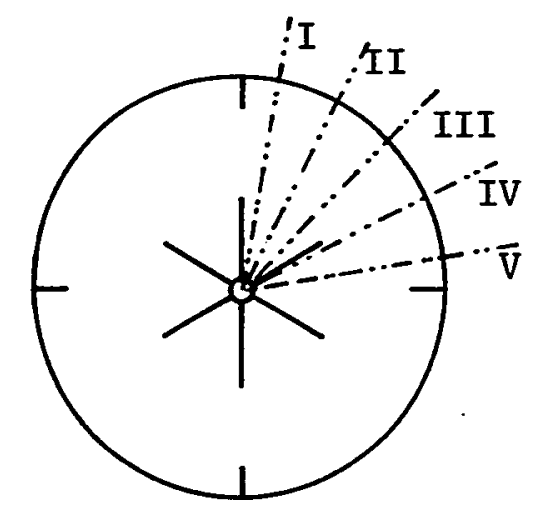

(a)

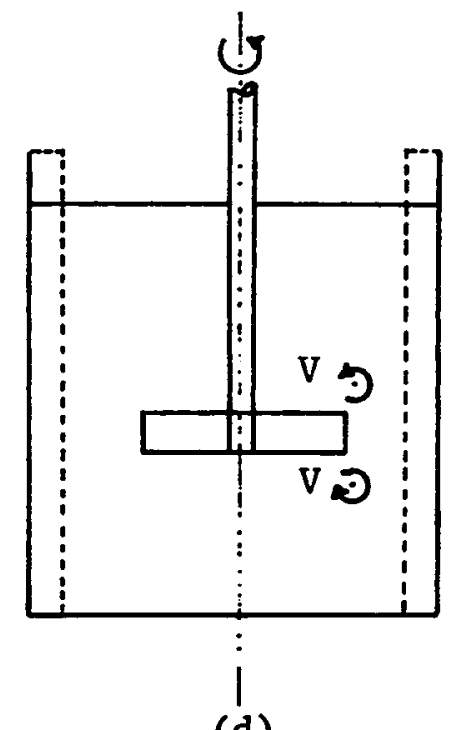

(d)

Section I

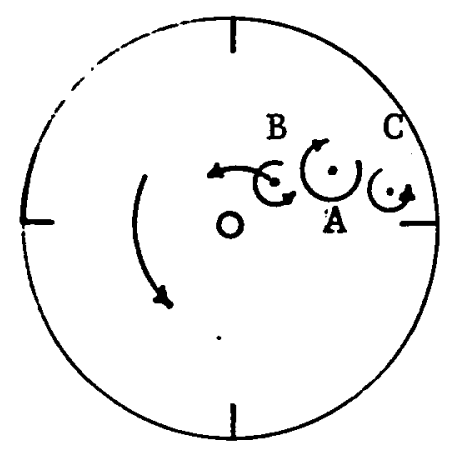

(b)

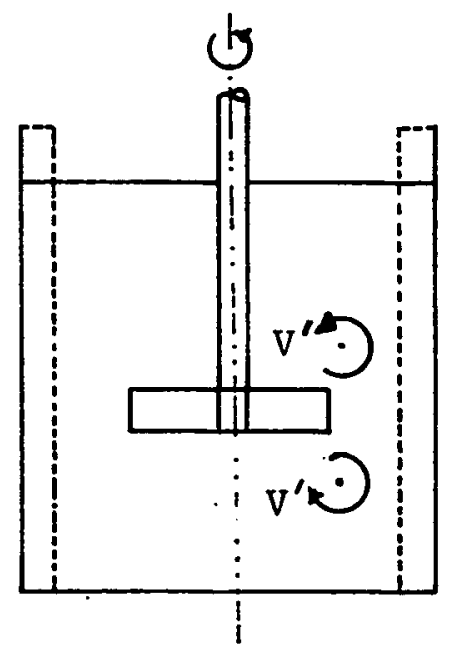

(e)

Section II

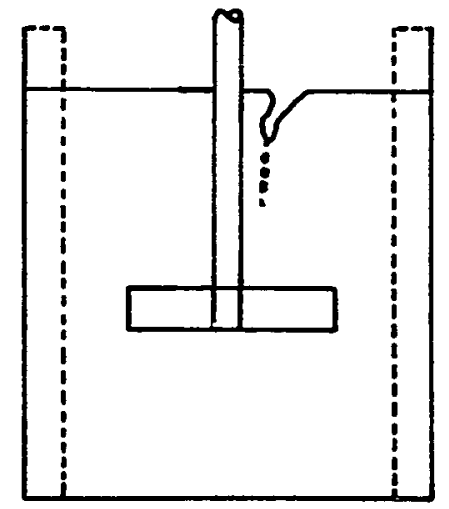

(c)

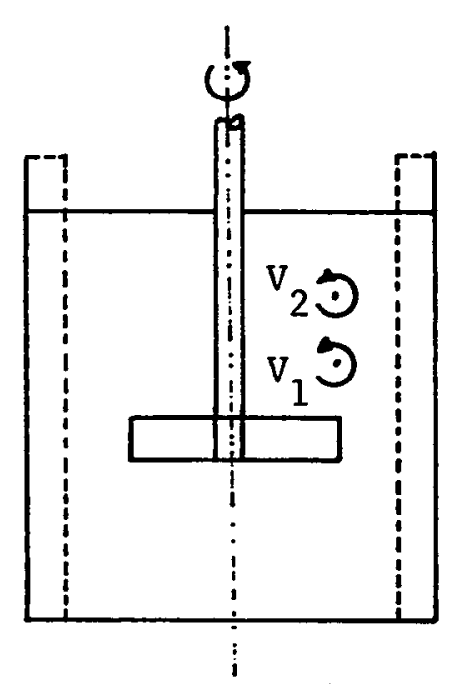

(f)

Section III-IV

Figure I-4. Schematic Diagram of Vortex Formation in Baffled Tanks from Nagata (19). 
In Figure I-5 the streamlines are shown in a radial plane which is typical of streamlines for all impellers in baffled tank. The center of circulation is continually fluctuating as noted in the preceding paragraph. For details of flow profiles in various planes the reader is referred to original papers or an excellent summary of them presented by Grey (38).

In calculating $Q_{1}$, the volumetric discharge from the impeller, Nagata found that there was a small variation in $Q_{1}$ depending on which radial plane the velocity profile was measured. This indicated that the velocity profile is not perfectly axially symmetrical. However, as the differences were not very large, $Q_{1}$ was reported as the average of measurements made in four different radial planes. Since $Q_{2}$ is the circulating flow hence $Q_{2} / Q_{1}$ will be the fractional entrainment. The value of $Q_{2} / Q_{1}$ ranges between 1.7 to 1.85 with an average value of 1.8 . As pointed out earliel $Q_{1}$ has been defined differently by other authors such as Sachs (28), and the figure of 1.8 as the amount of entrainment cannot be directly compared.

The conclusions arrived at by Nagata for baffled vessels were that even though baffles increased the circulation the power efficiency was lowered. This meant that considerably more power was required to get the increased circulation. He also concluded that impellers having excellant performance in unbaffled conditions also perform better under baffled conditions. Measurement of Velocity Profiles Near the Impeller by Sachs (28) The first quantative measurements of velocities in the region 


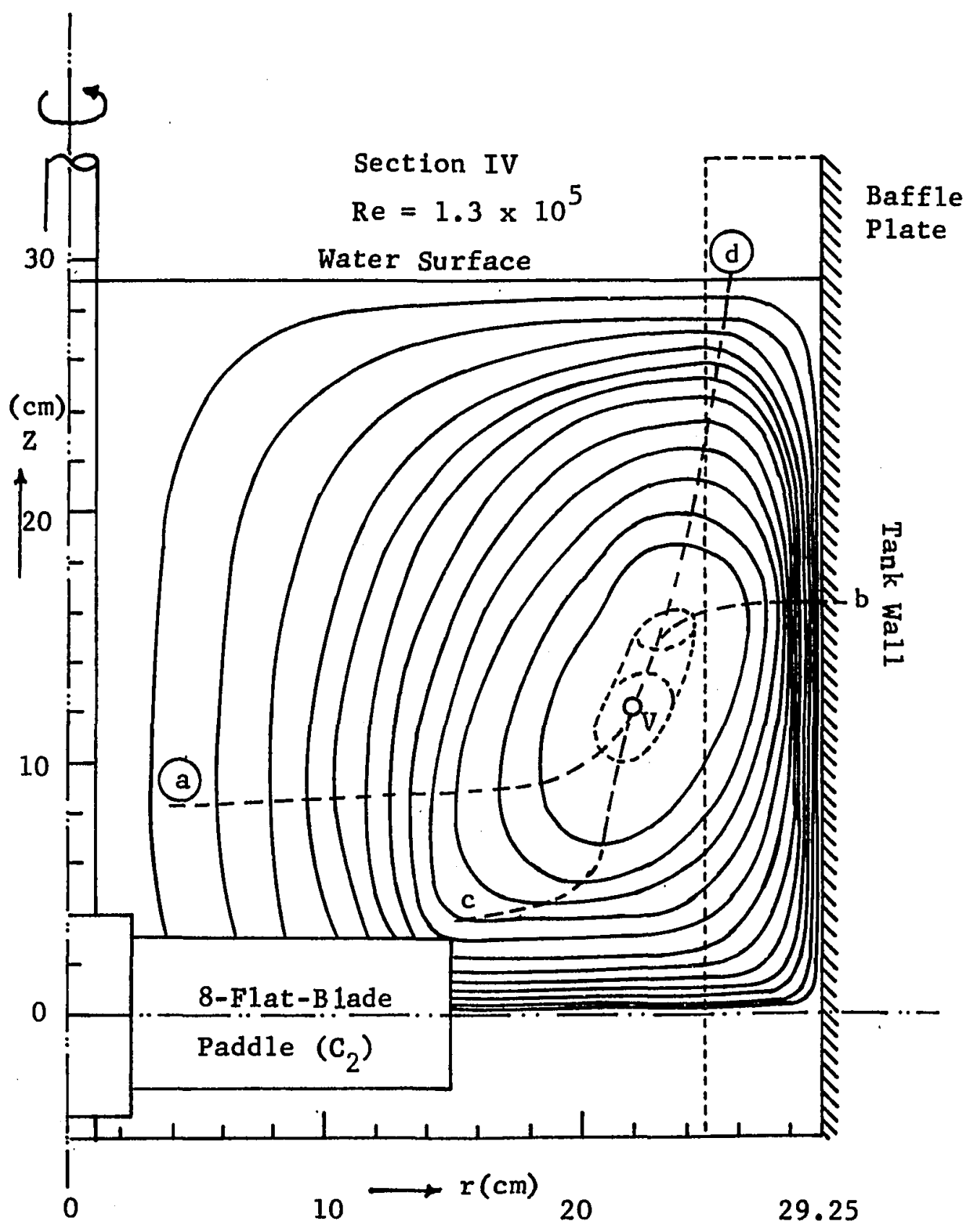

Figure I-5: Typical Streamlines in a Baffled Tank, From Nagata (19). 
of the impe11er was made by Sachs and Rushton (28). A 4.0 in. four blade type A impeller was used. Velocities were measured by the photographic method. Measurements were made at three impeller speeds and five radial positions. The velocity profiles were found to be bell-shaped and tended to flatten as the radial distance from the impeller increased. The ratio of the average velocity to maximum velocity $\left(\bar{v} / \bar{v}_{\max }\right)$ was found to be independent of impeller speed but to increase with radial distance. The volumetric flow from the impeller increased with increasing $r$ because of entrainment, and it reaches a maximum at $r=4.0$ inches. The amount of entrainment was reported as 1.96 with practically no variation for the three observations reported. This value of entrainment cannot be compared with Nagata as the discharge flow is defined as all the liquid emerging from the impeller.

Sachs observed that the radial velocity in between impeller blades was not uniform but reached a maximum at about $50^{\circ}$ ahead of the rotating blade. At this point the radial velocity was found to be $38 \%$ higher than the average and was an instantaneous value that should not be confused with average values of $\bar{v}_{r}$ at a point. This effect indicates that axial symmetry is only approximate, and depends upon the position of the impeller blade. This pulsating velocity distribution was found to persist as the radial distance was increased to about two-thirds of the distance to the wa11. After this point the flow appeared uniform or axially symmetrical. 
Sachs reported that the fluid leaves the impeller at an angle of $53^{\circ}$ indicating a considerable tangential component at the periphery of the impeller. This tangential component decreased to $22^{\circ}$ at $r=5.0$ inches indicating that the tangential component decreases to a small but finite value at the wall.

Sachs and Rushton made no attempt to postulate a mode1 for the flow from the impeller. They conclude that the volumetric flow is directly proportional to the impeller speed and that the flow from the impeller entrains a considerable quantity of fluid as it moves to the walls of the tank.

Nielson's Tangential Jet Model (21)

The fluid that emerges from the impeller is a narrow, high speed jet with a considerable tangential component. Nielson proposed a model of this tangential jet which replaced the impeller by a ring source of radius a, which is smaller than the radius of the impeller. The fluid emerges from this ring with a bell shaped velocity distribution, and the velocity vector $\vec{v}$ is tangent to the ring source at all points in the jet. This model not only accounts for an appreciable tangential velocity at the impeller periphery, but also the observation that the tangential component of $\vec{V}$ decreases as the tank wall is approached. A schematic drawing of the tangential jet is shown in Figure I-6.

Nielson time averaged the equations of motion, and simplified them using the boundary layer assumption to obtain the following equation for the tangential jet. 


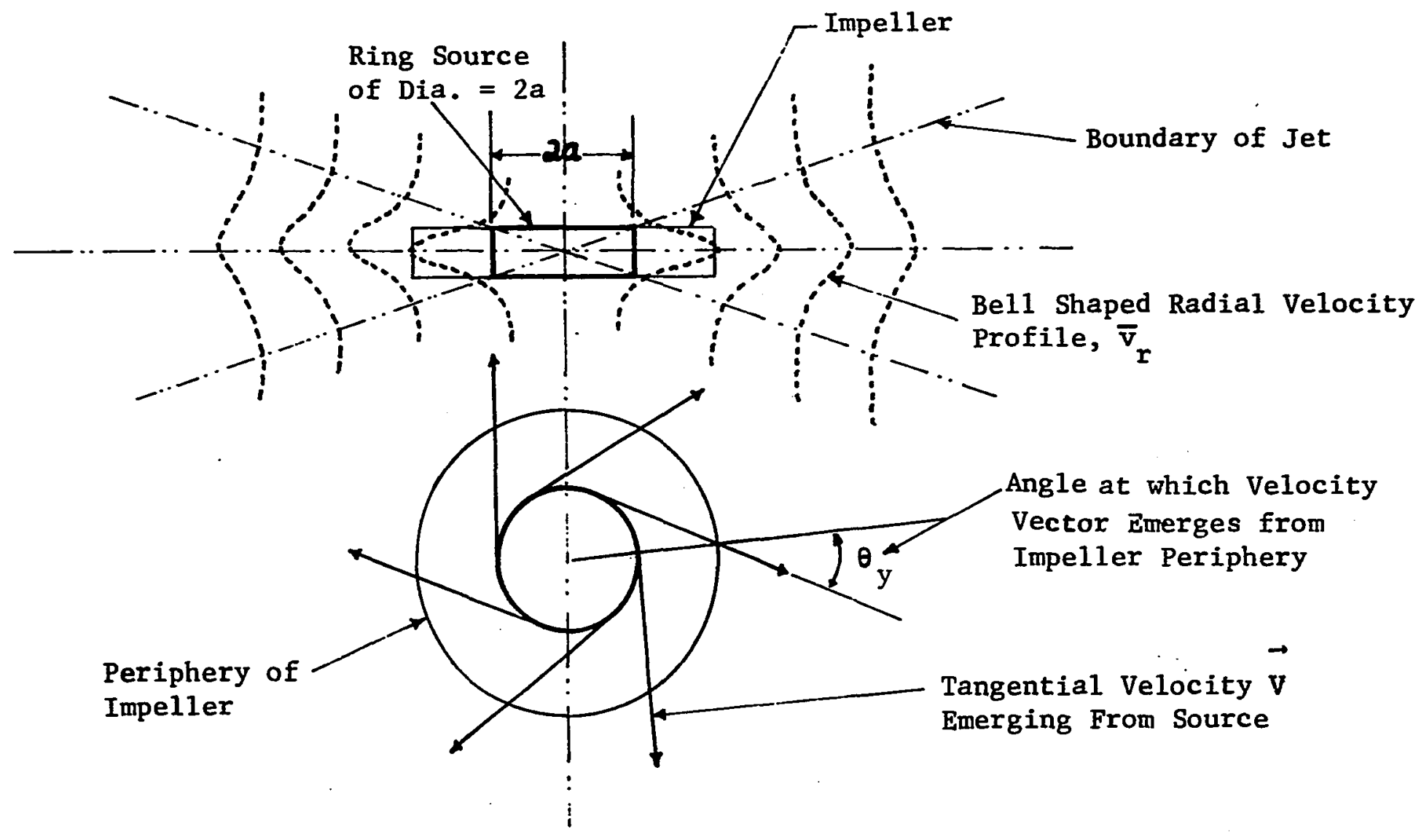

Figure I-6. Tangential Jet. 
$\bar{v}_{r} \frac{\partial \bar{v}_{r}}{\partial r}+\bar{v}_{z} \frac{\partial \bar{v}_{r}}{\partial z}-\frac{a^{2}}{r\left(r^{2}-a^{2}\right)} \bar{v}_{r} 2=-\frac{1}{\rho} \frac{\sqrt{r^{2}-a^{2}} \frac{\partial|\vec{\tau}|}{\partial z}}{r}$

Equation I-4 has been derived in Appendix A, since Nielson's model of the tangential jet is the best available model to describe the flow in the region of the impeller. In Equation $I-4, a$ is the radius of the ring source and $\vec{\tau}$ is the turbulent shear stress due to gradient of the velocity vector, $\vec{\nabla}$, and is equivalent to considering $\vec{V}$ as one-dimensional boundary layer flow.

Nielson used Prandtl's Mixing-length Theory to evaluate $|\vec{\tau}$.$| .$ The velocity profiles were assumed similar which means $\bar{v}_{r} /\left(\bar{v}_{r}\right)_{\max }$ is not a function of $r$. A similarity transformation of equation I-4 results in the ordinary differential equation

$$
\frac{d^{2} F}{d s^{2}}=\frac{d}{2 c^{2}} F \frac{d F}{d s}
$$

where $d$ is a constant and $c$ is the mixing-length constant. Other quantities are given by,

$$
\begin{aligned}
& s=z / b \\
& \bar{v}_{r} /\left(\bar{v}_{r}\right)_{\text {max }}=f(s) \\
& F(s)=\int_{0}^{s} f(s) d s
\end{aligned}
$$

In the above equation $f(s)$ is a dimensionless velocity profile, and states the condition of similarity. The variable $s$ is a dimensionless $z$ coordinate given by equation $I-6$, in which $b$ is the width of the jet. It should be noted that $b$ is a function of $r$ and is shown to be equal to, 


$$
b=\frac{d}{2} \sqrt{r^{2}-a^{2}}
$$

The velocity profile is given by the following equation

$$
\bar{v}_{r}=\frac{A}{r / b}\left(r^{2}-a^{2}\right)^{1 / 4} \frac{d F}{d s}
$$

In equation $\mathrm{I}-10, \mathrm{~A}$ is an arbitrary constant. It is evident that in order to obtain the velocity profile the function $F(s)$ has to be evaluated. This is done by solving the differential equation I-5 with appropriate boundary condition. Nielson obtained an implicit solution in parametric form for the differential equation I-5, and this is

$$
s=\frac{\sqrt{3}}{4 \pi} \quad \frac{\left(1-H+H^{2}\right)}{(1+H)}+\frac{3}{2 \pi} \tan ^{-1} \frac{(2 H-1)}{\sqrt{3}}+\frac{1}{4}
$$

where the parameter $H$ is given by

$$
H=\left(\frac{M^{3 / 2}}{1-M^{3 / 2}}\right)^{1 / 3}
$$

and $M$ is given by the following relationship

$$
\frac{\bar{v}_{r}}{\left(\bar{v}_{r}\right)_{\max }}=\frac{d F}{d s}=\frac{d M}{d t}=\left(1-M^{3 / 2}\right)^{2 / 3}
$$

A numerical method can be used to evaluate $\bar{v}_{r}$ as a function of $s$. This is done as follows; for a series of values of $M, H$ is calculated from I-12 which then enables the calculation of $\mathrm{s}$. A table of $M$ versus $s$ is thus obtained. From $I-13$ this table can be converted to a table of $s$ versus $\frac{d F}{d s}$. Then if the parameters $A$, 
$d$ and a are known, $\bar{v}_{r}$ can be evaluated as a function of $z$ for a fixed value of $r$ from I-9. Nielson solution is a three parameter model the parameters being $A, a$ and $d$.

By a trial and error method, Nielson was able to fit his velocity profile data to the model given by equations I-10 to I- 13 . From Table I-2 it can be observed that three impellers were studied at four different speeds. Since all other conditions in the tank are constant, this constitutes four different flow patterns. Nielson uses these four points to evaluate some of the constants from the experimental plots of different equations. The value of $d$ was found to be 0.782 . The constant $a$, was assumed to be proportional to the impeller diameter $\mathrm{D}$ and obtained as

$$
a^{2}=0.157 D^{2}
$$

The parameter A was not evaluated as such since it was not used directly.

Nielson presented equations for predicting $\left(\bar{v}_{r}\right)_{\max }$, the velocity at the impeller center line, as a function of $r$ and also the volumetric discharge $Q$ as a function of $r$. Both these quantities were found to be dependent on $\mathrm{D} / \mathrm{T}$, the impeller diameter ratio. These prediction equations for $\left(\bar{v}_{r}\right)_{\max }$ and $Q$ are not entirely conclusive since they are based on only four data points.

From Prandt's mixing length theory, and the velocity profiles Nielson obtained the following equation for the shear stress

$$
\tau_{z r}=\left(\frac{3}{4 \pi}\right)^{3} \frac{r \rho Q\left(\bar{v}_{r}\right)_{\max }}{\left(r^{2}-a^{2}\right)^{3 / 2}}\left|\frac{d^{2} F}{d s^{2}}\right| \frac{d^{2} F}{d s^{2}}
$$


Table I-2: Summary of Neilson's Investigation of Turbine Impellers

\begin{tabular}{|c|c|c|c|c|}
\hline $\begin{array}{l}\text { Impeller } \\
\text { Diametric Inches }\end{array}$ & $\begin{array}{c}\text { Radial Distance } \\
r \text { Inches } \\
\end{array}$ & $\begin{array}{l}\text { Fluid } \\
\text { Used }\end{array}$ & $\begin{array}{l}\text { Fluid } \\
\text { Temp. }{ }^{\circ} \mathrm{F}\end{array}$ & $\begin{array}{l}\text { Impeller } \\
\text { Speed RPM }\end{array}$ \\
\hline 2.0 & 1.0 & water & 83 & 600 \\
\hline 2.0 & 3.0 & water & 83 & 600 \\
\hline 2.0 & 4.0 & water & 83 & 600 \\
\hline 4.0 & 2.0 & water & 77 & 200 \\
\hline 4.0 & 2.0 & water & 77 & 200 \\
\hline 4.0 & 2.0 & corn-syrup & -- & 100 \\
\hline 7.0 & 3.5 & water & 83 & 75 \\
\hline
\end{tabular}


Figure I-7 shows Nielson's comparison of theoretical shear stress calculated from equation I-15 and the experimental value obtained from the turbulent components of the velocity $\bar{v}_{r}$ and $\bar{v}_{z}$. The corresponding turbulent fluctuating components will be denoted by $v_{x}^{\prime \prime} v_{z}^{\prime \prime}$ and the experimental shear stress $\left(\tau_{r z}\right)_{\text {exp }}$ is given by

$$
\left(\tau_{r z}\right)_{\exp }=-\rho \overline{v_{r z}^{\prime} v_{z}^{\prime \prime}}
$$

The bar over the $v_{r}^{\prime}$ and $v_{z}^{\prime}$ in equation $I-16$ signifies averaging. In Figure I-7 it is seen that the experimental values do not show any particular trend but might be interpreted as giving a maximum value of $\left(\tau_{r z}\right)$ exp smaller than the calculated $\tau_{r z}$ and at a distance $z$, closer to the impeller center 1 ine. The discrepancies in Figure I-7 may be because Nielson's photographic method of evaluating turbulent components $v_{r}^{\prime \prime}$ and $v_{z}^{\prime}$ is not sufficiently accurate. Velocity Profiles in Baffled and Unbaffled Tanks by Aiba (1) Aiba measured flow profiles using three different impellers in unbaffled and baffled tanks. The measuring device was a radioactive ball and a miniature Geiger-Muller counter. Both laminar and turbulent flow regions were investigated. The impellers used were: paddle (similar to type B with two blades), a turbine (six blades type A) and a three blade marine propeller.

Aiba found that the flow in a stirred tank was symmetrical with no variations existing in radial planes in between baffles. This observation was contrary to that of Nagata (19) and Sachs (28) both of whom report small variations in these velocity profiles to exist. Aiba presented his results as a plot of dimensionless 


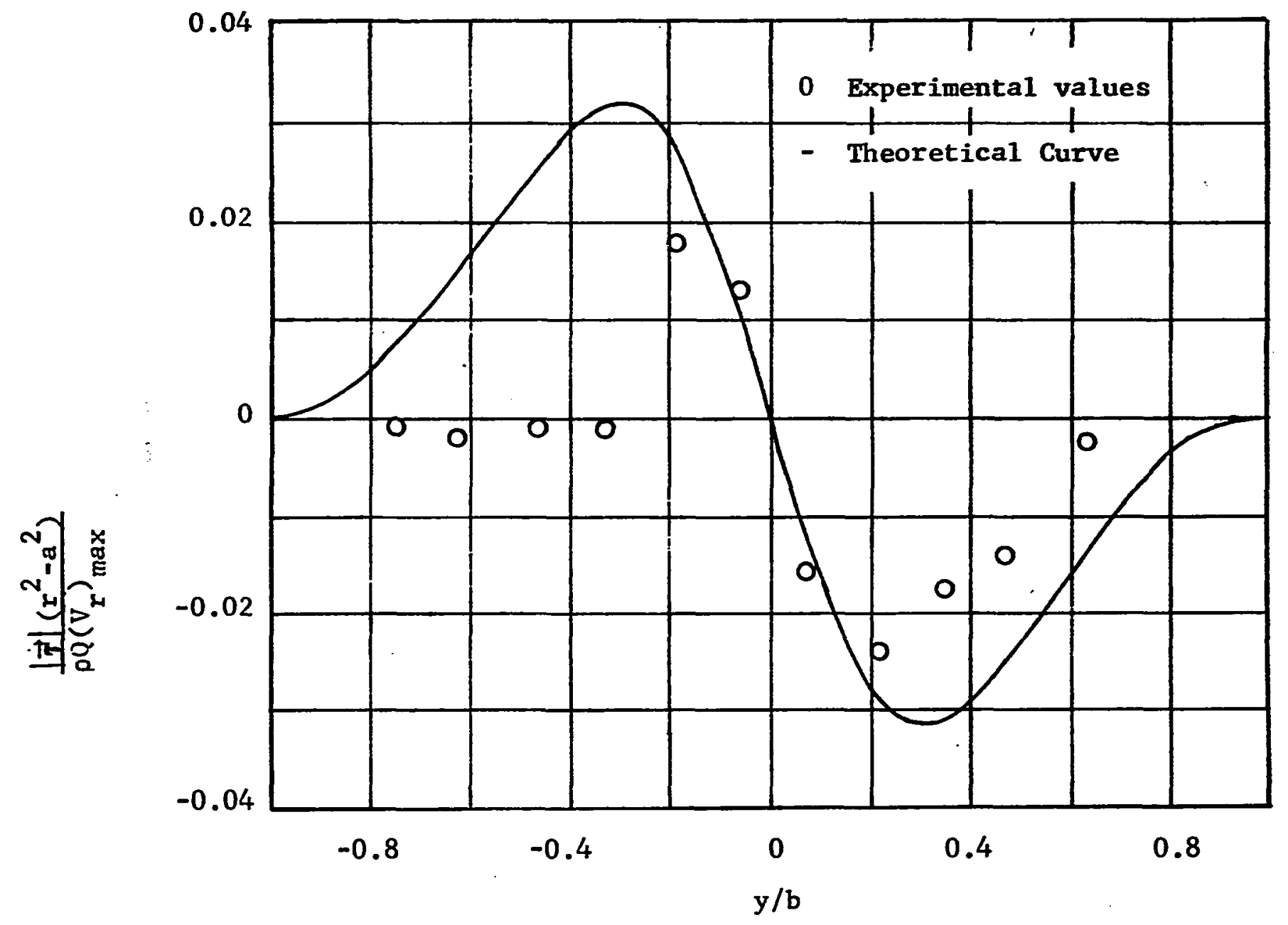

Figure I-7. Nielson's Comparison of Experimental and Theoretical Shear Stress at $r$ is equal to 4.0 inches (21). 
velocity, $v / v^{*}$ against a dimensionless radius $2 \mathrm{r} / \mathrm{T}$. The 1 iquid velocity is $v$, and $v^{*}$ is the impeller tip velocity. Aiba observed that the dimensionless plots were independent of the depth within the tank and the speed of the impeller. However, different plots were obtained for different impellers and for baffled and unbaffled tanks. In unbaffled tanks with glycerine solution of vicosity 108 centipoises it was observed that $\mathrm{v} / \mathrm{v}^{*}$ was dependent on speed. This indicated that viscosity has an effect on the flow patterns in unbaffled tanks although from a consideration of Reynolds number it would seem to have no effect. A representative plot at a depth of 7.0 inches was presented for the paddle impeller. The observation of dimensionless velocity being independent of impeller speed was in agreement with the findings of Sachs (28). No measurements were made in the vicinity of the impe11er.

Aiba characterizes the flow in unbaffled tanks as a solid cylinderical core of fluid rotating at the center of the tank. The radius of the core is smaller than the impeller diameter. This observation is in agreement with Nagata for unbaffled tanks. In baffled tanks the radius of the core is said to be larger. The flow outside the core is similar to a free vortex. This description is very close to that of an ideal circular vortex (15). It is not very clear what velocity Aiba refers to, since a vortex has flow in a tangential direction only. The dimensionless plot of velocity, $v$ looks very much like that for the resultant of $v_{\theta}$ and $v_{r}$. This is confirmed somewhat when Aiba's measuring device is 
examined (see Section 3.5 for details).

Measurement of Flow Profiles at the Impeller by Cutter (9)

Cutter measured flow profiles in the neighborhood of the impeller using an improved photographic method. The object of his work was to study energy dissipation in a stirred tank. A 11.5 inch diameter tank was used with covers on both the top and bottom of the tank, in other words no free surface was maintained. The distance between the top and bottom covers was 12.0 inches. The impeller used was 4.0 inch diameter type A impe1ler, placed 6.0 inches from the bottom of the tank. Velocity measurements were made at 200,400 and $600 \mathrm{rpm}$ in water. Both the mean velocity and the turbulent fluctuating components were measured. The ratio of fluid velocity to impeller tip velocity was found to be independent of impeller speed, as observed by Sachs (28) and Aiba (1). Cutter also reports that the ratio of the turbulent fluctuating velocity component to impeller tip speed was independent of the impeller speed.

The fluctuating velocity components were measured at the impeller center line only. Hence turbulent parameters such as the correlation coefficient and scale of turbulence could be calculated only at the impeller center 1 ine. The radial scale of turbulence was observed to be larger than the tangential scale. The tangential scale had a maximum, while the radial scale increased as the tank wal1 was approached.

The integral form of the equation of motion and energy were used to determine the energy destribution in the region of the 
impeller. The equations of motion and energy were time averaged and were simplified using the boundary layer approximation. The turbulent velocity components were simplified on the assumption that the turbulence behavior was approximately similar to turbulence in pipes. Observation from Laufer's well known study in pipes were then used to effect this simplification.

A torque table was used to obtain the input torque and also the energy input to the system. The momentum equation confirmed that all the angular momentum put into the tank could be accounted for in the impeller stream. This did not hold at the wall indicating that the flow. regime had changed. The flow was then no longer free turbulence with high shear but in stagnation flow. The assumption used to simplify the equation of motion for the jet hence no longer applies. The integral form of the energy equation was used to calculate the energy in the fluid stream at various radial distances from the impeller. It was estimated that $20 \%$ of the energy was dissipated in the impeller itself, $50 \%$ in the impeller stream and $30 \%$ in the rest of the tank. These values were in close agreement with that obtained by using Batchelor's emperical relationship for energy dissipation in turbulent flow.

Potential Flow Solution by Larson (14)

Larson (14) investigated flow patterns and flow profiles using the photographic method. He used a 12.0 inch diameter tank with four baffles 1.0 inch wide and $1 / 4$ inch thick. The, impeller was a Type A with four blades and was made of lucite. The diameter of the disk was 2-5/8 inches and the impeller blades had a dimension of 1.0 
by 1.0 inch. Fluids used were four different mixtures of corn syrup and water. This enabled studies to be made in the Reynolds number range of 31 . to 25,800 . The height of liquid in the tank was 12.0 inches and the impeller was placed 6.0 inches from the bottom of the tank.

Larson considered the flow in the entire tank to be irrotational flow. He admited that this was a weak assumption in the neighborhood of the impeller. The assumption of irrotational flow is equivalent to using inviscid flow theory. Irrotationality implies

$$
\nabla \times \vec{V}=0
$$

In cylinderical coordinated $1-7$ reduces to (43)

$$
\frac{\partial \bar{v}_{r}}{\partial z}-\frac{\partial \bar{v}_{z}}{\partial r}=0
$$

provided $\bar{v}_{\theta}=0$. This is probably true except in the vicinity of the impeller. Since the stream function $\Psi$ satisfies the continuity equation, it was introduced in appropriate form into I-18. The resulting equation is

$$
\frac{\partial^{2} \Psi}{\partial r^{2}}-\frac{1}{r} \frac{\partial \Psi}{\partial r}+\frac{\partial^{2} \Psi}{\partial z^{2}}=0
$$

Larson solved equation I-19 using the following boundary conditions

$$
\begin{aligned}
& \bar{v}_{r}(0, z)=\bar{v}_{r}(R, z)=\bar{v}_{z}\left(r, z_{1}\right)=0 \\
& \bar{v}_{z}\left(r, z_{2}\right)=f(r)
\end{aligned}
$$


Equation I-20 specifies the velocity components normal to the tank wall and normal to the tank surface to be zero. From the last statement it follows that $z_{1}$, is the height of the surface from the impeller centerline which is $z=0$. The boundary condition $I-21$ is a velocity profile in a plane of height $z_{2}$, and it was obtained experimentally. The plane $z_{2}$ was chosen at $z=1$ inch, as this was considered sufficiently far from the impeller centerline so that the flow could be considered as more nearly potential flow. The solution of I-19 with boundary conditions I-20 and I-21 had the form of an infinite series in terms of Bessels and Hyperbolic functions. Larson presents his data as velocity profiles of $\bar{v}_{r}$ and $\overline{\mathrm{v}}_{\mathrm{z}}$ at various points in the tank. The velocity data obtained in regions above and below the impeller are indistinguishable and was to be expected since the impeller was centrally located.

In laminar flow $\left(\mathrm{N}_{\mathrm{Re}}=62\right), \overline{\mathrm{v}}_{\mathrm{z}}$ is presented as a function of $r$ in several $\mathrm{z}$ planes at an interval of 0.5 inch appart. The $\bar{v}_{z}$ are negative, for $r$ smaller than 4.0 inches and positive for $r$ larger than 4.0 inches in all the plots. The velocities are presented scaled to 1 inch. On this scale for $r$ less than 4.0 inch, $\bar{v}_{z}$ theoretical is approximately 0.25 inch lower than experimental values in all plots shown. Beyond $r=4.0$ inch, which is the region near the wall, the theoretical equation does not hold at a11 and there were marked discrepancies. Similarly $\bar{v}_{r}$ is presented as flow profiles in radial planes 0.5 inches a part. The velocities again reported on a scale of 1.0 inch and on this basis the theoretical $\bar{v}_{r}$ is lower by about 0.25 
inch from the experimental values. For $z$ ranging between zero and 2.0 inches, the region of the impeller, the correlation breaks down.

In turbulent flow the experimental data show considerable scatter. On the basis of a full scale for the velocities of 1.0 inch the experimental data scatters in a region of 0.5 inch or $50 \%$ of scale. The theoretical predicted profile is again consistently lower than experimental data points, and the model breaks down in the region of the wall and the impeller. Larson admits that under turbulent conditions there was no agreement between theory and experiment.

Flow Pattern Studies by Schumm (32).

Schumm made qualitative studies of flow patterns with five different impellers. These impellers were Types A and B with variations such as perforated blades or alternated blades unperforated, etc. All impellers were 4.0 inches in diameter and mounted in a 12.0 inch diameter tank, 1.25 diameters off the tank bottom. Motion pictures of dye filiments and still pictures with ionexchange beads were made that follow the flow patterns. A11 impellers gave the characteristic radial flow pattern. Type B impeller when placed close to the bottom of the tank gave a predominant flow above the impeller as shown in Figure 1-8.

Schumm goes through an involved mathematical analys is to calculate a theoretical shear stress in the region of the impeller. This calculated shear stress was later used to obtain an empirical correlation of the half time of the reaction of oleic acid with sodium hydroxide. 


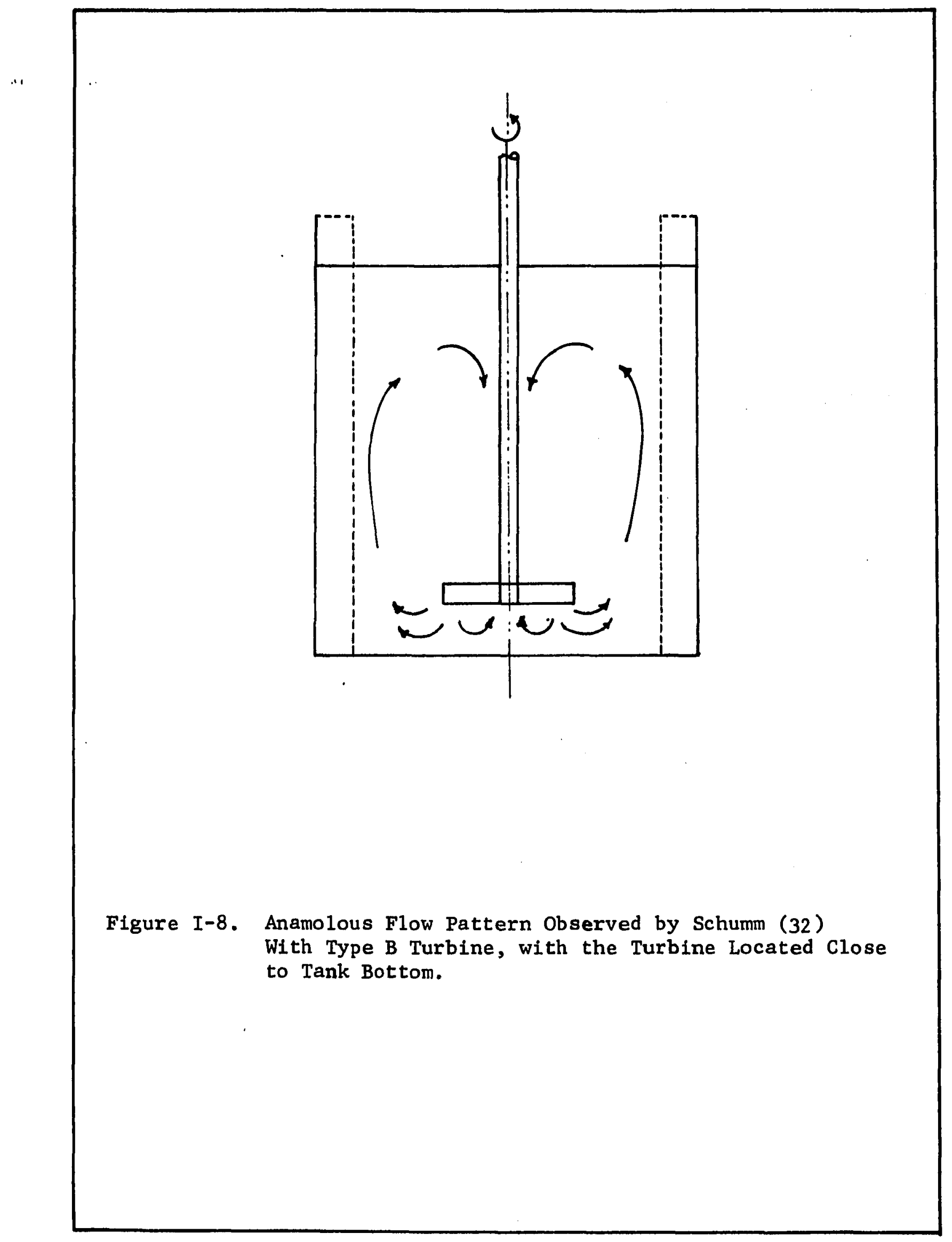


Velocity Measurements in the Region of the Impeller by Cooper (8) Cooper measured velocity profiles in a stirred tank with geometrically similar Type A impellers of varying diameter. With water as the fluid, a directional pitot tube was used, and with air as the fluid, a hot wire arenometer was used. The object of Cooper's work was to measure the flow profiles in the region of the impeller and determine pumping capacities. Measurements were made in a 15.0 inch diameter tank with four baffles. The impeller appeared to be centrally located. The height of fluid in the tank is not mentioned, but it was implied that it was the same as the diameter of the tank. Four geometrically similar Type A turbine impellers were used having diameters of $3.0,4.0,5.0$ and 6.0 inches. The effect of varying the blade width for the 4.0 inch diameter turbine was also studied. In addition measurements were made for an 8.0 inch diameter, three blade marine propeller.

Cooper made velocity profile measurements at the impeller preiphrey and also at three other values of the radial distance from the impeller axis. The effect of entrainement was thus measured quantatively. The profiles were bell shaped as observed by Sachs(28) and others $(4,20,21)$. Cooper also observed an angle profiles which was independent of impeller diameter and speed. The angle profile can best be understood by referring to Figure I- 6 which shows a schematic diagram of the tangential jet. The velocity vector $\vec{V}$ has a bell shaped, however the vector $\vec{V}$ does not emerge at the same angle $\theta_{y}$ at the impeller periphrey for different $z$ planes. The angle $\theta_{y}$ is thus a function of $z$ and is the angle 
profile referred to above. The value of $\theta_{y}$ was reported as $50^{\circ}$ at the impeller centerline and increased to a maximum of $70^{\circ}$ for both positive and negative values of increasing $z$. In other words if the flow from the impeller is considered a jet, then at the edge of the jet $\theta_{y}$ is $70^{\circ}$. The reason why this angle profile was not observed by the photographic method of Sachs (28) is because in the photogrphic method $\vec{v}$ is not measured but $\vec{v}_{r}$ and $\bar{v}_{\theta}$, the components of $\vec{v}$. Hence, in reconstructing $\vec{v}$, the angular dependence with $z$ could very well be lost.

Cooper developed a theoretical equation to predict the velocity profile. The model assumed a stagnant core of radius $k \mathrm{R}$, where $R$ is the impeller radius. In the region $(1-k) R$ the velocity $\bar{v}_{z}$ was assumed to have a finite value $\bar{v}_{z}$ and elsewhere $\bar{v}_{z}=0$. This is shown in Figure I-9. Taking a material balance at a point $z$ for an element of width $\Delta z$ gives

$$
-\left.\rho \bar{v}_{z} \pi\left(R^{2}-k^{2} R^{2}\right)\right|_{z+\Delta z}+\left.\rho \bar{v}_{z} \pi\left(R^{2}-k^{2} R^{2}\right)\right|_{z}-2 \pi R \Delta z z_{p} \bar{v}_{r}=0
$$

Taking the limit, as $\Delta z \rightarrow 0$, we get

$$
\frac{\overrightarrow{d v}_{z}}{d z}=\frac{-2 \bar{v}_{r}}{\left(1-k^{2}\right) R}
$$

Neglecting viscous forces, and taking a momentum balance on the element gives

$$
\begin{aligned}
& -\left.\rho \bar{v}_{z}^{2} \pi\left(R^{2}-k^{2} R^{2}\right)\right|_{z}+\left.\rho v_{z}^{2} \pi\left(R^{2}-k^{2} R^{2}\right)\right|_{z^{\prime}+\Delta z}+2 \rho \pi \bar{v}_{r}^{2} \Delta z \\
& =\rho \pi^{2} w^{2} R^{3}\left(1-k^{2}\right) \Delta z
\end{aligned}
$$




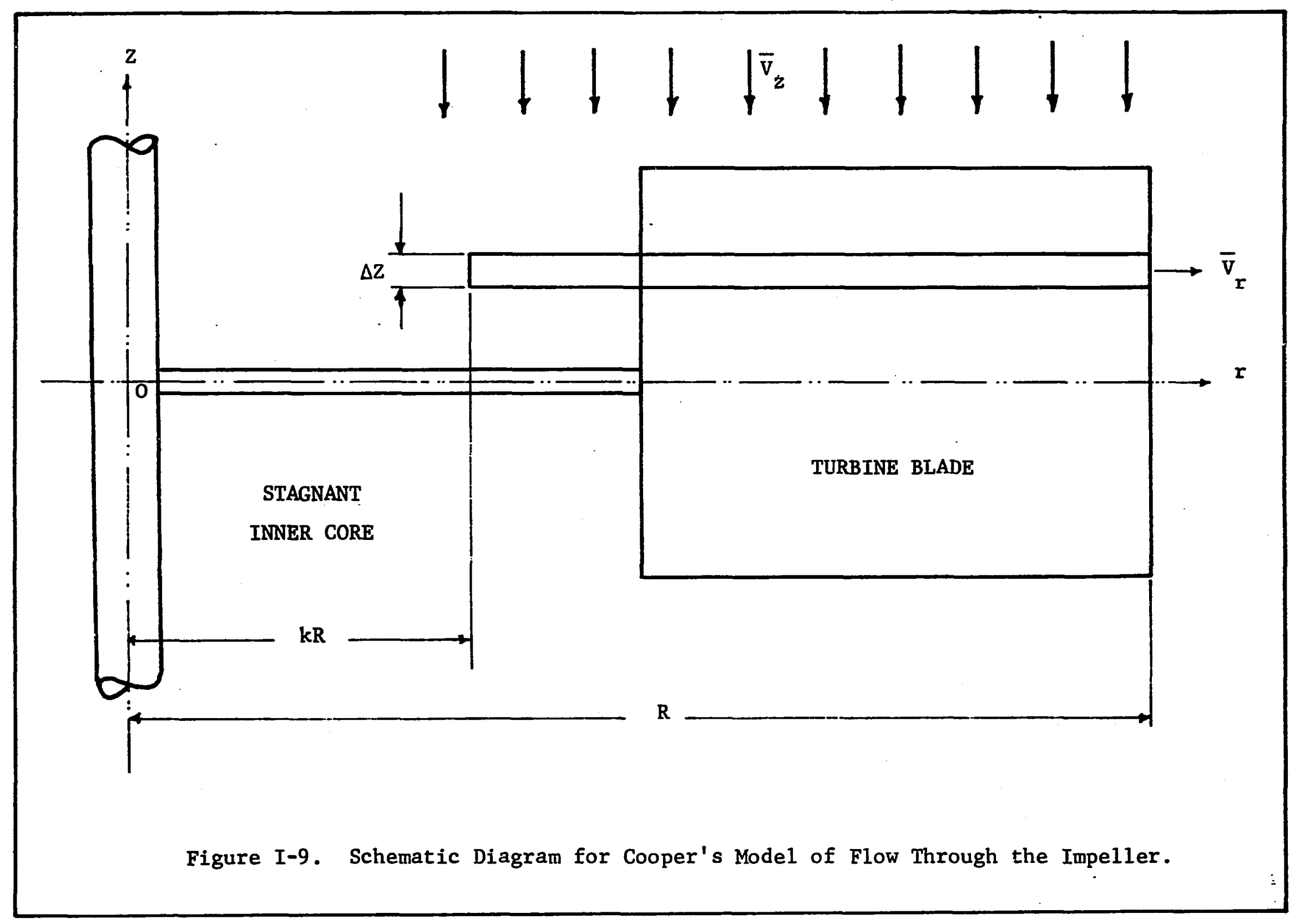


where $w$ is the angular velocity of the impeller. Equation I-24 as given by Cooper is in error since the rate of momentum is a vector. The momentum in the $\mathrm{z}$ direction has been added to the rate of momentum in the $r$ direction and the centrifugal force which also acts in the $r$ direction. Cooper takes the limit on $\Delta z$ in Equation I-24, then eliminates $\bar{v}_{z}$ using I-23 and solves the resulting second order differential equation for $\bar{v}_{r}$ with appropriate boundary conditions.

It is therefore not surprising that the experimental and theoretical values show no agreement. Cooper tries to salvage his theoretical equation for $\bar{v}_{\mathrm{r}}$ by introducing a correction factor which improves the fit somewhat, but it has no theoretical basis. From the above analysis, Cooper obtains the following equation for the angle $\theta_{y}$

$$
\tan \theta_{y}=\frac{1}{C_{1}} \tan \frac{z}{2 c_{1}^{2} R}+\frac{\pi}{4}
$$

In equation $I-25 C_{1}$ is a constant to be evaluated from experimental data. Equation I-25 shows that $\theta_{y}$ is a function of $z$ only and predicts $\alpha$ to range between $55^{\circ}$ and $71^{\circ}$, very close to the experimental values observed. This is a surprising result considering that the theory is incorrect.

Another surprising result observed by Cooper is that the velocity profiles for water and air in the region of the impeller is remarkably close. This indicates that in this region of the tank the flow is governed by turbulence, and the effect of viscosity is neg1 igible. 
Flow Patterns in Newtonian and Non-Newtonian Fluids by Metzner (16)

Metzner investigated flow patterns using the photographic method in baffled stirred tanks for Newtonian and non-Newtonia fluids. The laminar and transistion regions were investigated using geometrically similar impellers (Type A, diameter ranges from 2.0 to 6.0 in.) and two fluids: Karo syrup which is Newtonian and CMC, sodium carboxymethyl cellulose which is non-Newtonian. Several photographs show streak lines of tracer particles describing qualitatively the transistion of flow from laminar to turbulent flow. The velocity data obtained by the photographic method was differentiated to obtain the local shear rates. Power dissipation was calculated from a knowledge of the behavior of shear rates to shear stress from viscometric studies. The following conclusions were noted:

1. In both Newtonian and non-Newtonian fluids the shear rates and power dissipation was directly proportional to impeller speeds under all conditions studied. The: Reynolds number ranged from 2.1 to 450 .

2. Shear rates and power dissipation are high in the region of the impeller. In laminar flow the power dissipation is proportionately larger elsewhere in the vessel than it is in the region of the impeller. As the impeller speed is increased and turbulent flow results, the reverse becomes true: more power is dissipated in the region of the impeller. 
3. In laminar flow little mixing takes place with the circulation being confined close to the impeller.

4. At the start of the transistion range $\bar{v}_{r}$ increases rapidly with increasing impeller speed. The mixing is by transport and blending rather than turbulence in this range of $\mathrm{N}_{\mathrm{Re}}$.

5. At the middle of the transistion range $\left(\mathrm{N}_{\mathrm{Re}}=100\right)$ turbulence begins to appear and is first noticeable near or between the blades of the impellers. In pseudoplastics such as CMC local turbulence is rapidly damped out.

6. At the impeller centerline velocities increase slightly more than linearly with increasing impeller speed for Newtonian fluids. In non-Newtonian fluids this increase is more nearly exponential. The apparent viscosity of pseudoplastics decreases with increasing shear rate. Hence under conditions of high shear rates for the same apparent viscosity pseudoplastics will give a higher overall flow at a lower total power input than Newtonian fluids. The reverse was found to be true under conditions of low shear rates.

Turbulence Measurement in Stirred Tanks by Bowers (4) and Mujumdar (17)

Bowers measured average values and the turbulent quanties intensity and scale for Type B impellers, with two blades (paddles). Four blade and six blade turbines were also used. Design details were not specified. The measuring device was a hot wire anemometer 
and the tank was unbaffled. General profiles for the paddle impeller were presented as dimensionless velocities (ratio of average velocity to impeller tip speed) and were found to be independent of impeller speed.

Mujumdar also investigated turbulent parameters in stirred tanks. A single hot wire anemometer was used, and measurements were made in the plane of the impeller center line for the radial velocity component. A 6.0 inch diameter Type A impeller was used in a 15.0 inch diameter tank. The hot-wire signal shows a definite periodicity superimposed on the random velocity fluctuation, even at a distance from the impeller. He made no attempt to correlate the data. The data for average value of radial velocity is generally in agreement with the findings of Cutter (9).

Summary:

In Table I-3 a summary of the investigations of the turbine impellers reviewed in this section is presented, The work of Schumm and Metzner is not included as they were qualitative studies. The results of Mujumdar and Bowers were also not included since these articles predominantly deal with the study of turbulence in the impeller region of a stirred tank.

The symbols $T, D$, etc. in Table I-3 have the same meaning as shown in Figure I-1. All dimensions are in inches. Blanks in the table mean that the information was not reported. The column of impeller types gives the type of impeller used as shown in Figure I-2, and the number in brackets is the number of different 
Table I-3: Comparison of Impellers and Conditions Under Which Flow Patterns Were Examined. All Measurements reported are in inches.

\begin{tabular}{|c|c|c|c|c|c|c|c|c|c|c|c|c|c|c|}
\hline$\underline{T}$ & D & $\mathrm{H} / \mathrm{T}$ & $\begin{array}{l}a . s \\
h \\
\end{array}$ & $\mathrm{D} / \mathrm{T}$ & $h / D$ & $\begin{array}{l}\text { Impeller Type } \\
\text { and Number }\end{array}$ & $\begin{array}{l}\text { Baffle No. } \\
\text { and Size }\end{array}$ & $\begin{array}{l}\text { Flow ++ } \\
\text { Type }\end{array}$ & $\begin{array}{l}\text { Fluid + } \\
\text { Used }\end{array}$ & $\operatorname{Re} N o$. & RPM & $\begin{array}{l}\text { Distinct } \\
\text { Flow } \\
\text { Patterns }\end{array}$ & Comments & Ref. \\
\hline 23.0 & 11.8 & 1 & 11.5 & 0.513 & 1.05 & $A, B, C, D \quad(30)$ & & $\mathbf{L}$ & $W$ & $1 \times 10^{5}$ & & 1 & * & Nagata (18) \\
\hline 23.0 & 11.8 & 1 & .11 .5 & 0.513 & 1.05 & $A, B, C, D . \quad(6)$ & $8 \times 1.77$ & $\mathbf{T}$ & W & $1.3 \times 10^{5}$ & & 1 & * & Nagata (19) \\
\hline 11.8 & 5.9 & 1 & 5.9 & 0.5 & 1. & $A_{8}, B_{8}, C_{8}$ & & L & พ\&๐ & $6.7-1 \times 10^{5}$ & & 16 & $* *$ & Nagata $(20)$ \\
\hline 11.5 & 4.0 & 1.042 & 4.0 & 0.348 & 1. & $A_{4}(1)$ & $4 \times 1$. & $\mathrm{T}$ & W & & $\begin{array}{l}100 \\
150 \\
200\end{array}$ & 3 & $* * *$ & Sachs (28) \\
\hline 11.25 & $\begin{array}{l}2.0 \\
4.0 \\
7.0\end{array}$ & & 3.75 & & & $A_{6}(1)$ & $4 \times 1$ & $\mathrm{~T}$ & $\begin{array}{c}\text { W } \\
\text { W\&O } \\
\text { W }\end{array}$ & & $\begin{array}{r}600 \\
200 \\
75\end{array}$ & 4 & 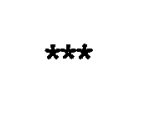 & Neilson (21) \\
\hline 11.5 & $\begin{array}{l}4-46 / 64 \\
3-15 / 12 \\
2-61 / 64\end{array}$ & 0.957 & $1-13 / 64$ & & & $\begin{array}{l}\text { Paddle } \\
\text { A }_{4}(1) \\
\text { Propeller }\end{array}$ & $4 \times 1-13 / 64$ & LET & W & $\begin{array}{l}1.47 \times 10^{4} \\
-4.2 \times 10^{4}\end{array}$ & & & $\star * * *$ & Aiba (1) \\
\hline 11.5 & 4. & 1.042 & 6. & 0.348 & 1.5 & $A_{6}(1)$ & $4 \times 1.2$ & $\mathbf{T}$ & $\mathrm{W}$ & $\begin{array}{l}200 \\
400 \\
600\end{array}$ & & 3 & $* t *$ & Cutter (9) \\
\hline 12.0 & 4.0 & 1.0 & 6. & 0.333 & 1.5 & $\mathrm{~A}_{4}(1)$ & $4 \times 1$ & L\&T & 0 & $\begin{array}{l}31 . \\
25,800\end{array}$ & & $H$ & $\star * * * t$ & Larson (14) \\
\hline 15.0 & $3 .-6$ & & & & & $\begin{array}{l}\mathrm{A}_{6}(9) \\
\text { Marine } \\
\text { Propeller(1) }\end{array}$ & $4 \times 1.5$ & $\mathbf{T}$ & $\mathrm{W}$ & & $\begin{array}{l}100 \\
600\end{array}$ & $H$ & $\star \star \star \star *$ & Cooper (8) \\
\hline
\end{tabular}

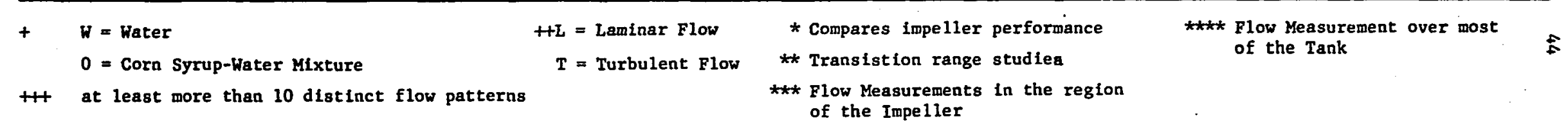


impellers studied. The column listing distinct flow patterns indicates the number of distinct or separated flow conditions studied. For a given impeller, tank and fluid, specifying the impeller speed will determine the flow pattern. For a given flow pattern quantities such as pumping capacity, eddy viscosity, and intensity of turbulence are thus constant in a particular region of a tank. Frequently in a review of the literature it appears that a large amount of effort has been expended to obtain a lot of data. However, if all conditions are kept fixed, such as in case of Nagata, this constitute on $1 y$ one flow pattern. In order to make predictions on flow patterns, a range of flow situations need to be studied. As seen in Table I-3 the only such studies are that of Cooper who used a Type A standard turbine and Larson who used a Type A four blade turbine.

\subsection{Pumping Capacities of Turbine Impellers}

Flow patterns and pumping capacities are invariably investigated together. The pumping capacity is a measure of the volumetric flow rate at a particular point, for example leaving the impeller. However the flow pattern indicates how the flow is distributed throughout the tank. From the work of Sachs (28), Nielson (21) and Nagata (19), it is clear that the flow from the impeller entrains considerable fluid as it approaches the wall. The entrainment is a function of radial distance from the impeller axis, and it increases as the radial distance increases. It reaches a maximum as shown in Figure $I-3$, at approximately $r_{0}$, the 
center of circulation of the upper and lower flow loops. Beyond $r_{0}$, the stream is in stagnation flow. The radial component progressively decreases reaching zero at the walls and the vertical component increases. The radial pumping capacities thus decreases in this region. Hence in reporting pumping capacities it is necessary to indicate the point at which such measurements were made. The most logical point is the impeller periphery, since this gives the flow resulting from the impeller motion alone, and is independent of entrained fluid. There are two approaches to correlating pumping capacities. These are:

1. Centrifugal pump approach first used by Van de Vusse (41)

2. Dimensionless pumping capacity $N_{Q}=\frac{Q}{N D} 3$ introduced by Nagata (18). $Q$ is the volumetric flow from the impeller.

It will be shown that both these methods are related under centain conditions. Grey (37) derives the equation for pumping capacity based on the flow from a curved blade impeller of a centrifugal pump. In this derivation it is assumed that the fluid entering the impeller has a zero momentum, friction losses are negligible, and the fluid leaving the impeller has a flat velocity profile. The last assumption is most questionable since a bell shaped profile has been observed by many investigators $(20,21,28)$ From the centrifugal pump approach, Grey obtained a relationship between $\mathrm{N}_{\mathrm{Q}}$ and the power number $\mathrm{N}_{\mathrm{P}}$ to be

$$
\mathrm{N}_{\mathrm{Q}}=\mathrm{N}_{\mathrm{p}} / \pi^{2} \mathrm{q}
$$


In equation I-26, $q$ is the ratio of the angular fluid velocity at the blade tip to the angular velocity of the impeller. It can be considered as the relative slip between the impeller and the fluid. Norwood (22) used this model to correlate the pumping capacity for a Type A turbine. The conditions under which the data were taken are: Impeller diameter ranging from $2.0-6.0$ inches, impeller centrally located, and viscosity of fluid ranging from 0.3624 to 7,780 centipoises (obtained with corn-syrup water mixture). A correlation was made of the total flow actoss the impeller stream, from the center of vortex above the impeller to the center of the vortex below the impeller. The correlation thus includes all the entrainment, a fact which is not sufficiently emphasized. Norwood's equation is

$$
Q=9.0 \times 10^{-6} \mathrm{ND}^{2} \mathrm{~b}\left(\frac{\mathrm{D}^{0.4} \mathrm{\rho}}{\mu}\right)^{1 / 2}\left(1-\mathrm{q}^{2}\right)^{1 / 2}
$$

where

$Q$ is in $f t^{3} / \mathrm{sec}$.

$\mathrm{N}$ is in rev/sec.

$D$ is impeller diameter, ft.

$\rho$ is density, 1b-mass/ft. ${ }^{3}$

$\mathrm{b}$ is impeller blade width, $\mathrm{ft}$.

In equation I-27 the only unknown quantity is $q$, the relative slip between the fluid and the impeller at the impeller periphery. Norwood recommended deleting this quantity as $q$ is experimentally observed to be a small value. Grey recommended that equation I-26 be used to estimate $q$. 
Equation $\mathrm{I}-27$ can be rearranged to read as

$$
\left.\frac{\mathrm{Q}}{\mathrm{ND}^{3}}=9 \times 10^{-6}\left(\frac{\mathrm{b}}{\mathrm{D}}\right) \mathrm{T}^{0.2}\left(\frac{\mathrm{D}}{\mathrm{T}}\right)^{0.4} \frac{\mathrm{Q}}{\mu}\right)^{1 / 2}\left(1-\mathrm{q}^{2}\right)^{1 / 2} \quad(\mathrm{I}-28)
$$

For geometrically similar impellers the ratio of blade width to impeller diameter $(b / D)$ is a constant. For a given tank, the tank diameter $\mathrm{T}$ is also constant. In practice the ratio, $\mathrm{D} / \mathrm{T}$, ranges from 0.2 to 0.6 . If Norwood's observation is accepted, that the factor $q$ can be deleted, then the quantity on the left hand side is approximately constant. Hence, the important result is that in given tank $\mathrm{N}_{\mathrm{Q}}$ is approximately constant for geometrically similar impellers.

This result can also be obtained, if the velocity profiles are considered similar. This condition can be mathematically stated as

$$
\frac{\bar{v}_{r}}{\left(\bar{v}_{r}\right)_{\max }}=f(n)
$$

Where $f(\eta)$ is the similar velocity profile and $\eta$ is a dimensionless variable which relates $r$ and $z$. In general there are many ways of specifying $\eta_{\text {. }}$. In Nielson's similarity solution it is specified by equation I-6. In our similarity solution $\eta$ is specified differently and is given by Equation B-2 of Appendix $B$, which gives $\eta$ as

$$
\eta=\sigma \frac{\mathbf{z}}{r}
$$


where $\sigma$ is a constant. In Appendix B, the relation for pumping capacity $Q$ has been calculated and is given by equation B- 26 . This equation for $Q$ is

$$
Q=4 \pi x \int_{0}^{\infty} \bar{v}_{r} d z
$$

For a constant value of $r$, combining I-29, I-30, and I-31 results

$$
\text { in } \quad \begin{gathered}
\int f(\eta) d \eta \\
Q=\frac{4 \pi^{2}}{\sigma}\left(\vec{v}_{r}\right)_{\max } \int_{0}^{\infty} f(\eta) d \eta
\end{gathered}
$$

The integral is a definite integral and hence has a finite and constant value which for convience is represented by $k$. From the observations of Sachs (28), Aiba (1), and Cutter (9) it is known that $\left(\bar{v}_{r}\right)_{\max }$ is proportional to the impeller tip speed, or

$$
\left(\bar{v}_{\mathrm{r}}\right)_{\max }=\mathrm{k}^{\prime} \mathrm{mDN}
$$

where $k^{\prime}$ is the constant of proportionality. Since pumping capacity is defined as the volumetric flow at the impeller periphery where $r=D / 2$, we have from I-32, I-33 and, that the integral is a constant $k$ that

$$
\mathrm{N}_{\mathrm{Q}}=\frac{\mathrm{Q}}{\mathrm{ND}^{3}}=\frac{\pi^{2}}{\sigma} \mathrm{kk}{ }^{\prime}
$$

$\mathrm{N}_{Q}$ has thus been proved to be a constant when the velocity profiles are similar.

The condition of similarity would be expected to hold when operating in a given fluid. It will be shown later that for geometrically similar impellers this is indeed the case. Cooper reported $\frac{Q}{N D^{3}}$ to range between 0.726 to 0.89 , showing a slight 
dependence on impeller diameter for geometrically similar turbines in water. Holmes (13) reported $N_{Q}$ to be 1.3 for water with Type A turbine. How the radial velocities were measured and a value of $\mathrm{N}_{Q}=1.3$ arrived at is not reported. Grey (39) summarizes values of $\mathrm{N}_{\mathrm{Q}}$ for a variety of turbines reported in the literature. The value of $\mathrm{N}_{\mathrm{Q}}$ ranges from 0.5 to 2.9 . This wide range reflects the variation in number of blades and blade geometry of the turbines used.

Larson (14) in his review of Aiba's work, indicated that Aiba also gave an equation for pumping capacity and it was $Q=\frac{N_{p}}{q} \frac{N_{\pi}}{} D^{3}$, where $q$ has the same meaning as in $I-26$. This will be recognized as equation I-26. While, Grey $\beta 7$ ) recommended this equation to estimate $q$, Aiba used this relationship to calculate $Q$. The value ' of $\mathrm{q}$ is estimated by trial and error from an emperical chart of $\mathrm{C}^{*}$ vs. $\mathrm{Re}^{*}$. This correlation is given in Reference (1) and the interested reader is referred to it for further details. Thus Aiba's correlation is not basically different from the others reviewed here.

\subsection{Velocity Measurements in Stirred Tanks}

A variety of methods have been used to measure velocities in the stirred tank. These generally fall under the categories of photographic methods, pitot tubes, hot wire anemometers and laser doppler meters. None of the methods reported in the literature is entirely satisfactory. All methods allow measurement of average velocity and some give estimates of the turbulent components. A 
satisfactory device for measuring velocities in a stirred tank is still to be developed, and this is one of the reasons why analysing the fluid mechanics in a stirred tank is so difficult.

The first measuring device to be developed was the photographic method of Sachs (28). This method introduced a small amount of immiscible liquid into the tank which immediately breaks up into fine droplets. A sheet of light about $3 / 16$ inch thick and 5 inches wide was used to illuminate the tank in any desired vertical or horizontal plane. A photograph was taken of the sheet of light at right angles to the light beam. The droplets of immiscible liquid appeared as streaks. The length of the streak, its direction, and the time of exposure of the photographic plate was used to determine the velocity.

Nielson (21) improved the photographic method by introducing a slotted wheel in between the tank and the sheet of light. The slots were alternately large and small. The wheel was rotated at constant speed by a synchronous motor. The streaks now appeared as dotted 1 ines of alternately small and large length corresponding to the length of the slots in the slotted wheel. This permitted more accurate determination of time interval of the streaks and the direction of motion of the tracer particle. The immiscible liquid was replaced by silver coated ion exchange bends. The ion-exchange beads used by Nielson were Dowex 50 in the size range of 100 mesh. Cutter (9) used the photographic method with some more modifications. The tracer particle was Lycopodium powder of average size 
30 microns. The light source was a flash tube of approximately 2 milliseconds duration. The film was exposed for $1 / 600$ of a second. Hence the start of the tracer streak was brighter and together with the slotted wheel gave better indication of the direction of motion of the tracer particle. A micro switch timed the flash to occur when the impeller blade was in a predetermined position and the slot in the slotted wheel was about to open. The flashing light source permitted taking photographs of all the tracer particles at the same time thus permitting calculations of Eulerian correlation coefficient from which the turbulence parameters of intensity and scale can be calculated.

Larson (14) used the photographic method with furthur modifications. The tank was illuminated by a strobe light. Multiple exposure of the tracer particles were photographed in two perpendicular planes simultaneously. One plane was that of the camera, and the other was the plane perpendicular to this plane is seen in a mirror placed at $45^{\circ}$ adjacent to the tank. The multiple exposure in two planes permitted reconstruction of the streamlines. The reconstructed stream lines in laminar flow were fairly regular and we11 defined. In turbulant flow the reconstructed streamlines move in irregular directions, and it was impossible to detect a definite pattern.

The method of Nielson and Cutter appeared to be the best design for the photographic method. Data from a number of photographs were averaged to yield an average velocity and a turbulent component. 
Another devise used to measure the velocity in a stirred tank was the Kiel tube. This is a pitot tube with a cylinderical shield. The shield acts as a vane to straighten the flow and permits velocity measurement independent of flow angle. The maximum flow angle (the angle which the velocity vector makes with the normal of the pitot tube) over which Kiel tube gives consistent results is $40^{\circ}$

Cutter also used a Kiel tube to compare the results of the photographic method. The Kiel tube gave values which appeared to be within $10 \%$ of the best fitting line drawn through the data obtained by the photographic method. The agreement can be considered fairly good considering that the photographic data had a larger spread about the best fitting line.

Nagata (18) used an elaborate pitot tube set-up that permited accurately evaluating the direction of the velocity vector at a point and then placing a Prandt 1 type pitot tube in the direction of the velocity vector. This is a true three dimensional set up for measuring velocities in three dimensional flow that occur in the stirred tank. The details of the design of the pitot tube is reported by Nagata in Reference (18). Nagata's data for velocities at actual points in the tank was not reported, and hence any furthur comment is conjecture. From the meager details presented this device seemed very suitable for measurement of velocities over most of the tank in the fully turbulent region.

Aiba (1) used an ingeneous device to measure velocities in a stirred tank. A ball containing radioactive cobalt was suspended 
from a platinum wire. About an inch away from the ball a miniature Geiger-Muller counter was placed. The counter monitored the radiation from the radioactive source. A calibration curve was made that related the deflection of the ball to the monitored radiation. The Geiger-Muller counter was thus used to determine the position of the ball. A force balance between the gravitational forces and the drag forces on the ball due to the moving fluid was made and this gave a relationship between deflection of the ball and velocity of the fluid. Knowing the position of the ball, and the drag coefficient of the ball the velocity was determined. From the reported analysis it was not clear how the direction of the velocity was determined. Aiba reported measurements of the vertical, radial and tangential components of velocity using this method. For high velocities a steel ball was used, and for low velocities the ball was made of an acrylic resin.

Cooper ( 8 ) used a three dimensional pitot tube made by United Sensors Corportation, for velocity measurements in water. In air he used a hot wire anemometer. The details of equipment used by Cooper is in general brief and sketchy. The fact that the angle of flow is measured more accurately by the directional pitot tube than the hot wire anemometer indicated that a single wire probe was used.

The hot-wire anemometer has been used by Bowers (4) and Mujumdar (17) in water. Both workers used single wire probes. The principle of the hot-wire anemometer is, briefly, based on cooling 
of long thin cylinders. If such a cylinder is placed in a moving fluid then the rate of cooling is a function of the velocity. component in a direction perpendicular to the axis of the cylinder. The hot-wire anemometer is a probe with a wire strung across two prongs. Effective length of wire in typical probe is about 0.05 inch, and its diameter is 0.00015 inch. The wire is thus effectively a long thin cylinder. A current is passed through the wire to heat it, and as the fluid flows past the wire it will be cooled. The wire has a coefficient of resistence which is a function of temperature, hence the resistance will change as its temperature changes. By means of a wheatstone bridge and suitable electronics the temperature of the wire can be maintained constant. The varying current to maintain the temperature constant is a function of the velocity and this functional relationship is non-linear. As the wire is very thin the response to changes in temperature will be almost immediate; and hence, the varying current almost exactly follows the varying velocity flowing past the wire. Two wires placed very close and at right angles but not in electrical contact can be used as a probe to measure velocity in two dimensional flow systems and also for measuring turbulence parameters.

In water the hot-wire anemometer encounters several problems. The overheat ratio is the ratio of the wire temperature to fluid temperature. This ratio has to be low in water since the temperature of the wire cannot exceed the boiling point of water. Should this occur then steam bubbles or bubbles of dissolved gases will form 
and the heat transfer will follow an entirely different functional relationship. High overheat ratios give better sensitivity and response and are hence desirable.

The water must be absolutely clean otherwise lint collects on the wire. Also dirt will adhere to it, and both of these will cause changes in the calibration of the system.

Water has a high inertia, and in high shear flows; it can and does break the delicate wire. This is a serious problem, and it has been reported by Bowers. This difficulty might be overcome by using a hot film anemometer. However hot film probes do not have directional properties, and hence cannot be used in two and three dimensional flow systems.

Finally ordinary water conducts electricity. Although the mechanism is not well understood, hot-wires coated with fused quartz have been used successfully in tap water by Del1'Osso (10) Coated probes are also called hot film probes and should not be confused with the film probes mentioned earlier. The film probe is a film of material deposited on a wedge shaped probe tip.

An ideal measuring instrument for velocities in a stirred tank is one that does not interfere with the flow field and permits not only velocity measurements at the walls of the tank but also the turbulent velocity components. Such a device may be the laserdoppler velocity meter. This device has been used by Goldstein (11) to measure laminar flow in a square $\operatorname{duct}(1.0 \mathrm{~cm} \mathrm{sq.})$. This device is based on the principle that the light beam from a laser which 
is scattered from a moving tracer particle undergoes a doppler shift. The tracer particle typically used is 0.5 micron polystyrene particles which is then diluted by one part solids to 50,000 part's by volume water. The scattered beam and original beam are brought together and optically hetrodyned. This gives rise to an alternate light and dark band that moves at the doppler frequency. The moving bands are monitored by a photomultipler and through suitable electronics a signal can be obtained of the doppler frequency. A functional relationship exists between the doppler frequency, the scattering angle and the velocity of the tracer particle. Thus the velocity of the particle can be computed and taken equal to the fluid velocity. Since the tracer particles are small the response of the device is expected to be high. The method requires precision components and an elaborate set up. It is still in the experimental stage. Rolfe and associates (25) have developed a three dimensional laser doppler velocity meter to measure gas velocities in a wind tunnel up to a velocity of Mach 2. Results obtained were in close agreement with that of a hot-wire anemometer. They presented an excellant summary of the capabilities of this measuring device.

\section{Conclusions}

In this Chapter justification for investigating the flow patterns in stirred tanks has been presented. Of the three basic flow patterns occurring in stirred tanks it is proposed to study the radial flow pattern generated by a turbine impeller. Several 
impeller designs are available, however the Type A design is considered to be standard and will be used in this work.

None of the investigators have been entirely successful in describing the fluid dynamics of the tank. However, some success has been achieved in certain regions of the tank. It was therefore decided to model the fluid dynamics of the tank by considering it to be made up of several regions. Each region will be treated separately using the best model available.

For example, in the region of the impeller, Nielson's tangential jet model appears to describe all the observed phenomena except the angle profile discovered by Cooper. However Nielson's solution is too involved and cumbersome to be useful. This is because of his use of Prandt1's Mixing Length Theory. The flow in the impeller region is essentially free turbulence, and in such cases Schlichtling (29) has shown that the use of Prandtl's second hypothesis can give an equivalent solution which is not only easier to obtain but also simpler in form. This will be apparent when Nielson's solution is compared with ours which is presented in Appendix B. Both theories are phenomenological, and the parameters in the solution are obtained by fitting experimental data. For free turbulence, Prandt1's second hypothesis is a simpler approach and hence favored.

The work of Larson shows that elsewhere in the tank potential flow might be applicable. The poor results obtained by Larson may well be because he considered the entire tank as one region. If 
potential flow is assumed, it seems easier to map streamline using the simpler approach of the aerodynamist: fit streamlines in a flow region using combination of sources, sinks and vortices. This approach will be used in Chapter II.

The flow from a turbine impeller is a narrow high velocity turbulent stream. This stream has been measured in a limited manner as indicated under the heading data points of Table I-3. The recent work of Cooper makes available considerable data on velocity profile measurements in this region. The data is not complete, and hence it will be necessary to make some more measurements. With the help of an adequate flow model it will be possible to characterize the flow in this region of the tank.

It is felt that before making turbulent studies a first step would be to define and predict the bulk flow. It is our objective to understand the flow rather than the detail mechanism of turbulence. The flow model will use a phenomenological theory, and this will permit calculating an eddy viscosity which will be of immediate practical use.

As a study of the turbulence in a stirred tank is not the objective of this work only average velocities need to be measured. From the literature review presented in Section 3.4 the directional pitot tube used by Cooper was selected for experimental measurements, The selection of this device was based on its simple construction and the remarkable consistency of the data obtained. It has its drawbacks in that velocities lower than $30 \mathrm{ft} / \mathrm{min}$. cannot be measured. 
CHAPTER II

THEORETICAL ANALYSIS OF THE FLUID DYNAMICS

IN THE STIRRED TANK

1. Development of a Mathematical Model for the Stirred Tank

In this chapter, a mathematical model will be developed to describe the flow pattern in the stirred tank. From the work of severa1 investigators reviewed in Chapter $I$, it appears that the stirred tank can be separated into two regions: one in the neighborhood of the impeller which has received considerable attention and the other the rest of the tank.

The flow in a baffled stirred tank with a turbine impeller can be described as follows, First a high speed, narrow, highly turbulent stream emerges from the impeller. This high speed stream entrains a considerable amount of fluid which causes it to broaden and slow down as it approaches the tank wall. On approaching the tank wall the radial component of the fluid velocity decreases rapidly reaching zero at the tank wall, while there is a correr sponding increase in the axial component of the velocity. The flow near the tank wall can be approximated by stagnation flow. The stagnation point occurs roughly at the point where the plane of the impeller center line intersects the walls of the tank. The stagnation flow results in the stream dividing into two equal parts giving rise to the clover leaf flow pattern as shown in Figure I-1, 
of Chapter I. The divided stream circulates in the rest the tank returning to the impeller region. Part of this stream enters the impeller the rest is the entrained by the impeller stream. In the upper and lower sections of the tank are two doughnut shaped regions. These regions form the centers of circulation for the circulating flow in the tank, mentioned above. The center of circulation is shom in Figure I-5. The above description of the flow field is based primarily on the work of Nagata (19).

In developing a mathematical model, the work of Larson (14) showed that an attempt to model the fluid dynamics of the stirred tank as entirely one flow region gave poor results. On the other hand Nielson (21) has had fair success in modeling the flow in the region of the impeller. This is not exactly surprising since the flow in the vicinity of the impeller has not only had the highest velocities but also most of the energy is dissipated in this part of the tank. This latter conclusion is based on the work of Cutter (9) and Bowers (4). They have shown that the neighborhood of the impeller is a zone of high mean and turbulent velocities, while the rest of the tank is relatively quiescent. Cutter estimates that $20 \%$ of the energy is dissipated in the impeller itself, $50 \%$ in the impe11er stream and $30 \%$ in the rest of the tank. Considering the relative volume of the rest of the tank to the region in the vicinity of the impeller it would appear that energy dissipation per unit volume in the rest of the tank is unimportant. We can thus conclude that the region of the impeller is a high shear region while in the rest of the tank shear forces are unimportant and hence potential flow could be applied. 
We thus have two regions to be modeled separately. The region in the vicinity of the impeller is modeled using Nielson's tangential fet model, and the flow in the rest of the tank is described by potential flow. In using potential flow, the method of the aerodynamists was adopted. This approach divided the rest of the tank into several regions, each region being fitted by a model that best appears to describe the flow pattern in that region. Nagata's flow pattern shown in Figure $I^{-5}$ was used as a guide for modeling the regions in the rest of the tank.

In Figure II-1 a computer drawn solution is shown of the stream lines in a $r$ - $z$ plane passing through the impeller axis, a line of symmetry of the system. This is from the solution of the model of the flow described in this chapter. In the figure the flow in the tank is divided into six regions. These regions are

I. The flow from the impeller, modeled by a tangential jet.

II. Impeller stream impinging on tank wall, modeled by stagnation flow.

III. Upper and lower corners of the tank, modeled by potentia 1 flow in a corner.

IV. Flow at the top and bottom of the tank axis, also modeled by potential flow in a corner.

V. Flow at the center of the tank axis, modeled by a circular jet.

VI. Two doughnut shaped regions, which have been modeled as dead water regions. 


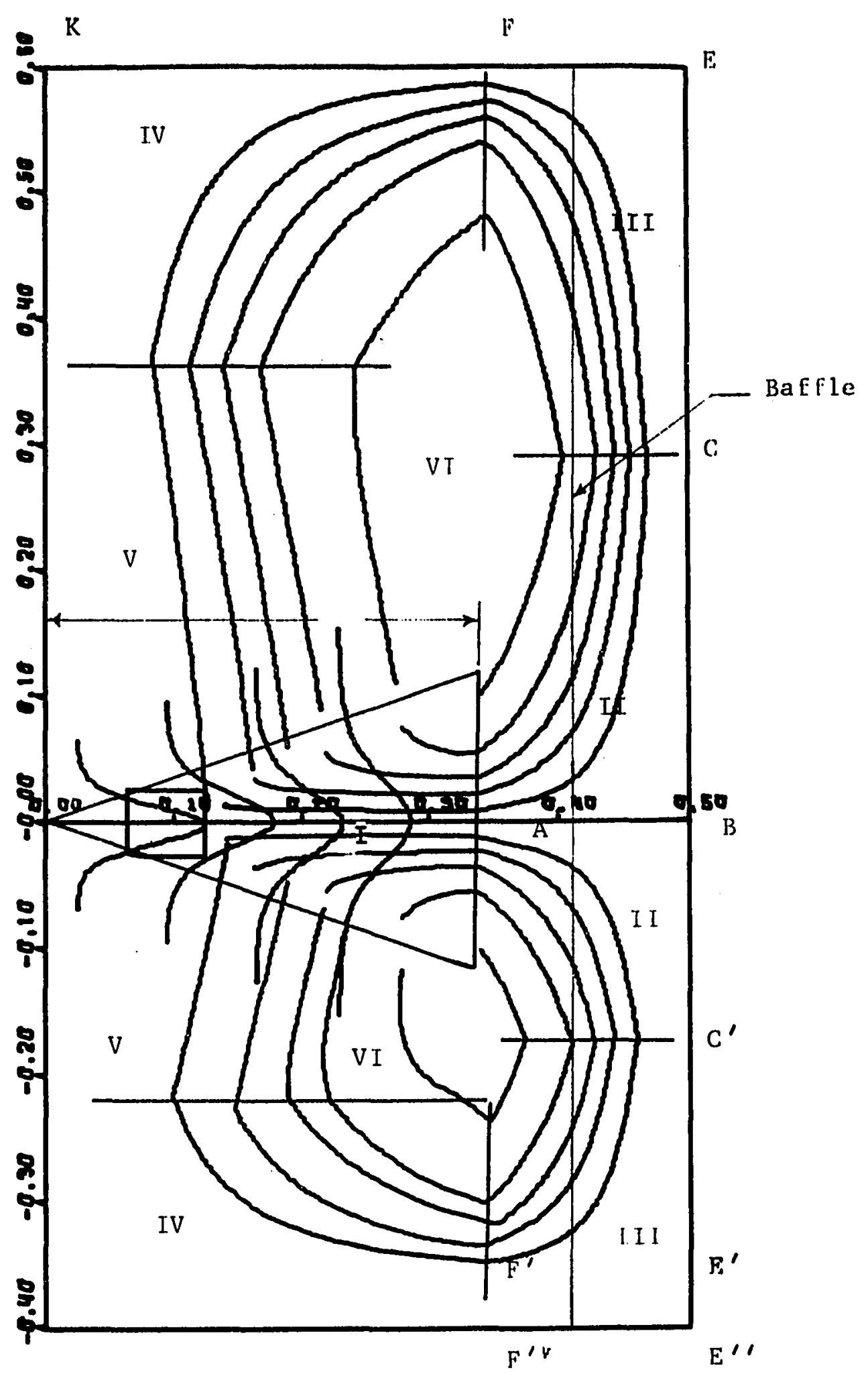

FigureII-1: Typical Computer Drawn Solution of Flow patterns Showing the Regions into which the Tank is Divided 
Regions shown in the figure have the same fluid dynamics model are given the same number. However, the parameters in the model of, region $V$; for example; shown above and below the impeller are not the same. Each of these regions will be examined in detail and the merits of the model chosen will be discussed. 1.1. Region I - Neighborhood of the Impeller

The Tangential Jet: This region is the area around the impeller. At a very early stage it was recognized that a free jet model would give a satisfactory description of flow in this region. The radial plane jet was first developed and tested on Nielson's data. The model seemed inadequate. The jet width was either too wide or too narrow and depended on where the origin of the jet was located. On examining Cooper's data ( 8 ) and in our own investigation of the problem it was apparent that a sizeable tangential velocity component existed near the impeller and should be taken into account. The tangential component decreases at increasing distances from the impeller and reached a small but non-zero value near the tank wall.

Nielson has accounted for this tangential component by considering a ring jet in which fluid emerged tangentially from the periphery of a cylinder of radius a and height $2 \mathrm{~b}$. A physical picture of the model consists in replacing the impeller by a cylinder of diameter $2 a$ and height $2 b$. The fluid leaves this cylinder of diameter $2 a$ on a tangent with an average velocity of $\vec{q}$. Consider a point, $A$, on the periphery of the source as shown in FIgure II-2. The fluid leaves at a tangent to the source, and the yaw angle $\theta_{y}$ at $A$ is $90^{\circ}$. The yaw angle $\theta_{y}$ is defined as the 


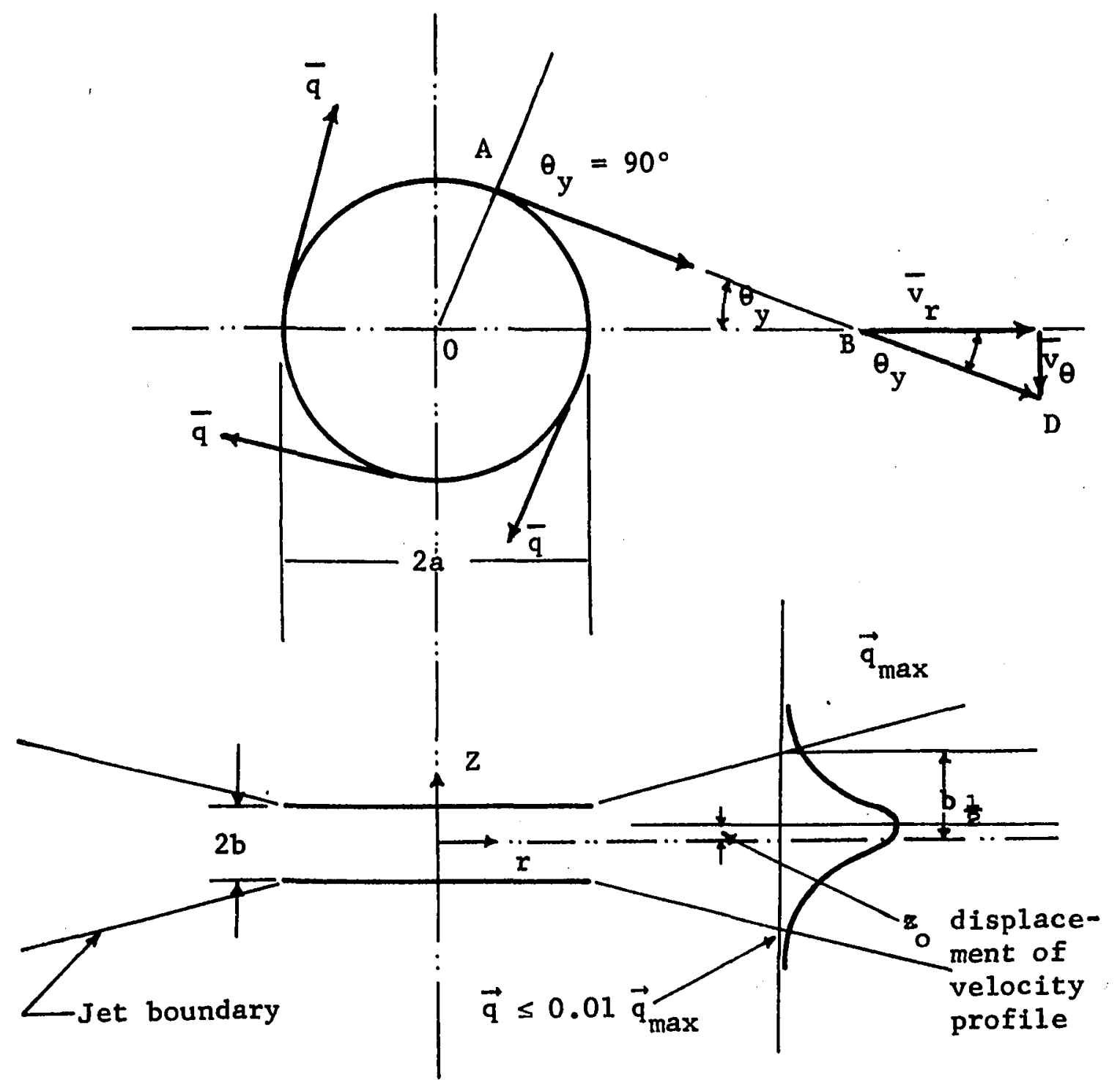

Figure II-2. The Tangential Jet. 
angle between the velocity vector and the radius vector. Once the fluid leaves the cylinderical source, the baffles seem to freeze the flow, and fluid continues to move in the direction $\vec{q}$ it had as it left the ring source. Although the direction of $\vec{q}$ does not change, its magnitude decreases because of entrainment. At a point $B$ far from the source the yaw angle $\theta_{y}$ has decreased and is less than $90^{\circ}$. It is thus clear that at $r=a$, the yaw angle $\theta_{y}=90^{\circ}$, decreasing to a small value near the tank wall, From the geometry of Figure II-2 it follows that $\bar{v}_{r}$ and $\bar{v}_{\theta}$ is given by,

$$
\begin{aligned}
& \bar{v}_{r}=|\vec{q}| \cos \theta_{y} \\
& \bar{v}_{\theta}=|\vec{q}| \sin \theta_{y}
\end{aligned}
$$

In equation II-1 and II-2, the velocities, $\bar{v}_{x}, \bar{v}_{\theta}$ are time average values. A bar over a variable signifies a time average quantity. A vector is denoted by an arrow over the variable as for $\vec{q}$ in Equation II-1 and II-2.

The pumping capacity of the impeller depends on $\bar{v}_{\mathbf{r}}$. From equation II-1, $\bar{v}_{r}$ has a limiting value of zero when $\theta_{y}=90^{\circ}$, and $r=a$. Hence, for $\bar{v}_{r}$ to be finite, $\theta_{y}$ must be less than $90^{\circ}$. As all impellers have a finite pumping capacity it follows then, that the radius of the ring source, a must be less than the impèller radius $\mathrm{D} / 2$.

Equations of Continuity and Momentum for the Tangential Jet: In Appendix A, the equations of motion for turbulent flow have been simplified for the assumptions of isothermal, incompressible flow and the boundary layer approximations. The resulting time 
averaged equations that describe the flow in the turbulent tangential jet are

Continuity Equation:

$$
\frac{\partial}{\partial r}\left(r \bar{v}_{r}\right)+\frac{\partial}{\partial z}\left(r \bar{v}_{r}\right)=0
$$

Equation of Motion:

$$
\bar{v}_{r} \frac{\partial \bar{v}_{r}}{\partial r}+\bar{v}_{z} \frac{\partial \bar{v}_{r}}{\partial z}-\frac{a^{2}}{r\left(r^{2}-a^{2}\right)} \bar{v}_{r}^{2}=-\frac{1}{\rho} \frac{\sqrt{r^{2}-a^{2}}}{r} \frac{\partial|\vec{\tau}|}{\partial z}
$$

These equations can be reduced to a single partial differential equation by introducing the stream function $\psi$, and into an ordinary differential equation by the similarity principle. The similarity principle assumes that the velocity profile to be similar in the direction of fluid motion.

In Appendix A, it was shown that the tangential jet is a one dimensional flow problem resulting in the single equation of motion A-22. As a result, the stress tensor $\overline{\bar{T}}$ was reduced to a vector $\vec{\tau}$. A phenomenological theory is used to define $\vec{T}$ since in this work; time average velocities are measured.

From the several theories available, Prandt1's Second Hypothesis (Schlichtling (29)) was selected as it was best suited to our problem. This is because the turbulence in the tangential jet is not due to a wall or boundary, and is hence essentially a case of free turbulence. It is a region of extreme velocity gradients, the velocity $\vec{q}$ is a maximum at the center and reaches zero at the boundaries of the jet which can be seen in Figure II-1. 
Prandtl's Second Hypothesis (29) gives the value of $\vec{T}$ for flow in the $\vec{q} d i r e c t i o n$ as:

$$
\vec{\tau}=\rho k b_{\frac{z}{z}}\left|\left(\vec{q}_{\max }-\vec{q}_{\min }\right)\right| \frac{d \vec{q}}{d z}
$$

where $\rho$ is the density, and $k$ a constant, $b_{\frac{1}{2}}$ is the half width of the jet shown in Figure II-2 and is defined in Appendix B. Since $\vec{q}_{\text {min }}$, the velocity at the boundary of the jet is zero,

$$
\vec{\tau}=\rho k b_{\frac{1}{2}}\left|\vec{q}_{\max }\right| \frac{d \vec{q}}{d z}
$$

Defining $\epsilon$ as the virtual kinematic viscosity,

$$
\epsilon=\mathrm{kb}_{\frac{1}{\mathrm{z}}}\left|\overrightarrow{\mathrm{q}}_{\max }\right|
$$

and hence

$$
\vec{\tau}=\rho \in \frac{d \vec{q}}{d z}
$$

Substituting for $\cos \theta_{y}$ from A-14 into II-1 gives

$$
|\vec{q}|:=\frac{r}{\sqrt{r^{2}-a^{2}}} \vec{v}_{r}
$$

Introducing II-7 in II-6 and noting that $\bar{v}_{r}$ is not a function of $\mathbf{r}$ which will be emphasized by using partial derivatives we have

$$
|\vec{\tau}|=\rho \epsilon \frac{r}{\sqrt{r^{2}-a^{2}}} \frac{\partial \bar{v}_{r}}{\partial z}
$$

Substituting for $|\vec{T}|$ from II-8 into A-22 gives

$$
v_{r} \frac{\partial v_{r}}{\partial r}+\bar{v}_{z} \frac{\partial \bar{v}_{r}}{\partial z}-\frac{a^{2}}{r\left(r^{2}-a^{2}\right)} v_{r}^{2}=-\epsilon \frac{\partial^{2} \bar{v}_{r}}{\partial z^{2}}
$$


It should be noted the $\epsilon$, the virtual kinematic viscosity is a function of $b_{\frac{1}{z}}$ and $\bar{q}_{\max }$ which are functions of $r$ and not $z$. Solutions of the Tangential Jet Equations: A similarity solution of Equation II-9, subject to continuity Equation A-3 and boundary conditions $A-28$ and $A-29$, is detailed in Appendix $B$. The equations for the velocity profile are given by B-24 and B-26 which are reproduced below.

$$
\begin{aligned}
& \bar{v}_{r}=\left(\bar{v}_{r}\right)_{\max }\left[1-\tanh ^{2}(\eta / 2)\right] \\
& \bar{v}_{z}=-\frac{\left(\bar{v}_{r}\right)_{\max }}{\sigma}\left[\frac{2 r^{2}-a^{2}}{r^{2}-a^{2}} \tanh (\eta)-\eta\left(1-\tanh ^{2}(\eta / 2)\right)\right](B-26)
\end{aligned}
$$

where

$$
\begin{aligned}
& \left(\vec{v}_{r}\right)_{\max }=\frac{A}{2}\left(\frac{\sigma}{r^{3}}\right)^{\frac{1}{2}}\left(r^{2}-a^{2}\right)^{\frac{1}{4}} \\
& \eta=\sigma \frac{z}{r}
\end{aligned}
$$

It should be noted that the above equations $B-24$ and $B-26$ are obtained from exactly the same basic analysis as presented by Nielson ( 21 ). The difference lies in the definition of $\vec{\tau}$ the shear stress. Nielson used Prandtl's mixing length theory which results In a cumbersome solution as described in Chapter $I$. Both theories result in three parameter models and give an excellent fit of the velocity. profile data. However the velocity profile as obtained by Nielson is not in explicit form but has to be evaluated from equations $I-10$ to $I-13$ and are thus harder to fit. In contrast, Equation B-24 is not only simpler to derive but gives the velocity profile directly. Another reason for selecting 
Prandtl's Second Hypothesis is because it is a well tested approach to describing free turbulence, examples of which are jet and wakes; and these have been solved in Schlichtling (29).

Results of Tangential Jet Solution: In obtaining velocity profile data, as will be seen in Chapter III, neither $\bar{v}_{r}, \bar{v}_{z}$, or $\bar{v}_{\theta}$ is measured but the resultant of $\vec{v}_{r}$ and $\bar{v}_{\theta}$ which is $\vec{q}$. The velocity $\vec{q}$ is shown in Figure II-2, and its relation with $\vec{v}_{r}$ and $\bar{v}_{\theta}$ is given by Equations II-1 and II-2.

Substituting for $\vec{v}_{r}$ from B-24 and B-25 into II-7 gives,

$$
\bar{q}=\frac{A}{2}\left(\frac{\sigma}{x}\right)^{\frac{1}{2}} \frac{1}{\left(x^{2}-a^{2}\right)^{\frac{1}{4}}}\left[1-\tanh ^{2}(\eta / 2)\right]
$$

Equation II-10 is non-linear, and as it has been explained earlier that a, should not exceed $D / 2$.

It has been observed by Nielson (21) and from our analysis of Cooper's ( 8 ) data that $\vec{q}_{\max }$ does not coincide with the impeller center line but is displaced by a small. amount, $z_{0}$ as shown in :.' Figure II-2. Experimental data is made to fit equation II-10 using the least square criteria. An optimization technique known as Pattern Search has been found satisfactory to evaluate the four parameters $\sigma, A, a$, and $z_{0}$ subject to constraint on $a$. An out1ine of the procedure and the computer program is presented in Appendix C. The kinematic eddy viscosity $\epsilon$ has been evaluate in Appendix $B$, Equation $B-15$, and is

$$
\epsilon=\frac{A}{2} \frac{1}{\left(\sigma^{3} r\right)^{\frac{1}{2}}} \frac{2 r^{2}-a^{2}}{\left(r^{2}-a^{2}\right)^{3 / 4}}
$$


Comparing $B-15$ and $I I-5$ and substituting for $q_{\max }$ from $I I-10$, the half width of the jet $b_{\frac{1}{6}}$ and the constant $k$ can be calculated, a factor $k^{\prime}$ is included in the calculations giving

$$
\begin{aligned}
& b_{t}=\frac{\left(2 r^{2}-a^{2}\right)}{\left(r^{2}-a^{2}\right)^{\frac{1}{2}} k^{\prime}} \\
& k=\frac{k^{\prime}}{\sigma^{2}}
\end{aligned}
$$

The factor $k^{\prime}$, it is seen cancels out and thus has no effect on $\epsilon$. It is included to make $b_{\frac{1}{2}}$ as defined in II-12 identical to that in B-34 and will be discussed in Chapter IV.

It will be observed that the velocity profile and expression for eddy viscosity contain three arbitrary parameters $A, a$, and $\sigma$. These were evaluated by a least square fit of experimenta1ly obtained velocity profile data, as will be described in Chapter IV. 1.2. Regions II, III, and IV

Regions II, III, and IV will be modeled together. The reason why this is so will be apparent as we proceed. In these regions extreme velocity gradients do not exist, and the flow is considered to be in potential flow. This is confirmed somewhat by Larson's (14) work and also from the observation of Cutter (9) that energy dissipation in these regions are small. All of these regions are bounded by the walls except the surface.

Near the tank wall a turbulent boundary layer exists. This has been observed by Askew ( 2 ) who has investigated the flow profile close to the tank wall. The thickness of the boundary layer is of the order of 0.1 inch and does not exceed this value in all cases investigated. Askew's results were obtained using distilled 
water with the following impellers: a $90^{\circ}$ Turbine, a $45^{\circ}$ Turbine (design details unknown) and a marine propeller. Impeller speed ranged from 0 . to 18.75 revolutions per second (rps). For the $90^{\circ}$ Turbine (presumably similar to the type used in this work) only one graph is presented at 9;0 rps for velocity profiles at various 10cations along the wall in regions II and III. From this work it appears that the boundary layer at the tank walls in regions II and III is very sma11, at least beyond the precision of the measuring instruments used in this work. The boundary layer effect can be accounted for by excluding the boundary layer region from the potential flow region. This follows from the definition of boundary layer which assumes that the region outside the boundary layer is In potential flow. From the discussion above it will be seen that 0.1 inch, the thickness of the boundary, is so small in comparison with the width of regions II and III that it can be neglected.

Regions II, III and IV are essentially two-dimensional flows, as the tangential component is assumed to have a small value in these regions and can thus be neglected. The flow is thus in the $r$ - $z$ plane and the velocities of interest are $\bar{v}_{z}$ and $\bar{v}_{r}$. Two dimensional flows are conveniently handled by using the stream function $\psi$ which also makes the evaluation of streamlines easy. In Appendix $D$ it was shown that the stream function given by Equation D-9 is a possible potential flow solution that satisfies the boundary conditions of Regions II, III and IV. Equation D-9 is given by 


$$
\psi=x^{2} z
$$

and will be used to evaluate the streamlines.

In Figure II-3 (a) and (b) two solutions of equation D-9 are shown. It is noticed that Figure II-3 (b) is a part of Figure II-3 (a). This is a valid solution since by definition, a streamline is a line across which no flow occurs, and hence a streamline can be replaced by a boundary. The need for replacing a streamline by a boundary becomes clear on comparing Figure II-1 and II-3. It is seen that Figure II-3 (a) corresponds to Region II, while Figure II-3 (b) corresponds to regions III and IV. It is now apparent why regions II, III and IV are considered together. A11 these regions use the same equation, D-9, for the stream function; however, the origin 0 of Figure II-3 has a different location in different regions. In other words, each of the above regions uses that part of Figure II-3 (a), whose streamlines correspond to the experimentally observed streamlines.

The units on the stream function $\psi$ is $\mathrm{ft}^{3} /(\min )(\mathrm{ft})$. It thus represents the volumetric flow between two streamlines. To give the streamlines a value, a zero streamline must be defined. In our problem, this is the streamline along which the velocity $\bar{v}_{r}$ is a maximum. From Figure II-2, this will correspond to a line parralled to the impeller centerline and displaced from it by an amount $z_{0}$. It thus follows that the equation of the streamline is obtained by giving $\psi$ a suitable numerical value in Equation D-9. This will be more clear when the stream lines of Figure II-1 are developed in the Section 2,2. 


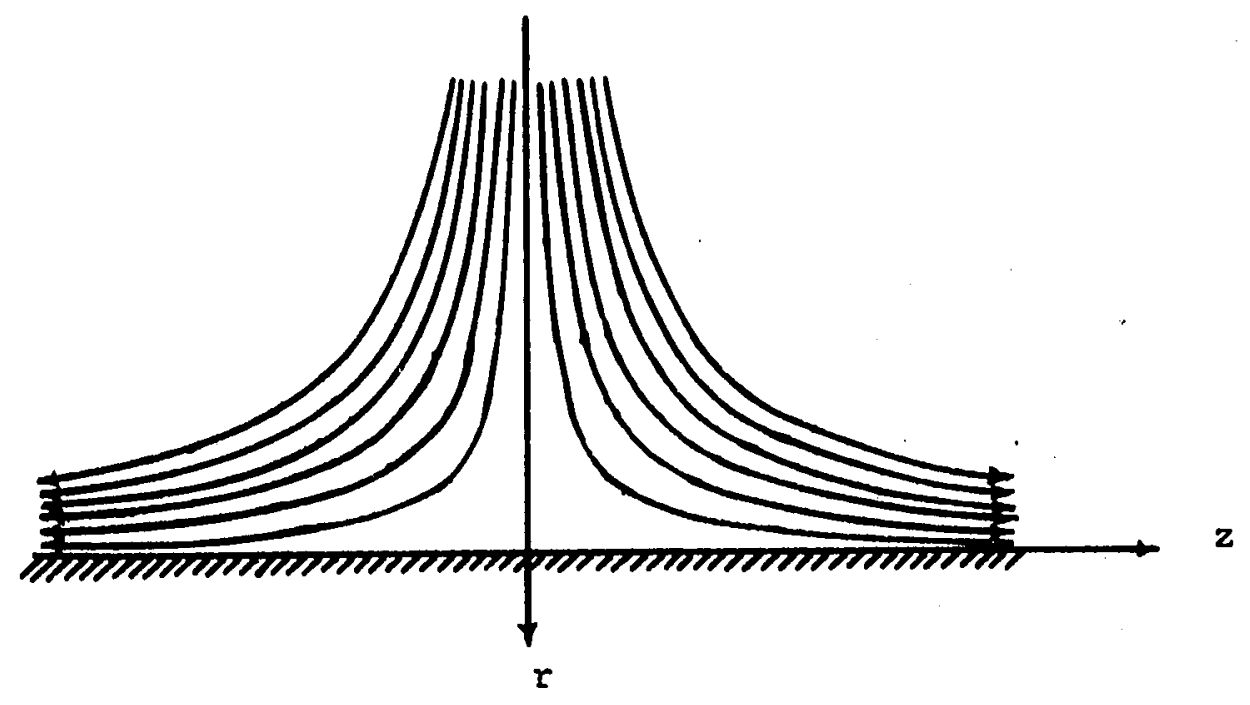

(a) Stagnation Flow

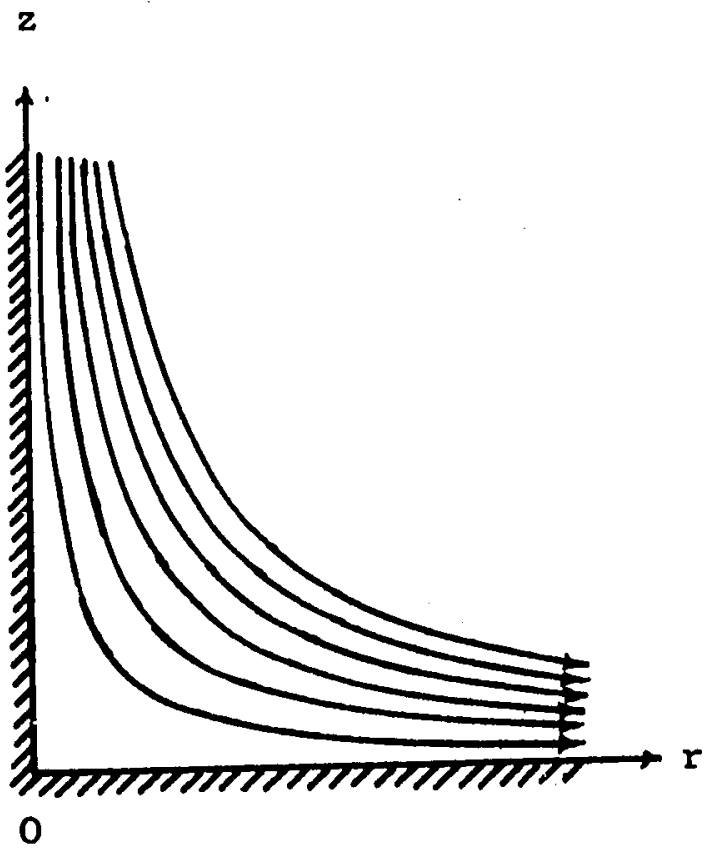

(b) Flow in a Corner

Figure II-3: Potential Flow Solution of Equation D-9 


\subsection{Region V}

This region is modeled as a circular jet. In Figure II-4 is a sketch of a circular jet. The velocity profile and other quantities are shown in the figure. The fluid dynamics of the circular jet is well known. Schlichtling (31) gives a very good treatment of the circular jet using Prandt1's Second Hypothesis for the eddy viscosity. The equations as obtained by Schlichtling for the veloprofile and other pertinent data on the circular jet is summarized below.

The velocity in the $z$ direction $\bar{v}_{z}$ is given by

$$
\bar{v}_{z}=\frac{\epsilon_{0}}{\left(z+z_{0}\right)^{\theta}} \cdot \frac{2 \gamma^{2}}{\left(1+\frac{1}{4} \xi^{2}\right)^{2}}
$$

The entrainment of fluid by the jet is due to the r-component, $\bar{v}_{r}$ and is given by.

$$
\bar{v}_{r}=\frac{\bar{\epsilon}_{0}}{z+z_{0}} \quad y \frac{\xi-\frac{1}{4} \xi^{3}}{\left(1+\frac{1}{4} \xi^{2}\right)^{2}}
$$

where $\xi$ is a dimensionless coordinate normal to the direction of flow and is given by

$$
\xi=\gamma \frac{r}{z+z_{0}}
$$

where $\gamma$ is the jet spreading parameter for circular jet defined above, and $z_{0}$ is coordinate of origin of jet and is shown in Figure II-4. Other important quantities of the circular jet are the pumping capacity $Q_{c}$ and momentum $J_{c}$ and are 


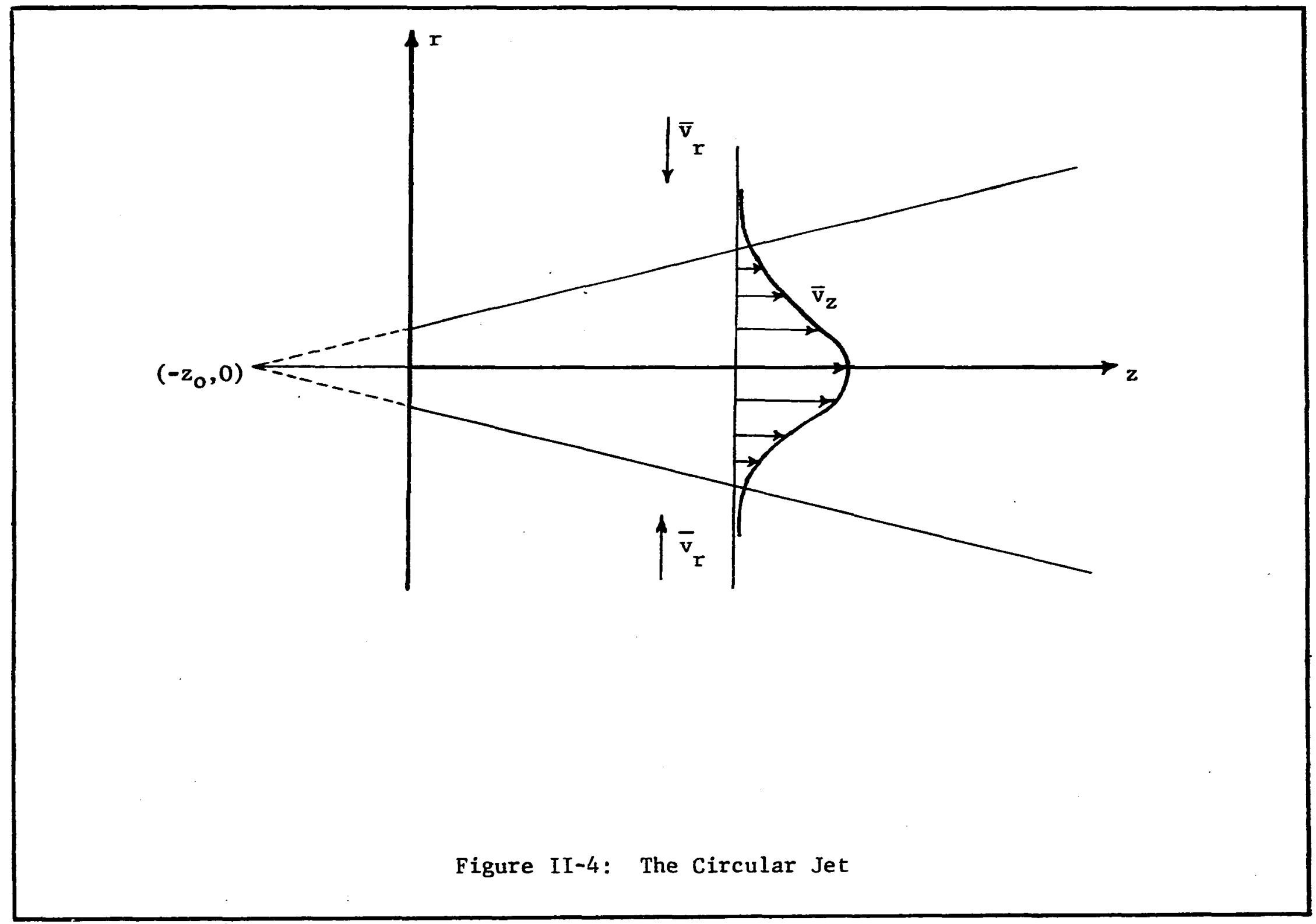




$$
\begin{aligned}
& Q_{c}=8 \pi \epsilon_{o} \mathrm{x} \\
& J_{c}=\frac{16}{3} \pi p \gamma^{2} \epsilon_{o}^{2}
\end{aligned}
$$

Equations II-14 to II-18 describe the circular jet and will be used in Section 2,4 to develop the streamlines in Region $V$.

\subsection{Region VI}

This region occupies two doughtnut shaped spaces, one above and one below the impeller as shown in Figure II-1. In the course of investigating several different models for fitting this region that of a pair of confined ring vortices appeared as a strong possibility. This was based on two observations from the literature (a) that a center of circulation existed as reported by Nagata (19),

(b) that the tangential component of velocity $\vec{v}_{\theta}$ is small and can be neglected.

In order to obtain a more quantitative feel for the problem, velocity measurements were made in three horizontal planes that cuts across regions II, III, IV, V and VI. These measurements are discussed in Chapter IV. It was observed that velocity in general are extremely small in these regions and of the order of $0.5 \mathrm{ft} / \mathrm{sec}$. It required a high impeller speed of $500 \mathrm{rpm}$ in water (impeller diameter 3.0 in., and $\mathrm{N}_{\mathrm{Re}}$ was 54,000 ) for the three dimensional probe used in this work to barely respond. This is because the lowest velocity to which the probe will respond is $0.5 \mathrm{ft} / \mathrm{sec}$. The impeller speed is considered high because beyond $500 \mathrm{rpm}$, excessive entrainment of air occured. The air entrainment not 
only adds another dimension to the problem, but is also not normal practice in the use of the stirred tank. It was observed that $\bar{v}_{\theta}$ was of the same order of magnitude as $\bar{v}_{r}$ and $\bar{v}_{z}$ and thus could not be neglected. The stagnation region described by Nagata (19) and shown in Figure $I-5$ is fictitious, existing only in the $r-z$ plane when velocities components $\bar{v}_{r}$ and $\bar{v}_{z}$ are considered. The flow is three dimensional having a fairly constant value for the overall velocity $\vec{V}$. Thus, in the so called stagnation region the flow is predominantly tangentiäl.

In order to arrive at a suitable model more adequate data on velocity profiles is needed. The fact that outside of the impeller we have a three dimensional flow field adds to the complexity of the problem. As a proposed model could not be adequately tested, and the flow is quiescent at low impeller speeds this region was modeled as a stagnation or deadwater region.

2. Development of Computer Program to Draw the Flow Pattern

In the previous section the stirred tank was divided into several regions and the fluid dynamics of each region was examined separately. In this section the models developed for each region will be tied together and the theoretical basis of the computer program for drawing the streamlines will be presented. The computer program itself is reproduced in Appendix E. It consists of two sections or subprograms called PLOTER and STREAM. The subprogram PLOTER is responsible for ploting the actual streamlines, the outline of the tank, the outline of the impeller and four velocity profiles in Region $I$. It reads in values of the parameters needed 
for drawing the steamlines and prints out pertinent information on the plot. The program is designed to plot one quadrant at a time. Since the axis of the impellex is a line of symmetry only one half of the $x-z$ plane need be plotted as shown in Figure II-1.

The plotting is done on a Calcomp Plotter Model 750 plotting unit made by California Computer Products, Inc. The device works as follows. The desired plot is scaled and all coordinate are converted into inches of plotter pen movements; the movement is from a reference zero on the plot which may or may not conincide with the zero of the coordinates on the plot. The Calcomp Plotter has a number of built in subroutines such as AXIS, PLOTS, PLOT, and LINE which permit calculating coordinates of the variables being plotted in proper form using any suitable computer. In the present case an IBM $360 / 65$ and IBM 7040 were used. The output from the computer is obtained on tape which is then transferred to the Calcomp Plotter to make the desired plot.

The subroutine STREAM calculates the coordinates of one complete streamline as it passes through the various regions. Each streamline encloses a definite amount of fluid. In the sections that follow the equations used for evaluating the streamlines in subroutines STREAM will be detalled. 2.1. Equations for Streamlines in Region I

This region is modeled by a tangential jet. ' Combining Equations B-9 and B-21 gives the value of the stream function $\psi$ as

$$
\psi=A(r / \sigma)^{\frac{1}{2}}\left(r^{2}-a^{2}\right)^{\frac{1}{4}} \tanh (\eta / 2)
$$


For a given streamline, $\psi$ has a constant value expressed as $\mathrm{ft}^{3} /(\mathrm{min})$ (ft) Equation II-19 does not have the proper units as will be seen by considering the equation for pumping capacity in the tangential jet Equation $\mathrm{B}-30$, which is

$$
Q=4 \pi A(r / \sigma)^{\frac{t}{2}}\left(r^{2}-a^{2}\right)^{\frac{1}{4}} \tanh (\eta / 2)
$$

The numerical difference between Equation II-19 and B-30 is the

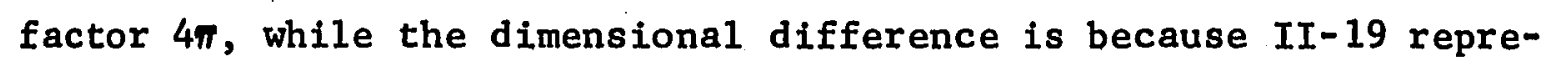
sents a streamline in the $r-z$ plane while B-30 is the fluid flowing out of an area of radius $2 \pi r$ and width $2 \eta$. If axial symmetry is assumed, meaning that the streamlines are considered. identical in all radial planes (and in particular in between baffles) then B-30 can be considered as a stream surface. It then follows that $\psi$ is equal to $Q$ with dimension of $\mathrm{ft}^{3} / \mathrm{min}$ and is given by

$$
\psi=4 \pi A(r / \sigma)^{\frac{1}{2}}\left(r^{2}-a^{2}\right)^{\frac{1}{4}} \tanh (\eta / 2)
$$

In Equation II-20 $\psi$ has the desired units of $\mathrm{ft}^{3} / \mathrm{min}$ and represents the flow in Region I between two stream surfaces that are a distance $\eta$ apart. Now for $\eta=0, \tanh (\eta / 2)=0$ and the zero streamline occurs at $\eta=0$. From Appendix $B, \eta$ is given by

$$
\eta=\sigma \frac{z}{r}
$$

However, it was observed in the previous section that the tangential jet is displaced in the axial direction by an amount $z_{0}$ as shown in Figure II-2, hence $\eta$ should be given by 


$$
\eta=\sigma \frac{z-z_{0}}{r}
$$

The value of $z_{0}$ is in no case larger than $0.01 \mathrm{ft}$ which is a very small quantity. This displacement is so small that it cannot be shown on Figure II-1. Hence for purpose of plotting $z_{0}$ is neglected and $\eta$ will be given by $B-2$.

We are now in a position to evaluate the equation of the stream1ine. Let $Q_{0}$ be the incremental value of $\psi$ between any two stream surfaces. Then in general $\psi$ will be given by

$$
\psi=\mathrm{kQ}_{\mathrm{o}}
$$

where $k$ is some integral multiple of $Q_{0}$ and represents the $k^{\text {th }}$ stream surface. Combining II-20 and II-22

$$
\tan h(\eta / 2)=\frac{k Q_{0}}{4 \pi \mathrm{A}} \cdot \frac{(\sigma r)^{\frac{1}{2}}}{\left(r^{2}-a^{2}\right)^{\frac{1}{4}}} \cdot \frac{1}{r}
$$

From the property of hyperbolic tangents and Equation II-20 it can be shown that

$$
z=\frac{r}{\sigma} \ln \left(\frac{r+k f}{r-k f}\right)
$$

where

$$
f=\frac{Q_{0}(\sigma r)^{\frac{1}{2}}}{4 \pi A\left(r^{2}-a^{2}\right)^{\frac{3}{4}}}
$$

Equation II-24 gives the streamline in terms of $z$ as a function of $r$ and $k$. The parameter $k$ takes on values of $1,2,3, \ldots$, and gives rise to pairs of stream surface on either side of the 
impeller centerline that encloses fluid having a volumetric flow of $\mathrm{kQ}_{\mathrm{o}}$.

Figure IV-5 shows the streamline and velocity profiles for the tangential jet in Region $I$. Three streamlines are drawn, first with $\psi$ having a value less than $Q$ the impeller discharge. This streamline it is seen exists for $r$ less than the impeller diameter. In drawing such a streamline it is started a 1ittle before $r=D / 2$ as shown in Figure II-1. The second streamline has a value of $\psi$ equal to $Q$, the impeller discharge. The streamline is asymptotic to $\psi=D / 2$ and intersects the velocity profile at $b_{\frac{1}{2}}$ as shown in Figure IV-5. The third streamline has a value of $\psi$ greater than Q. This streamline will be asymptotic to some radial distance $\mathbf{r}_{c}$ which is larger than $\mathrm{D} / 2$. To find $r_{c}$, we substitute $k Q_{0}$ (greater than Q) for $\psi$ in $I I-20$ and solve for $r_{c}$. Since Equation II-23 is not explicit in $r, r_{c}$ is evaluated by a Golden section search procedure (42), Once $r_{c}$ is located subsequent points on the streamline is located for finite increments of $r$ larger than $r_{c}$ from Equation II-20 with $\psi$ equals the appropriate value of $k_{0} \cdot A$ description of the Golden section section search procedure and a listing of the subroutine GOLD which performs the search, is given in Appendix E.

\subsection{Region II, III, IV}

In Region II the jet does divide, since the fluid is incompressible and assumed to be in potential flow it is reasonable to consider the width of the jet entering and leaving this region as the same. In other words the jet ceases to grow or expand but turns 


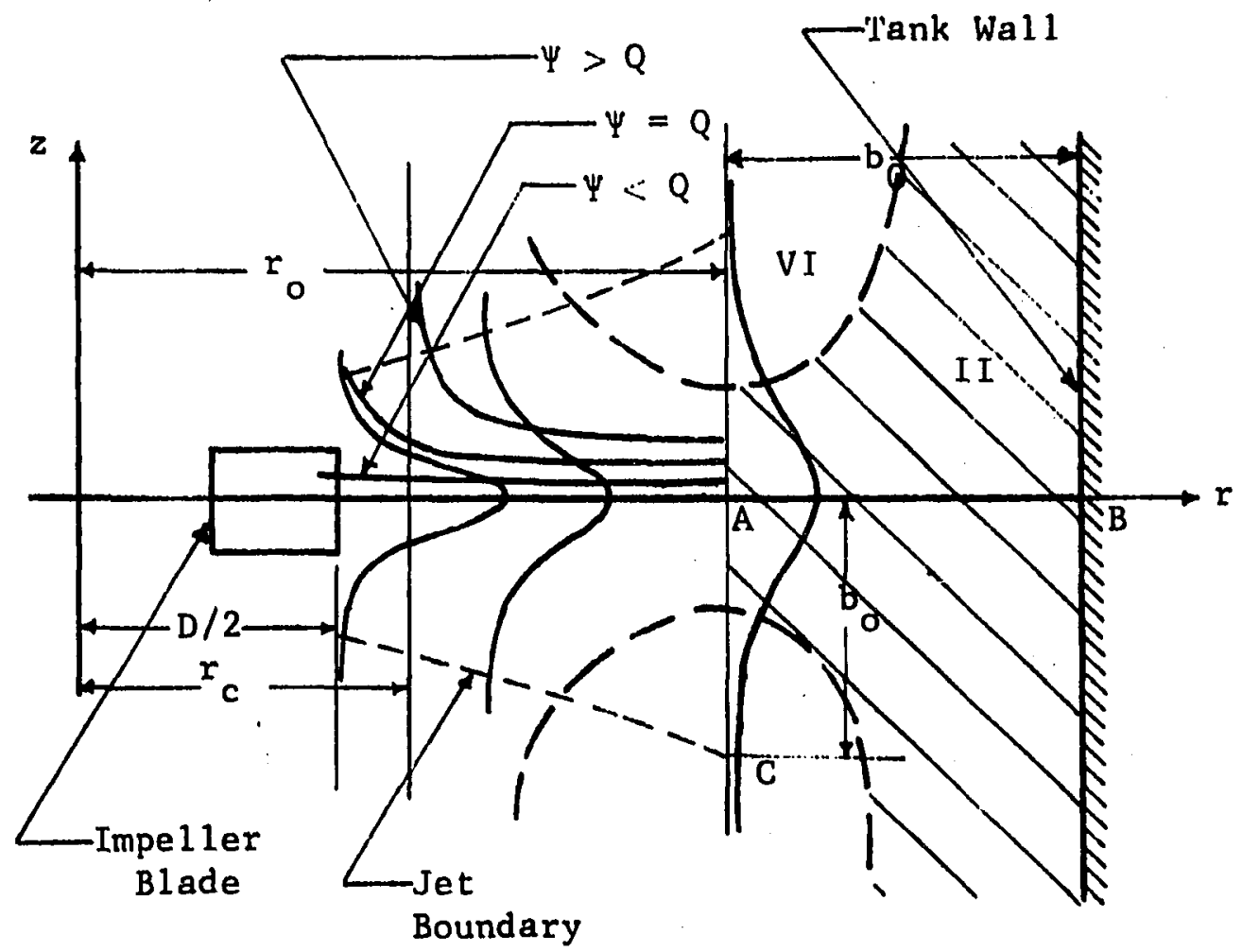

Figure II-5: Tangential Jet Model in Region I 
around the corner intact. If $r_{0}$ is the point at which this occurs, point $A$ in Figure II-5, then from Equation B-34 of Appendix B, the half width of the jet $b_{\frac{1}{2}}$ at this point is

$$
b_{\frac{1}{2}}=\frac{6 x_{0}}{\sigma}
$$

From the above reasoning and Figure II-5 it follows that $A B$ must equal $b_{0}$. From the geometry of Figure II-5, $b_{0}$ equals $\left(T / 2-r_{0}\right)$ hence,

$$
\frac{T}{2}-r_{0}=\frac{6 r_{0}}{\sigma}
$$

where, T in II-27 is the tank diameter. Simplifying

$$
r_{0}=\frac{T \sigma}{12+2 \sigma}
$$

Equation II-28 says that beyond $r_{0}$ the jet no longer expands but is in stagnation flow. The radial component will decrease and the vertical component increases. Thus if the volumetric flow is measured in the jet with increasing $r$, then at $r_{0}$ the volumetric flow should reach a maximum and this has been observed experimentally. The stream function for Regions II, III, and IV is given by Equation D-9 of Appendix $D$ and is

$$
\psi=r^{2} z
$$

Equation D-9 is strictly applicable for a uniform flow approaching a wall, or turning a corner. The jet at $x_{0}$, the start of Region II, has a flat bell shaped profile due to entrainment as seen in Figure II-5. For regions not far removed from the center of the jet the flow can be considered as approximately uniform and hence Equation D-9 
can be used. The regions far removed from the center of the jet is essentially a part of Region VI and is thus modeled separately.

These remarks can be made clear by looking at Figure IV-5. Three velocity profile are shown. In the figure, the scale for $\bar{q}$ on the first profile is arbitrary. The other two profiles are drawn on the same scale as the first and hence correctly pictures the distortion of the flow profile due to entrainment. For the three streamlines shown, the flow enclosed by these streamlines is approximately uniform, beyond $r_{0}$. At the edges of the jet velocities get rapadily smaller and cannot be included in the analysis. To emphasize this point the extent of Region II has been crosshatched in Figure IV 5 .

Also shown in Figure IV-5 is an area where Region I and VI overlap. The velocities due to the jet in this region are sma11 as can be seen in the jet profile that protrudes into this area. The boundary of the jet has been sketched in Figure IV-5 and is approximately linear as predicted by Equation II-12. 2.3. Mechanics of Plotting Streamlines in Regions I, II, III and IV As mentioned earlier, to produce a trace of a line with the Calcomp Plotter the $x$ and $y$ coordinates are required at small increments $\Delta x$ and $\Delta y$ along the 1ine. This is accomplished by subroutine-STREAM which generates arrays PX and PY in which are stored the coordinates $r$ and $z$ or a complete streamline at sma11 intervals $\Delta r$ and $\Delta z$ appart. The subroutine STREAM is called by the main program PLOTER each time a streamline is desired. The command to plot is executed by the program PLOTER. 
From Section 2.1 it will be recalled that a streamline starts in Region $I$. Hence the number of streamlines plotted depends on the volumetric flow in the jet. Since extrainment stops at $r_{0}$, the extent of the jet, the maximum flow will occur at this point. The number of streamlines is thus, maximum flow divided by $Q_{0}$, the incremental flow rate on the streamline. It is observed in Figure IV-1 that there are five streamlines. It is also seen that the last streamline begins very near to $r_{0}$, hence the entrainment in the jet beyond this point is less than $Q_{0}$.

This section will detail the equations used and the steps involved in calculating a streamline as it proceeds from region to region and is the mathematical basis for the subroutine STREAM. FøRTRAN symbols will be used to denote all variables in this section as the discussion is closely related to the flow diagram and listing of program PLOTER given in Appendix E. The FøRTRAN symbols will be recognized as containing more than one upper case characters. To avoid confusion mathematical operations with these variables will also be written in FøRTRAN. For convenience the FØRTRAN symbols used in the text is listed separately. In the section devoted to nomenclature.

Region I

As pointed in Section 2.1 and the illustration shown in Figure IV-5 two cases exists. The first case is when $k_{0}$ is less than $Q$ the impeller discharge. In this case the streamline is started at the Impeller periphery and the coordinates of the streamline is calculated from Equation II-24 by incrementing $x$ in increments of $0.005 \mathrm{ft}$. The calculated coordinates are stored in the 
arrays of $P X$ and $P Y$. When $k Q_{0}$ is larger than $Q$, then $r_{c}$ is evaluated as outlined in Section 2.1 and the streamline started t0.01 ft from $r$. If the streamline is started at $r_{c}$ the $z$ coordinate obtained will be excessively large as the streamlines are asymptotic to $r_{c}$. When $r$ equals $r_{0}$ (calculated from Equation II-28), Region II is reached and the streamline is now given by Equation D-9. $\underline{\text { Region II }}$

In subroutine STREAM it will be noticed that in each region the calculations are based on a local $\mathrm{X}, \mathrm{Y}$ axis which are then transformed into $r, z$ corrdinates of the overall plot. Thus in Region II the origin of the local $X, Y$ axis is point $B$ in Figure II-1. Equation $D-9$ is then used in the local frame of axis giving rise to the stagnation flow pattern shown in Figure II-3 (a). In Equation D-9 a value of $\psi$ is needed to evaluate the stream function. Since local coordinates are used a value for $\psi$ in this frame of axis has to be calculated. This is possible since the entry point to Region II is a point on the streamline. Accordingly, if the last point in Region $I$ is $x_{n}, y_{n}$, this is an entry point to Region. II and thus a point on Equation D-9, hence

$$
\psi=\mathrm{x}_{\mathrm{n}}^{2} \mathrm{y}_{\mathrm{n}}
$$

The equation of the streamline with the point B of Figure II-1 as the origin is then obtained from D-9 and II-29 as

$$
x=\left(\frac{x_{n}^{2} y_{n}}{y}\right)^{\frac{1}{2}}
$$


The value of the streamlines in terms of the original $r, z$ coordinates are from the geometry of Region II,

$$
\begin{aligned}
& z=y \\
& r=T / 2-\left(\frac{x_{n}^{2} y_{n}}{y}\right)^{\frac{1}{2}}
\end{aligned}
$$

The unanswered question is how far does Region II extend. This was determined by making a parameter study. Scale Used for Plotting

In the process of making these plots it was found convenient to scale the entire plot in terms of $T$ the tank diameter. A scale of unity was chosen for $\mathrm{T}$ equal $1.0 \mathrm{ft}$. Thus a $11.5 \mathrm{in}$. diameter tank will on this scale be represented as 0.96. All other dimensions of the tank are reported as fractions of $\mathrm{T}$ i and are shown in Figure II-6, and defined in Table II-1. On the axis of the computer solution is printed a scale as seen in Figure II-1. The units on the scale is $T$ equals $1.0 \mathrm{ft}$. The reason for choosing a scale of $\mathrm{T}$ equals $1.0 \mathrm{ft}$. is to permit generalization of solution to any size tank by use of a suitable scale factor. Thus for example the scale on the $z$ axis shows the bottom most corner as -0.4 in Figure II- 1 . This means that the impeller is immersed to $60 \%$ of the depth of fluid $H$ in the tank and it is also evident that $H$ equals $T$. Figure II- 6 shows the boundary of the regions into which the tank is divided. AA is the boundary between Regions IV and V and has two possible locations 1 and 2 . $B B$ is the boundary between Regions III and IV and CC is the boundary between Regions II and III. 


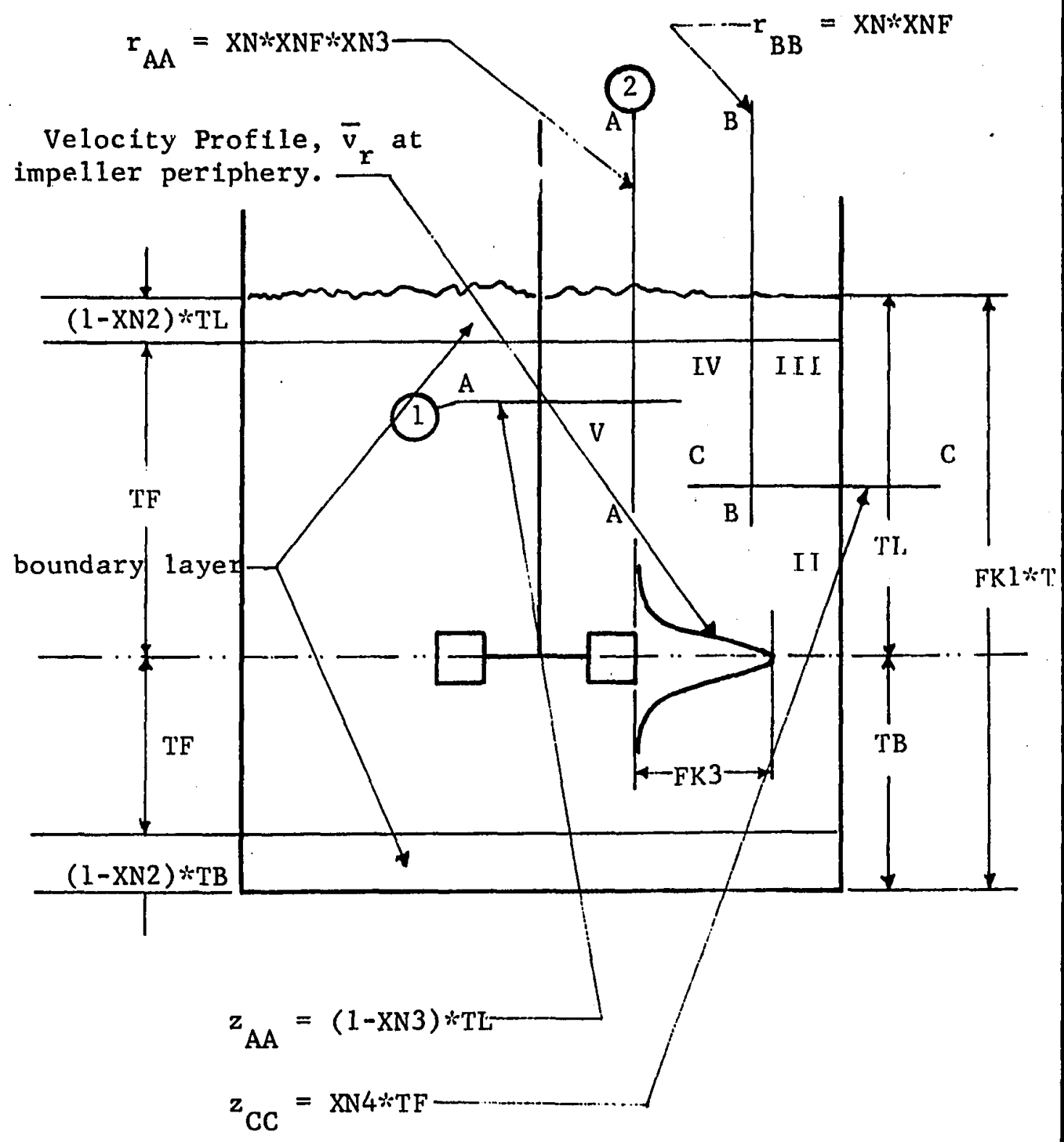

Figure II-6: Dimensionless Ratios Used for Obtaining the Theoretical Streamlines by Program PLOTER. Note all Symbols and Mathematical Operations are in FORTRAN. 
TABLE II-1

LIST AND DEFINITION OF SYMBOLS USED IN FIGURE II-6

\begin{tabular}{|c|c|c|}
\hline Symbol & Defining Equation & $\begin{array}{c}\text { Effect on the Theoretical } \\
\text { Flow Patterns }\end{array}$ \\
\hline FK1 & $\mathrm{FK} 1=\mathrm{H} / \mathrm{T}$ & Controls depth of fluid \\
\hline FK2 & $\mathrm{FK} 2=\mathrm{h} / \mathrm{T}$ & $\begin{array}{l}\text { Controls height of impeller } \\
\text { above tank bottom }\end{array}$ \\
\hline FK3 &. & $\begin{array}{l}\text { Scales } \bar{v}_{\mathrm{r}} \text {, for velocity } \\
\text { profile } \mathrm{p} \text { lot in Region } \mathrm{I}\end{array}$ \\
\hline TB & $\mathrm{TB}=\mathrm{FK} 2 * \mathrm{~T}$ & Depth of fluid below impëller \\
\hline TL & $\mathrm{TL}=(\mathrm{FK} 1-\mathrm{FK} 2) * \mathrm{~T}$ & Depth of fluid above impeller \\
\hline TF & $\begin{array}{l}\text { (1) } \mathrm{TF}=\mathrm{XN} 2 * \mathrm{~TB} \\
\text { (2) } \mathrm{TF}=\mathrm{XN} 2 * \mathrm{TL}\end{array}$ & $\begin{array}{l}\text { Effective depth of fluid } \\
\text { above or below the impeller } \\
\text { considered in potential } \\
\text { flow solution }\end{array}$ \\
\hline $\mathrm{XN}$ & $\mathrm{XN}=\mathrm{r}_{0}$ & Extent of tangential jet \\
\hline $\mathrm{XNF}$ & $r_{B B}=X N * X N F$ & $\begin{array}{l}\text { Controls } r_{B B}, r \text { coordinate } \\
\text { of boundary } B B \text { between regions } \\
\text { III and IV }\end{array}$ \\
\hline $\mathrm{XN} 2$ & $\begin{array}{l}\text { (1) } t=(1-\mathrm{XN} 2) * \mathrm{TL} \\
(2) \mathrm{t}=(1-\mathrm{XN} 2) * \mathrm{~TB}\end{array}$ & $\begin{array}{l}\text { Controls } t \text {, thickness of } \\
\text { boundary layer }\end{array}$ \\
\hline \multirow[t]{2}{*}{$\mathrm{XN} 3$} & (1) ${ }^{z_{A A}}=(1-\mathrm{XN} 3) * \mathrm{TL}$ & $\begin{array}{l}\text { Controls } z_{A A}, z \text { coordinate } \\
\text { of boundary AA between Regions } \\
\text { IV and } V\end{array}$ \\
\hline & (2) $r_{\mathrm{AA}}=\mathrm{XN} * \mathrm{XNF} * \mathrm{XN} 3$ & $\begin{array}{l}\text { Controls }{ }_{A A}, r \text { coordinate } \\
\text { of boundary AA between Regions } \\
\text { IV and } V\end{array}$ \\
\hline $\mathrm{XN4}$ & ${ }_{\mathrm{CC}}=\mathrm{XN} 4 * \mathrm{TF}$ & $\begin{array}{l}\text { Controls }{ }^{z} \mathrm{CC}^{\prime} \mathrm{z} \text { coordinate } \\
\text { of boundary between Regions } \\
I \text { and II }\end{array}$ \\
\hline
\end{tabular}


The dimensions of the tank, the coordinates of the boundary between the regions and their defining equations are given in Table II- 1 . The symbols and mathematical operations in Table II-1 and Figure II-6 are in FØRTRAN as noted above.

Provisions for a boundary layer exist and is the thickness of fluid removed from the potential flow solution. Since the subroutine STREAM calculates one complete streamline it makes no difference whether this streamline is above or below the impeller. Hence the boundary layer is shown at the top and bottom of the tank in Figure II-6. The boundary layer at the sides of the tank is small and hence neglected as seen from the work of Askew (2) which was discussed earlier. Physically a boundary layer is conceivable only at the bottom of the tank and this boundary layer is implemented in Figure II-1. The thickness of the boundary layer is controlled by XN2 which is a fraction of TL or TB the depth of fluid above and below the impeller. The variable $\mathrm{TF}$ is the depth of fluid after removing the boundary layer if any, and thus constitutes the effective depth of fluid in the potential flow solution. FK3 is a scale factor on the velocity profile $\bar{v}_{r}$, which is drawn at four equally spaced points between the impeller periphery and $r_{0}$.

The boundaries of the various regions are controlled by the parameters XN4, SNF and XN3. Thus the extent of Region II is fixed by $\mathrm{XN} 4$ which is in terms of a fraction of $\mathrm{TF}$ the effective depth of the potential flow region above of below the impeller centerline. Region III and IV

The streamline in Regions III and IV are evaluated in a similar 
manner as Region II. The local origin for Region III is point E while that of Region IV is point $\mathrm{K}$ of Figure II-1. As for Region II, $x_{n}, y_{n}$ is the coordinates of the entry point to these regions and the streamline in the local frame of reference is given by II-30. The extent of Region IV is given by $\mathrm{T} / 2-\mathrm{XNF} * \mathrm{XN}$, where $\mathrm{XN}$ is the program symbol for $\dot{r}_{0}$. The boundary between Region III and IV can thus be varied by varying the parameter XNF.

Region IV can be terminated in two ways: either by a line parallel to the axis or a line parallel to the $r$ axis. Both these limits are shown in Figure $I V-6$ and marked 1 and 2 respectively. The extent of Region IV is in the first case given by $\mathrm{XN} * \mathrm{XNF} * \mathrm{XN} 3$ and in the second case by $T L * X N 3$. Region $I V$ can thus be varied by varying the parameter $\mathrm{XN} 3$. This point will be discussed in more detail in Section 3.1 .

\subsection{Region V}

In Appendix B, the equation for the stream function $\psi$ is given for cylinderical coordinates. As $z$ is the direction of flow, $\psi$ is evaluated from Equation $B-7$, and not $B-6$ as in the case of the tangential jet. Integrating B-7 gives $\psi$ as

$$
\psi=2 \pi \int_{0}^{r} \bar{v}_{r} r d r
$$

where the additional $2 \pi$ is necessary since $\psi$ is a stream surface. The sign in Equation $B-7$ is taken as positive since $\bar{v}_{z}$ is positive and $\bar{v}_{r}$ is negative for the circular jet as can be seen in Figure II-3. Substituting for $\bar{v}_{z}$ and $r$ from Equation II-14 and II-16 and simplifying

$$
\psi=2 \pi \epsilon_{0}\left(z+z_{0}\right) \int_{0}^{\xi} \frac{2 \xi d \xi}{\left(1+\frac{1}{4} \xi^{2}\right)^{2}}
$$


Integrating Equation II-34 results in

$$
\psi=\frac{2 \pi \epsilon_{0}\left(z+z_{0}\right) \xi^{2}}{1+\frac{1}{4} \xi^{2}}
$$

Equation II-35 is the desired equation of the streamline for a circulate jet. Ordinarily there are a number of parameters to be evaluated in II-35. However, these have been reduced to two parameters $Y$ the jet width and: $z_{o}$, the displacement of jet origin.

As before, let $x_{\mathbb{I}}, z_{n}$ be the end points of the previous region and the starting points for Region $V$. Then from Equation II-16.

$$
\xi_{0}=\gamma \frac{r_{n}}{z_{n}+z_{0}}
$$

$\Psi$ can now be calculated at the point $r_{n}, z_{n}$ from II-36 and II-35. This calculated value of $\psi$ is called $\mathrm{CK}$ in the program PLOTER, thus

$$
\mathrm{CK}=\frac{2 \pi \xi\left(z_{\mathrm{n}}+z_{\mathrm{o}}\right) \xi_{\mathrm{o}}^{2}}{1+\frac{1}{4} \xi_{0}^{2}}
$$

Since $\psi$ has a value $\mathrm{CK}$, Equation II-35 is solved for $\xi$. On substituting for $\xi$ from Equation II-16 results in

$$
r=\frac{z+z_{0}}{r}\left(\frac{1}{\frac{2 \pi \epsilon_{0}\left(z+z_{0}\right)}{C K}-\frac{1}{4}}\right)^{\frac{1}{2}}
$$

Substituting for $\mathrm{CK}$ from II-37 gives 


$$
r=\frac{z+z_{0}}{\gamma}\left(\frac{1}{\left(z+z_{0}\right)\left(\frac{1+\frac{2 \pi}{4} \xi_{0}^{2}}{\left(z_{n}+z_{0}\right) \xi_{0}^{2}}-\frac{1}{4}\right)}\right)^{\frac{1}{z}}
$$

Equation II-39 gives the streamlines for the circular jet in terms of $r$ as a function of $z$. There are two parameters to be determined: $z_{0}$ the displacement of the jet origin from the base of the potential flow region (see Figure II-4) and $\gamma$ the jet width parameter. The best value of $z_{0}$ is determined by making a parameter study, and this will be discussed in Section 3 .

The jet width parameter, $\gamma$, controls the slope of the stream1ines. In Figure II-1, the ends of the streamlines do not meet and are joined by dotted 1ines. $Y$ is chosen such that the lengths of the dotted lines is minimized. This is done by calculating a $\gamma$ that makes the lengths of the dotted lines zero for each individual streamline. Obviously a circular jet cannot have a different $\gamma$ for each streamline, hence an average value of $\gamma$, for the individual streamline is selected for the final plot. This makes the ends of the streamlines in Region $V$, under shoot the start of the streamlines in Region I in some cases, and overshoot in others. This can be seen in Figure II-1. A sample calculation of the above procedure for evaluating $\gamma$ is given in Appendix E. 


\section{Theoretical Flow Patterns}

In the previous sections the theoretical basis of the computer program to draw the flow patterns were discussed. This section is devoted to a discussion of what the program can and cannot do. The program requires a number of parameters to be specified and this section will indicate how some of these parameters can be determined.

The main parameters are that of the tangential jet model: $\sigma$ the jet width parameter, A the volumetric flow parameter and, a, the radius of source parameters. These will be obtained from experimental correlations which will be treated in Chapter IV. The values of $\sigma, A$ and a used in the flow patterns of Figures II-5 through II-9 are from Cooper's ( 8 ) data (Profile 5 Table G-16 of Appendix G). The pertinent tank parameters are given in Table II-2 and are common to all the flow patterns. The value of $Q$, the impeller discharge, and $\epsilon$ the eddy viscosity are calculated from Equations B-30 and B-15. Table II-2 gives the angle $\theta_{y}$ at which the velocity vector $\overrightarrow{\mathrm{q}}$ leaves the impeller periphery. This angle is calculated from Equation A-14. Finally the value of the incremental flow rate on the streamline $Q_{0}$ is also given in Table II-2.

The parameters to be determined are the relative sizes of the six regions into which the stirred tank has been divided. There are five parameters which determine the relative extent of these regions. A large number of profiles were studied, with varying values of these parameters. From these studies, twelve different combinations of parameters were selected. These combinations illustrate the effect of varying the parameters on the solution and 
TABLE II-2

OPERATING CONDITIONS AND PERTINENT DATA ON THE STIRRED TANK WHOSE THEORETICAL PROFILES ARE DRAWN IN FIGURES II-5 TO II-9

\begin{tabular}{|c|c|c|}
\hline Tank Diameter $\mathrm{T} *$ & $=1.0$ & ft. \\
\hline Depth of Fluid $\mathrm{H}^{*}$ & $=1.0$ & ft. \\
\hline Impeller Diameter $\mathrm{D} *$ & $=3.0$ & in. \\
\hline Height of Impeller of Tank Bottom $h$ & $=0.4$ & ft. \\
\hline Impeller Speed & $=600$ & RPM \\
\hline $\mathrm{N}_{\mathrm{Re}}$ & $=64,780$ & \\
\hline Eddy Viscosity at Impeller Periphery & $=0.79$ & $\mathrm{ft}^{2} / \mathrm{min}$ \\
\hline Impellex Discharge $Q$ & $=7.42$ & $\mathrm{ft} / \mathrm{sec}$. \\
\hline Increment on Streamline & $=5.0$ & $\mathrm{ft} \mathrm{t}^{3} / \mathrm{min}$ \\
\hline Jet Width Parameter $\sigma$ & $=12.62$ & \\
\hline Volumetric Flow Parameter A & 22.5 & $\mathrm{ft}^{3} / \mathrm{min}$ \\
\hline Radius of Source, a & $=0.1038$ & ft. \\
\hline Yaw Angle at Impeller Periphery $\theta_{y}$ & $=56.1^{\circ}$ & \\
\hline Extent of Tangential Jet $r_{0}$ & $=0.339$ & ft. \\
\hline
\end{tabular}


the basis for selecting the best combination of these parameters. The parameters are varied one at a time and their effect on the flow patterns is discussed in the following sections.

The flow patterns in Figures II-7 through II-11 are drawn with the impeller immersed to $60 \%$ of the fluid depth. This is to illustrate the distortion that results by reducing the potential flow area below the impeller centerline. Also shown in these flow patterns is a boundary layer at the bottom of the tank. The thickness of this layer is $10 \%$ of the depth of fluid below the impeller center1ine. The inclusion of the boundary layer in these flow patterns reduces the depth of the potential flow region adding to distortion of the flow patterns located below the impeller.

The scale shown in Figures II-7 through II-11 is based on $T$ equal $1.0 \mathrm{ft}$. All other dimensions are in terms of fractions of $\mathrm{T}$ as shown in Figure II- 8 .

\subsection{Extent of Region IV}

The factor XN3 is used to control the effect of Region IV in two different ways. It can be limited in a horizontal plane as shown by , the lines AA in Figure II-7, or in a cylinderical plane as shown by ..the lines AA in Figure II-8. The program PLOTER is operated in two different modes depending on whether Figures II-7 or II-8 is desired.

In the first mode of operation shown in Figure II-7, the boundary between Regions IV and $V$ is fixed by the $z$ coordinate of the line AA is given by TL*XN3. TL is the depth of fluid above the impeller 

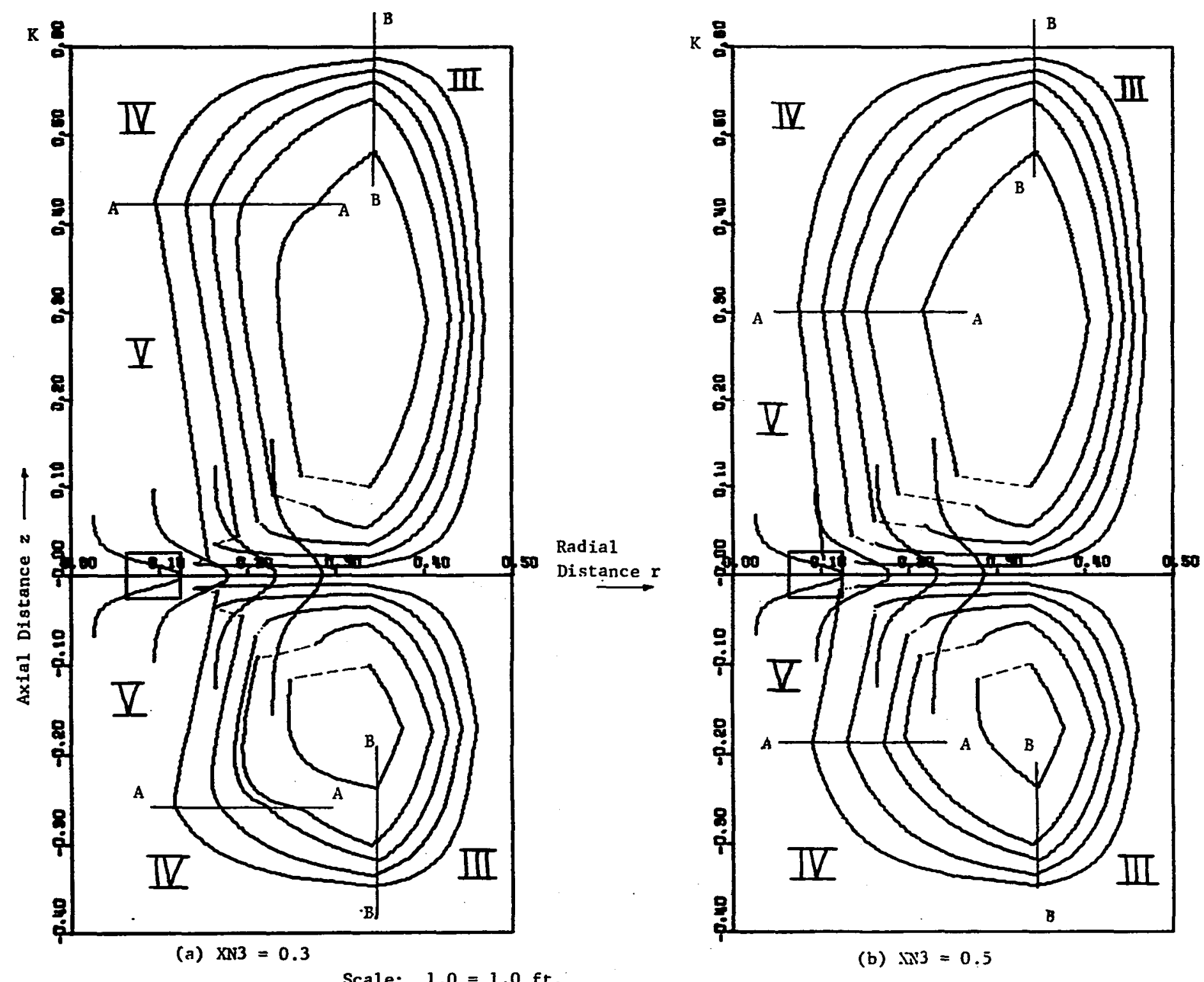

Scale: $1.0=1.0 \mathrm{ft}$

(b) $\sin 3=0.5$

Figure II-7: Effect of Varying the Boundary Between Regions $I V$ and $V$ in a Horizontal Plane on the Theoretical Flow Patterns. 

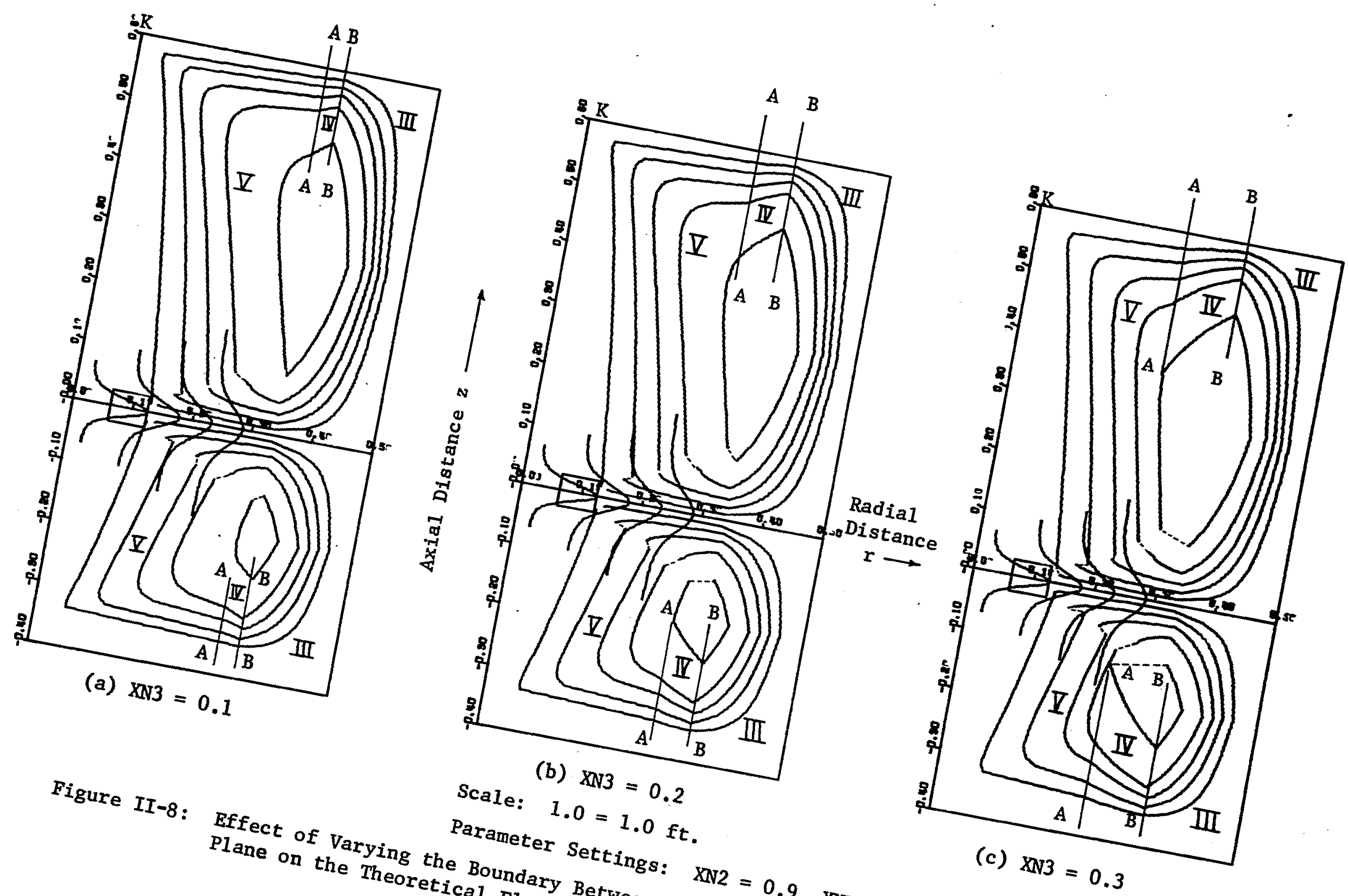

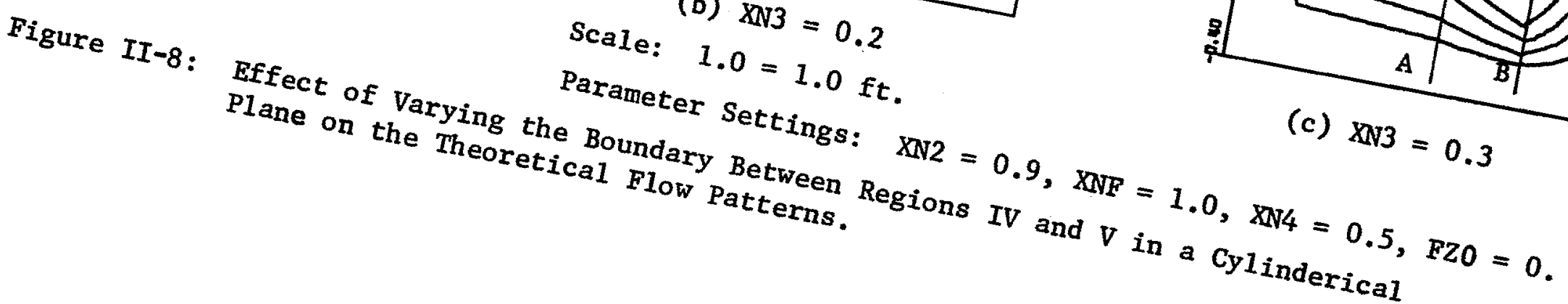


centerline. Similarly below the impeller centerline the $\mathrm{z}$ coordinate of $\mathrm{AA}$ is given by $\mathrm{TB} * \mathrm{XN} 3$. Two values of $\mathrm{XN} 3 ; 0.3,0.5$ are used, resulting in the flow patterns of Figure II-7 (a) and II-Y (b). These plots show that the ends of the streamlines do not meet, also for large values of XN3 the boundary between Regions IV and V show a discontinuity. It is noticed that the reduction of the potential flow region causes distortion of the streamline. The maximum distortion occurs for the fifth streamline below the impelier.

In Figure II-8 is shown the other mode of operation in which Region IV is restricted by a line parallel to the $z$ axis. The extent of Region IV in this case is controlled by the coordinate of the 1 ine $A A$, which is given by $X N * X N F * X N 3$. By varying $X N 3$ the position of the line AA can be changed. The coordinates of AA also depends on XNF which controls the coordinates of the line BB that marks the boundary between Regions III and IV. The coordinates of $\mathrm{BB}$ is given by $\mathrm{XN} * \mathrm{XNF}$.

In Figure II-8 the coordinates of the line BB is kept constant and three values of $\mathrm{xN} 3$ are examined. These are $0.1,0.2$ and 0.3 . Increasing XN3 pushes the line AA to the left. In Figure II-8 (c) it is seen that if $\mathrm{XN} 3$ is increased beyond 0.3 then the last streamline will be deleted from the solution in Region $V$. This occurs in Figure II-7 (b) for the fifth streamline, below the impeller. Thus 0.3 is an upper limit for XN3.

Since point $K$ in Figure II-8 is the origin for the local frame of reference in Region IV it would appear that the truncated Region IV of Figure II-8 is unnecessary. However on comparing Figures II-7 
and II-8 it is seen that by locating AA as in Figure II-8 causes the ends of the streamline to more nearly approach each other. Hence truncating Region IV as in Figure II-8 is better than extending Region IV as in Figure II-7. In future plots $A A$ is located as in Figure II-8. 3.2. Boundary Between Regions III and IV

The boundary between Regions III and IV is marked by the line $\mathrm{BB}$ in Figure II-8. The $r$ coordinte of $\mathrm{BB}$ is given by $\mathrm{XN} * \mathrm{XNF}$, where $\mathrm{XN}$ is the computer symbol for $\mathrm{r}_{0^{\circ}}$. In Figure II-9 (a), XNF = 0.9 while in Figure II-9 (b), XNF $=1.1$. It is seen that increasing the value of $\mathrm{XNF}$ pushes $\mathrm{BB}$ to the right. This causes a discontinuous transistion of the streamlines from Region III to Region IV, particularly in the inner streamlines. Large values of XNF causes the streamlines in Region IV to move appart as seen in Figure II-9 (b).

In Figure II-9 (c) is shown a proper combination of XN3 and $\mathrm{XNF}$. It is seen that the ends of the streamlines approach each other and the streamlines are fairly smooth at the boundaries of the various regions. The distortion of the streamlines below the impeller is also smaller than in Figures II-9 (a) or II-9 (b). 3.3. Locating the Origin of the Circular Jet

The origin of the circular jet is varied by the parameter $z_{0}$ of Equation II-35. The computer symbol for $z_{0}$ is FZO. In Figures II-7 to II-10, FZO equals zero. In these Figures the origin of the circular jet is at the point $k$, the intersection of the impeller axis with, the surface of the fluid above the impeller and the bottom surface of the tank below the impeller. In Figure II-11(a) and II-1I(b) is shown the effect of varying FZO. Two values of FZO 


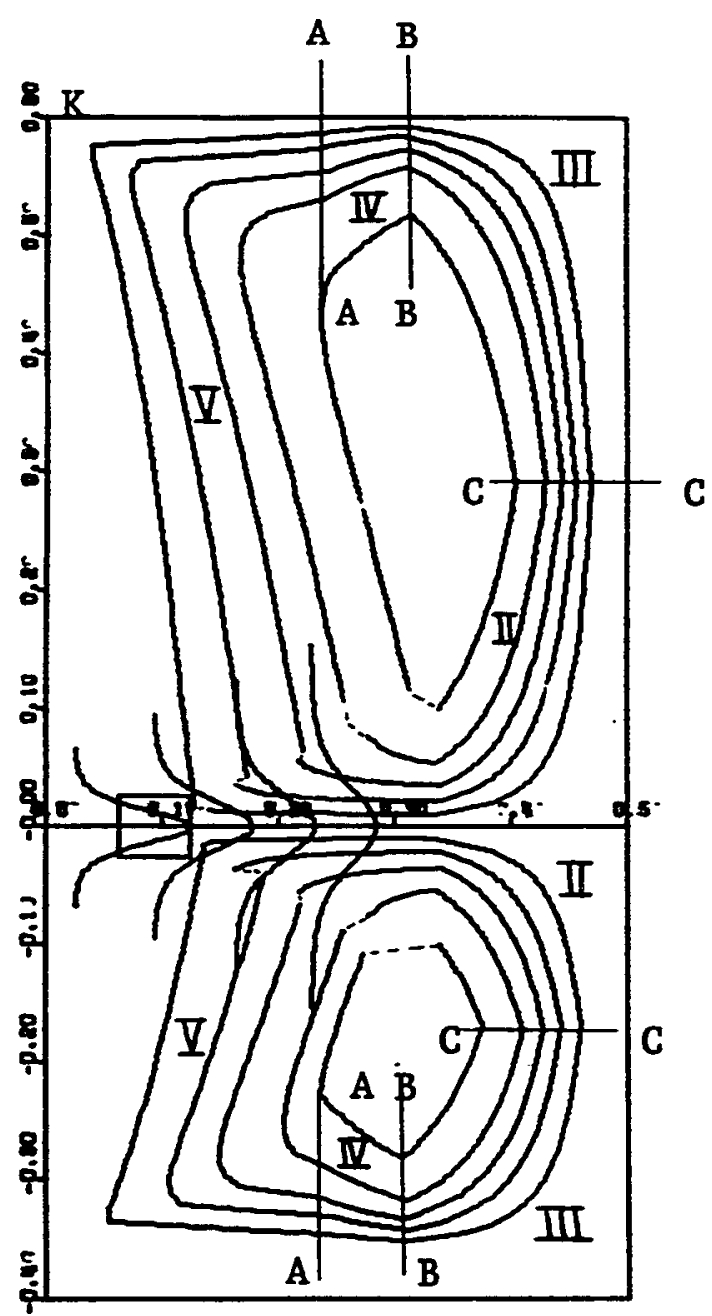

(a) $\mathrm{XNF}=0.9$

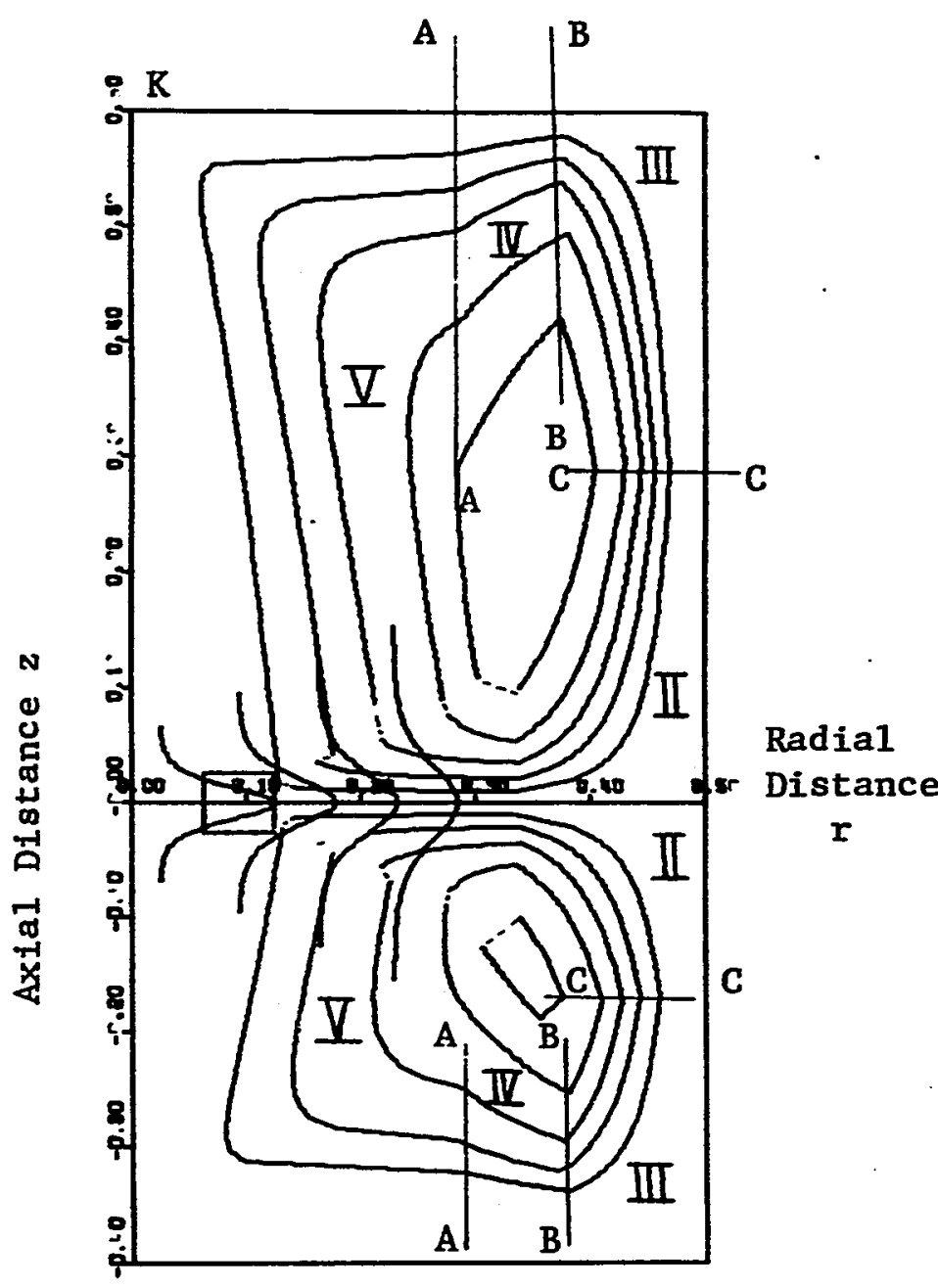

(b) $\mathrm{XNF}=1.1$

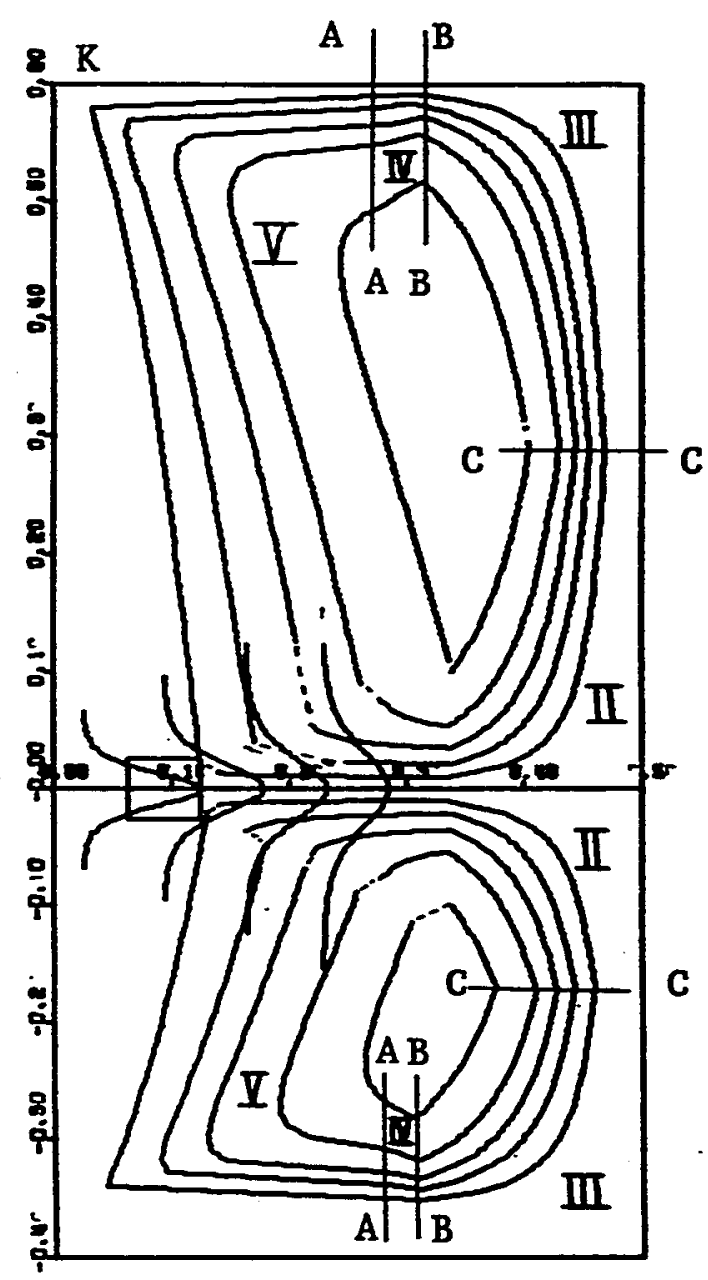

(c) $\mathrm{XN} 3=0.1, \mathrm{XNF}=0.9$

Scale: $1.0=1 \mathrm{ft}$.

Parameter Settings: $\mathrm{XN2}=0.9, \mathrm{XN} 3=0.25, \mathrm{XN} 4=0.5, \mathrm{FZO}=0$ Figure II-9: Effect of Varying the Boundary Between Regions III and IV on the Theoretical Flow Patterns. 


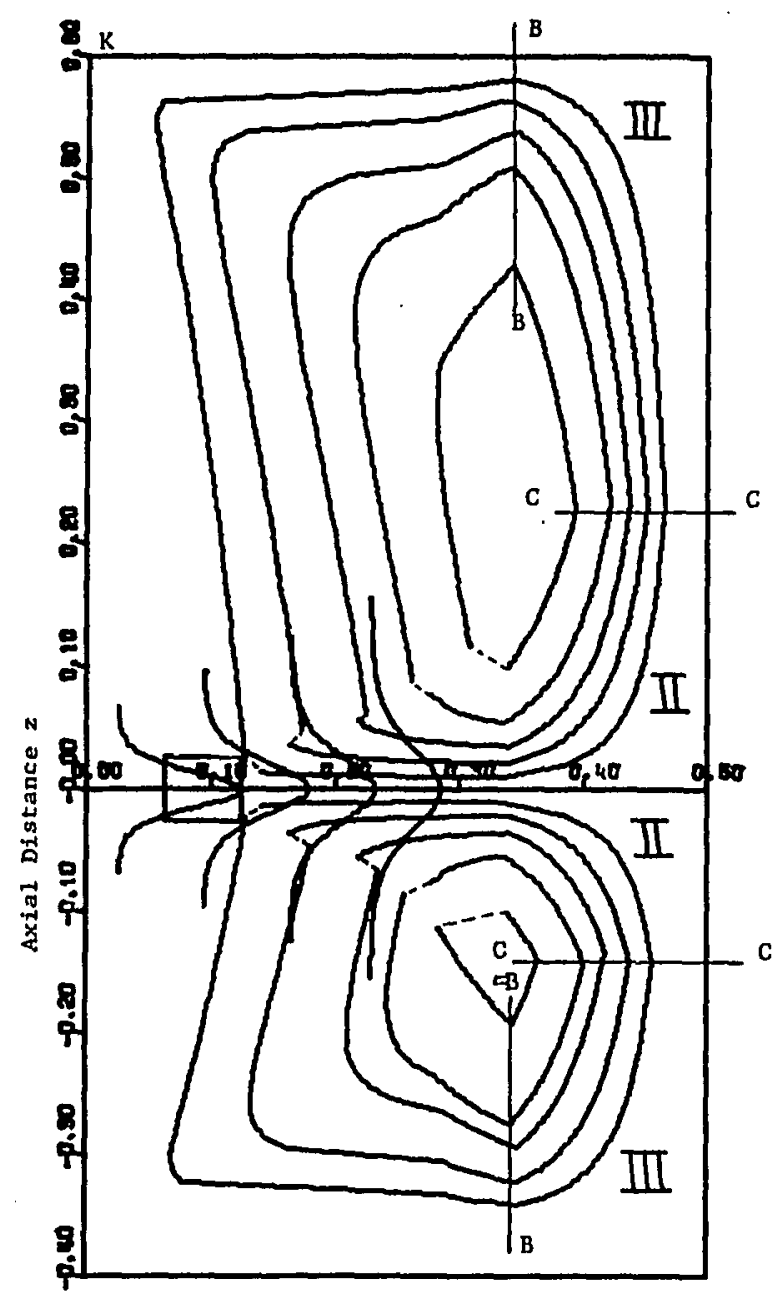

(a) $\mathrm{XN} 4=0.4$

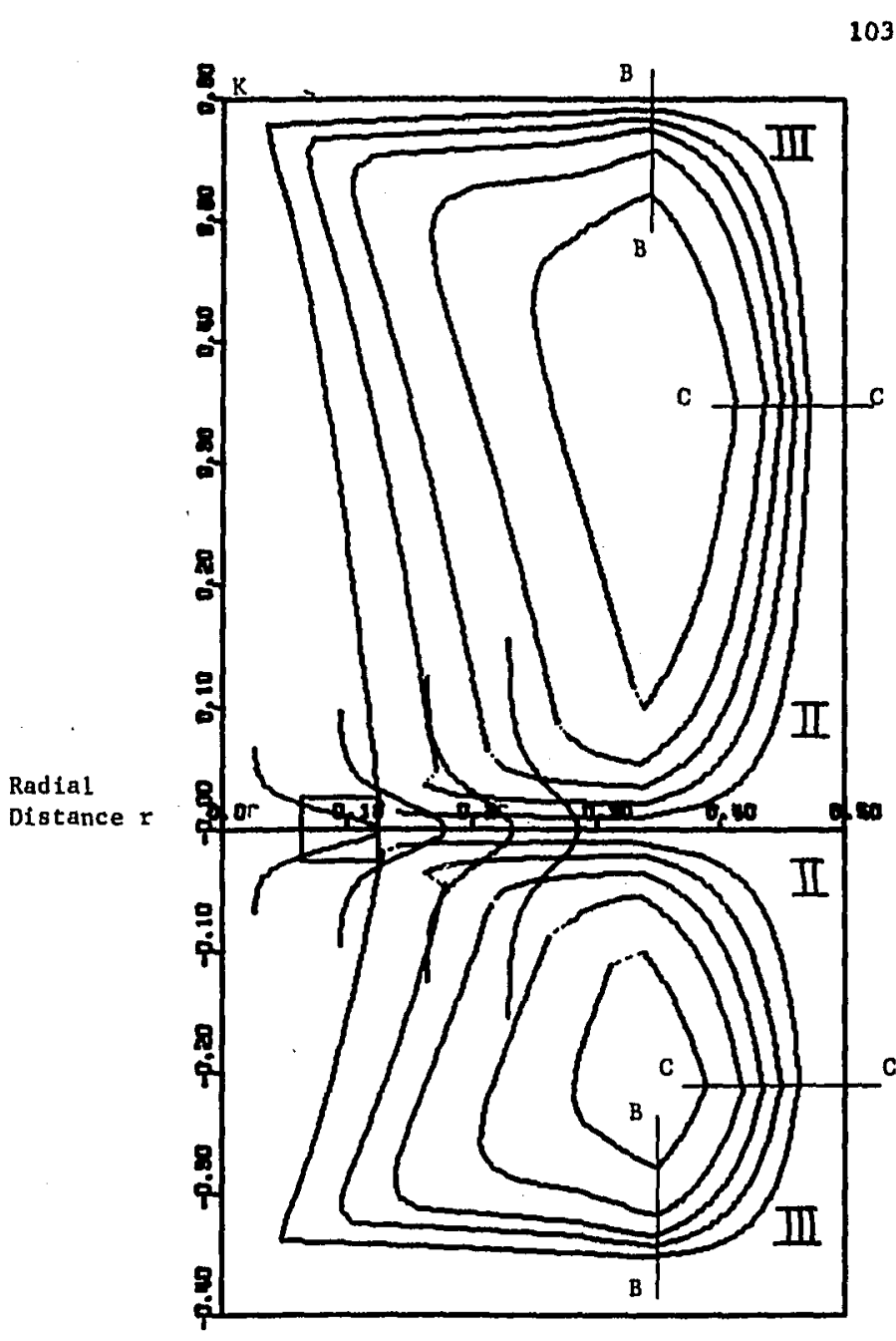

(b) $\mathrm{xN} 4=0.6$

Scale: $1.0=1.0 \mathrm{ft}$.

Parameter Settings: $\quad \mathrm{XN} 2=0.9, \quad \mathrm{XN} 3=0.2, \mathrm{XNF}=1.0, \mathrm{~F} 70=0$

Figure II-10. Effect of Varying the Boundary Between Regions II and III on the Theoretical flow patterns. 
are chosen: 0.1 and 0.2 . These values displace the point $\mathrm{K}$ away from the impeller centerline by the amount FZO. Negative values of FZO would bring the point $\mathrm{K}$ towards the impeller centerline. A large negative FZO will cause the knee or bend in the stream lines of the circular jet to be pushed towards the impeller resulting in an abrupt discontinuity. This effect is visible in the fourth streamline below the impeller in Figure $\operatorname{II}-7$ (a). The exact location of $K$ is shown for the potential flow solution above the impeller in Figure II-11.

Figure II-11 shows that increasing FZO tends to pull the flow patterns closer together, and away from the impeller axis. As it is more desirable to have the flow pattern fill the entire field, the best value of FZO is zero.

\subsection{Desirable Values of Boundary Parameters}

From examining more than thirty theoretical flow patterns, illustrative examples of which are given in Figures II-7 through II-10, it is concluded that Figure $\operatorname{II}-8$ (c) is the best. In this figure the streamlines are smooth and they fill the entire flow field. Furthermore their ends almost meet, and material balances are satisfied. From this figure it appears that the best values for the boundary determining parameters are:
(1) $\mathrm{XN} 4=0.5$
(2) $\mathrm{XNF}=0.9$
(3) $\mathrm{XN3}=0.1$
(4) $\mathrm{FZO}=0$

The above values are recommended values and they are relatively insensitive to impeller speed and diameter. The program is flexible 


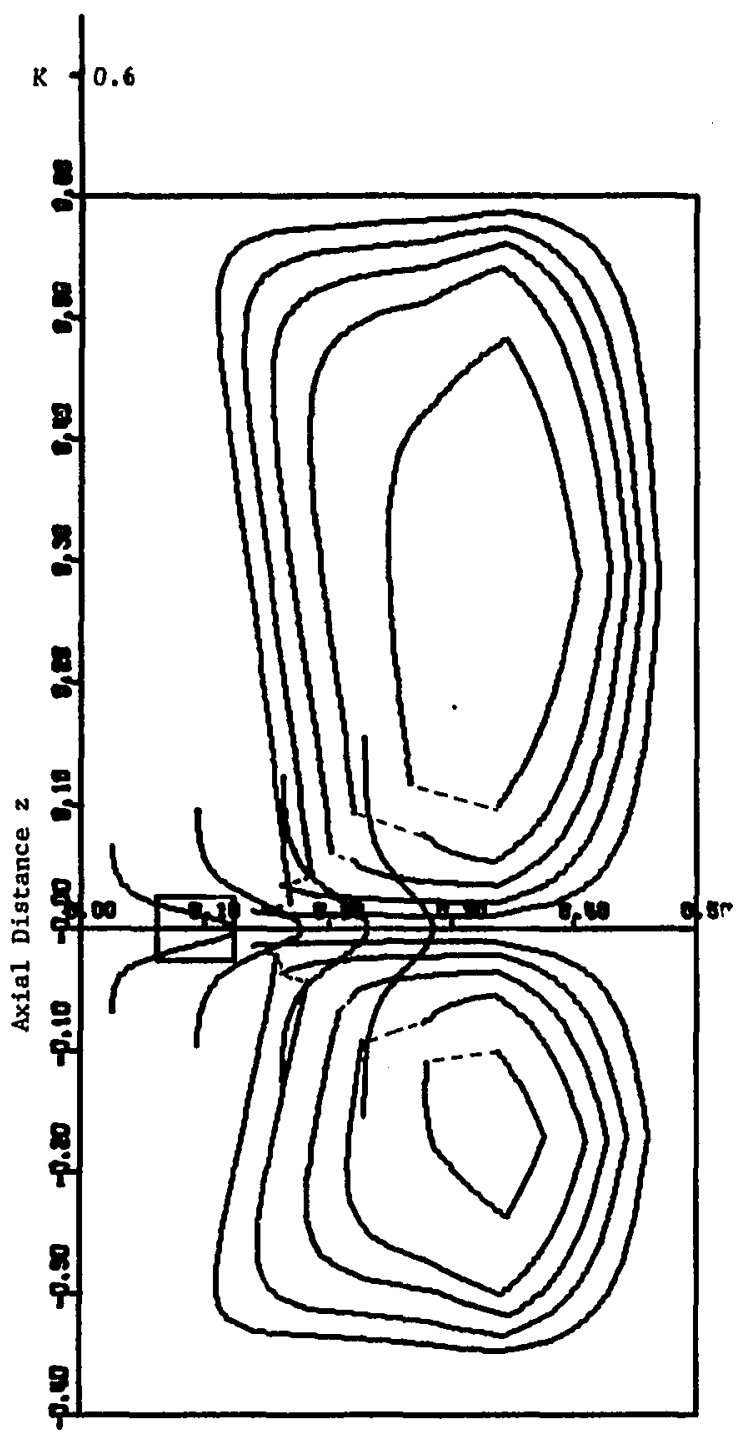

(a) F20 $=0.1$

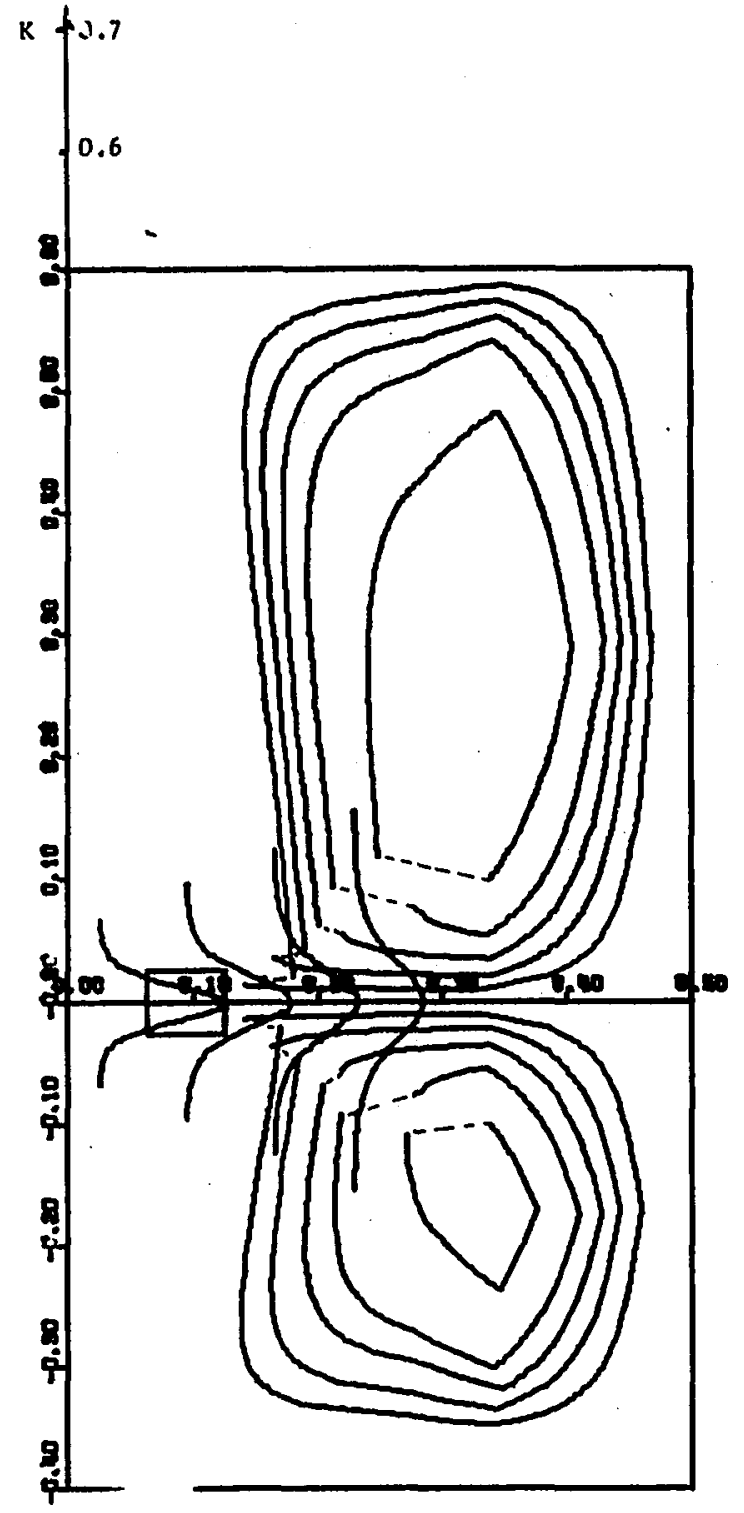

(b) $\mathrm{FZO}=2.0$

Scale: $1.0=1.0 \mathrm{ft}$.

Parameter Settings: XN2 $=0.9, X_{N} 3=0.2$, XNF $=1.0$, XN4 $=0.5$

Figure II-11. Effect of Varying the Origin, $k$ of the Circular Jet on the Theoretical Flow patterns. 
enough to take any desired value for the above boundary parameters. The tangential jet parameters are obtained from correlations given in Chapter IV. The flow pattern shown in Figure II-1 is for illustration purpose only and hence does not conform to the above recommended values, but is similar to that shown in. Figure II-7. The reason for this is that in Figure II-1, Region IV is well defined and not truncated as in Figure II-9 (c). 4. Summary and Conclusions

In this chapter the theoretical basis for drawing flow patterns was developed in Section 1. In Section 2 the basis for the computer program that enables a Calcomp Plotter to draw the flow patterns was discussed. Several theoretical flow patterns were drawn in Section 3. The limits and capabilities of the mathematical model are thus shown. The boundaries of the various regions are shown to be flexible and suitable limits for these boundaries have been recommended.

The flow pattern as drawn by the program is not exactly similar to the one given by Nagata (19). First in Region $\mathrm{V}$ because of the circular jet model selected the streamlines are pulled towards the axis of the tank and are made somewhat square rather than rounded. This is clearly visible when Figures II-9 (c) and I-5 are compared. Second, the inner most streamlines suffer the maximum distortion, tending to have corners rather than being round and smooth. The reason for this can be seen if the profile of $\bar{v}_{r}$ in Region $I$ is examined. These profiles have been scaled relative to the profile at the impeller periphery. The relative flattening of the profile with increasing $r$ is thus visible. It will be recalled from Section 
2.2 that Equation D-9, the potential flow model used in Regions II, III and IV is valid for uniform flow. If the tangential jet profile is examined in Figure II-7 through II-11, it is seen that at Region II although the jet has broadened, the uniform flow can be considered to hold approximately for three of the streamlines and is questionable for the rest of the streamlines. These inner streamlines are really a part of Region VI which has been modeled as a dead water region. This is not too severe a restriction since the velocities beyond the third streamline is less than half $q_{\max }$ evaluated at $r_{0} \cdot$ From the shape of the velocity profile, beyond this point the velocity falls rapidly to zero. This value of $q_{\max }$ in Figure II-7 (c) is approximately $135 \mathrm{ft} / \mathrm{min}$. This means that velocities at the inner streamlines is at the most $60 \mathrm{ft} / \mathrm{min}$ which is relatively small, compared to the maximum velocity of $238.5 \mathrm{ft} / \mathrm{min}$ at the impeller periphery. From the theoretical profiles it seems that Region VI occupies a considerable portion of the tank and the bulk flow is limited to the periphery of the tank. Examining Nagata's plot of representative streamlines for baffled tanks shown in Figure I-5 of Chapter I it is seen that the streamlines are close and crowned at the interface of Regions II and III, indicating that the bulk flow does occur at the periphery of the tank. 
CHAPTER III

EXPERIMENTAL APPARATUS AND OPERATING PROCEDURE

In this chapter, the experimental apparatus used will be described. The procedures and steps involved in taking the data will be detailed. Some problems were encountered in operating the equipment. The method employed in solving these problems together with the limitations on the apparatus used will be discussed in the sectlons that follow.

\subsection{Mixing Vesse1}

Two mixing vessels were used in this work. The first vessel used was a 12.5 in. i.d., by 18 in. pyrex glass vessel as shown in Figure III-1. The bottom of this vessel had a small crown. In order to have a truly flat bottom vessel a 1.0 in. thick plastic sheet was placed at a depth of 15.0 in. The tank was fitted with four baffles. The baffles were made of AISI 316 stainless stee 1 flat, measuring $1.0 \mathrm{in}$. by $1 / 8 \mathrm{in.} \mathrm{thick.} \mathrm{These} \mathrm{baffles} \mathrm{are} \mathrm{with-}$ in the recommended baffle width range of $1 / 12$ to $1 / 10$ of the tank diameter, being closer to $1 / 12$ of the tank diameter.

The tank was fitted at the top with a $1 / 2$ in. thick steel cover. In order that the cover should not slide it was recessed into the tank by $1 / 4 \mathrm{in}$. The baffles were mounted by welding them to the tank cover at the top and fitting into slots in the plastic 


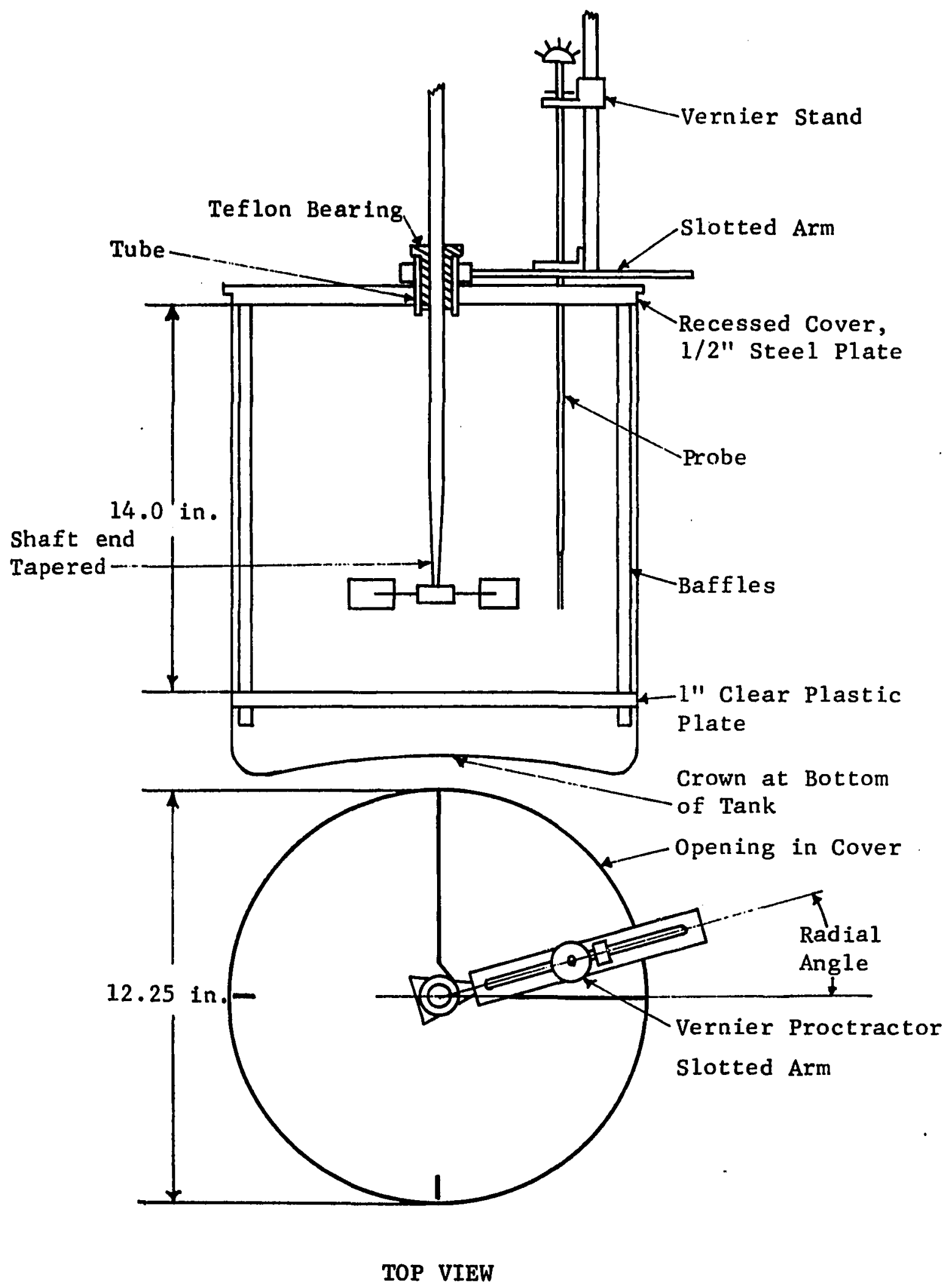

Figure III-1. Details of Stirred Tank and Probe Assembly. 
sheet at the bottom. The tank cover had a $90^{\circ}$ quadrant removed so as to have access to a region between two adjacent baffles. It was not necessary to have access to the rest of the tank since the flow is symmetrical in between adjacent baffles. The tank cover also had a cylinderical tube fitted exactly at the center to faciliate centering the impeller shaft by means of a simple teflon bearing. The tube also acts as a bearing for the probe assembly. The above details are shown in Figure III-1 which is not drawn to scale.

To measure velocity profiles in the region outside of the impeller, three holes were drilled in the sides of the tank wall as shown in Figure III-2. As this could not be done on the pyrex glass tank, a new tank was built out of plexiglass. A 11.5 in., inside diameter by 18.0 in. high, plexiglass pipe was used to make this tank. The diameter of the new tank was determined by the diameter of plexiglass pipe available which was closest to the diameter of the glass tank.

The velocity outside the impeller region reaches a maximum at approximately half the depth of the fluid above or below the impeller centerline. Since the impeller was centrally located the flow is symmetrical and the upper half of the tank was selected for measuring velocities outside the impeller region. Accordingly, three holes were drilled into the sides of the plexiglass tank at 8.0, 9.0 and $10.0 \mathrm{in}$. from the inner surface of the tank bottom. The 9.0 in. hole corresponds to the $z$-plane at which the velocities are a maximum. The other two holes are an inch apart above and below this hole to further explore the velocity field. A $5 / 8$ in. diameter by $3.0 \mathrm{in.}$ long nylon rod was glued to these holes. This 


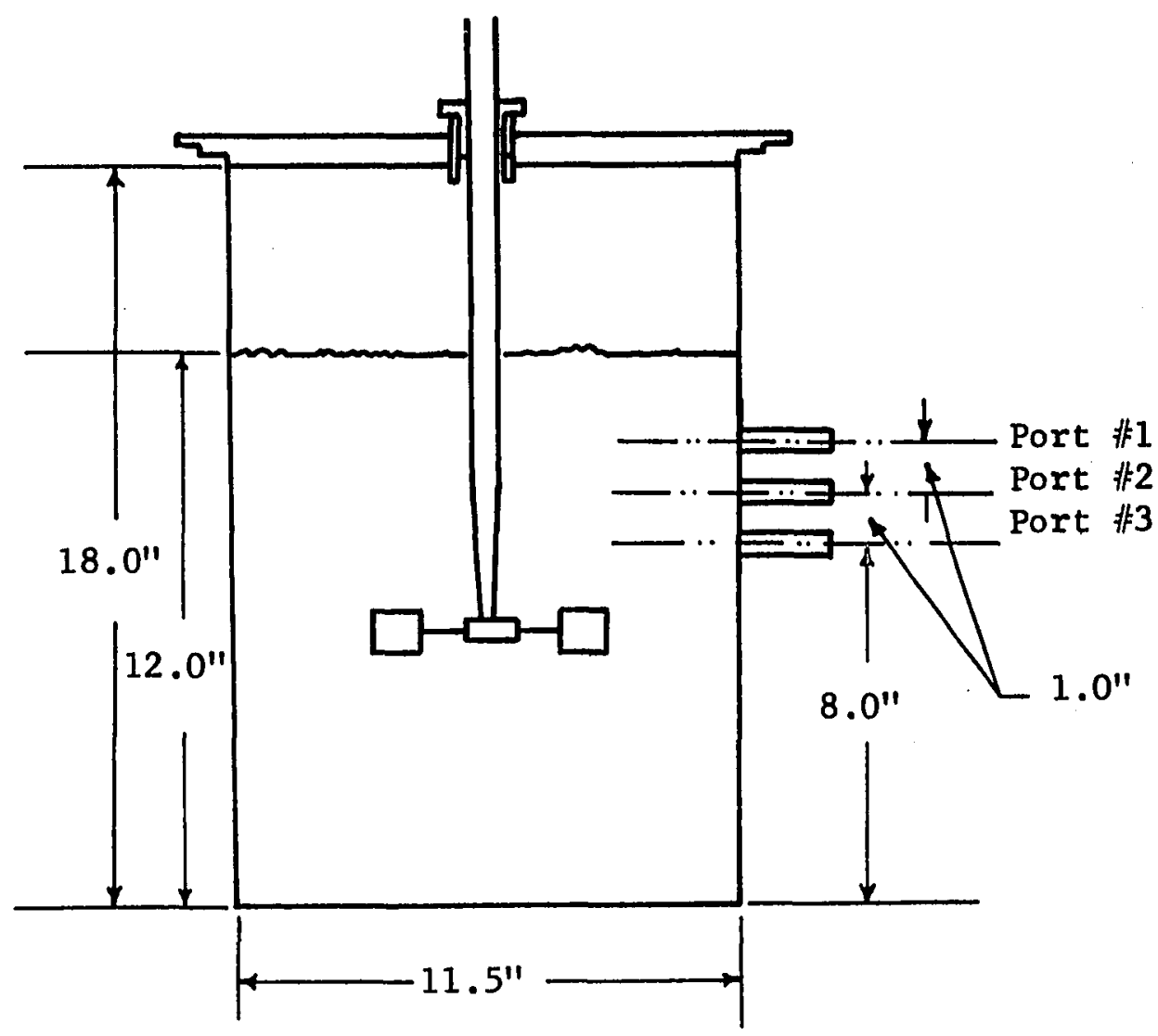

(a) Plexiglass Tank Showing Location of Horizontal Parts.

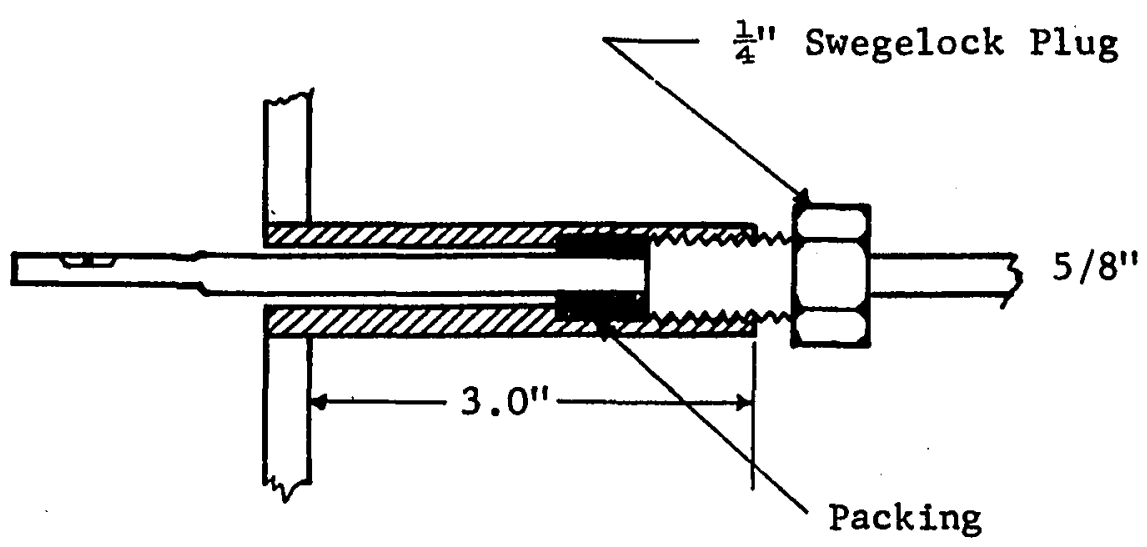

(b) Details of Port Assembly

Figure III-2. Plexiglass Tank. 
rod was drilled and tapped such that it acted as a guide, support and sealing gland for the probe. The details of this assembly is also shown in Figure III-2. These holes are referred to as ports and are numbered 1,2 and 3 as shown in Figure III-2.

The tank cover was machined down to $11.5 \mathrm{in.} \mathrm{so} \mathrm{that} \mathrm{the} \mathrm{rest}$ of the tank assembly could be used with the plexiglass tank, The same baffles 1.0 in. wide as in the glass tank were used.

\subsection{Impeller and Impe1ler Drive Assembly}

A 3.0 in. Type A turbine made of AISI 316 stainless stee 1 was used as the impeller. The blade dimensions are width, $b$, equal to 0.6 in., length of blade, 1 , equal to 0.75 in. and diameter of disk, $\mathrm{d}_{1}$, equal to 2.0 in. The impeller shaft was made of $5 / 8$ in. AISI 316 stainless stee 1 . The impeller shaft was tapered from $5 / 8$ in. to $1 / 2 \mathrm{in.}$ for the 1 ast $6.0 \mathrm{in.}$ of the shaft, and the bottom most $1 / 2$ in. was turned to $5 / 16 \mathrm{in.}$ so as to fit into the hub of the impeller where it was held fast by two screws.

The drive used was a Bench Scale Equipment Company (Dayton, Ohio) Model $4 \mathrm{R}$ Lab drive unit. It has continuously variable speed from 100 RPM to 1000 RPM. A higher speed range could be obtained by using a different chuck. The above range was adequate for this work. The entire drive was mounted on a $5 \mathrm{ft}$. vertical pipe which permitted raising and lowering the impeller. The drive mounting can be seen in Figure III-3 which is an overall photograph of the experimental apparatus. Figure III-4 is a close-up photograph of the glass tank and drive mechanism. In Figure III-5 is shown a close-up photograph of impeller and probe in the tank. 


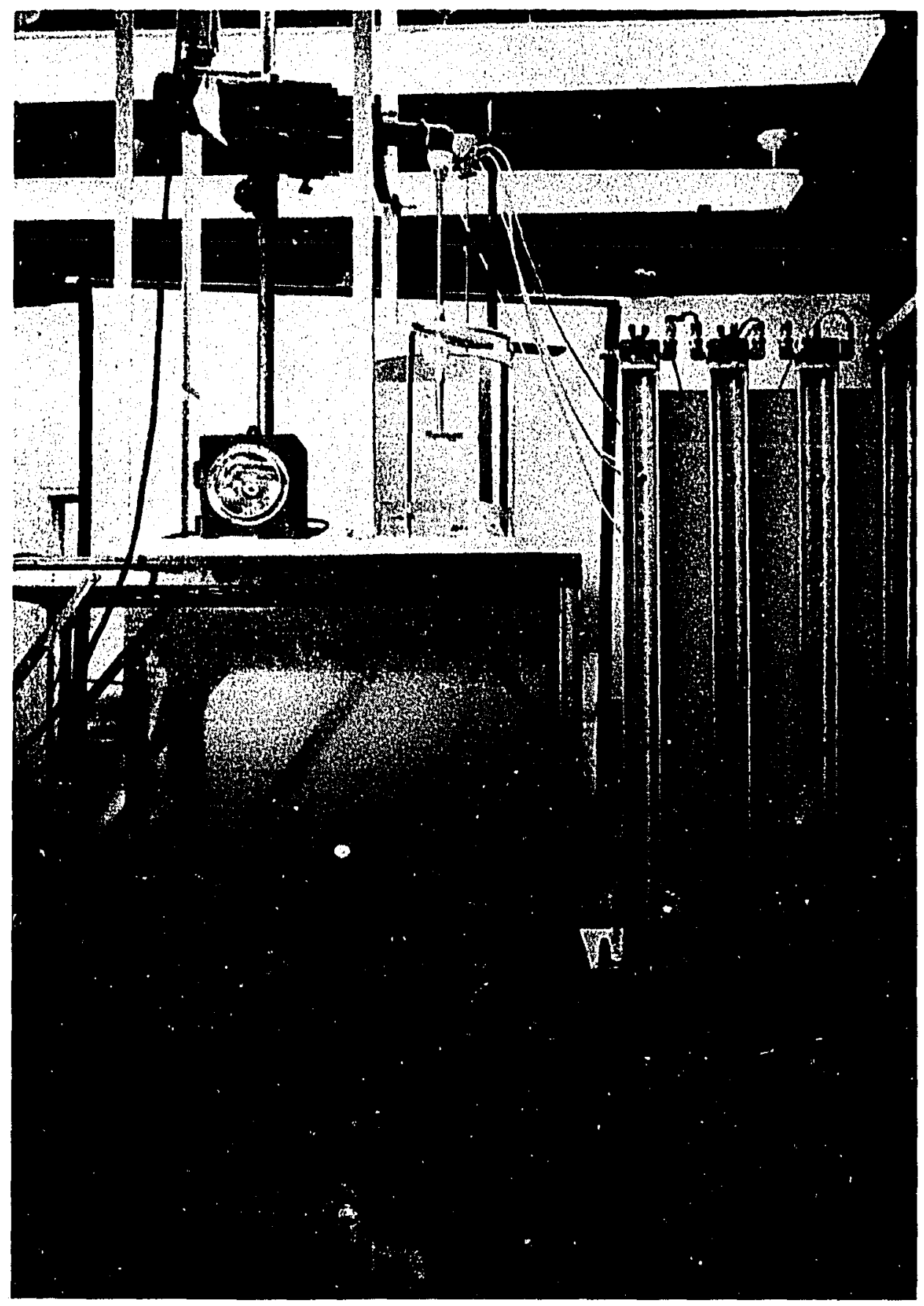

Figure III-3

Overa11 Views of the Experimental Apparatus 


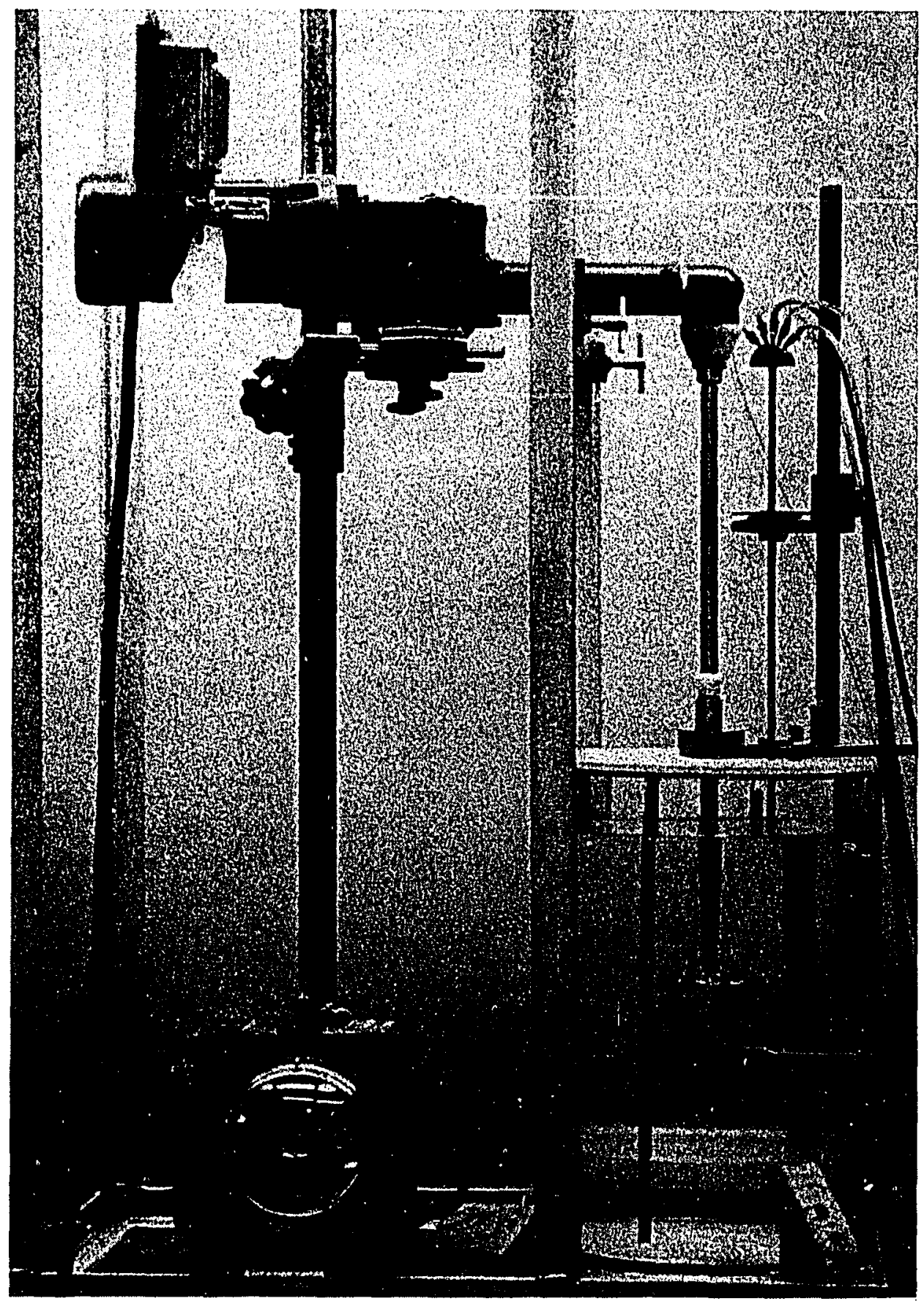

Figure III-4

Close-up View Showing Tank Drive,

Vernier Stand, Strobotac and Tank 


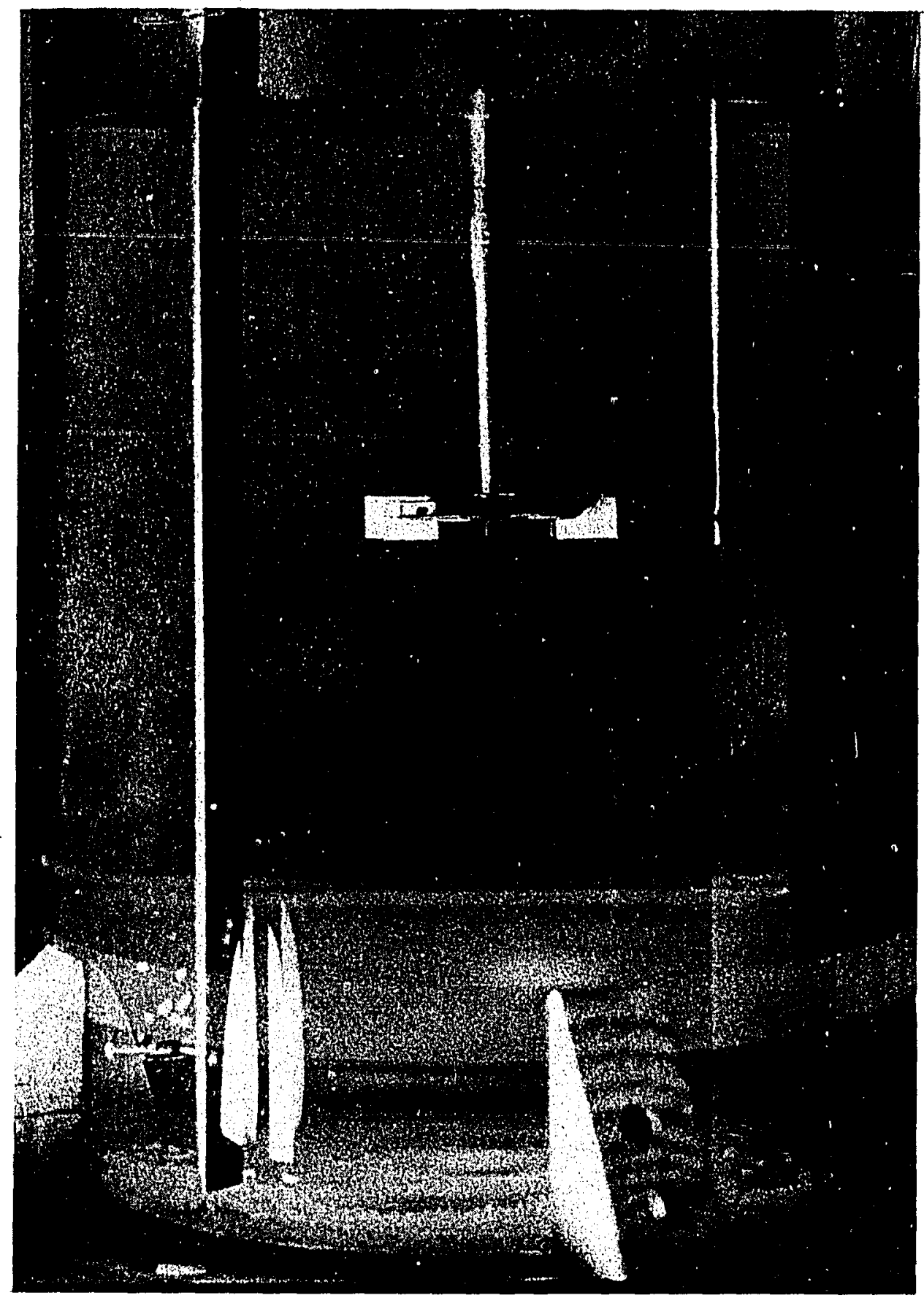

Figure III-5

Close-up Showing Impeller and Probe Tip 


\subsection{Velocity Measuring Probe and Probe Assembly}

Velocities in the neighborhood of the impeller were measured with a three dimensiona1 probe Model DA 187 made by United Sensor Corporation, Watertown, Massachusetts. The probe consists of a $3 / 16$ in. tube ending in a $1 / 8$ in. measuring tip. In the $1 / 8$ in. diameter measuring section of the tube is a prism shaped section in which five pressure taps are located. The probe and the prism shaped section is shown in Figure III- 6. Three of the pressure. taps $P_{1}, P_{4}$, and $P_{5}$ are located in the same plane, but $P_{4}$ and $P_{5}$ are located in a slight overhang above and below $P_{1}$. The prism face on which $P_{1}$ is located is so fashioned that the tap $P_{1}$ does not move when the probe is rotated. Individual connections are made from each tap to five nipples located at the top of the probe. The nipples are labeled $P_{1}, P_{2}$, etc.

The pressure is measured by four manometers and the manometer connections are shown in Figure III-7. All connections are made through $1 / 4$ in. polystyrene tubing with Swedgelock fittings. The manometers used are Merriam Instrument Company, Mode1 10AA25WM, 50 in. range manometers.

The probe mounting assembly consists of a slotted arm that rotates in a bearing about the tube mounted in the tank cover. On the slotted arm is mounted a vernier stand. The probe fits into a vernier protractor mounted on the stand. The entire assembly permits the following movements.

1. Rotating the slotted arm allows measurements in any desired radial plane.

2. The vernier stand slides in the slot of the slotted arm, 


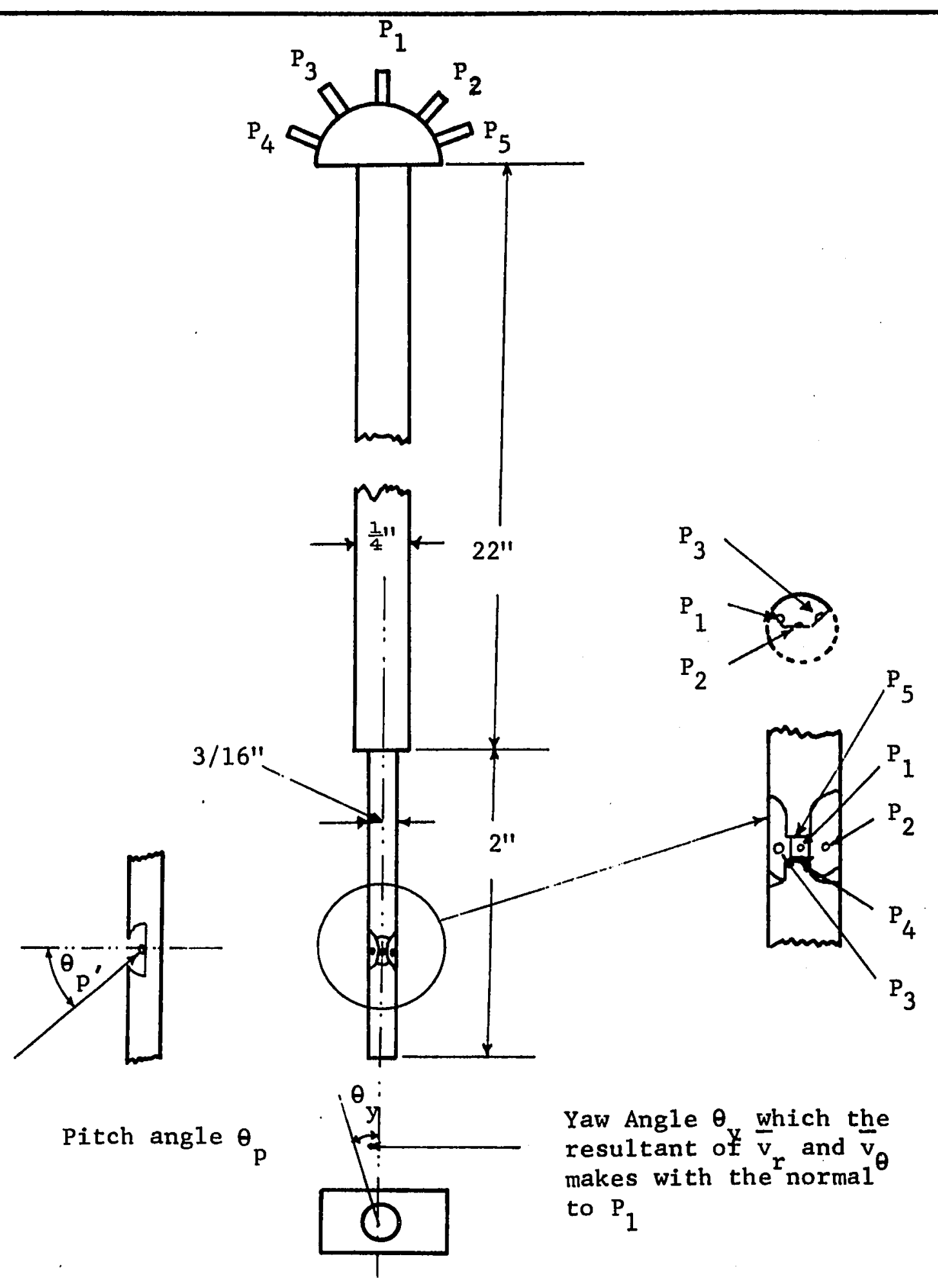

Figure III-6. Details of Three Dimensional Pitot Tube. 


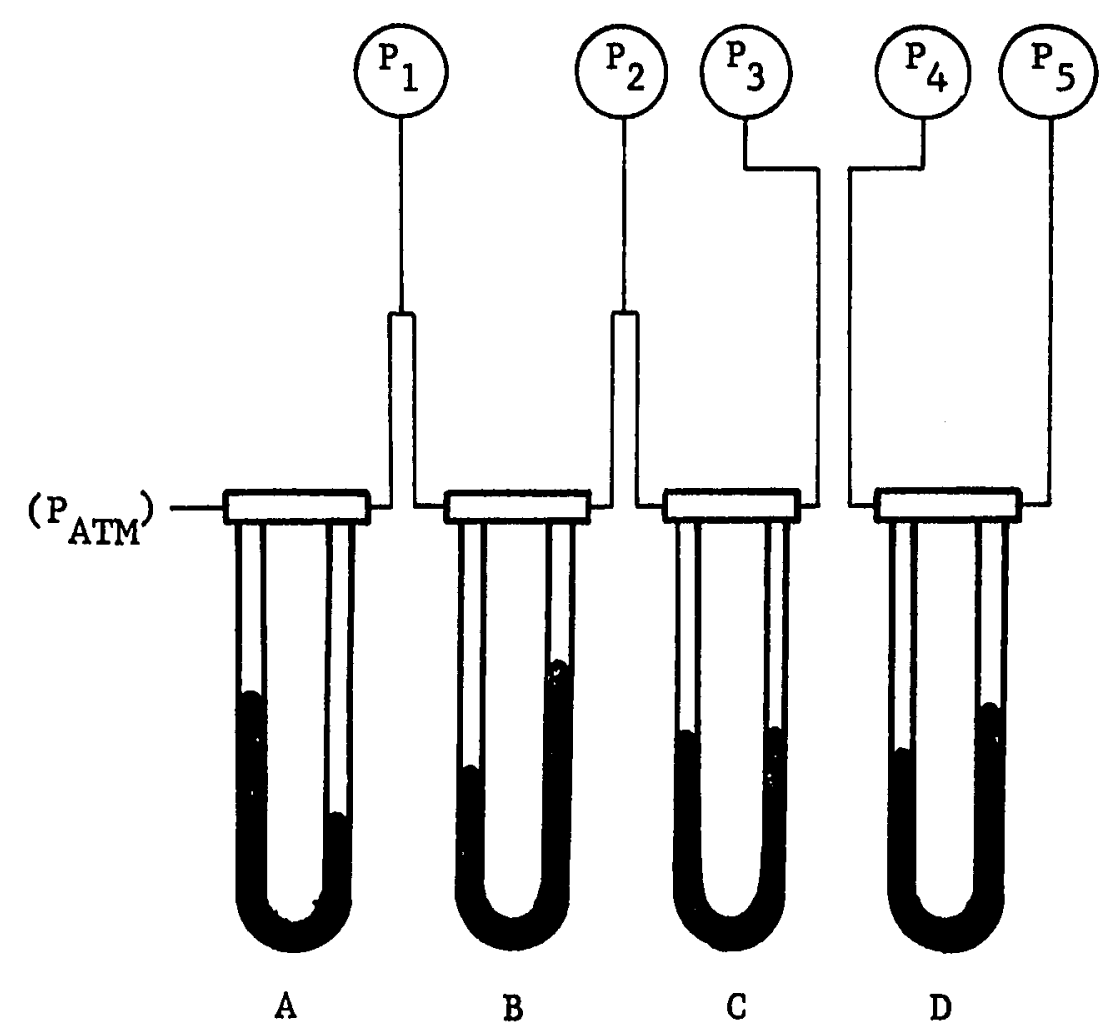

Figure III-7. Manometer Connections to Three-Dimensional Pitot Tubes. 
thus allowing the probe to be placed at any desired radius in a given $r-z$ plane.

3. The vernier stand permits locating the pressure tap $P_{1}$ at any desired level $\mathrm{z}$ from some reference level. This reference level is the impeller centerline where $z$ is taken as zero. The vernier permits measurements to within 0.01 inch.

4. The vernier protractor is used to measure rotation of the probe and is accurate to 0.2 of a degree. This permits measurements of the yaw angle, $\theta_{y}$, shown in Figure III-6. To obtain $\theta_{y}=0$, the pressure tap $P_{1}$ is aligned with the center of the impeller by using the impe1ler blades as guides. 3.4. Measurement of Velocities

The probe is set for measurement by first fixing the slide arm in the desired radial plane. Two radial planes were selected for making measurements. These were $5.5^{\circ}$ and $44.1^{\circ}$, the angles being measured relative to a baffle as shown in Figure III-1. The pressure tap $P_{1}$ is placed at the desired $r, z$ coordinates by suitable movement of the stand and the probe (motions 2 and 3 of section 3.3). The probe is now ready for making measurements.

In order to understand how the probe is used to measure the velocity, consider Figure III-6 in which the details of probe measuring section is shown. The pressure taps $\mathrm{P}_{2}$ and $\mathrm{P}_{3}$ are in the same plane as $P_{1}$ located on prism faces on either side of $P_{1}$. From the manometer connections shown in Figure III-7, it is seen that the taps $\mathrm{P}_{2}$ and $\mathrm{P}_{3}$ are connected to manometer $\mathrm{C}$. If the probe is rotated such that manometer $C$ is balanced, then the fluid velocity is affecting the pressure taps $P_{2}$ and $P_{3}$ equally and the normal to the 
pressure tap $P_{1}$ is identical with the direction of the fluid velocity. Manometer $B$ which is connected between $P_{1}$ and $P_{2}$ will thus act as a Pitot tube with $P_{2}$ and $P_{3}$ being the static tap and $P_{1}$, the dynamic tap, this differential pressure is measured by manometer $B$ and referred to as $P_{1}-P_{2}$. In case of two dimensional flow field with probe vertical and $\bar{v}_{z}=0, P_{1}-P_{2}$ measures the dynamic head due to the resultant velocity $\bar{q}$ (resultant of $\bar{v}_{r}$ and $\bar{v}_{\theta}$ ). In case of three dimensional flows the dynamic head does not give $\bar{q}$ since it is affected by $\bar{v}_{z}$. In this case the pressure tap $P_{3}$ and $P_{4}$ connected to manometer $D$ will register a pressure drop, $P_{4}-P_{5} \cdot A$ manufacturers calibration chart relates the ratio $\left(P_{4}-P_{5}\right) /\left(P_{1}-P_{2}\right)$ with $\theta_{p}= \pm 40^{\circ}$. Another calibration curve relates $\theta_{p}$ to the dimensionless pressure ration $\left(\mathrm{P}_{t}-\mathrm{P}_{s}\right) /\left(\mathrm{P}_{1}-\mathrm{P}_{2}\right) \cdot\left(\mathrm{P}_{\mathrm{t}}-\mathrm{P}_{\mathrm{s}}\right)$ is the true dynamic head due to the resultant velocity $\vec{v}$ whose components are $\bar{v}_{r}, \bar{v}_{\theta}$ and $\bar{v}_{z}$. From $\left(P_{t}-P_{s}\right), \vec{v}$ can be calculated. Knowing $\theta_{y}, \theta_{p}$ and the geometry of the flow field the component velocities can be calculated. Since the flow field geometry varies, the details of each individual case is worked out, as needed in Chapter IV.

\subsection{Manometers}

From the manometer connections shown in Figure III-7 and the discussion in sections 3.3 and 3.4 the following conclusion can be drawn.

1. Manometer $\mathrm{A}$ is for measuring stagnation or total pressure at pressure tap $P_{1}$.

2. Manometer $B$ measures the resultant of the component velocities $\bar{v}_{r}$ and $\bar{v}_{\theta}$ which is $\vec{q}$ for two dimensional flows in the $r, \theta$ plane. For three dimensional flows the manufacturers calibration 
charts must be used.

3. Manometer $\mathrm{C}$ is used to locate the yaw angle $\theta_{\mathrm{y}}$, which is shown in Figure III-6. This is done by rotating the probe until manometer $\mathrm{C}$ is balanced. The protractor attached to the probe then gives the yaw angle $\theta_{\mathrm{y}}$. When manometer $\mathrm{C}$ is balanced manometer $B$ measures $\vec{q}$ exactly in two dimensional flow fields and the yaw angle is used to determine $\bar{v}_{r}$ and $\bar{v}_{\theta}$.

4. Manometer $D$ evaluates the pitch angle $\theta_{p}$ from the probe manufactures calibration curve, as explained in section 3.4 .

The manometer fluid used is Merriam No. 3 of specific gravity 2.95 in manometer A. In Manometers B and C, Merriam No. D-7878 fluid was used having a specific gravity of 1.2. At first, in manometers $B$ and $C, \alpha$ - chloronapthalene having a specific gravity of 1.2 was used. $\alpha$-chloronapthalene is a colorless fluid, hence a dye, sudan brown of unknown origin, was used to colour it. Merriam Fluid No. D-7878 has a similar smel1 as $\alpha$ - chloronapthalene and appears identical. The additives in the Merriam fluid make it a better manometer fluid hence it was used in all but the first runs. In manometer $D$, the pressure drops were found to be very small hence a mixture of $90 \% \alpha$ - chloronapthalene and $10 \%$ n-hexane was used, giving a resultant mixture of specific gravity 1.09 which was fiound to be satisfactory.

The densities of the manometer fluid was obtained by first filling a clean dry manometer with manometer fluid to the desired leve1. Water was then poured into cne arm. The ratio of the column of manometer fluid that is balanced by the column of water then gives the manometer fluid density. The column of manometer fluid was 
kept larger than 10 in. since at lower values the density obtained was not consistent. At least five readings were taken for each fluid. A t-test was used to obtain the 0.95 confidence 1 imit on the average. The results are:

1. Density of fluid in manometer $A=2.93 \pm 0.0014$

2. Density of fluid in manometer $B=1.1957 \pm 0.00000622$

3. Density of fluid in manometer $D=1.0953 \pm 0.00000588$ Manometer $\mathrm{C}$ used the same fluid as manometer $\mathrm{B}$. To interpret the manometer readings obtained by manometer D consider Figure III-8, in which is shown the pressure connections of pressure taps $P_{4}$ and $\mathrm{P}_{5}$ of the probe connected to manometer $\mathrm{D}$. Taking a force balance about plane $A$, the level of the manometer fluid in the right hand arm of manometer $D$, gives

$$
\rho_{m} \Delta H_{m}+\rho_{w} h_{a}+P_{4}=P_{5}+\rho_{w} h_{b}+\rho_{w} \Delta H_{w}
$$

In equation III-1, $\rho_{m}$ and $\rho_{\mathrm{w}}$ are the densities of the manometer fluid and water. $\Delta H_{m}, h_{a}, h_{b}$ are the heights above plane $A$ as shown in Figure III-8. Rearranging III-1 to evaluate $\mathrm{P}_{4}-\mathrm{P}_{5}$ gives

$$
P_{4}-P_{5}=\rho_{w}\left(h_{a}-h_{b}\right)-\Delta H_{m}\left(\rho_{m}-\rho_{w}\right)
$$

Equation III-2 gives the pressure drop $\mathrm{P}_{4}-\mathrm{P}_{5}$ in terms of the manometer differential $\Delta \mathrm{H}_{\mathrm{m}}$ and the distance between the pressure taps in the probe $h_{a}-h_{b} \cdot \Delta H_{21}$ is the manometer reading of manometer $B$, it is related to velocity by the pitot tube equation (44), which is 


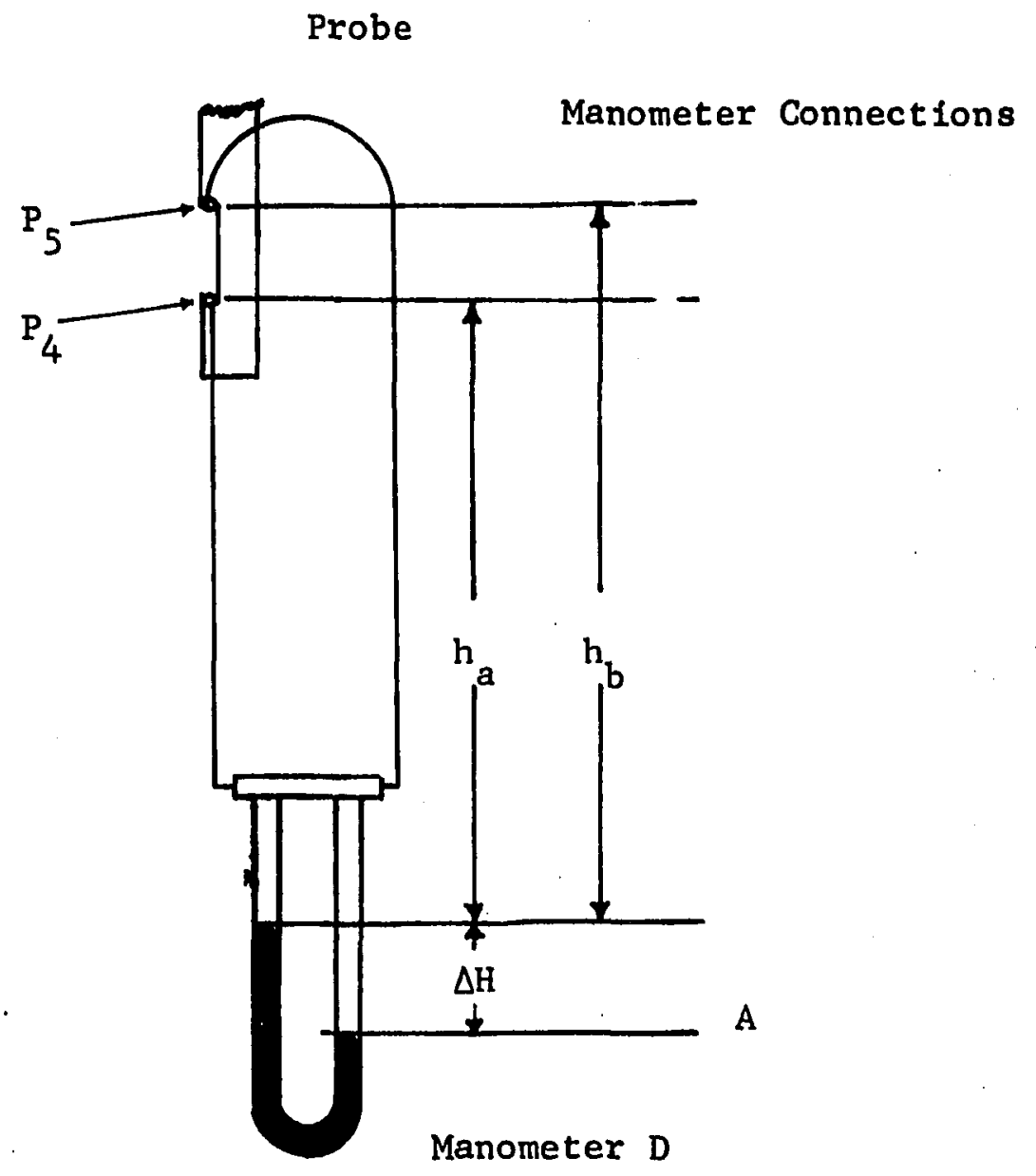

Figure III-8. Pressure Measurement of Pressure Taps $\mathrm{P}_{4}$
and $P_{5}$ in High Shear Flows. and $\mathrm{P}_{5}$ in High Shear Flows. 
given by

$$
\overline{\mathrm{q}}=\left(2 \mathrm{~g} \Delta \mathrm{H}_{21}\left(\frac{\rho_{\mathrm{m}}-\rho_{\mathrm{W}}}{\rho_{\mathrm{w}}}\right)\right)^{1 / 2}
$$

Equations III-2 and III- 3 are used to calculate the pressure drops and velocity in Chapter IV.

\subsection{Measurement of the Impe 11er Speed}

The impeller speed was measured by a Strobotac made by a General Radio Company, Cambridge, Massachusetts. The instrument normally reads in the speed range of 600 to $14,500 \mathrm{RPM}$. This speed range is not in the range of speeds used in this work. However, as the impeller has six-fold symmetry the impeller could be stopped at six times its actual speed thus permitting use of this instrument. The strobotac was found very useful in checking speeds above $300 \mathrm{RPM}$, in which range of speeds the drive was observed to wander by $\pm 10 \mathrm{RPM}$. Hence, before recording a measurement the speed was checked and also at frequent intervals to insure that the RPM was maintained at the desired value.

\subsection{Problems Encountered}

In order to prevent air bubbles from being entrapped in manometer connections it was found necessary to raise the tank such that the level of fluid in the tank was above the top of the manometers. This can be seen in the photograph of the overall equipment shown in Figure III-2. It was also found convenient to install stop cocks on the manometer heads which helped in bleeding out entrapped air. 
The manometers had to be cleaned frequently due to rust deposits from the cast iron manometer heads. This was prevented by coating the manometer heads with an epoxy paint. The tank cover was also painted with epoxy paint to prevent rusting and thus contaminating the distilled water in the tank.

The vessel was found to move with the torque generated by the impeller and to prevent this it had to be fixed firmly by four wooden blocks which are visible in Figure III-4. Since the tank was fixed the drive had to be made movable so as to vary the depth of the impeller in the tank. This was accomplished by mounting the drive on a pipe which was in turn mounted in an angle iron cage. Some residual vibration due to small misalignments in mounting the impeller shaft still remained. The vibrations when severe were observed to be transferred to the probe causing the measuring end of the probe to vibrate and hence move a considerable amount. Severe vibrations are thus undesirable and steps were taken to keep the vibrations a minimum.

\subsection{Summary}

In this chapter the experimental apparatus is described. Details of the three dimensional pitot tube probe used for measuring velocities in the tank were presented. Finally some observations on the problems involved in making the entire experimental set up work was also discussed. 
CHAPTER IV

DISCUSSION AND ANALYSIS OF RESULTS

In Chapter II, the stirred tank was divided into several regions and a model was developed to describe the flow field in these regions. It will be recalled, that the computer solutions developed to draw the velocity profiles required several parameters to be specified. In this chapter the experimental data will be analysed to obtain these parameters. The mathematical models will also be examined to evaluate how good they predict the experimental data.

The experimental data is divided into two parts, that taken in the vicinity of the impeller and that in the rest of the tank. Following this, ig detailed analysis of the experimental profiles :: will then be examined and a comparison made between this work and the results of investigators reviewed in Chapter I. The results of this comparison show that the tangential model gave a good prediction of the velocity profiles in the vicinity of the impeller.

The tangential jet model has four parameters, these are $\sigma$ the jet width, A the volumetric flow parameter $a_{1},:$ the radius of source; and $\sigma_{\mathbf{z}}$, the jet displacement:" It will be shown that $\sigma$ the jet widthrparameter is a constant, independent of tank diameter, Impeller speed, the fluid used, and within limits the impeller location. The last observation is based on the fact that the jet width was found to be a constant resulting in a narrow stream of maximum width equal to $0.16 \mathrm{~T}$. 
Within limits this width is constant independent of the height of the impeller above the tank bottom. The width of the jet was also found to be independent of impeller diameter for geometrically similar impellers.

The volumetric flow parameter $A$ was found to be dependent on tank diameter; the best correlation for $A$ was with $N D^{3} /\left((D / 2)^{2}-a^{2}\right)^{\frac{1}{4}}$. The impeller discharge $Q$, was however found to be a function of $\mathrm{ND}^{3}$ and independent of tank diameter or the fluid in the tank. The radius of source, $a$, was found to be dependent on both tank diameter and impeller diameter. Finally the jet displacement showed no trend and its only purpose is to shift the theoretical profile so that a better fit is obtained. It does not affect the properties of the jet since the velocity profile which determine these properties is symmetrical, and the shape of the profile is not altered by varying $z_{0}$

4.1. Velocity Profiles in the Region of the Impeller

The three dimensional pitot tube described in Chapter III was used to measure velocity profiles under different conditions to establish the validity of the tangential jet model. Velocity profile measurements were made with a 3.0 in diameter, type A impeller at two different impeller depths $h$, these are; (a) with impeller p laced one impeller diameter off the bottom of the tank, which is the minimum recommended in the literature and (b) with the impeller centrally located. Two different radial planes were selected, one close to the baffle $\left(5.5^{\circ}\right)$ and the other in between baffles $44.1^{\circ}$. 
These angular measurements are referred to as radial angles and are measured with respect to a baffle as shown in Figure III-1. Four different radial distances $I$ were selected to evaluate the effect of this parameter on the jet profile.

\section{Analysis of Velocity Profiles}

A program FLOWANL was written to analyzed velocity profiles measured in the neighborhood of the impeller. Each velocity profile consists of a series of measurements of $\vec{q}$, the resultant of $\bar{v}_{r}$ and $\bar{v}_{\theta}$, as a function of $z$ at constant $r$. These measurements are a result of making a vertical pass with the probe across the impeller stream. The number of data points in a profile depends on the thickness of the impeller stream. The closer the probe is to the impeller periphery the smaller the width of the stream resulting in a fewer number of data points since each data point is taken at a. minimum spacing of 0.1 in, apart.

The analysis consists of evaluating the best values for the parameters in the tangential jet model so as to predict the experimentally determined velocity profiles. The correlation coefficient $R$ is used to measure the goodness of fit and is defined by

$$
R^{2}=1-\frac{\Sigma\left(\vec{q}_{\text {exp }}-\overrightarrow{\mathrm{q}}_{\text {mode } 1}\right)^{2}}{\Sigma\left(\overrightarrow{\mathrm{q}}_{\text {exp }}-\left(\overrightarrow{\mathrm{q}}_{\text {exp }}\right)_{\text {avg }}\right)^{2}}
$$

In Equation IV-1, the numerator of the second term on the right is the sum of squares of deviation between the experimental velocity $\bar{q}_{\exp }$ 
and the velocity predicted by the model $\bar{q}_{\text {model }}$. As this term is referred to frequently in the text it is called sum of squares sS and is identical to Equation C-2 of Appendix C. The denominator of the second term on the right of Equation IV-1 is the sum of squares of the deviation of $\bar{q}_{\exp }$ from its average value $\left(\bar{q}_{\text {exp }}\right)_{\text {avg }}$. For perfect agreement the deviation between the experimental value and the model given by SS will tend to zero and $\mathrm{R}$ will tend to one. In the case of a poor agreement or no agreement, the second term on the right of Equation IV-1 will tend to one, and R!will thus tend to zero. In the present work, $R$ values of 0.98 to 0.99 have been obtained indicating that the deviation of $\bar{q}_{\exp }$ from the model is sma11 and the prediction by the model is hence very good.

Of the four parameters $\sigma, A, a$ and $z_{0}$ that describe the tangential jet, three are obtained by a nonlinear least square fit. These are $\sigma$ the jet width, A the volumetric flow and $z_{0}$ the jet displacement. The fourth parameter, a, is obtained by a weighted average value of the yaw angle profile as explained in the next section.

The nonlinear least square fit uses Pattern Search to minimize the sum of squares $S S$ resulting in a maximum value for $R$ and thus the best possible fit of the data. Pattern Search is a procedure that perturbs the parameters a small amount, such that each small change in the parameters results in a reduction in the sum of square SS, eventually leading to maximum value of $R$. A theoretical discussion of Pattern Search and the subroutine PATERN that was written to 
perform this search procedure is given in Appendix C. In future discussions a least square fit will refer to above procedure using Pattern Search.

In all, 39 velocity profiles were measured under varying conditions, summarized in Table IV-1. A detailed analysis of a velocity profile is given in Section 4.4. The analysis gives four pages of computer printout for each profile. This amount of information is needed to examine and evaluate a profile analysis in detail as done in Section 4.4. From this detail analysis seventeen items were considered as important factors that determine the character of the analysis. These are presented in tables and reported in Appëndix A. In subsequent sections, pertinent information will be taken from Appendix G to support observations and conclusions that result from this velocity profile analysis.

Radius of Source Parameter a

It will be recalled from Chapter I That the velocity profile stream exhibited an angle profile $\left(\theta_{y}\right.$ as a function of $z$ ), and this was first observed by Cooper ( 8 ). The tangential jet model on the other hand which is used in this work to predict the experimental data predicts a constant angle profile. This inconsistency was resolved by using an average angle for $\theta_{\mathrm{y}}$. Several averaging schemes were tried, the most successful was the weighted average $\theta_{y}$, the weights being the velocity and is defined by,

$$
\bar{\theta}_{y}=\frac{\Sigma \bar{q}_{i}\left(\theta_{y}\right)_{i}}{\Sigma \bar{q}_{i}}
$$


TABLE IV-1

CONDITIONS UNDER WHICH VELOCITY PROFILES WERE MEASURED

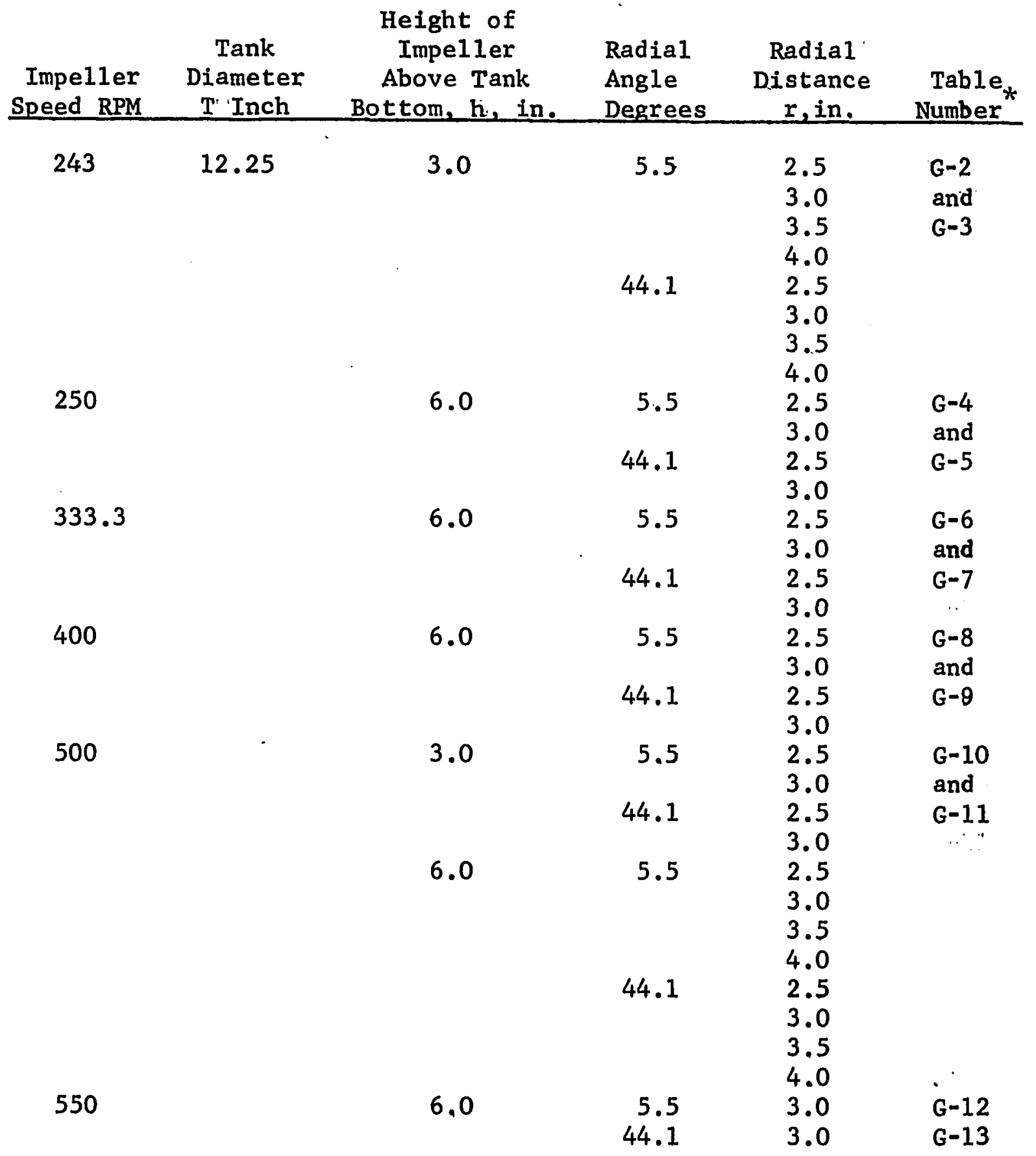


TABLE IV-1 CONTINUED

\begin{tabular}{|c|c|c|c|c|c|}
\hline $\begin{array}{r}\text { Impeller } \\
\text { Speed RPM } \\
\end{array}$ & $\begin{array}{l}\text { Tank } \\
\text { Diameter } \\
\text { T Inch } \\
\end{array}$ & $\begin{array}{c}\text { Height of } \\
\text { Impeller } \\
\text { Above Tank } \\
\text { Bottom, } h \text {, in. }\end{array}$ & $\begin{array}{l}\text { Radial } \\
\text { Angle } \\
\text { Degrees }\end{array}$ & $\begin{array}{c}\text { Radial } \\
\text { Distance } \\
\text { r. in. }\end{array}$ & $\begin{array}{l}\text { Table } \\
\text { Number }\end{array}$ \\
\hline 333.3 & 11.5 & 6.0 & $\begin{array}{r}5.5 \\
44.1\end{array}$ & $\begin{array}{l}2.5 \\
3.0 \\
2.5 \\
3.0\end{array}$ & $\begin{array}{l}G-14 \\
\text { and } \\
G-15\end{array}$ \\
\hline
\end{tabular}

* Corresponds to table numbers in Appendix $G$ where a summary of the detailed analysis at constant impeller speed is given. 
The yaw angle $\theta_{y}$ determines the direction of the velocity vector $\vec{q}$ and is a function of $r$ and the radius of source, $a$. This follows from Equation A-14 which gives $\theta_{y}$ as

$$
\cos \theta_{y}=\left(\frac{r^{2}-a^{2}}{r^{2}}\right)^{\frac{1}{2}}
$$

The value of; $a$, can now be determined from A-14 by using $\left(\bar{\theta}_{y}\right)$ for the yaw angle $\theta_{y}$. In order to have some flexibility in selecting $a$, the radius of source, a velocity factor WT is defined as

$$
W T=\frac{\bar{q}_{i}}{q_{\max }}
$$

In Equation $I V-3 \bar{q}_{i}$ is the velocity below which the angle profile is truncated in calculating the weighted average angle $\bar{\theta}_{\mathrm{y}}$ from Equation IV-2. This is illustrated in Figure IV-1 (b) which is a rectangular plot of $\bar{q}$ versus $\theta_{y}$ for Run Number 29 . It is seen that the angle profile is symmetrical above and below the impeller. $\bar{q}_{i}$ was calculated from Equation IV-3 as $66.2 \mathrm{ft} / \mathrm{min}$ for Run Number 29, with WT equal to 0.5 and $q_{\max }$ equal to $132.3 \mathrm{ft} / \mathrm{min}$. A line is thus drawn : through: this point as shown in Figure IV-1 (b). In calculating $\bar{\theta}_{y}$ from Equation IV-2 only experimental points located above this line, indicated by the arrow, are used. Increasing WT raises the line reducing the number of points used for evaluating $\theta_{y}$. The converse occurs when WT is lowered.

In Figure IV-1 (a) is shown a polar plot of $\bar{q}$ versus $\theta_{y}$. The angle profile for the range of velocities measured lie in a narrow 


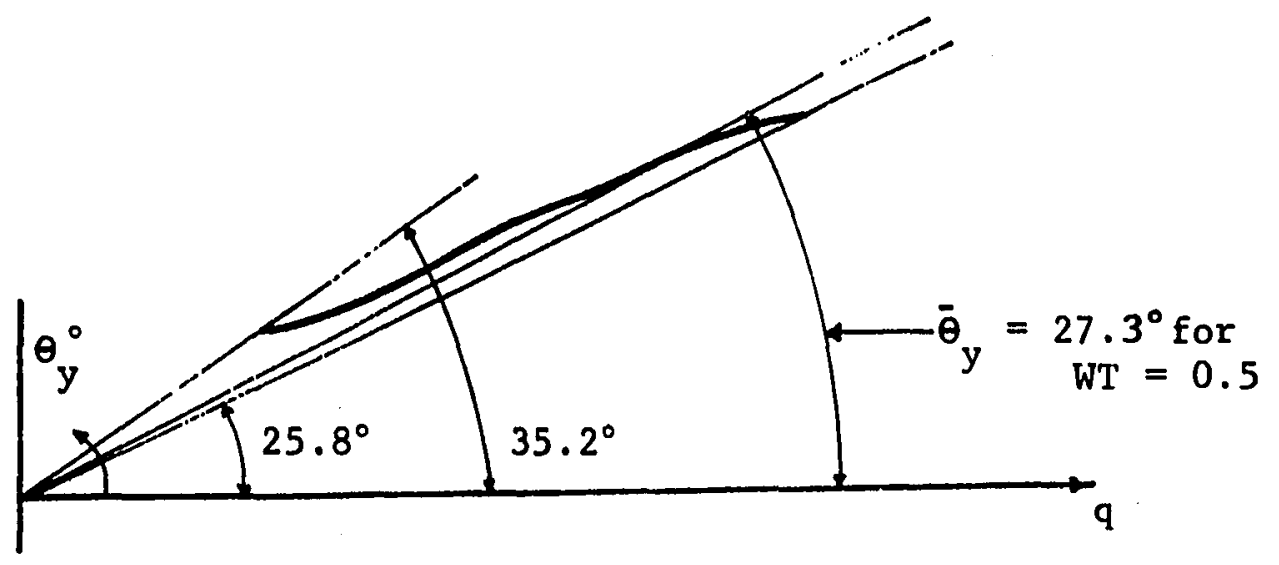

(a) Angle profile in Polar Coordinate

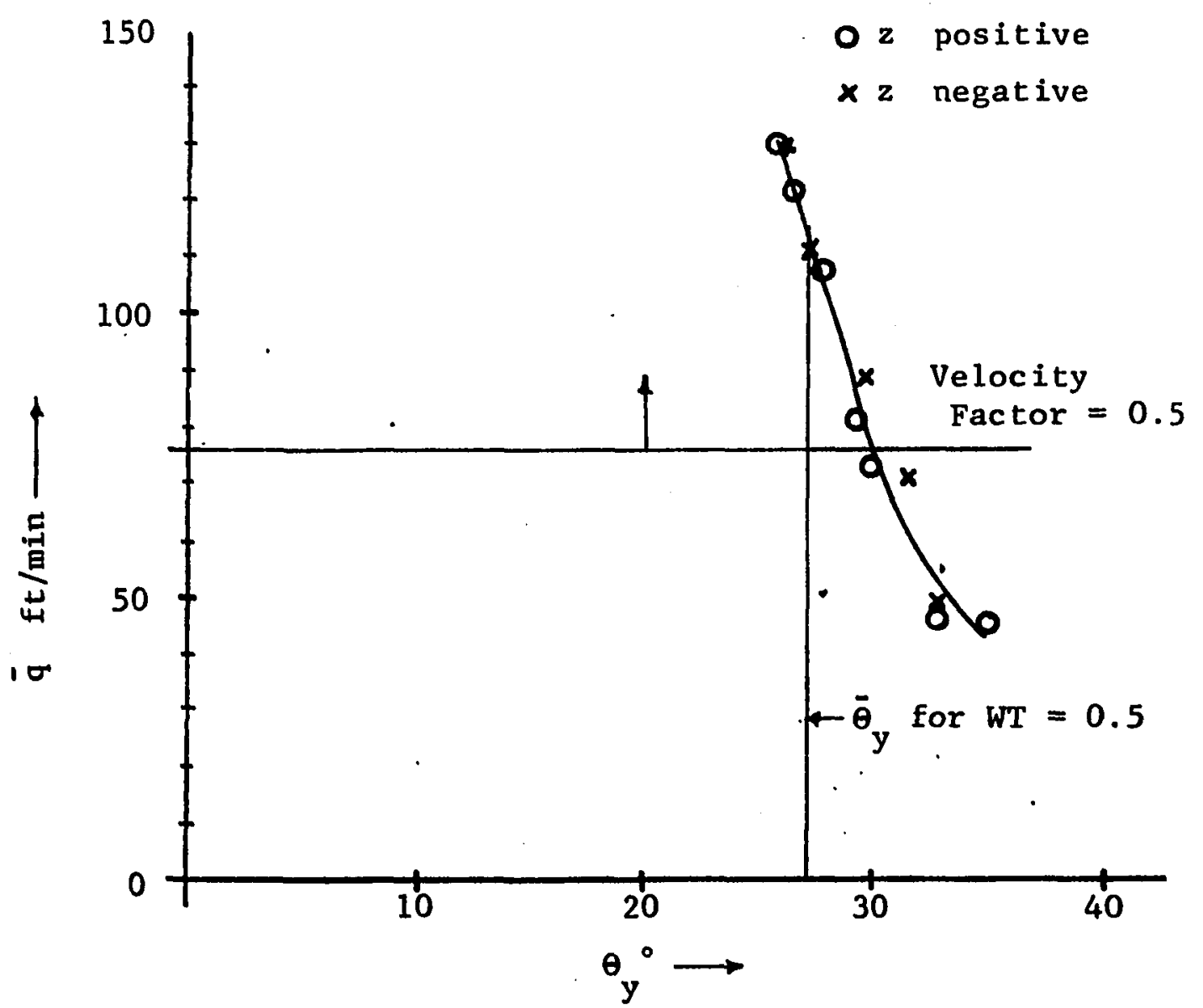

(b) Angle Profile in Rectangular Coordinates.

Figure IV-1: Angle Profile for Run Number 29, Illustrating the Definition of Velocity Factor 
band of $10^{\circ}$, and is typical of the velocity profiles measured in this work. Also shown in the diagram is $\bar{\theta}_{y}$ the weighted average angle for WT equal to $0.5 . \bar{\theta}_{y}$ is seen to lie closer to $\theta_{y}$ corresponding to $\bar{q}_{\max }$ and is a result of the weights being larger at the center, than at the ends of the velocity profile. $\bar{\theta}_{\mathrm{y}}$ was found to increase with increasing .

Various values of the velocity factor were tried and WT equal to 0.5 was found to give the best prediction of the velocity profile in all cases. This is illustrated in Table IV-2 which shown four velocity profiles measured at constant impeller speed of $333.3 \mathrm{RPM}$ and varying radial distance and radial angle. Three different velocity factors are selected and these are $0.6,0.5$ and 0.4 .

Table IV-2 shows the effect of the velocity factor WT on the sum of square SS and the correlation coefficient $R$ for both $\vec{q}$ and $\overline{\mathrm{v}}_{\mathrm{r}}$. Equations similar to $\mathrm{C}-2$ and $I V-1$ can be written to define SS and $R$ for $\bar{v}_{r}$. The velocity profile for $\bar{v}_{r}$ is obtained from Equation A-20 which is

$$
\bar{v}_{r}=|\vec{q}| \cos \theta_{y}
$$

The need for examining SS and $R$ for $\bar{v}_{r}$ in Table IV-2 is because $\bar{v}_{r}$ determines the pumping capacity and it is desirable that this quantity be also predicted as accurately as possible.

Examining Table IV-2 it is seen that the velocity factor WT has very little effect on SS for $\bar{q}$, for a given profile. For example, Profile Number 1 has a constant value of SS equal to 1167.41 for $\bar{q}$. 
TABLE IV-2

EFFECT OF VELOCITY FACTOR ON LEAST SQUARE FIT OF A VELOCITY PROFILE FOR A 3.0 INCH DIAMETER IMPELLER AT 333.3 RPM IN A 12.25 INCH DIAMETER TANK

(a) Velocity Factor $=0.6$

\begin{tabular}{|c|c|c|c|c|c|c|}
\hline \multirow{2}{*}{$\begin{array}{l}\text { Profile } \\
\text { Number }\end{array}$} & \multirow[b]{2}{*}{$\begin{array}{l}\text { Radial } \\
\text { Angle }\end{array}$} & \multirow{2}{*}{$\begin{array}{l}\text { Radial } \\
\text { Distance } \\
\mathrm{r} \text { Inch }\end{array}$} & \multicolumn{2}{|c|}{ Sum of Squares SS } & \multicolumn{2}{|c|}{$\begin{array}{l}\text { Correlation } \\
\text { Coefficient } R\end{array}$} \\
\hline & & & $\vec{q}$ & $\bar{v}_{r}$ & $\vec{q}$ & $\bar{v}_{r}$ \\
\hline 1 & 44.1 & 2.5 & 1167.41 & 1044.30 & 0.9782 & 0.9756 \\
\hline 2 & 5.5 & 2.5 & 2155.45 & 1512.04 & 0.9669 & 0.9701 \\
\hline 3 & 44.1 & 3.0 & 123.27 & 126.96 & 0.9935 & 0.9925 \\
\hline 4 & 5.5 & 3.0 & 235.64 & 215.69 & 0.9919 & 0.9915 \\
\hline \multicolumn{7}{|c|}{ (b) Velocity factor $=0.5$} \\
\hline 1 & 44.1 & 2.5 & 1167.41 & 1030.68 & 0.9782 & 0.9759 \\
\hline 2 & 5.5 & 2.5 & 2155.45 & 1512.04 & 0.9669 & 0.9701 \\
\hline 3 & 44.1 & 3.0 & 123.27 & 126.96 & 0.9935 & 0.9925 \\
\hline 4 & 5.5 & 3.0 & 235.63 & 214.30 & 0.9919 & 0.9915 \\
\hline \multicolumn{7}{|c|}{ (c) Velocity factor $=0.4$} \\
\hline 1 & 44.1 & 2.5 & 1167.41 & 1021.85 & 0.9782 & 0.9761 \\
\hline 2 & 5.5 & 2.5 & 2155.45 & 1499.11 & 0.9669 & 0.9703 \\
\hline 3 & 44.1 & 3.0 & 123.26 & 128.95 & 0.9935 & 0.9924 \\
\hline 4 & 5.5 & 3.0 & 235.63 & 214.08 & 0.9919 & 0.9915 \\
\hline
\end{tabular}


Small changes are seen in the sum of squares for $\bar{v}_{r}$. In general decreasing the velocity factor decreases the sum squares for $\bar{v}_{r}$ and increases the magnitude of the weighted average angle $\bar{\theta}_{y}$. In one profile (not shown in Table IV-2) $\bar{\theta}_{y}$ was increased to where it gave a value of a, the radius of source, just larger than the impeller radius. From Table IV-2 it is seen that the difference in sum squares. for $\bar{v}_{r}$ between velocity factors of 0.5 and 0.4 are not very large. In some cases (for example Profile Number 3) the sum square is smaller with a velocity factor of 0.5 . The trends noted above apply to all the profiles investigated, hence a velocity factor of 0.5 was chosen for all the profiles. The summary of results reported in Appendix G are based on this value of velocity factor.

In Table IV-2, the correlation coefficient reported for $\bar{v}_{r}$ of Profile Number 2 is slightly larger than that of $\vec{q}$. This is an exceptional case. In the majority of profiles it was found that the correlation coefficient was of the order of 0.98 to 0.99 for $\vec{q}$. The correlation coefficient for $\bar{v}_{x}$ was found to be slightly lower than that of $\vec{q}$ but very close as shown in Table IV-2. Effect of Impellex Depth, Radial Angle, and Radial Distance on Tangential Jet Parameters

The tank geometry is an important consideration in the design of stirred tanks. With a veiw of finding the most important variables the impeller depth, radial angle and radial distance were varied at constant impeller speed. The impeller depth, $h$, is defined in this 
work as the distanc $h$ from the tank bottom to the impeller center1ine. Two values of $h$ were chosen; the impeller centrally located, $h=6.0$ inches and the impeller at maximum depth of one impeller diameter off the tank bottom recommended in the literature, $h=3.0$ inches.

Velocity profile measurements were made in two radial planes at radial angles of $5.5^{\circ}$ and $44.5^{\circ}$. The angles are measured with respect to a baffle as shown in Figure III-1. These measurements were made to test the assumption of angular symmetry.

At fixed impeller depth and radial angle, velocity profile measurements were made at four different radial distance $r$ from the impeller axis. The purpose of these measurements was to explore the radial distance to which the tangential jet model was valid.

In Table IV-3 are shown values of the tangential jet parameters $\sigma, A$ and $a$ for varying $h$, radial angle and radial distance $r$. Profiles $3,4,7$ and 8 are measured at $h=6.0$ in. The rest of the profiles are measured at $h=3.0$ in. Table IV-3 is extracted from Table G-1; the radius of source, a is evaluated with a velocity factor of 0.5 .

The volumetric parameter, A, and the radius of source parameter, a, show very little variation in Table IV-3 indicating that at constant RPM. These two parameters are not affected by varying $h, r$, or radial angle. This is more closely brought out when the average value variance and confidence limit of $A$ for the twelve profiles is 
TABLE IV-3

EFFECT OF IMPELLER DEPTH, RADIAL ANGLE, AND RADIAL DISTANCE ON TANGENTIAL JET PARAMETERS AT IMPELLER SPEED OF 500 RPM

\begin{tabular}{|c|c|c|c|c|c|c|}
\hline $\begin{array}{c}\text { Profile } \\
\text { No. }\end{array}$ & $\begin{array}{l}\text { h } \\
\text { in. }\end{array}$ & $\begin{array}{l}\text { Radial } \\
\text { Angle } \\
\ldots \text {.. Deg... }\end{array}$ & $\begin{array}{l}\text { Radial } \\
\text { Distance } \\
\ldots \mathbf{r}, \text {,in.... }\end{array}$ & $\sigma$ & A & $a$ \\
\hline 1 & 3.0 & 44.1 & 2.5 & 12.2 & 27.4 & 0.116 \\
\hline 2 & & 5.5 & & 11.9 & 27.4 & 0.119 \\
\hline 3 & 6.0 & 44.1 & & 14.3. & 27.3 & 0.117 \\
\hline 4 & & 5.5 & & 13.5 & 29.7 & 0.119 \\
\hline 5 & 3.0 & 44.1 & 3.0 & 11.2 & 27.7 & 0.114 \\
\hline 6 & & 5.5 & & 10.9 & 27.7 & 0.117 \\
\hline 7 & 6.0 & 44.1 & & 11.3 & 28.9 & 0.104 \\
\hline 8 & & .5 .5 & & 10.9 & 29.3 & 0.112 \\
\hline 9 & 3.0 & 44.1 & 3.5 & 10.3 & 28.6 & 0.106 \\
\hline 10 & & 5.5 & & 10.5 & 28.3 & 0.110 \\
\hline 11 & & 44.1 & 4.0 & $9: 7$ & 29.2 & 0.097 \\
\hline $12 \ldots$ & $\ldots$ & 5.5 & & 9.8 & 29.3 & 0.1 .10 \\
\hline
\end{tabular}

Average Variance Confidence Limit

$\begin{array}{llll}\sigma & 11.4 & 1.98 & \pm 0.9\end{array}$

A $28.4 \quad 0.75 \quad \pm 0.6$.

$\begin{array}{llll}a & 0.112 & 0.00004 & \pm 0.004\end{array}$ 
examined at the bottom of Table IV-3. It is seen that both the variance and confidence limit are small indicating the low scatter or deviation of $A$ about its mean value.

The variance $s_{x}^{2}$ reported at the bottom of Table IV-3 is defined by

$$
s_{x}^{2}=\frac{1}{N-1} \sum_{i=1}^{n}\left(x_{i}-\bar{x}\right)^{2}
$$

where $\bar{x}$ is the average value of $x$. The confidence limit, DEVIATION, is calculated from the following equation:

$$
\text { DEVIATION }=t_{0.95, N-1} s_{\bar{x}}^{2}
$$

where $t_{0.95, N-1}$ is obtained from a $t$-table at a $95 \%$ confidence level and $\mathrm{N}-1$ degrees of freedom. $\mathrm{S}_{\overline{\mathrm{x}}}^{2}$ in IV-5 is the standard deviation on the mean and is given by

$$
s_{\bar{x}}^{2}=s_{x}^{2} / N
$$

Table IV-3 also shows that, $a$, the radius of source is a constant equal to $0.112 \pm 0.004$ having a small value of variance and confidence limit.

The jet width $\sigma$ in Table IV-3 has a high value for Profile. Numbers 3 and 4 and a low value for Profile Numbers 11 and 12. The rest of the profiles are within the confidence limit of the average given as $11.4 \pm 0.9$ at the bottom of Table IV-3. It will be shown later that Profile Numbers 11 and 12 were taken at an $r$ value close to $r_{0}$ the limit of the tangential jet region. These two profiles 
could hence be considered as not representative of the tangential jet region, and the low values of $\sigma$ obtained could be the result of interference caused by the proximity of the stagnation flow region that follows the tangential jet. The high value of $\sigma$ in Profile Number 3 and 4 could be due to the effect of $h$ on the velocity profile. In general the velocity profiles gave a slightly higher value of $\sigma$ for smaller values of $r$ and this trend is clearly noticed in Table IV-3. A case for $\sigma$ being considered a constant is plausible although from Table IV-3 this conclusion is not as clear as in the case of $A$ and $a$.

From the tangential jet model we have that the dimensionless velocity profile $\eta$ is given by

$$
\eta=\sigma \frac{\mathbf{z}}{\mathbf{r}}
$$

At constant $r$ from $B-2$ it is seen that $\sigma$ is scale factor on $z$, hence the name jet width parameter for $\sigma$. If $\sigma$ is constant it follows that the width of the jet is a constant. If the width of the jet is constant then within limits it appears that it should not be affected by varying $h$.

To test this assumption the impeller speed was reduced by approximately half to 243 RPM and $h$ was kept at its minimum value of one impeller diameter (3.0 in.). The resulting values of $\sigma, A$ and $a$, are shown in Table IV-4. It is seen in Table IV-4 that A and $a$ are constant given by $13.5 \pm 0.2$ and $0.114 \pm 0.004$ respectively. In Table IV-4, Profile Numbers 8 and 9 for $r$ equal to 4 show a low 
TABLE IV-4

EFFECT OF RADIAL DISTANCE ON TANGENTIAL JET PARAMETERS

FOR A 3.0 IN. IMPELLER AT 243 RPM AND IMPELLER DEPTH OF $3.0 \mathrm{IN}$.

\begin{tabular}{|c|c|c|c|c|c|}
\hline $\begin{array}{c}\text { Profile } \\
\text { No. }\end{array}$ & $\begin{array}{l}\text { Radia } 1 \\
\text { Angle } \\
\text { Deg. }\end{array}$ & $\begin{array}{l}\text { Radial } \\
\text { Distance } \\
\mathbf{r} \text { in. } \\
\end{array}$ & $\sigma$ & A & a \\
\hline 1 & 44.1 & 2.5 & 11.5 & 13.5 & 0.114 \\
\hline 2 & 44.1 & & 11.6 & 13.3 & 0.116 \\
\hline 3 & 5.5 & & 11.1 & 13.4 & 0.112 \\
\hline 4 & 44.1 & 3.0 & 10.8 & 13.0 & 0.115 \\
\hline 5 & 5.5 & & 10.5 & 13.4 & 0.120 \\
\hline 6 & 44.1 & 3.5 & 10.4 & 13.4 & 0.107 \\
\hline 7 & 5.5 & & 10.3 & 13.5 & 0.116 \\
\hline 8 & 44.1 & 4.0 & 8.7 & 14.0 & 0.103 \\
\hline \multirow[t]{2}{*}{9} & 5.5 & 4.0 & 9.7 & 13.9 & 0.115 \\
\hline & Average & Variance & \multicolumn{3}{|c|}{ Confidence Limit } \\
\hline$\sigma$ & 10.5 & 0.839 & \multicolumn{3}{|c|}{0.7} \\
\hline A & 13.5 & 0.093 & \multicolumn{3}{|c|}{0.2} \\
\hline$a$ & 0.114 & 0.00003 & \multicolumn{3}{|c|}{0.004} \\
\hline
\end{tabular}


for $\sigma$ indicating that this point is close to the stagnation region. As in the case of Table IV-3 for small $r, \sigma$ is found to be larger in magnitude. The average value of $\sigma$ in Table IV-4 is seen to be within the confidence limit of $\sigma$ reported in Table IV-3. It is thus concluded that an argument for $\sigma$ being a constant exists. Since $h$ has been varied in the profiles investigated in Tables IV- 3 and IV-4 without significant effect on the jet parameters it was concluded that the impeller depth $h$ had no effect and hence in subsequent runs the impeller was centerally located with $h$ equal to 6.0 inches.

It is thus concluded that the tangential jet parameters are a function of impeller speed. In order to get a reasonable estimate of an average value, four profiles were taken at constant impeller speed. The four profiles were taken at two radial angles, $5.5^{\circ}$ and $44.1^{\circ}$ and two values of $r, 2.5 \mathrm{in}$, and $3.0 \mathrm{in}$. Larger values of $r$ were not selected since as noted above the value of $\sigma$ was noticed to fall markedly being influenced by the flow in the next region. The summary of the analysis of these velocity profiles are given in Appendix G. Tables IV-5 to IV-7 give the average values of $\sigma$, $A$ and $a$ at varying impeller speeds and are obtained from Appendix G. Item 1-6 in these tables are taken in a 12.25 in. diameter tank, while Item 7 is taken in a 11.5 diameter tank.

\section{Effect of Impeller Speed on Tangential Jet Parameter}

The observations made and conclusions drawn for the tangential jet parameters obtained from profile measurements taken at constant 
TABLE IV -5

AVERAGE VALUE OF JET WIDTH PARAMETER $\sigma$ FOR

A 3.0 INCH DIAMETER IMPELLER

\begin{tabular}{cccccc}
$\begin{array}{c}\text { Item } \\
\text { Number }\end{array}$ & $\begin{array}{c}\text { Impeller } \\
\text { Speed } \\
\text { (RPM) }\end{array}$ & $\begin{array}{c}\text { Average Value } \\
\text { of } g\end{array}$ & Variance & $\begin{array}{c}\text { Confidence } \\
\text { Limit }\end{array}$ & $\begin{array}{c}\text { Number of } \\
\text { Profiles }\end{array}$ \\
\hline 1 & 243 & 10.5 & 0.838 & 0.7 & 9 \\
2 & 250 & 10.0 & 2.750 & 2.6 & 4 \\
3 & 333.3 & 11.8 & 0.448 & 1.1 & 4 \\
4 & 400 & 11.8 & 0.7 .19 & 1.3 & 4 \\
5 & 500 & 11.4 & 1.982 & 0.9 & 12 \\
6 & 550. & 11.6 & 0.064 & 2.3 & 2 \\
7 & 333.3 & 10.7 & 0.773 & 1.4 & 4 \\
Tank diameter $=11.5$ inches & & & & \\
\end{tabular}


TABLE IV-6

AVERAGE VALUE OF VOLUMETRIC FLOW PARAMETER

A, FOR A 3.0 IN. DIAMETER IMPELLER

\begin{tabular}{cccccc}
$\begin{array}{c}\text { Item } \\
\text { No. }\end{array}$ & $\begin{array}{c}\text { Impeller } \\
\text { Speed } \\
\text { RPM }\end{array}$ & $\begin{array}{c}\text { Average } \\
\text { Value of } \\
\text { A }\end{array}$ & Variance: & $\begin{array}{c}\text { Confidence } \\
\text { Limit }\end{array}$ & $\begin{array}{c}\text { Number of } \\
\text { Profiles }\end{array}$ \\
\hline 1 & 243 & 13.5 & 0.093 & 0.2 & 9 \\
2 & 250 & 14.3 & 0.204 & 0.7 & 4 \\
3 & 333.3 & 18.6 & 0.062 & 0.4 & 4 \\
4 & 400 & 22.7 & 0.014 & 0.2 & 4 \\
5 & 500 & 28.4 & 0.750 & 0.6 & 12 \\
6 & 550 & 31.6 & 0.119 & 3.1 & 2 \\
$7^{*}$ & 333.3 & 17.9 & 0.170 & 0.6 & 4 \\
& & & &
\end{tabular}


TABLE IV-7

AVERAGE VALUE OF RADIUS OF SOURCE a FOR A 3,0 INCH DIAMETER IMPELLER IN A 12.5 INCH DIAMETER TANK

\begin{tabular}{cccccc}
$\begin{array}{c}\text { Item } \\
\text { Number }\end{array}$ & $\begin{array}{c}\text { Impeller } \\
\text { Speed }\end{array}$ & $\begin{array}{c}\text { Average } \\
\text { Value of a }\end{array}$ & Variance & $\begin{array}{c}\text { Confidence } \\
\text { Limit }\end{array}$ & $\begin{array}{c}\text { Number of } \\
\text { Profilés }\end{array}$ \\
\hline 1 & 243 & 0.114. & 0.000029 & 0.004 & .9 \\
2 & 250 & 0.119 & 0.000024 & 0.008 & 4 \\
3 & 333.3 & 0.115 & 0.000004 & 0.003 & 4 \\
4 & 400 & 0.117 & 0.000012 & 0.005 & 4 \\
5 & 500 & 0.112 & 0.000045 & 0.004 & 12 \\
6 & 550 & 0.112 & 0.0000006 & 0.007 & 2 \\
7 & 333.3 & 0.122 & 0.000001 & 0.002 & 4 \\
\hline
\end{tabular}

${ }^{*}$ Tank diameter $=11.5$ inches 
Impeller speed of 500 and 243 RPM in the previous section are also valid at other impeller speeds. In Table IV-5 is shown the average values, variance and confidence for $\sigma$ the jet width at different impeller speeds. The average value of $\sigma$ is seen to fall in a narrow range indicating that $\sigma$ is independent of impeller speed and tank diameter. The later observation is made from Item 7 of Table IV-5 which is taken in a different tank diameter. The constancy (1ow scatter) in the average value of $\sigma$ is misleading since the confidence limit of these average values are rather large. The maximum confidence limit is 2,6 which is about $25 \%$ of the magnitude of $\sigma$. This means that assuming the model is correct the measurement made by the probe are not as accurate as desirable.

In Table IV-6 is shown the average value, variance and deviation for the volumetric flow parameter $A$, at varying impeller speed. Both variance and confidence limit are small indicating that the average value is a good estimate of this parameter. The only exception is Item 6 which will be discussed in the next section.

On examining Table IV-7 it is evident that the radius of source, a, is a constant independent of impeller speed but not of tank diameter. For the $12.25 \mathrm{in.} \mathrm{diameter} \mathrm{tank} \mathrm{the} \mathrm{average} \mathrm{value} \mathrm{is} 0.115 \pm$ 0.008. The confidence limit is taken as the largest deviation of Items 1 to 6 in Table IV-5. This is because the average value is based on components which are error bound themselves: A t-test taken on the average of the average values will reflect the variation between the averages, but will not account for the error in 
in each component. In this case the t-test gave a confidence limit of 0.003 which is smaller than the confidence limits of individual averages reported in Table IV-7.

Effect of High Impeller Speed on Velocity Profiles Measurements

In Table IV-5, the rather large deviation for A shown in Item 6 is due partly because the sample size (number of profiles) is small and partly because the manometer $B$ was observed to fluctuate erratically making it difficult to record the true pressure drop. The amplitude of the fluctuation ranged from 0.5 to as much as $2 \mathrm{cms}$, the larger value being observed for large $\Delta \mathrm{H}_{\mathrm{m}}$. The period of the fluctuations was not fixed and varied in a random manner from 30 seconds to 2 minutes. Since the manometers have high inertia, such large fluctuations indicates that at this high impeller speed the intensity of turbulence is very high, and could be as much as $100 \%$.

At this high speed with the impeller centrally located it was observed that considerable amount of air was entrained. The air remained in the system as large bubbles. The entrainment of air in the system was observed to begin at a little below 500 RPM. The air entrainment is due to the rotating vortex at the surface shown in Figure $\mathrm{I}-4$ (c) and first observed by Nagata (19). It was noticed that the vortex is enhanced by high speed, sma11 tank diameter, and large D/T ratio. However, if the liquid level is increased sufficiently it was observed that the vortex was reduced in strength and air entrainment eliminated. Lowering of the impeller (i.e., decreasing $h$ ) would also have the same effect. It is possible that the fluctuations of the manometer may be due to the excessive air entrainment. 
The velocity profile data at this impeller speed reported in Appendix $G$ is based on an average value of manometer differential $\Delta \mathrm{H}_{\mathrm{m}}$. The average value of $\Delta \mathrm{H}_{\mathrm{m}}$ being eyed in since it fluctuated in an exratic manner as described above. In spite of these rough estimates of $\Delta \mathrm{H}_{\mathrm{m}}$, the parameters are rather close for the two profiles measured as can be seen in Appendix G. This is reflected in a rather low value for the variance of the tangential jet parameters given in Tables IV-5 to IV-7 for Item 6 . Two profiles were taken at this speed at two radial angles and $r=3.0$. At $r=2.5$ in $(1.0$ in. from impeller periphery), the probe was observed to vibrate excessively due to the large velocity and high intensity of turbulence at that point.

\section{Effect of Tank Diameter}

To measure velocity profiles in the rest of the tank, a tank was assembled from plexiglass having a diameter of 11.5 inches. A single impeller speed of 333.3 RPM was investigated to compare the effect of change in tank diameter. As in the other cases four profiles were measured. The results are in agreement with conclusions made earlier, namely that the tangential jet parameters are independent of radial angle and radial distance. The impeller depth was not varied. The results of this analysis is reported in Appendix $G$ and the average value of the jet parameters are reported as Item 7 in Tables IV-5 to IV-7. From Table IV-5 it is seen that $\sigma$ has the same order of magnitude and confidence limit as in the glass tank. This means that the width of the jet is approximately the 
same and hence impeller depth should have no effect on the jet. In Table IV-6 comparing the average value of $\mathrm{A}$ at $333.3 \mathrm{RPM}$ for the two tanks (Item Numbers 3 and 7), A is smaller in the smaller diameter tank. The difference in the two values is out of the range of their respective confidence 1 imits and it is thus concluded that the diameter of the tank has an effect on the parameter $A$ and hence the pumping capacity of the impeller. In Table IV-7 it is seen that while the average value of $a$ is almost constant for the glass tank, its value for the plexiglass tank is much.larger being very close to the impeller radius of $0.125 \mathrm{ft}$. It is thus concluded that the diameter of the tank has an effect on a, the radius of source.

\section{Correlation of Tangential Jet Parameters}

From Tables IV-5 to IV-7 and the discussion in the previous section the tangential jet parameters can be considered as independent of impeller depth, radial angle and radial distance ( $r$ should be less than 4.0 in.). These tables also indicate that the average values at constant impeller speed are good estimates of the parameters. They are a function of impeller speed, impelier diameter and tank diameter.

In Table IV-8, the average values of the parameter from Tables IV-5 to IV-7 are collected, and reported together with other information on the impeller stream. These are $Q$, the volumetric flow at the impeller periphery, calculated from Equation B-31 with $r=D / 2$ and is given by,

$$
Q=4 \pi A\left(\frac{D}{2 \sigma}\right)^{\frac{1}{2}}\left((D / 2)^{2}-a^{2}\right)^{\frac{1}{2}}
$$


TABLE IV-8

SUMMARY OF RESULTS OF TANGENTIAL JET PARAMETERS FOR A 3.0 INCH DIAMETER IMPELLER IN A 12.25 INCH DIAMETER TANK

\begin{tabular}{|c|c|c|c|c|c|c|c|c|}
\hline $\begin{array}{c}\text { Item } \\
\text { Number }\end{array}$ & $\sigma$ & A & $\mathbf{a}$ & $\begin{array}{l}\text { Impeller } \\
\text { Speed } \\
\text { RPM }\end{array}$ & $\mathbf{Q}$ & $Q / N D^{3}$ & $\bar{\theta}_{y}$ & $\mathrm{ND}^{3}$ \\
\hline 1 & 10.5 & 13.5 & 0.114 & 243.0 & 4.18 & 1.10 & 65.8 & 3.80 \\
\hline 2 & 10.1 & 14.3 & 0.119 & 250.0 & 3.92 & 1.00 & 72.2 & 3.91 \\
\hline 3 & 11.8 & 18.6 & 0.115 & 333.3 & 5.28 & 1.01 & 67.3 & 5.21 \\
\hline 4 & 11.8 & 22.7 & 0.117 & 400.0 & 6.18 & 0.988 & 69.3 & 6.25 \\
\hline 5 & 11.4 & 28.4 & 0.112 & 500.0 & 8.87 & 1.14 & 63.3 & 7.81 \\
\hline 6 & 11.6 & 31.6 & 0.112 & 550.0 & 9.81 & 1.41 & 63.1 & 8.59 \\
\hline $7^{*}$ & 10.7 & 17.9 & 0.122 & 333.3 & 4.13 & 0.79 & 76.6 & 5.21 \\
\hline
\end{tabular}

Correlating Equation

$$
\begin{aligned}
& A=3.47\left(\mathrm{ND}^{3}\right)^{1.024} \\
& Q=0.903\left(\mathrm{ND}^{3}\right)^{1.09} \\
& \mathrm{~A}=0.0004\left(\mathrm{~N}_{\mathrm{Re}}\right)^{1.024} \\
& \mathrm{Q}=0.0001\left(\mathrm{~N}_{\mathrm{Re}}\right)^{1.09}
\end{aligned}
$$

Correlation Coefficient $\mathbf{R}$

$$
\begin{aligned}
& 0.9996 \\
& 0.9897 \\
& 0.9996 \\
& 0.9897
\end{aligned}
$$

\section{Average}

$\sigma$
$\begin{array}{ll}\text { a } & 0.115 \\ \mathrm{Q}_{\text {ND }}{ }^{3} & 1.06\end{array}$
0.003
0.07

11.2

0.8

* Tank diameter 11.5 inches 
Also given is the dimensionless pumping capacity $N_{Q}=Q / N D^{3}$, and the weighted average yaw angle, $\bar{\theta}_{y}$ at the impeller periphery. The angle $\bar{\theta}_{y}$ is calculated by inverting Equation A-14 with $r=D / 2$ and is

$$
\bar{\theta}_{y}=\cos ^{-1}\left(\left[(D / 2)^{2}-r^{2}\right]^{\frac{1}{2}} / r\right)
$$

In Figure IV-2 is shown a $10 \mathrm{l}-10 \mathrm{~g}$ plot of $\mathrm{ND}^{3}$ versus $\sigma$; $\mathrm{ND}^{3}$ versus $A$; and $N D^{3}$ versus $Q$. The best correlation for $\sigma$, the jet width parameter is a horizontal straight line drawn at the average value of $11.2 \pm 2.6$ for all seven points. This is shown in Figure IV-2. The deviation on $\sigma$ is taken as the same as the largest deviation in Table IV-5 which is Item 2 . It should be noted that tank diameter has no effect on $\sigma$ as seen in Table IV-8.

Figure IV-2 shows that both $A$ and $Q$ correlate very well with $N^{3}$ for the 12.25 in diameter tank. The correlation coefficient $R$ for both these plots is 0.99 . Item 7 of Table IV-8 has been plotted in Figure IV-2 as a cross. The deviation of this point from the regression line for $A$ and $\sigma$ is small showing that they correlate reasonably well. The point for $Q$ shows a large deviation from the regression line indicating that the tank diameter has an effect on Q. The reason for this is that the radius of source parameter a is significantly different as can be seen in Table IV-8.

In Figure IV-2 it is seen that the slopes of $A$ and $Q$ are almost equal. This suggests that $A$ is a scaled value of $Q$, hence the name volumetric flow parameter. In Table IV-8 is also given the regression 


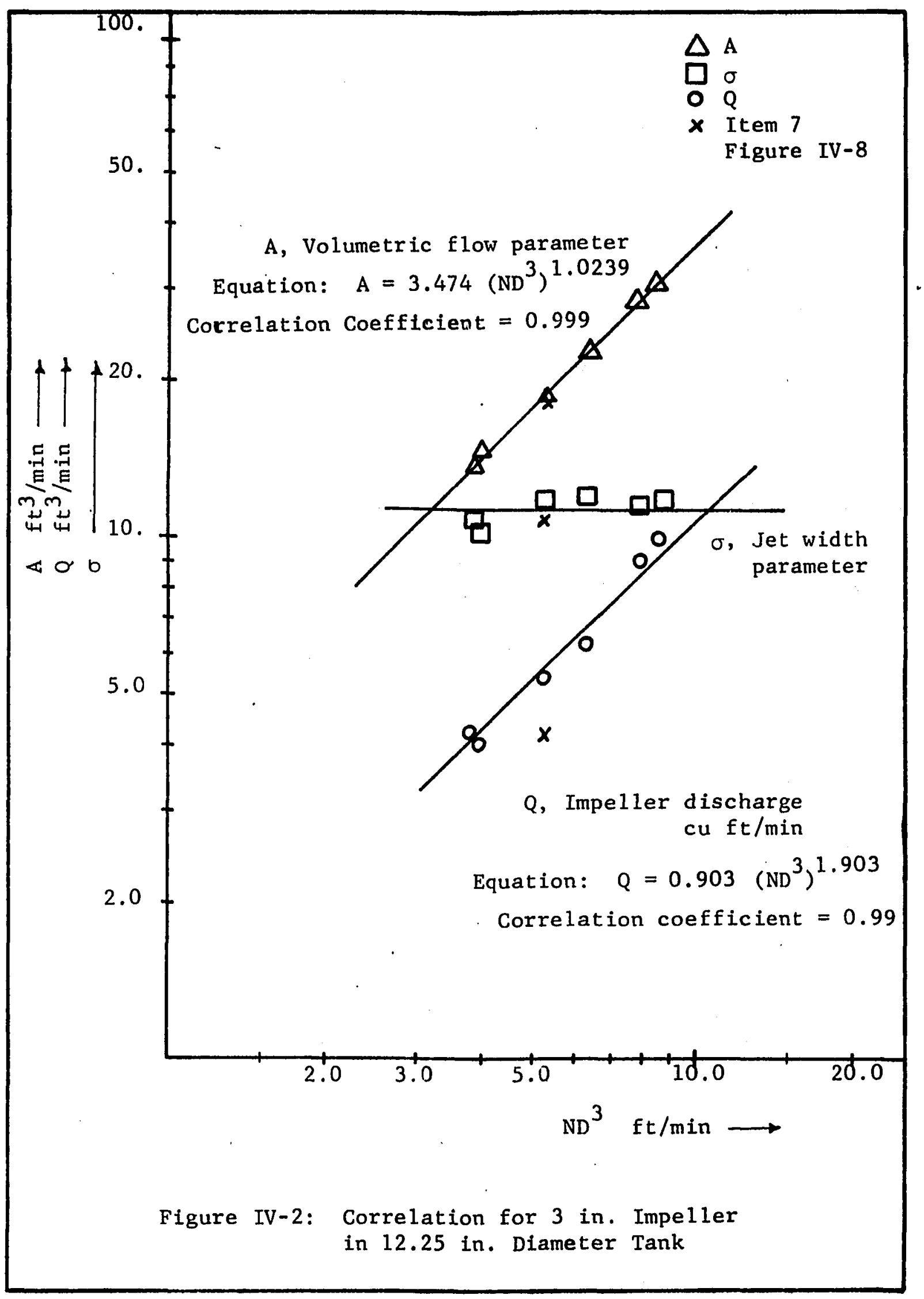


equations for the $\log -\log$ plot. For convenience these are also reported in Figure IV-2. The equations reported in Table IV-8 are for the 12.25 in. diameter tank.

Log-log least square fits of $A$ and $Q$ versus $N_{R e}$ the Reynolds number were also tried. The results for the se equations are given in Table IV-8. Since $N_{R e}=\mathrm{ND}^{2} \rho / \mu$ and, $\rho$ and $\mu$ are constant for $a$ given fluid, this in effect is a correlation with ND ${ }^{2}$. Table IV-8 shows the correlation coefficient for $\mathrm{N}_{\mathrm{Re}}$ or in effect $\mathrm{ND}^{2}$, to be the same as for the correlation with $\mathrm{ND}^{3}$.

In Table IV-8 it is seen that $\theta_{y}$ varies from $63.13^{\circ}$ to $72.18^{\circ}$ whereas the corresponding values of, a, vary only in the third significant place for the 12.25 in. diameter tank. This indicates the relative insensitivity of the tangential jet model to the angle profile and hence the good prediction of experimental velocity profiles by using a weighted average value of $\theta_{y}$.

4.2. Effect of Constant Jet Width and Radius of Source on the Analysis

Since a reasonable estimate of $\sigma$, the jet width and, $a$, the radius of the tangential source were found to be overall average values, the entire analysis was repeated keeping $\sigma$ and a constant at

$$
\begin{aligned}
& \sigma=11.2 \pm 2.6 \\
& a=0.115 \pm 0.008
\end{aligned}
$$

The results of this analysis is also given in Appendix G. The result of fixing the parameters $\sigma$ and a was to increase the sum of the squares SS by a small amount and thus decrease the 
correlation coefficient R. A typical case is illustrated in Table IV-9 which shows a comparison of sum of squares $S S$ and $R$ the correlation coefficient for velocity profiles measured at a constant impeller speed of 243 RPM. The information is extracted from Tables G-2 and G-3 of Appendix G. The increase in SS in Table IV-9 is seen to result in a much larger change in $R$ for $\bar{v}_{r}$ than for $\bar{q}$. This trend of small changes in $R$ for $\bar{q}$ and slightly larger changes in $R$ for $\bar{v}_{r}$ is seen in all the Tables of Appendix $G$. In those profiles where $R$ has a relatively low value, the change in $R$ will be proportionately larger. This is clearly seen in case of Profile Number 8 of Table IV-9 where R drops from 0.917 to 0.841 .

In Table IV-10 the volumetric flow parameter $A$ and $z_{0}$ are presented to see the effect of keeping $\sigma$ and a as constant. Both parameters $A$ and $z_{0}$ show very. 1ittle variation as can be seen in Table IV-10. The fact that there is no variation in $z_{0}$ is not significant, its only purpose is to shift the coordinates as pointed out at the beginning of this chapter. This trend is the case for all the profiles analyzed as can be verified in Appendix G. It is thus concluded that the parameters $\sigma$ and a can be treated as constants independent of impeller speed, radial angle or impeller depth. In addition $\sigma$ is independent of tank diameter.

The reason for the above behavior is a peculiarity of nonlinear least square fits. In general the larger the number of parameters the better the fit. However if one of these parameters is a constant the nonlinear least square fit will treat it as a variable 


\section{, TABLE IV-9}

EFFECT OF CONSTANT $\sigma$ AND, $a$, ON THE SUM OF SQUARES SS, AND CORRELATION COEFFICIENT FOR $\bar{q}$ AND $\bar{v}_{\mathrm{r}}$ AT

IMPELLER SPEED OF 243 RPM

\begin{tabular}{|c|c|c|c|c|c|c|}
\hline \multirow[b]{2}{*}{$\begin{array}{l}\text { Profile } \\
\text { Number }\end{array}$} & \multirow[b]{2}{*}{$\begin{array}{c}\text { Radial } \\
\text { Distance } \\
\text { in. }\end{array}$} & \multirow[b]{2}{*}{$\begin{array}{l}\text { Radial } \\
\text { Angle }\end{array}$} & \multicolumn{2}{|c|}{$\begin{array}{c}\text { Sum of Square } \\
\text { SS } \\
\end{array}$} & \multicolumn{2}{|c|}{$\begin{array}{c}\text { Correlation } \\
\text { Coefficient } \\
\end{array}$} \\
\hline & & & $\bar{q}$ & $\bar{v}_{r}$ & $\bar{q}$ & $\bar{v}_{r}$ \\
\hline
\end{tabular}

(a) $\sigma$ and, a, constant

$\begin{array}{rrrrrrr}1 & 2.5 & 44.1 & 201 & 319 & 0.988 & 0.979 \\ .2 & & 44.1 & 77 & 279 & 0.996 & 0.982 \\ 3 & & 5.5 & 23 & 212 & 0.999 & 0.988 \\ 4 & 3.0 & 44.1 & 52 & 36 & 0.995 & 0.996 \\ 5 & & 5.5 & 114 & 59 & 0.989 & 0.994 \\ 6 & 3.5 & 44.1 & 51 & 49 & 0.990 & 0.989 \\ 7 & & 5.5 & 85 & 55 & 0.986 & 0.990 \\ 8 & 4.0 & 44.1 & 635 & 537 & 0.841 & 0.862 \\ 9 & & 5.5 & 110 & 78 & 0.960 & 0.971\end{array}$

(b) $\sigma$ and, a, obtained by least square fit

$\begin{array}{rrrrrrr}1 & 2.5 & 44.1 & 179 & 222 & 0.990 & 0.985 \\ 2 & & 44.1 & 39 & 146 & 0.998 & 0.991 \\ 3 & & 5.5 & 23 & 168 & 0.999 & 0.990 \\ 4 & 3.0 & 44.1 & 41 & 47 & 0.996 & 0.995 \\ 5 & & 5.5 & 64 & 73 & 0.994 & 0.992 \\ 6 & 3.5 & 44.1 & 21 & 26 & 0.996 & 0.994 \\ 7 & & 5.5 & 29 & 41 & 0.995 & 0.993 \\ 8 & 4.0 & 44.1 & 344 & 316 & 0.917 & 0.921 \\ 9 & & 5.5 & 19 & 26 & 0.993 & 0.990\end{array}$


TABLE IV-10

EFFECT OF CONSTANT $\sigma$ AND, a, ON THE VOLUMETRIC FLOW

PARAMETER A AND JET DISPLACEMENT PARAMETER

$z_{0}$ AT IMPELLER SPEED OF 243 RPM

\begin{tabular}{|c|c|c|c|c|}
\hline \multirow[b]{2}{*}{$\begin{array}{l}\text { Profile } \\
\text { Number }\end{array}$} & \multicolumn{2}{|c|}{$\begin{array}{c}\sigma \text { and } a \\
\text { Constant }\end{array}$} & \multicolumn{2}{|c|}{$\begin{array}{l}\sigma \text { and, } a \text {, obtained } \\
\text { By Least Square Fit }\end{array}$} \\
\hline & $\begin{array}{c}\mathrm{A} \\
\mathrm{ft}\end{array}$ & $z_{0}, f t$ & $\begin{array}{c}\mathrm{A} \\
\mathrm{ft}{ }^{3} / \mathrm{min} \\
\end{array}$ & $z_{0}, f t$ \\
\hline 1 & 13.5. & 0.000457 & 13.5 & .0 .00048 \\
\hline 2 & 13.4 & -0.000731 & 13.3 & -0.00071 \\
\hline 3 & 13.5 & 0.001305 & 13.4 & 0.00131 \\
\hline 4 & 12,9 & 0.001954 & 13.0 & 0.00198 \\
\hline 5 & 13.4 & 0.001570 & 13.4 & 0.00160 \\
\hline 6 & 13.2 & 0.003100 & 13.4 & 0.00325 \\
\hline 7 & 13.4 & 0.005407 & 13.5 & 0.00542 \\
\hline 8 & 13. & 0.006688 & 14.0 & 0.00621 \\
\hline 9 & 13.6 & 0.005964 & 13.9 & 0.00612 \\
\hline
\end{tabular}


to reduce the scatter in the data as measured by sum of squares SS about the regression 1 ine. It therefore becomes necessary to exercise proper judgement to get meaningful results from nonlinear least square fits.

The result of considering $\sigma$ and a as constants has more revelance when the volumetric flow at the impeller periphery and at the point of measurement $r$ is examined in Table IV-11. The volumetric flow at the impellex periphery (impeller discharge) exhibits some scatter when $\sigma$ and $a$ are free. For Profiles 8 and 9 where $r$ is 4.0 in., the volumetric flow appears to be larger. This erratic behavior disappears when $\sigma$ and a áre taken as a constant. The volumetric flow at radial distance $r$ is not affected appreciably up to $r$ equal to $3.5 \mathrm{in}$. There is a significant difference in volumetric flow for $r$ equal to 4.0. If Equation $\mathrm{IV}-7$ is examined it is seen that keeping $\sigma$ and a constant does not make the impeller discharge constant (at fixed impeller speed) unless A was a constant too.

In Table IV-12 is shown the average value of $A$, averaged over profiles obtained at a constant impeller speed. The number of profiles over which this average is taken is given in the last column. The variance and confidence limit in this table has small values. This indicates that $A$ has a constant value at constant impeller speed, and this is the reason for constant $Q$ values in Table IV-11 noted above. The large confidence 1 imit in Item 6 is because of small sample size. The conditions under which the velocity profiles (Item 6) were obtained are questionable and were discussed in an 
TABLE IV- 11

EFFECT OF CONSTANT $\sigma a$ AND a ON VOLUMETRIC FLOW AT IMPELLER PERIPHERY AND AT RADIAL DISTANCE $r$ FOR A CONSTANT IMPELLER SPEED OF 243 RPM

\begin{tabular}{|c|c|c|c|c|c|c|}
\hline \multirow[b]{2}{*}{$\begin{array}{l}\text { Profile } \\
\text { Number }\end{array}$} & \multirow[b]{2}{*}{$\begin{array}{l}\text { Radial } \\
\text { Distance } \\
\mathbf{r} \text { in. } \\
. \quad \ldots \quad \ldots \ldots\end{array}$} & \multirow[b]{2}{*}{$\begin{array}{l}\text { Radia } 1 \\
\text { Angle }\end{array}$} & \multicolumn{2}{|c|}{$\begin{array}{l}\sigma \text { and a Obtained } \\
\text { by Least Sq. Fit }\end{array}$} & \multicolumn{2}{|c|}{$\sigma$ and a Constant } \\
\hline & & & $\begin{array}{l}\text { Flow at } \\
\text { Impe 1ler } \\
\text { CEM } \\
\end{array}$ & $\begin{array}{l}\text { Flow at } \\
\text { Radia } 1 \\
\text { Distance } \\
r \text {, CFM }\end{array}$ & $\begin{array}{l}\text { Flow at } \\
\text { Impe11er } \\
\text { CFM } \\
\ldots\end{array}$ & $\begin{array}{l}\text { Flow at } \\
\text { Radial } \\
\text { Distance } \\
\text { r. CFM }\end{array}$ \\
\hline 1 & 2.5 & 44.1 & 3.9 .81 & 9.50 & 4.001 & 9.68 \\
\hline 2 & & 44.1 & 3.741 & 9.30 & 3.970 & 9.59 \\
\hline 3 & & 5.5 & 3.373 & 9.51 & 4.022 & 9.71 \\
\hline 4 & 3.0 & 44.1 & 3.869 & 11.67 & 3.839 & 11.48 \\
\hline 5 & & 5.5 & 3.502 & 12.19 & 3.975 & 11.89 \\
\hline 6 & 3.5 & 44.1 & 4.684 & 14.71 & 3.925 & 13.93 \\
\hline 7 & & 5.5 & 4.004 & 14.78 & 3.969 & 14.08 \\
\hline 8 & 4.0 & 44.1 & 5.561 & 19.29 & 3.946 & 16.17 \\
\hline 9 & & 5.5 & 4.445 & 18.13 & 4.031 & 16.52 \\
\hline
\end{tabular}




\section{TABLE IV-12}

EFFECT OF CONSTANT $\sigma$ AND a ON THE AVERAGE

VALUES OF THE VOLUMETRIC FLOW PARAMETER A

\begin{tabular}{cccccc}
$\begin{array}{c}\text { Item } \\
\text { No. }\end{array}$ & $\begin{array}{c}\text { Impeller } \\
\text { Speed } \\
\text { RPM }\end{array}$ & $\begin{array}{c}\text { Average } \\
\text { Value } \\
\text { of A }\end{array}$ & Variance & $\begin{array}{c}\text { Confidence } \\
\text { Limit }\end{array}$ & $\begin{array}{c}\text { Number } \\
\text { of } \\
\text { Profiles }\end{array}$ \\
\hline 1 & 243 & 13.4 & 0.039 & 0.2 & 9 \\
2 & 250 & $14.1:$ & 0.196 & 0.7. & 4 \\
3 & 333.3 & 18.6 & $0.084:$ & $0.5:$ & 4 \\
4 & 400 & 22.9 & $0.084:$ & 0.5 & 4 \\
5 & 500 & 28.3 & $0.539:$ & 0.5 & 12 \\
6 & 550 & 31.6 & $0.144:$ & $3.4:$ & 2 \\
7 & 333.3 & 17.8 & 0.236 & 0.8 & 4 \\
* Tank diameter $=11.5$ inches & & &
\end{tabular}


earlier section. Comparing Tables IV-12iand IV-6 the value of $A$ are not significantly different being within the estimates of their error bounds as reported by the deviation. Hence keeping $\sigma$ and a constant has not materially affected the least square fit of the data.

Width of the Tangential Jet

An important conclusion that resülts from considering $\sigma$, the jet width parameter, constant can be obtained from considering Equation $B-25$ which is

$$
\bar{v}_{x}=\left(\bar{v}_{-x}\right)_{\max }\left[\tanh ^{2}(\eta / 2)\right]
$$

This equation can be rewritten as:

$$
\frac{\bar{v}_{r}}{\left(\bar{v}_{r}\right)_{\max }}=f(\eta)
$$

where

$$
\eta=\sigma \frac{\mathbf{r}}{\mathbf{z}}
$$

Equation IV-11 is a restatement of the original assumption that the velocity profiles are similar. From IV-12 it is evident that since $\sigma$ is independent of impeller speed, hence the width of the jet is also independent of impeller speed, and $\eta$ is a function of $r$ and $z$ only. From IV-11 it then follows that $\bar{v}_{r} /\left(\bar{v}_{r}\right)_{\max }$ is independent of the impeller speed and $a$ function of $r$ and $z$ only. This result has been observed by Sachs (28) and Aiba (1) and Cutter ( 9 ), thus confirming the conclusion that $\sigma$ is a constant. 
In Chapter II, Section 2.2 it was shown that the maximum radial distance $r_{0}$, that the tangential jet model holds is given by Equation II-28,

$$
r_{0}=\frac{T \sigma}{12+2 \sigma}
$$

Substituting $T=12.25 \mathrm{in}$. and $\sigma$ from $I V-9$, gives $r_{0}=4.2 \mathrm{in}$. In Table IV-3 profiles 11 and 12 are runs made at $r$ equals 4 in., a radial distance close to $\mathbf{r}_{0}$, where the tangential jet model breaks down. It is thus not surprising that the values of $\sigma$ is much lower than the average value of $\sigma$. This effect was also noticed in profiles 8 and 9 of Table IV-4. It is for this reason that in subsequent runs the maximum value of the radial distance $r$ is limited to $r$ equal to 3.0 in., well below $r_{0}$.

Jet Dispłacement Parameter

The parameter $z_{0}$ is a measure of the displacement of the jet from the impeller centerline. It is obtained under all cases by a least square fit of the data and reported in the Tables of Appendix $G$ under the column $P(4)$. The value of $z_{0}$ is extremely small ranging from 0.003 to $0.009 \mathrm{ft}$. and can be either positive or negative. In Table IV-10 where profiles 1 and 2 are replicate measurements made at exactly the same points, $z_{0}$ has a positive value for Profile 1 and negative value for profile 2. It is more often positive than negative and most cases shows about twice the displacement for profile measurements made near the baffle than in between baffles, The value of $z_{o}$ is not affected appreciably by fixing $\sigma$ and a at 
their average values. This is because $z_{0}$ can only cause a shift or displacement in the profile, it cannot alter the shape of the profile. Hence it does not affect the properties of jet which are der termine by the shape of the velocity profile. The only anomalous result is in Brofile 3 of Table G-11 (impeller speed 500 RPM) where $z_{0}$ has an excessively high value of $-0.94: i n i$. The correlation coefficient is rather low for this profile casting further doubts on this particular measurement.

4.3. Velocity Profile Measurements in the Rest of the Tank

Figure IV-3 shows a computer drawn solution, drawn by the program PLOTER described in Chapter II. This is a solution for a 3.0 in. diameter impeller, centrally located in a 11.5 in. diameter tank, depth of fluid is $12.0 \mathrm{in}$. and the impeller speed is 500 RPM. The values of the parameters needed for this solution are (a) the tangential jet parameters and (b) the geometric parameters that set the boundaries of the different flow regions into which the tank has been divided as explained in Chapter II. The tangential jet parameters have been evaluated in Section 4.2 and are; $\sigma$ and a given by Equations IV-9 and IV-10 and the volumetric parame ter A obtained from Figure IV-2. The geometric parameters are taken as that for Figure II-9 (c) with one exception. XN4 was set at 0.55 instead of 0.5 , as this solution was considered the best representation of the flow field.

In Figure IV-3 it is seen that the streamlines in the region outside the impeller tend to stay close to the tank wall and the axis of the impeller. This is in agreement with Nagata's profile as can 


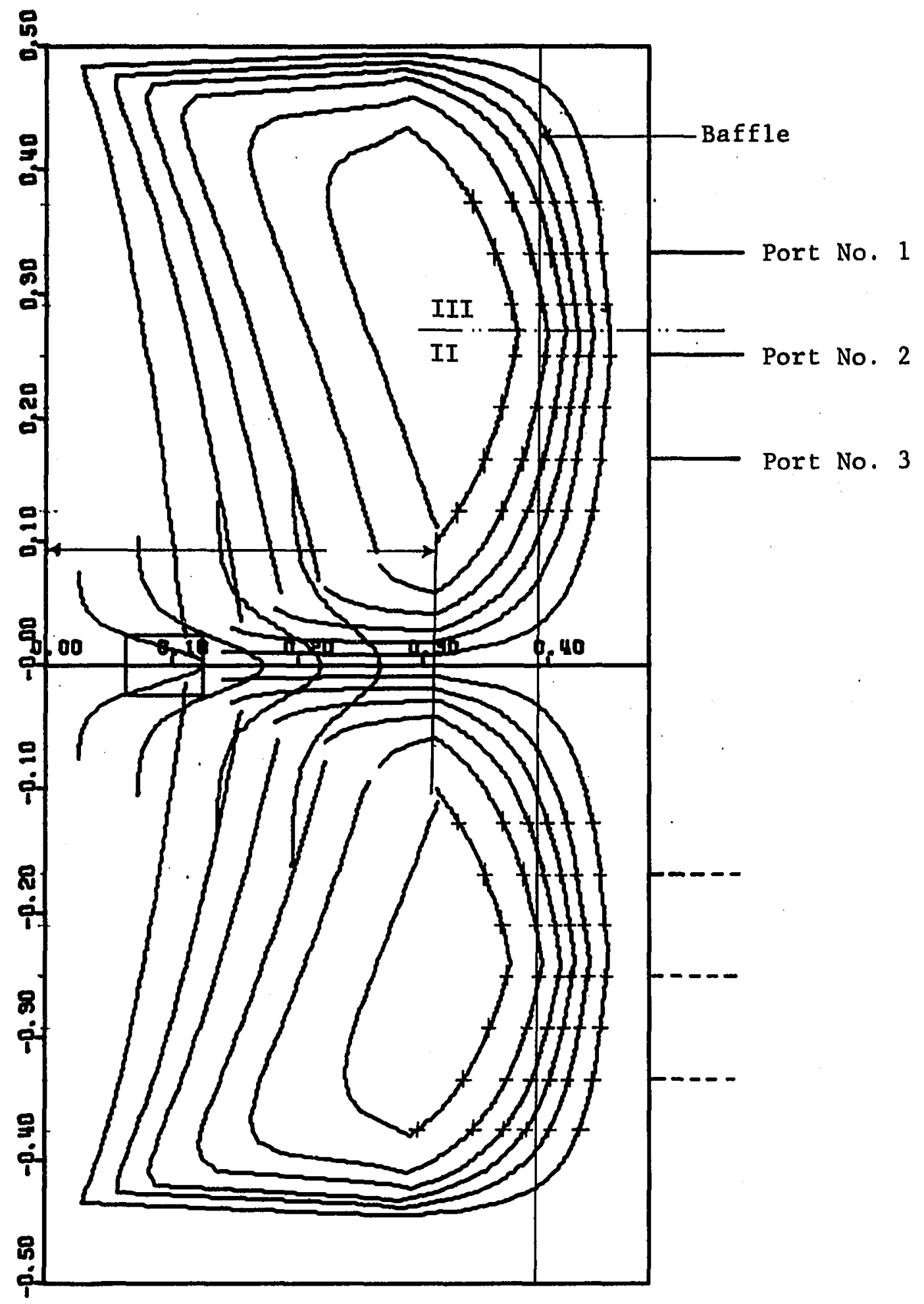

Figure IV-3:

Theoretical streamlines for a $3.0 \mathrm{in.} \mathrm{Impeller} \mathrm{at} 500$ RPM Values of Parameters are $=11.192, \mathrm{~A}=28.24, \mathrm{a}=0.1147$, $\mathrm{h}=6.0$. Depth of Water $12.0 \mathrm{in}$. and Tank diameter $=11.5 \mathrm{in}$. Each Pair of Streamlines Fnrlnse $5 \mathrm{ft}^{3} / \mathrm{min}$ or Water. This Theoretical Profile Illustrates a Boundary Layer at the Tank Bottom having a Width of $10 \%$ of the Fluid Depth. 
be seen in Figure I-5. This is not exactly surprising since up to the radial distance $r_{0}$ (the point where the tangential jet model ends) the flow in the rest of the tank is into the impeller and the jet. The peripherial region $T / 2-r_{0}$ is thus left for the upward and downward flow out of the tangential jet. In our case, $r_{0}$ was estimated to be 3.98 inches (see Section 4.2), hence the thickness of the upward and downward flow from the stagnation region is restricted to a narrow peripherial region of 1.9 inches. The flow as it leaves Region II tends to stay close to the wall as noticed by the crowding of the streamlines. The maximum crowding of the streamlines and thus the minimum width of the flow occurs roughly half way between the impeller center line and the liquid surface. The minimum width is of order of one baffle width thick, as seen in Figure IV-3 where the vertical line represents a 1 inch baffle. The theoretical solution shown in Figure IV-3 is for a 11.5 in diameter tank with impeller speed of 500 RPM. This represents the condition under which velocity profiles were measured outside the impeller region which will now be described. In order to get a quantative idea of the velocity profiles in the region outside of the impeller, three ports where drilled into a plexiglass tank 11.5 in. in diameter at 10,8 and 9 inches from the bottome of the tank. These ports are numbered 1, 2, and 3 and their locations are marked on Figure IV-3. The three dimensional pitot tube was used to make a horizontal pass through these ports to measure velocity profiles. The flow is symmetrical and hence only one quadrant need be considered. Also 
Port Number 2 is located where the velocities would be a maximum and hence the probe should give a maximum response. Ports 1 and 3 were placed 1.0 in. above and below Port 2 so as to further explore the velocity field.

\section{Velocity Measurements in the Rest of the Tank}

As noted in Chapter III, probe response is a function of flow geometry. In Figure IV-4 (a) is shown schematically the probe response close to the tank wall. $\theta_{p}$ is the pitch angle and $\theta_{y}$ is the yaw angle. The yaw angle $\theta_{y}$ is obtained by rotating the probe t111 the manometer $\mathrm{C}$ balances as explained in Chapter III. $\theta_{\mathrm{y}}$ is taken as zero when the probe points vertically upwards. With this as a reference point, the component velocities can be calculated with their correct sign if in case (a) where the flow is upward

$$
\theta_{y}=\left(\theta_{y}\right)_{\exp }+90^{\circ}
$$

and in case (b) where the flow is downward

$$
\theta_{y}=180-\left(\theta_{y}\right) \exp
$$

The above equations follow from the geometry of Figure IV-4 (a) and $\operatorname{IV}-4$ (b).

The pitch angle $\theta_{y}$ is obtained from the manufactures calibration plot which is shown in Figure IV-5. Curve $A$ is an empirical plot of $\left(P_{4}-P_{5}\right) /\left(P_{1}-P_{2}\right)$ versus $\theta_{p}$; where $\left(P_{4}-P_{5}\right)$ is the pressure drop recorded by manometer $D$ and $\left(P_{1}-P_{2}\right)$ is the pressure drop from manometer $B$. Curve $B$ is an empirical plot of $\left(P_{t}-P_{s}\right) /\left(P_{1}-P_{2}\right)$ versus $\theta_{p}$, 


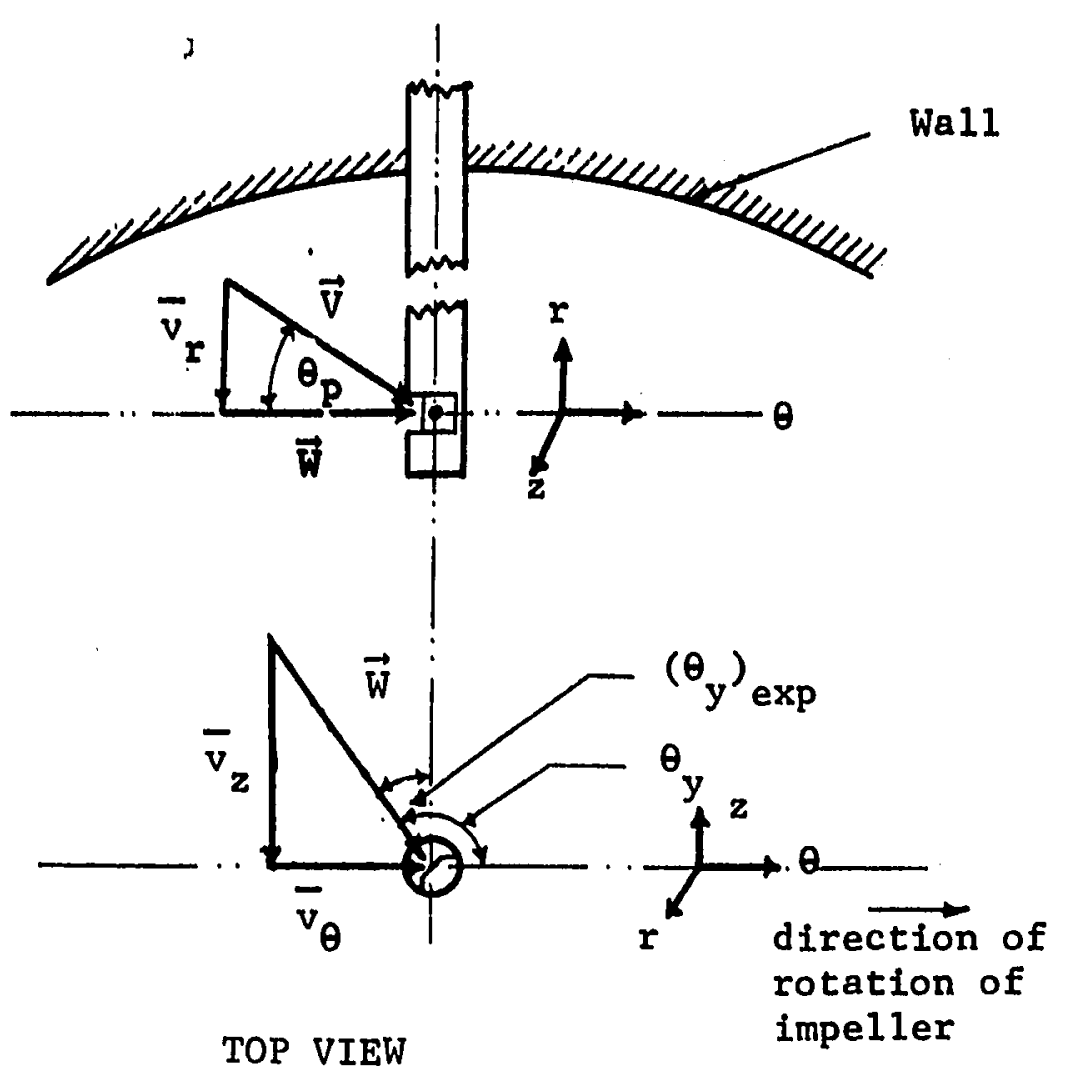

(a) Far from Tank Wall

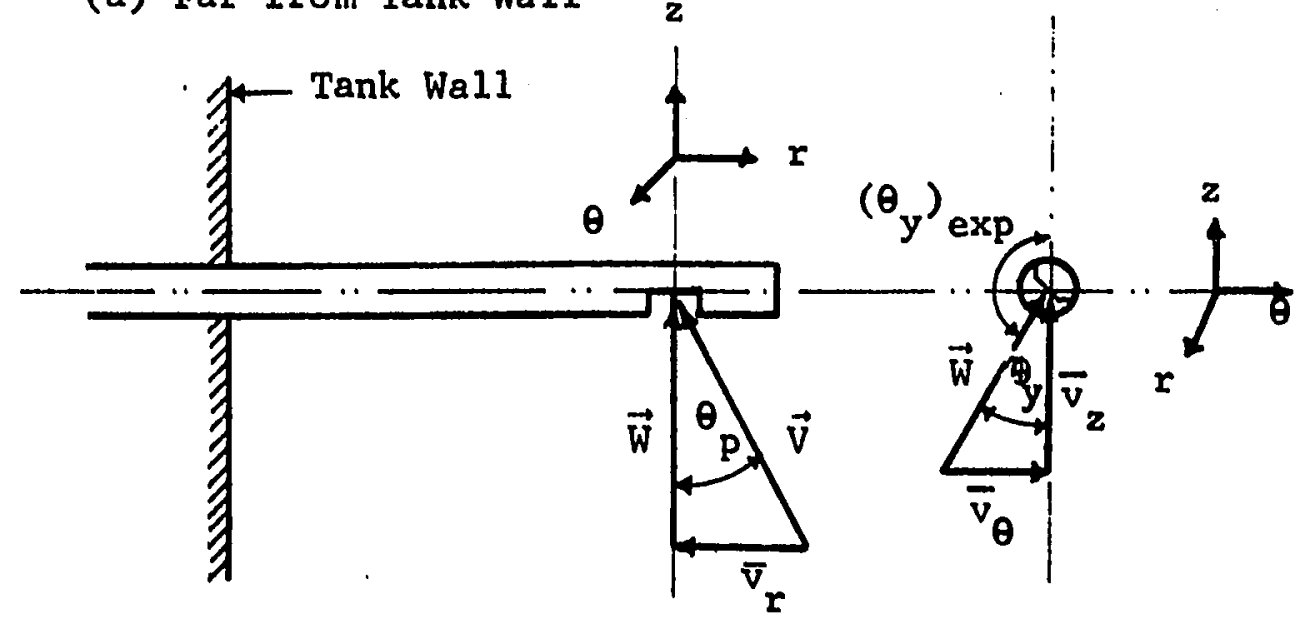

END VIEW

(b) Close to Tank Wall

Figure IV-4: Resolution of Velocity into its Components When the Three Dimensional Probe is in a Horizontal Position. 


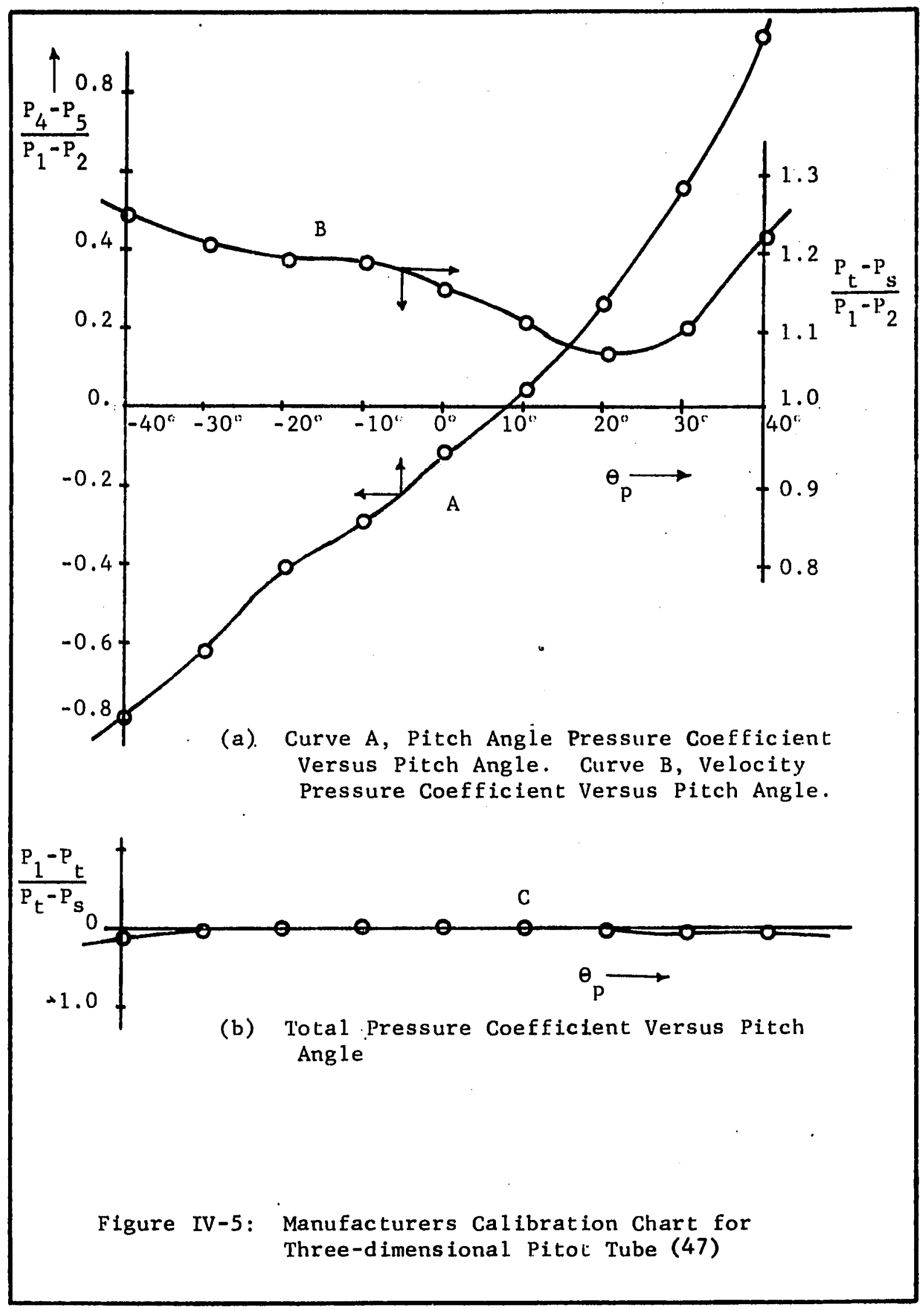


where $\left(P_{t}-P_{s}\right)$ is the true kinetic head and $\left(P_{1}-P_{2}\right)$ is the actual kinetic head recorded by the instrument. Curve $C$ indicates that the kinetic head $\left(\mathrm{P}_{t}-\mathrm{P}_{\mathrm{s}}\right)$ is the true kinetic head provided that $\theta_{p} \leq$ $\pm 40^{\circ}$

A program called TANKANL was written that analyses the data obtained from the horizontal pitot tube runs. The program and the input data are given in Appendix F. The program uses Equations IV-13 and IV-14 to give correct value of $\theta_{y}$. It next calculates $\left(\mathrm{P}_{4}-\mathrm{P}_{5}\right)$, $\left(P_{1}-P_{2}\right)$ and the ratio of $\left(P_{4}-P_{5}\right) /\left(P_{1}-P_{2}\right)$. Curve $A$ is then interpolated by a three point Lagrange interpolation subprogram OMEGA to give $\theta_{\mathrm{p}}$. OMEGA is used again with $\theta_{\mathrm{p}}$ as input to obtain $\left(\mathrm{P}_{t}-\mathrm{P}_{s}\right) /$ $\left(P_{1}-P_{2}\right)$ from curve $B$. A logical trap omits calculations when the ratio $\left(P_{4}-P_{5}\right) /\left(P_{1}-P_{2}\right)$ is less than -0.8 or greater than 0.8 as these values are outside the range of the calibration chart. Let $\alpha$ be the values obtained from the calibration chart using curve $B$, then

$$
\alpha=\frac{\mathrm{P}_{\mathrm{t}}-\mathrm{P}_{\mathrm{s}}}{\mathrm{P}_{1}-\mathrm{P}_{2}}
$$

The velocity $\vec{V}$ recorded by the three dimensional probe is then

$$
\vec{V}=\left(2 g \alpha\left(P_{1}-P_{2}\right)\right)^{\frac{1}{2}}
$$

From Figure IV-3 and from geometry, the component velocities can be written as

$$
\begin{aligned}
& \bar{v}_{\dot{r}}=|\vec{v}| \sin \theta_{p} \\
& \bar{v}_{z}=|\vec{v}| \cos \theta_{p} \sin \theta_{y}
\end{aligned}
$$




$$
\bar{v}_{\theta}=|\vec{v}| \cos \theta_{p} \cos \theta_{y}
$$

where $\theta_{y}$ in IV-18 and IV-19 are given by Equations IV-3 or IV-14. The above equation gives the component velocities with their correct sign. $\dot{\vec{v}}_{\theta}$ is considered positive in the direction of rotation of the impeller, and the positive values of $\bar{v}_{z}$ and $\bar{v}_{r}$ are shown in Figure IV-4.

\section{Experimental Velocity Profiles}

The results of the experimental velocity profiles are calculated by program TANKANL and are given in Appendix $\mathrm{H}$. These velocity profiles are summarized here as plots of $\bar{v}_{r}, \bar{v}_{\theta}$ and $\bar{v}_{z}$ versus the distance from the tank wall; $T-r$ in Figures $I V-6$ to $I V-8$. The data points for these plots are taken from tables given in A'ppendix $J:$ In Figure IV-6 is shown a plot of radial velocity $\bar{v}_{\mathrm{r}}$ for Port Numbers 1,2 , and 3. The velocity profiles of Port Numbers 2 and 3 are approximately of the same magnitude, that of Port Number 2 being higher near the wall, while Port Number 3 is higher at the center of the tank. This behavior is reasonable since near the wall Port Number 3 is still in the stagnation region, while near the tank center, Port Number 3 is closer to the impeller and the fluid begins to turn and accelerate due to the impeller suction and entrainment in the jet. The profiles take a dip at 1.5 inches from the wall and this point corresponds to the stagnation region observed by Nagata. The $\bar{v}_{r}$ velocity profile for Port Number 1 shows peculiar behavior, showing a positive value between 0.5 and 1 inch. This behavior is possibly due to erratic probe response. 


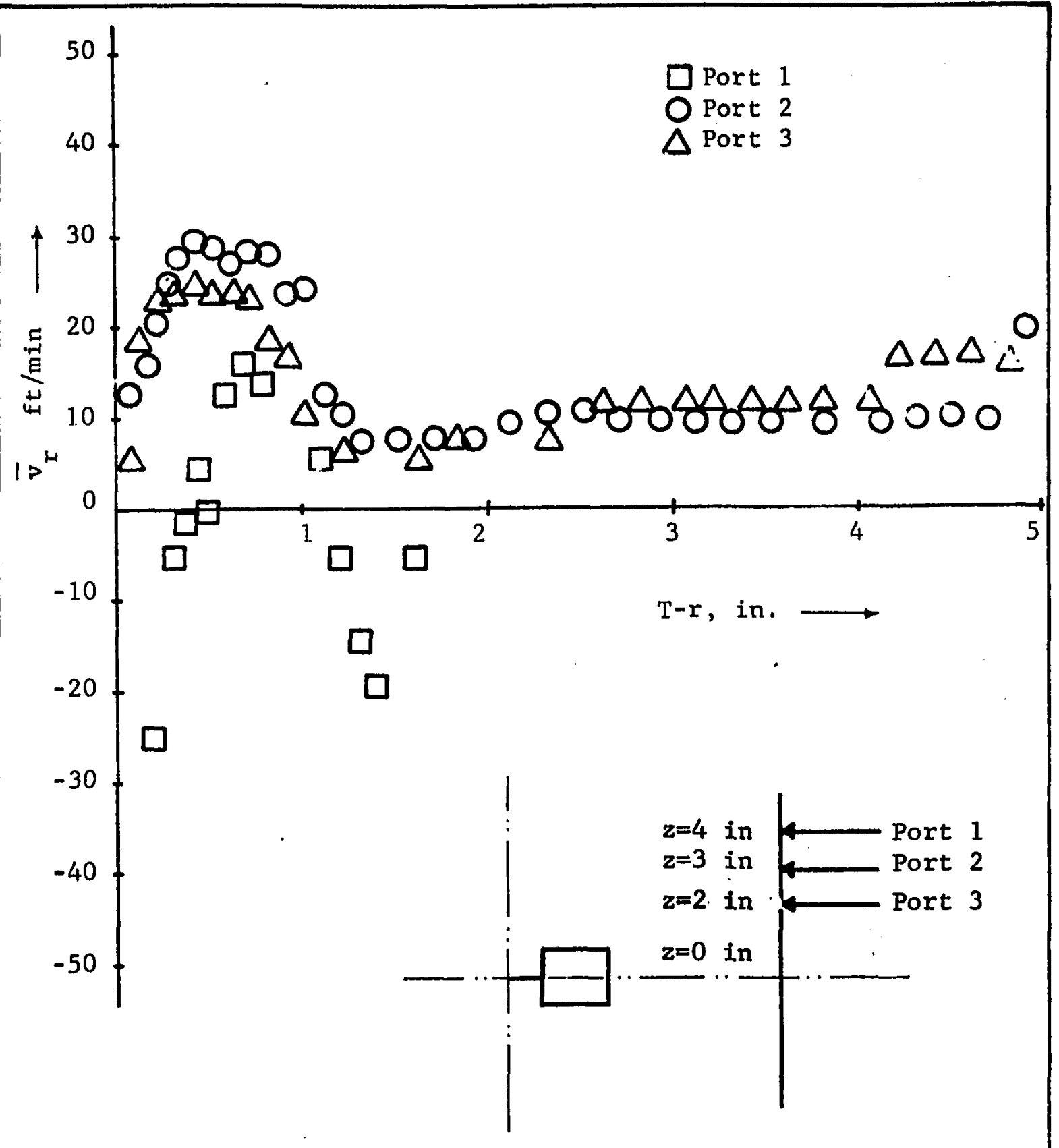

Figure IV-6: Experimental Velocity Profiles of $\bar{v}_{\mathbf{r}}$ for Ports 1, 2, and 3 


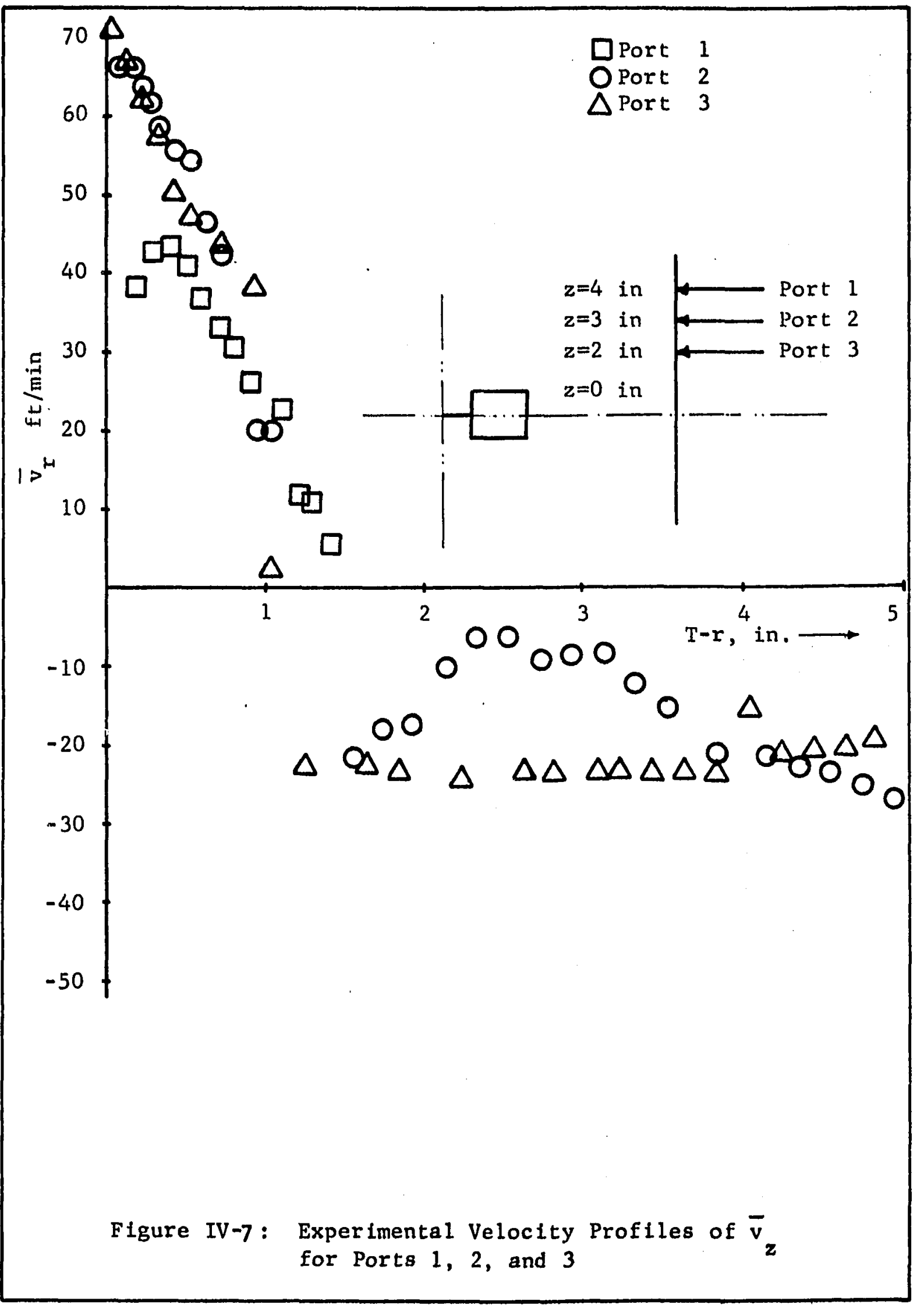




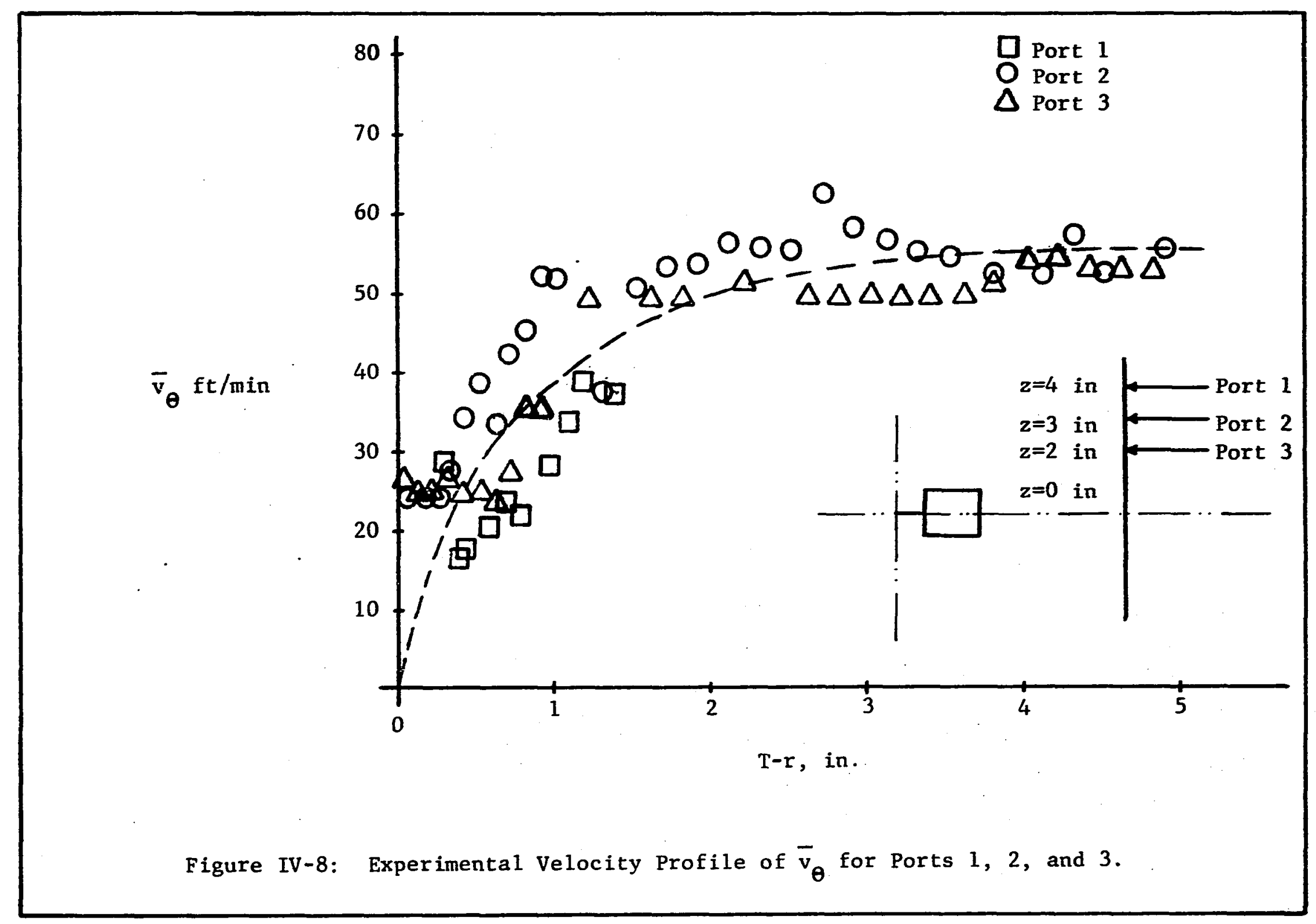


In Figure IV -7 is shown the vertical velocity profile $\vec{v}_{z}$. The velocity profile for Ports 2 and 3 are virtually indentically near the wal1. For Port Number 1 (located 4.0 inches from impeller centerline), $\bar{v}_{z}$ is much smaller close to the wall and shows a manimum, at $T-r$ of 0.4 inch. This Indicates that at Port Number 1 the fluid is beginning to turn. Beyond $\mathrm{T}-\mathrm{r}$ of $1.6 \mathrm{in}$. only one negative value at $T-r$ equal to 1.6 coủld be analyzed. This is because the pitch angle $\theta_{\mathrm{p}}$ was larger than $40^{\circ}$.

For T-r larger than 1.0 in., $\bar{v}_{2}$ for Port Numbers 2 and 3 deviate from one another. This is because Port Number 3 is closer to jet while port Number 2 crosses the center of circulation. At the center of circulation the flow is largerly tangential, $\bar{v}_{\theta}$ being larger than $\bar{v}_{r}$ or $\bar{v}_{z}$. This can be seen in Figure IV-8 which shows $\bar{v}_{\theta}$ to have reached near its maximum value at $T-r=2.5 \mathrm{in}$. At this point $\bar{v}_{z}$ for Port Number 2 reaches its smallest value thus accounting for the stagnation point observed by Nagata in the $r-z$ plane. From Figures $\mathrm{IV}-6$ and $\mathrm{IV}-7$ the stagnation point could be estimated at $T-x$ of 1.5 to 2.0 inkhes. In our theoretical model, this point can be calculated from Equation II-28. and is found to have a value of $3.98 \mathrm{in.}$, or $\mathrm{T}-\mathrm{r}=1.9 \mathrm{in}$. Considering the precision of the velocity measurements the calculated and experimental value of the circulation point are not significantly different. It will be recalled that Equation II-28 was obtained on the assumption that the tangential jet model breaks down at $r_{0}$, the $r$ coordinate of the center of circulation. The above evidence supports this assumption. 
In Figure IV-8 is shown the plot of $\overline{\mathrm{v}}_{\theta}$ versus $\mathrm{T}-\mathrm{r}$. In this plot $\bar{v}_{\theta}$ decreases as it approaches the tank wall and reaches a maximum limiting value at 4.0 inches from the tank wall. $\bar{v}_{\theta}$ for all three ports have the same order of magnitude and a dotted line has." been drawn through the data points as shown in Figure IV-8. This means that within the region covered by the three parts, $\bar{v}_{\theta}$ is independent of $z$ and a function of $x$ only. Figure IV-8 indicates that $\bar{v}_{\theta}$ is not small in the region outside the impeller. Comparing its magnitude with that of $\vec{v}_{r}$ in Figure IV-6 it is seen that $\bar{v}_{\theta}$ is larger than $\bar{v}_{r}$ except in a narrow region near the tank wall. In the case of $\bar{v}_{z}, \bar{v}_{\theta}$ is smaller than $\bar{v}_{z}$ near the tank wall and larger than $\bar{v}_{z}$ for from the tank wall. These observations indicate that $\bar{v}_{\theta}$ has an appreciable magnitude and thas cannot be neglected in comparison with $\bar{v}_{x}$ and $\bar{v}_{z}$. The flow field is thus three dimensional.

A clearer idea of the three dimensional structure of the flow field in the region outside the impeller is obtained on examining the values of $\vec{V}$ the resultant velocity in the tables given in Appendix $\mathrm{H}$. It was found that for Port $1, \overrightarrow{\mathrm{V}}$ ranges from $56 \mathrm{ft} / \mathrm{min}$ near the wall steadily decreasing to $36 \mathrm{ft} / \mathrm{min}$ far from the wall. For Port Number 2 this range is $71 \mathrm{ft} /$ min near the wall decreasing to $58 \mathrm{ft} / \mathrm{min}$ far from the wall. In the case of Port Number 3 $\vec{V}$ is $56 \mathrm{ft} / \mathrm{min}$ near the wall increasing to $74 \mathrm{ft} / \mathrm{min}$ far from the wa11. The increase in velocity far from the wall for Port Number 3 reflects the acceleration of the fluid by impeller suction and jet entrainment. 
It should be noted that probe readings very close to the tank wall are not rellable. This is because the probe interacts with the flow field tending to report higher values of velocity. The probe manufactureres recommends that readings be taken beyond 4 to 5 probe diameters for a 4.0 inch diameter duct. Since the tank is 11.5 In., in diameter this restriction could be relaxed somewhat, hence readings taken for $T-r$ less than 0.5 inches are considered as biased.

\section{Theoretical Velocity Profiles}

For comparison, theoretical: profiles at Port Numbers 1, 2, and 3 were also calculated and these are shown in Figures IV-9 and IV-10. No values for $\bar{v}_{\theta}$ exist as it was assumed to be zero in the theoretical model. Since the theoretical model as drawn by the program FOWANL and given in Figure IV-3, does not give the stream function explicitly it had to be calculated. For this purpose the program FLOWANL was made to punch out the value of $\psi$ and the corresponding radial position $r$ for seven different constant $z$ values. These are shown by plus marks on the streamline in Figure IV-3. Since no additional effort is required to obtain the corresponding points in the bottom of the tank, these were also analyzed. These points are alsol marked in Figure IV-3. The answers will of course be different since the profile in bottom half of the tank was drawn with a boundary layer.

The program VELPRO was written to calculate $\bar{v}_{r}$ and $\bar{v}_{z}$ from the following definitions of $\psi$ for stream surfaces 


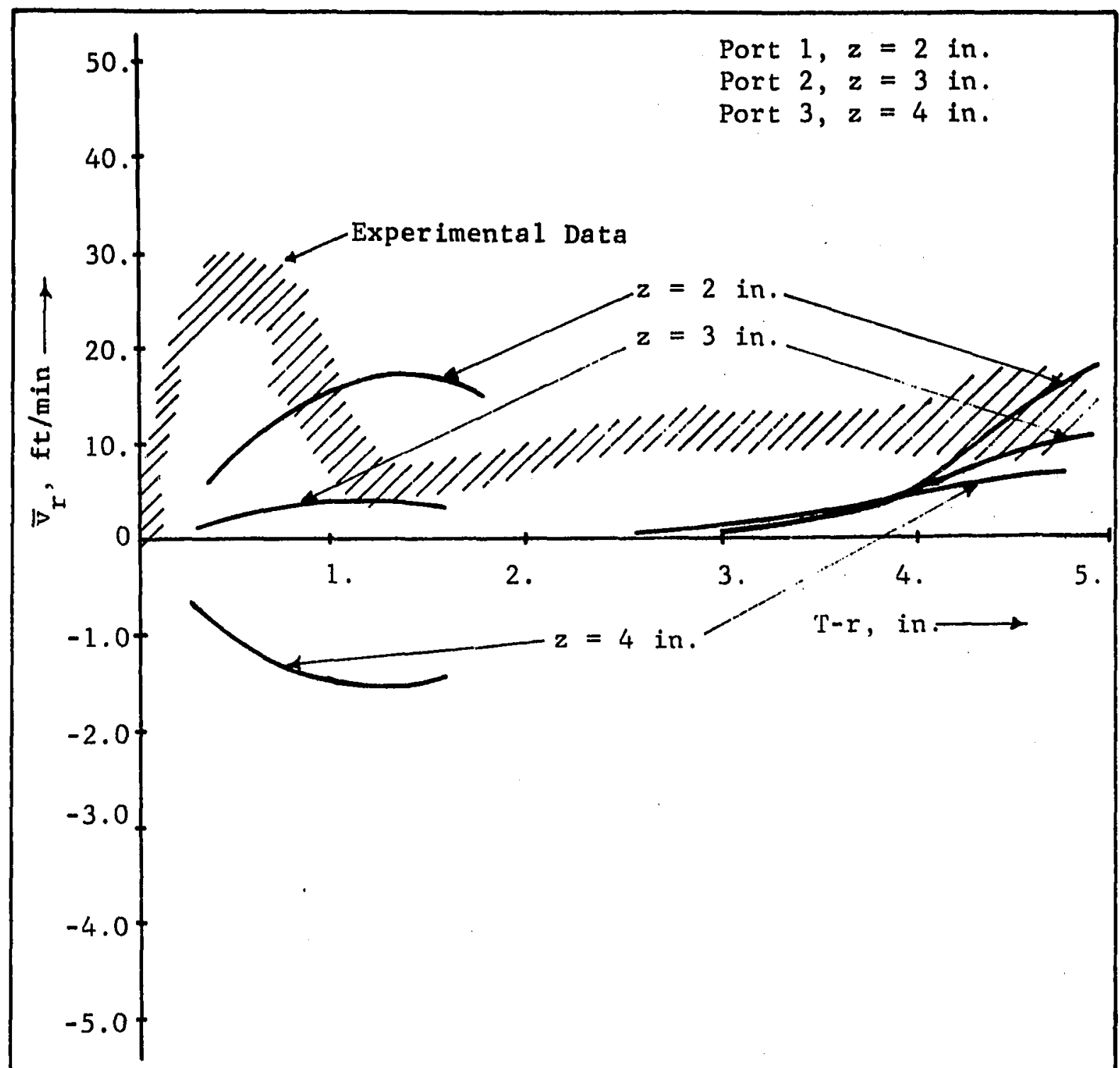

Figure IV-9: Comparison between Experimental and Theoretical Velocity Profiles for $\bar{v}_{r}$ for Ports 1, 2, and 3 


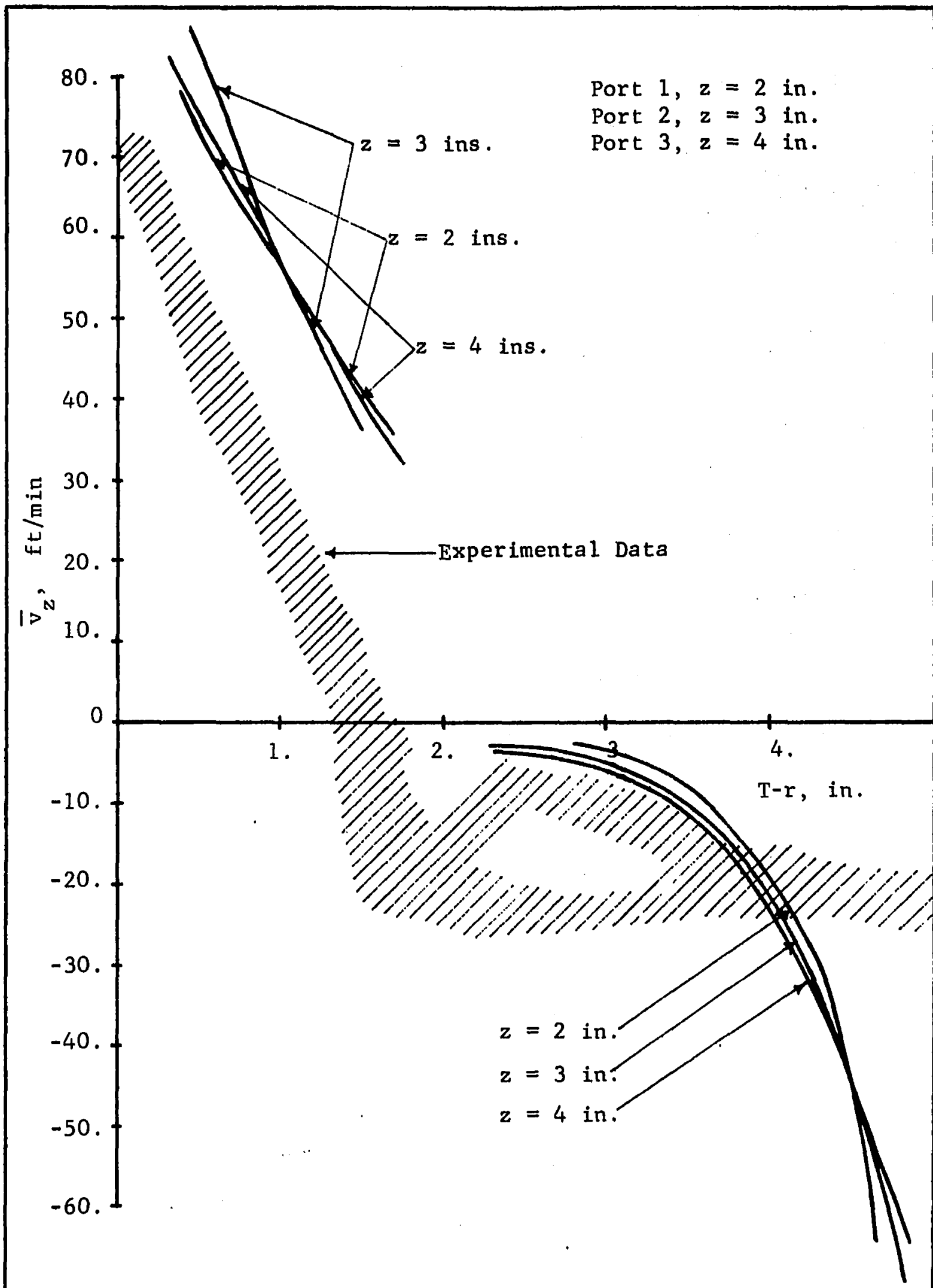

Figure IV-10: Comparison between Experimental and Theoretical Velocity Profiles for Ports 1, 2, and 3 


$$
\begin{aligned}
& \bar{v}_{r}=\frac{1}{2 \pi r} \frac{\partial \psi}{\partial z} \\
& \bar{v}_{z}=-\frac{1}{2 \pi r} \frac{\partial \psi}{\partial r} .
\end{aligned}
$$

Program VELPRO and its input data is given in Appendix F. As a : streamline in Figure IV-3 is given by $r=f(z)$ at constant $\psi$, the derivatives of $\psi$ in Equations IV-20 and IV-21 are obtained by a numerical method. This is done by fitting a third order polynomial through a row of points having a constant $z$ value. Seven such rows are shown in Figure IV-2. The bottom most row is 1.0 in. from the impeller centerline (i.e., $z=1.0$ inch). Each succeeding row is at a distance of 0.5 inch apart. Differentiating this polynomial gives $\frac{\partial y}{\partial x}$ and from Equation Iv-21, $\bar{v}_{z}$ is calculated.

To calculate $\bar{v}_{r}$, first we calculate $\psi=f(r)$ at constant $z$ by fitting a third order polynomial for each of the seven $z$-planes shown in Figure IV-3. Next, these seven polynomials are used to obtain seven values of $\psi$ as a function of $z$ at constant $r$. The constant value of $\mathrm{r}$ is determined by the coordinates of the point at which $\bar{v}_{r}$ is desired. A third order polynomial is then fitted through these seven points to obtain $\psi=f(z)$ at constant $r$. $\frac{\partial \psi}{\partial z}$ can now be calculated and hence $\bar{v}_{r}$ from Equation IV-20, The goodness of fit for $\psi=f(r)$ at constant $z$ was greater than 0.99 , while that for $\psi=f(z)$ at constant $r$ was greater than 0.98. Both these values indicate a good estimate of $\psi$. The final results 
obtained by the program VELPRO is given in Table IV-13 for the upper quadrant while that for the lower quadrant is shown in Table IV-14. The crossover point in Figure IV-3 from Regions II to III was changed : so that $\bar{v}_{r}$ shows a positive value rather than a negative value for Port Number 2. This is clearly seen in Figure IV-3, where Port Number 2 in the lower quadrant is located in Region III and hence $\bar{v}_{r}$ is negative as shown in Table IV-14 for $z$ greater than 3.0 inches. The crossover p $₫$ int was changed in the upper quadrant by increasing XN4, one of the boundary parameters, from 0.5 to 0.55 . The probable range of the experimentally determined values of $\bar{v}_{r}$ and $\bar{v}_{z}$ are shown in Figure IV-9 and IV-10 as a crosshatch band. This permits comparison between experimental and theoretical velocity profiles. It is seen in Figure IV-9 that $\bar{v}_{z}$ theoretical for all three Ports are fairly close together having the same slope as the experimental values but are much larger in magnitude and thus appear to be displaced. The values of $\vec{v}_{r}$ are much lower and show little resemblance" to the experimental profiles. This indicates that the analysis needs to include $\bar{v}_{\theta}$, which would lower the value of $\bar{v}_{z}$ and increase that of $\bar{v}_{r}$. for a constant value of $\vec{q}$.

Theoretical values of $\bar{v}_{r}$ and $\bar{v}_{z}$ in Region $v$ were also obtained from the circular jet model used in this section of the tank. However it will be recalled from the analysis of Chapter II that the eddy viscosity $\epsilon_{0}$ was not needed for obtaining $\psi=f(r, z)$. This can be clearly seen when Equations II-38 and II-39 are examined. However 


\section{TABLE IV-13}

THEORETICAL VELOCITY PROFILES CALCULATED BY PROGRAM VELPRO FOR REGIONS II AND III ABOVE THE IMPELLER

Velocity Profile at $z=2 \mathrm{in.}$

\begin{tabular}{lccc} 
T-r(in.) & $\bar{v}_{\mathbf{z}}(\mathrm{ft} / \mathrm{min})$ & $\overline{\mathrm{v}}_{\mathrm{r}}(\mathrm{ft} / \mathrm{min})$ & $\overrightarrow{\mathrm{V}}(\mathrm{ft} / \mathrm{min})$ \\
\hline 0.5 & 73.9 & 9.7 & 74.5 \\
0.7 & 67.1 & 12.5 & 68.3 \\
0.8 & 62.2 & 14.2 & 63.8 \\
1.0 & 57.0 & 15.6 & 59.0 \\
1.2 & 50.2 & 16.7 & 52.9 \\
1.6 & 38.5 & 16.5 & 41.9
\end{tabular}

Velocity Profile at $z=3$ in.

0.4

0.6

0.7

0.8

1.0

1.3

0.6

0.8

0.9

1.1

1.5
88.9

80.2

7.3 .9

67.1

58.7

44.3
1.9

2.7

3.1

3.5

3.8

3.9
88.9

80.2

73.9

67.2

58.8

44.5

Velocity Profile at $z=4$ in.

$0.4 \quad 79.6$

72.1

66.7

61.2

53.5

40.4
$-8.7$

$-11.3$

$-12.8$

$-14.0$

$-15.0$

$-14.9$
80.0

73.0

68.0

62.8

55.5

43.1 


\section{TABLE IV-14}

THEORETICAL VELOCITY PROFILE CALCULATED BY PROGRAM VELPRO FOR REGIONS II AND III BELOW IMPELLER. SHOWS EFFECT OF CONSIDERING A BOUNDARY IAAYER IN THE THEORETICAL MODEL.

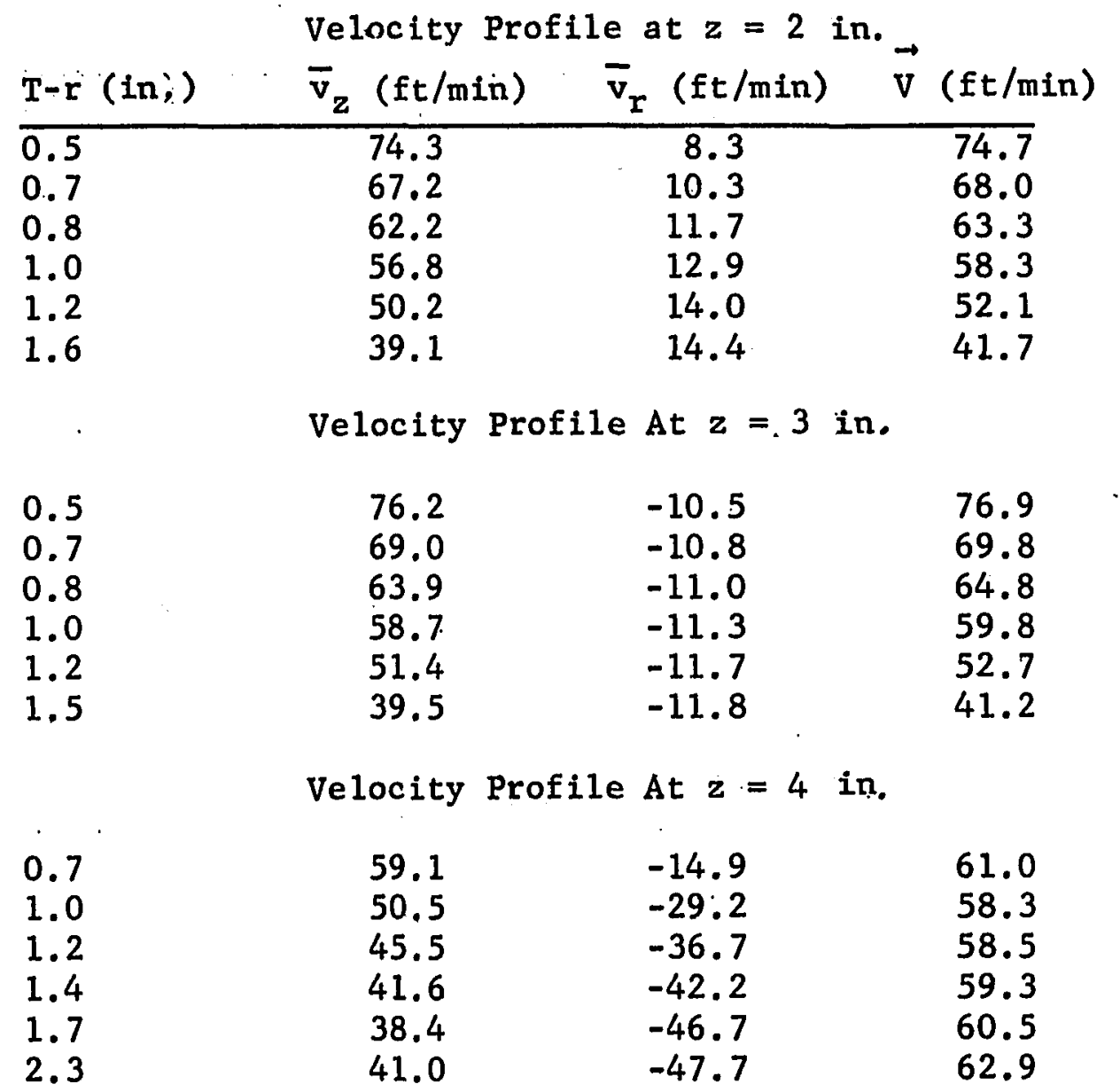


in order to calculate $\bar{v}_{x}$ and $\bar{v}_{z}$, Equations II-14 and II-15 are used which are given below and require a value for $\epsilon_{0}$.

$$
\begin{aligned}
& \bar{v}_{z}=\frac{\epsilon_{0}}{z+z_{0}} \frac{2 y^{2}}{\left(1+\frac{1}{4} \xi^{2}\right)^{2}} \\
& \bar{v}_{r}=\frac{\epsilon_{0}}{z+z_{0}} \quad \gamma \frac{\xi-\frac{1}{4} \xi^{2}}{\left(1+\frac{1}{4} \xi^{2}\right)^{2}}
\end{aligned}
$$

To evaluate $\epsilon_{0}$, as a first approximation the momentum in the circular jet was equated to that of the tangential jet. This appears reasonable, since both these jets are connected in series and energy dissipation outside the impeller stream is sma11. The momentum in the tangential jet $J_{t}$ is obtained from Equation A-32 which after simplication gives

$$
J_{t}=\frac{4}{3} \pi \rho A^{2}
$$

The momentum in the circular jet $J_{c}$ is given by Equation II-18 and is

$$
J_{c}=\frac{16}{3} \pi p r^{2} \epsilon_{o}^{2}
$$

However to allow for some dissipation of the momentum in the tangential jet, a factor $E_{c}$ was included where $E_{c}$ is defined as

$$
E_{c}=\frac{J_{c}}{J_{t}}
$$

It follows from the definition of $E_{c}$, that $E_{c}$ is the fraction of the momentum transferred to the circular jet. Combining IV-22, II-18 and IV-23 gives 


$$
\epsilon_{0}=E_{c} \frac{A}{2 \gamma}
$$

The program FLOWANL was again used to evaluate $\bar{v}_{z}$ and $\bar{v}_{r}$ using Equations II-14, II-15, and IV-26, The result of these calculations are shown in Table IV-15 and are plotted on Figure IV-9 and IV-10 for comparison. The theoretical value of $\vec{v}_{\mathbf{r}}$ are very small while those of $\bar{v}_{z}$ are grossly incorrect. $\bar{v}_{z}$ shows an extremely high value for $T-r$ greater than 4.5 inches and being extremely low below 4.5 inches. Varying $\mathrm{E}_{c}$ the fraction of momentum transferred to the circular jet, doesi not alter the shape of the profile but merely scales the value of the velocities; large values of $\mathrm{E}_{\mathrm{c}}$ gives large values of $\epsilon_{0}$ which in turn gives larger values of $\bar{v}_{z}$ and $\bar{v}_{r}$. The theoretical velocities shown in Figures IV-9 and IV-10 have been calculated with $E_{c}=0.6$. Also shown in Table IV-15 is $\vec{v}$ the resultant velocity of $\bar{v}_{r}$ and $\bar{v}_{\theta}$, and is not constant but decreases as $r$ increases.

The above analysis suggests that although the streamlines appear reasonable the velocity profiles obtained from them need not necessarily reflect a desired velocity profile. The flow in the region outside the impeller appears to have an apprbximately uniform resultant velocity which could be represented in potential flow. However this flow is a three dimensional flow field. Since in potential flow the Laplace equation holds, the superposition principle could be applied. Thus subtracting $\bar{v}_{\theta}$ from the total velocity 


\section{TABLE IV-15}

\section{THEORETICAL VELOCITY PROFILES IN REGION V} OBTA INED FROM THE CIRCULAR JET

\begin{tabular}{lccc}
\multicolumn{4}{c}{ Velocity Profile at $z=2$} \\
T-r (in.) & $\bar{v}_{z}(\mathrm{ft} / \mathrm{min})$ & $\bar{v}_{\mathrm{r}}$ (ft/min) & $\overrightarrow{\mathrm{v}}$ (ft/min) \\
\hline 5.0 & 98.8 & 18.6 & 100.5 \\
4.6 & 54.1 & 14.1 & 55.9 \\
4.3 & 29.7 & 9.0 & 31.0 \\
4.0 & 16.0 & 5.4 & 16.9 \\
3.5 & 7.8 & 2.8 & 8.2 \\
3.0 & 3.5 & 1.1 & 3.7
\end{tabular}

Velocity Profile at $z=3$ in.

$\begin{array}{rrrr}4.8 & 67.7 & 10.5 & 68.6 \\ 4.4 & 38.3 & 8.3 & 39.2 \\ 4.0 & 21.7 & 5.6 & 22.4 \\ 3.7 & 12.1 & 3.6 & 12.7 \\ 3.2 & 6.4 & 2.1 & 6.8 \\ 2.6 & 3.3 & 1.1 & 3.4\end{array}$

Velocity Profile at $z=4$ in.

4.6
4.2
3.8
3.4
2.9
2.3

51.5

29.6

17.0

9.7

5.3

2.8

6.9

5.6

3.9

2.5

1.6

1.0
52.0 30.1 17.4 10.0

5.5

3.0 
$\vec{V}$ would then result in two dimension flow field which could be modeled by the methods suggested in this section. In order to evaluates the results of this model a probe is needed that is not only sensitive to low velocity fields but also has directional properties such that the component velocities $\bar{v}_{r}, \bar{v}_{\theta}$ and $\bar{v}_{z}$ can be evaluated. A cross-wire, two channel, hotwire anemometer could be used for this purpose. Two different probes will be needed, one set of wires crossed in a horizontal plane to give $\bar{v}_{r}$ and $\bar{v}_{\theta}$ and another set crossed in a vertical plane to give $\bar{v}_{r}$ and $\bar{v}_{\mathbf{z}}$.

4.4. Detailed Analyses of a Velocity Profile in the Neighborhood of the Impel1er

In this section a velocity profile analysis will be presented in detail. The results of the analysis was performed by the program FLOWNAL. A description of the program, its FORTRAN source listing and a flow diagram is given in Appendix F. At the end of the program is given the raw data which is the input to the program.

A complete analysis of a single profile result in four pages of computer printout. To illustrate a typical printout, Run Number 29 was analyzed and the results are given in Tables $\mathrm{H}-1$ to $H-4$ of Appendix $H$.

It will be recalled from Chapter II that the tangential jet model is a one dimensional flow in the direction of the velocity $\vec{q}$. This model was also found to give extremely small values of $\bar{v}_{z}$ of the order of $0.1 \mathrm{ft} / \mathrm{min}$ in a region of \pm 0.5 inches about the impeller centerline. The flow in the region of the impeller 
is thus essentially one dimensional in the direction of $\vec{q}$. However a velocity probe is needed to locate the direction of $\vec{q}$. The three dimensional pitot tube locates this direction from a yaw angle measurement $\theta_{y}$. As $\bar{v}_{z}$ is very small, the pitch angle $\theta_{p}$ is essentially zero, and hence from a description of the probe in Chapter III the manometer $D$ should show no response. However the manometer $D$ was observed to give a measurable pressure drop indicating that the pitch angle $\theta_{p}$ was not zero. In Section 4.5 the reading from the manometer $D$ will be analyzed and it will be shown the manometer $D$ measures $\Delta q^{2}$ and not $\theta_{p}$.in this high shear flow field.

The flow in the impeller stream is thus two dimensional as far as the probe is concerned. The only measurable velocities are $\bar{v}$ and $\bar{v}_{\theta}$. Hence no additional information is obtained from measuring $\Delta \mathrm{q}^{2}$ and a two dimensional probe can beised." This type of probe has been used by Cooper (8) for obtaining velocity profiles in the region of the impeller.

The program FLOWANL converts pressure drop readings of manometer B into velocities using Equation III-3. This procedure results in an experimental velocity profile of $\vec{q}_{\exp }$ as a function of $z$. Table IV-16 shows the results of this calculation. In the first column is given values of $z ;$, in the next columns are given the corresponding value of $\vec{q}_{\exp }$ and the yaw angle $\theta_{y} \cdot$ It is noticed that $\theta_{y}$ is a function $z$ and is plotted in Figure IV-1. This angle profile was first observed by Cooper (8).

$$
\begin{aligned}
& \text { A predicted value of } \bar{q}_{c a l c} \text { is obtained from a least square fit } \\
& \text { of } \bar{q}_{\exp } \text { as a function of } z \text { by: adjusting } \sigma, a \text {, and } A \text { as explained in }
\end{aligned}
$$


TABLE IV-16

TYPICAL RESULTS OF VELOCITY PROFILE ANALYSIS IN THE NEIGHBORHOOD OF THE IMPELLER FROM PROGRAM FLOWANL AND ILLUSTRATED FOR

EXPERIMENTAL DATA FROM RUN NUMBER 29

\begin{tabular}{cccc}
$\begin{array}{c}z \\
\mathrm{ft}\end{array}$ & $\begin{array}{c}\overline{\mathrm{q}}_{\exp } \\
\mathrm{ft} / \mathrm{min}\end{array}$ & $\begin{array}{c}\bar{\theta}_{\mathrm{y}} \\
\text { Degrees }\end{array}$ & $\begin{array}{c}\bar{q}_{\text {calc }} \\
\mathrm{ft} / \mathrm{min}\end{array}$ \\
\hline 0.058 & 44.8 & 35.2 & 36.9 \\
0.050 & 45.6 & 32.8 & 50.2 \\
0.042 & 62.8 & 30.0 & 66.5 \\
0.033 & 80.9 & 29.4 & 85.0 \\
0.025 & 108.4 & 28.0 & 103.9 \\
0.017 & 122.0 & 26.6 & 120.1 \\
0.008 & 130.2 & 26.4 & 130.2 \\
0.000 & 130.5 & 25.8 & 131.8 \\
-0.008 & 124.4 & 26.6 & 124.4 \\
-0.017 & 111.8 & 27.4 & 109.9 \\
-0.025 & 89.2 & 29.8 & 91.5 \\
-0.033 & 72.2 & 31.6 & 72.6 \\
-0.042 & 50.3 & 32.8 & 55.4 \\
-0.050 & 48.8 & 32.8 & 41.0
\end{tabular}

Arithemetic average,

$$
\begin{aligned}
\bar{\theta}_{\mathrm{y}} & =29.7^{\circ} \\
\bar{\theta}_{\mathrm{y}} & =27.8^{\circ} \\
\mathrm{WT} & =0.5 \\
\mathrm{R} & =0.99 \\
\sigma & =11.28 \mathrm{ft} \\
\mathrm{A} & =18.54 \mathrm{ft} / \mathrm{min} \\
\mathrm{a} & =0.116 \mathrm{ft} \\
z_{\mathrm{o}} & =0.0027 \mathrm{ft} \\
\mathrm{b}_{\frac{z}{z}} & =1.6 \mathrm{in}
\end{aligned}
$$$$
\text { Jet parameters }
$$

Half width of jet, 
Section 4.1. $\bar{q}_{\text {calc }}$ is given by Equation II-10 and is reproduced here

$$
\bar{q}_{\text {calc }}=\frac{A}{2}\left(\frac{\sigma}{r}\right)^{\frac{1}{2}} \frac{1}{\left(r^{2}-a^{2}\right)^{\frac{1}{4}}}\left[1-\tanh ^{2}(\eta / 2)\right]
$$

where $\eta$ is given by

$$
\eta=\sigma \frac{\mathbf{z - z _ { 0 }}}{\mathbf{r}}
$$

As explained in Section 4.1, the parameters $\sigma^{\prime}, A$ and $z_{0}$ are obtained by a least square fit and $a$, the radius of source, is calculated from a weighted average angle and a velocity factor. The resulting parameters and other pertinent information obtained from Tables J-1 to $\mathrm{J}-4$ are given in Table IV-16.

The theoretical velocity profile $\bar{q}_{\text {calc }}$ is also given in Table IV-16. In Figure IV-11 is shown a plot of $\bar{q}_{c a l c}$ versus $z$. Also shown on the plot is $\bar{q}_{\text {exp }}$. The correlation coefficient $R$ is 0.99 and as can be seen in Figure IV-11 the agreement between experimental and theoretical values of $\mathrm{q}$ is excellant. Also shown on the plot is the jet displacement parameter $z_{0}$ and the half width of the jet. $b_{\frac{1}{2}}$. A quantity BHALF is also shown and should not be confused with $b_{\frac{1}{2}}$, the half width of the jet. BHALF is the $z$ coordinate of $\frac{1}{2} \bar{q}_{\max }$. It is noticed in Figure IV-11 that only two data points are measured for $z$ greater than BHALF. This is because the probe has reached a velocity near $30 \mathrm{ft} / \mathrm{min}$ below which it will not respond. It is noticed that for $z$ larger than BHALF the velocity profile falls off rapidly, the rate of fall is then gradual reaching $1 \%$ of $\bar{q}_{\max }$ at $z$ equal to $b_{\frac{1}{2}}$, the half width of the jet. 


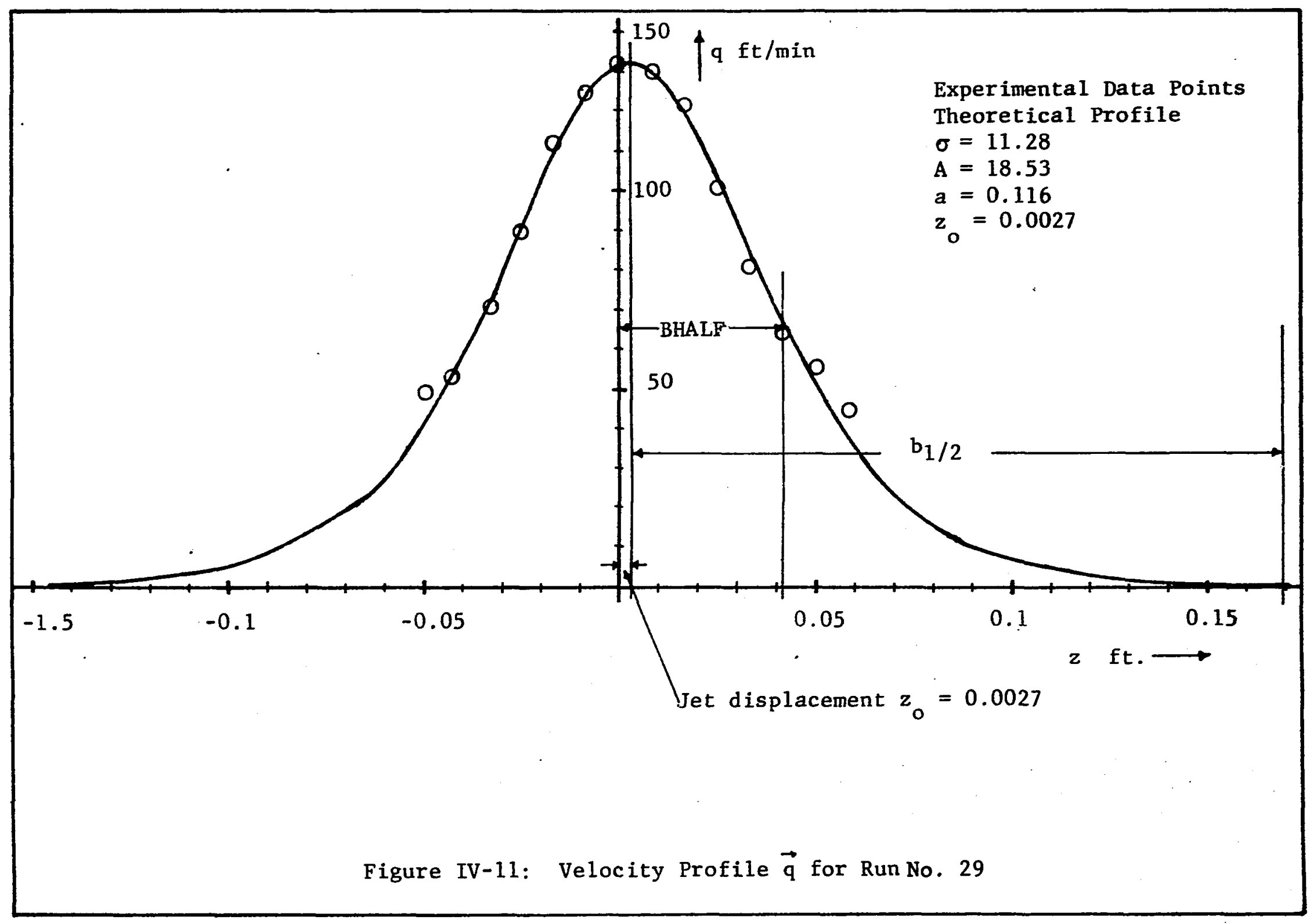




\section{Radial Velocity Profile Analysis}

The experimental value of the radial velocity is obtained from Equation A-20 which is

$$
\left(\bar{v}_{r}\right)_{\exp }=\bar{q}_{\exp } \cos \theta_{y}
$$

Table IV-17 1ists the value of $\left(\bar{v}_{r}\right)_{\text {exp }}$ for Run Number 29 calculated from A-20. The theoretical value of the radial velocity $\left(\bar{v}_{r}\right)_{c a l c}$ is obtained from Equation B-22,

$$
\left(\bar{v}_{r}\right)_{c a l c}=\frac{A}{2}\left(\frac{\sigma}{3}\right)^{\frac{1}{2}}\left(r^{2}-a^{2}\right)\left[1-\tanh ^{2}(\eta / 2)\right]
$$

In Table IV-7, $\left(\bar{v}_{x}\right)_{\text {calc }}$ is obtained from B-22, using the values of $\sigma, A, a$ and $z_{0}$ from Table IV-16. A correlation coefficient $R$ is also calculated from an equation similar to Equation IV-1 written for $\vec{v}_{r}$. The value of $R$ is 0.99 indicating an excellant prediction of the data.

\subsection{Interpretation of Manometer D}

In Section 4.5, the experimental data obtained from the three dimensional pitot tube was analyzed on the basis that $\theta_{p}$ the pitch angle equals zero. In this section it will be shown that this is indeed the case and that the readings from manometer $D$ must be interpreted as measuring $\Delta q^{2}$ because of the large velocity gradients in the impeller stream.

In Chapter III it was shown that the pressure difference between the pressure taps $\mathrm{P}_{4}$ and $\mathrm{P}_{5}$ is given by

$$
P_{4}-P_{5}=\rho_{w}\left(h_{a}-h_{b}\right)-\Delta H_{m}\left(\rho_{m}-\rho_{w}\right)
$$


TABLE IV-17

TYPICAL RESULTS OF RADIAL VELOCITY PROFILE ANALYSIS. ILLUSTRATED FOR EXPERIMENTAL DATA FROM RUN NUMBER 29.

\begin{tabular}{|c|c|c|}
\hline $\begin{array}{r}z \\
f t \\
\end{array}$ & $\begin{array}{l}\left(v_{r}\right) \exp \\
\mathrm{ft} / \mathrm{min}\end{array}$ & $\begin{array}{l}\left(v_{r}\right) \\
\text { ft } / \mathrm{min}\end{array}$ \\
\hline 0.058 & 36.6 . & 32.7 \\
\hline 0.050 & 38.4 & 44.4 \\
\hline 0.042 & 54.4 & 58.9 \\
\hline 0.033 & 70.5 & 75.3 \\
\hline 0.025 & 95.7 & 92.0 \\
\hline 0.017 & 109.2 & 106.4 \\
\hline 0.008 & 116.7 & 115.4 \\
\hline 0.000 & 117.5 & 116.8 \\
\hline-0.008 & 111.2 & 110.2 \\
\hline-0.017 & 99.3 & 97.3 \\
\hline-0.025 & 77.4 & 81.1 \\
\hline-0.033 & 61.5 & 64.4 \\
\hline-0.042 & 42.2 & 49.1 \\
\hline-0.050 & 41.0 & 36.4 \\
\hline
\end{tabular}


where $h_{a}-h_{b}$ is the linear distance between taps $P_{4}$ and $P_{5}$. This linear distance is :small and hard to measure, however it is recognized that $\rho_{w}\left(h_{a}-h_{b}\right)$ is the static head recorded by the manometer under no flow conditions. This static head is called $\Delta \mathrm{H}_{s}$ and was found equal to $1.0 \mathrm{in}$. of manometer fluid. In terms of the static head $\Delta \mathrm{H}_{s}, \mathrm{~h}_{\mathrm{a}}-\mathrm{h}_{\mathrm{b}}$ is given by

$$
h_{a}-h_{b}=\Delta H_{s}\left(\rho_{w}-\rho_{m}\right) / \rho_{w}
$$

Combining IV-25 and III-3 gives

$$
\mathrm{P}_{4}-\mathrm{P}_{5}=\left(\Delta \mathrm{H}_{\mathrm{s}}-\Delta \mathrm{H}_{\mathrm{n}}\right)\left(\rho_{\mathrm{m}}-\rho_{\mathrm{w}}\right)
$$

Hypothesize that: the: pressure drọ $P_{4}-P_{5}^{11}$ is'the pressùre differential due to the vertical componentiof yelocity $\bar{v}_{z}$. Then as'in Section $4: 3$, Figure IV-12 gives the geometry of the three dithensional flow field acting on the probe، The resultant velocity $\overrightarrow{\vec{v}}$ ap $=$ proaches the probe at a pitch angle of $\theta_{p}$ and a yaw angle of $\theta_{\frac{7}{3}}$; From the geometry of Figure IV-12 it follows that

$$
\begin{aligned}
& \bar{v}_{z}=|\vec{v}| \sin \theta_{p} \\
& \bar{v}_{r}=|\vec{v}| \cos \theta_{p} \cos \theta_{y} \\
& \bar{v}_{\theta}=|\vec{v}| \cos \theta_{p} \sin \theta_{y}
\end{aligned}
$$

As in the case of Section 4.3, Equation IV-15 is used to calculate the pressure ratio $\alpha$. Curves $A$ and $B$ of Figure IV-5 are then interpolated to evaluate the pitch angle $\theta_{p}$ and a corrected value of the pressure $\left(\Delta \mathrm{P}_{12}\right)$ corrected. Equation IV-16 is then used to 


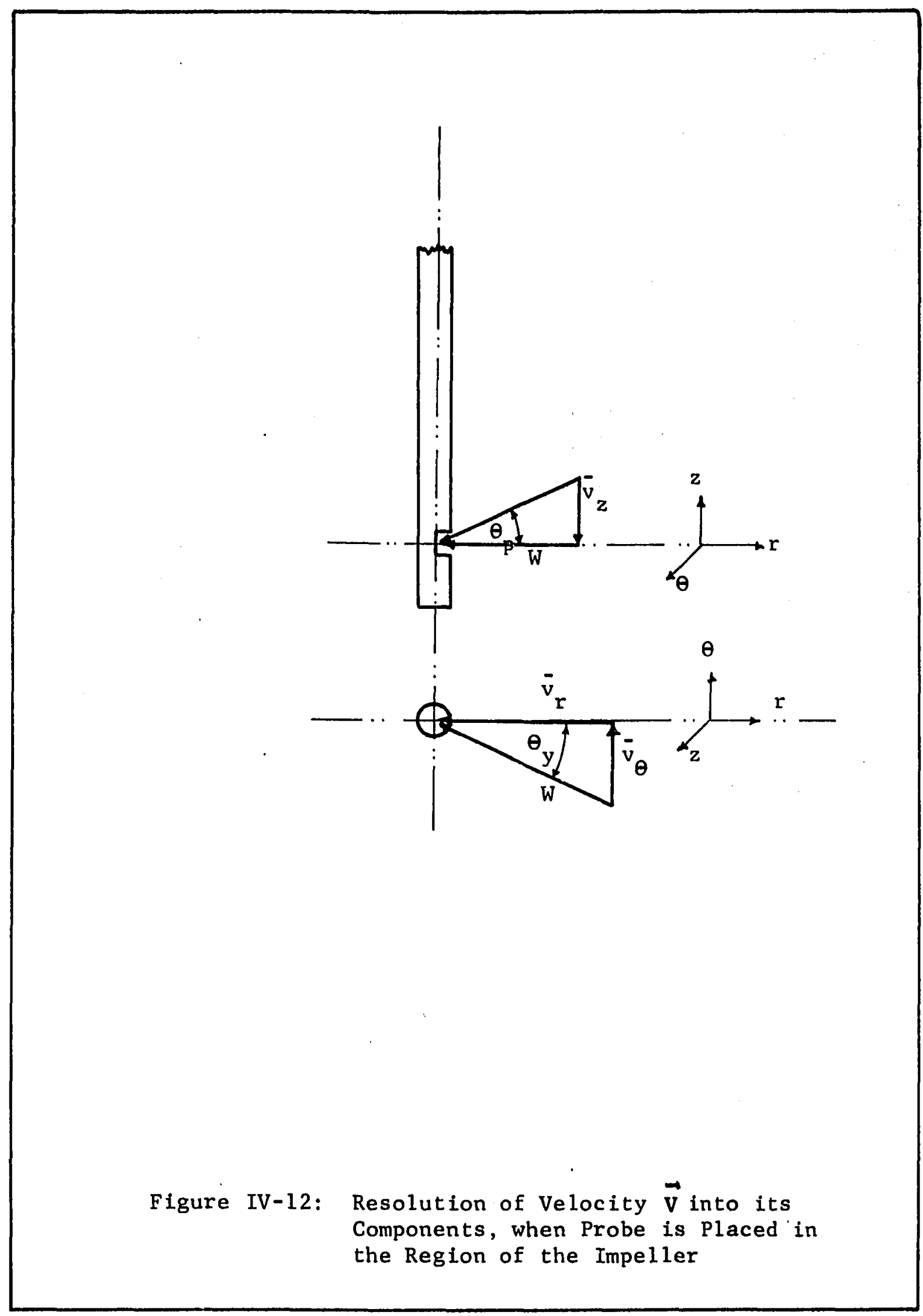


evaluate $\vec{v}$. The component velocities $\bar{v}_{r}, \vec{v}_{\theta}$ and $\bar{v}_{z}$ is then evaluated from Equations IV-27 to IV-29. These calculations are performed by the program YAWANL. The results of the calculations by YAWANL is given in Table IV-18 and is typical of the analysis obtained for a velocity profile measurement. Table IV-18 illustrates the results by analyzing the experimental data from Run Number 29 .

Two things are evident in Table IV-18, (a) five of the points cannot be analyzed indicating that the pitch angle is larger than $\pm 40^{\circ}$, (b) the valve of $\bar{v}_{z}$ is large and about the same magnitude as $\bar{v}_{\theta}$. Also $\bar{v}_{z}$ decreases as the magnitude of $z$ increases. This behavior is inconsistent with the jet model which assumes $\bar{v}_{z}$ is small and that $\bar{v}_{z}$ increases with increasing magnitude of $z$. It is also physically impossible for the fluid in the impeller stream to have a pitch angle $\theta_{\mathrm{p}}$ larger than $40^{\circ}$. The conclusion is that this hypothèsis 'is not'válid and some "other' phenomena 'is' the cause 'of' the pressure drop recorded by manometer $D$.

Since $\mathrm{P}_{4}-\mathrm{P}_{5}$ is a kinetic head caused by the motion of the fluid, it was concluded that the pressure drop is due to the high velocity gradient present in the tangential jet. This is expected since the tangential jet is aihigh shear flow and the boundary. layer assumptions hold.

Because of the velocity gradient, the velocities at the pressure taps $P_{4}$ and $P_{5}$ are different. Let these velocities be $\vec{q}_{4}$ and $\vec{q}_{5}$. If $P_{S}$ is the stagnation pressure then from pitot tube theory (44) we can write the following relation 


\section{TABLE IV -18}

RESULTS OF PROGRAM YAWANL WHICH TREATS THE TMPELLER REGION AS.' A THREE-DIMENSIONAL FLOW FIELD, AND ILLUSTRATED FOR EXPERIMENTAL DATA FROM RUN NUMBER 29

$\begin{array}{rrrrr}\begin{array}{c}\text { Item } \\ \text { No. }\end{array} & \text { z in } & \Delta \mathrm{P}_{12} * & \Delta \mathrm{P}_{4} 5^{*} & \begin{array}{r}\left(\Delta \mathrm{P}_{12}\right) * \\ \text { Corrected }\end{array} \\ 1 & 0.7 & 1.35 & -0.8 & 1.6 \\ 2 & 0.6 & 1.40 & -4.1 & * * * \\ 3 & 0.5 & 2.65 & -7.6 & * * \\ 4 & 0.4 & 4.40 & -8.8 & * * 5 \\ 5 & 0.3 & 7.90 & -9.8 & 11.9 \\ 6 & 0.2 & 10.00 & -7.1 & 12.9 \\ 7 & 0.1 & 11.40 & -1.1 & 12.2 \\ 8 & -0.0 & 11.45 & 5.6 & 11.3 \\ 9 & -0.1 & 10.40 & 10.0 & 9.5 \\ 10 & -0.2 & 8.40 & 11.4 & * * \\ 11 & -0.3 & 5.35 & 10.8 & * * \\ 12 & -0.4 & 3.50 & 8.2 & 1.8 \\ 13 & -0.5 & 1.70 & 4.7 & \\ 14 & -0.6 & 1.60 & 2.4 & \end{array}$

\begin{tabular}{|c|c|c|c|c|c|c|c|}
\hline $\begin{array}{l}\text { Item } \\
\text { No. }\end{array}$ & $z$ in. & $\begin{array}{c}\bar{V} \\
\mathrm{ft} / \mathrm{min} .\end{array}$ & $\bar{v}_{r}$ & $\begin{array}{c}\bar{v}_{z} \\
\mathrm{ft} / \mathrm{min}\end{array}$ & $\begin{array}{c}\bar{v}_{\theta} \\
\mathrm{ft} / \mathrm{min}\end{array}$ & $\begin{array}{c}\text { Pitch } \\
\text { Anglè } \theta_{p}^{\circ}\end{array}$ & Angle $\theta_{y}^{\circ}$ \\
\hline $\begin{array}{r}1 \\
5 \\
6 \\
7 \\
8 \\
9 \\
10 \\
14\end{array}$ & $\begin{array}{l}0.7 . \\
0.3 \\
0.2 \\
0.1 \\
-0.0 \\
-0.1 \\
-0.2 \\
-0.6\end{array}$ & $\begin{array}{r}48.7 \\
119.0 \\
133.0 \\
138.4 \\
135.0 \\
129.5 \\
118.8 \\
52.4\end{array}$ & $\begin{array}{r}39.3 \\
92.2 \\
115.6 \\
123.7 \\
114.9 \\
102.8 \\
88.1 \\
35.8\end{array}$ & $\begin{array}{r}-7.9 \\
-57.1 \\
-31.1 \\
11.0 \\
44.2 \\
59.6 \\
65.3 \\
30.4\end{array}$ & $\begin{array}{l}27.7 \\
49.0 \\
57.9 \\
61.3 \\
55.5 \\
51.5 \\
45.7 \\
23.1\end{array}$ & $\begin{array}{r}-9.4 \\
-28.7 \\
-13.5 \\
4.5 \\
19.1 \\
27.4 \\
33.4 \\
35.5\end{array}$ & $\begin{array}{l}35.2 \\
28.0 \\
26.6 \\
26.4 \\
25.8 \\
26.6 \\
27.4 \\
32.8\end{array}$ \\
\hline
\end{tabular}

* Inches Manometer fluid

** Yaw Angle $\theta_{p}$ greater than $40^{\circ}$ 


$$
\bar{q}_{4}^{2}-\bar{q}_{5}^{2} \stackrel{\doteq}{=} \frac{2 g}{\rho_{w}^{\prime}}\left[\left(P_{4}-P_{s}\right)-P_{5}-P_{s}\right]
$$

Since the pressure taps $P_{4}$ and $P_{5}$ are close together (about 0.1 in. apart): the stagnation pressure $P_{s}$ is approximately constant. Lumping all the approximations in a coefficient of performance $c_{1}$ and noting that $q_{4}^{2}-q_{5}^{2}$ is a change in $q^{2}$ in the $z$-direction we have

$$
\Delta \bar{q}^{2}=c_{1} 2 g\left(P_{4}-P_{5}\right) / \rho w
$$

The coefficient of performance $c_{1}$ can be defined by

$$
d_{1}=\frac{\Delta q_{\exp }^{2}}{\Delta q_{\text {calc }}^{2}}
$$

where $\Delta q^{2}$ exp is given by

$$
\Delta \bar{q}^{-2} \exp =2 g\left(P_{4}-P_{5}\right) / p_{w}^{i}
$$

$\Delta \bar{q}^{2}$ calc is obtained by calculating $\bar{q}$ from Equation II-10 at a distance $\eta \pm \Delta \eta$ where $\Delta \eta$ is given by

$$
\Delta \eta=\frac{h_{a}-h_{b}}{2 r}
$$

$\eta$ is the value of the dimensionless velocity profile coordinate at the mid-point of the pressure taps $P_{4}$ and $P_{5} \cdot \Delta n$ in Equation IV-30 then locates the pressure taps $P_{4}$ and $P_{5}$ relative to the dimensionless coordinate $\eta \cdot \Delta \bar{q}_{\text {calc }}^{2}$ can now be written as

$$
\Delta \bar{q}_{\text {calc }}^{2}=\bar{q}_{\max }^{2}\left[\left(1-\tanh ^{2}(\eta+\Delta \eta) / 2\right)^{2}-\left(1-\tanh ^{2}(\eta-\Delta \eta) / 2\right](I V-31)\right.
$$


From a plot of $c_{1}$ versus $1 / \bar{q}^{2}$ exp for a few points it appeared that a correlation exist. Hence a fourth order polynomial was fitted through the point $c_{1}$ versus $1 / \bar{q}^{2}$ exp using a: Share Library program CURVEF. Next points which were located more than three times the standard error (which is an estimate of the standard deviation) were removed from the correlation. The remaining points gave an average value of 0.5216 and a variance of 0.0413 indicating that $c_{1}$ is a constant. The original data had 471 points the above proc edure reduced the number of points to 440 . Since such a large number of points were considered the variance will be considered as the best estimate of the standard deviation, hence with a 0.95 probability, and assuming a normal distribution the best estimate for $c_{1}$ is

$$
c_{1}=0.52 \pm 0.02
$$

This value of $c_{1}$ was used in Equation IV-28 to obtain $\Delta \bar{q}^{2}$ which is shown in Table IV-19. The agreement between the experimental and theoretical values of $\Delta \bar{q}^{2}$ is fairly good with a deviation of \pm 0.2 as seen in the last column which shows the difference between experimental and calculated values of $\Delta \bar{q}^{2}$. Both experimental and theoretical values show a maximum for $z= \pm 0.025 \mathrm{ft}$, a strong evidence that the manometer $\mathrm{D}$ is recording $\Delta \bar{q}^{2}$. The value of $\bar{v}_{z}$ as obtained from the tangential jet model is also shown for comparison and are seen to be extremely small. The results of the above analysis which is shown in Table IV-19 is calculated by the subroutine YAW. This subroutine is part of program FLOWANL that analyses the experimental velocity profile data. 


\section{TABLE IV-19}

RESULT OF ANALYSIS BY SUBROUTINE YAW THAT TREATS THE REGION OF THE IMPELLER AS A REGION OF HIGH SHEAR, FOR

EXPERIMENTAL DATA OF RUN NUMBER 29

\begin{tabular}{cccccc}
$\begin{array}{c}\text { Item } \\
\text { Number }\end{array}$ & $\begin{array}{c}z \\
\mathrm{ft} .\end{array}$ & $\mathrm{ft}^{2} / \mathrm{min}^{2}$ & $\begin{array}{c}\Delta \mathrm{q}^{2} \mathrm{cal} \\
\mathrm{ft}^{2} / \mathrm{min}^{2}\end{array}$ & $\begin{array}{c}\left(\bar{v}_{\mathrm{z}}{ }^{2} \mathrm{calc}\right. \\
\mathrm{ft} / \mathrm{min}\end{array}$ & $\begin{array}{c}\Delta \mathrm{q}^{2}-\Delta \mathrm{q}_{\mathrm{ca}}^{2} \mathrm{c} \\
\mathrm{ft}^{2} / \mathrm{min}^{2}\end{array}$ \\
\hline 1 & 0.058 & -0.23 & -0.24 & -0.24 & -0.01 \\
2 & 0.050 & -0.57 & -0.42 & -0.15 & 0.15 \\
3 & 0.042 & -0.94 & -0.66 & -0.12 & 0.28 \\
4 & 0.033 & -1.07 & -0.91 & -0.08 & 0.16 \\
5 & 0.025 & -1.18 & -1.05 & -0.04 & 0.13 \\
6 & 0.017 & -0.89 & -0.91 & -0.02 & -0.02 \\
7 & 0.008 & -0.26 & -0.44 & -0.00 & -0.18 \\
8 & 0.000 & 0.44 & 0.22 & 0.01 & -0.22 \\
9 & -0.008 & 0.90 & 0.79 & 0.01 & -0.11 \\
10 & -0.017 & 1.05 & 1.04 & 1.03 & -0.02 \\
11 & -0.025 & 0.99 & 0.98 & 0.06 & -0.01 \\
12 & -0.033 & 0.71 & 0.75 & 0.10 & 0.04 \\
13 & -0.042 & 0.25 & 0.50 & 0.14 & 0.15 \\
14 & -0.05 & 0.11 & 0.30 & 0.18 & 0.19
\end{tabular}


4.6. Accuracy of the Three-Dimensional Pitot Tube as a Measuring

\section{Device in Turbulent Flow Fields}

On examining a recent dissertation by Rao (24), it was pointed out that the readings obrained by the three dimensional probe was in error. The error is due to distortion of the turbulence field; (a) by the probe (called nose effect) and (b) by suppresstion of the normal velocities in the neighborhood of the pitot tube surface (called surface effect). The distortion of the turbulence is also a function of the size of the eddies (defined by the macro-scale) and the radius of the Pitot tube. Ani analysis of these factors is given by Toomre (24), and a summary of the analysis is given by Rao. Rao. checked the results of the pitot tube by a hot film anemometer.

The system in which Rao made his measurements consists of a 11 5/8 in. diameter pyrex glass cylinder. The bottom of the tank is a $10 \mu$ porous stainless steel plate that acts as a distributor for an upward flow of water at 6.625 liters per minute. The tank is baffled and the overflow water is collected by a weir, at the top of the tank. The reason for this upward draft of water is to sweep out the dye introduced into the tank for turbulence measurements. The impeller used is a Type B, 4.0 in. diameter turbine. At the bottom of the impeller is notched a $1.5 \mathrm{in.diameter}$ recess, $0.4 \mathrm{~cm}$ thick, which serves to introduce the dye directly into the impeller at a rate of 1.753 liters per minute.

A three dimensional pitot tube similar to the one used in this work was used to measure velocity profiles in the neighborhood of 
impeller. The impeller was run at a single speed of 300 RPM. The various pressure differentials from the five pressure taps were measured by a single differential pressure transducer, Model P7 (The Pace Engineering Company) using a system of three way stop cocks. The stop cocks permit connecting the transducer to any two pressure taps. An auxilliary manometer was used for calibration. This three dimensional pitot tube was used to locate yaw angle $\theta_{y}$ and the pitch angle $\theta_{p}$, the pitch angle was obtaine from the manufacturers calibration chart of $\left(\mathrm{P}_{4}-\mathrm{P}_{5}\right) /\left(\mathrm{P}_{1}-\mathrm{P}_{2}\right)$ versus $\theta_{y}$ as explained in Section 4.3. The hot film probe was then introduced into the system at exactly the same point and directed along the yaw angle. A linearized signal $E$, in volts was obtained from the instrument and was a linearized form of King's law. As described in Chapter $I$, the hot film (or wire) is sensitive to the normäl component of velocity. If $\vec{V}$ is the true velocity having a pitch angle $\theta_{p}$ then the anemometer will respond to the velocity normal to it namely $\bar{V} \cos \theta_{p}$ and from King's law we have

$$
\bar{V} \cos \theta_{p}=\bar{A} \bar{E}+B
$$

$\bar{E}$ is the linearized average voltage from the anemometer, and $A$ and $B$ are calibration constants of the instrument. It can be shown that if if $e^{\prime}$ is the fluctuating voltage that, the intensity of turbulence $v^{\prime}$ is given by

$$
v^{\prime} \cos \theta_{p}=A e^{\prime}
$$


$\vec{V}$ is thus obtained from IV-33. Knowing $\theta_{p}$ and $\theta_{y}, \bar{v}_{r}, \bar{v}_{\theta}$ and $\bar{v}_{z}$ can be calculated from the geometry of the system. $\theta_{p}$ was evaluated by using the three dimensional pitot tube.

Using the above method, Rao obtained a value of $\bar{V}$ from IV-33 which is lower than $\bar{V}$ from the pitot tube by about $50 \% . \bar{V}$ from the pitot tube was calculated from

$$
\overline{\mathrm{V}}=\sqrt{2 \mathrm{g \Delta \Delta}} \mathrm{H}_{12}
$$

where $\Delta \mathrm{H}_{12}$ is the pressure drop across pressure taps $\mathrm{P}_{1}$ and $\mathrm{P}_{2}$. Apparently Rao uses the manufacturers calibration chart to calculate $\theta_{p}$, however he did not use these charts to get a corrected $\Delta \mathrm{H}_{12}$ as described in Section 4.3. Nor does he examine the flow geometry as was done in Figure IV-12.

On examining the velocity profile measured at four values of $\left(2.5,3.0,3.5\right.$, and 4.0 inches) it was found that $z_{0}$ the displacement of the jet ranged from 0.031 to $0.038 \mathrm{ft}$. above the impeller centerline. In our jet $z_{0}$ is of the order of 0.003 and in only one case has an excessively large value of 0.079 . The large value of $z_{0}$ obtained by Rao is attributed to the upward flow of water and dye.

The quantity BHALF, the point at which the velocity equals half the maximum velocity was also calculated for these four prom files and was found to range from 0.038 to 0.11 feet. The value of BHALF is calculated relative to the maximum velocity where $z$ is taken equal to zero. Our values for the tangential jet ranges 
from 0.033 to 0.067 . This indicates that the type B turbine exhibits a broader jet. The tangential jet model could thus st111 apply.

It was shown in Section 4.6 that for a tangential jet $\vec{v}_{z}$ is very small for $z$ less than BHALF. Rao's profiles were measured a little beyond BHALF. Hence the pressure differential, $\mathrm{P}_{4}-\mathrm{P}_{5}$, is as was shown in the previous section due to $\Delta \bar{q}^{2}$; and the pitch angle which has been calculated is thus erroneous. Since $\cos \theta_{p}$ is needed in Equation IV-33 as a correction factor, it is bound to give lower values of $\bar{V}$, for $\theta_{\mathrm{p}}$ greater than zero. As the raw data is not given, it was not possible to check the above conclusions quantatively.

Rao used Toomre's analysis to estimate a correction factor for the pitot tube. The nose effect was assumed zero. Using a maximum possible value for the surface effect. Rao obtained a correction that was $10 \%$ lower than the experimental value. This calculation cannot be repeated for our work since it requires a knowledge of $\mathrm{v}^{\prime}$; the intensity of turbulence.

It is possible that the surface effect could have an effect on the pitot tube reading and is perhaps a maximum of $10 \%$ as shown by Rao using Toomre's method (which is itself is an approximate method). We agree with Rao that it is necessary to know more quantatively the effect of turbulence on pitot tube measurements. In absence of any concrete evidence it was thought best to not include a correction 
factor on the velocity profiles since it is sma11 and may very weIl be within the precision of the subsequent analysis using the tangential jet model.

\subsection{Comparison with Coopers (8) Data}

From the review of Cooper's work in Chapter I, it will be recalled that Cooper used a similar measuring device as in this work and has measured velocity profiles only in the region of the impeller. Cooper however did not present his raw data, nor indicated by way of a sample calculation how the resulting velocity profiles $\vec{q}$, were obtained. Tables are presented of $\vec{q}$ in (in./sec.) for various cases together with the corresponding yaw angle $\theta_{y^{*}}$ These tables have been conveniently punched on cards for making a nonlinear least square fit to the tangential jet model. The analysis was done by reading the data for a single table as a matrix and analyzing the several flow profiles in the table by the program COOPER. The program COOPER is very similar to FLOWANL, the difference is mainly in data processing. Since Cooper's data exists as a velocity profile, several profiles at constant impeller diameter are read in and stored. The program COOPER then analysed one profile at a time using the same subroutines as FLOWANL to obtain a least square fit of the data for the tangential jet model. The subroutines used are, PATERN, PROC, BOUNDS, and AVG. The program COOPER is given in Appendix $\mathrm{F}$ together with the input data.

To obtain the velocity profile data, Cooper used a two dimensional probe. This probe is similar to the probe used in this 
TABLE IV-20: SUMAARY OF TANGENTIAL JET ANALYSIS FOR DATA

OBTAINED WITH A DIRECTIONAL PITOT TUBE

Data set Item Number No.

1

2

3

6

7

1
2
3
4
5

9

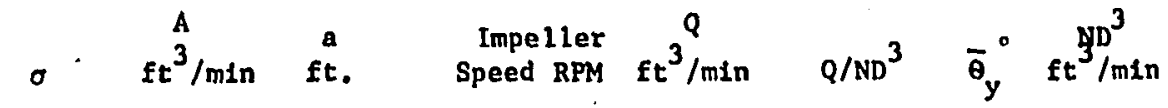
$T=15.0 \mathrm{IN} ., \mathrm{D}=3.0 \mathrm{IN}$, FLUID USED IS WATER, COOPER (8)

$\begin{array}{lrllllll}11.5 & 9.8 & 0.105 & 200.0 & 3.33 & 1.067 & 57.2 & 3.13 \\ 11.8 & 14.1 & 0.105 & 300.0 & 4.74 & 1.012 & 57.0 & 4.69 \\ 11.6 & 15.0 & 0.109 & 400.0 & 4.88 & 0.780 & 60.2 & 6.25 \\ 14.4 & 19.2 & 0.101 & 500.0 & 6.11 & 0.782 & 53.9 & 7.81 \\ 12.5 & 22.5 & 0.104 & 600.0 & 7.46 & 0.795 & 56.2 & 9.38\end{array}$

$T=15.0$ IN., D $=4.0$ IN., FLUID USED IS WATER, COOPER (8)

$\begin{array}{rrrrrrrr}14.1 & 7.9 & 0.139 & 100.0 & 3.26 & 0.880 & 56.6 & 3.70 \\ 12.1 & 11.9 & 0.142 & 150.0 & 5.20 & 0.935 & 58.5 & 5.56 \\ 13.6 & 17.1 & 0.140 & 200.0 & 7.12 & 0.961 & 57.3 & 7.41 \\ 11.3 & 20.6 & 0.145 & 250.0 & 8.98 & 0.969 & 60.7 & 9.26 \\ 11.7 & 23.0 & 0.146 & 300.0 & 9.82 & 0.883 & 60.9 & 11.11\end{array}$

$T=15.0 \mathrm{IN} ., \mathrm{D}=5.0 \mathrm{IN} .$, FLUID USED IS WATER, COOPER (8)

$\begin{array}{rrrrrrrr}12.5 & 12.8 & 0.173 & 100.0 & 7.06 & 0.975 & 56.2 & 7.23 \\ 12.7 & 20.1 & 0.171 & 150.0 & 11.13 & 1.025 & 55.4 & 10.85 \\ 12.8 & 26.5 & 0.169 & 200.0 & 14.83 & 1.025 & 54.2 & 14.47 \\ 12.6 & 34.0 & 0.170 & 250.0 & 19.04 & 1.053 & 54.7 & 18.08 \\ 12.7 & 37.1 & 0.171 & 275.0 & 20.63 & 1.037 & 55.1 & 19.89 \\ & \end{array}$

$T=15.0$ IN., $D=6.0$ IN., FLUID USED IS WATER, COOPER (8)

$\begin{array}{rrrrrrrr}11.7 & 14.2 & 0.202 & 50.0 & 9.96 & 1.593 & 54.0 & 6.25 \\ 11.8 & 19.5 & 0.203 & 100.0 & 13.67 & 1.093 & 54.3 & 12.50 \\ 11.7 & 24.5 & 0.203 & 125.0 & 17.12 & 1.095 & 54.4 & 15.63 \\ 12.6 & 29.4 & 0.204 & 150.0 & 19.86 & 1.059 & 54.6 & 18.75 \\ 12.3 & 33.7 & 0.201 & 170.0 & 23.47 & 1.104 & 53.4 & 21.25\end{array}$

$T=12.25$ IN., $D=3.0$ IN., FLUID USED IS WATER, THIS WORK

$\begin{array}{llllllll}10.5 & 13.5 & 0.114 & 243.0 & 4.18 & 1.101 & 65.8 & 3.80 \\ 10.1 & 14.3 & 0.119 & 250.0 & 3.92 & 1.002 & 72.2 & 3.91 \\ 11.8 & 18.6 & 0.115 & 333.3 & 5.28 & 1.014 & 67.3 & 5.29 \\ 11.8 & 22.7 & 0.117 & 400.0 & 6.18 & 0.988 & 69.3 & 6.25 \\ 11.4 & 28.4 & 0.112 & 500.0 & 8.87 & 1.135 & 63.3 & 7.81 \\ 11.6 & 31.6 & 0.112 & 550.0 & 9.81 & 1.141 & 63.1 & 8.59\end{array}$

$\mathrm{T}=11.5 \mathrm{IN}, \mathrm{D}=3.0 \mathrm{IN}$, , FLUID USED IS WATER, THIS WORK

$\begin{array}{llllllll}10.7 & 17.9 & 0.122 & 333.3 & 4.13 & 0.793 & 76.6 & 5.21\end{array}$

$T=15.0$ IN., $D=4.0$ IN., FLUID USED IS AIR, COOPER (8)

$\begin{array}{rrrrrrrr}13.7 & 13.3 & 0.143 & 200.0 & 5.40 & 0.729 & 58.9 & 7.41 \\ 13.0 & 19.1 & 0.143 & 300.0 & 7.95 & 0.715 & 59.0 & 11.11 \\ 14.3 & 26.4 & 0.143 & 366.0 & 10.49 & 0.774 & 58.8 & 13.56 \\ 15.2 & 35.8 & 0.141 & 500.0 & 14.05 & 0.758 & 57.8 & 18.52 \\ 14.6 & 43.9 & 0.141 & 600.0 & 17.51 & 0.788 & 57.9 & 22.22\end{array}$

$T=15.0$ IN., D $=5.0$ IN., FLUID USED IS AIR , CHOPER (8)

$\begin{array}{llllllll}12.9 & 20.5 & 0.169 & 200.0 & 11.43 & 0.790 & 54.1 & 14.47 \\ 13.6 & 31.3 & 0.169 & 300.0 & 17.04 & 0.785 & 54.0 & 21.70 \\ 14.1 & 43.8 & 0.169 & 400.0 & 23.36 & 0.807 & 54.0 & 28.94 \\ 14.0 & 57.2 & 0.167 & 500.0 & 30.95 & 0.856 & 53.1 & 36.17 \\ 14.0 & 67.6 & 0.169 & 600.0 & 36.29 & 0.836 & 54.0 & 43.40\end{array}$

$T=15.0$ IN., $D=6.0$ IN., FLUTD USED IS AIR, COOPER (8)

$\begin{array}{llllllll}11.9 & 18.1 & 0.202 & 100.0 & 12.68 & 1.014 & 53.7 & 12.50 \\ 12.6 & 34.0 & 0.200 & 200.0 & 23.41 & 0.937 & 53.0 & 25.00 \\ 12.9 & 46.6 & 0.200 & 300.0 & 31.64 & 0.844 & 53.0 & 37.50 \\ 13.7 & 81.6 & 0.200 & 500.0 & 53.69 & 0.859 & 53.0 & 62.50 \\ 13.6 & 95.3 & 0.200 & 600.0 & 63.09 & 0.841 & 53.0 & 75.00\end{array}$


work with the taps $\mathrm{P}_{4}$ and $\mathrm{P}_{5}$ removed. No serious loss of information results from using this probe as can be seen from our analysis of pitch angles in Section 4.5 and the discussion on velocity measurements in the impeller stream in section 4.4. (The two dimensional probe is placed within a tenth of an inch off the impeller periphery, and hence the velocity profile measured can be considered as a good estimate of the velocity profile at the impeller periphery.)

As in the case of our data, a velocity factor was used to obtain, a, the radius of the tangential jet source. A velocity factor of 0.5 gave the best fit for the radial velocity profile. However the difference between the correlation coefficients of $\vec{q}$ and $\bar{v}_{r}$ is much larger for Cooper (8) than in ours. The correlation coefficient for $\vec{q}$ ranges from 0.97 to 0.99 while that of $\vec{v}_{\mathbf{r}}$ range from 0.92 to 0.97 . This indicates that the tangential jet model gives a good predication of the experimental data. As in our data, the velocity factor had very little effect on $\sigma$ but caused small changes in $A$ the volumetric factor and, $a$, the jet radius.

In Appendix $G$ is given a detailed summary of the analysis of Cooper's data. Table IV-20 gives a summary of the results from Appendix G, and consists of nine sets of data, each set at a constant impeller diameter. Its purpose is to facilitate comparison. of al1 Pitot tube measurements.

Examining these nine sets of data, some of the items are found to be approximately constant. These are $\sigma, a, N_{Q}$ and $\bar{\theta}_{y}$. These quantities have been averaged and a 0.95 confidence limit using a t-test have also been calculated. The results are reported in 
Table IV-21. The confidence limits are reported under the heading $\Delta \sigma$, $\Delta a$ etc. The impeller diameter and tank diameter for which these average values correspond and the fluid used are also given in Table IV-21.

\section{Jet Width $\sigma$}

Various correlations were tried to relate the data presented in Table IV-20. In Figure IV-13 is shown a $\log -\log$ plot of $\sigma$ versus $\mathrm{ND}^{3}$. The data correlates very well along a line drawn through the average value of $\sigma$, indicating that $\sigma$ is a constant. Since the data consists of two fluids, air and water this means that turbulence and not viscosity is the significant variable in the impeller stream. The average value of $\sigma$ for all the data in Table IV-20 is

$$
\sigma=12.621 \pm 0.066
$$

The confidence limit of $0.066^{\prime}$ on $^{\prime} \sigma$ mightibei:misleading since the confidence limit on our data is'rather large', equal to 2.66 (see Equation IV-9.): It should be noted that Cooper reports only one'profile lfor each :condition' investigated, hence it is not possible:to estimate the confidence limit on the parameters obtained by the least square fit of a velocity profile from his data.

If the average value of $\sigma$ at constant impeller diameter is examined in Table IV-21!, these values are found to fall into three groups; Items 1 to 4 are Coopers data for water, Items 5 to 6 are, our data in water and Items 7 to 9 which are Coopers data in air. In each group the value of $\sigma$ appears approximately constant. The 
TABLE IV-21: AVERAGE VALUES OF JET WIDTH $\sigma$, RADIUS OF SOURCE a, DIMENSIONLESS PUMPING CAPACITY $\mathrm{N}_{0}$ AND WEIGHTED AVERAGE YAW ANGLE $\bar{\theta}_{\mathrm{y}}$ EXTRACTED FOR DATA SETS OF TABLE IV-20

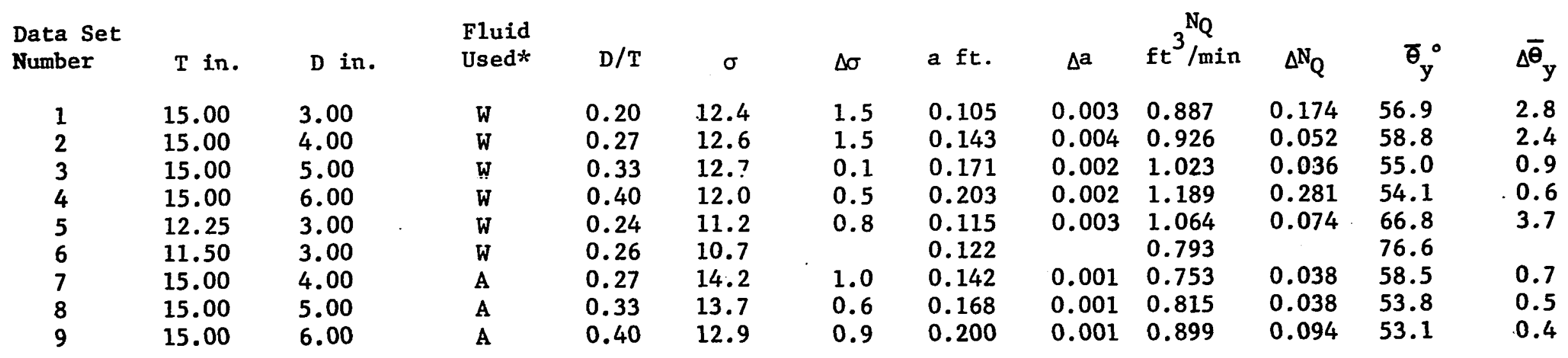




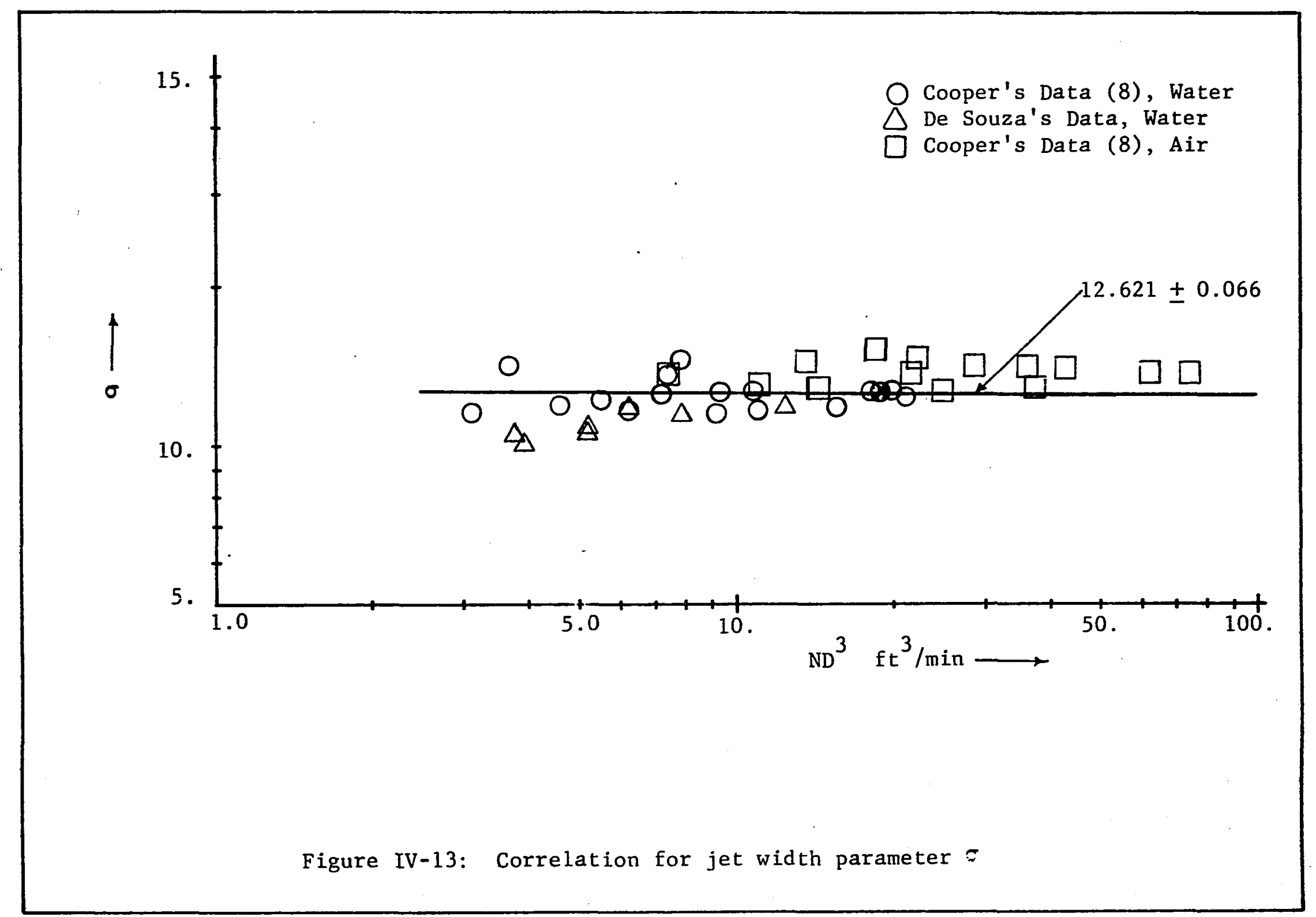


confidence limits for these nine items are also larger than that of the overall average given in Equation IV-36. These groupings are not visible when the individual data points are plotted in Figure IV-13. This suggests more exhaustive measurements are needed to conclude that $\sigma$ is independent of tank diameter and fluid in the tank. The data presented here suggests that $\sigma$ is a universal constant independent of tank geometry, impeller speed, and fluid used in the tank.

\section{Radius of Source a}

In Table IV-20 the radius of source, a, is reported for a number of profiles. It is observed that in each set of data at constant impeller diameter, $a$, is approximately constant. This is seen to be the case when the average value and confidence limit is examined in Table IV-21. The confidence limit is of the order of 0.003 indicating that, $a$, is constant to two significant figures.

From Table IV-20, a, is seen to be a function of both impeller diameter and tank diameter. In Figure IV-14 is shown a log-log plot of, $a$, versus $D / T$ also shown is the regression line calculated by program GRAPH (for listing see Appendix F). The plot shows that, a, for both air and water are almost.identical. However the points obtained by using a smaller tank does not correlate very we11. The value predicted by the regression line for our data is found to be larger than $\mathrm{D} / 2$ the impeller radius. This violates the constraint on, a, which was discussed in Chapter II.

It thus appears that $\mathrm{D} / \mathrm{T}$ is not a good criteria for correlating, a. As the flow occurs in the region between the periphery of the 


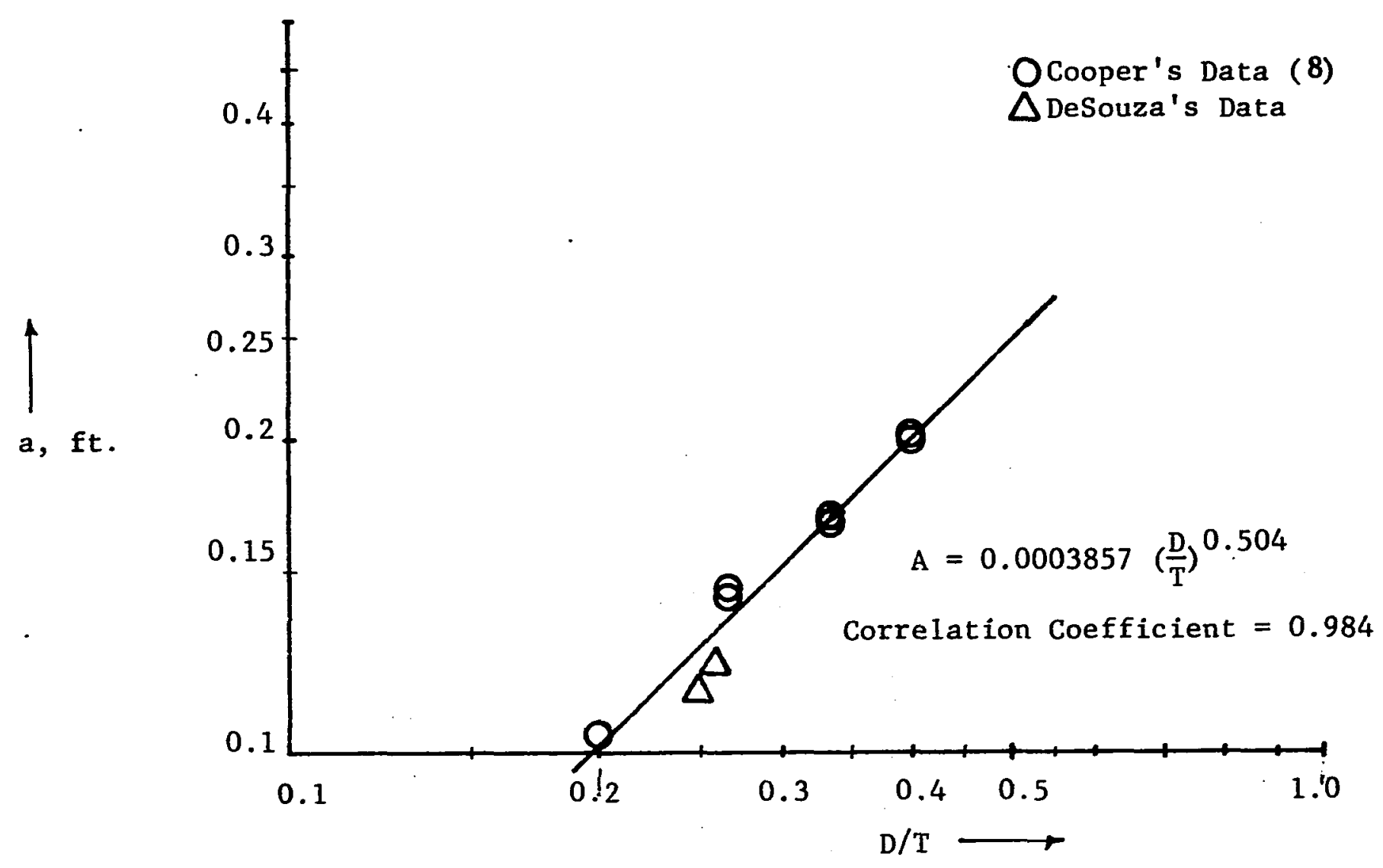

Figure IV-14: Correlation for Radius of Source a with D/T 
impeller and the tank wall, changing the tank diameter would alter the length of this region which is measured by $T-D$. Accordingly Figure IV-15 shows a plot of a versus $(D-T) / T$. In this plot it is seen that the points at constant impeller diameter correlate better separately. The results are two separate correlations. One at constant impeller diameter of 3.0 inches given by

$$
a=0.06924 \frac{T-D}{T}^{-1.837}
$$

and the second at constant tank diameter of 15.0 inches $-1.7281$

$$
a=0.08354 \quad \frac{T-D}{T}
$$

This relationship can be seen more clearly in Table IV-22 where the value of, a, from Table IV-21 and the predicted value using one of the appropriate Equations IV-37 and IV-38 are also shown. The above two equations are thus useful for interpolating the value of a given in Table IV-20.

Equations IV-37 and IV-38 were used to calculate, a, for Nielson's data analyzed in Section 4.8. The tank diameter used by Nielson was $11.25 \mathrm{in.}$ and is the smallest size tank investigated. It was found that both Equations IV-27 and IV-38 gave.values of, a, larger than $\mathrm{D} / 2$, the impeller radius for all three impeller used by Nielson. The equations are thus not recommended for tank diameters smaller than 11.5 inches.

From the above discussion it will be necessary to investigate more fully the effect of tank diameter on the parameter, a. The data that is presently available is not sufficient to make a 


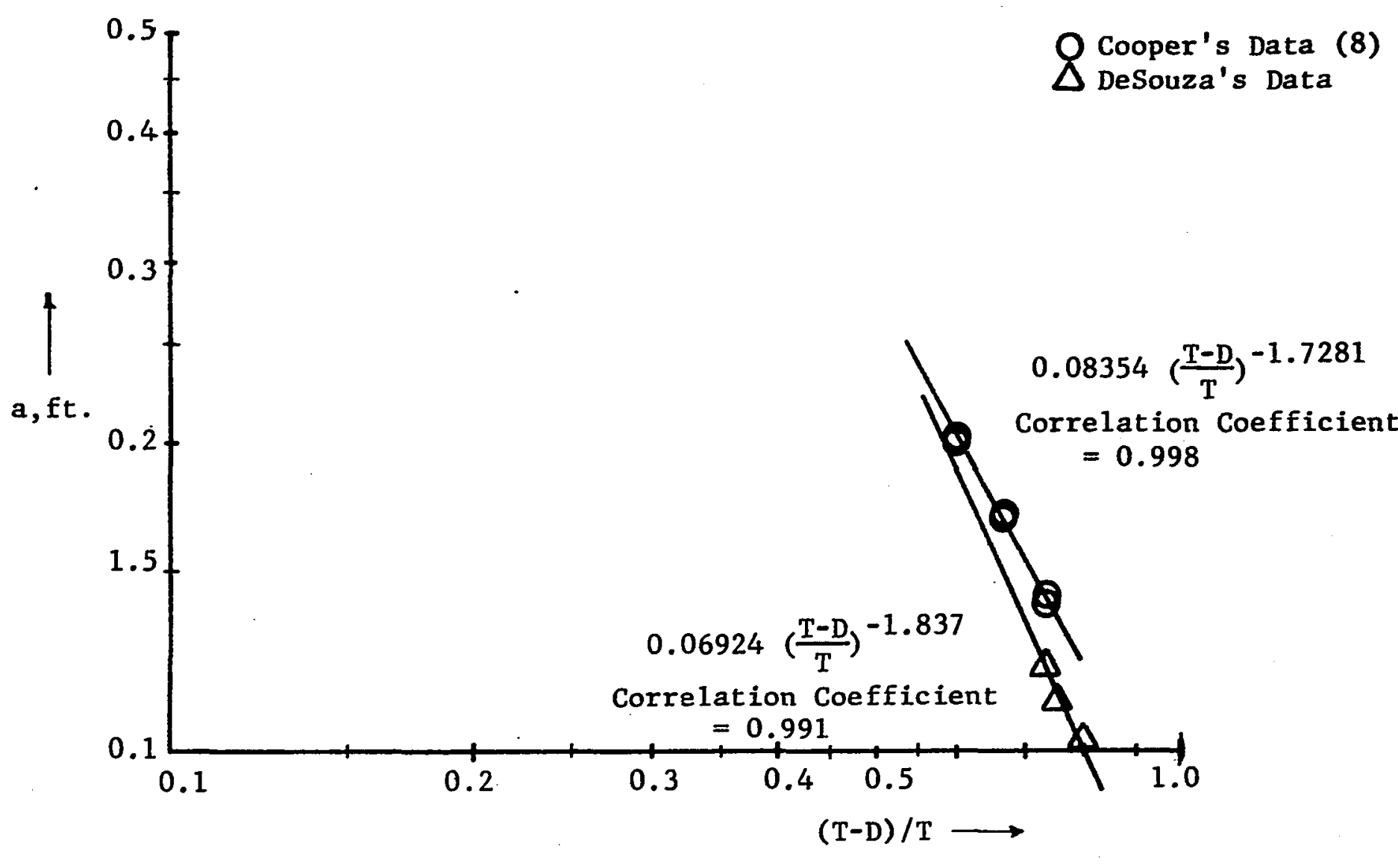

Figure IV-15: Correlation of Radius of suurce a with (T-D)/T. 
TABLE IV-22

PREDICTED AND EXPERIMENTALLY DETERMINED VALUES

OF a, THE RADIUS OF SOURCE

a, ft.

\begin{tabular}{|c|c|c|c|c|c|c|}
\hline \multirow[b]{2}{*}{$\begin{array}{c}\text { Item } \\
\text { Number }\end{array}$} & \multirow[b]{2}{*}{$\begin{array}{c}\text { D } \\
\text { In: }\end{array}$} & \multirow[b]{2}{*}{$\begin{array}{c}\mathrm{T} \\
\mathrm{In} .\end{array}$} & \multirow[b]{2}{*}{$(T-D) / T$} & \multicolumn{3}{|c|}{$a, f t}$. \\
\hline & & & & $\begin{array}{l}\text { Obtained } \\
\text { From } \\
\text { Table } \\
\text { IV-21 } \\
\end{array}$ & $\begin{array}{c}\text { Calculated } \\
\text { From Equation } \\
\text { IV-36 }\end{array}$ & $\begin{array}{c}\text { Calculated } \\
\text { From Equation } \\
\text { IV-37 } \\
\end{array}$ \\
\hline 1 & 3.0 & 15.0 & 0.8 & 0.105 & 0.104 & \\
\hline 2 & 4.0 & 15.0 & 0.73 & 0.143 & & 0.143 \\
\hline 3 & 5.0 & 15.0 & 0.67 & 0.171 & & 0.168 \\
\hline 4 & 6.0 & 15.0 & 0.6. & 0.203 & . & 0.202 \\
\hline 5 & 3.0 & 12.25 & 0.76 & 0.115 & 0.116 & \\
\hline 6 & 3.0 & 11.5 & 0.74 & 0.122 & 0.121 & \\
\hline 7 & 4.0 & 15.0 & 0.73 & 0.142 & & 0.143 \\
\hline 8 & 5.0. & 15.0 & 0.67 & 0.168 & & 0.168 \\
\hline 9 & 6.0 & 15.0 & 0.6 & 0.200 & & 0.202 \\
\hline
\end{tabular}


conclusive statement for predicting the behavior of, a. Dimensionless Pumping Capacity $\mathrm{N}_{\mathrm{Q}}$

The dimensionless pumping capacity $\mathrm{N}_{\mathrm{Q}}=\mathrm{Q} / \mathrm{ND}^{3}$ appears to be constant for a given impeller and tank diameter as seen in Table IV-21. The confidence limit of Data Sets 1 and 4 are large. Figure IV-16 shows a $\log -\log$ plot of $\mathrm{N}_{\mathrm{Q}}$ versus $\mathrm{D} / \mathrm{T}$. No apparent correlation exists. The line drawn through the points is an average value. The confidence limit for the average values is 0.01 which is much smaller than the confidence limits of the individual points given in Table IV-21. The best estimate of $N_{Q}$ is thus an average value with the confidence limit of \pm 0.28 corresponding to that of Data Set 4 of Table IV-21. Hence,

$$
\mathrm{N}_{\mathrm{Q}}=0.93 \pm 0.28
$$

This conclusion is in agreement with Equation I-34, Section 3.4 of Chapter I where it was shown that for geometrically similar impellers $\mathrm{N}_{\mathrm{Q}}$ is a constant.

Pumping Capacity $Q$

It should be noted that there is a marked difference in the pumping capacity that was calculated by different investigators. $(8,19)$ This is illustrated by consulting Figure IV-17 which shows the theoretical and experimental values of the radial velocity $\bar{v}_{r}$ for Run Number 29. This profile has been discussed in Section 4.4. The experimental values are from Table IV-17, and the theoretical line 


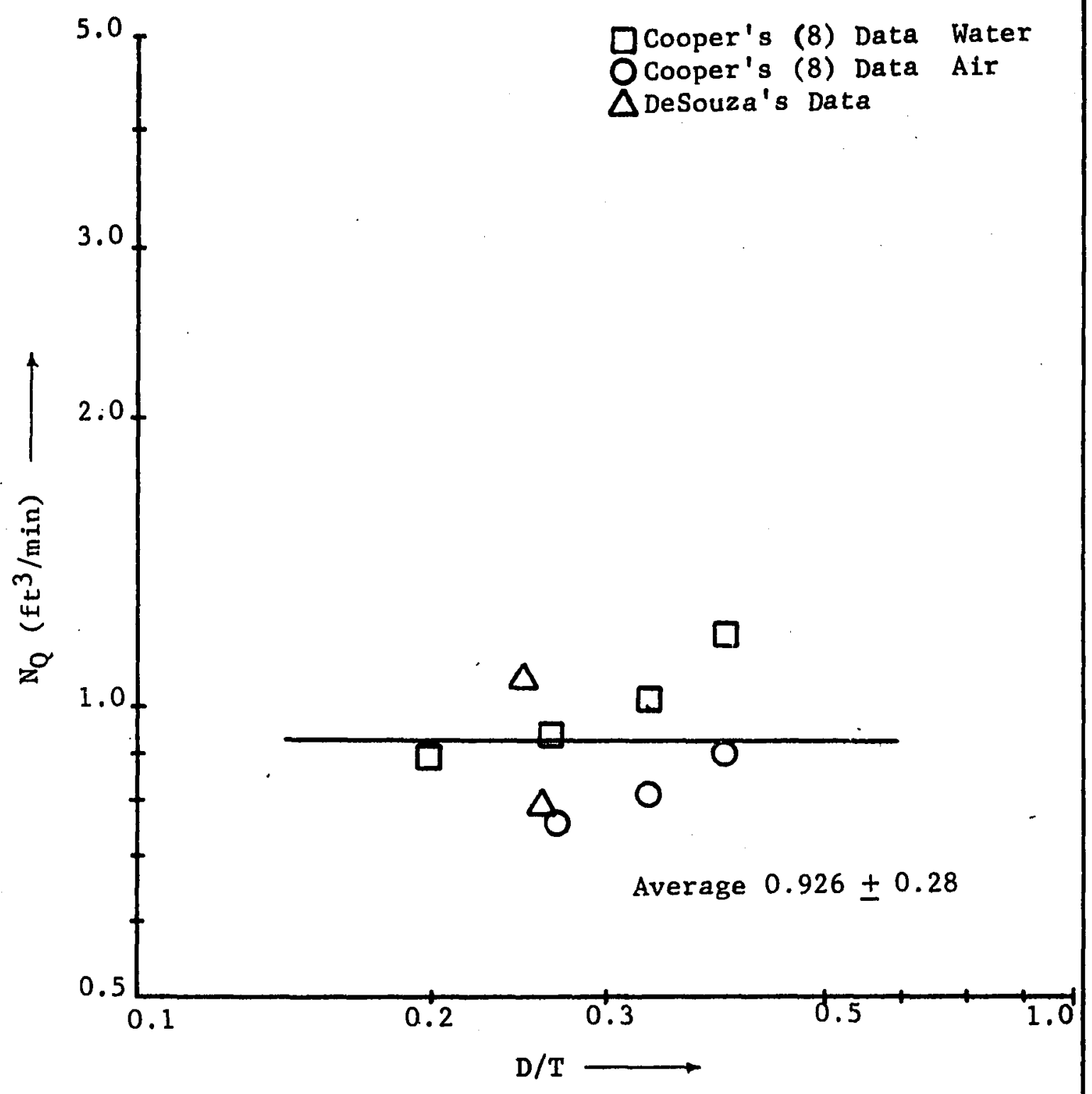

Figure IV-16: Correlation for Dimensionless Pumping Capacity $\mathrm{N}_{Q}$. 


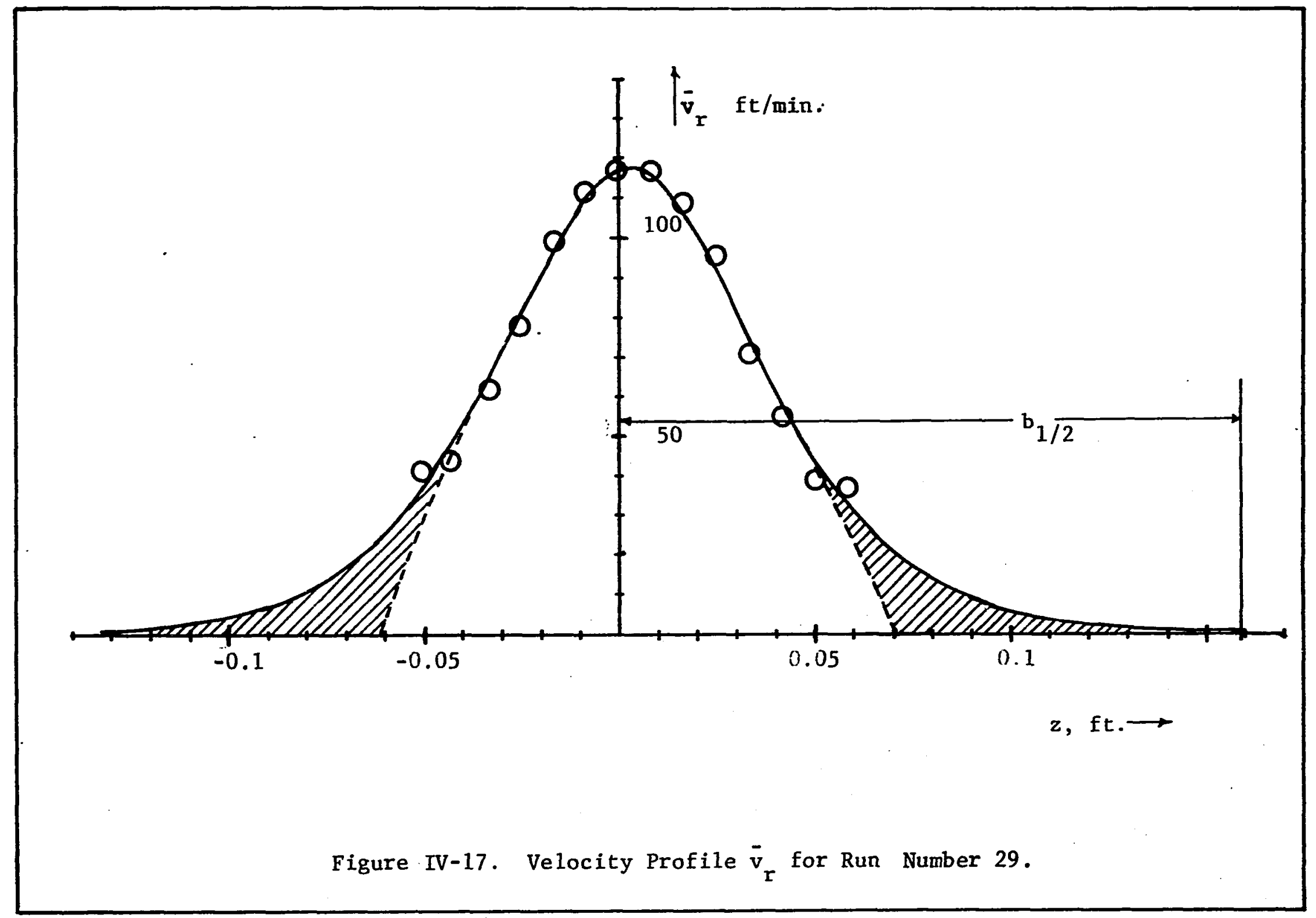


line was drawn by using Equation B-22. The tangential jet parameters in Equation B-22 are taken from Table IV-17. Table IV-17 gives a correlation coefficient of 0.99 for $\bar{v}_{\mathbf{r}}$ indicating that the fit is good and is verified by the plot in Figure IV-17. The fit, like that for $\vec{q}$ in Figure IV-11 deteriorates for low velocities.

Cooper in presenting his profiles extropolates the velocities to zero as shown by the dotted lines. As no sample calculations are given it is presumed that the crosshatched areas are omitted in calculating $Q$ the impeller discharge. This is brought out by comparing our values for $\vec{q}$ with those reported by Cooper (8). In all cases our estimation of $Q$ is larger than that of Cooper by 0.5 to as much as $5.0 \mathrm{ft}^{3} /$ min. The discrepancy is large when $\bar{v}_{r}$ is large as in the case of the 6.0 inch impeller.

No investigators reviewed by us have measured velocity pro$f$ iles very far beyond BHALF of Figure IV-11. This is also true of velocity profiles measured by the light streak method to be analyzed in the next section. The value of $Q$ reported in Table IV-20 is calculated using Equation IV-7. This equation includes the crosshatched area of Figure IV-17. Although Equation IV-7 involves integrating $\bar{v}_{\mathbf{r}}$ from $-\infty$ to $+\infty$, it is evident from Figure IV-17 that the contribution to $Q$ beyond $b_{\frac{7}{2}}$ is negligible. $b_{\frac{1}{2}}$ in this case is $0.169 \mathrm{ft}$. Figure IV-18 shows a plot of $Q$ versus $\mathrm{ND}^{3}$. The correlation coefficient for the plot is 0.99 , indicating that the correlation is good as' can be seen by the low scatter in Figure IV-18. The regres- . sion line from program GRAPH is 


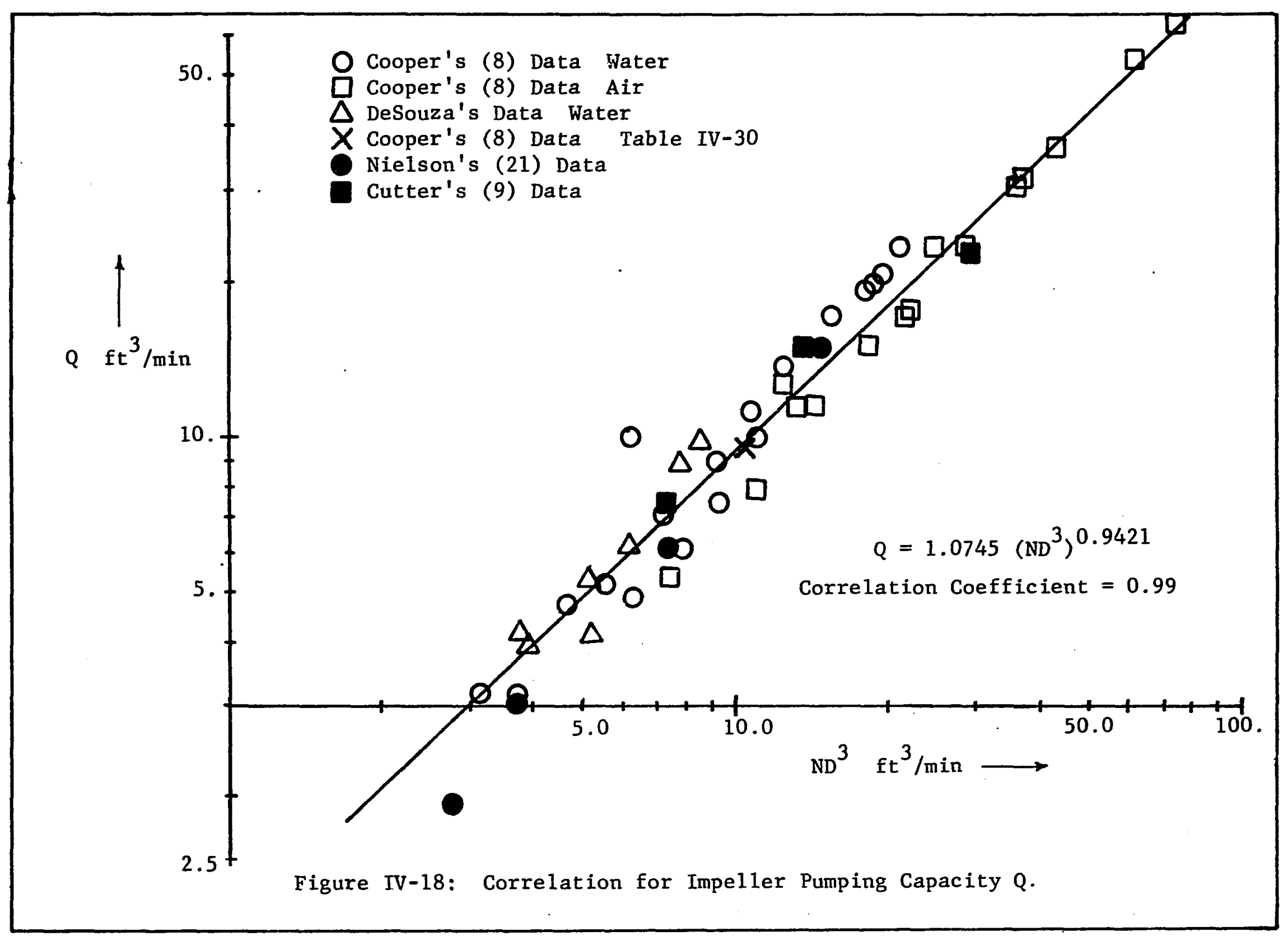




$$
Q=1.075\left(N D^{3}\right)^{0.942}
$$

\section{Volumetric Flow Parameter A}

The volumetric flow parameter reported in Table IV-20 for several profiles is plotted in Figure IV-19. This is a $\log -\log$ plot of A versus $\mathrm{ND}^{3}$. Also shown in the figure is the regression line whose correlation coefficient is 0.958 . There is some scatter in the data which is the cause of a low correlation coefficient as compared to that for Q. Our data shown by triangles could be correlated by a separate line roughly parallel to Cooper's data. This shows that $A$ is a function of tank diameter in addition to impeller diameter and impeller speed. We have noticed in Figure IV-2 that $A$ is a scaled value of $Q$. Since $Q$ correlates very well as shown in Figure IV-18, a logical choice for correlating $A$ would be $\mathrm{ND}^{3} /\left((\mathrm{D} / 2)^{2}-\mathrm{a}^{2}\right)^{\frac{1}{4}}$. This follows from combining Equations IV-40 and IV-7. In Figure $\mathrm{IV}-20$ is shown a $\log -\log$ plot of a versus $\mathrm{ND}^{3} /\left((\mathrm{D} / 2)^{2}-\mathrm{a}^{2}\right)^{\frac{1}{4}}$. The value of, a, used in the above correlation is from Table IV-20. The regression line as calculated by program GRAPH is

$$
A=1.144\left(\frac{N D^{3}}{\left((D / 2)^{2}-a^{2}\right)^{\frac{1}{4}}}\right)^{0.834}
$$

The correlation is improved as measured by the correlation coefficient which has increased from 0.958 in Figure IV-19 to 0.986 in Figure IV-20. 


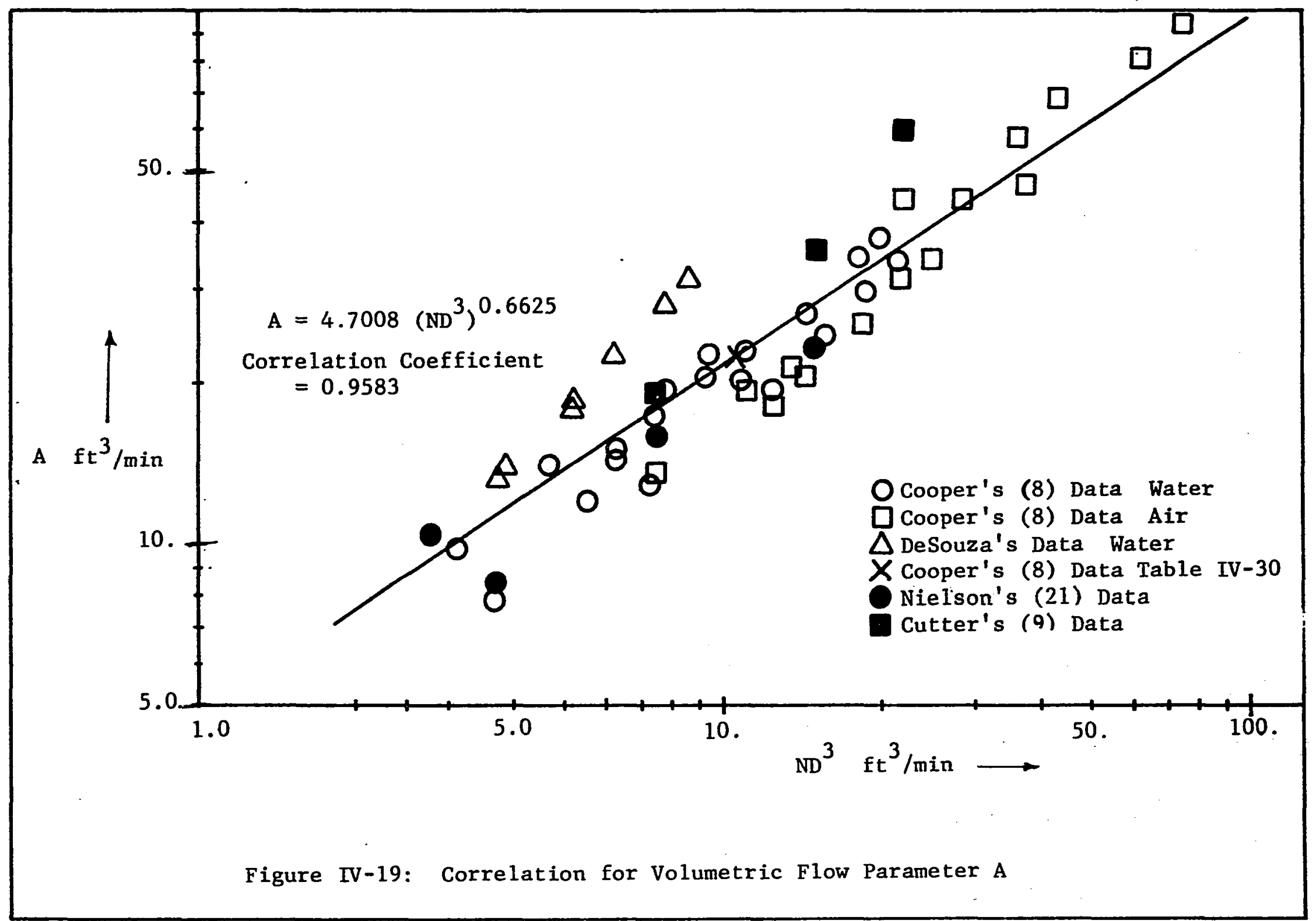




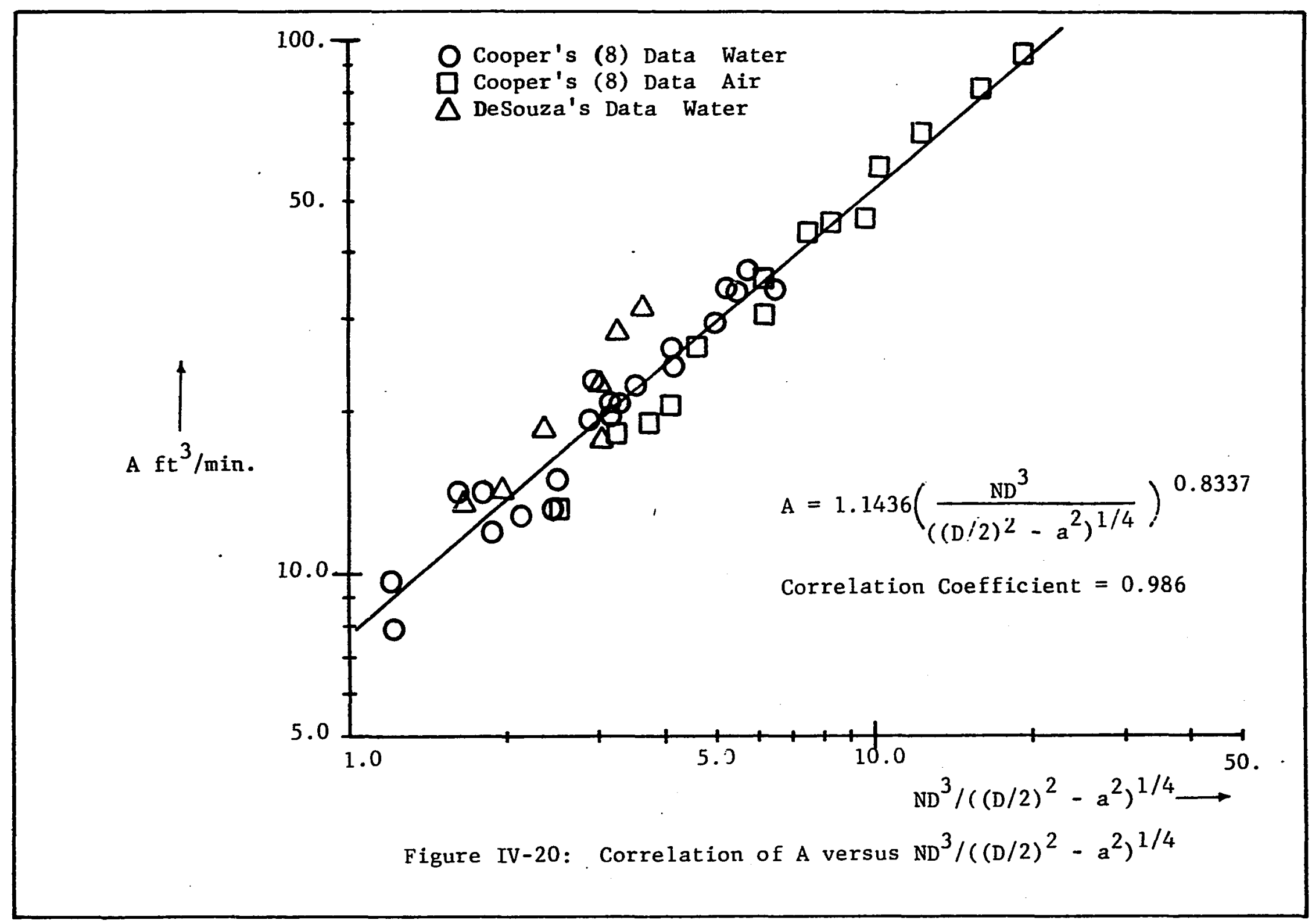

N 


\section{Results of Correlating Tangential Jet Parameters}

To test the usefulness of the correlations of the tangential jet parameters, these correlations were used to evaluate the impeller discharge. $Q$ given by Equation $I V-7$ and the eddy viscosity given by Equation B-15. A program RESULT was written for this purpose, For a listing of the program RESULT and the calculated output see Appendix F.

In Figure IV-21 is plotted $Q_{\text {calc }}$ versus $Q . Q_{c a l c}$ is obtained from Equation B-15 using Equation $I V-36$ for $\sigma$, one of the appropriate Equation IV-37 or IV-38 for, a, and Equation IV-41 for, A. The value of $Q$ is calculated from the experimentally determined values of the jet parameters $\sigma, A$ and $a$ and given in Table IV-20. If $Q_{\text {calc }}$ accurately predicts $Q$ then the data should fall along a straight line inclined at $45^{\circ}$ to the horizontal, It is seen that the data for air and water fall closely about this 1ine with a low scatter. The prediction of $Q$ by the correlated values of the tangential jet parameters is very good.

A similar calculation is performed for the eddy viscosity, $\epsilon$, using Equation B-15. The predicted value $\epsilon_{c a l c}$ and experimentally determined value $\epsilon$ is given in Table IV-23. In Figure IV-22 is plotted $\epsilon_{\text {calc }}$ versus $\epsilon$. It is seen that there is a relatively large scatter about the predicted line indicating that the prediction of the eddy viscosity is not as good as for the impeller dischange $Q$. The eddy viscosity is thus sensitive to small changes in the magnitude of the tangential jet parameters. 

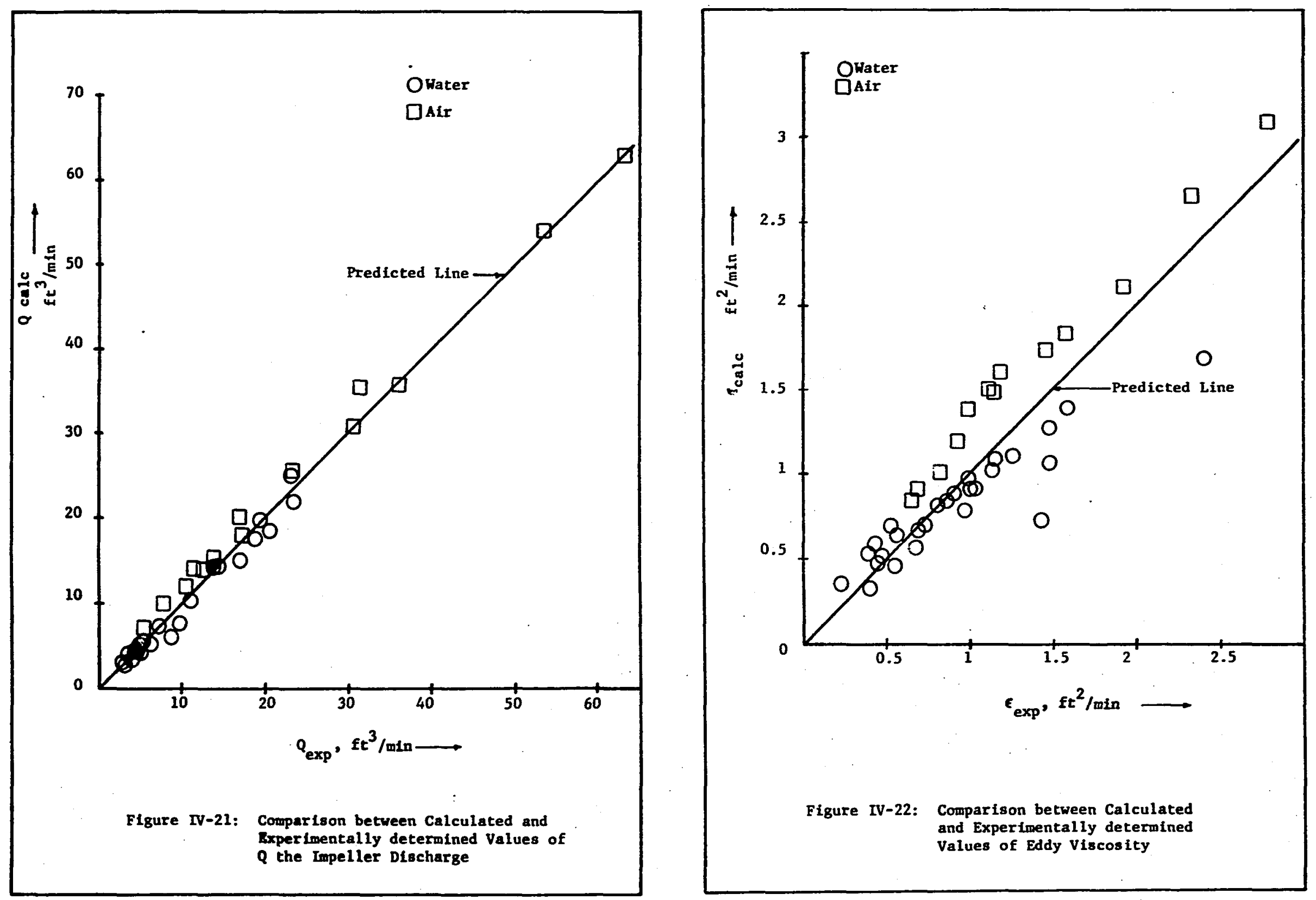
TABLE IV-23

EDDY VISCOSITY $\epsilon$. CALCULATED FROM PREDICTED VALUES OF $\sigma$, A AND a

\begin{tabular}{|c|c|c|c|c|c|c|}
\hline $\begin{array}{c}\text { Item } \\
\text { Number }\end{array}$ & I, inch & $D_{2}$ inch & $\begin{array}{c}\text { Impeller } \\
\text { ¿. Speed } \\
\text { RPM }\end{array}$ & $\mathrm{ft}^{2} / \mathrm{min}$ & $\begin{array}{r}\text { calc } \\
\mathrm{ft} / \mathrm{min} \\
\end{array}$ & Difference \\
\hline 1 & 15.00 & 3.00 & 200.0 & 0.407 & 0.321 & 0.086 \\
\hline 2 & & & 300.0 . & 0.557 & 0.450 & 0.107 \\
\hline 3 & & & 400.0 & 0.676 & 0.572 & 0.105 \\
\hline 4 & & & 500.0 & 0.523 & 0.688 & -0.165 \\
\hline 5 & & & 600.0 & 0.802 & 0.801 & 0.001 \\
\hline 6 & & & 100.0 & 0.237 & 0.361 & -0.124 \\
\hline 7 & & & 150.0 & 0.478 & 0.506 & -0.028 \\
\hline 8 & & & 200.0 & 0.552 & 0.643 & -0.092 \\
\hline 9 & & & 250.0 & 0.979 & 0.775 & 0.204 \\
\hline 10 & & & 300.0 & 1.048 & 0.902 & 0.146 \\
\hline 11 & & 5.00 & 100.0 & 0.455 & 0.474 & -0.009 \\
\hline 12 & & & 150.0 & 0.687 & 0.665 & 0.022 \\
\hline 13 & & & 200.0 & 0.866 & 0.845 & 0.021 \\
\hline 14 & & & 250.0 & 1.149 & 1.018 & 0.132 \\
\hline 15 & & & 275.0 & 1.260 & 1.102 & 0.158 \\
\hline 16 & & 6.00 & 50.0 & 0.526 & 0.389 & 0.138 \\
\hline 17 & & & 100.0 & 0.729 & 0.693 & 0.036 \\
\hline 18 & & & 125.0 & 0.919 & 0.835 & 0.085 \\
\hline 19 & & . & 150.0 & 1.000 & 0.972 & 0.028 \\
\hline 20 & & & 170.0 & 1.159 & 1.078 & 0.081 \\
\hline 21 & 12.25 & 3.00 & 243.0 & 0.879 & 0.697 & 0.181 \\
\hline 22 & & & 250.0 & 1.447 & 0.714 & 0.733 \\
\hline 23 & & & 333.3 & 1.099 & 0.908 & 0.192 \\
\hline 24 & & & 400.0 & 1.489 & 1.057 & 0.432 \\
\hline 25 & .. & & 500.0 & 1.481 & 1.273 & 0.208 \\
\hline 26 & . & & 550.0 & 1.588 & 1.378 & 0.210 \\
\hline 27 & 11.50 & & 333.3 & 2.412 & 1.677 & 0.735 \\
\hline $28^{*}$ & 15.00 & 4.00 & 200.0 & 0.446 & 0.643 & -0.197 \\
\hline 29 & & & 300.0 & 0.693 & 0.902 & -0.209 \\
\hline 30 & & & 366.0 & 0.825 & 1.065 & -0.240 \\
\hline 31 & & & 500.0 & 0.999 & 1.381 & -0.382 \\
\hline 32 & & & 600.0 & 1.298 & 1.608 & -0.310 \\
\hline 33 & & 5.00 & 200.0 & 0.660 & 0.845 & -0.185 \\
\hline 34 & & & 300.0 & 0.934 & 1.185 & -0.251 \\
\hline 35 & & & 400.0 & 1.22 .8 & 1.506 & -0.278 \\
\hline 36 & & & 500.0 & 1.587 & 1.814 & -0.227 \\
\hline 37 & & & 600.0 & 1.929 & 2.112 & -0.183 \\
\hline
\end{tabular}


TABLE IV-23 CONTINUED

\begin{tabular}{|c|c|c|c|c|c|c|}
\hline $\begin{array}{c}\text { Item } \\
\text { Number }\end{array}$ & $\mathrm{T}$, inch & D, inch & $\begin{array}{c}\text { Impell ex } \\
\text { Speed } \\
\text { RPM } \\
\end{array}$ & $\mathrm{ft}^{2} / \mathrm{min}$ & $\begin{array}{r}\epsilon_{c a l c} \\
\mathrm{ft} / \mathrm{min} \\
\end{array}$ & Difference \\
\hline $\begin{array}{l}38 \\
39 \\
40 \\
41 \\
42\end{array}$ & . & $6.00^{\circ}$ & $\begin{array}{l}100: 0 \\
20060 \\
300.0 \\
500.0 \\
600.0\end{array}$ & $\begin{array}{l}0.653 \\
1.115 \\
1.468 \\
2.333 \\
2.780\end{array}$ & $\begin{array}{l}0.693 \\
1.235 \\
1.732 \\
2.651 \\
3.086\end{array}$ & $\begin{array}{l}-0.040 \\
-0.120 \\
-0.264 \\
-0.318 \\
-0.306\end{array}$ \\
\hline
\end{tabular}


Examining the eddy viscosity in Table IV-23 it is seen that at constant impeller diameter it increases with impeller speed. At constant impeller diameter reducing the tank diameter results in an increase in eddy viscosity. This is clear on examining Items 2,23 and 27 of Table IV-23. In Figure IV-22 it is seen that the predicted value of eddy viscosity is smaller for water and larger for air. On further examining of Table IV-23 it is observed that for a given impeller speed at constant tank diameter, increasing the diameter of the impeller increases the eddy viscosity. For example in Item 1 and 13 the RPM is 200 and the impeller diameters are 3.0 inches and 5.0 inches. The eddy viscosity is doubled when the diameter change form 3.0 inches to 5.0 inches. However, the pumping capacity was found to increase by 4.4 times. This means that the momentum transfer per unit volume is lower by a factor of two. Hence the well known fact that a large impeller at low speeds gives more circulation and less mixing, while a small impeller at high speeds gives a low circulation but intense mixing in the impeller stream. The eddy viscosity gives the rate ofmomentum transfer and its value can now be predicted and fixed at any desired level. 4.8. Analysis of Cooper's (8) Data at Varying Radial Distance and Varying Impeller Blade Width

Cooper has also reported velocity profiles for the 4.0 inch Type A impeller at $280 \mathrm{RPM}$ and five different points along the radius. These are: $2.0,2.5,3.0,4.5$, and 5.0 inches. He has in addition reported velocity profiles at 100 RPM and 200 RPM for a 4.0 inch Type A impeller with five different blade widths $b$. The 
dimension $b$ is the length of the blade in the axial direction as hown in Figure I-2. These varying blade widths are $1.6,1.4,1.2$, 1.0 and 0.6 inches. The normal value of $b$ for $a 4.0$ inchimpeller is 0.8 inch. This impeller has been used in the data reported in the previous section. Hence, six different blade widths at constant impeller diameter were investigated.

Analysis at Varying Radial Distance

In Table IV-24 (b) is shown the results of the analysis of Cooper's data for varying radial distance. In this Table $\sigma$, and A were obtained by a least square fit and the radius of source, a, from the weighted average angle $\bar{\theta}_{y}$. The velocity factor was 0.5 . The fit on the data is excellent as noted by the high correlation coefficient of 0.99 for $\bar{q}$ and 0.96 to 0.98 for $\bar{v}_{r}$. The only exception is Profile Number 5 where requals 5.0 inches. This profile does: not correlate at all and was suspected to be located near $r_{0}$. This was indeed the case as $r_{0}$ calculated from Equation II-28 with $\mathrm{T}$ equal to 15 inches and $\sigma$ equal to 12.621 , is found to be 5.08 inches.

Table IV-24 (b) shows a widely varying value for $\sigma$ and the radius of source, a, increases with $r$. In Table IV-24 (a) are shown the analysis on the same data wïth $\sigma$ kept constant at its universal value given in Equation IV-36, and, a, obtained from Equation IV-38. The correlation coefficient is smaller in Table IV-24 (a) than in IV-24 (b) for both $\bar{q}$ and $\bar{v}_{r}$. The lowering in the correlation coefficient is not large and the correlation is thus still very good. 


\section{TABLE IV-24}

ANALYSIS OF COOPER'S DATA WITH VARYING RADIAL DISTANCE, SHOWING EFFECT OF $\sigma$

(a) $\sigma=12.621$, constant Correlation Coefficient

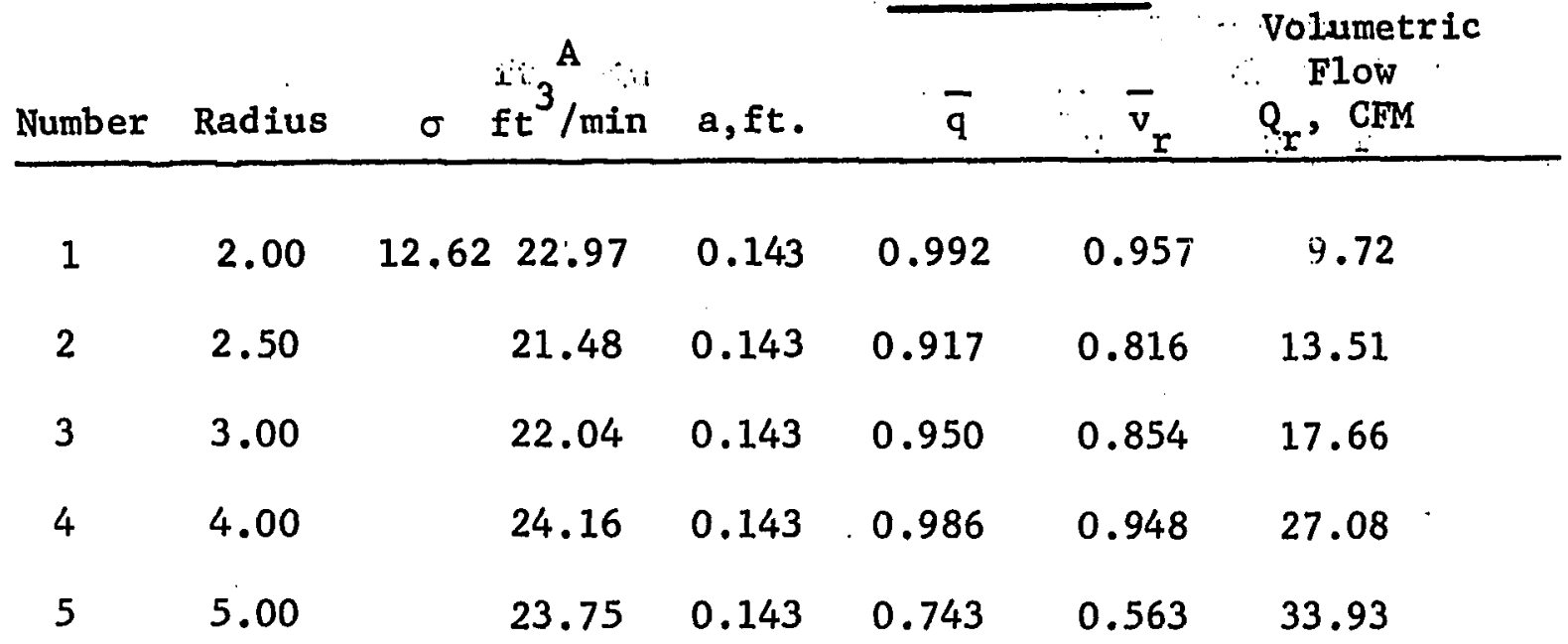

(b) $\sigma$ obtained by least square fit

\begin{tabular}{llllllll}
1 & 2.00 & 12.38 & 23.52 & 0.140 & 0.993 & 0.955 & 10.29 \\
2 & 2.50 & 17.03 & 20.34 & 0.156 & 0.982 & 0.967 & 10.49 \\
3 & 3.00 & 15.77 & 20.59 & 0.165 & 0.997 & 0.981 & 14.12 \\
4 & 4.00 & 13.37 & 23.38 & 0.164 & 0.991 & 0.983 & 25.00 \\
5 & 5.00 & $\ldots$ & $\ldots$ & 0.188 & $\ldots$ & $\ldots$ & $\ldots$ \\
\hline
\end{tabular}


Profile Number 5 is also correlated indicating that a correct choice for $\sigma$ and a was made.

The correlation coefficient on $\vec{v}_{r}$ is low, indicating that the profile is no longer in the tangential jet region as noted above, This shows that keeping $\sigma$ constant is a valid argument and that Equation IV-38 predicts the correct value for a. The volumetric parameter $A$ in Table IV-24 (a) is seen to be more nearly constant and independent of $r$, which is in agreement with the tangential jet model. The average value of $A$ is 22.9 and is very close to the correlating line when plotted in Figure IV-19. The value of $Q$ is also found to correlate well on Figure IV-18.

The complete results corresponding to Tables IV-24 (a) and IV-24 (b) are given in Appendix G, Tables G-30 and G-31. Analysis at Varying Blade Widths

In Table IV-25 is shown the analysis for varying blade widths at 100 and 200 RPM. Profile Number 5 in this Table is for a normal 4.0 inch impeller and the results are reported from Table G-18. At 100 RPM the value of $\sigma$ is seen to be fairly constant while at 200 RPM it is seen to increase with decreasing blade width. The value of A, the volumetric parameter decreases with decreasing blade width indicating that the impeller discharge is a function of the blade width. The radius of source parameter, a, shows a slight increase with decreasing blade width. Its value has the same order of magnitude at corresponding blade widths for the two impeller speeds. 
TABLE IV-25

ANALYSIS OF COOPER'S DATA AT VARYING BLADE WIDTH AND RPM

(a) $100 \mathrm{RPM}, 4.0$ inch diameter impeller

\begin{tabular}{cccccccc}
$\begin{array}{l}\text { Profile } \\
\text { Number }\end{array}$ & $\begin{array}{c}\text { Biade } \\
\text { Width }\end{array}$ & $\sigma$ & $\mathrm{ft}^{3}$ /min & & \multicolumn{2}{c}{$\begin{array}{c}\text { Correlation } \\
\text { Coefficient }\end{array}$} & \multicolumn{2}{l}{$\begin{array}{l}\text { Impeller } \\
\text { Discharge } \\
\text { Q, CFM }\end{array}$} \\
\hline 1 & 1.6 & 10.6 & 11.6 & 0.115 & 0.937 & 0.914 & 6.32 \\
2 & 1.4 & 10.6 & 10.9 & 0.107 & 0.953 & 0.923 & 6.1 .2 \\
3 & 1.2 & 12.5 & 10.7 & 0.106 & 0.981 & 0.982 & 5.58 \\
4 & 1.0 & 12.3 & .8 .4 & 0.134 & 0.987 & 0.962 & 3.88 \\
5 & 0.8 & 14.1 & 7.9 & 0.139 & 0.993 & 0.975 & 3.26 \\
6 & 0.6 & 11.8 & 7.8 & 0.142 & 0.996 & 0.887 & 3.45
\end{tabular}

(b) 200 .PPM, 4.0 inch diameter Impe11er

$\begin{array}{llllllll}1 & 1.6 & 8.5 & 23.7 & 0.112 & 0.938 \ldots & 0.902 \ldots \ldots 13.98 \\ 2 & 1.4 & 11.0 & 22.3 & 0.105 & 0.938 & 0.929 & 12.43 \\ 3 & 1.2 & 11.8 & 21.7 & 0.107 & 0.963 & 0.964 & 11.58 \\ 4 & 1.0 & 12.4 & 17.8 & 0.132 & 0.993 & 0.982 & 8.27 \\ 5 & 0.8 & 13.6 & 17.1 & 0.140 & 0.994 & 0.963 & 7.12 \\ 6 & 0.6 & 13.0 & 14.3 & 0.141 & 0.996 & 0.922 & 6.11\end{array}$


Table IV-26 gives the results for keeping $\sigma$ constant at its universal value of 12.621 . In Table IV-26 (a), the radius of source, a, is predicted from IV-38. This value of a has a small effect on $\vec{q}$ as measured by the correlation coefficient being slightly smaller. Its effect on $\bar{v}_{r}$ is disasterous. Profile Number 1 of Table IV-25 shows no correlation for $\vec{v}_{r}$, while Profile Numbers 2 and 3 give an unacceptably low value for the correlation coefficient. Profile Numbers 4 and 6 are fairly good correlations. Thisimeans that the radius of source is sensitive to blade width and this factor should be included in the correlation for, a.

In Table IV-26 (b) the value of, $a$, is obtained from the weighted average angle $\bar{\theta}_{y}$. The correlation is excellant for Profile Numbers 4, 5, and 6, and deteriates: for Profile Numbers 1 and 2. The indication is that $\sigma$ is less sensitive for small variations in b. From Table IV-26 we conclude that the universal value of $\sigma$ given by Equation :IV-36 is valid for b ranging from 0.6 to 1.0 inch ( $\pm 33 \%$ change). The value of, a, given by Equation IV-38 could be used for this range of $b$ but is not as good for predicting the data as in the case of $\sigma$. For this reason the detail analysis in Appendix G, Tables G-32 and G-33 are reported for $\sigma, A$ and $z_{0}$ obtained by a least square fit and, $a$, from the weighted... average angle $\bar{\theta}_{y}$.

Figure IV-23 shows a log-log plot of $Q$ theimpeller discharge versus the blade width $b$. The value of $Q$ is taken from Table IV-25 and is a good estimate of $Q$ as measured by the large value of correlation coefficient on $\bar{v}_{r}$. In Figure IV-22, $Q$ increases almost 


\section{TABLE IV-26}

EFFECT OF CONSTANT $\sigma$ EQUAI; TO 12.621 ON VARYING BLADE WIDTH AT 100 RPM AND IMPELLER DIAMETER OF 4.0 INCH

(a) Radius of Source a, from Equation IV-38

\begin{tabular}{|c|c|c|c|c|c|}
\hline \multirow[b]{2}{*}{ Number } & \multirow[b]{2}{*}{$\begin{array}{l}\text { Blade } \\
\text { Width }\end{array}$} & \multirow[b]{2}{*}{$\mathrm{ft}^{3} / \mathrm{min}$} & \multirow[b]{2}{*}{$a, f t$} & \multicolumn{2}{|c|}{$\begin{array}{l}\text { Correlation } \\
\text { Coefficient }\end{array}$} \\
\hline & & & & $\vec{q}$ & $\bar{v}_{r}$ \\
\hline 1 & 1.6 & 9.8 & 0.143 & 0.8983 & 0.0000 \\
\hline 2 & 1.4 & 8.8 & . & 0.9145 & 0.0996 \\
\hline 3 & 1.2 & 8.7 & & 0.9806 & 0.5093 \\
\hline 4 & 1.0 & 7.9 & & 0.9862 & 0.8887 \\
\hline 5 & 0.8 & 7.6 & & 0.9830 & 0.9414 \\
\hline 6 & 0.6 & 7.7 & & 0.9893 & 0.9144 \\
\hline
\end{tabular}

(b) Radius of Source a, From Weighted Average Angle

$\begin{array}{llllll}1 & 1.6 & 11.4: & 0.115 & 0.8983 & 0.8462 \\ 2 & 1.4 & 10.7 & 0.107 & 0.9145 & 0.8900 \\ 3 & 1.2 & 10.7 & 0.106 & 0.9806 & 0.9813 \\ 4 & 1.0 & 8.4: & 0.134 & 0.9862 & 0.9687 \\ 5 & 0.8 & 7.9 & 0.139 & 0.9830 & 0.9459 \\ 6 & 0.6 & 7.8 & 0.142 & 0.9893 & 0.9169\end{array}$




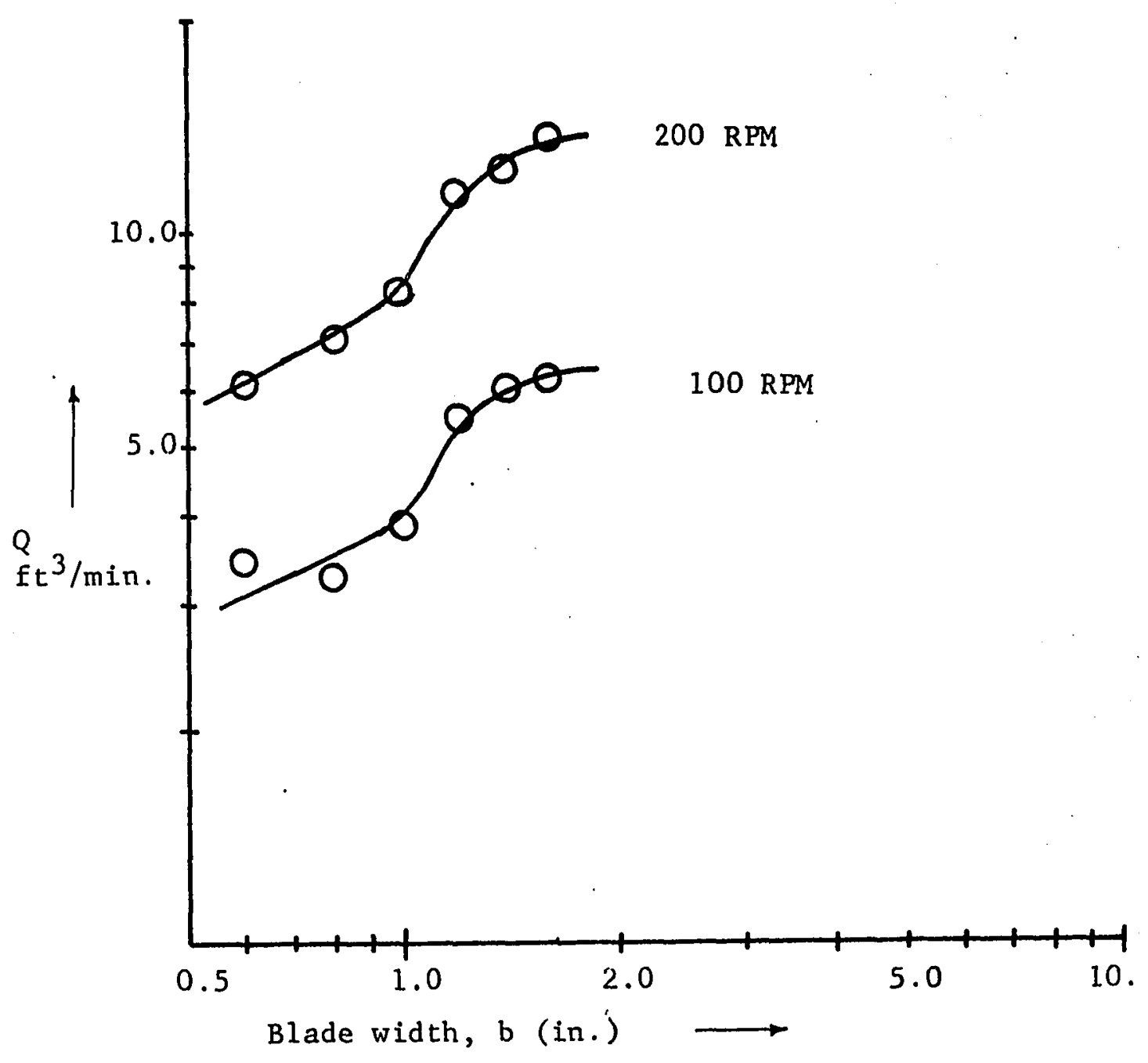

Figure IV-23: Effect of Blade Width on the Impeller Discharge $Q$ for a 4.0 in. Impeller 
linearly till b reaches 1.0 inch. After this point there is a sudden increase in $b$. This is the reason why $\sigma$ the jet width and a are different from that obtained for the standard impeller with $b$ equal 0.8 inches.

From the point of view of optimum value of $Q, b$ equal to 1.0 in. appears a good choice. However othercconsiderations such as power input which have not been determined, might be a significant factor in determining the optimum blade width. It is noticed that changing the impeller speed.merely shifts the curve up indicating that $Q$ is proportional to $\mathrm{N}$, the impeller speed. The blade width is thus not an interacting factor with the impeller speed. It thus appears that Equation IV-40 could still be used to calculate $Q$ for impellers have varying blade widths with a suitable scaling factor to account for $b$. 4.9. Analysis of Nielson's (21) and Cutter's (9) Data

Both Nielson and Cutter used the light streak method described in Chapter I to obtain velocity profiles in the neighborhood of the impeller. The experimentally determined velocity profiles were fitted to the tangential jet model. The results of this analysis is reported in this section.

The velocity profiles reported by Nielson and Cutter are for $\bar{v}_{r}$. From Chapter II, the equation for $\bar{v}_{r}$ is

$$
\bar{v}_{\mathrm{r}}=\frac{\mathrm{A}}{2} \frac{\sigma^{\frac{1}{2}}}{\dot{\mathrm{r}}^{3}}\left(\mathrm{r}^{2}-\mathrm{a}^{2}\right)^{\frac{2}{2}}\left[1-\tanh ^{2}(\eta / 2)\right]
$$

In analyzing our data and that of Cooper, the value of, a, was fixed by a weighted average value of the angle profile. In the light streak method the angle profile was not detected nor is an average 
angle given. Hence in analyzing Nielson's and Cutter's data an average angle $\bar{\theta}_{j}$ was estimated to evaluate $a$. It was noted earlier that Equations IV-37 and IV-38 could not be used to predict a, as they gave a value for, $a$, larger than $D / 2$.

As a first trial $\bar{\theta}_{\mathrm{y}}$ was selected using $\bar{\theta}_{\mathrm{y}}$ given in Table IV-21 as a guide. It is noted in Table IV-21 that $\bar{\theta}_{y}$ for Cooper's data in a 12.0 inch diameter tank ranges from $53.0^{\circ}$ to $59.0^{\circ}$. Our data in 12.25 inch and 11.5 inch diameter tanks gave $\bar{\theta}_{\mathrm{y}}$ as $66.8^{\circ}$ and $76.6^{\circ}$. From these observations a trend is noticed in which $\bar{\theta}_{\mathrm{y}}$ increases as tank diameter decreases. As Nielson and Cutter used a 11.25 inch and 11.5 inch diameter tanks respectively, three values of $\bar{\theta}_{y}$ were tried. They are $60^{\circ}, 65^{\circ}$ and $70^{\circ}$.

The analysis of Nielson's and Cutter's data is done by the program NIELCUT. The listing of the program together with a listing of the raw data is given in Appendix F. The program NIELCUT is essentially the same as FLOWANL. The difference 1ies in processing the raw data, the way, a, the radius of source is calculated, and the regression is for $\bar{v}_{r}$ and not $\bar{q}$.

Analysis of Nielson's Data

Nielson measured seven velocity profiles. Profile Numbers 1,2 and 3 are at three different radial distances $r$, for a 2.0 inch impeller in water at 600 RPM. Profile Numbers 4, 5, and 6 are with. a 4.0 inch impeller. Profiles 4 and 6 are with water at 200 RPM, while Profile Number 5 is in corn syrup (viscosity 10 centipoise) 
at 100 RPM. Finally Profile Number 7 is for a 7.0 inch diameter impeller at 75 RPM.

Table IV-27 shows the effect of varying $\bar{\theta}_{y}$ on Nielson's data. Comparing Tables IV-27 (a) and IV-27 (b) it is seen that changing $\bar{\theta}_{y}$, (from which a is calculated) results in a change in $A$ the volumetric parameter only. No change is noticed in $\sigma$, the sum square SS and the correlation coefficient for the corresponding profiles in these tables.

Table IV-27 also gives a widely varying value for $\sigma$ at constant impeller diameter. From the analysis presented in the previous section it was shown that $\sigma$ is a universal constant. Hence in Table IV-28 is shown the result for Nielson's data with $\sigma$ a constant given by Equation IV-36. The result of keeping $\sigma$ a constant is to increase the sum of squares about the regression line and decrease the correlation coefficient. The decrease in correlation is not excessive and can be verified by comparing Tables IV-27 and IV-28. The value of $A$ however is not affected. Here again it is seen that $\sigma$ can be considered as a universal constant, without drastically affecting the fit.

Table IV-29 shows the final result of analyzing Nielson's data. In this table, the value of $\bar{\theta}_{y}$ was fixed at $70^{\circ}$ for impeller diameter of 2.0 inches, since this gave a more nearly constant value of $\mathrm{A}=$ 10.67 for the Profile Numbers 1,2 , and 3 taken at different radial distances. Similarly for Profiles 4,5 , and $6 \bar{\theta}_{y}$ was fixed at $60^{\circ}$ as this gave a nearly constant value for $A$ in Profile Numbers 4 and 6. These two profiles it will be recalled are for a 4.0 inch 
TABLE IV-27

EFFECT OF $\bar{\theta}_{y}$ ON THE ANALYSIS OF NIELSON'S DATA

Impeller

Profile Speed Radial

Number

RPM

Distance

Correlation

Sum Square Coefficient

(a) $\bar{\theta}_{y}=60^{\circ}$

$\begin{array}{llllllll}1 & 600 & 1.0 & 22.4 & 8.6 & 0.072 & 3282 & 0.938\end{array}$

$\begin{array}{lllllll}2 & 3.0 & 12.1 & 10.5 & 0.072 & 49 & 0.989\end{array}$

$\begin{array}{lllllll}3 & 4.0 & 10.2 & 10.9 & 0.072 & 16 & 0.978\end{array}$

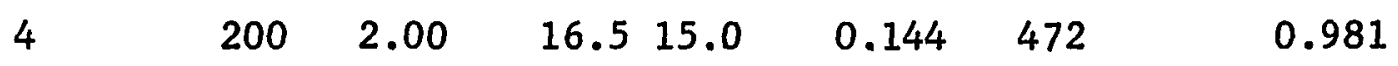

$\begin{array}{llllllll}5 & 100 & 2.00 & 17.5 & 7.7 & 0.144 & 97 & 0.987\end{array}$

$\begin{array}{llllllll}6 & 200 & 4.00 & 13.4 & 15.1 & 0.144 & 30 & 0.998\end{array}$

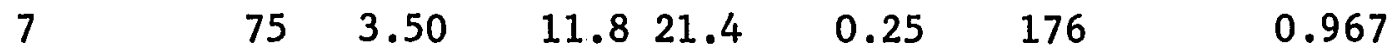

(b) $\bar{\theta}_{y}=65^{\circ}$

$\begin{array}{lrrrrrrr}1 & 600 & 1.00 & 22.4: 9: 4 & 0.076 & 3282 & 0.938 \\ 2 & & 3.00 & 12.2 & 10.6 & 0.076 & 49 & 0.989 \\ 3 & & 4.00 & 10.1 & 10.9 & 0.076 & 16 & 0.978 \\ 4 & 200 & 2.00 & 16.5 & 16.4 & 0.151 & 472 & 0.981 \\ 5^{*} & 100 & 2.00 & 17.5 & 8.3 & 0.151 & 97 & 0.987 \\ 6 & 200 & 4.00 & 13.4 & 15.23 & 0.151 & 30 & 0.998 \\ 7 & 75 & 3.50 & 11.8 & 23.22 & 0.264 & 176 & 0.967\end{array}$

* Profile measurements made in Corn Syrup, viscosity 10 centipoises. 


\section{TABLE IV-28}

EFFECT OF KEEPING $\sigma$ A CONSTANT VALUE OF 12.621

ON NIELSON'S DATA. $\bar{\theta}_{\mathrm{y}}=65^{\circ}$

\begin{tabular}{|c|c|c|c|c|c|c|}
\hline Number & $\begin{array}{c}\text { Radial } \\
\text { Distance } \\
\text { i.in:i... } \\
\end{array}$ & $\sigma$ & $\begin{array}{c}\mathrm{ft}^{3} / \mathrm{min} \\
\mathrm{A}\end{array}$ & $a, f t$. & $\begin{array}{c}\text { Sum Square } \\
\text { SS } \\
\end{array}$ & $\begin{array}{c}\text { Correlation } \\
\text { Coefficient } \\
\mathrm{R}\end{array}$ \\
\hline 1 & 1.00 & 12.621 & 9.9 & 0.076 & 9221 & 0.814 \\
\hline 2 & 3.00 & & 10.5 & 0.076 & 55 & 0.814 \\
\hline 3 & 4.00 & & 10.5 & 0.076 & 84 & 0.879 \\
\hline 4 & 2.00 & & 16.4 & 0.151 & 1586 & 0.934 \\
\hline $5^{*}$ & 2.00 & $\cdot$ & 8.3 & 0.151 & 609 & 0.919 \\
\hline 6 & 4.00 & & 15.2 & 0.151 & 63 & 0.995 \\
\hline 7 & 3.50 & & 23.0 & 0.264 & 203 & 0.962 \\
\hline
\end{tabular}

${ }^{*}$ For corn syrup 


\section{TABLE IV-29}

ANALYSIS OF NIELSON'S DATA RESULTING FROM AN

$$
\text { OPTIMUM SELECTION OF } \overline{\boldsymbol{\theta}}_{\mathrm{y}}
$$

\begin{tabular}{ccccccc}
$\begin{array}{c}\text { Profile } \\
\text { Number }\end{array}$ & $\begin{array}{l}\text { Radial } \\
\text { Distance } \\
\text { r, in. }\end{array}$ & $\sigma$ & \multicolumn{2}{c}{$\mathrm{ft}^{3} / \mathrm{min}$} & $\begin{array}{c}\text { Correlation } \\
\mathrm{A}, \mathrm{ft} .\end{array}$ & $\begin{array}{c}\bar{\theta}_{\mathrm{y}} \\
\begin{array}{c}\text { Coefficient } \\
\mathrm{R}\end{array}\end{array}$ \\
\hline 1 & 1.00 & 12.621 & 11.0 & 0.078 & 70 & 0.814 \\
2 & 3.00 & & 10.6 & 0.078 & 0.987 \\
3 & 4.00 & 10.5 & 0.078 & 0.879 \\
4 & 2.00 & 15.1 & 0.144 & 60 & 0.934 \\
$5^{*}$ & 2.00 & 7.6 & 0.144 & & 0.919 \\
6 & 4.00 & 15.2 & 0.144 & & 0.995 \\
7 & 3.50 & 23.1 & 0.264 & 70 & 0.962 \\
\hline
\end{tabular}

* For corn syrup 
diameter impeller in water at 200 RPM. In case of Profile Numb er 7 the value of $\bar{\theta}_{y}$ was fixed at $70^{\circ}$ since this gave a value of $A$ that came very close to the regression line of Figure IV-18. The above observations can be checked by comparing Tables IV-28 and IV-29. In Table IV-28, $\bar{\theta}_{\mathrm{y}}$ was selected equal to $65^{\circ}$ so as to make this comparison possible and thus show the effect of a suitable choice in $\bar{\theta}_{y}$. The values of A reported in Table IV-34 are plotted in Figure IV-18. It is seen that the result for Profile Number 5 which is for corn syrup is not very much different, lending further support to the observation that the physical properties of the fluid is not an important variable in analyzing the flow in the stirred tank. A summary of the detailed analysis from program NIELCUT for Tabie.IV-29 is given in Appendix G. Tables for $\sigma$ free and fixed by Equation IV-36 are included for comparison. The value of a, in these Tables are at the optimum values shown in Table IV-29.

\section{Analysis of Cutters Data}

Cutter reports average values of $\bar{v}_{r}$ for only positive values of $z$, or half a velocity profile. Velocity profiles are for a single 4.0 inch diameter impeller, and at several different radial distances, The data is obtained at three different impeller speeds. As for Nielson data a value of $\bar{\theta}_{y}$ had to be selected in order to calculate a, the radius of source.

In Table IV-30 is shown the analysis of Cutter's data at 200 RPM with two values of, $a$. The radius of source is calcula ted from $\bar{\theta}_{y}$ set at $65^{\circ}$ and $70^{\circ}$. It is observed that changing, a, has a very 


\section{TABLE IV-30}

ANALYSIS OF CUTTER'S DATA AT 200 RPM FOR A 4.0 INCH IMPELLER SHOWING EFFECT OF VARYING $\bar{\theta}_{\mathrm{y}}$

(a) $\bar{\theta}_{y}=65^{\circ}$

\begin{tabular}{|c|c|c|c|c|c|c|}
\hline Number & $\begin{array}{l}\text { Radial } \\
\text { Distance } \\
r \text {, in. }\end{array}$ & o & $\begin{array}{r}\mathrm{At}^{3} / \mathrm{min} \\
\end{array}$ & a, $\mathrm{ft}$ & $\begin{array}{c}\text { Sum Square } \\
\text { SS } \\
\end{array}$ & $\begin{array}{c}\text { Correlation } \\
\text { Coefficient } \\
\mathrm{R} \\
\end{array}$ \\
\hline 1 & 2.00 & 9.7 & 33.7 & 0.151 & 112 & 0.995 \\
\hline 2 & 2.75 & 18.1 & 12.0 & & 315 & 0.976 \\
\hline 3 & 3.38 & 13.7 & 18.5 & & 196 & 0.985 \\
\hline 4 & 4.13 & 6.5 & 24.0 & & 166 & 0.896 \\
\hline 5 & 4.38 & 23.6 & 15.3 & & 114 & 0.87 .7 \\
\hline 6 & 5.13 & 7.8 & 24.8 & & 177 & 0.647 \\
\hline
\end{tabular}

(b) $\bar{\theta}_{y}=70^{\circ}$

$\begin{array}{rrrrrrr}1 & 2.00 & 9.5 & 40.1 & 0.157 & 112 & 0.995 \\ 2 & 2.75 & 18.4 & 12.0 & & 314 & 0.976 \\ 3 & 3.38 & 14.0 & 18.2 & 195 & 0.985 \\ 4 & 4.13 & 6.9 & 22.0 & 168 & 0.894 \\ 5 & 4.38 & 23.6 & 15.3 & 114 & 0.877 \\ 6 & 5.13 & 6.8 & 28.7 & 172 & 0.661\end{array}$


small effect on $\sigma$, sum of squares about the correlation and the correlation coefficient. It has a proportionately larger effect on the volumetric parameter A. The parameter $\sigma$ is seen to vary in an irregular manner. The tank diameter is 11.5 inches, taking $\sigma$ at 12.621 , $r_{0}$ from II-28 is found to be 3.9 inches. Hence profiles 4,5 , and 5 are outside the tangential jet region as their values of $r$ is larger than 3.9 inches. This is verified by the comparatively lower values of the correlation coefficient for these profiles. This trend is the profiles measured at 400 and 600 RPM and can be verified in Appendix $G$ where a detail summary of the profiles are given.

In Table IV-31 is shown the velocity profiles measured at 200 RPM with $\sigma$ constant and equal to 12.621 . The results are presented for two values of, a, calculated from $\bar{\theta}_{y}$ set at $65^{\circ}$ and $70^{\circ}$. Comparing this analysis with the previous analysis given in Table IV-30, it is seen that the sum of squares has increased and the correlation coefficient has been lowered a small amount. It is observed that the results with $\bar{\theta}_{y}$ equal to $65^{\circ}$ gives a more nearly equal value for $A$ in Profile. Numbers 1,2 , and 3 than that obtained with $\bar{\theta}_{\mathrm{y}}$ equal to $70^{\circ}$. Only Profile Numbers 1,2 and 3 are considered since it has been noted above that these are the only profiles for which the tangential jet model is valid. The rest of the profiles are for $r$ greater than 3.9 inches and are thus in the stagnation region, Region II. This observation is supported by the fact that $Q$ decreases for $r$ larger than 3.9 as seen in Tables IV-32 and IV-33. 
TABLE IV-31

ANALYSIS OF CUTTER'S DATA AT 200 RPM FOR A 4.0 INCH IMPELLER, WITH $\sigma$ CONSTANT AND VARYING $\bar{\theta}_{\mathrm{y}}$

(a) $\bar{\theta}_{y}=65^{\circ}$

\begin{tabular}{|c|c|c|c|c|c|c|}
\hline $\begin{array}{l}\text { Profile } \\
\text { Number }\end{array}$ & $\begin{array}{l}\text { Radial } \\
\text { Distance } \\
r\end{array}$ & $\sigma$ & $\begin{array}{c}\mathrm{ft}^{3} / \mathrm{min} \\
\mathrm{A}\end{array}$ & $a, f t$. & $\begin{array}{c}\text { Sum Square } \\
\text { SS }\end{array}$ & $\begin{array}{c}\text { Correlation } \\
\text { Coefficient } \\
\text { R } \\
\end{array}$ \\
\hline 1 & 2.00 & 12.621 & 19.9 & 0.151 & 205 & 0.991 \\
\hline 2 & 2.75 & & 17.2 & & 493 & 0.962 \\
\hline 3 & 3.38 & & 20.5 & & 205 & 0.984 \\
\hline 4 & 4.13 & & 12.9 & & 266 & 0.827 \\
\hline 5 & 4.38 & & 19.7 . & & 275 & 0.667 \\
\hline 6 & 5.13 & & 17.7 & & 213 & 0.548 \\
\hline
\end{tabular}

(b) $\bar{\theta}_{y}=70^{\circ}$

\begin{tabular}{|c|c|c|c|c|c|c|}
\hline 1 & 2.00 & 12.621 & 22.1 & 0.157 & 205 & 0.991 \\
\hline 2 & 2.75 & & 17.5 & & 493 & 0.962 \\
\hline 3 & 3.38 & & 20.5 & & 204 & 0.984 \\
\hline 4 & 4.13 & & 13.0 & & 266 & 0.827 \\
\hline 5 & 4.38 & & 19.8 & & 275 & 0.668 \\
\hline 6 & 5.13 & & 17.8 & & 213 & 0.548 \\
\hline
\end{tabular}


TABLE IV-32

RESULTS OF ANALYSIS OF CUTTER'S DATA AT 400 RPM, FOR A 4.0 INCH IMPELLER, $\sigma$ CONSTANT AND $\bar{\theta}_{\mathrm{y}}=65^{\circ}$

\begin{tabular}{|c|c|c|c|c|c|c|}
\hline Number & $\begin{array}{r}\text { Radial } \\
\text { Distanc } \\
x \text {, in. } \\
\end{array}$ & $\sigma$ & $\begin{array}{c}\mathrm{ft}^{3} / \mathrm{min} \\
\mathrm{A}\end{array}$ & $a, \mathrm{ft}$. & $\begin{array}{l}\text { Correlation } \\
\text { Coefficient }\end{array}$ & $\begin{array}{l}\text { Volumetric } \\
\text { Flow at } \\
\text { Corresponding } \\
\text { r, cfm } \\
\end{array}$ \\
\hline 1 & 2.00 & 12.621 & 42.9 & 0.151 & 0.963 & 16.4413 \\
\hline 2 & 2.81 & & 35.5 & & 0.984 & 25.6676 \\
\hline 3 & 2.86 & & 39.7 & & 0.985 & 29.4310 \\
\hline 4 & 3.44 & & 31.6 & & 0.934 & 29.5016 \\
\hline 5 & 3.56 & & 29.5 & & 0.982 & 28.7167 \\
\hline 6 & 4.19 & & 31.8 & & 0.892 & 37.3338 \\
\hline 7 & 4.75 & & 21.8 & & 0.885 & 29.3451 \\
\hline 8 & 5.56 & & 9.4 & & 0.673 & 15.0565 \\
\hline
\end{tabular}


TABLE IV-33

RESULTS OF ANALYSIS OF CUTTER'S DATA AT 600 RPM, FOR

A 4.0 INCH IMPELLER, $\sigma$ CONSTANT AND $\bar{\theta}_{y}=65^{\circ}$

\section{Radial}

Volumetric Flow

Distance

Number $r$, in.

$2 \quad 2.75$

$\begin{array}{ll}3 & 3.38\end{array}$

54.230

$0: 978$

38.1213

$4 \quad 4.13$

59.044

0.951

54.0455

$5 \quad 4: 38$

72.044

0.958

83.1381

$6 \quad 5.13$

62.255

0.889

76.6890

37.850

0.848

55.3585 
The above observations were also noticed in the profiles measured at 400 and 600 RPM. The best estimate of, a, was obtained by setting $\bar{\theta}_{y}$ equal to $65^{\circ}$. Tables IV -32 and IV-33 show the results of profiles measured at 400 and 600 RPM with the parameter $\sigma$ chosen at 12.621 and, $a$, calculated from $\bar{\theta}_{y}$ equal to $65^{\circ}$. These values of $\sigma$ and a are the best estimates for these parameters.

From Tables IV-31 through IV-33, the average value of A for $r$ less than 3.9 inches (value of $r_{0}$ ) was calculated and given in Table IV-36. In Appendix $G$ is given the detailed summary for these profiles. The radius of source is obtained from $\bar{\theta}_{y}$ and the results for $\sigma$ free and a constant are given for comparison.

4.10. Summary of Velocity Profiles obtained by the Light Streak Method

In Table IV-34 is given the best estimates of the tangential jet parameters for Nielson's and Cutter's data. Item Numbers 1 to 4 were taken by Nielson and Profile:-Numbers 5 to 7 by Cutter. Item Numbers 2 and 5 are at the same impeller speed but different tank diameters. As in the case of our measurements the smaller tank gave lower values of $A$ and $Q$. This is seen from our data in Table IV-20 Data'Set, 5,: Ittem 3 and Dața Set:6; Item $1 . \cdots$ Data. Set 6 is'. taken in the smallex diameter tank.

The value of $Q$, the impeller discharge reported in Table IV-34 is obtained from $\sigma, A$ and a reported in the Table and using Equation IV-7. In Figure IV-18, $Q$ has been plotted versus $\mathrm{ND}^{3}$. Although there is some scatter in the data it is not excessive except at low 


\section{TABLE IV-34}

SUMMARY OF RESULTS FOR THE TANGENTIAL JET BY THE

LIGHT STREAK METHOD

\begin{tabular}{|c|c|c|c|c|c|c|c|c|}
\hline $\begin{array}{c}T \\
\text { No. in. } \\
\end{array}$ & $\begin{array}{c}D^{I} \\
\text { in. }\end{array}$ & $\begin{array}{l}\text { mpeller } \\
\text { Speed } \\
\text { (RPM) }\end{array}$ & $\sigma$ & $\mathrm{ft}^{3}{ }^{\mathrm{A}} / \mathrm{min}$ & $\begin{array}{r}a \\
\mathrm{ft} \\
\end{array}$ & $\begin{array}{r}\mathrm{Q} \\
\mathrm{ft}{ }^{3} \min \\
\end{array}$ & $\begin{array}{c}N^{3} \\
\mathrm{ft} t^{3} / \mathrm{min} \\
\end{array}$ & Reference \\
\hline 1. 11.25 & 2.0 & 600 & 12.621 & 10.3 & 0.0755 & 1.98 & 2.78 & 21 \\
\hline 2 & 4.0 & 200 & & 15.83 & 0.1511 & 6.06 & 7.41 & 21 \\
\hline $3^{*}$ & 4.0 & 100 & & 8.31 & 0.1511 & 3.18 & 3.7 & 21 \\
\hline 4 & 7.0 & 75 & & 23.084 & 0.2643 & 15.48 & 14.89 & 21 \\
\hline $\begin{array}{ll}5 & 11.5\end{array}$ & 4.0 & 200 & & 19.198 & 0.1511 & 7.35 & 7.41 & 9 \\
\hline 6 & 4.0 & 400 & & 35.82 & 0.1511 & 13.72 & 14.81 & 9 \\
\hline 7 & 4.0 & 600 & & 59.82 & 0.1511 & 22.91 & 22.22 & 9 \\
\hline
\end{tabular}


values of $\mathrm{ND}^{3}$. In Figure IV-19 is plotted the values of A versus $\mathrm{ND}^{3}$. In this plot the scatter is larger than for $Q$. However it is within the scatter of the other points on the plot and thus would be acceptable for correlation. It should be noted that Profile Number 3 was measured by Nielson in corn syrup. This data point is also seen to correlate well confirming the observation made earlier that viscosity is not an important factor in the stirred tank. The correlation IInes given in Figures IV-17 and IV-18 were calculated without using Nielson's and Cutter's data. This was because, the parameters $\sigma$ and a were estimated and not obtained from the measured data.

The analysis presented in this section shows that the tangential jet model successfully predicts the data of Nielson and Cutter.

\subsection{Extent of the Tangential Jet}

Equation II-28 gives $r_{0}$ the extent of the tangential jet as

$$
r_{0}=\frac{\sigma T}{12+2 \sigma}
$$

Substituting for $\sigma$ equal to 12.621 from Equation IV-36 gives

$$
r_{0}=0.339 \mathrm{~T}
$$

In terms of radius of the tank this reduces to

$$
r_{0}=0.678(T / 2)
$$

Equation IV-42 gives the extent of the tangential jet as $67.8 \%$ of the tank radius. This leaves $32.2 \%$ of the tank radius for the return 
flow at the perphery of the tank. Velocity profile measurements in Section 4.3 show this to be true as can be seen in the radial velocity profile for $\bar{v}_{r}$ given in Figure IV-6 and the axial velocity for $\bar{v}_{z}$ given in Figure IV-7. The maximum value of $\bar{v}_{z}$ and $\bar{v}_{r}$ occurs well beyond $r_{0}$ in these figures. This indicates that the bulk of the flow at the periphery of the tank occurs in a region less than $32 \%$ of the radius of the tank.

We can now determine the width of the baffle. The function of the baffle is to interfere with the flow and reduce the tangential component. Since the flow is restricted to the periphery of the tank its position should thus be at the tank periphery. Its width should be the thickness of the return flow which is estimated as maximum of $32 \%$ of the tank radius. In the literature the recommended baffle width is $10 \%$ of the tank diameter, i.e., $20 \%$ of the tank radius. As noted above the flow as it leaves the stagnation region, Region II, is reduced in width and is thus amply covered by a $10 \%$ baffle. This is the reason why at fully baffled conditions, increasing the baffle width or giving it a pitch has very little effect on the flow (36)

\section{Jet Width}

In Appendix B, the jet width was defined on the basis of the point where the velocity profile is less than $1 \%$ of $\bar{q}_{\max }$, and given by

$$
b_{\frac{1}{2}}=\frac{6 r}{\sigma}
$$

The tangential jet model also defines a half width of the jet, and this value of $b_{\frac{1}{2}}$ is given by 


$$
b_{\frac{1}{2}}=\frac{2 r^{2}-a^{2}}{\left(r^{2}-a^{2}\right)^{\frac{1}{2}} k^{\prime}}
$$

Since, a, the radius of the jet source $D / 2$, it is clear that for large $r, I I-12$ reduces to

$$
b_{\frac{1}{6}}=\frac{2 r}{k^{\prime}}
$$

Comparing Equations IV -43 and $B-34$ it follows that

$$
k^{\prime}=\frac{3}{\sigma}
$$

Hence II-12 becomes

$$
b_{\frac{1}{2}}=\frac{3}{\sigma} \frac{\left(2 r^{2}-a^{2}\right)}{\left(r^{2}-a^{2}\right)^{\frac{1}{2}}}
$$

Equation IV-45 and B-34 are thus made identical for large $r$ at the impeller periphery, it was found that Equation IV-45 with $\sigma$ equal to 12.621 and, a, reported in Table IV-21 gave a slightly larger value of $b_{\frac{1}{2}}$ as compared to Equation B-34. As and example, for our data in a 12.25 diameter tank $b_{\frac{1}{2}}$ as calculated from Equation IV-45 is 0.077 feet as compared to 0.067 feet from Equation B-34. 4.12. Summary

In this chapter experimental evidence is presented and interpreted in terms of the mathematical model developed in Chapter II. Measurements were made with a three dimensional pitot tube in the vicinity of the impeller and in the region outside the impeller. It was shown that the tangential jet model adequately describes the flow 
in the region of the impeller. The angle profile is not predicted by the tangential jet model. The model however is relatively insensitive to the angle profile and a weighted average angle was found to give satisfactory results.

The significant velocity in the neighborhood of the impeller centerline was shown to be $\vec{q}$ the resultant of $\bar{v}_{r}$ and $\bar{v}_{\theta}$. It was also shown that the three dimensional pitot tube is not sensitive enough to make measurements beyond the point where $\vec{q}=\frac{1}{2} \bar{q}_{\max }$. This was because for $z$ larger than that corresponding to $\frac{1}{2} \bar{q}_{\max }$ the velocity falls rapidly to less than $30 \mathrm{ft} / \mathrm{min}$. This velocity is the lower limit of probe response. It was also found that the contribution to volumetric flow beyond this point is small. However for completeness it is desirable to obtain measurements in this region.

The readings from the three dimensional pitot tube was shown to be dependent on the flow geometry. It was pointed out by Rao (24) that the readings given by the pitot tube in a turbulent flow field are subject to error. However, these errors if any are small being of the order of less than $10 \%$ and could be neglected.

The tangential jet model was successfully fitted to the data taken by Cooper ( 8 ) and to a lesser extent by Nielson (21) and Cutter ( 9 ). In the latter case some of the parameters had to be estimated. The model has three parameters; these are: $\sigma$, the jet width parameter, A the volumetric flow parameter and, $a$, the radius 
of source parameter. It was shown that the results for Cooper and our data for $\sigma$ results in a constant value of $\sigma$. The data analyzed includes measurement made in air and water. The data also spans varying impeller diameter, tank diameter and impeller speeds. This means that for geometrically similar impellers $\sigma$, the jet width parameter is a universäl constant. It follows that in such cases" the width of the jet is also constant. The analysis of Cooper's data for a single impeller and varying blade widths showed that $\sigma$ is dependent on the impeller blade width in the case of impellers that are not geometrically similar.

A correlation with $\mathrm{ND}^{3} /\left((\mathrm{D} / 2)^{2}-\mathrm{a}^{2}\right)^{\frac{1}{4}}$ was found satisfactory for $A$. In the case of the radius of source $a$, it was observed that the tank diameter had a significant effect. Two separate correlations are given for $a$, which are satisfactory for the purpose of interpolating the results analyzed in this work. The data is not enough to make a sweeping statement to cover all possible cases. The results of the correlation was shown to successfully predict the impeller discharge $Q$ and to a lesser extent the eddy viscosity E. The impeller discharge $Q$ was found to correlate satisfactorily with $\mathrm{ND}^{3}$.

It was also found that the dimensionless pumping capacity $\mathrm{N}_{\mathrm{Q}}$ was a constant for geometrically similar impellers. The eddy viscosity as defined by Equaton B-15 was shown to increase with both impeller diameter and impeller speed. However as the impeller discharge $Q$, increased at a much faster rate with increasing speed 
and impeller diameter, the net effect is a low momentum transfer per unit volume of fluid with a larger impeller at constant speed.

In the rest of the tank the model presented in Chapter II was shown to be inadequate. The model in Chapter II was based on a two dimensional potential flow. The measurements with the pitot tube was possible only at high impeller speed. The results of the analysis at three different horizontal planes showed that the flow was a three dimensional low velocity flow field. The stagnation zone shown by Nagata is not a true velocity flow field. The stagnation zone shown by Nagata is not a true stagnation zone, but appears to exist when the streamlines are plotted in the $x-z$ plane.

It was also shown that the return flow of the impeller stream occupies a narrow region at the tank walls which ranges from $32.2 \%$ of the tank radius, at the Impeller centerline and reduces to about $20 \%$ of the tank radius half way between the impeller centerline and the fluid surface. This was the reason for having a baffle width of $10 \%$ of the tank diameter. 


\section{CHAPTER V}

\section{CONCLUSIONS}

In the preceding chapters the stirred tank with a Type A turbine impeller was investigated. The investigation reveals that the study of the stirred tank consists of two parts; first, selecting and evaluating a suitable probe to measure three dimensional velocity profiles and second to arrive at a suitable model to predict the measured flow field. Partial success has been achieved in both these areas.

\subsection{Three-Dimensional Velocity Probe}

A three-dimensional pitot tube probe has been used in this work and found to be a satisfactory device in the region of the impeller. The lower limit of probe response is $0.5 \mathrm{ft} / \mathrm{sec}$ and a maximum pitch angle $\theta_{p}$ of $40^{\circ}$. With this probe near the impeller conterline the predominant velocity was shown to be $\vec{q}$, the resultant of $\bar{v}_{r}$ and $\bar{v}_{\theta}$. The velocity profile $\vec{q}$ is a function of $z$ and $r$ and is symetrical about a line parallel to the impeller centerline and displaced from it by approximately. \pm 0.036 in. An angle profile on $\vec{q}$ was measured and a typical profile is shown in Figure IV-1. The 1imits of measurement of the profile is a little beyond $\bar{z}_{\max }$, at which point the velocity profile decreases rapidly to 1 ess than $0.5 \mathrm{ft} / \mathrm{sec}$. 
The region of the impeller is a high velocity, high shear layer with the important velocities being $\bar{v}_{x}$ and $\bar{v}_{\theta}$. It should be noted that a single 3.0 inch diameter impeller was used in water. With this Impeller the lowest impeller speed for which the probe gave an adequate response was 250 RPM. A lower 1imit of 200 RPM was reported by Cooper ( 8 ). At 200 RPM the Reynolds number is 21,600. The probe is thus suitable for measuring velocities only in fully turbulent flow.

In the region outside the impeller, measurements were made in three horizontal planes at $2.0,3.0$, and 4.0 inches above the Impeller centerline, (the depth of fluid was 12.0 inches and the impeller was centerally located). The probe was found to respond for an impeller speed of $500 \mathrm{RPM}\left(\mathrm{N}_{\mathrm{Re}}=54,000\right)$. At higher impeller speeds excessive air entrainment occured which is not a normal operating condition. The flow was found to be three dimensional and fairly quiescent. The resultant velocity ranged between 30 to $70 \mathrm{ft} / \mathrm{min}$. In the horizontal plane 4.0 inches above the impeller centerline, in some areas the probe could not be used as the pitch angle were larger than $40^{\circ}$. It is thus concluded that in the region outside the impeller, the three dimensional pitot tube probe is a poor measuring device. In this region a probe is required which is not only sensitive to low velocity fields but permits true three dimensional velocity proflle measurements with large pitch angles. A quartz coated cross-wire hot wire anenrometer is recommended for this purpose. Two such probes will be needed. A probe 
with wires crossed in a horizontal plane will permit measuring $\bar{v}_{\boldsymbol{r}}$ and $\bar{v}_{\theta}$, and a second probe with wires crossed in a vertical plane to measure $\bar{v}_{r}$ and $\bar{v}_{z}$. The hot wire anemometer is not recommended for measuring velocities in the region of the impeller since the high shear layer will damage the wires. Other devices such as thermister beads are available for measuring low velocity fields; however these do not have directional properties and hence cannot be used to measure three-dimensional flow fields. 5.2. Flow Model for the Stirred Tank

In modeling the flow field generated by the stirred tank, it was convenient to devide the flow field into two regions. One region is the neighborhood of the impeller modeled by a tangential jet and the other is the rest of the tank modeled by potential flow.

\section{Tangential Jet}

In the region of the impeller the flow was represented by a tangential jet. It consists of a circular source of radius, $a$, smaller than the impeller diameter from which the fluid emerges at a tangent to the periphery of the source. The tangential jet model was first proposed by Nielson (21). The jet is a one dimensional flow in the direction of the tangent to circular source. An analytical solution giving the velocity $\vec{q}$ in explicit form was obtained from solving the equation of motion and using Prandtl's Second Hypothesis as a model for the turbulent shear stress. The resulting equations are

$$
\bar{q}=\frac{A}{2}\left(\frac{\sigma}{r}\right)^{\frac{1}{2}} \frac{1}{\left(r^{2}-a^{2}\right)^{\frac{1}{4}}}\left[1-\tanh ^{2}(\eta / 2)\right]
$$


where $\eta$ is a dimensionless $z$ coordinate and is given by

$$
\eta=\sigma \frac{z-z_{0}}{r}
$$

Equation II-10 is a four parameter model, the parameters are $\sigma$ the jet width, A the volumetric flow, a, the radius of source and $z_{0}$ the jet displacement. The model does not predict the angle profile and a weighted average angle was found to be satisfactory to evaluate, a, the radius of source. The other three parameters were obtained by a nonlinear least square regression analysis, and the fit on the data was excellent. It should be noted that since velocity profiles are measured for a little beyond $\frac{1}{2} q_{\text {max }}$, the model could not be checked for accuracy at the tail ends of the profile.

Cooper (8) had measured velocity profile in water and air using essentially the same probe. His data was found to be compatible with the tangential jet model and the results were in agreement with the data taken by us. Cooper's and our data were thus combined together for the purpose of correlating the results. The combined data covers four geometrically similar type A turbines and three different tank diameters. Cooper's data is for air and water, thus the data includes two different flulds. The Reynolds number $\mathrm{N}_{\mathrm{Re}}$ range is 19,000 to 358,000 . The higher values for $\mathrm{N}_{\mathrm{Re}}$ is for air.

The conclusions from data are:

1. The jet width is a constant, since $\sigma$ was found to be a constant, independent of radial angle, radial distance and height of impeller off tank battom, $h$, The minimum value 
of $h$ recommended is twice the maximum jet width $b_{0}$ and is given by Equation II-28 (since $b_{0}$ is equal to $r_{0}$.)

2. A weighted average angle was found to give a value of $a$, the radius of source, which satisfactorily predicted the measured velocity profile.

3. The volumetric flow parameter, $A$, was found to be a scaled value of the impeller discharge Q.

The conclusions from the combined data of ours and that of Cooper's are:

1. The jet width $\sigma$ was found to be a constant.:. Its value is 12.6: \pm 1.5 . It is thus also independent of tank diameter and the physical properties of the fluid. The independency with tank diameter is not conclusive; however, in the absence of adequate data this analysis indicates it to be approximately true.

2. The radius of source parameter, $a$, was found to be dependent on tank and impeller diameter. Two separate correlations were found that could satisfactorily interpolate the data, one at constant tank diameter and one at constant impeller diameter.

3. The impeller discharge, $Q$, was found to correlate well with $\mathrm{ND}^{3}$, as the correlation coefficient was found to be 0.99 . The equation for $Q$ is

$$
Q=1.075\left(\mathrm{ND}^{3}\right)^{0.942}
$$

4. The volumetric flow parameter $A$ was found to be dependent on the tank diameter it was found to correlate well with 
$N D^{3} /\left((D / 2)^{2}-a^{2}\right)^{\frac{1}{4}}$. The equation for $A$ is

$$
A=1.144\left(\frac{N D^{3}}{\left((D / 2)^{2}-a^{2}\right)^{\frac{1}{4}}}\right)^{0.834}
$$

and 'the correlation coefficient was found to be 0.986 .

5. The extent of the tangential jet was successfully predicted as $34 \%$ of the tank diameter.

6. Cooper also measured velocity profiles for a 4.0 inch diameter impeller with varying blade widths, b. These impellers are not geometrically similar. The jet width $\sigma$ is insensitive to small changes in b but in general is a function of $b$. The radius of source $a$ is strongly dependent on $b$. Finally the impeller discharge $Q$ was found to be proportional to a function of $b$. This means that the functional relationship with $b$ does not interact with that of the impeller speed.

The tangential jet model was also tested on the velocity profile data of Nielson (21) and Cutter (9). This data was obtained by the light streak method and does not show an angle profile, Since geometrically similar impellers were used the results of the correlation were used to estimate $\sigma$. The correlation gave an erroneous value of a, the radius of source, indicating that a is strongly dependent on the tank diameter. The value of a was thus estimated. These estimates of a,gave a satisfactory fit of the velocity profile data, and the results of the analysis was in agreement with Cooper's and our data. 
The tangential jet model gave a value for the eddy viscosity $\epsilon$

as

$$
\epsilon=\frac{A}{2} \frac{1}{\left(\sigma^{3} r\right)^{\frac{1}{2}}} \frac{2 r^{2}-a^{2}}{\left(r^{2}-a^{2}\right)^{3 / 4}}
$$

The correlations for $\sigma, A$ and a was found to give a satisfactory prediction for the eddy viscosity and impeller discharge $Q$. The correlations for the tangential jet parameters are thus good estimates of these parameters.

The tangential jet model is a satisfactory model for predicting the flow in the region of the impeller. It does not predice the angle profile and is thus not a completely satisfactory model.

\section{Potential Flow}

Outside the impeller region two dimensional potential flow was used to model the flow field. The field was divided into five regions as shown in Figure II-1 and are:

1. Impeller stream impinging on tank wall, modeled by stagnation flow.

2. Upper and lower corners of the tank modeled by potential flow in a corner.

3. Flow at the top and bottom of the tank axis, also modeled by potential flow in a corner.

4. Flow at the center of the tank axis, modeled by a circular jet.

5. Two doughnut shaped regions on either side of the impeller stream modeled as dead water regions. 
A Calcomp plotter was used to draw the flow patterns and is a useful method for obtaining flow patterns.

The agreement between the model and experimental data is poor because of the assumption of two dimensional flow. Velocity profile measurements indicate that a three dimensional flow fleld exists. Comparison of $\bar{v}_{z}$ between model and experimental data show a close resemblance in the shape but not in magnitude of the velocity profile in Regions II and III. There is no agreement between the model and experimental profiles for $\bar{v}_{r}$. It is thus concluded that potential flow is a valid flow model in this region. Since potential flow is a linear model, the inclusion of $\overline{\mathrm{v}}_{\theta}$ in the model would scale the $\bar{v}_{z}$ profile correctly and bring an agreement of the profile for $\bar{v}_{r}$. No attempt was made to test this theory as adequate data could not be taken with the three dimensional probe:

In Region $V$ the circular jet model was shown to be a poor representation of the velocity field. This model illustrates that the agreement in flow pattern by streamlines need not necessary give an agreement in velocity profiles as well.

The flow outside the impeller was shown to be a maximum at the walls of the tank. The width of this stream was shown to be the width of the $10 \%$ baffle used. This clearly shows that the width and location of the baffle was correctly determined for the purpose of modifying the flow.

\section{$\underline{\text { Recommendation }}$}

The following are the recommendations based on the evaluation 
and analysis of the experimental data presented in this dissertation, and the data available in the literature.

1. Hot wire anemometer is recommended to measure flow fields outside the impeller region.

2. Measurements of velocity profiles are needed near the boundary of the tangential jet region to test the validity of the model in this region. If the model is inadequate, it is not a serious error since this region can be modeled with Region VI, and the tangential jet could be used to model the present range of the velocity profile.

3. Velocity profile measurements are needed with varying tank diameters to adequately predict the radius of source a, and the effect if any on $\sigma$.

4. Exhaustive velocity profile measurements with at least one fluid other than air or water are needed to test conclusively the independence of the tangential jet parameters on the fluid used.

5. In the region outside the impeller a potential flow model is recommended. Since potential flow is a linear model it would be valid to subtract $\bar{v}_{\theta}$ from the resultant velocity $\vec{v}$ of the three dimensional flow field. $\bar{v}_{\theta}$ could be then modeled seperately as a vortex in combination with a solid rotating cylinder at the impeller axis. This model is suggested from the velocity profile for $\bar{v}_{\theta}$ given in Figure IV-9. The remaining two velocity components $\bar{v}_{r}$ and $\bar{v}_{z}$ could be handled as a two dimensional flow field as done in this work. 


\section{NOMENCLATURE}

In the case of vectors and tensors, the symbols given in this nomenclature represents their magnitudes. Vectors and Tensors are distinguished in the text by overmarks and are given below under the heading overmarks,

Symbol

A

a

$\therefore$

b

$b_{\frac{1}{2}}$

$\mathrm{c}_{1}$

c

D

d

$d_{1}$

E

\section{Description}

Volumetric flow parameter

Parameter in Equation I-10

Radius of source of the tangential jet

Constant Equation I-1

Parameter Equation I-10

Width of impeller blade. (Figure I-2)

Width of jet (Equation I-9)

Half width of the jet (Equation B-32)

Constant (Equation I-25)

Mixing length constant (Equation I-5)

Constant (Equation B-3)

D Impeller diameter

Constant (Equation I-9)

Diameter of Type A Impeller disc

(Figure I-2)

Voltage
Units

$f t^{3} / \mathrm{min}$

ft.

none

none

in.

ft.

ft.

none

none

ft.

none

in.

volts 


\begin{tabular}{|c|c|c|}
\hline$E_{c}$ & $\begin{array}{l}\text { Fraction of momentum transferred, } \\
\text { Equation IV-23 }\end{array}$ & none \\
\hline$e^{\prime}$ & Fluctuating component of $\mathrm{E}$ & volts \\
\hline $\mathbf{F}$ & Function defined by Equation $B-9$ & none \\
\hline $\mathbf{f}$ & Function defined by Equation B-5 & none \\
\hline$g_{c}$ & $\begin{array}{l}\text { Standard acceleration of gravity } \\
\left(32.174 \mathrm{ft} / \mathrm{sec}^{2}\right)\end{array}$ & $\mathrm{ft} / \mathrm{sec}^{2}$ \\
\hline \multirow[t]{2}{*}{$\mathrm{H}$} & Depth of fluid in tank & ft. \\
\hline & $\begin{array}{l}\text { Also used as length of column of mano- } \\
\text { meter fluid in manometer calculations }\end{array}$ & ins. \\
\hline $\mathrm{h}$ & Height of impeller of tank bottom & ins. \\
\hline$h_{1}$ & $\begin{array}{l}\text { Height of blade above impeller plane } \\
\text { (see Figure I-2) }\end{array}$ & ins.: \\
\hline $\mathrm{J}$ & Momentum & $\left(1 b_{m}\right)(f t) / s e c$ \\
\hline $\mathrm{k}$ & Constant & \\
\hline$k^{\prime}$ & $\begin{array}{l}\text { Constant defined by Equation IV }-44 \\
\text { Constant, Equation I-33 }\end{array}$ & \\
\hline 1 & Length of impeller blade (Figure I-2) & in. \\
\hline $\mathbf{N}$ & Impe1ler speed & RPM \\
\hline $\mathrm{N}_{P}$ & Power number $\mathrm{Pg}_{c} / \mathrm{N}^{3} \mathrm{D}^{5}$ & none \\
\hline $\mathrm{N}_{\mathrm{Q}}$ & $\begin{array}{l}\text { Dimensionless pumping capacity } \\
\mathrm{Q} / \mathrm{ND}^{3}\end{array}$ & none \\
\hline $\mathrm{N}_{\mathrm{Re}}$ & $\begin{array}{l}\text { Reynolds number for stirred tanks } \\
\rho \mathrm{ND}^{2} / \mu\end{array}$ & none \\
\hline $\mathbf{P}$ & Power & $\left(I b_{f}\right)(f t) / s e c$ \\
\hline & Pressure & $1 b_{f} / \mathrm{ft}^{2}$ \\
\hline$P_{t}$ & Total Pressure & $1 b_{f} / f t^{2}$ \\
\hline$P_{8}$ & Stagnation pressure & $1 b_{f} / f t^{2}$ \\
\hline
\end{tabular}




\begin{tabular}{|c|c|c|}
\hline Q & Volumetric flow & $\mathrm{ft}^{3} / \mathrm{min}$ \\
\hline$Q_{0}$ & $\begin{array}{l}\text { Volumetric flow increment on a stream- } \\
\text { line }\end{array}$ & $\mathrm{ft}^{3} / \mathrm{min}$ \\
\hline $\overrightarrow{\mathrm{q}}$ & Velocity, resultant of $v_{r}, v_{\theta}$ & $\mathrm{ft} / \mathrm{min}$ \\
\hline$q$ & $\begin{array}{l}\text { Relative slip in velocity between the } \\
\text { fluid and impeller at impeller periphery, } \\
\text { Equation I-27 }\end{array}$ & none \\
\hline$r, \theta, z$ & Lengths, in cylinderical coordinates & ft. \\
\hline \multirow[t]{2}{*}{$\mathbf{R}$} & Impeller radius & ft. \\
\hline & $\begin{array}{l}\text { Correlation Coefficient defined by Equation } \\
\text { IV-1 }\end{array}$ & none \\
\hline$x_{0}$ & Extent of tangential jet model & ft. \\
\hline \multirow[t]{2}{*}{$r_{c}$} & $\begin{array}{l}\text { Starting coordinate of a streamline } \\
\text { in Region I. Also } r \text { coordinate of } \\
\text { center of circulation (Figure I-3) }\end{array}$ & ft. \\
\hline & $\begin{array}{l}\text { Dimensionless coordinate for velocity } \\
\text { profile defined by Equation I- } 6\end{array}$ & none \\
\hline$s_{x}^{2}$ & Variance (see Equation IV-4) & none \\
\hline s & Dimensionless $z$ coordinate, (Equation $I-6$ ) & none \\
\hline$T$ & Tank diameter & ft. \\
\hline $\mathbf{t}$ & Boundary layer thickness (Figure II-6) & ft. \\
\hline $0.95,(\mathrm{~N}-1)$ & $\begin{array}{l}\text { t-test parameter } 0.95 \text { probability with } \\
\text { (N01) degrees of freedom }\end{array}$ & none \\
\hline $\mathrm{V}$ & Velocity & $\mathrm{ft} / \mathrm{min}$ \\
\hline$v_{r}, v_{\theta}, v_{z}$ & $\begin{array}{l}\text { Velocity components, cylinderical } \\
\text { coordinates }\end{array}$ & $\mathrm{ft} / \mathrm{min}$ \\
\hline$x, v_{y}, v_{z}$ & Velocity components, cartesian coordinates & $\mathrm{ft} / \mathrm{min}$ \\
\hline W & Velocity, resultant of $v_{z}$ and $v_{\theta}$ & $\mathrm{ft} / \mathrm{min}$ \\
\hline$w$ & Angular velocity & radians/sec \\
\hline
\end{tabular}


$x, y, z \quad$ Lengths, in cartesian coordinates ft

$x_{n}, y_{n} \quad$ Local coordinates of boundary point ft.

between two regions, defined in

Section 2.3

zo Displacement of tangential jet profile ft.

from impeller centerline

$z$ coordinate of center of circulation

(Figure I-3)

Displacement of circular jet relative

to tank bottom

Greek

$\alpha$

Anglè

degrees

Pressure ratio defined by Equation IV-15

none

Circular jet width parameter, defined by

Equation II-19

$\Delta \mathrm{H}$. Pressure drop in inches of manometer fluid

$\Delta \mathrm{H}_{\mathrm{s}} \quad$ Static head

in.

Eddy viscosity, tangential jet

in.

$\epsilon$

Eddy viscosity, circular jet

$f t^{2} / \min$

$\epsilon_{0}$

$\eta$

Dimensionless velocity profile caordinate for tangential jet defined by Equation

II-13

$\theta$

Angle

degrees

$\mu \quad$ Viscosity

$1 b_{m} /(f t)(s e c)$

$\xi$

Dimensionless velocity profile coordinate

for circular jet defined by Equation II-19

none

Density

$1 b_{m} /(f t)^{3}$

Tangential jet width parameter defined by Equation II-13

none 


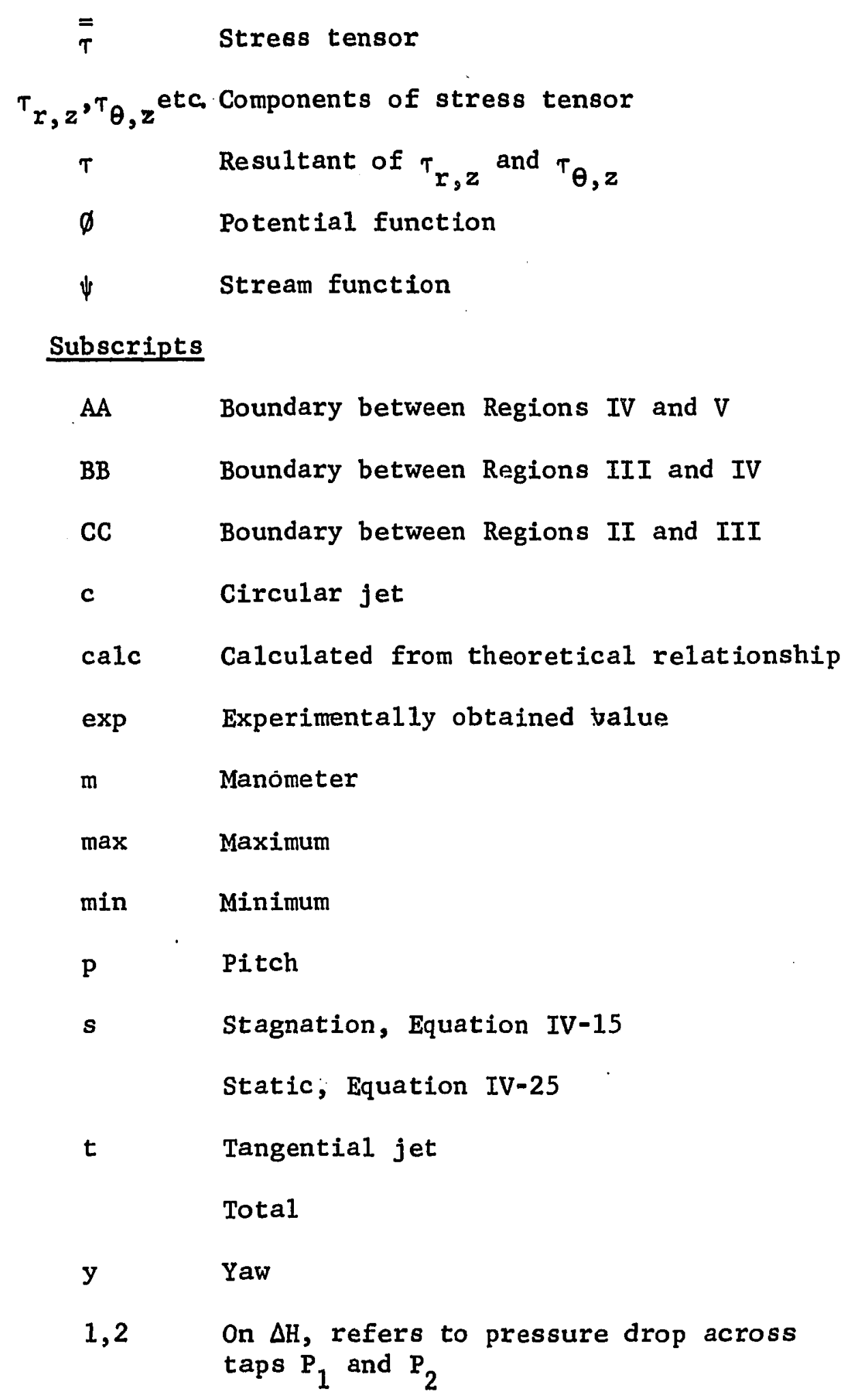

Super Scripts

Derivative

- Angular measurement or temperature in degrees

$1 b_{f} / f t^{2}$

$1 b_{f} / f t^{2}$

$1 b_{f} / f t^{2}$

$\mathrm{ft}^{3} / \mathrm{min}$ 
- Fluctuating component

Overmarks

- Time average quantity

$\begin{array}{ll}\rightarrow & \text { Vector } \\ = & \text { Tensor }\end{array}$

List of FøRTRAN Symbols Used in Text

A A, volumetric flow parameter

AA a, radius of source parameter

ANGLE $\bar{\theta}_{\mathrm{y}}$, weighted average angle

AVERAGE Arithmetic average angle

AVG Subroutine for calculating averages (Lișt F-1)

AVG ANGLE ' Arithmetic average of $\theta_{y}$

BOUNDS Subroutine required by PATERN (List $\mathrm{F}-1$ )

BCALC $\quad z$ coordinate corresponding to $\frac{1}{2} q_{\max }$ obtained by interpolating

experimental data

BHALF $\quad z$ coordinate conresponding to $\frac{1}{\mathrm{max}}$ from Equation $\mathrm{H}-1$

CFM Volumetric flow at $r$, calculated from Equation B-31

Impeller dischange calculated from Equation IV-1

$\cos (\mathrm{PHI}) \quad \cos \bar{\theta}_{\mathrm{y}}$

CORR COEF Correlation coefficient defined by Equation IV-1

COOPER Main program, to analysis Cooper's data (List F-5) 


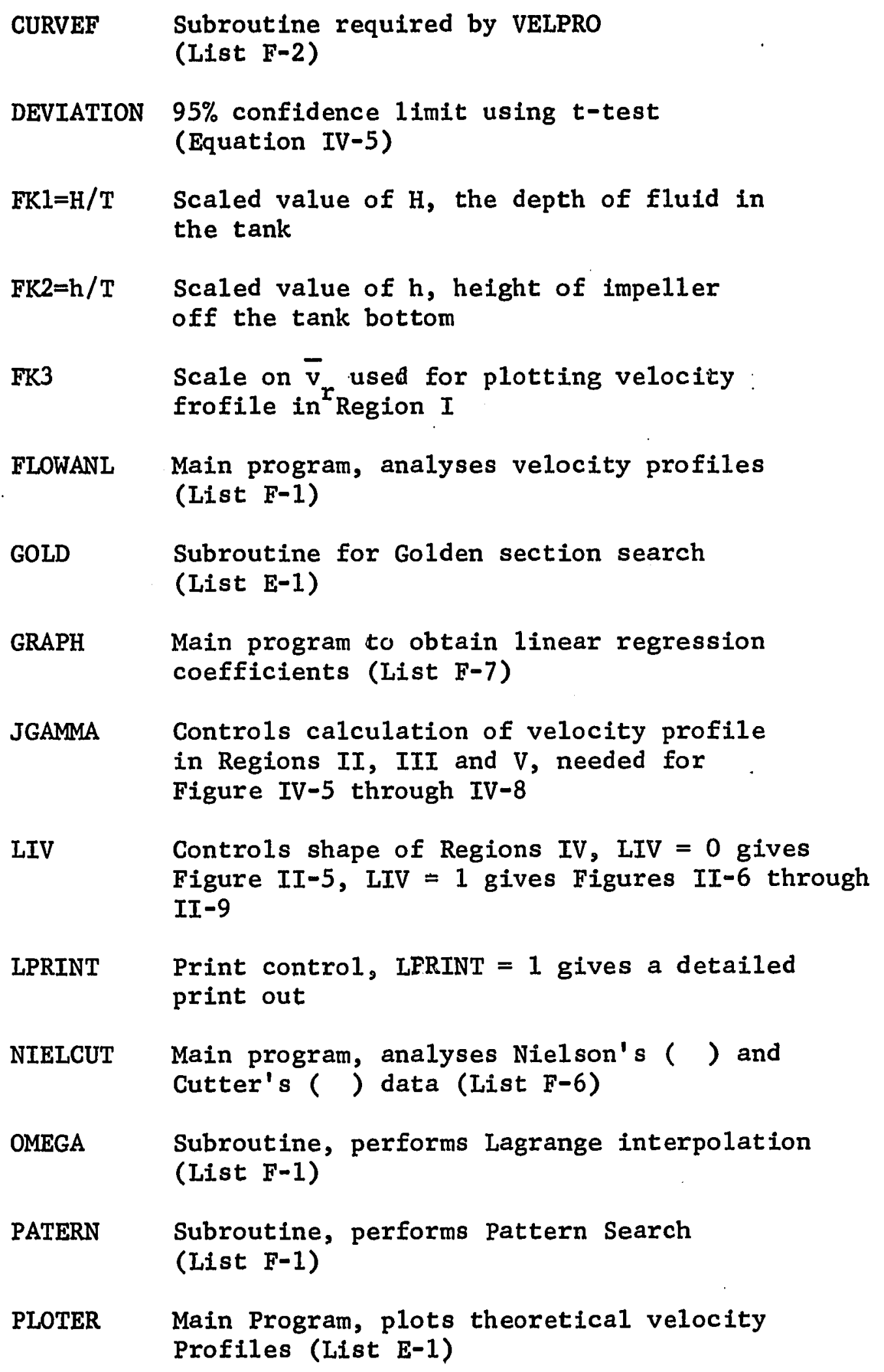


PROC Subroutine, minimization criteria function for PATERN (List $\mathrm{F}-1$ )

POLY Subroutine, performs polynomial least square fit (List $\mathrm{F}-2$ )

PX, PY Array, stores $\mathrm{r}$ and $\mathrm{z}$ coordinates of a complete streamline

$P(4) \quad z$, displacement parameter of tangential jet

RRX

Stores $r$ coordinate of velocity profile analyses from theoretical streamlines of Regions II, III and V

SOLVE Subroutine, needed by GRAPH (List F-6)

SPRINT Subroutin, needed by PLOTER (List E-1)

SS Sum of Squares about predicted line defined by Equation $\mathrm{C}-2$

STREAM Subroutine, calculates coordinates of a streamline (List $\mathrm{E}-1$ )

SY Stores values of $\psi$ in velocity profile analysis from theoretical streamlines of Regions II, I.II and V

T Tank diameter, ft.

TANKANL Main program, analyses three dimensional flow data from Pitot tube in the region outside the impeller (List $\mathrm{F}-3$ )

$\mathrm{TB}=\mathrm{FK} 2 * \mathrm{~T}$ Height of impeller of tank bottom

$\mathrm{TF}$

Effect depth of fluid in potential flow region above or below impeller centerline (see Figure IV-6)

$\mathrm{TL}=(\mathrm{FK} 1-\mathrm{KF} 2) * \mathrm{~T}$

Depth of fluid above impeller centerline 


\begin{tabular}{|c|c|}
\hline UHALE & $\frac{1}{2} q_{\max }$ \\
\hline VARIANCE & Variance, $s_{x}^{2}$ (Equation $I V-4$ ) \\
\hline VR, VZ & $\begin{array}{l}\text { Stores } \bar{v}_{r} \text { and } \bar{v}_{\text {of velocity profile. }} \text { of } \\
\text { analysis from theoretical streamlines } \\
\text { in Regions. II, III and IV }\end{array}$ \\
\hline WT & Velocity factor (EquationlIV-3) \\
\hline$X, Y$ & Local frame of axis \\
\hline $\mathrm{XN}$ & Extent of tangential jet solution $x_{0}$ \\
\hline $\mathrm{XN2}$ & $\begin{array}{l}\text { Parameter, determined thickness of } \\
\text { boundary layer given by XN } 2 * \mathrm{~TB}\end{array}$ \\
\hline $\mathrm{XN} 3$ & $\begin{array}{l}\text { Parameter, determines boundary between } \\
\text { Regions IV and } V \text {. }\end{array}$ \\
\hline $\operatorname{xin} 4$ & $\begin{array}{l}\text { Parameter, determines boundary between } \\
\text { Regions II and III }\end{array}$ \\
\hline $\mathrm{XNF}$ & $\begin{array}{l}\text { Parameter, determines boundary between } \\
\text { Regions III and IV }\end{array}$ \\
\hline YAW & $\begin{array}{l}\text { Subroutin, calculates results from } \\
\text { manometer D (List } F^{-} 1 \text { ) }\end{array}$ \\
\hline YAWANL & $\begin{array}{l}\text { Main program, analyses three dimensional } \\
\text { flow data from Pitot tube in the region } \\
\text { of the impeller (List } F-4 \text { ) }\end{array}$ \\
\hline
\end{tabular}




\section{BIBLIOGRAPHY'}

1. Aiba, S. "Flow Patterns of Liquids in Agitated Vessels", A.I.Ch.E. J. , 4 , 485 (1958).

2. Askew, Waren S., Beckman, Robert B., "Velocity Profiles and Transfer Operations at the Wall of an Agitated Vesse1", I \& EC Process Design and Development, 5 (3), 268 (1966).

3. Bates, Robert L., Fondy, Philip L., Corpstein, Robert R., "An Examination of Some Geometric Parameters of Impeller Power", Ind., Eng. Chem., Process Design and Development, 2, 310 (1963).

4. Bowers, R.H., "An Investigation of Flow Phenomena in Stirred Liquids", A.T.Ch.E. - T. Chem. E., Symposium Series 10, London (1965).

5. Bird, R. B., Stewart, W. E., Lightfoot, F. N., Transport Phenomena, John Wiley and Sons Inc., New York, p. 159 (1962).

6. Ibid, p. 83,85 .

7. Camps, Jorge A., Mixing Model and Measurements of Heptane-Sulfuric Acid Emulsions in a Continuous Flow Stirred Tank Reactor, M.S. Thesis, Louisiana State University (1968).

8. Cooper, R. G., Velocity Profiles and Pumping Capacities of Turbine Type Impel lers, M. S. Thesis, McGill University (1966).

9. Cutter, L. A., Flow and Turbulence in a Stirred Tank, Ph.D. Thesis, Columbia University (1960).

10. Dell'osso, Luino Jr., Turbulence Measurement in Water in an Open Channel with the Hot-film Anemometer, Ph. D. Thesis, Rice University (1966).

11. Goldstein, R. J., Kreid, D. K., "Measurement of Laminar Flow Development in a Square Duct Using a Laser-Doppler Flowmeter", J. Applied Mech., Trans. of ASME, Paper No. 67, APM-37, (1967).

12. Holland, F. A., Chapman F. S., Liquid Mixing and Processing in Stirred Tanks, Reinhold Publishing Corporation, New York (1966).

13. Holmes, D. B., Voncken, R. M., Dekker, J. A., "Fluid Flow in Turbinestirred, Baffled Tanks", Chem. Eng. Sc. 19, 201. (1964). 
14. Larson, Paul Z., Flow Patterns and Velocity Profiles in an Agitated Vesse1, Ph. D. Thesis, University of Delaware (1965).

15. Milne-Thompson, I.M., Theoretical Hydrodynamics, 5th Edition $(1968)$, p. 351 , Macmillian, New York.

16. Metzner, A. B.., Taylar, J. S., "Flow Patterns in Agitated Vessels," A.I.Ch.E.J., $\underline{6}$ (1), 109 (1960).

17. Mujumdar, A.A. Huang, B., Wolf, D., Weber, M. E., Douglas, W. J. M., "Turbulence Parameters in a.Stirred Tank", Preprint 25f, Chemical Engineering Conference, Triportite Meeting, Montreal Canada (Sept. 22-25, 1968).

18. Nagata, S., Yamamoto, K., Ujihara, M., "Flow Patterns of Liquids in a Cylinderical Mixing Vessel without Baffles", Mem. Fac. Eng., Kyoto University, 20, 336 (1958).

19. Nagata, S., Yamamoto, K., Hashimoto, K., Naruse, Y., "Flow Patterns of Liquids in a Cylinderical Mixing Vessel with Baffles", Mem. Fac. Eng., Kyoto University 21, 260 (1959).

20. Nagata, S., Yamamoto, K., Hashimoto, K., Naruse, Y., "Studies on the Flow Patterns of Liquids in a Cylinderical Mixing Vesse1, Over a Wide Range of Reynolds Numbers", Mem. Fac. Eng., Kyoto University 22, 68 (1960).

21. Nielson, Hugo J., Flow and Turbulence form a Flat Blade Turbine Mixing Impeller, Ph. D. Thsis, Illinois Institute of Technology (1958).

22. Norwood, K. W., Metzner, A. W., "Flow Patterns and Mixing Rates In Agitated Vessels", A.I.Ch.E.J. 6, (3), 432 (1960).

23. Oldshue, J..Y., "Fermentation Mixing Scale-up Techniques", Biotechnology and Bioengineering, Vo1. VIII, pp. 3-24, John Wiley and Sons, Inc., New York (1966).

24. Rao, A. M., Turbulence and Mixing in a Continuous Flow Stirred Tank, Ph. D. Thesis, The Ohio State University (1969).

25. Rolfe, E., Silk, J. K., Booth, S., Melster, K., Young, R. M., Laser Doppler Velocity Instrument, NASA CR-1199 (Dec. 1968).

26. Rushton, J. H., Costich, E. W., Everett, H.J., "Power Characteristics of Mixing Impellers", Chem. Eng. Prog. 46,(395) 467 (1950).

27. Rushton, J. H., Oldshue, J.Ỵ., "Mixing of Liquids", Chem. Eng. Prog. 55 (25), 181 (1959). 
28. Sachs, J.P., Rushton, J.H., "Discharge Flow from Turbine-Type Mixing Impellers", Chem. Eng. Prog., 50, (12), 597 (1954).

29. Schlichtling, H., Boundary Layer Theory, 4 th Edition, p. 482 McGrw-Hi11 Book Co, Inc., New York, (1960).

30. Ibid. p. 604-609.

31. Ibid. p. 182,607 .

32. Schumm, Jr., Brooke, A Mathematical Model for Wake-Type Fluid Flow from Turbine-Tube Impellers, Ph. D. Thesis, University of Rochester (1966).

33. Sterbacek, Z., Tausk, P., "Mixing in the Chemical Industry," Permagon Press Inc., New York (1965).

34. Uh1, V. W., Grey, J. B., Mixing, Theory and Practice, Vols. I and II, Academic Press, New York (1966).

35. Ibid., Vol I, p. 132 .

36. Ibid., Vol I, p. 137, 157.

37. Ibid., Vol I, p. 181 .

38. Ibid., Vo1 I, p. 187-200.

39. Ibid., Vo1 I, p. 207.

40. Ibid., Vo1 I, p. 209 .

41. Van de Vusse, J. G., "Mixing by Agitation of Miscible Liquids", Chem. Eng. Sc. 4, 178, 209 (1955).

42. Wilde, D. J., Optimum Seeking Methods, Prentice-Ha11, Inc., New Jersey $(1964)$.

43. Yaun, S. W., Foundations of Fluid Mechanics, Prentice-Hal Inc. New Jersey, p. 74, (1967).

44. Ibid., p. 158 .

45. Ibid., p. 200 .

46. Ibid., p. 207, 208.

47. United Sensor and Control Corporation, 85 School Street, WaterTown, Massachusetts 02172. Calibration Data Sheet for USC-1086, probe type Da-187-24-F-22-CD, Seria1 No. A-402. 
APPENDIX A

\section{TANGENTIAL OR RING JET}

\section{Derivation of Differential Equation}

This appendix will derive the equations that describe the motion of the tangential or ring jet. The approach used, is to simplify the time averaged continuity equation and equation of motion subject to geometric constraints and observations. on the behavior of the flow. The time averaged equation of continuity and equation of motion are used to describe the energy dissapation due to turbulence.

The flow in the jet is similar to boundary layer flow in that both flows have high velocity gradients perpendicular to the direction of flow. The turbulence in the jet is free turbulence and will be modeled by using Prandt1's Second Hypothesis. The assumptions and constraints that apply to the tangential jet are:

1. Pressure gradients are negligible. The only pressure gradient that exists is in the $z$-direction due to gravity and does not influence the flow of the fluid.

2. Axial symmetry about $z$, axis; hence $\bar{v}_{r}, \bar{v}_{\theta}, \bar{v}_{z}$ are independant of $\theta$.

3. Steady State.

4. Incompressible isothermal flow, hence the density $\rho$ is constant. 5. $\bar{v}_{r}, \bar{v}_{\theta}$ are of the same order of magnitude, $\bar{v}_{z}$ is of a lower 
order. This will be recognized as the boundary layer approximation. Furthermore, the velocity gradient in the $z$ direction is larger than in the r., and $\theta$ directions. This is because the flow is in the $r, \theta$ plane and in the $r$ direction. The flow does not change rapidly with increasing $r$. However, the change in the velocity profile in the $\mathrm{z}$ direction is comparatively larger. The time averaged equation of continuity in vector form (5) for an incompressible fluid and steady state conditions is

$$
\vec{\nabla} \cdot \vec{V}=0
$$

The velocity $\vec{V}$ is the resultant of $v_{r}, v_{\theta}$, and $v_{z}$. The arrow over $v$ signified that it is a.vector. The bar over $\bar{v}_{r}$ for example, indicates that it is a time averaged velocity. Writing. $A-1$ in cylinderical coordinates,

$$
\frac{i}{r} \frac{\partial}{\partial r}\left(r \bar{v}_{r}\right)+\frac{1}{r} \frac{\partial \bar{v}_{\theta}}{\partial \theta}+\frac{\partial \bar{v}_{z}}{\partial z}=0
$$

The second term in equation $A-2$ is zero from Assumption Two, rearranging $A-2$ gives

$$
\frac{\partial}{\partial r}\left(r \vec{v}_{r}\right)+\frac{\partial}{\partial z}\left(r \bar{v}_{z}\right)=0
$$

Equation $A-3$ is the continuity equation for the tangential jet.

The time averaged equation of motion in vector form (5) for an incompressible fluid is

$$
\rho \frac{\mathrm{D} \vec{V}}{\mathrm{Dt}}=-\vec{\nabla} \cdot \overrightarrow{\mathrm{P}} \cdot[\vec{\nabla} \cdot \bar{\tau}(1)]-\left[\vec{\nabla} \cdot \frac{\bar{\tau}}{(t)}\right]+\rho \overrightarrow{\mathrm{g}}
$$

where, $\overline{\bar{T}}(1)$ and $\overline{\bar{T}}(t)$ are the laminar and turbulent momentum fluxes. In fully turbulent flow. $\bar{\tau}(t)$ is much larger than $\bar{\tau}(1)$, hence $\bar{\tau}^{(1)}$ can be neglected in comparison. In future discussion the superscript on 
$\overline{\bar{T}}$ will be dropped, since it is clear that $\bar{\tau}$ refers to $\overline{\bar{\tau}}(t)$. From Assumption one, the pressure $\bar{p}$ is constant hence $A-4$ reduces to

$$
\rho \frac{\mathrm{DV}}{\mathrm{Dt}}=-\vec{\nabla} \cdot \overline{\bar{\tau}}+\rho \overrightarrow{\mathrm{g}}
$$

Equation A-5 can be simplified further by writing it in cylinderical coordinates. The r-component is (6) given by,

$$
\begin{aligned}
& \rho\left(\frac{\partial \bar{v}_{r}}{\partial t}+\bar{v}_{r} \frac{\partial \bar{v}_{r}}{\partial r}+\frac{\bar{v}_{\theta}}{r} \frac{\partial \bar{v}_{r}}{\partial \theta}-\frac{v_{\theta}^{2}}{r}+v_{z} \frac{\partial \bar{v}_{r}}{\partial z}=\right. \\
& \left.-\left(\frac{1}{r} \frac{\partial}{\partial r}\left(r r_{r r}\right)+\frac{1}{r} \frac{\partial \tau}{r \theta} \frac{\tau_{\theta \theta}}{\partial \theta}+\frac{\partial \tau r z}{\partial z}\right)\right)
\end{aligned}
$$

In equation A-6 the first term on the left hand side is zero because of steady state and the third term is zero because of axial symmetry. From the boundary layer assumption, Assumption Five, it follows that $\tau_{r z}$ is much larger in comparison with $\tau_{r r}$ and $\tau_{r \theta}$, and hence the terms containing these quantities can be deleted from the equation. In the light of the above remarks A-6 reduces to

$$
\bar{v}_{r} \frac{\partial \bar{v}_{r}}{\partial r}-\frac{\bar{v}_{\theta}{ }^{2}}{r}+\bar{v}_{z} \frac{\partial \bar{v}_{r}}{\partial z}=-\frac{1}{\rho} \frac{\partial \tau}{\partial z}
$$

The $\theta$-component of equation A-5 is

$$
\begin{aligned}
& \rho\left(\frac{\partial \bar{v}_{\theta}}{\partial t}+\bar{v}_{r} \frac{\partial \bar{v}_{\theta}}{\partial r}+\frac{\bar{v}_{\theta}}{r} \frac{\partial \bar{v}_{\theta}}{\partial \theta}+\frac{\bar{v}_{r} \bar{v}_{\theta}}{r}+\bar{v}_{z} \frac{\partial \bar{v}_{\theta}}{\partial z}\right)= \\
& -\frac{1}{\rho}\left(\frac{1}{r^{2}} \frac{\partial}{\partial r}\left(r^{2} \tau_{r \theta}\right)+\frac{1}{r} \frac{\partial \tau_{\theta \theta}}{\partial \theta}+\frac{\partial \tau_{\theta z}}{\partial z}\right)
\end{aligned}
$$


In equation $A-9$, the first term on the left hand side is zero because of steady state. Since $\bar{v}_{\theta}$ is not a function of $\theta$ the third term is zero. Examining the right had side of A-8 it is seen on using Assumption Five that $\tau_{\theta z}$ is much larger than $\tau_{\theta \theta}$ and $\tau{ }_{\tau \theta}$ and terms containing them could thus be deleted. Equation A-8 thus simplifies to

$$
\bar{v}_{r} \frac{\partial \bar{v}_{\theta}}{\partial \theta}+\frac{\bar{v}_{r} \bar{v}_{\theta}}{r}+\bar{v}_{z} \frac{\partial v_{z}}{\partial z}=-\frac{1}{\rho} \frac{\partial \tau_{\theta z}}{\partial z}
$$

The $z$-component of $A-5$ is given by

$$
\begin{aligned}
& \rho\left(\frac{\partial \bar{v}_{z}}{\partial t}+\bar{v}_{r} \frac{\partial \bar{v}_{z}}{\partial r}+\frac{\bar{v}_{\theta}}{r} \frac{\partial \bar{v}_{z}}{\partial r}+\bar{v}_{z} \frac{\partial \bar{v}_{z}}{\partial z}\right)= \\
& -\frac{1}{\rho}\left(\frac{1}{r} \frac{\partial}{\partial r}\left(r r_{r z}\right)+\frac{1}{r} \frac{\partial \tau_{\theta z}}{\partial \theta}+\frac{\partial \tau}{\partial z}\right)+\rho g_{z}-\frac{\partial p}{\partial z}
\end{aligned}
$$

In equation A-10 al1 terms on the left hand side are small being functions of $\bar{v}_{z}$ and hence can be neglected from Assumption Five. The turbulent shear stresses are also functions of $\bar{v}_{z}$ and can be neglected. The two extra terms have been included in Equation A-10. These are the static pressure $P$ and the acceleration due to gravity. These terms are a result of Assumption One. Equation A-10 thus simplifies to

$$
-\frac{\partial p}{\partial z}+\rho g=0
$$

For convenience, the continuity equation and the equation of motion which have been simplified above are rewritten below. 


$$
\begin{aligned}
& \frac{\partial}{\partial r}\left(r \bar{v}_{r}\right)+\frac{\partial}{\partial z}\left(r \bar{v}_{z}\right)=0 \\
& \bar{v}_{r} \frac{\partial \bar{v}_{\theta}}{\partial r}+\bar{v}_{z} \frac{\partial \bar{v}_{r}}{\partial z}-\frac{\bar{v}_{\theta} 2}{r}=-\frac{1}{\rho} \frac{\partial \tau}{\partial z} \\
& \bar{v}_{r} \frac{\partial \bar{v}_{\theta}}{\partial r}+\bar{v}_{z} \frac{\partial \bar{v}_{\theta}}{\partial z}+\frac{\bar{v}_{r} \bar{v}_{\theta}}{r}=-\frac{1}{\rho} \frac{\partial \tau_{\theta z}}{\partial z} \\
& -\frac{\partial P}{\partial z}+\rho g=0
\end{aligned}
$$

The above equations can also be used to describe axiallysymmetric two dimensional boundary layer flows. These equations are partial differential equations and as such cannot be solved directly. Further simplification is possible when the tangential jet is examined. In Figure $A-1$ is shown the tangential jet. 0 is the center of the tangential source of radius a and height $2 b$. The fluid flows out of the source in a direction which is a tangent to the periphery of the source. When the fluid leaves the periphery such as from a point $A$ in figure $A-1$ it has a magnitude, $\vec{q}$, and a fixed direction. Consider any arbitrary point $B$. The angle which the velocity of the fluid makes with the radius vector is $\theta_{y^{\circ}}$ In chapter $1 \mathrm{~V}$, this was referred to as a yaw angle. It is easily seen that $\theta_{y}=90^{\circ}$ at $r=a$ and decreases as $r$ increases. The radial and tangetial components are $\bar{v}_{r}$ and $\bar{v}_{\theta}$ and are shown in the figure. From the geometry of Figure $A-1$ it is clear that

$$
\tan \theta_{y}=\frac{\bar{v}_{\theta}}{\bar{v}_{r}}
$$




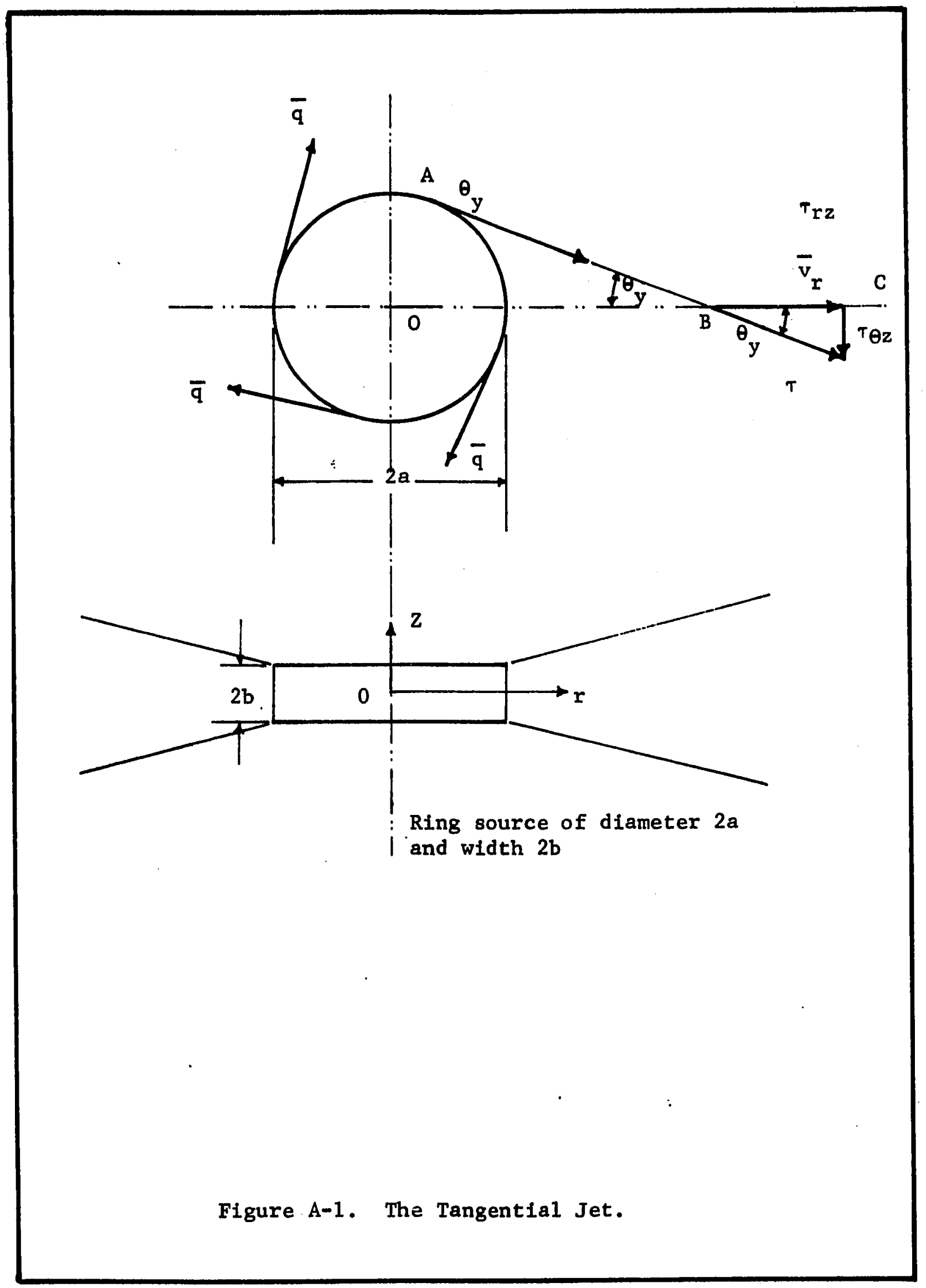


Since $O B=r$ and $O A=a$, from the geometry of the figure it follows that

$$
\begin{aligned}
& \tan \theta_{y}=\frac{a}{\sqrt{r^{2}-a^{2}}} \\
& \cos \theta_{y}=\frac{\sqrt{r^{2}-a^{2}}}{r} \\
& \sin \theta_{y}=\frac{a}{r}
\end{aligned}
$$

In the equations of motion, $A-7$ and $A-9$, the process of simplification using Assumptions One to Five have reduced the stress tensor $\overline{\bar{\tau}}$ from nine components to two, $\tau_{r z}$ and $\tau_{\theta z}$. The stress tensor has thus been reduced to a vector $\vec{\tau}$. From Figure $A-1$ it is seen that the tangetial jet is clearly a one dimension flow in the direction of $\vec{q}$. The shear stress $\vec{\tau}$ is thus a result of the flow $\vec{q}$ and hence $\tau_{r z}$ and $T_{\theta z}$ are components of $\vec{T}$, as shown in Figure $A-1$.

$$
\begin{aligned}
& \tau_{\mathrm{rz}}=|\vec{\tau}| \cos \theta_{\mathrm{y}} \\
& \tau_{\theta z}=|\vec{\tau}| \sin \theta \mathrm{y}
\end{aligned}
$$

Substituting for $\operatorname{Cos} \theta_{y}$ from $A-14$ and $\sin \theta_{y}$ from $A-15$ gives 


$$
\tau_{r z}=\frac{\sqrt{r^{2}-a^{2}}}{r}|\vec{T}|
$$

and

$$
\tau_{\theta z}=\frac{r}{a}|\vec{\tau}|
$$

From the geometry of Figure $A-1$ it follows that

$$
\begin{aligned}
& \bar{v}_{r}=|\vec{q}| \cos \theta_{y} \\
& \bar{v}_{\theta}=|\vec{q}| \sin \theta_{y}
\end{aligned}
$$

We can now show that the two equations of motion, A-7 and A-9 are identical, reducing to a single equation. This is done as follows. Substituting for $\tau_{r z}$ from Equation $A-18, \bar{v}_{\theta}$ from $A-21$ into $A-7$ and simplifying gives 
$\bar{v}_{r} \frac{\partial \bar{v}_{r}}{\partial r}+\bar{v}_{z} \frac{\partial \bar{v}_{r}}{\partial z}-\frac{a^{2}}{r\left(r^{2}-a^{2}\right.} \quad \bar{v}_{r}^{2}=-\frac{1}{p} \frac{\sqrt{r^{2}-a^{2}}}{r} \frac{\partial|\vec{\tau}|}{\partial z}$

Similar1y substituting ${ }^{\top} \theta_{z}$ from $A-19$ and $\bar{v}_{\theta}$ from A-21 into A-9 and simplifying gives

$\bar{v}_{r} \frac{\partial \bar{v}_{r}}{\partial r}+\bar{v}_{r} 2\left[\frac{1}{r}-\frac{r}{r^{2}-a^{2}}\right]+\bar{v}_{z} \frac{\partial \bar{v}_{r}}{\partial z}=\frac{1}{\rho} \frac{\sqrt{r^{2}-a^{2}}}{r} \frac{\partial|\vec{\tau}|}{\partial z}$

On examining equations $\mathrm{A}-22$ and $\mathrm{A}-23$ we see they are identical. We thus have a single equation of motion, which is expected since the problem is a one dimensional flow field. The above analysis for a tangential jet was first obtained by Nielson (21).

Momentum of Tangential Jet.

Equation of motion $A-22$, the continuity equation $A-3$ and the boundary conditions are sufficient to obtain a solution for the tangential jet. An analytic solution will be obtained using the similarity principle. However in order to do this the momentum in the jet has to be evaluated. This is done by first considering the following identity from calculus.

$$
\bar{v}_{z} \frac{\partial \bar{v}_{r}}{\partial z}=\frac{\partial\left(\bar{v}_{r} \bar{v}_{z}\right)}{\partial z}-\bar{v}_{r} \frac{\partial \bar{v}_{z}}{\partial z}
$$


Introducing the continuity equation $A-3$ into $A-23$,

$$
\bar{v}_{z} \frac{\partial \bar{v}_{r}}{\partial z}=\frac{\partial\left(\bar{v}_{r} \bar{v}_{z}\right)}{\partial z}+\bar{v}_{r} \frac{\partial \bar{v}_{r}}{\partial r}+\frac{\bar{v}_{r}^{2}}{r}
$$

Combining $A-22$, the equation of motion, and $A-25$ and rearranging,

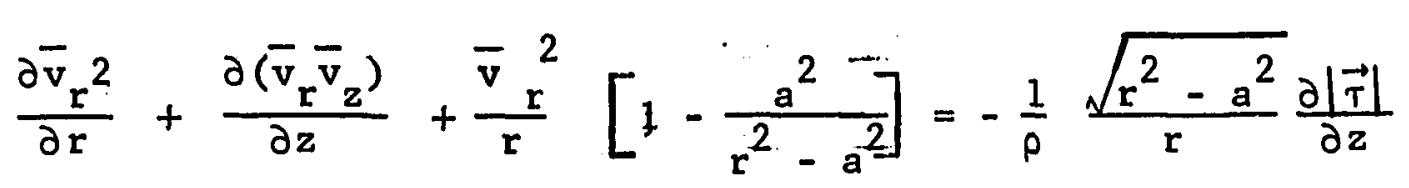

Equation A-26 can be shown to reduce to

$$
\frac{\partial\left(\frac{r^{2} \bar{v}_{r}^{2}}{\sqrt{r^{2}-a^{2}}}\right)}{\partial r}+\frac{r^{2}}{\sqrt{r^{2}-a^{2}}} \frac{\partial\left(\bar{v}_{r} \bar{v}_{z}\right)}{\partial z}=-\frac{r}{\rho} \frac{\partial|\vec{\tau}|}{\partial z}
$$

The boundary conditions for the tangential jet are, at $z= \pm \infty$ the radial velocity is zero and so is the velocity gradient $\frac{\partial \bar{v}_{r}}{\partial z}$. At the centerline of the jet the radial. velocity $\bar{v}_{r}$ is a maximum and $\bar{v}_{z}=0$. These can be expressed mathematically as

$$
z= \pm \infty \quad \bar{v}_{r}=0, \quad \frac{\partial \bar{v}_{r}}{\partial z}=0
$$




$$
z=0 . \quad \frac{\partial \bar{v}_{r}}{\partial z}=0, \quad \bar{v}_{z}=0
$$

Integrating A-27 with respect to $z$ from $-\infty$ to to

$$
\int_{-\infty}^{\infty} \frac{\frac{r^{2} v_{r}^{2}}{\sqrt{r^{2}-a^{2}}}}{\partial r} d z+\frac{r^{2}}{\sqrt{r^{2}-a^{2}}} \bar{v}_{r} \bar{v}_{z}=-\frac{r}{\rho}|\vec{\tau}|
$$

Applying the boundary conditions and noting that $|\vec{\tau}|$ is proportional to $\frac{\partial \vec{q}}{\partial z}$ which quantity is zero at $z= \pm \infty$, we have

$$
\int_{\infty}^{\infty} \frac{r^{2} \cdot \bar{v}_{r^{2}}}{\sqrt{r^{2}-a^{2}}} \frac{d z=0}{\partial r} d z=0
$$

Integrating once again but with respect to $r$ gives,

$$
\frac{r^{2}}{\sqrt{r^{2}-a^{2}}} \int_{-\infty}^{\infty} \bar{v}_{r}^{2} d z=c
$$

Where $c$ is a constant of integration.

The quantity under the integral sign is the momentum. Equation A-32 indicates that the total momentum in the jet is a constant. This should be so, since the only momentum received by the jet is that obtained from the impeller. 


\section{APPENDIX B \\ SIMILARITY SOLUTION FOR A TANGENTIAL JET}

In Appendix A the equation of continuity and motion were developed that describe the flow in the tangential jet. In this appendix, these equations will be solved using the assumption of similarity of velocity profiles. Sachs (28), Aiba (1), and other workers have observed that the flow in a stirred tank can be described by a dimensionless velocity which is independent of impeller speed. This dimensionless velocity is the ratio of actual velocity to impeller tip speed. Cooper ( 8 ) plots $\bar{v}_{r}, \bar{v}_{r \text { max }}$ against. $z$ and finds that for a given turbine impeller this quantity is independent of impeller speed. If $z$ is also normalized by dividing by the width of the jet then the flow profile data was found by Cooper to be independent of impeller size for geometrically similar impellers. These observations suggest that the flow profiles are similar and can be stated mathematically as

$$
\frac{\bar{v}_{r}}{\left(\bar{v}_{r}\right)_{\max }}=f(\eta)
$$

where $\eta$ is given by

$$
\eta=\sigma \frac{z}{r}
$$


The maximum velocity of jet $\left(\bar{v}_{r}\right)_{\max }$ occurs at $z$ equal to zero. The jet spreading parameter is $\sigma$ and, a large value of $\sigma$ means a narrow jet. In Appendix A, the momentum of the tangential jet was evaluated as

$\frac{r^{2}}{\sqrt{r^{2}-a^{2}}} \int_{-\infty}^{\infty} \frac{-2}{v} d z=c$

where $c$ is a constant.

Substituting for $\bar{v}_{r}$ from $B-1$ and $z$ from $B-2$ in $A-32$ results in

$$
\frac{r^{3}\left(\bar{v}_{r}\right)_{\max }^{2}}{\sigma \sqrt{r^{2}-a^{2}}} \int_{-\infty}^{\infty} f(\eta) d \eta=c
$$

Since $f(\eta)$ is a similar velocity profile independent of the coordinates of the system, the integral must be a constant. Defining $A^{2}$ as

$$
A^{2}=c / \int_{-\infty}^{\infty} f(\eta) d \eta
$$

and introducing $A^{2}$ into $B-3$ gives,

$$
\left(\bar{v}_{r}\right)_{\max }^{2}=\frac{A^{2} \sigma\left(r^{2}-a^{2}\right)^{\frac{1}{2}}}{r^{3}}
$$


or

$$
\left(v_{r}\right)_{\max }=\frac{A \sigma^{\frac{1}{2}}\left(r^{2}-a^{2}\right)^{\frac{1}{4}}}{r^{3 / 2}}
$$

Substituting for $\left(\bar{v}_{\mathbf{r}}\right)_{\max }$ in $B-1$,

$$
\bar{v}_{r}=\frac{A \sigma^{\frac{1}{2}}\left(r^{2}-a^{2}\right)^{\frac{1}{4}}}{r^{3 / 2}} f(\eta)
$$

The velocity $\vec{v}_{r}$ has thus been evaluated in terms of a dimensionless velicity profile $f(\eta)$.

As the tangential jet is a two-dimensional problem, the introduction of the stream function $\$$ into the equation of motion A-22 automatically insures that the continuity equation A-3 is satisfied. The appropriate form of $\psi$ in cylinderical coordinates (46) is

$$
\begin{aligned}
& \bar{v}_{r}=\frac{1}{r} \frac{\partial \psi}{\partial z} \\
& \bar{v}_{z}=-\frac{1}{r} \frac{\partial \psi}{\partial r}
\end{aligned}
$$

From B-6 it follows that

$$
\psi=\int_{0}^{z} r \bar{v}_{r} d z
$$

Substituting for $\bar{v}_{r}$ and $z$ from $B-4$ and $B-2$ 


$$
\psi=A\left(\frac{r}{\sigma}\right)^{\frac{1}{2}}\left(r^{2}-a^{2}\right)^{\frac{1}{4}} \int_{0}^{\eta} f(\eta) d \eta
$$

if we let

$$
F(\eta)=\int_{0}^{\eta} f(\eta) d \eta
$$

then,

$$
\psi=A\left(\frac{r}{\dot{\sigma}}\right)^{\frac{1}{2}}\left(r^{2}-a^{2}\right)^{\frac{1}{4}} F(\eta)
$$

From B-6, B-7, and B-9 the following quantities can be evaluated in terms of the function $F$ and its derivatives

$$
\begin{aligned}
& \bar{v}_{r}=\frac{A \sigma^{\frac{1}{2}}}{r^{3 / 2}}\left(r^{2}-a^{2}\right)^{\frac{1}{4}} F^{\prime} \\
& \bar{v}_{z}=-\frac{A\left(r^{2}-a^{2}\right)^{\frac{1}{4}}}{2 \sigma^{\frac{1}{2}} \cdot r^{3 / 2}}\left(F+\frac{r^{2}}{r^{2}-a^{2}} F-2 \eta F^{\prime}\right)
\end{aligned}
$$

$$
\frac{\partial \bar{v}_{r}}{\partial r}=\frac{A}{2} \frac{\sigma^{\frac{1}{2}}}{r^{5 / 2}} \quad\left(r^{2}-a^{2}\right)^{\frac{1}{4}}\left[-3 F+\frac{r^{2}}{r^{2}-a^{2}} \quad F^{\prime}-2 \eta F^{\prime \prime}\right]
$$

$$
\frac{\partial \bar{v}_{r}}{\partial z}=A \frac{\sigma^{3 / 2}}{r^{5 / 2}}\left(r^{2}-a^{2}\right)^{\frac{1}{4}} F^{\prime \prime}
$$




$$
\frac{\partial^{2} \bar{v}_{r}}{\partial z}=A \frac{\sigma^{5 / 2}}{r^{7 / 2}}\left(r^{2}-a^{2}\right)^{\frac{1}{4}} F^{\prime \prime \prime}
$$

In the above equations $F$ is the same as $F(\eta)$ and $F^{\prime}=\frac{\partial F}{\partial \eta}, F^{\prime \prime}=\frac{\partial^{2} F}{\partial \eta^{2}}$ etc. All quantities of Equation II-9, the equation of motion, have been evaluated above in terms of the dimensionless quantity $F(\eta)$. Equation II-9 is reproduced here for convenience.

$$
\bar{v}_{r} \frac{\partial \bar{v}_{r}}{\partial r}+\bar{v}_{z} \frac{\partial \bar{v}_{r}}{\partial z}-\frac{a^{2}}{r\left(r^{2}-a^{2}\right)} \quad \bar{v}_{r}^{2}=-\epsilon \frac{\partial^{2} \bar{v}_{r}}{\partial z^{2}}
$$

Introducing equations $\mathrm{B}-10$ to $\mathrm{B}-14$ into II-9 and rearranging gives

$$
\begin{aligned}
& \frac{A^{2}}{2} \frac{\sigma}{r^{4}}\left(r^{2}-a^{2}\right)^{\frac{1}{2}} F^{\prime}\left(-3 F^{\prime}+\frac{r}{r^{2}-a^{2}} F^{\prime}-2 \eta F^{\prime \prime}\right) \\
& -\frac{A^{2} \sigma}{r^{4}}\left(r^{2}-a^{2}\right)^{\frac{1}{2}} \frac{a^{2}}{r^{2}-a^{2}}\left(F^{\prime}\right)^{2} \\
& -\frac{A^{2} \sigma}{2 r^{4}}\left(r^{2}-a^{2}\right)^{\frac{1}{2}} F^{\prime \prime}\left(F+\frac{r^{2}}{r^{2}-a^{2}} F-2 \eta F^{\prime}\right) \\
& =-A \epsilon\left(r^{2}-a^{2}\right)^{\frac{1}{4}} \frac{1}{r^{7 / 2}} \sigma^{5 / 2} F^{\prime \prime \prime}
\end{aligned}
$$


Dividing through by $\frac{A^{2} \sigma}{2 x^{4}}\left(x^{2}-a^{2}\right)^{\frac{1}{2}}$, and simplifying we get

$$
\left(F^{\prime}\right)^{2}+F F^{\prime \prime}+\frac{2 \epsilon}{A} \frac{\left(r^{2}-a^{2}\right)^{3 / 4}}{\left(2 r^{2}-a^{2}\right)}\left(\sigma^{3} r\right)^{\frac{1}{2}} F^{\prime \prime \prime}=0
$$

Since $\epsilon, A$, $a$ and $\sigma$ are arbitrary constants to be set by boundary conditions, there is no loss in generality, if in the above third order differential equation we let

$$
\epsilon=\frac{A}{2} \frac{1}{\left(\sigma^{3} r\right)^{\frac{1}{2}}} \frac{2 r^{2}-a^{2}}{\left(r^{2}-a^{2}\right)^{3 / 4}}
$$

We still have three independent constants $A, \sigma$ and a which will be determined by three boundary conditions of the resulting third order differential equation.

$$
\mathrm{F}^{\prime 2}+\mathrm{FF}^{\prime \prime}+\mathrm{F}^{\prime \prime \prime}=0
$$

The boundary conditions are given by Equation A-28 and A-29 of Appendix $A$ and is reproduced here for convenience.

$$
\begin{aligned}
& z= \pm \infty \quad \bar{v}_{r}=0, \frac{\partial \bar{v}_{r}}{\partial z}=0 \\
& z=0 \quad \frac{\partial \bar{v}_{r}}{\partial z}=0, \quad \bar{v}_{z}=0
\end{aligned}
$$

From Equations B-2 and B-10 to B-14, it can be easily shown that the boundary conditions A-28 and A-29 transform to

$$
\begin{aligned}
& F(0)=F^{\prime \prime}(0)=0 \\
& F^{\prime}(\infty)=0
\end{aligned}
$$

Equation B-16 can be rearranged to 


$$
\frac{d}{d \eta}\left(F F^{\prime}\right)+\frac{d}{d \eta}\left(F^{\prime \prime}\right)=0
$$

which on integrating and applying boundary condition $B-17$ reduces to

$$
F F^{\prime}+F^{\prime \prime}=0
$$

Integrating once more results in

$$
\frac{7}{2} F^{2}+F^{\prime}=C
$$

Since $\sigma, A$ and $a$ are independent constants to be determined from experimental data, the constant $C$ in $B-20$ can be given any value without loss of generality. Let $C=\frac{1}{2}$, this is equivalent to saying $F(\infty)=1$. Hence,

$$
F^{\prime}=\frac{1}{2}\left(1-F^{2}\right)
$$

The above equation integrates to yield

$$
F=\tanh (\eta / 2)
$$

The constant of integration is zero since $F(0)=0$. We can now find $\bar{v}_{r}$ and $\bar{v}_{z}$ from $B-10$ and $B-11$ since the value of $F$ is now known.

$$
\begin{aligned}
& \bar{v}_{r}=\frac{A}{2}\left(\frac{\dot{\sigma}}{r^{3}}\right)^{\frac{1}{2}}\left(r^{2}-a^{2}\right)^{\frac{1}{4}}\left[1-\tanh ^{2}\left(\frac{\eta}{2}\right)\right] \\
& \bar{v}_{z}=-\frac{A\left(r^{2}-a^{2}\right)^{\frac{1}{2}}}{2 \sigma^{\frac{1}{2}} r^{3 / 2}} \quad \frac{2 r^{2}-a^{2}}{r^{2}-a^{2}} \tanh \left(\frac{\eta}{2}\right)-\eta\left(1-\tanh ^{2}\left(\frac{\eta}{2}\right)\right)
\end{aligned}
$$

Since at $\eta=0, \tanh (\eta)=0, \bar{v}_{r}$ has a maximum value at $\eta=0$. B-22 can hence be written as

$$
\bar{v}_{r}=\left(\bar{v}_{r}\right)_{\max }\left[1-\tanh ^{2}\left(\frac{\eta}{2}\right)\right]
$$

where 


$$
\left(\bar{v}_{r}\right)_{\max }=\frac{A}{2}\left(\frac{\sigma}{r^{3}}\right)^{\frac{1}{2}}\left(r^{2}-a^{2}\right)^{\frac{1}{4}}
$$

Equation B-23 can also be written as

$$
\bar{v}_{z}=-\frac{\left(\bar{v}_{r}\right)_{\max }}{\sigma}\left(\frac{2 \dot{r}^{2}-a^{2}}{r^{2}-a^{2}} \tanh (\eta)-\eta\left(1-\tanh ^{2}\left(\frac{\eta}{2}\right)\right)\right)
$$

Properties of the Tangential Jet

In setting up a computer program to draw the streamlines in the stirred tank it is necessary to evaluate some of the properties of the tangential jet. These properties will be evaluated in this section.

\section{(1) Pumping Capacity}

The pumping capacity is the net flow of fluid out of the impeller. Consider a cylinderical surface of radius $r$ ( $r$ larger than $D / 2$ ) and of infinite length, surrounding the impeller. Then the net amount of fluid that flows out of this surface can be obtained by integrating the velocity normal to the surface. Hence $Q$ the pumping capacity or the volume of fluid flowing out of the cylinderical surface of radius $r$ is

$$
Q=\int_{-\infty}^{\infty} 2 \pi r \bar{v}_{r} d z
$$

Since $\bar{v}_{r}$ is an even function and a function of $z$ only,

$$
Q=4 \pi r \int_{0}^{\infty} \bar{v}_{r} d z
$$

Substituting for $\bar{v}_{\mathrm{r}}$ from $\mathrm{B}-22$ and $\mathrm{z}$ from $\mathrm{B}-2$ gives, 


$$
Q=4 \pi \mathrm{A}\left(\frac{r}{\sigma}\right)^{\frac{1}{2}}\left(\mathrm{r}^{2}-\mathrm{a}^{2}\right)^{\frac{1}{2}} \int_{0}^{\infty}\left(1-\tanh ^{2}\left(\frac{\eta}{2}\right)\right) \mathrm{d}\left(\frac{\eta}{2}\right)
$$

which integrates to,

$$
Q=4 \pi \mathrm{A}\left(\frac{r}{\sigma}\right)^{\frac{1}{2}}\left(\mathrm{r}^{2}-\mathrm{a}^{2}\right)^{\frac{1}{4}} \tanh \left(\frac{\eta}{2}\right)
$$

Equation B-30 gives the flow out of the tangential jet for a given value of $r$ between the limits $\pm \eta$, since the lower limit of $B-29$ is zero. For $\eta=\infty$ we have the total pumping capacity of the jet which is

$$
Q=4 \pi A\left(\frac{r}{\sigma}\right)^{\frac{1}{2}}\left(r^{2}-a^{2}\right)^{\frac{1}{2}}
$$

From $B-31$ it is seen that for $r$, considered far from the source of the jet, $Q$ is approximateiy proportional to $r$.

(2) Half Width of the Tangential Jet

The width of the jet is determined by the boundary of the jet. In order to determine the jet boundaries consider Equation B-24. From the properties of hyperbolic tangents it is seen from B-24 that $\bar{v}_{r}$ rapidly goes to zero for large $\eta$. It can be verified from a table of hyperbolic tangents that when $\eta=6, \tanh ^{2}(\eta / 2)=0.99102$ and hence from $B-24, \bar{v}_{r} /\left(\bar{v}_{r}\right)_{\max }$ is equal to 0.00998 . As in the case of boundary layers we can define the boundary of the jet as the point where $\bar{v}_{r} /\left(\bar{v}_{r}\right)_{\max }$ is less than 0.01 . From the above discussion this is obviously given by

$$
\eta= \pm 6
$$


Substituting for $\eta$ from $B-2$ and rearranging

$$
z=\frac{6 r}{\sigma}
$$

The above value of $z$ is the half width of the jet and to avoid confusion is called $b_{\frac{1}{2}}$, hence

$$
b_{\frac{1}{2}}=\frac{6 r}{\sigma}
$$

From the above discussion it follows that the boundary of the jet is given by

$$
z=b_{\frac{1}{2}}
$$

It should be noted that $b_{\frac{1}{2}}$ as defined by Equation B-34 is not the same as that defined by II-12. In Section 4.11 it is shown that by defining $k^{\prime}$ by Equation IV-44, Equation II-12 and B-34 are identical for values of $r$ of the order of $r_{0}$ or larger. 


\section{APPENDIX C}

NON-LINEAR LEAST SQUARE FIT OF EXPERIMENTAL VELOCITY PROFILE

In this appendix the procedure that will be used to obtain the best values of the parameters of Equation. II-13 from experimental data will.be developed. The best fit of experimental velocity profile data will be considered one in which the least square criteria is satisfied. If $q_{i}$ is the experimentally determined velocities and $\hat{q}_{i}$ is the corresponding velocity obtained from Equation II-10, the sam of squares, SS of the deviation between $q_{i}$ and $q_{i}$ is given by

$$
\text { SS }=\sum_{i=1}^{N}\left(\hat{q}_{i}-q_{i}\right)^{2}
$$

Substituting for $\hat{q}_{i}$ from Equation II-10:

$$
S S=\Sigma\left[\frac{A}{2}\left(\frac{\sigma}{r}\right)^{\frac{1}{2}} \frac{1}{\left(r^{2}-a^{2}\right)^{\frac{1}{2}}}\left(1-\tanh ^{2}\left(\frac{\sigma\left(z_{i}-z_{o}\right)}{r}\right)\right)-q_{i}\right]
$$

In equation $\mathrm{C}-2$ it is understood that the summation of $i$ is from 1 to $n$, where $n$ is the number of experimental observations or data points. The least square criteria requires that the parameters $\sigma, A, a$ and $z_{0}$ be chosen such that $S S$ has a minimum value. Equation C-2 is non-linear with four parameters, one of which has a constraint. 
The constraint is

$$
-\mathrm{D} / 2<\mathrm{a}<\mathrm{D} / 2
$$

An optimization technique know as Pattern Search was used to obtain the parameters of Equation $\mathrm{C}-2$ subject to constraint $\mathrm{C}-3$. The constraint feature of Pattern Search was not needed since the parameter a was evaluated from the weighted average angle $\bar{\theta}_{\mathrm{y}}$ as . described in Chapter IV, Section 4.1. Wilde has given an excellent account of how pattern search works. His comment that D. Himmelblau, of the University of. Texas, found Pattern Search as an excellent least-square minimization technique is confirmed from the innumerable runs that were made using this method.

The least square criteria equation $\mathrm{C}-2$ raises the power of the parameters to at the most two. This is equivalent to generating a surface in multidimension coordinates. Pattern Search treats the parameters of the model as an $n$-dimensional vector $\vec{P}$ that moves from point to point on this least square surface by perturbing $\vec{P} \cdot$ till a minimum is reached. Since in multidimension surfaces several local minimas may. exist, the minimum value obtained by this method (or by any optimization method) will depend on the initial value of $\vec{P}$ selected. Briefly, Pattern Search starts with an arbitrary point $P_{1 j} \cdot$ The first subscript refers to the point under consideration, the second subscrip to the dimension of the vector $\vec{p}$. Let $\Delta p_{i}$ be the amount by which each vector component of $\vec{P}$ (orparameter) will be perturbed. Consider perturbing $\mathrm{P}_{11}$ by $\Delta \mathrm{p}_{i}$. The sum of the square SS (also known as the criteria function) is calculated. If SS decreases then this new value of $P_{11}=P_{11}+\Delta p_{1}$ is accepted. If SS does not 
decrease, $P_{11}$ is perturbed in the negative direction or $P_{11}=P_{11}$ - $\Delta \mathrm{p}_{i}$. If perturbing $\mathrm{p}_{11}$ does not give a decrease in SS, $\mathrm{p}_{11}$ is. left unchanged and the next parameter is perturbed. When all parameters are perturbed point $P_{2 i}$ is reached. The above logic is easily generalized and the algorithim for the i-th parameter is

$$
P_{2 i}=\begin{aligned}
& P_{1 i}+\Delta p_{1} \\
& P_{1 i}-\Delta p i \\
& P_{1 i}
\end{aligned} \quad \text { if }\left(\begin{array}{l}
\operatorname{SS}\left(P_{1 i}+\Delta p_{i}\right)<S S\left(P_{1 i}\right) \\
S S\left(P_{1 i}-\Delta p_{i}\right)<S S\left(P_{1 i}\right) \\
S S\left(P_{1 i}\right)<M i n\left[S S\left(P_{1 i}+\Delta p_{i}\right), S S\left(P_{1 i}-\Delta p_{i}\right)\right]
\end{array}\right)
$$

At point $\mathrm{P}_{2 \mathrm{i}}$ the algorithim $\mathrm{C}-4$ is not repeated but an acceleration step is taken. This is based on the reasoning that the point $P_{2 i}$ is in the direction of a trend or pattern. The point $P_{3 i}$ is obtained by moving a linear distance equal to $P_{1 i} P_{2 i}$ in the direction $P_{1 i}$ to $P_{2 i}$. Vectorially this is given as

$$
P_{3 i}=P_{2 i}+F\left(P_{2 i}-P_{1 i}\right)
$$

The factor $\mathrm{F}$ as indicated above is unity. However if a constraint on $\vec{P}$ is violated, then $F$ is reduced by a suitable amount. In our program this amount is in steps of 0.1 . The minimum value of $F$ is of course zero (i.e. acceleration step is omitted).

In Figure $\mathrm{C}-1$, the above reasoning is illustrated for the two dimensional or two parameter case. $P_{11}$ is perturbed by $-\Delta p_{1}$ to give a decrease in the criteria function SS. A further decrease in 


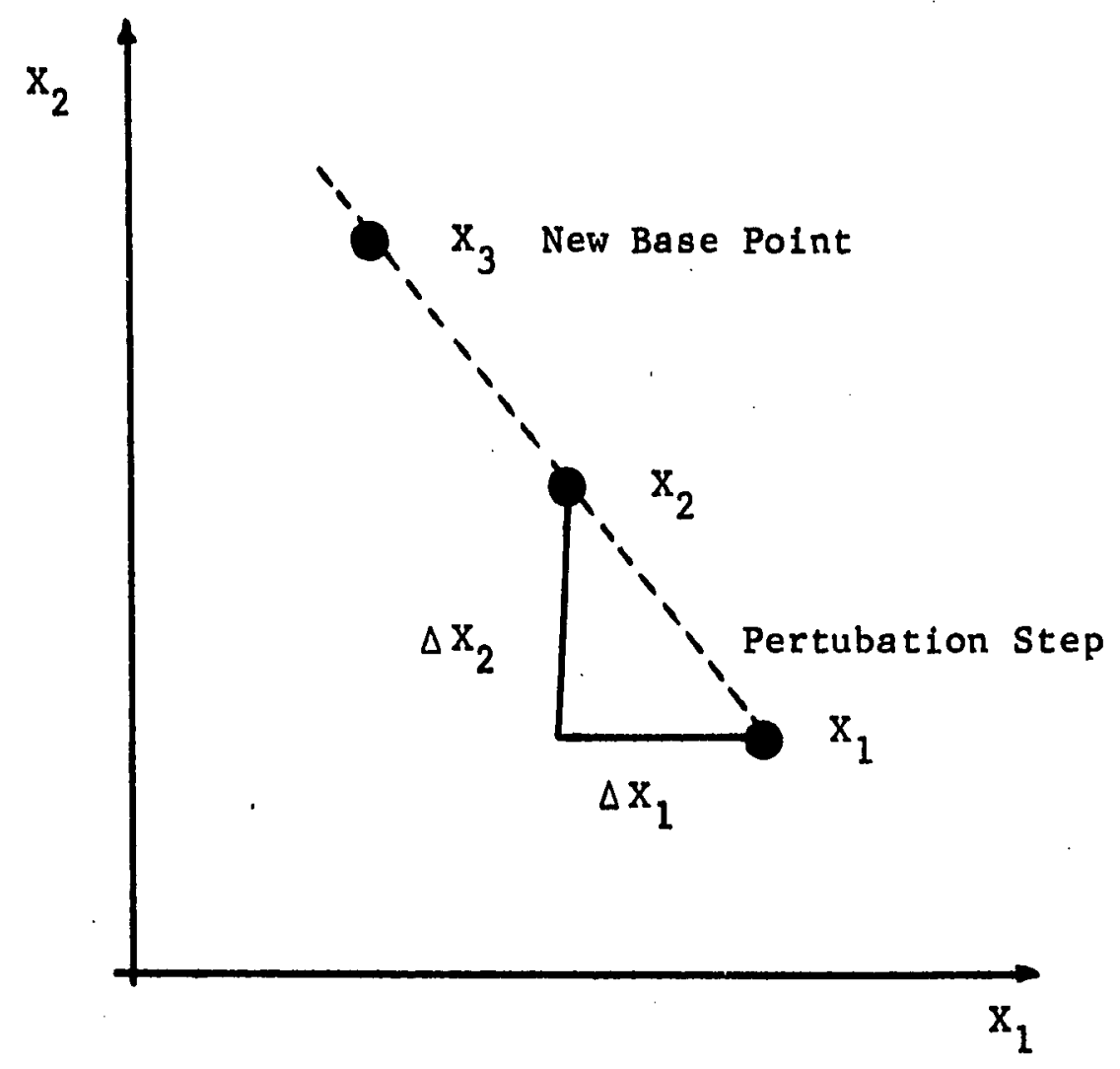

Figure C-1. Pattern Search in Two Dimension. 
sS is obtained by perturbing $\mathrm{P}_{12}$ by $\Delta \mathrm{p}_{2}$. We thus arrive at $\mathrm{P}_{2}$. The acceleration step is then taken to arrive at $\mathrm{P}_{3}$. The procedure is then repeated until no futher decrease in SS is observed.

If the initial step size $\Delta \mathrm{p}_{i}$ are large, the above prodedure will quickly bring the search in the region of the minimum. However for a more precise evaluation of the minimum, the pattern search. prodedure is repeated with a smaller step size. In the program used by us, the initial step size is decreased by a factor of 10 , four times. The number of times is purely arbitrary. In spite of this excessive reduction, the running time for fitting a single velocity profile in no case exceeded 30.0 seconds on the IBM 7040 Computer. This figure does not include the compiling time. The time is also dependant on the initial value selected. After some experience it is not too difficult to select an initial value close to the desired optimum.

In Figure $\mathrm{C}-2$ is presented a flow diagram of the Pattern Search subroutine. The flow diagram outlines a general flow of information. In the program itself several other steps are included so as to make the program work under al1 possible conditions. The Pattern Search subroutine was written as a general optimization program and to do this it requires two other subroutines that depend on the problem being solved. These sub-programs are PROC in which the criteria function, $\mathrm{C}-2$, is specified and BOUNDS in which the constraints, if any are specified. To illustrate the general nature of the program, it. could have been used to evaluate the four parameters using some other criteria. For example to minimize the absolute value of the deviation $\left(\hat{q}_{i}-q_{i}\right)$. The details of the actual program used for evaluating the 
parameters and other pertinent information from experimental data is treated in Chapter IV. 


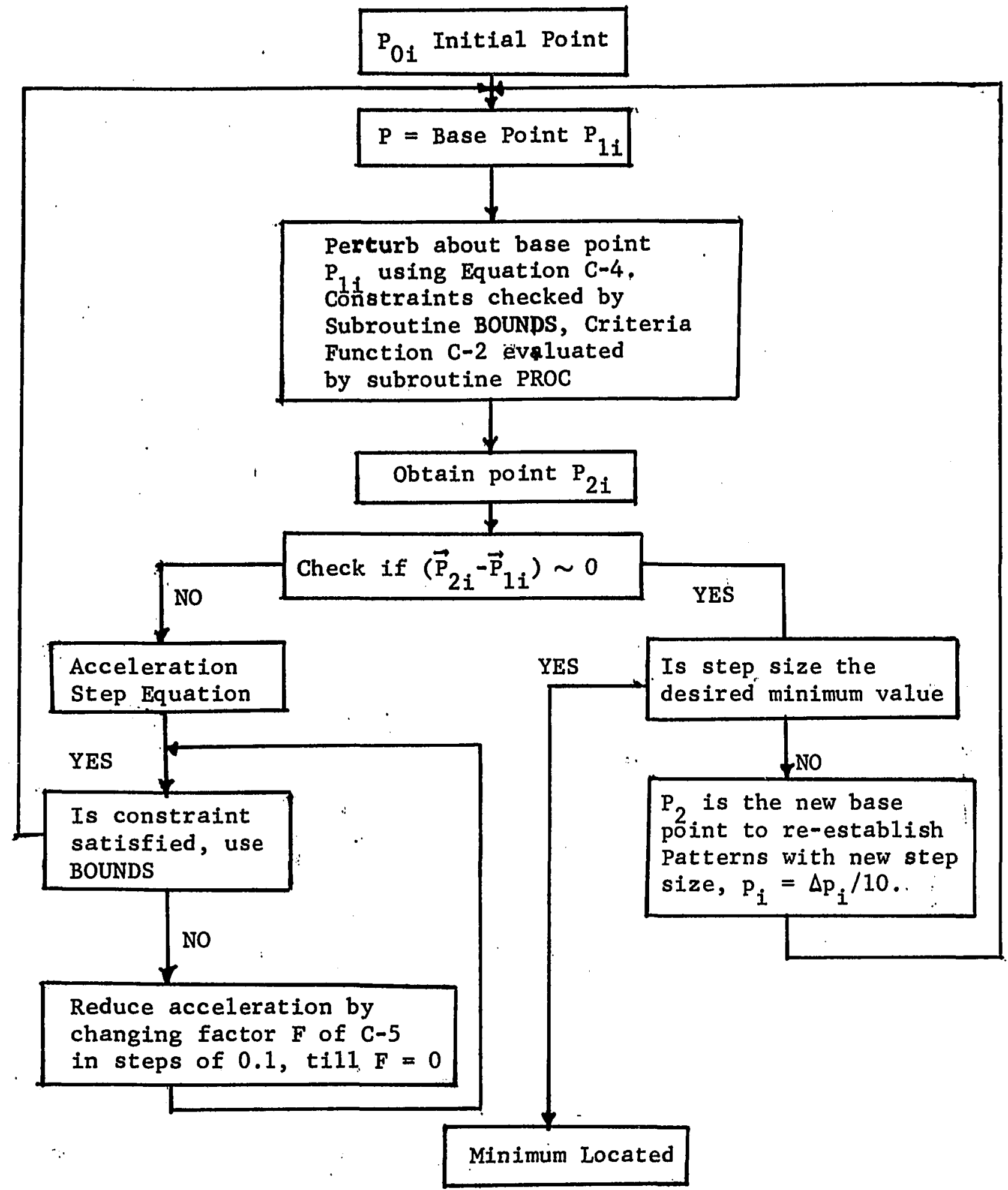

Figure C-2. Flow Diagram for Pattern Search Subroutine. 


\section{APPENDIX D}

TWO DIMENSIONAL POTENTIAL FLOW IN $\mathrm{r}-\mathrm{z}$ PLANE

In this appendix the stream function for two dimensional potential flow in cylinderical coordinates for the $\mathrm{r}-\mathrm{z}$ plane will be derived. In potential flow the föllowing assumptions hold which are: viscous forces are not important, and the flow is irrotational. In the stirred tank the above conditions are approximately true, except in the region of the impeller and at the walls of the tank. In addition the flow. in the stirred tank is in steady state.

The continutty equation thus reduces to,

$$
\vec{\nabla} \cdot \vec{\nabla}=0
$$

Neglecting the viscous forces in Equation $A-4$, the equation of motion reduces to

$$
(\overrightarrow{\mathrm{V}} \cdot \vec{\nabla}) \overrightarrow{\mathrm{V}}=-\frac{1}{\rho} \vec{\nabla} \mathrm{P}+\frac{1}{\rho} \overrightarrow{\mathrm{g}}
$$

In potential flow a potential function $\emptyset$ is defined as,

$$
\vec{V}=\vec{\nabla} \emptyset
$$

The existence of $\emptyset$ stems from the condition of irrotationality Combining $D-1$ and $D-3$ results in the Laplace equation

$$
\nabla^{2} \emptyset=0
$$

The problem reduces to finding a suitable function $\emptyset$ which satisfies Equation D-4 and the boundary conditions. The velocity field can 
then be evaluated from $D-3$, and pressure field from $D-2$.

In the present case it is more convenient to use the stream function $\psi$ than the potential function $\emptyset$. These are related by the following equations (46) in cylindrical coordinates.

$$
\begin{aligned}
& \frac{\partial \emptyset}{\partial z}=\frac{1}{r} \frac{\partial \psi}{\partial r} \\
& \frac{\partial \emptyset}{\partial r}=-\frac{1}{r} \frac{\partial \psi}{\partial z}
\end{aligned}
$$

The stream function is a more useful quantity since it can be used to obtain the streamlines directly. The relationship between velocity and streamlines in the $r-z$ plane is

$$
\begin{aligned}
& \bar{v}_{z}=\frac{1}{r} \frac{\partial \psi}{\partial r} \\
& \bar{v}_{r}=-\frac{1}{r} \frac{\partial \psi}{\partial z}
\end{aligned}
$$

In the literature several solutions to the potential function are available. One such solution in terms of the stream function $\psi$ is (45)

$$
\psi=r^{2} z .
$$

Equation D-9 was chosen because it represents stagnation flow in cylinderical coordinates. This is evident when the streamlines are drawn for Equation D-9 as in Figure II-3(a) that Equation D-9 satisfies the boundary conditions in Region II of Figure II-1.

Since a streamline is a line across which no flow takes place, it can thus be replaced by a solid boundary. In Figure II-3(b) is shown the streamlines for Equation D-9 with OR now a solid boundary. 
The flow is now seen to take place in a conner and could thus represent the flow in Regions III and IV of Figure II-1. 


\section{APPENDIX E}

DESCRIPTION OF PROGRAM PLOTER: USED FOR PLOTTING STREAMLINES

This appendix presents the flow diagram for the computer program PLOTTER. The program is written in FORTRAN IV compatible with the IBM Systems/360. Originally the program was written for the IBM 7040 computer and was converted to the IBM Systems/360 with minor additions. The additions are print statements which are not compatible with the IBM 7040 machine.

Figure E-1 shows a flow diagram for the main program PLOTER, Figure E-2 is the flow diagram for the subroutine STREAM and Figure E-3 is the flow diagram for the subroutine GOLD. In List E-1 is a printout of the entire program with a sample output. The program contains a print control variable LPRINT. A value of LPRINT =' 1 gives detailed printout. In the program given in List E-1, LPRINT $=0$ which gives a minimum printout. The symbols used in the program are explained in comment cards at the beginning of the main program and each subprogram.

Golden Section Search. Golden section is a one dimensional search procedure and is used to locate $r_{c}$ the start of the streamline in Region I. For a detalled analysis of the theory the reader is referred to Wilde (42). The method is well suited for locating single roots of an equation in a known interval. It can also be used 
for locating maxima and minima of functions that have a unique optimia in a given interval. The subroutine GOLD has been written to locate roots in a given interval.

To obtain an idea: of how the method works, consider Figure E-4 (a). In the Figure $\mathrm{X} 1$ and $\mathrm{X} 2$ are the boundaries of the given interval. The points DG1 and DG2 are located by the following equations:

$$
\begin{aligned}
& \mathrm{DG} 1=\mathrm{X} 1+(\mathrm{X} 2-\mathrm{X} 1) / 1.618032 \\
& \mathrm{DG} 2=\mathrm{X} 1+(\mathrm{DG} /-\mathrm{X} 1) / 1.618034
\end{aligned}
$$

In Equation E-1 and E-2, the constant 1.618034 is the Golden section ratio.

The value of the function of $D G 1$ and $D G 2$ are $f(D G 1)$ and $f(D G 2)$. These values are compared with $\mathrm{kQ}_{0}$ the desired root. Since both these values are larger than $\mathrm{kQ}_{0}$ it is obvious that the root does not 1 ie in the interval between $\mathrm{X} 2$ and $\mathrm{DG} 1$. This interval is then eliminated by shifting X2 to DG1 as shown in Figure E-4 (b). The crossed out section indicates intervals eliminated.

New values of DGI and DG2 are calculated using Equations E-1 and E-2. These points are shown in Figure E-4 (b). The quantities of $f(D G 1)$ and $f(D G 2)$ are then computed. These values are compared with $\mathrm{kQ}_{0}$ and found to be on either side of it. It follows then that the root lies within the interval $D G 1$ and DG2. In two iterations the original interval $\mathrm{X} 1, \mathrm{X} 2$, of Figure $\mathrm{E}-4$ (a) have been reduced to DG1, DG2 of Figure E-4 (b). By repeating the above procedure the final interval can be made as small as desired. In our program the final interval is reduced to 0.001 of $(\mathrm{X} 2-\mathrm{Xl})$ the original interval 
in seven iterations. The subroutine GOLD is written in a general manner to cover all possible cases including if the function $Q=f(r)$ is decreasing in the interval $\mathrm{X} 1$ to $\mathrm{X} 2$ rather than increasing as shown in Figure E-1. With slight modifications it could be used to locate an extremum for a unimodal function.

Evaluation of Velocity Profiles. In Chapter IV velocity profiles from the theoretical solution were needed for the purpose of comparison with the experimentally determined profile. These are also obtained from the program PLOTER. Small modifications were made in the subroutine STREAM to permit calculating and storing the values of $\bar{v}_{r}$ and at constant $z$. These values are stored in the arrays VR, SY, VZ, RRX, RVZ. The subroutine SPRINT permits printing the results in a suitable tabular form. This could not be done at the moment of calculation since subrouitne STREAM evaluates one streamline at a time. The fixed point variable JGAMMA controls operation or deletion of velocity profile calculations. To delete this option JGAMMA is set equal to zero as shown in sample list in Table E-1. Sample Printout from PLOTER. At the end of the program is given the input data necessary for plotting Figure II-7 (a). It should be noted that the parameter LIV controls truncation of Region IV. This is because two different calculation procedures are needed to obtain Figure II-7 and Figures II-8 through II-11. To obtain Figure II-7 put LIV equal to zero, otherwise equal to one. Also given in the listing is the sample printout. This printout is the minimum desirable information. If LPRINT, print control parameter is put equal to 1 a detailed printout of practically every step in 
the program will be given. This was found useful in debugging. It should be noted that Figure E-3 showing the flow diagram of subroutine STREAM does not include the details of branching to obtain Figure II-7 as opposed to Figures II-8 through II-11. It also does not include the details of the output to program SPRINT for velocity profile data from the streamlines. Both these changes are minor and can be better seen in the program listing itself where it is clearly pointed out by comment statements.

The printout gives first the print control, a value of less than 5 means program should be recalled for another plot. Next, the input parameters and data are printed. This is followed by a list of important variables that give information about the flow such as Reynolds number RE, eddy viscosity EDVIS etc. Next is given TF, TB etc. defined in Figure II-6 which are overall scaled dimensions of the quadrant being plotted. This is followed by the iteration sequence that determines $\gamma$, and discussed in Section 2.4. A starting value of $\gamma$ equal to 1.87 is selected. With this value of $\gamma$, the $\gamma$ for each streamline is then calculated and 1isted. The numbers below $\gamma$ are the number of points evaluated for each streamline required for plotting. The average value of $\gamma$ for all the streamlines is then taken and called new value of $\gamma$ which is 1.048 . This value is used as a new starting value to calculate another average value of $\gamma$. In two iteration the average value of $\gamma$ has converged tu 1.01 (within $1 \%$ of the previous value) and is used for plotting the streamlines in Region $\mathrm{V}$. 
The next series of print statements is for plotting in the quadrant above the impeller centerline. The iteration sequence for $\gamma$ is given and it should be noted that since the quadrant dimension are different, (see Figure II-7 (a)), the final value of $\gamma$ is also different. 
INPUT: $K$, RHO, RPM, D, POISE, T, $Q$, YL, WB, WL, FK1, FK2, FK3, FAC, XN2, XN3, XN4, GAMMA, FZO. T.T. SIGMA, A, AA. (For interpreting above symbol see comment cards at the beginning of the program.)

CALCULATE: (1) Scale factors for plot DX, DY

(2) $r$, extent of tangential jet

(3) Angle of discharge, $\theta_{y}$

(4) $\epsilon$, eddy viscosity

(5) Reynodls number

(6) Discharge flow from impeller cu $\mathrm{ft} / \mathrm{min}$

(7) Dimensional quantities TE, TL, TB of Figure II-6

Overall scale of plot set by FAC

Draw $X$ and $Y$ axis

Draw Tank outline

Draw Impeller outline

Write label of appropriate quantities

Draw four profiles of tangential jet, first profile at impeller periphery. The rest spaced equally at a distance $1 / 4\left(r_{0}-D / 2\right)$ appart.

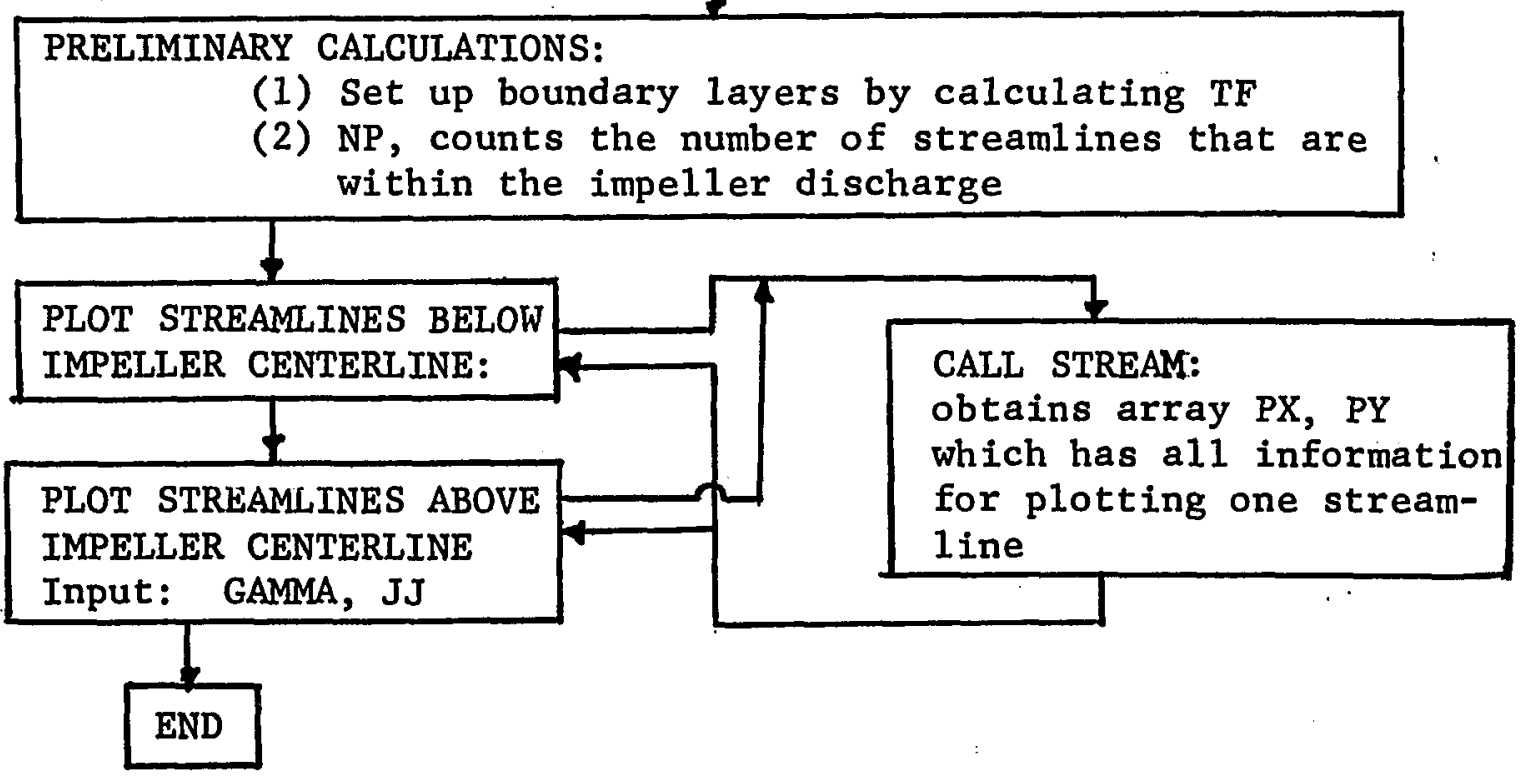

Figure E-1. Flow Diagram for Main Program PLOTER. 


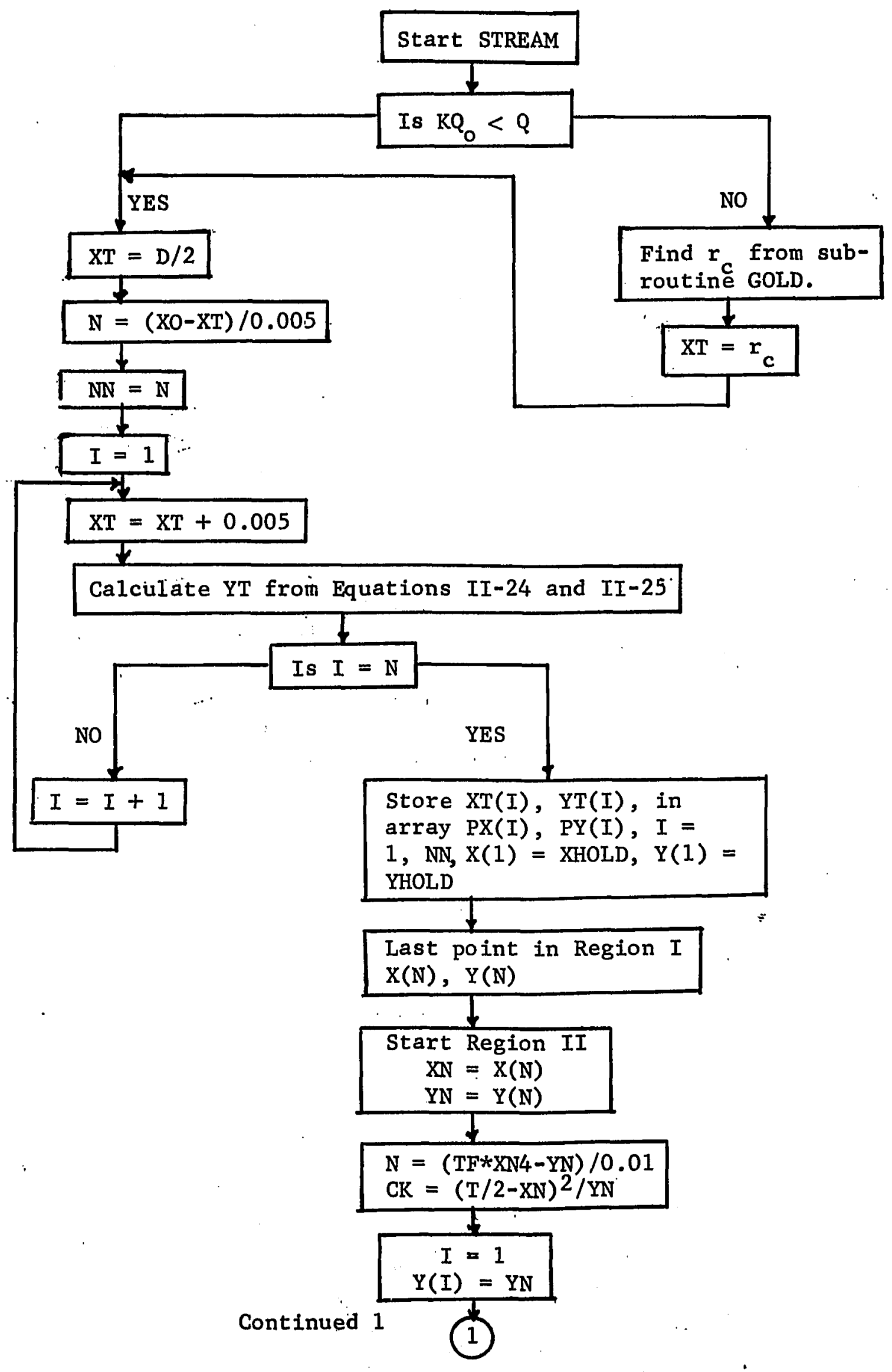




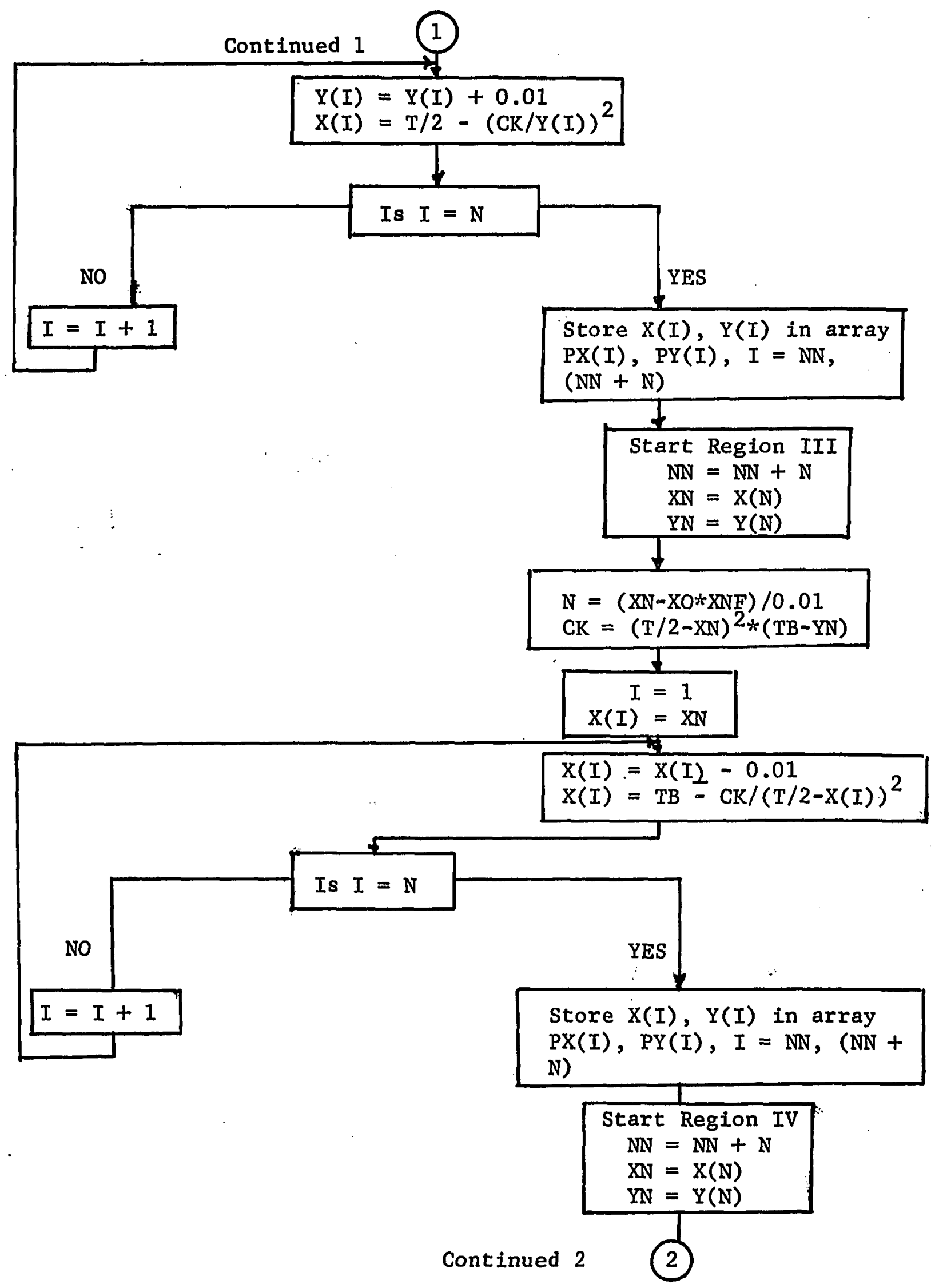




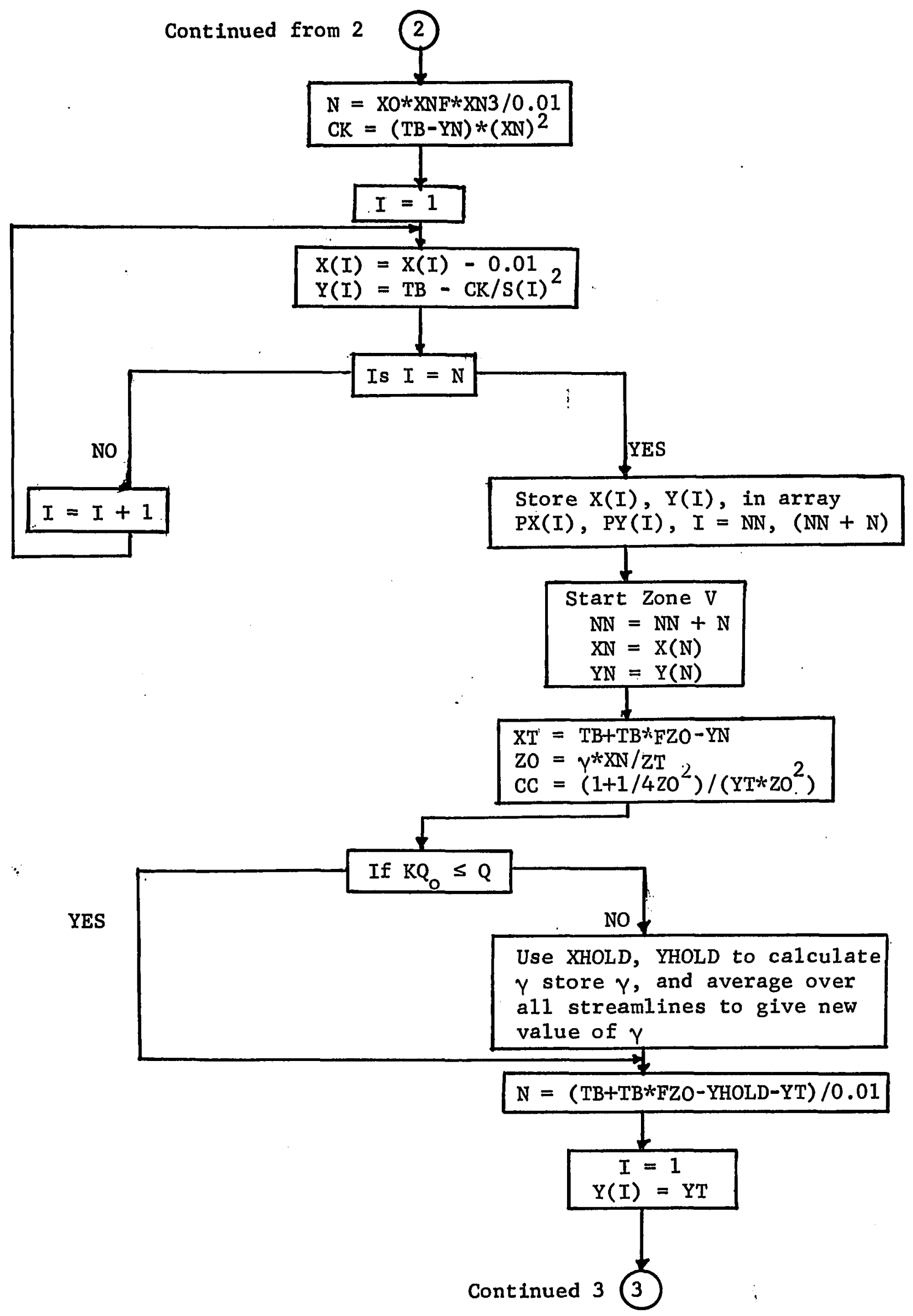




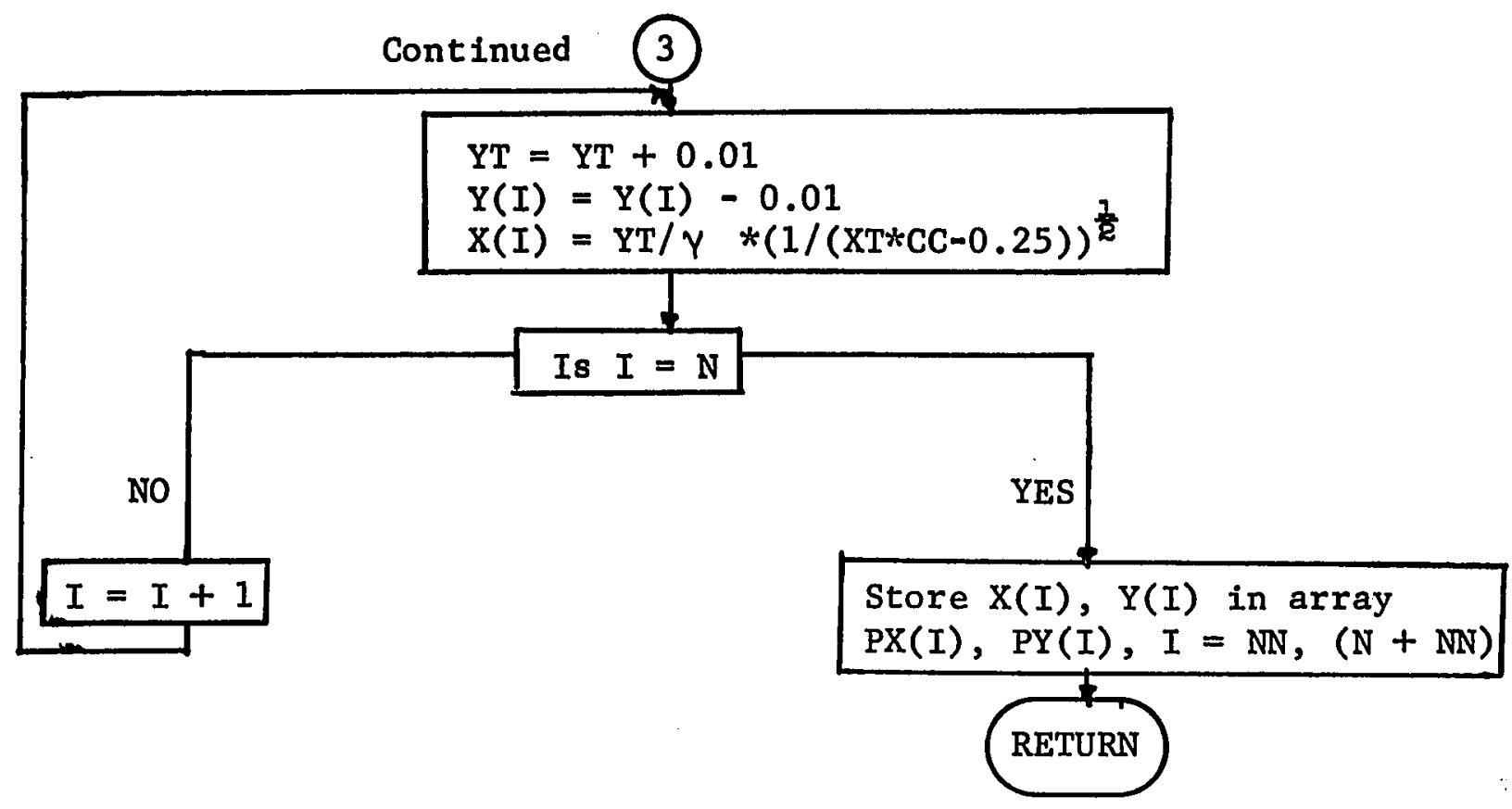

Figure E-2. Flow Diagram for Subroutine STREAM. 


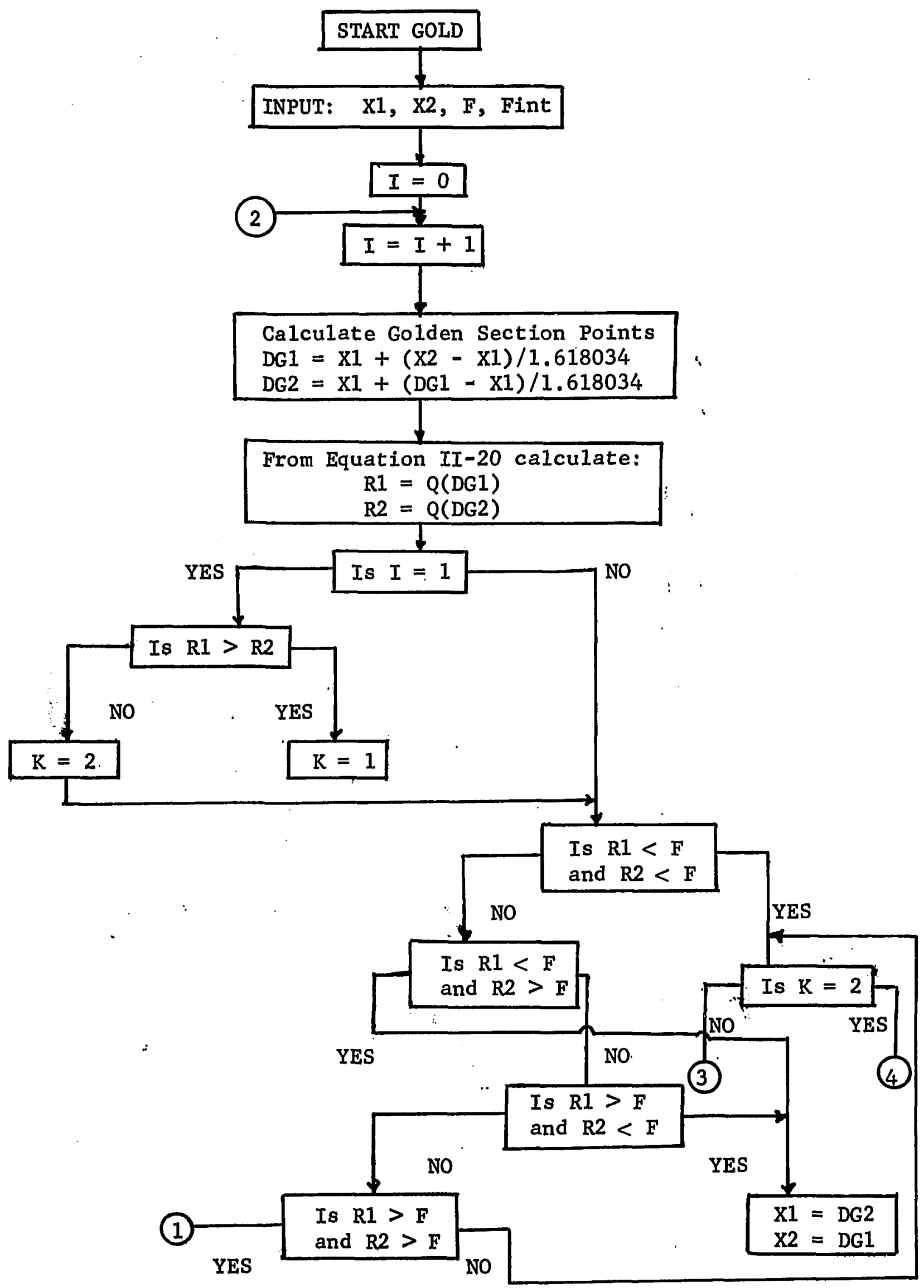


Continued from 1

1

(3)

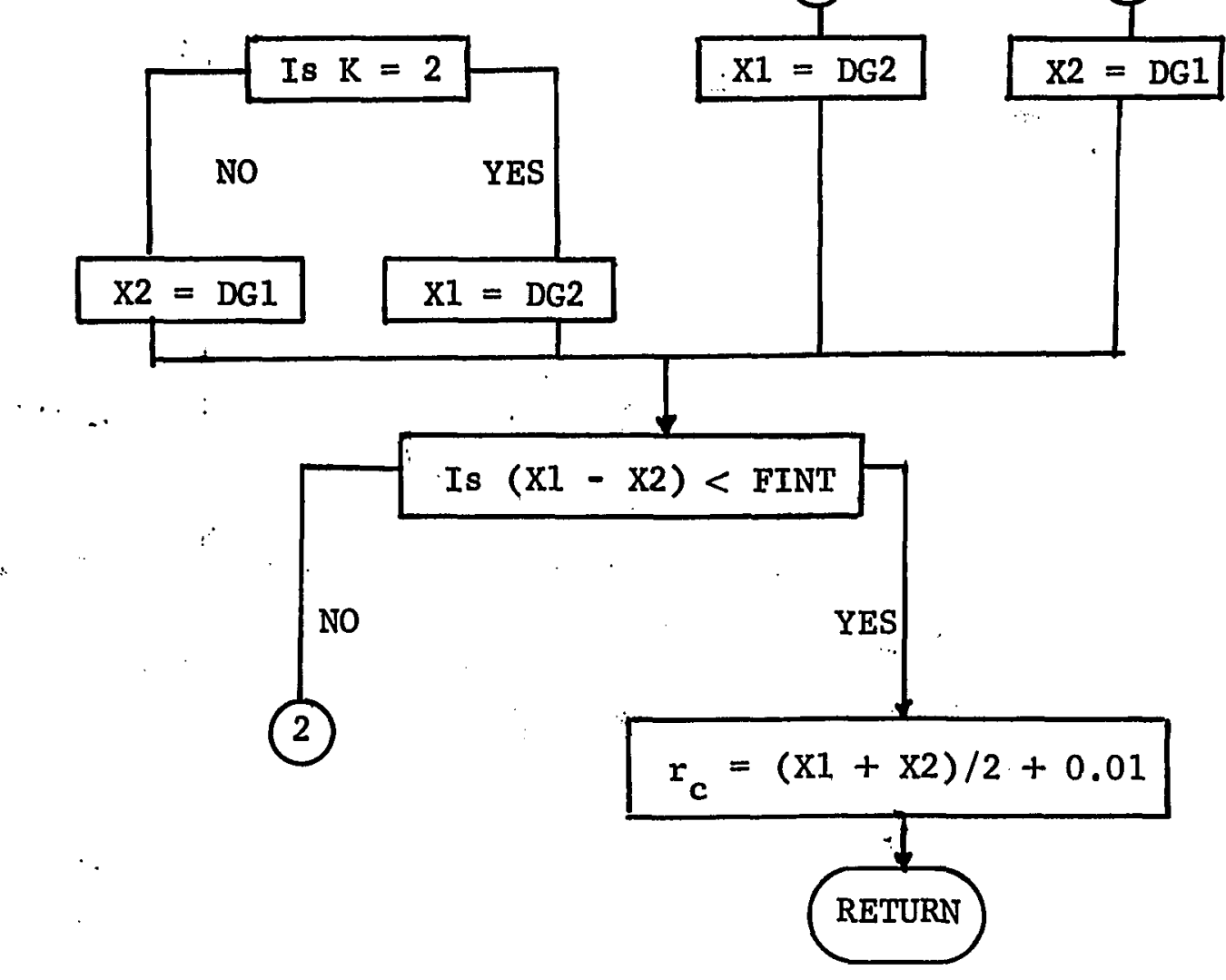

Figure E-3. Flow Diagram for Subroutine GOLD. 


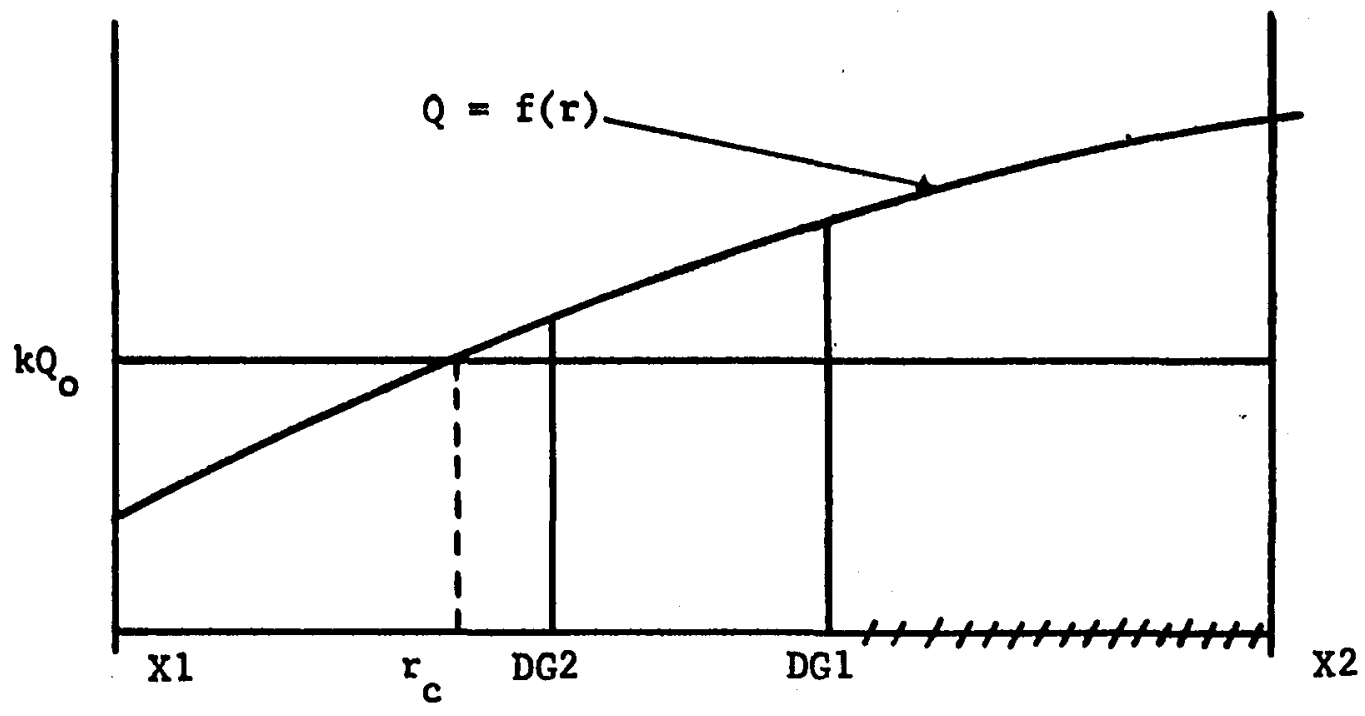

(a) First Iteration for Golden Section Search

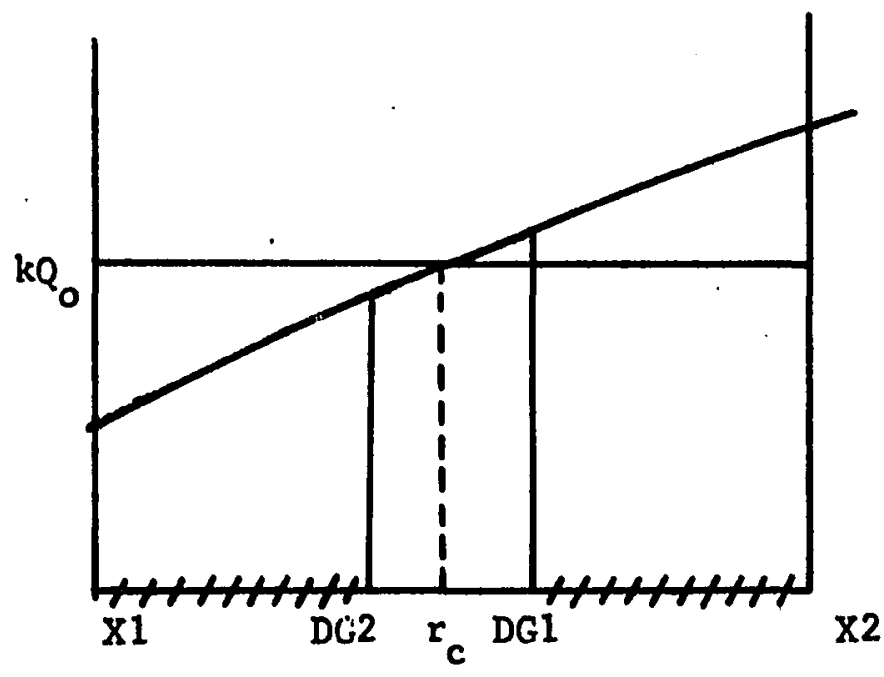

(b) Second Iteration for Golden Section Search

Figure E-4. Illustration of Golden Section Search Procedure 
LIST E-1

FORTRAN LIST OF PROGRAM PLOTER, WITH SUPPORTING SUBROUTINES STREAM, GOLD AND SPRINT, AND SAMPLE OUTPUT

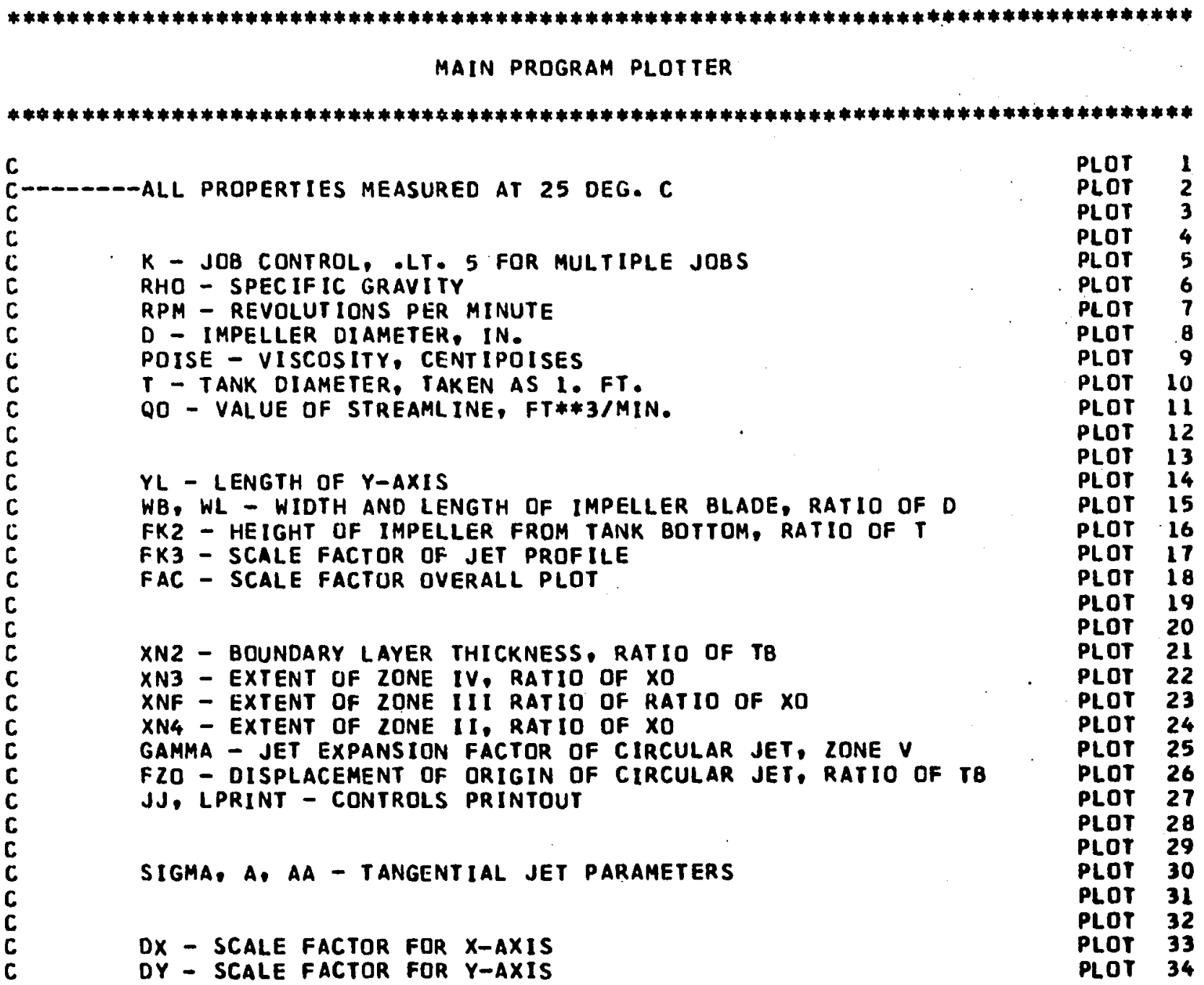




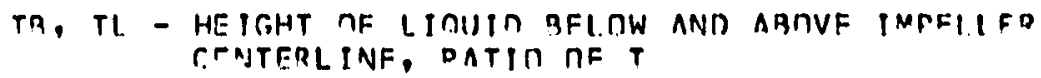

ก.า 35

oint in

Dist it

DLIT 30

nint 3 in

DI ?

DI.רT 41

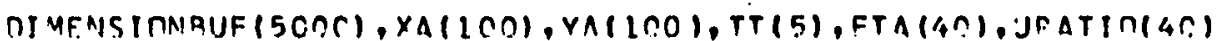
$220 \times$, LFNGTH OF IMP BLADE $=1$ OF5.?.T5ח, FI.UIN DFPTH RATIN $=1$ PLTT 78 3,F5.? 120X, 'IMPELI.FR DEPTH = I,F5.?,T50, 'SCALF FACTOR .JFT DROFILF = 'PLDT 70 4,F5.2/20X, 'SCALE FACTOR OF PI.NT $=1, F 5.2, T 50$, BUUNMARY I.AYFQ THICKNPLOT B? SFSS RATIN $=$,F5.2I

WRITF $(6,402)$ XN3, XNF, XN4

PLOT 81

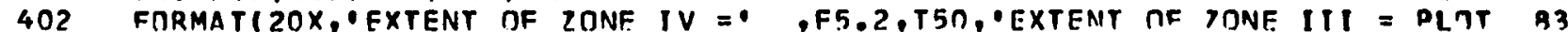

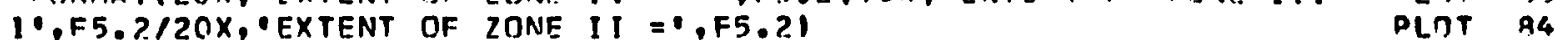
READIS,110ISIGMA,A, AA

110 FORMAT $(3 F 10.21$ DAYT $=0 / 12$. 


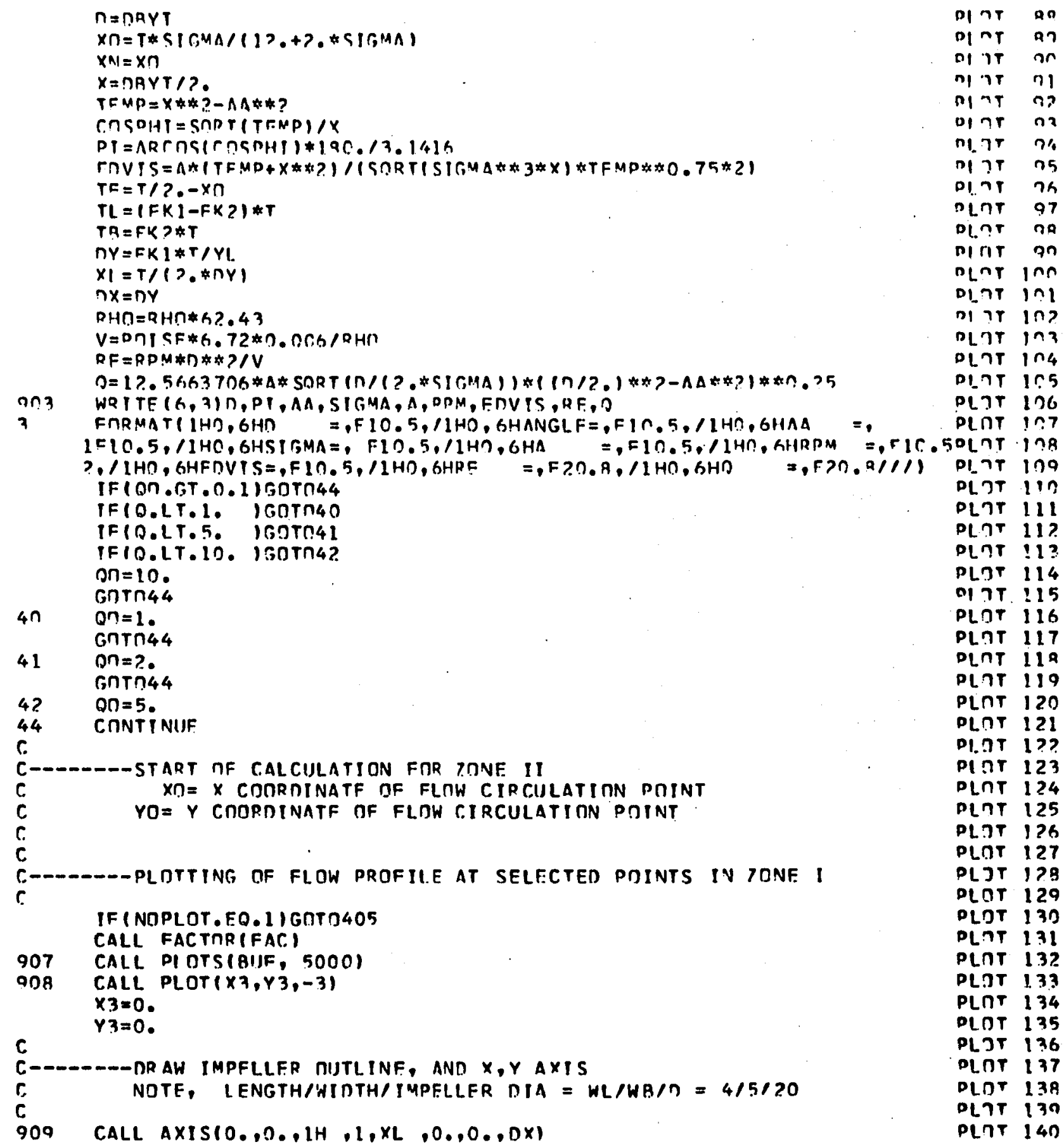

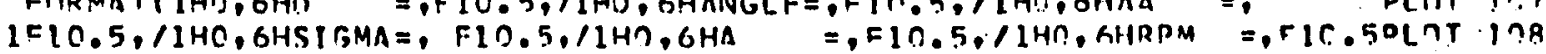

Dint an

Dint an

D i on

ח

niat as

oine no

ni.je nis

ก।

pl.ar nh

Dint 97

DL?T

nint on

olner ino

Dint int

ni in in?

nLTt in?

dint ina

काาT I.5

peTt ino

DLT IIn

DLרT 111

PL.? 11 ?

oLnT II3

plot 114

ol 7T :15

PLOT 116

PLnT 117

DLnT IL

plot 119

PLnT 120

plnt 121

PI.AT 1??

PInT 123

PLnT 124

PLnT 125

PLIT I?G

PLAT 127

PLJT 128

PLot 129

DLDT 130

PLרT 131

plnt 132

PL.TT 133

PLnT 134

DLnT 135

PLJT 136

Dlnt 137

PLOT 138

Dlot izn

plat 140 
CALL $\Lambda X I S(O, .-F K ? * T / \cap Y, I H, 1, Y L, 00, .-T K 2 * T, \cap Y)$

DLTT 141

oir r.ALL pI nTID..-Th/DY, 3)

CALL OINT(XI., -TA/NY, 2$)$

DI CALL PLOT(XL. TL/NV.2)

CALL PINTIO.. TL/NY, ZI)

$r$

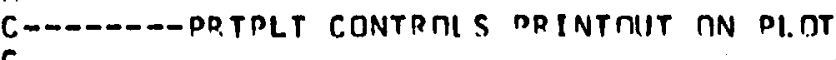

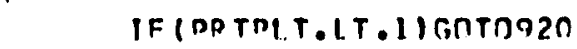

$012 \quad A X=X 1+2.5$

$A Y=-T A / 2$.

OI3 CALL SYMAMI (AX,RY, D.?,SA,C.,15)

BY $=$ RY -0.3

CAI.L. SYMBNL (AX,BY, .,2,SA,0.015)

$714 \quad R Y=R Y-0.3$

CALL SYMAOLIAK, AY , 0.2, SC, 0.,15)

AY $=$ RY -0.3

CAI.L SYMBNLIAX,BY ,0.?,SD,N...15)

$B Y=3 Y-0.3$

915 CAI.L SYMBOL (AX,BY ,0.2,SE.0..15)

$A Y=B Y-0.3$

CAI L. SYMBOL. (AX,BY, 0.2, SF,,., 15$)$

$R Y=B Y-0.3$

916 C.ALI SYMBOLIAX,BY, 0.2.,SG,0..15)

$B Y=R Y-0.3$

C.ALL SYMBOLIAX,BY, $0.2, S H, 0.115)$

BV $=$ RY -0.3

CALL SYMBOL (AX,BY $, 0.2, S I, 0 ., 15)$

AY $=B Y-0.3$

CALL SYMBחLIAX,AY, 0.2, SJ,0.,15)

$A X=A X+3.5$

$917 \quad R Y=-T A / 2$.

CALL NUMBFRIAX,BY $, 0.2,0.0 .04)$

$B Y=B Y-0.3$

CALL NUMBER(AX,BY $, 0.2, R E, 0 ., 4)$

$B Y=B Y-0.3$

CALL NUMBER (AX,BY $, 0.2, R P M, 0 ., 4)$

$A Y=A Y-0.3$

CALL NUMBER (AX,BY, 0.2, EDVIS, $0 ., 4)$

91 A $B Y=B Y-0.3$

CALL NUMBER(AX,BY $0.0 .2,0 \quad 00.41$

$B Y=A Y-0.3$

CALL NUMBER (AX,BY $, 0.2,0$ O, 0.04 )

$B Y=B Y-0.3$

CALL NUMBER (AX, BY $.0 .2,5$ IGMA,0..4)

$B Y=B Y-0.3$

919 C.ALL NUMBFR(AX,BY ,0.2.A, 0.04$)$

$B Y=B Y-0.3$

CALL NUMBER (AX,BY, $0.2 .4 A \quad .0 .04)$

$A Y=R Y-0.3$

CALL NUMBER (AX,BY $0.2,01 \quad, 0 . .4)$

920 CALL PLNT( D/(2.*DX),D/(WB*DY*2.1,3)

CALL PLOT ( $(0 / ? .-n / W L / / O x, n /(w \theta * n Y * 2.1 .2)$

PI.าT 140

PIกT 14?

DLT 14\%

PI 7 T 145

DI if 1 i4

PI.าT 147

D) 149

DLTT 1:?

PI.กT IIn

PI. 151

PLIT 15 ?

DI.jT 153

PLnT 154

PI 755

DLרT 156

Bi.nr ist

PLnT 159

olft jag

plot lao

PInT inl

PLnT l6?

Dlnt laz

plot jak

DLTT 165

PLnT IGA

PLnT 167

PLDT 1GR

Plnt ikt

plnt itn

pint 171

plot 17 ?

plat 173

Plnt 174

plor 175

PLnT 175

plat 177

Plnt 178

olge 179

plat ian

plor lal

Plnt 1 az

Plot 1 B3

Dlot 194

plat Ias

Dlnt lab

PLDT 197

PLOT 1 AR

Plat 199

plite ian

PLJT 191

plat laz

PLaT 193 


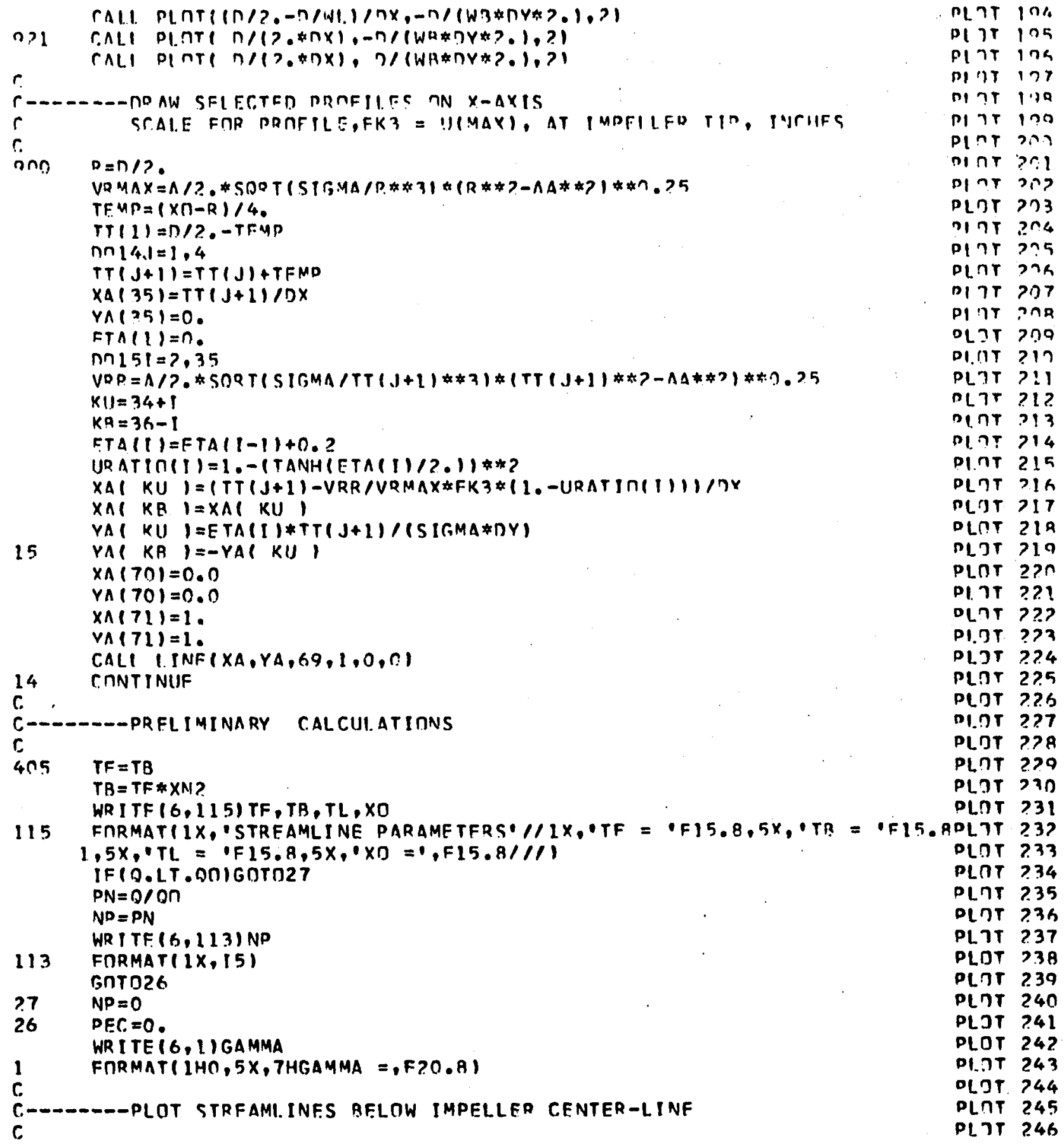


$.1=0$

$[F(J), F Q .11) J=1$

ITAMMA $=J$

$71 \quad T R A M=r$.

I PRINT $=$ J

DEC, $=0$.

NDP $=0$

nח $161=1,20$

CALL STRFAM

IF I XHOLN.GF EXNIGOTR?A

IFI J.EO.OIGRTIIG

IFINN.GT . 3nOIGOTO2L

$P \times(N N+1)=0$.

$\operatorname{PY}(N N+1)=0$.

$P X(N N+2)=n X$

$\operatorname{Pr}(N N+2)=n Y$

$D O 13 L=1, N N$

13 PY $(L)=-P Y(L)$

IF I NOPL OT EEO.IIGOTDI

C.ALL LINE (PX,PY,NN, $1,0, n)$

LG CONTINUE.

2R IFIJ.EO.1)GDTO?Q

IF (ARSITGAM-GAMMA) - LE . $(0.01$ \&GAMMA ) I GOTO30

GAMMA = TGAM

$30 \quad J=1$

GOTD31

JGAMMA $=$ J

GAMMAETGAM

GOT031

C

C.

29 READ (5,37 IGAMMA, XN2,JJ

IF I JGAMMA.EQ. I ICALL SPRINT

37 FORMAT 2 2F $10.2,12)$

WR ITE $(6,36)$

36 FORMATIIX, $/ / / 2 X, T L 5$, STREAMLINES ABOVE CFNTFR-LINE,$/ / / I$ $T A=T L * X N 2$

WRITE $(6,115) \mathrm{TF}, T B, T L, X O$

WR I TE $(6,1)$ GAMMA

$J=0$

IF (JJ.EQ. II J $=1$

JGAMMA $=J$

$34 \quad$ TGAM $=0$.

LPRINT $=J$

PEC $=0$.

$N P P=0$

$D 0171=1.20$

CALL STREAM

IF (XHOLD.GE, XN) GOT032

IFI J.EQ.01GOTOIT

IF (NN.GT. 300)GOTOLI

PX $(N N+1)=0$.

BI 247

DLIT ?:4

DI.?T 749

DinT 250

PLTT 25 !

DIกT 75 ?

DLרT 752

กI.าT 254

กi ? 355

D! it 5 का,

DLרT 257

DLIT 259

PLרT 259

Dा गת

DLרT ?aI

DLnT ?a?

חIT 26 ?

DLDT PG4

plat 365

pint okh

PI.กT 267

DLרT ?AQ

DInT 260

PLOT ?

DInT ?7I

PLחT ?.7?

PLDT 273

PLDT 274

PLDT 775

PINT ? TA

PLDT ? 77

PLDT 278

PLDT 279

PLJT 290

PLDT 2RI

DI Tा 2.R2

PLDT 283

PLTT $>84$

PLOT ?.85

PLOT 786

PLOT 287

PLOT $28 B$

PLIT ?.99

plot 290

PLIT 2.91

PLOT 292

PLOT 293

PLחT 294

PLDT $>95$

PI.กT 296

PLET 297

PLTT 299

PLJT 299 
$\operatorname{Pr}(N N+1)=0$

nLTt $3 n n$

$n \times(N N+2)=n X$

PY $(N N+2)=n Y$

IF INNPL RT . FO. L I GOTnIT

C.AL.L LINF (PX,PY,NN, $1,0,0)$

17 C.NNTINUE

3? IFI.I.FO.1)GRTR10

IF (ARSITGAM-GAMMA) I E. 10.01 *GAMMA I GOTO33

TAMMMA = TGAM

gกrn34

$33 \quad J=1$

JFAMMA $=J$

GAMMA $=$ TrAAM

GOTn34

$? 1$ WR ITF $(6,1)$ INN

12 FQRMATIIHO,5X,29HNN IS GRFATER THAN 300, AND $=, 141$

In IFI JGAMMA.FQ.1)CALLSPRINT

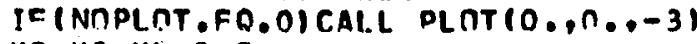

$X 3=\times 3+X L+2 \cdot 5$

19 IFIK.LT.5IGOTng

22 IFINOPLOT.EQ.OICALL PLOTI0.. N..999)

WR TTE $(6,114) \mathrm{K}$

STOP

ENn

DLit 301

Dint 30 ?

p)

DLOT $3 \cap 4$

BLר 3 ins

DI.าT $30 \%$

PLite 3n?

Dint zon

plot zona

PLרT 310

DLTT 311

plne 31?

pLOT 313

PLOTT 314

DLרT 315

PLרT 316

PLJT 317

DLIT 319

plnt 310

plat $2>0$

DI.TT 3?

PLIT 3??

PLIT 323

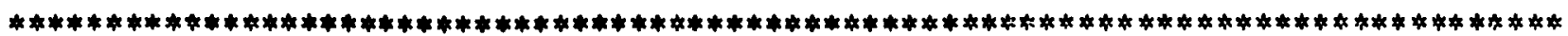
SUBRDUTINF STREAM

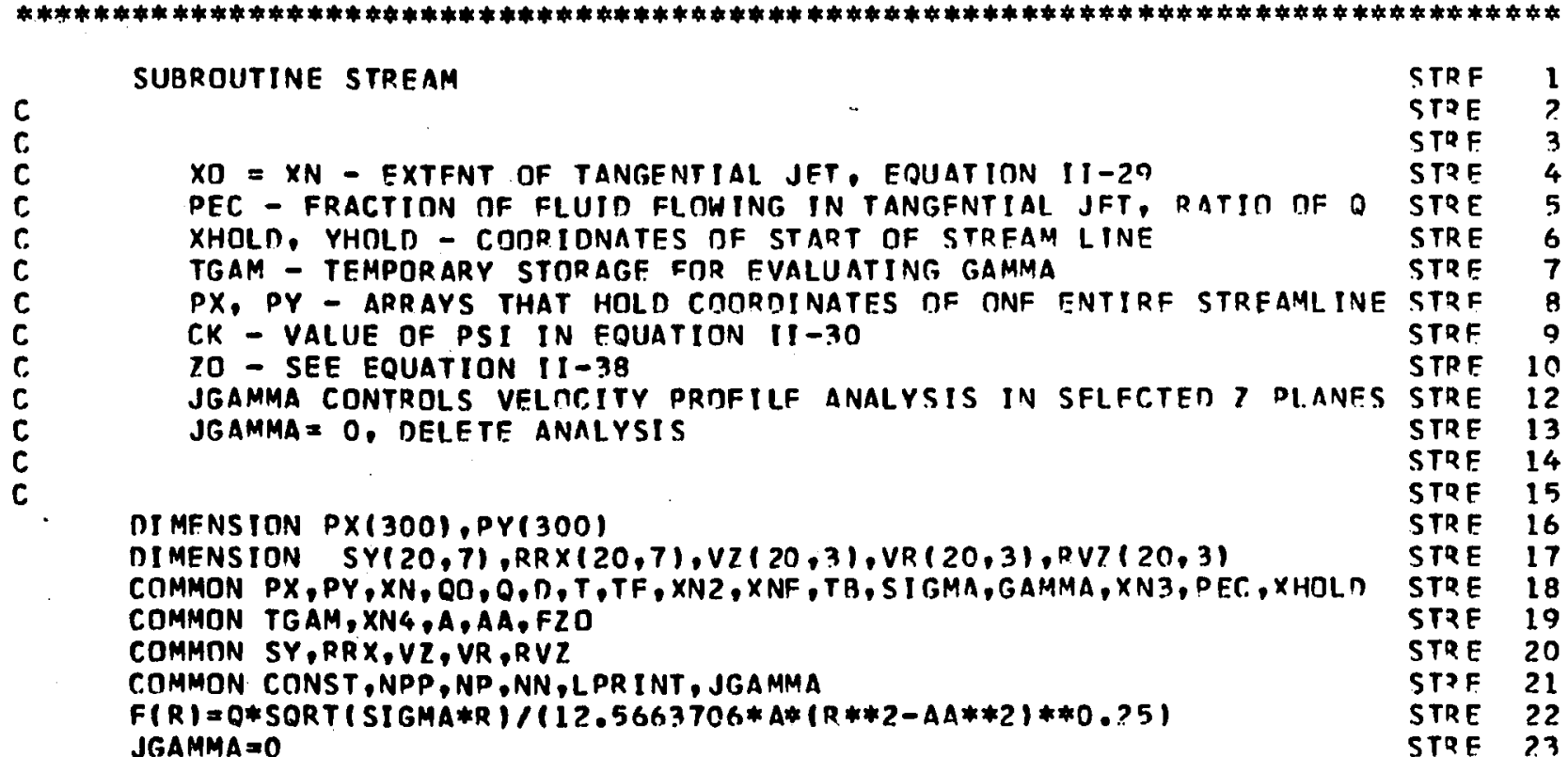


$I P=1$

I PR INT $=0$

IF(NPP.FO. 0$) 27=2$.

$n n=n / ?$.

$\mathrm{JC}=\mathrm{n}$

$\partial \partial=$ ดก

$X \cap=X N$

IF (NPP . GF .NP IGNTRPA

$N D P=N \cap P+1$

$P F C=P F C+O O / Q$

$D X=P E C$.

$x T=n / 2 .+n .01$

$n K=0 \times * F(X Y)$

gntחio

2ก

$N P P=N P P+1$

$P N=N P P$

$O X=Q \cap * P N$

C.ALI. ROLDEND, XO,OX, OOOL, RRI

$X T=R R$

$B X=0 X / 0$

$n K=0 X * F(X T)$

$19 \quad T \cap P=X T+D K$

AOT $=X T-D K$

$X H \cap L D=X T$

IF I XHOL.

$Y T=X T / S I$ (IMA*ALOG (TDP./BOT)

YHNI.D $=Y T$

IFILPR INT,EQ. IIWRITE $16,1001 \times$ XHLD, YHOL, DK

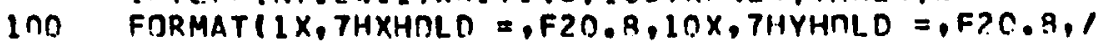

$11 X, 7 H \cap K$

$=, F 20.81$

TEMP $=(X N-X T \cdot) / .005$

$N=$ TEMP

$N N=N$

$D \cap 17 J=1, N$

$X T=X T+.005$

$n K=Q \times * F(X T)$

$T D P=X T+D K$

$A O T=X T-D K$

$Y T=X T / S I G M A * A L D G(T \cap P / B O T)$

$\operatorname{PX}(J)=X T$

PY $(J)=Y T$

17 CONTINUE

c.

C-D- - -PLOT STREAM LINES OF ZRNF II

C.

$X=X T$

$Y=V T$

$T F=T / 2 .-X N$

$c$

$C K=(T / 2,-X) * * 2 * Y$

C-m-n-CALCILATE, R AND STREAM FUNCTION IN SELEC.TED 7 PLANES C TO EVALUATF VFLOCITY PRIFILFS

C

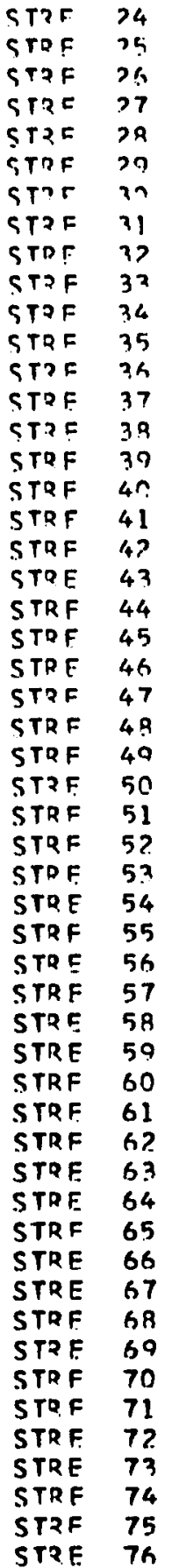


IF( JOAMMA.EQ.0)GRTOZI

STRF 77

$S C A L . F R=11.5 / T$

TIN $=$ TA/ 12 . *XN? $)$

THQ $=0$. G*TA/XN? - 4.*T INC

$T O N=Q D / 2$. \#NPP

$n \cap 3 \cap J K=1.7$

$T R A=T D R+T I N C$

$T E M D=T P R * X N P * 6 . / T B$

IF (LPR INT . FO.I)WR I TF(40B) TFMP

4nG FDPMATI2OX, TRR = F 15.21

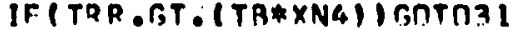

$R R \times\left(N P P_{,}, J K\right)=S Q R T\left(C K / T R R_{,}\right) * S C_{,} A L F R$

$30 \quad S Y\left(N P P_{. J K}\right)=T P R$

31 IFILPRINT.FQ.1)WRITF(6.106) ICK

IDG FIRMATIIX, 4HCK $=, F ? 0.8,1 / 1$

$T F M D=(T B * X N 4-Y) / O$. त)

$N=T F M P$

$n \cap\{$ I $=1, N$

$Y=Y+0.01$

$X=T / ? .-(C K / Y) * * 0.5$

$J N=J+N N$

$\operatorname{PX}(J N)=X$

PY $(J N)=Y$

11 CONNTINUE

C C- TONF II
T.

C $\quad N N=N N+N$

$X \Pi=X N * X N F$

$X R=T / 2 .-X$

$C . K=X R * * 2 *(T B-Y)$

ST?F 79

STOE 70

STRF

STrF 91

STRE क?

STRE 97

STRF भद

STDF 45

STRF 96

STRF RT

STOF $A R$

STRF QD

STRF Oก

STRF $O 1$

STRF 7 ?

STOF $n 2$

STRF 94

STRE 95

STRF OR

STRF 97

STOF 79

ST?E 00

STRE IN?

STRE 101

STOE IO?

STPE 103

STRE 104

STRE 105

STRF IO6

STRE 107

STRF INA

STRF 109

C.

C------CONTINUE R AND STRFAM FUNCTION C.ALCULATINNS IN SELECTFN, DLAVFSTRE IIO

C

IF ( JGAMMA. EQ.01GOTn35

STRE 111

STRE 112

STRE 113

STRE 114

$T R R=T R R-T I N C$

$D \cap 32 J K=J L, T$

$T R P=T R R+T I N C$

$T F M P=T R R * X N 2 * 6 . / T B$

$T F P=T B * X N 2-T R R$

IF (LPR INT FO.1) WR I TE (406) TFMP

$P R \times(N P P, J K)=S Q R T(C K / T E P) * S C A L F R$

32 SY(NPP,JK) $=T D O$

IF (L.ORINT.EQ.0)GOTO35

IPR $=7$

HR I TE $16,4031 \mathrm{NPP}$

403 FORMAT(1X/19X, 13/)

HR ITE (IPR, 400$)(S Y(N P P, J K), J K=1,7)$

400 FORMAT $(1 \times, 7 F 10.2)$

HR ITF (IPR, 401) (RRX (NPP, JK), JK= I, 7)

STPF 115

STRF 116

STRE 117

STRE 118

STRF 119

STRE 120

STRF $1 ? 1$

STRF 12 ?

STRF 123

STRF 124

STRE 125

STPF 126

STRE 12.7

STOE 128

401

FORMAT $(1 X, T F 10.5)$

STRE 120 
STQF 131

STOF 13?

STIF 133

STPF 136

ST?F, 35

STRC 136

STPF 137

STIF 132

STPF 139

STOF 142

STQF 141

STRF 14?

STRF 143

STPF 144

STDE 145

STRF 146

STRE 147

STRF 148

STRF 149

STRE 150

STRE 151

STRE 15?

STRE 153

STRE 154

ST2E 155

STRE 156

STRF 157

STRE 159

STRF 159

STOF 150

STRE ISI

STRF 16?

STRF 163

STRE 164

STPE 165

STRE 166

STQE 167

STPE 168

STPE 169

STRF 170

STRE 171

STRF 172

STRE 173

STRE 174

STRE 175

STRE 176

STRE 177

STRE 178

STRF 179

STRE ISO

STRE IRI

STRF I A? 


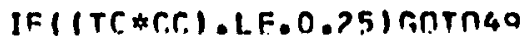

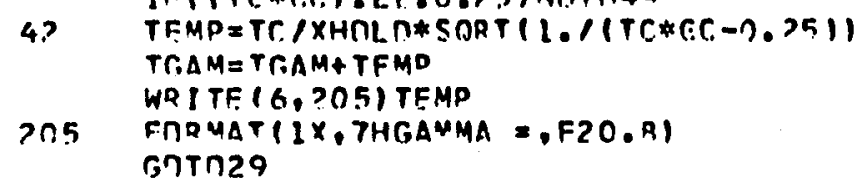




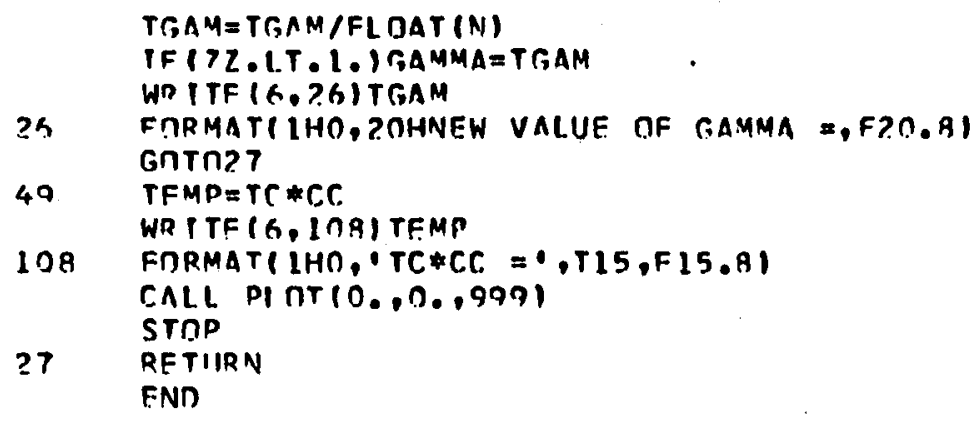

STQF $>36$ STOF 937

ST?F ?39

STRT $>30$

STPE 740

STRF ?41

STOF ?4?

STPF 247

STzR ?44

STQF 245

STOF ?:4,

STRT ?:7

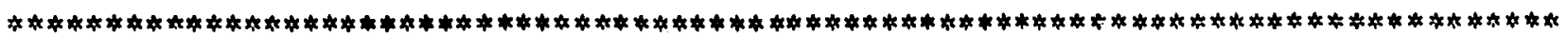
SUAPחUTINF GII $n$

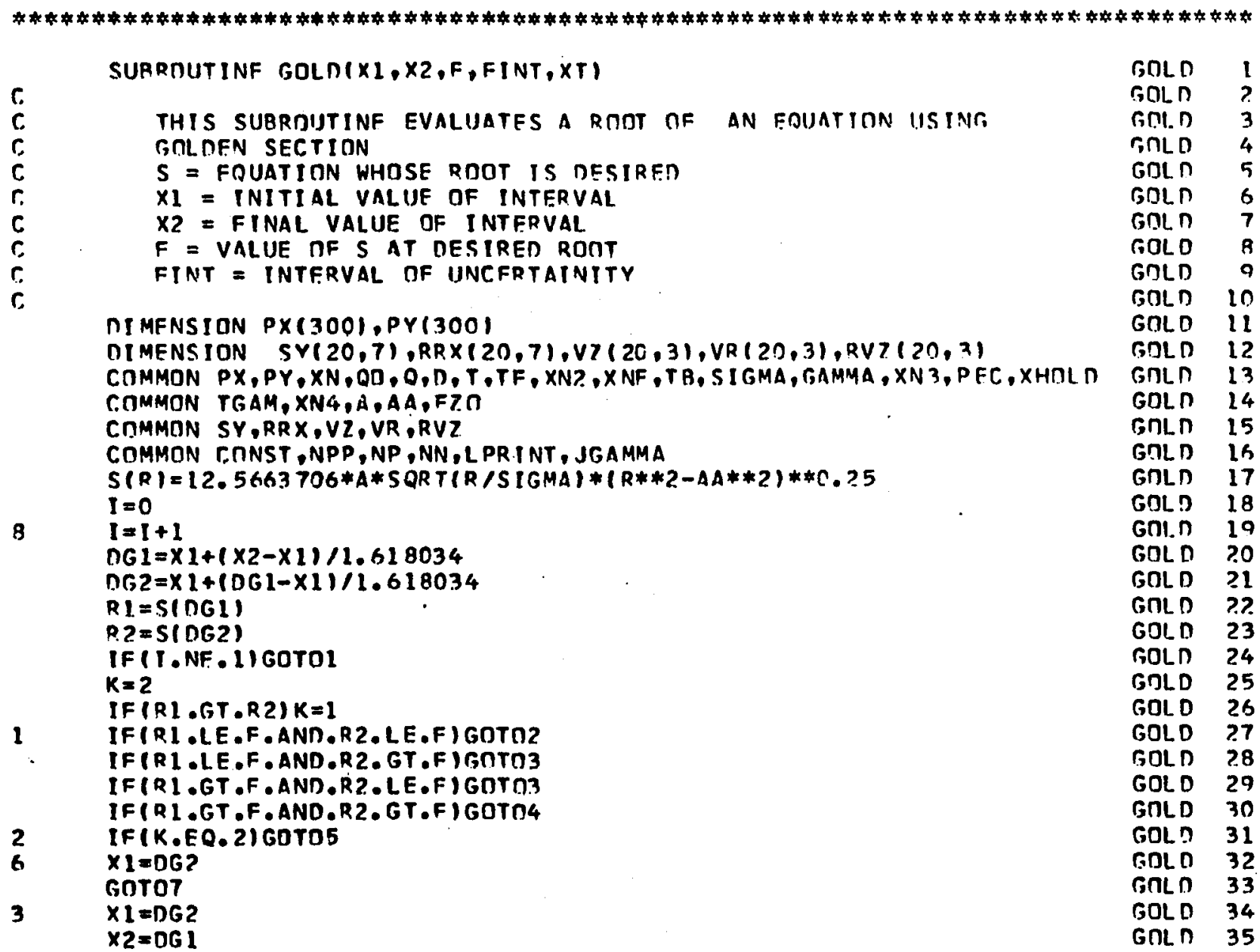




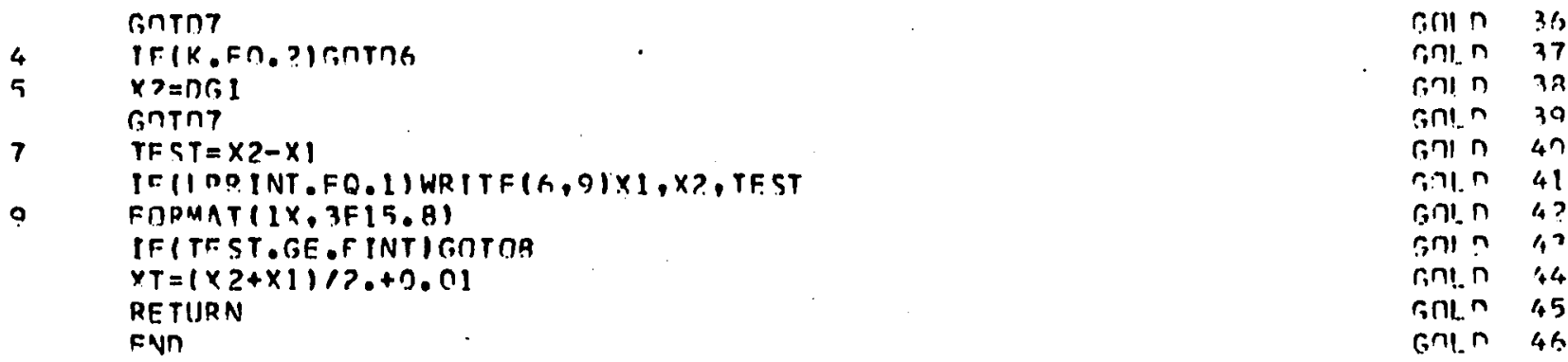

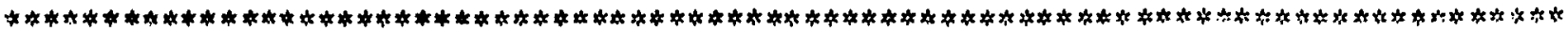

SIJRPOIITINE SPHINT

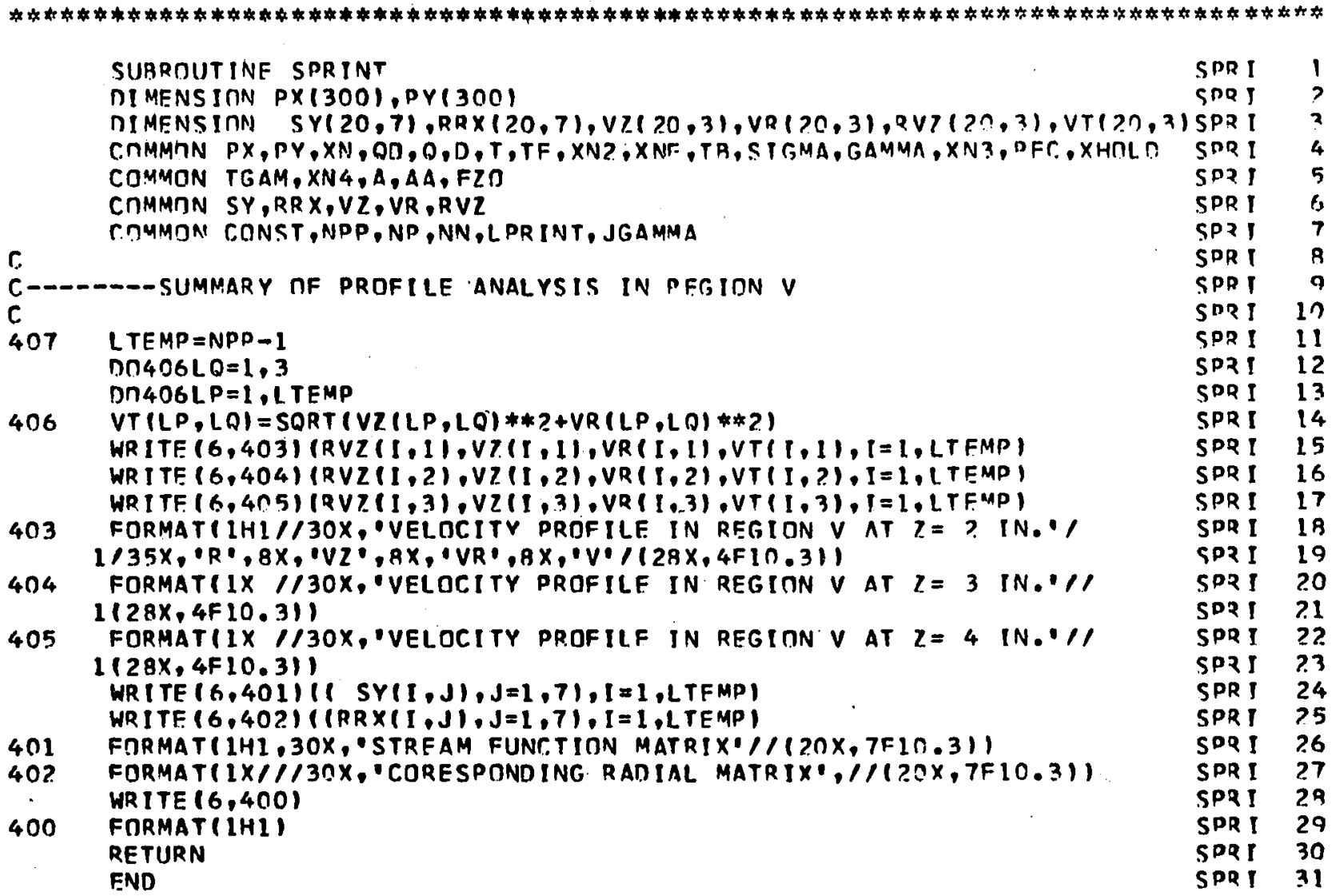




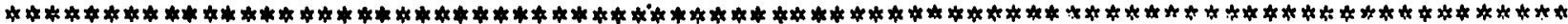

\begin{tabular}{|c|c|c|c|c|c|c|}
\hline $1 r .997 n 8$ & 500. & 3. & 0.8937 & 1. & 5. & \\
\hline $\begin{array}{l}10 . \\
1.9 \\
1 ? .521 \\
? .5\end{array}$ & $\begin{array}{l}5.0 \\
0.3 \\
19.7 .73 \\
1 .\end{array}$ & $\begin{array}{l}4.0 \\
1.0 \\
0.1009 \\
0\end{array}$ & $\begin{array}{l}1 . \\
n .5\end{array}$ & $\begin{array}{l}0.4 \\
1.87\end{array}$ & $\begin{array}{l}1.0 \\
n .0\end{array}$ & 0.5 \\
\hline
\end{tabular}

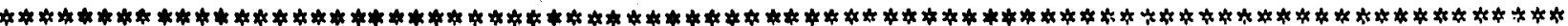

$$
\text { INPIIT DATA FOR FIGURE II-5(A) }
$$

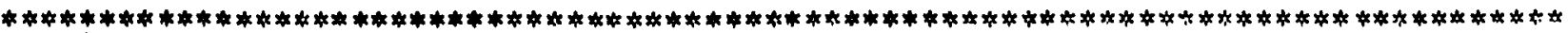

\begin{tabular}{|c|c|c|c|c|c|c|}
\hline 90.99708 & 500. & 3. & 0.9937 & 1. & 3 & \\
\hline $\begin{array}{l}10 . \\
0.9 \\
12.621 \\
? .5\end{array}$ & $\begin{array}{l}5.0 \\
0.5 \\
19.273 \\
1 .\end{array}$ & $\begin{array}{l}4.0 \\
1 . \\
0.1009 \\
0\end{array}$ & 1.5 & $\begin{array}{l}0.4 \\
1.87\end{array}$ & $\begin{array}{l}1.0 \\
0.0\end{array}$ & 3.5 \\
\hline
\end{tabular}


SAMPLE OUTPUT

RRINT CONTRDL =

9

INPUT PARAMFTERS

FLUTD ANN TANK C.ONSTANTS

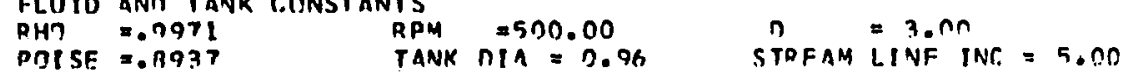

IMPELLEP AND ROUNRARY DARAMFTERS

LFNGTH DF $X$ AXIS=10.00 WIDTH OF IMPELLER BLANE $=5.00$

LFNGTH DF IMP AL.ADF $=4.00$ FI.UID DEPTH RATIN $=1.04$

IMPEI LER NFOTH = 0.5? SCALF FAC.TOR JET OD OFILF = 0.10

SCALE FACTIRR OF PLOT $=0.70$ BRUNDARY L.AYFR THICKNESS RATID $=0.90$

EXTFNT OF ZONE IV $=0.10$ FXTENT DF ZOMF III $=0.90$

EXTENT MF TINF II $=0.55$

$n \quad .0 .25000$

ANGIE $=66.57747$

$A A=0.11470$

SIGMA= 11.19200

$A=28.23950$

RPM $=500.00000$

EOVIS: 1.74240

RF $=53983.52343750$

- B. 35951233

(a) Streamlines Above Impeller Centerline

STRFAMLINE PARAMETFRS

$T F=0.50083190 \quad T A=\quad 0.45074868 \quad T L=0.4994 A 782 \quad \times n=0.31248027$

1

GAMMA $=\quad 1.86999989$

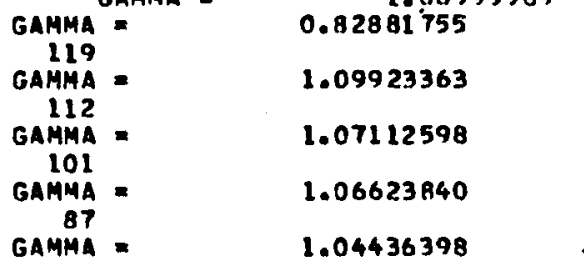




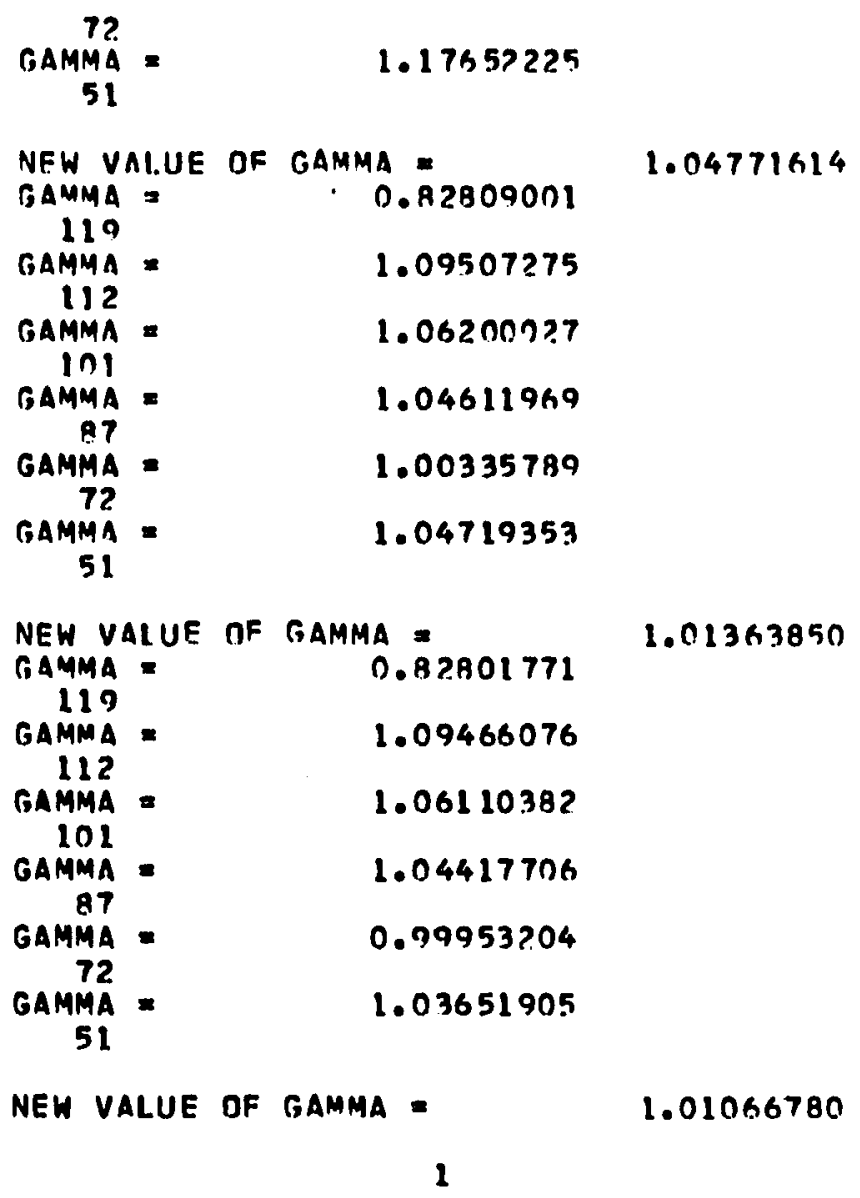

119

2

112

3

101

4

87

5

72 
51

NFW VaLUE nF Gamma $=0.0$ 
VELOCITY PROFII.F IN RFGION $V A T T=$ ? IN.

$\begin{array}{cccc}R & V 7 & V R & V \\ 4.921 & 94.289 & 18.683 & 96.12 ? \\ 4.548 & 51.676 & 14.095 & 53.567 \\ 4.261 & 28.379 & 9.071 & 29.794 \\ 3.86 R & 14.762 & 5.375 & 15.710 \\ 3.481 & 7.543 & ? .873 & 9.07 ? \\ 2.783 & 3.275 & 1.064 & 3.443\end{array}$

VEL OCITY PROFILF IN RFGION V AT $7=3$ IN.

$\begin{array}{rrrr}4.742 & 64.711 & 10.557 & 65.567 \\ 4.306 & 36.601 & 8.317 & 37.534 \\ 3.968 & 20.748 & 5.609 & 71.493 \\ 3.527 & 11.381 & 3.675 & 11.944 \\ 3.112 & 6.191 & 2.184 & 6.565 \\ 2.415 & 3.078 & 1.171 & 3.293\end{array}$

VELOCITY PROFILE IN REGION $V$ AT $Z=4$ IN.

$\begin{array}{rrrr}4.590 & 49.235 & 6.984 & 49.728 \\ 4.088 & 28.275 & 5.615 & 29.828 \\ 3.715 & 16.271 & 3.866 & 16.724 \\ 3.727 & 9.144 & 2.591 & 9.504 \\ 2.777 & 5.113 & 1.632 & 5.367 \\ 2.043 & 2.687 & 0.972 & 2.857\end{array}$


STREAM FINCTION MATRIX

$\begin{array}{rrrrrrr}2.500 & 2.500 & 2.500 & 2.500 & 2.500 & ? .500 & ? .500 \\ 5.000 & 5.000 & 5.000 & 5.000 & 5.000 & 5.000 & 5.000 \\ 7.500 & 7.500 & 7.500 & 7.500 & 7.500 & 7.500 & 7.500 \\ 10.000 & 10.000 & 10.000 & 10.000 & 10.000 & 10.000 & 10.000 \\ 12.500 & 12.500 & 12.500 & 12.500 & 12.500 & 12.500 & 12.500 \\ 15.000 & 15.000 & 15.000 & 15.000 & 15.000 & 15.000 & 15.000\end{array}$

CIRFSPANDING RADIAL MATRIX

$\begin{array}{lllllll}0.540 & 0.468 & 0.418 & 0.457 & 0.535 & 0.672 & 1.039 \\ 0.785 & 0.680 & 0.608 & 0.666 & 0.779 & 0.980 & 1.515 \\ 0.963 & 0.834 & 0.746 & 0.816 & 0.955 & 1.200 & 1.955 \\ 1.155 & 1.000 & 0.895 & 0.968 & 1.132 & 1.423 & 2.199 \\ 1.399 & 1.212 & 1.084 & 1.182 & 1.382 & 1.738 & 2.686 \\ 1.821 & 1.577 & 1.410 & 1.533 & 1.793 & 2.255 & 3.485\end{array}$


(b) STREAML INFS ABRVE CENTFP-L INF

STRFAML INE PARAMFTERS

$T F=0.50083190 \quad T A=\quad T l=0.49948797 .0 .49948787 \quad \times n=0.31249077$

\begin{tabular}{|c|c|c|}
\hline & FAMMA = & 2.5000 \\
\hline $\begin{array}{r}\text { r.AMMA } \\
126 \\
\text { r.AMMA }\end{array}$ & $\begin{array}{l}= \\
=\end{array}$ & $\begin{array}{l}0.86653042 \\
1.15571308\end{array}$ \\
\hline $\begin{array}{r}120 \\
\text { TAMMMA }\end{array}$ & $=$ & 1.13208771 \\
\hline $\begin{array}{r}1090 \\
\text { GAMMA }\end{array}$ & $=$ & 1.07725239 \\
\hline $\begin{array}{r}97 \\
\text { SAMMA }\end{array}$ & $=$ & 1.12177372 \\
\hline $\begin{array}{r}80 \\
\text { GAMMA }\end{array}$ & $=$ & 1.22035503 \\
\hline
\end{tabular}

NEW VALUF DF GAMMA

SAMMA $=\quad 0.86574781$

126

TAMMA =

120

GAMMA $=$

109

GAMMA =

97

GAMMA =

$B O$

GAMMA
60

1.15118217

1.12202644

1.05829620

1.07476425

1.08933163

NEW VALUE DF GAMMA

GAMMA =

126

GAMMA $=$

120

GAMMA =

109

GAMMA $=$

97

GAMMA $=$

BO

GAMMA $=$

1.09561825

0.86568201

1.06022453

1.15080357

1.12119293

1.05674939

1.07107830

1.08015060 
60

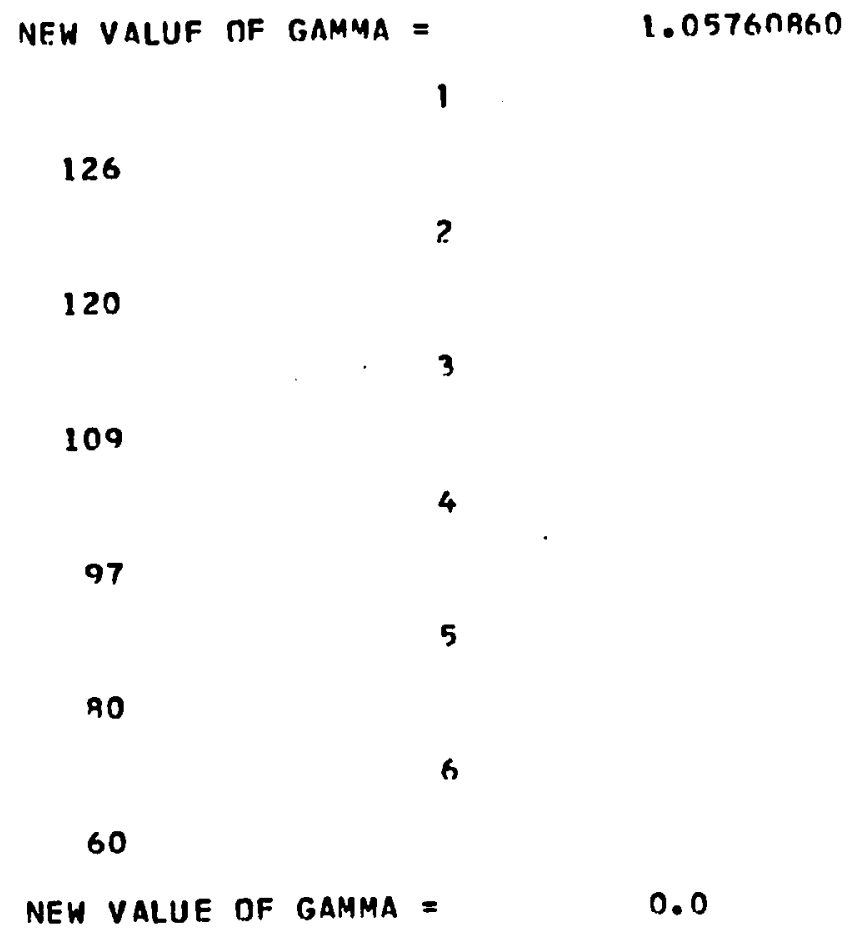


VELOCITY PROFILF IN RFGION V AT T=? IN.

$\begin{array}{cccc}R & V Z & \text { VP. } & V \\ 4.965 & 98.772 & 18.597 & 100.507 \\ 4.607 & 54.112 & 14.087 & 55.915 \\ 4.327 & 29.664 & 9.074 & 31.021 \\ 4.079 & 15.973 & 5.441 & 16.875 \\ 3.548 & 7.751 & 2.870 & 8.249 \\ 7.961 & 3.495 & 1.107 & 3.666\end{array}$

VEL DCITY PROFILE IN RFGION V AT $7=3$ IN.

$\begin{array}{rrrr}4.795 & 67.763 & 10.503 & 69.573 \\ 4.371 & 38.318 & 8.306 & 39.208 \\ 4.048 & 21.697 & 5.614 & 2 ? .411 \\ 3.723 & 12.160 & 3.591 & 12.679 \\ 3.195 & 6.408 & 2.178 & 6.768 \\ 2.609 & 3.256 & 1.187 & 3.464\end{array}$

VELOCITY PROFILE IN REGION V AT $Z=4$ IN.

$\begin{array}{rrrr}4.652 & 51.549 & 6.947 & 52.015 \\ 4.169 & 29.598 & 5.607 & 30.125 \\ 3.806 & 17.017 & 3.871 & 17.452 \\ 3.446 & 9.715 & 2.544 & 10.047 \\ 2.872 & 5.309 & 1.636 & 5.555 \\ 2.255 & 2.833 & 0.975 & 2.996\end{array}$




\section{STREAM FINCTION MATRTX}

$\begin{array}{rrrrrrr}2.500 & 2.500 & 2.500 & 2.500 & 2.500 & 2.500 & 2.500 \\ 5.000 & 5.000 & 5.000 & 5.000 & 5.000 & 5.000 & 5.000 \\ 7.500 & 7.500 & 7.500 & 7.500 & 7.500 & 7.500 & 7.500 \\ 10.000 & 10.000 & 10.000 & 10.000 & 10.000 & 10.000 & 10.000 \\ 12.500 & 12.500 & 12.500 & 12.500 & 12.500 & 12.500 & 12.500 \\ 15.000 & 15.000 & 15.000 & 15.000 & 15.000 & 15.000 & 15.000\end{array}$

CORESPONDING RADIAL MATRIX

$\begin{array}{lllllll}0.541 & 0.469 & 0.419 & 0.383 & 0.388 & 0.434 & 0.501 \\ 0.786 & 0.681 & 0.609 & 0.556 & 0.566 & 0.633 & 0.730 \\ 0.965 & 0.835 & 0.747 & 0.682 & 0.693 & 0.775 & 0.895 \\ 1.157 & 1.002 & 0.896 & 0.818 & 0.323 & 0.920 & 1.062 \\ 1.401 & 1.213 & 1.085 & 0.991 & 1.004 & 1.172 & 1.796 \\ 1.823 & 1.579 & 1.412 & 1.289 & 1.303 & 1.456 & 1.68 ?\end{array}$




\section{APPENDIX F}

LISTING AND DESCRIPTION OF COMPUTER PROGRAMS

This Appendix contains a listing of all the programs used in Chapter IV. The listing consists of the FORTRAN IV source list. compatible with a IBM $360 / 65$ computer. At the end of the source list is given sets of imput data read into the program and a sample output. Each set of data is for one pass through the program and is in the order called for by the source list. For example in list F-1 is presented the program. FLOWANL. Two types of initial input data is shown for two modes of operation. Next. is given data sets at constant impeller speed. Each data set contains a number of. velocity profiles all taken at the same impeller speed.

In presenting the source list, subroutines are sometimes used in more than one program. In such cases, the listing of the subroutines is given only in the first program that it appears. The subroutine CURVEF performs a polynomial fit and is obtained from the LSU Computer Center Share Library. A similar program is assumed readily available (see for example IBM Scientific Subroutine Package) and is hence not listed.

A flow diagram of the flow of information in the computer is. also provided were it is considered necessary. In other programs the comment cards in the listing are descriptive of the calculations performed, hence flow diagrams were not needed. The flow diagrams are closely associated with the comment cards that are liberally 
distributed throughout the program. In the listing, only those statements with the first four letters of the program name followed by a serial number are a part of the program. Other statements are there to help make the listing more readable.

Program FLOWANL: This program is given in List F-1 and the flow diagram in Figure F-1. The program output is in two modes (a) To obtain a detail printout of complete profile analysis, and to print out a summary of. the important pieces of information of all profiles run at constant impeller speed (b) To printout only the summary of the profile analysis at constant impeller speed.. FLOWANL reads in the raw data, converts this data.into $\bar{q}$ versus $z$, (where $z=0$ is the impeller centerline), performs a nonlinear least square fit on $\bar{q}$ using the Tangential jet model and prints the result in desired format. The nonlinear fit is done by pattern search program subroutine PATTERN, with its supporting subroutines PROC and BOUNDS. The flow diagram and theoretical treatment of subroutine PATTERN is given in Appendix $C$. The flow diagram for FLOWANL is given in Figure $F=1$.

In Figure $\mathrm{F}-2$ is given the flow diagram for subroutine YAW, which analyses the information from manometer $D$. The flow diagram is a direct application of Equations IV-31, IV-32 and IV-28(b). Subroutine BOUNDS is self explanatory. Subroutine PROC is again straight forward although it might appear complex. It calculates a criteria for minimization which is Equation C-2. It has two additional features (1) Allows keeping $\sigma$ and a constant though the variable P1 whose value is controlled by FLOWANL (2) Gives a printout if desired of every 30th evaluation of the criteria function. The 
second feature permits following the search procedure as it proceeds to locate the minimum of the.sum of squares..

Subroutine OMEGA is a Lagrange in terpolation Program. It was obtained from the Share Llbrary; a listing is given since one was available. A flow diagram will not be given as this is a library program and not written by the author. The program is designed to handle unequal step size and the number of points desired for interpolation is specified by NPTS. In the present case NPTS $=3$, resulting in the three closest points being used for interpolation. It was found that use of this program is equivalent to drawing a smooth curve through the data and interpolating with the help of this curve.

The flow diagram for subroutine AVG is given in Table F-3. The subroutine is designed to take-a one dimensional array and calculate an average value and variance, From the variance it calculates a 0.96 confidence limit for the average using a t-test.

Program VELPRO. This program calculates velocity profiles from the theoretical model in Regions. II and III as explained in Section 4. Figure $F-4$ gives the flow diagram for VELPRO. Supportin subroutines are POLY and CURVEF. Subroutine POLY is a service program that was written to throw out points whose diviation from the regression line is larger than three times the standard error. This has no effect on the imput data to VELPRO, since the data correlates very well. It calls ISU Share Library program CURVEF that performs the polynomial fit and prints out detall results useful in debugging. 
Program TANLANL and YAWANL. Programs TANKANL and YAWANL calculate the component velocities $\bar{v}_{r}, \bar{v}_{\theta}$, and $\bar{v}_{z}$ from the manometer readings recorded by the three dimensional pitot tube. The resulting analysis of the data it is recalled, is dependent on the flow geometry. The flow diagram is identical for both programs. The difference lies in the final equations used to calculate $\bar{v}_{\mathbf{r}}, \bar{v}_{\mathbf{z}}$, and $\bar{v}_{\theta}$. TANKANL computes component velocities from data obtained from Ports $1,2,3$. YAWANL analyses data obtained in the neighborhood. of the impeller. Program COOPER and NIELCUT. Program COOPER analyses Cooper's (8) data, while Program NIELCUT analyses Nielson's (21) and Cutter's (9) data. The flow diagram is identical to FLOWANL hence no flow diagram is given. The difference lies in reading th input data and initial calculation of $\bar{q}=f(z)$. In the case of NIELCUT the least square anlysis is performed on $\bar{v}_{r}=f(z)$. This causes a change in the criteria function hence subroutine PROC is different and was rewritten. The comment statements in the program closely follows the flow diagram of Figure F-1 and notes the differences as they occur. Program GRAPH. This program correlates the parameters of the tangential jet model. The main program is mainly data processing. Provision exists for getting correlations at constant impeller diameter or lumping all the data together. The regression is done by subroutine SOLVE. Subroutine SOLVE is designed to obtain three types of plots.
(1) $\log -\log$
(2) Semilog
(3) Rectangular

No flow diagram is given for GRAPH or SOLVE since the program 
is not complicated and is mainly used for processing the data. The reason for including a listing is to serve as a sample calculation so that the results quoted could be.checked if desired.

The following log-log correlations are obtained from GRAPH, and quoted in Chapter IV

(1) $\mathrm{ND}^{3}$ versus $A$

(2) $\mathrm{ND}^{3} /\left((D / 2)^{2}-a^{2}\right)^{\frac{1}{4}}$ versus $A$

(3) $\mathrm{N}_{\operatorname{Re}}$ versus $A$

(4) $\mathrm{ND}^{3}$ versus $Q$

(5) $\mathrm{N}_{\mathrm{Re}}$ versus $\mathrm{Q}$

Program RESULT: . Like Program GRAPH, RESULT processes data. It calculates experimental and calculated values of impeller discharge $Q$ and eddy viscosity $\epsilon$. Again a listing is given to serve as sample calculations. 


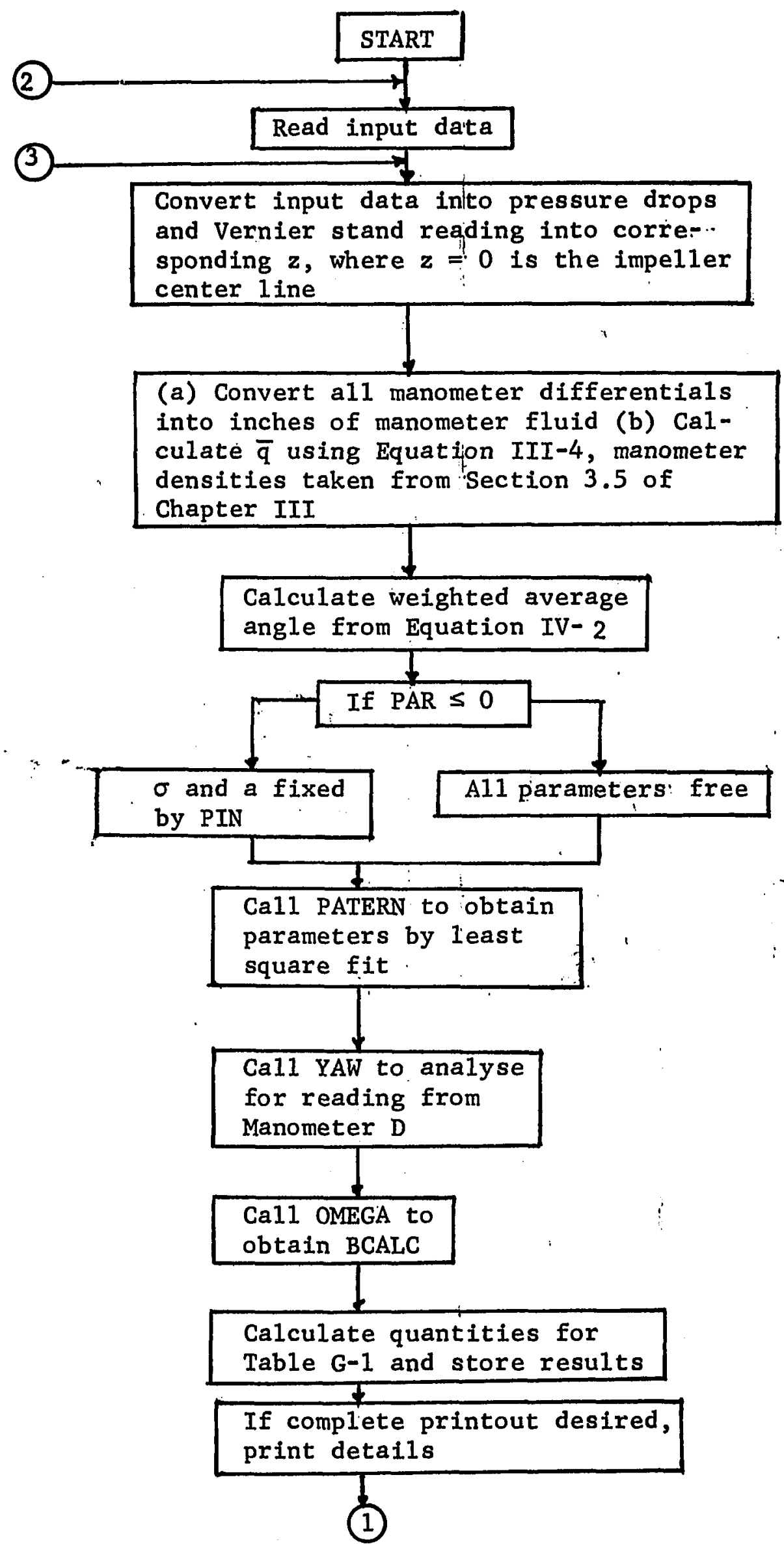




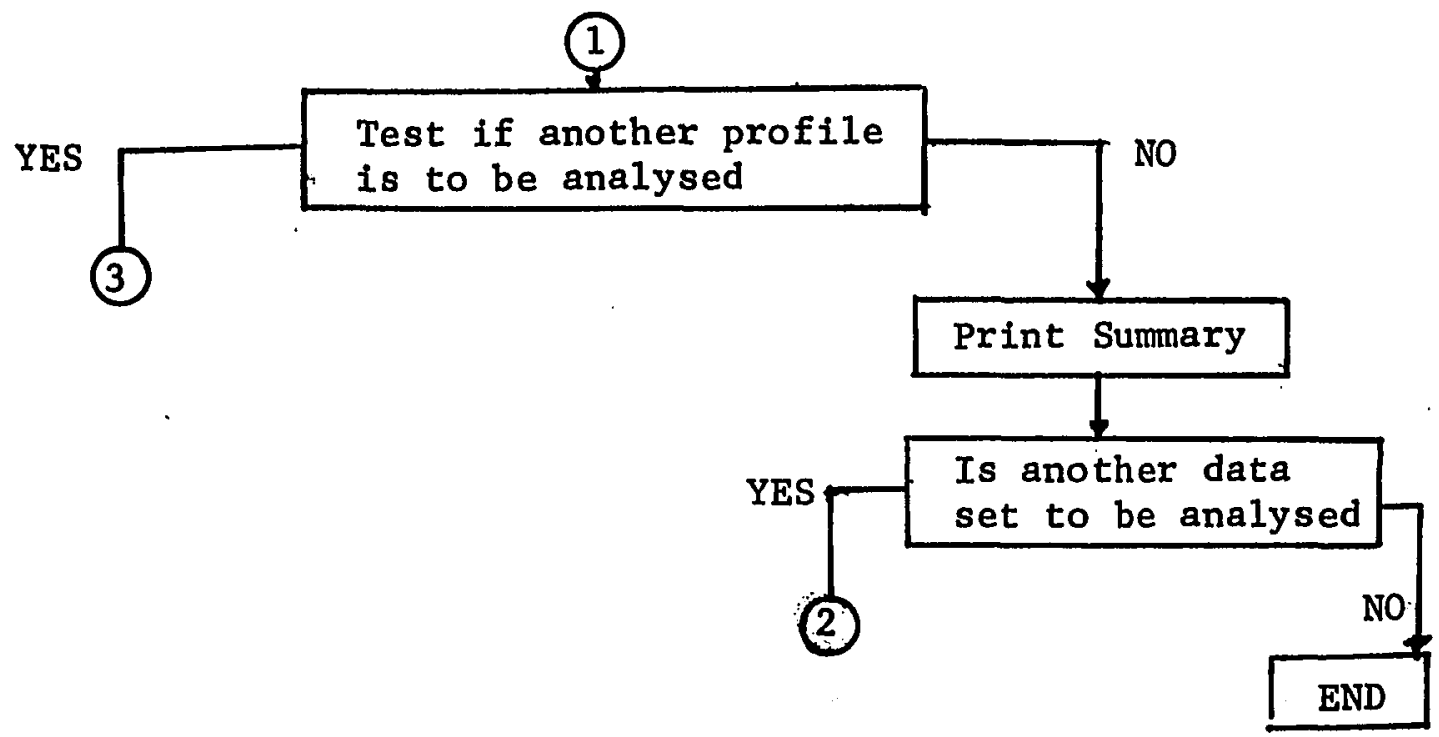

Figure F-1. Flow Diagram For Program FLOWANL. 


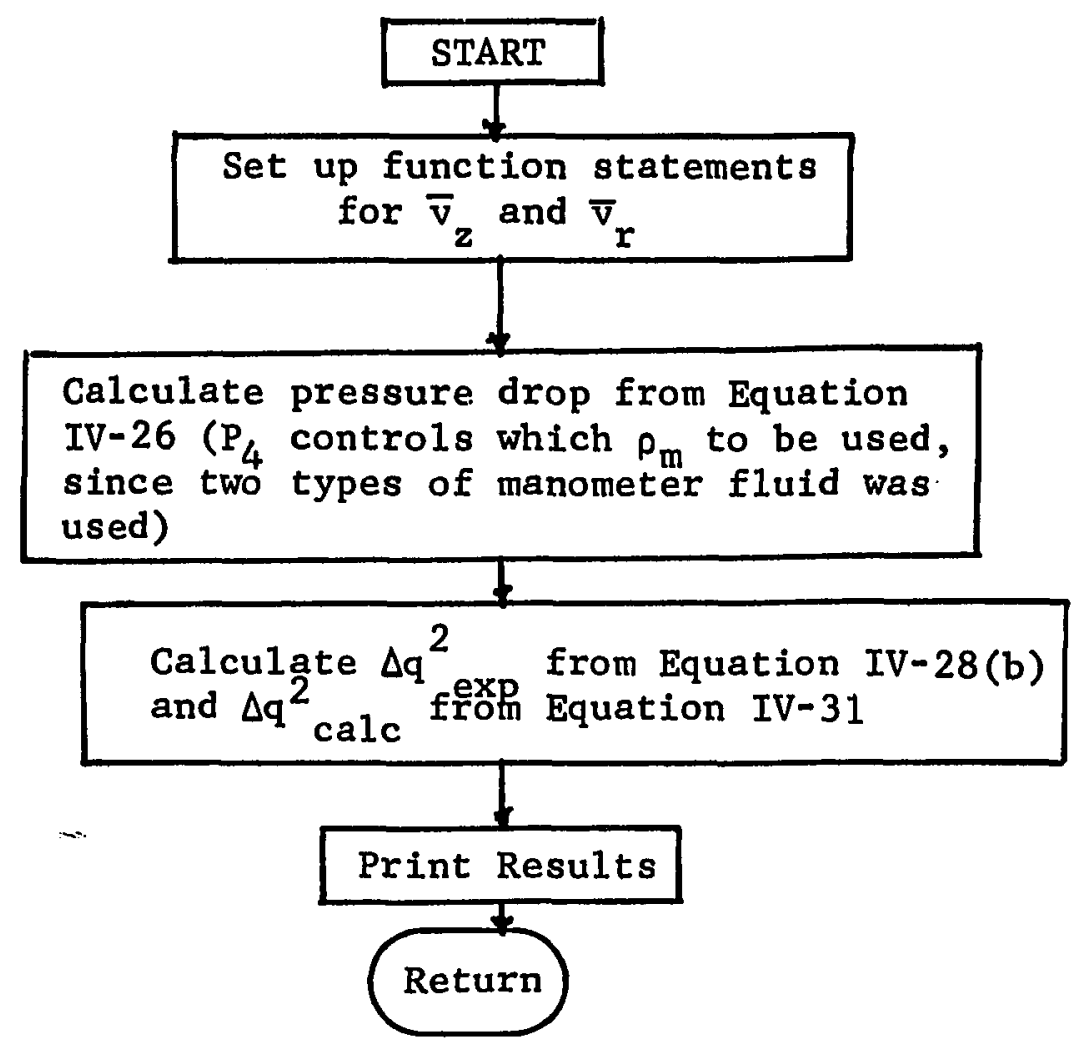

Figure F-2. Flow Diagram For Program YAW. 


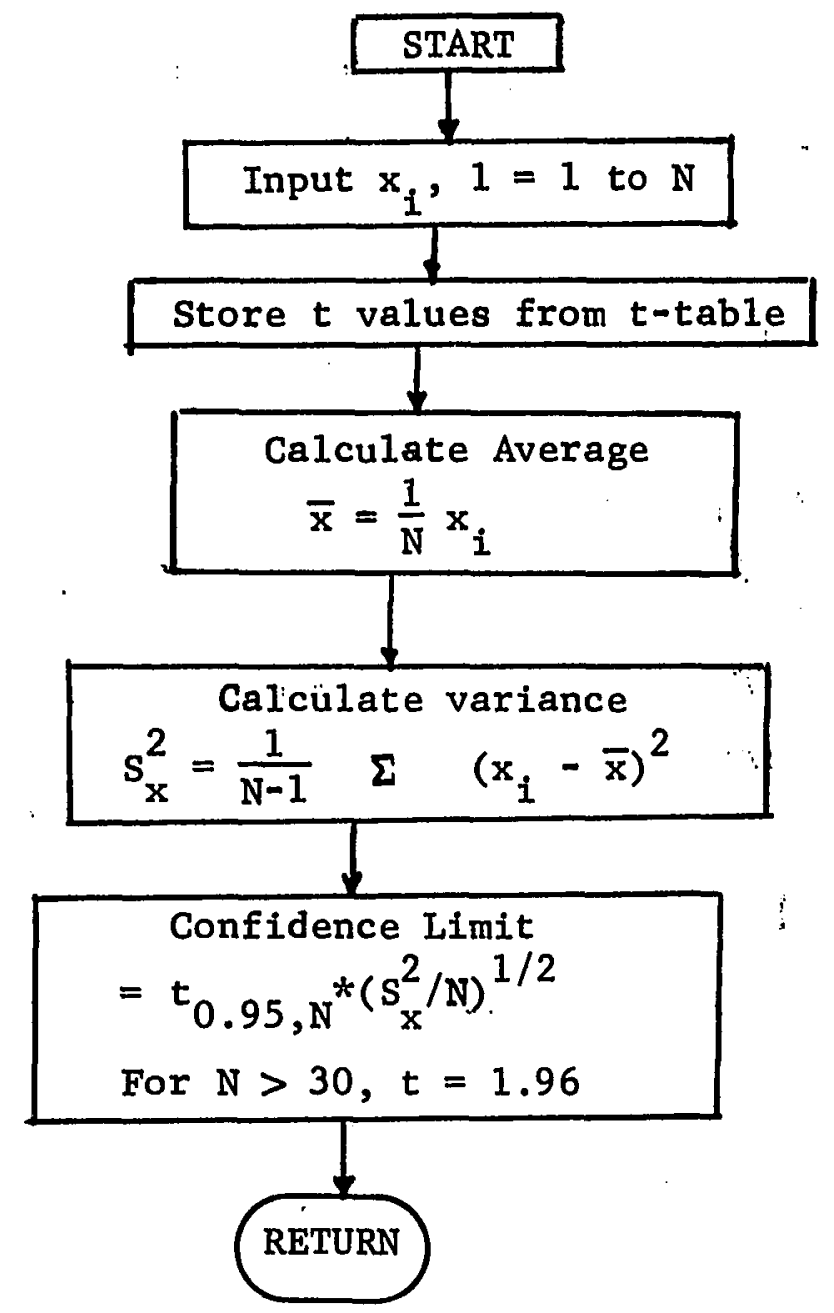

Figure F-3. Flow Diagram for Subroutine AVG. 
Read $\psi$ and corresponding $r$ at constant z. Store $\psi$ and $r$ for each profile in matrix $Q Q R R$, rowwise

Call POLY and obtain coefficients of polynomial of $\psi=f(r)$ at constant $z$ for each profile

Store coefficients of Polynomial in $C O$ and coefficients of polynomial derivative in $D R$ for each profile

Use DR to calculate $\bar{v}_{\text {}}$ at $r$ given in $R R$ ( $z$ constant) from Equätion IV-20. Store results for Port 1 in V1, Port 2 in V2 and Port 3 in V3.

$=2$ Selects z-plane corresponding to Ports 1, 2, and 3

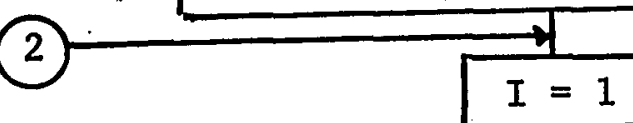

Calculate $\psi(R R(I))$ from $\mathrm{CO}$, store $\psi$ in $Q Q$ and corresponding $z$ of each profile in $\mathrm{ZZ}$.

Ca11 POLY obtain coefficients of polynomial $\psi=f(z)$ at constant $r$, store coefficients in $\mathrm{CZ}$ and coefficients of polynomial deriviative in $\mathrm{DZ}$

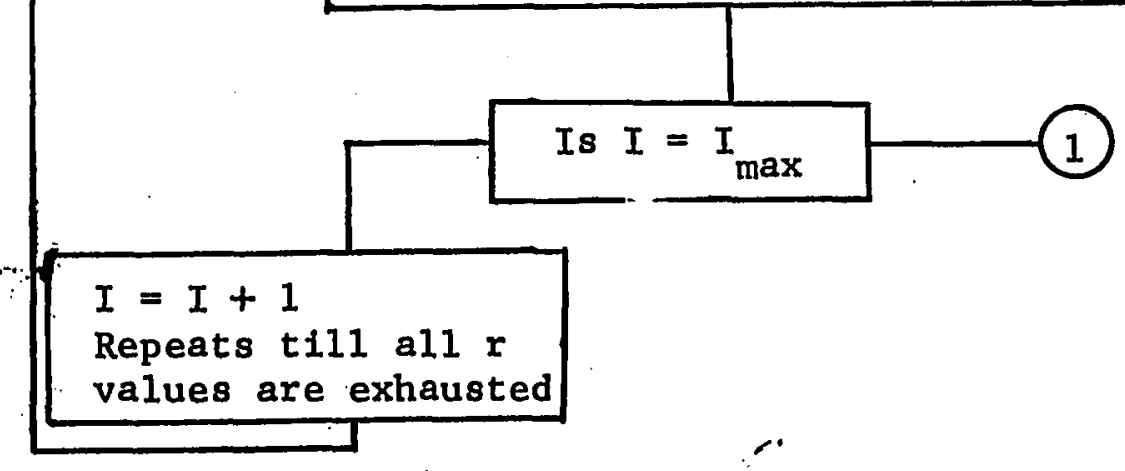




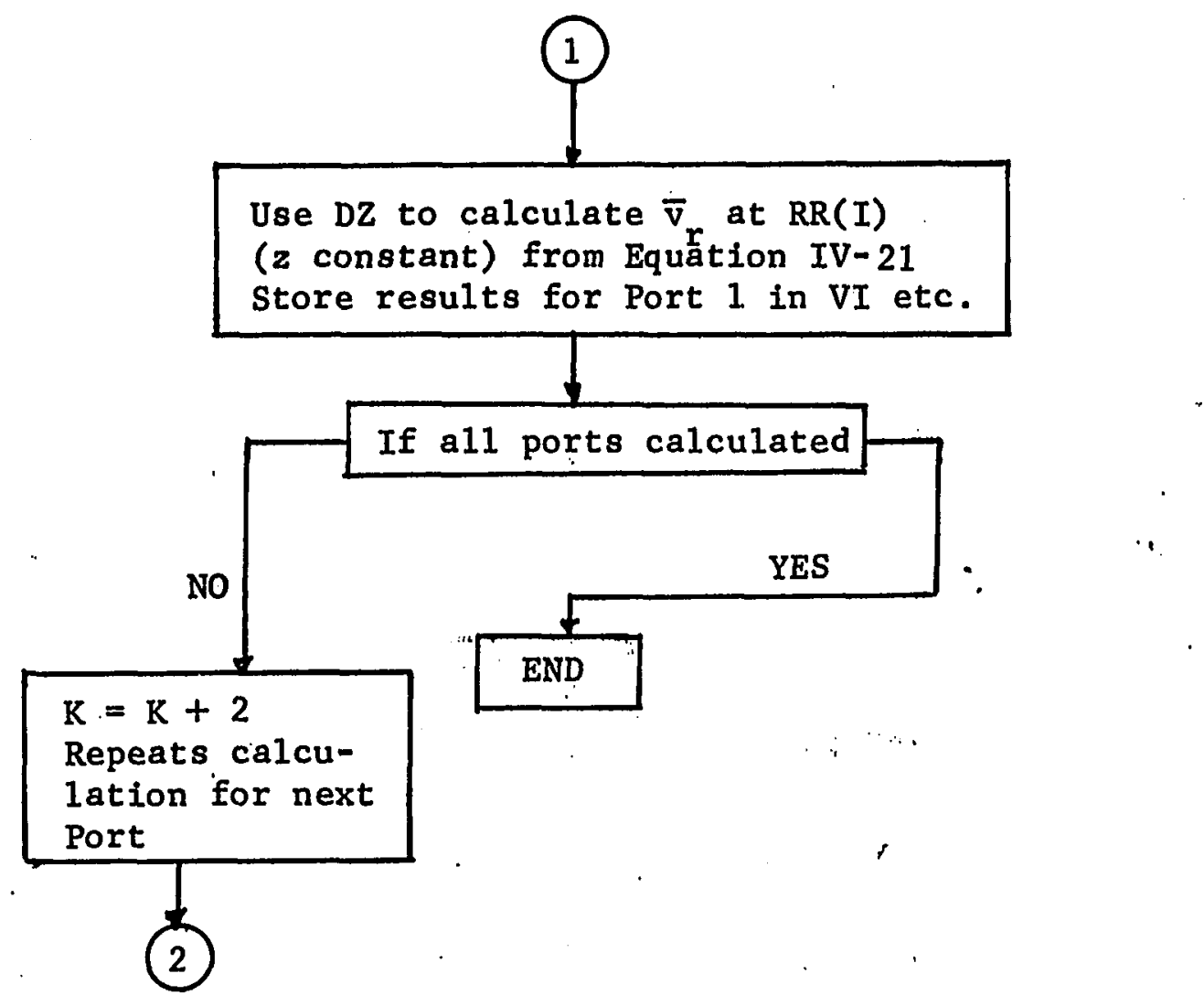

Figure F-4. Flow Diagram For Program VELPRO. 


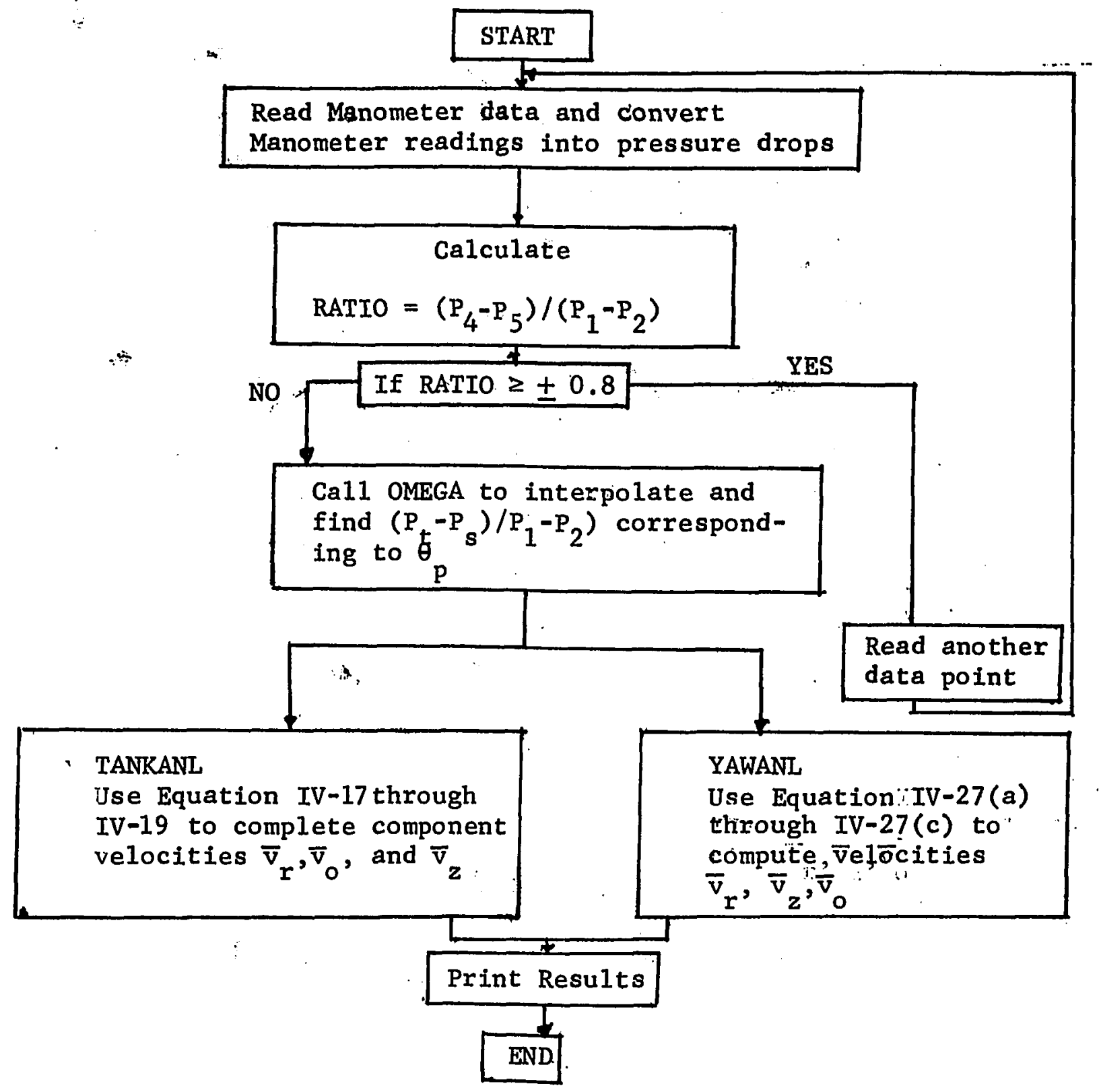

Figure F-5: Flow Diagram for Program TANKANL and YAWANL 
LIST $F-1$

FORTRAN LISTING PROGRAM FLOWANL WITH SUPPORTING SUBROUTINES YAW, AVG, PROC, BOUNDS, OMEGA

AND PATERN. THIS LISTING ALSO GIVES RAW DATA WHICH IS THE INPUT TO THE PROGRAM AND A

DETAILED SAMPLE OUTPUT FOR RUN NUMBER 29.

MAIN PROGRAM FLOWANL.

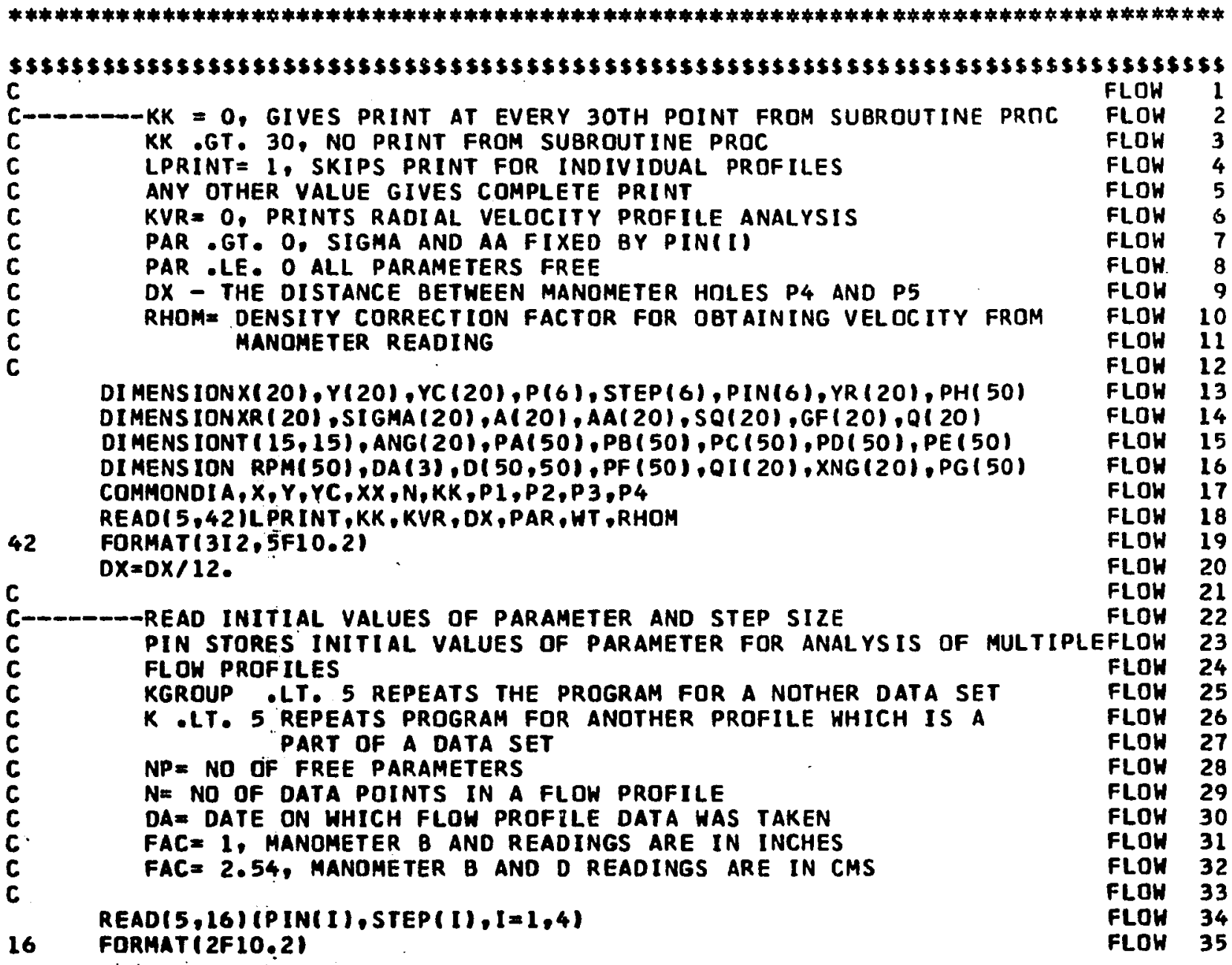




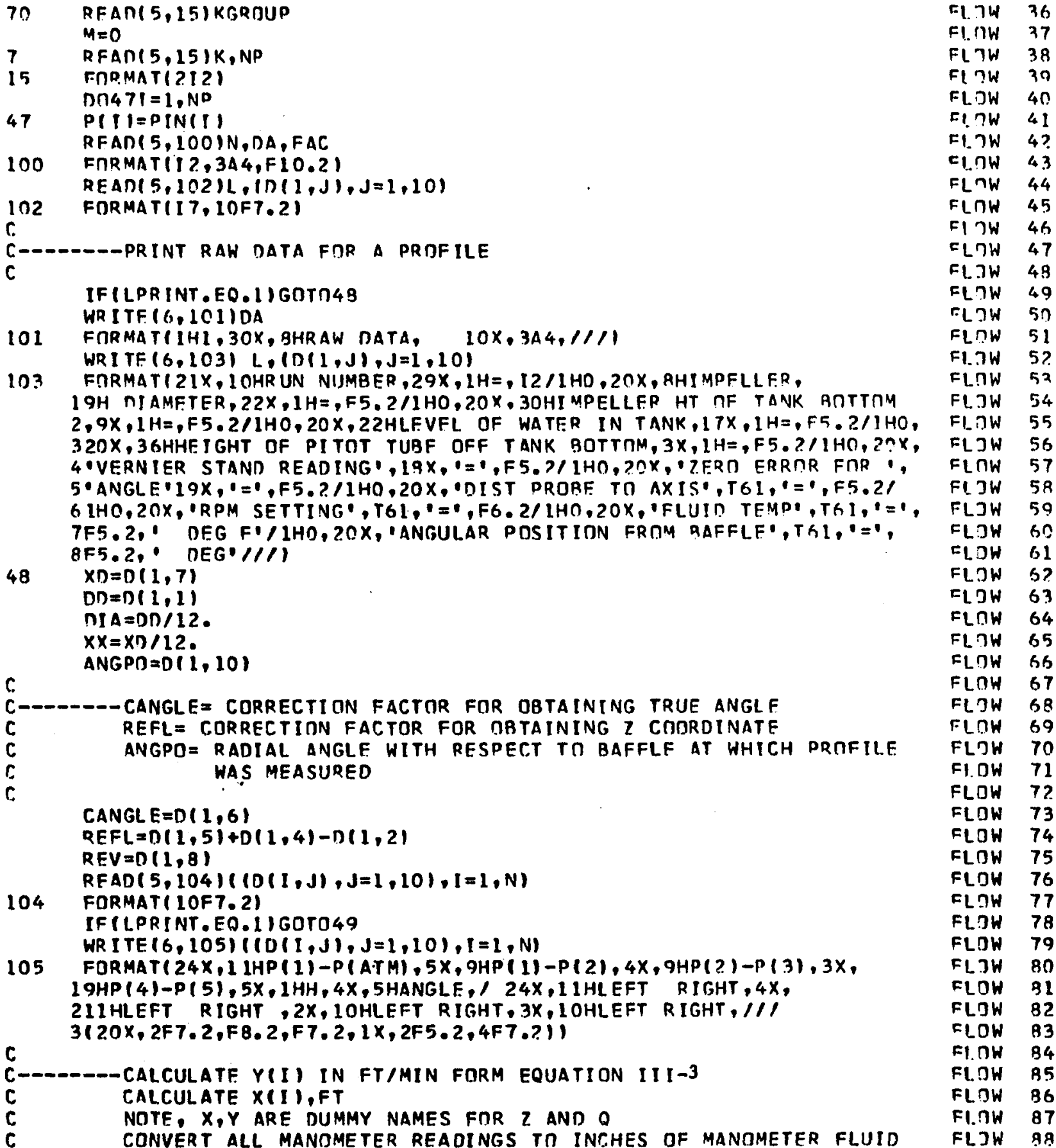


C.

$49 \quad D \Pi 106 I=1, N$

$X(I)=(R E F L-D(I, 9)) / 12$.

$D(I, 10)=D(I, 10)-C$.ANGI E

DFL $Z=n(1,4)-D(I, 3)$

IFIFAC .FA - 1 . IGOTRIOG

DEL $Z=D E L T . / F A C$

$D(1,3)=D(1,3) / F A C$

$n(1,4)=D(1,4) / F A C$

$D(1,7)=D(1,7) / F A C$

$D(1, B)=D(1,8) / F A C$

$106 Y$ YII $=$ SART $(2 . * 32.174 *$ DFL $7 *$ RHOM $/ 12.1 * 60$.

c.

r.-----CALCULATE WEIGHTEO AVERAGE ANGLF AND AVERAGE ANGLE

C WT = RATIO OF VELOCITIES FOR WHICH WEIGHT IS ZFRO, CHOSFN AS 0.7

C YM= AVERAGE VALUE DF $Y$

C.

$Y M=0$

$\triangle N G L E=0$.

$N K=N /$ ?

$\triangle N G L=0$.

$Y M M=0$.

$M=M+1$

$\operatorname{Do2} 2 \mathrm{I}=1, \mathrm{~N}$

IFIIYIII/YINKI) -LT.WT IGOTO34

$A N G L=A N G L+D(I, 10) \neq Y(I)$

$Y M M=Y M M+Y(I)$

$34 \quad$ ANGLE $=A N G L E+D(1,10)$

2?. $Y M=Y M+Y(I)$

ANGLE = ANGLE /FL NAT (N)

$Y M=Y M / F L$ MAT $(N)$

$A N G L=A N G L / Y M M$

C

C-D-DIGIC. BRANCH FOR ONE DIMENSIONAL SEARCH ON PARAMETER P(?)

C OTHFR PARAMETERS FIXED THROUGH PIN

C

IFIPAR I51,51,52

$52 \quad P I=P I N(1)$

$P 2=P I N(2)$

$P 3=P[N(3)$

$P 4=P$ IN $(4)$

51 G0T053

$51 \quad P 1=0$.

$P 3=\quad X X * S I N(A N G L * 3.1416 / 180.1$

IF (P3 .GT \& ( DIA/2 \&) ) GOTD60

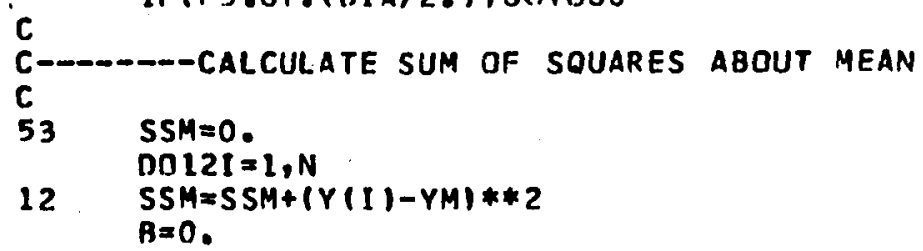

FL.TW 89

FInW on

CLDW 91

FInW 9?

CI.?W 93

FL?W 94

FLJW 95

FINW 96

FL.TW 97

FLIN 98

FL.TW 90

FLJW ID?

FIONW InI

FLOW 102

FLOW 103

FlOW 104

FLnW 105

FLOW 106

FIOW InT

FLOW 108

FLOW 109

FLDW 110

FLOW 111

FLDW 112

FLOW 113

FL:IW 114

FLOW 115

FL.OW 116

FLDW 117

FLOW 119

FLOW 119

FLOW 120

FLOW 121

FLOW 122

FLTW 123

FLOW 124

FLDW 125

FLDW 126

FLDW 127

FLOW 128

FLOW 129

FI. OW 130

FLDW 131

FLOW 132

FLOW 133

FLOW 134

FLDW 135

FLOW 136

FLOW 137

FLOW 138

FLDW 139

FLDW 140

FLOW 141 


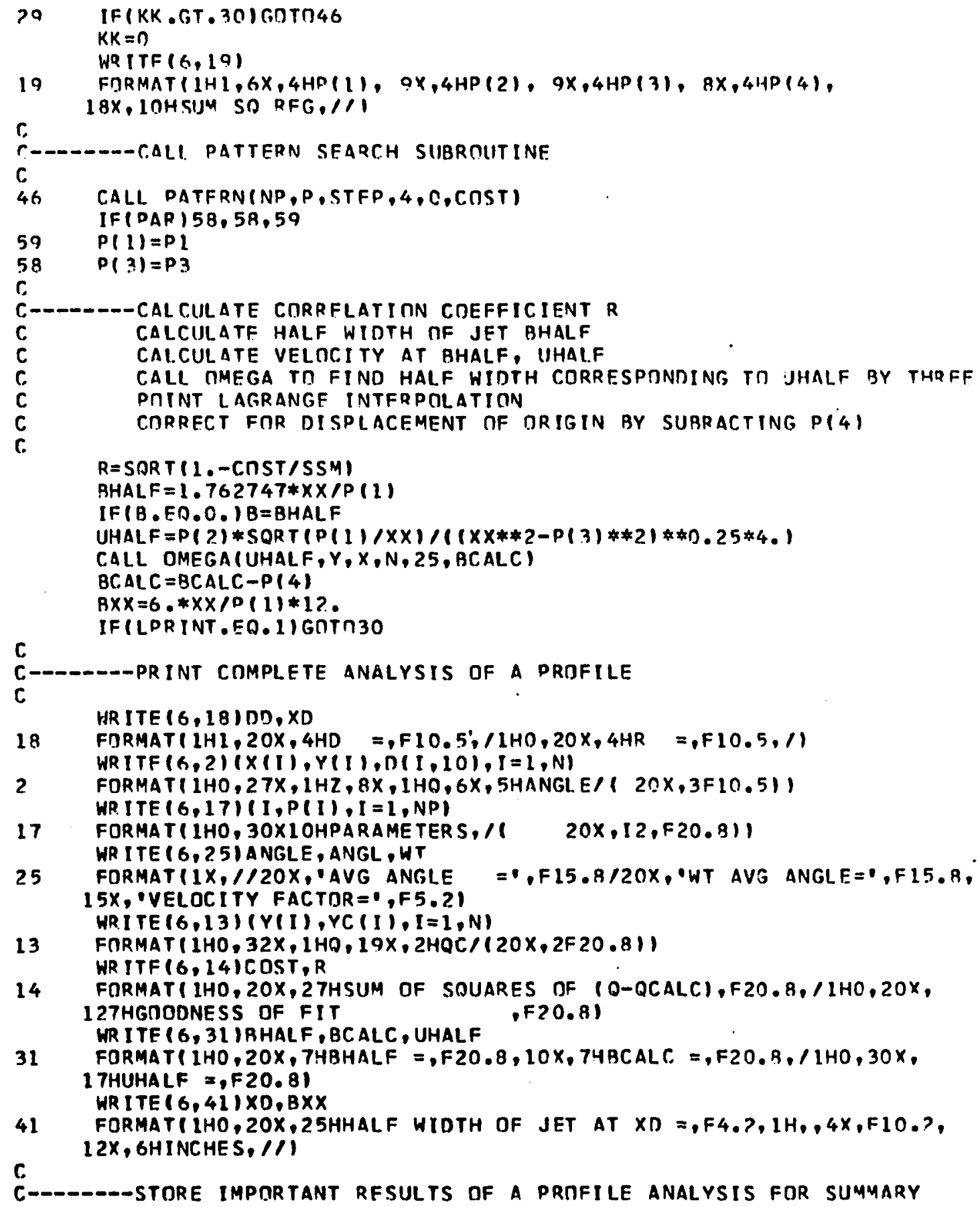

FLJW 14?

FI. 7 W 143

FI 194144

FLOW 145

FLDW 146

ELTW 147

FLOW 149

FLNW 147

FLOW 150

FI.7W 151

FLDW 152

FI.NW 153

FL TW 154

FI. TW 155

FLר! 156

FLIN 157

FLOW 158

FLnW 159

FLOW 160

FL.JW 161

FLOW 16?

FLnW 163

FLDW 164

FLnW 165

FLJW 166

FLTW 167

FLOW 168

FLDW 160

FLOW 170

FLNW 171

FLNW 172

FLOW 17?

FLOW 174

FLOW 175

FLOW 176

FLJW 177

FLOW 178

FLOH 179

FL. 180

FL.OW 181

FLOW 192

FLDW 183

FLOW 184

FLOH 185

FLJW 186

FLnH 187

FLDH 188

FLOH 189

FLDW 190

ELDW 191

FLJW 192

FL.DW 193

FLnW 194 
$r$

$30 \quad A N G(M)=\operatorname{SQR} T(x \times * 2-D(3) * 2) / X X$

$P A(M)=A R C D S(A N G(M)) * 180.13 .1416$

$37 \quad Q(M)=4.3 .1416 * P(?) *(X X * * 2-P(3) * 2) * 0.25 * S \cap R T(X X / P(1))$

$X P .|M|=\times n$

$\operatorname{SIGMA}(M)=P(1)$

$A(M)=P(?)$

$A A(M)=P(3)$

$S Q(M)=C N S T$

GF $(M)=R$

$P B(M)=P(4)$

$P C(M)=$ BHALF

$R P M(M)=R E V * D[A * 3$

$P D(M)=B C . A L C$.

$\operatorname{DF}(M)=A N G L F$

$P F(M)=L H A L F$

$X N G(M)=A N G P O$

$X T=D I A / 2$.

TEMP $=4 * 3.1416 * P(2) *(X T * 2-P(3) * 2) * 0.25 * S \cap R T(X T / P(1))$

$Q I(M)=T F M P$

$P 4=K$

C

C- - - CALL YAW TO ANALYSE FDR DATA FRTM MANDMETER D$$
\text { C }
$$$$
\text { C }
$$$$
\text { IF (LPR INT - EQ. } 1 \text { I GOTO62 }
$$

C CALL YAWID,P,DXI

C

62 VRMAX $=P(2) / 2 *$ SQRT $(P(1) / X X * * 3) *(x \times * * 2-P(3) * 2) * * 0.25$ TFMP $=P(1) /(2 \cdot * X X)$ $D 063 I=1, N$

$A L P=D(I, 101 * 3.1416 / 180$.

$Y(I)=Y(I) * C \cap S(A L P)$

6.3 YR(I) $=$ VRMAX*(1.-(TANH $(T E M P *(X(I)-P(4))) * * 2)$

CAIL AVGIY,N,YM, AR,BBI

$S S M=0$.

$S S R=0$.

D064I $=1, N$

$S S R=S S R+(Y R(I)-Y(I)) * 2$

64 SSM $* S S M+(Y(I)-Y M) * * 2$.

COST $=S S R$

IFISSR.GT.SSM)GOTO6:

$R=S Q R T(1 .-S S R / S S M)$

$68 \quad R=0$.

67 FORMAT(IHO, 20X,'SSR = , F 15.8/20X, SSM = , F $15.8 /)$

$69 \quad P G(M)=R$

$P H(M)=S S R$

IF (KVR.EQ. I) GOT044

WR ITE $(6,65)$

65 FDRMATPIHI, $/ / / /, 30 X$, RADIAL VELOCITY ANALYSIS $/ / 1$
FI.7W 195

rinW Ios

FI NW 197

FLITW 199

FLJW 193

FI IN ? OO

FI.רW ?? I

finW ?ח?

FLJW 2!?

FI TW 204

FLOW 205

FI. TW 206

FLJW ?0?

FI.TW 209

Fl. DW ? 09

FL.JW ? 10

cinW 211

FLOW 21 ?

FLDW $2: 13$

FL ?W ? 14

FLnW 215

FI. OW ? 16

FLOW 217

FLJW ?218

FLOW $>19$

FI. JW 2?

FLOW 221

FLnW 22 ?

FLDW ??3

FLOW ?.24

FLTH 2.25

FLIN 2.26

FLOW 2.27

FI.OW 228

FLOW ??29

FLTW 230

FLnW 231

FLOW 232

FLOW 233

FLDW 234

FLIN 235

FLOW ?36

FLON 237

FLOW 238

FLnW 239

FLDW 240

FLOW 241

FLOW 2.42

FLnW 243

FLOW 244

FI. TW 245

FLOW 246

FI.NW 247 


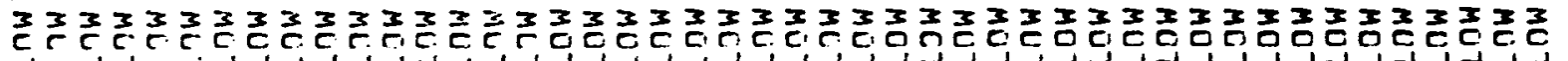

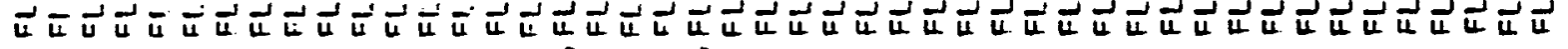

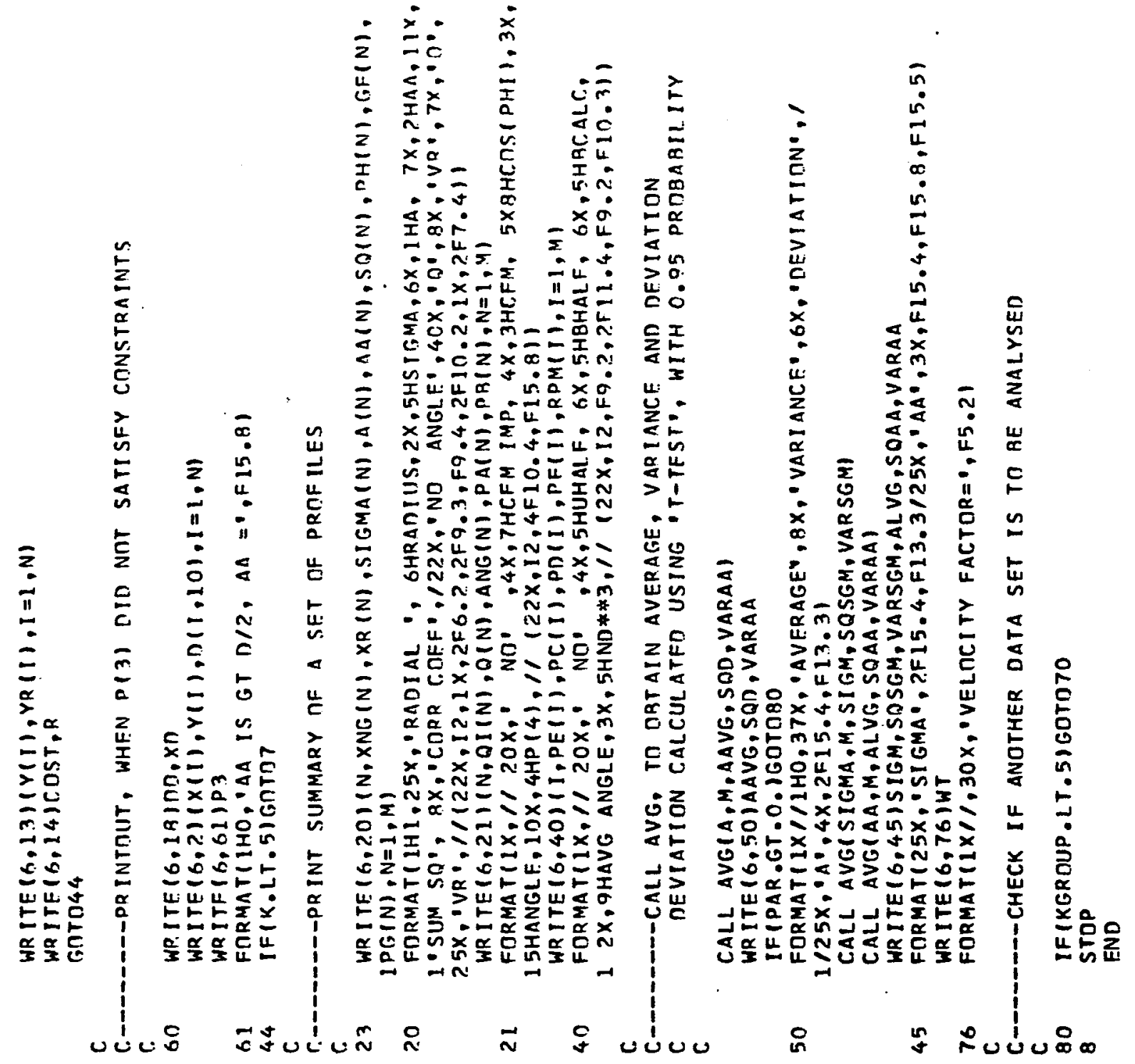

$$
\text { a d o d T }
$$


SUBPIUTINE YAW(D,P,DX) Y YAW 1

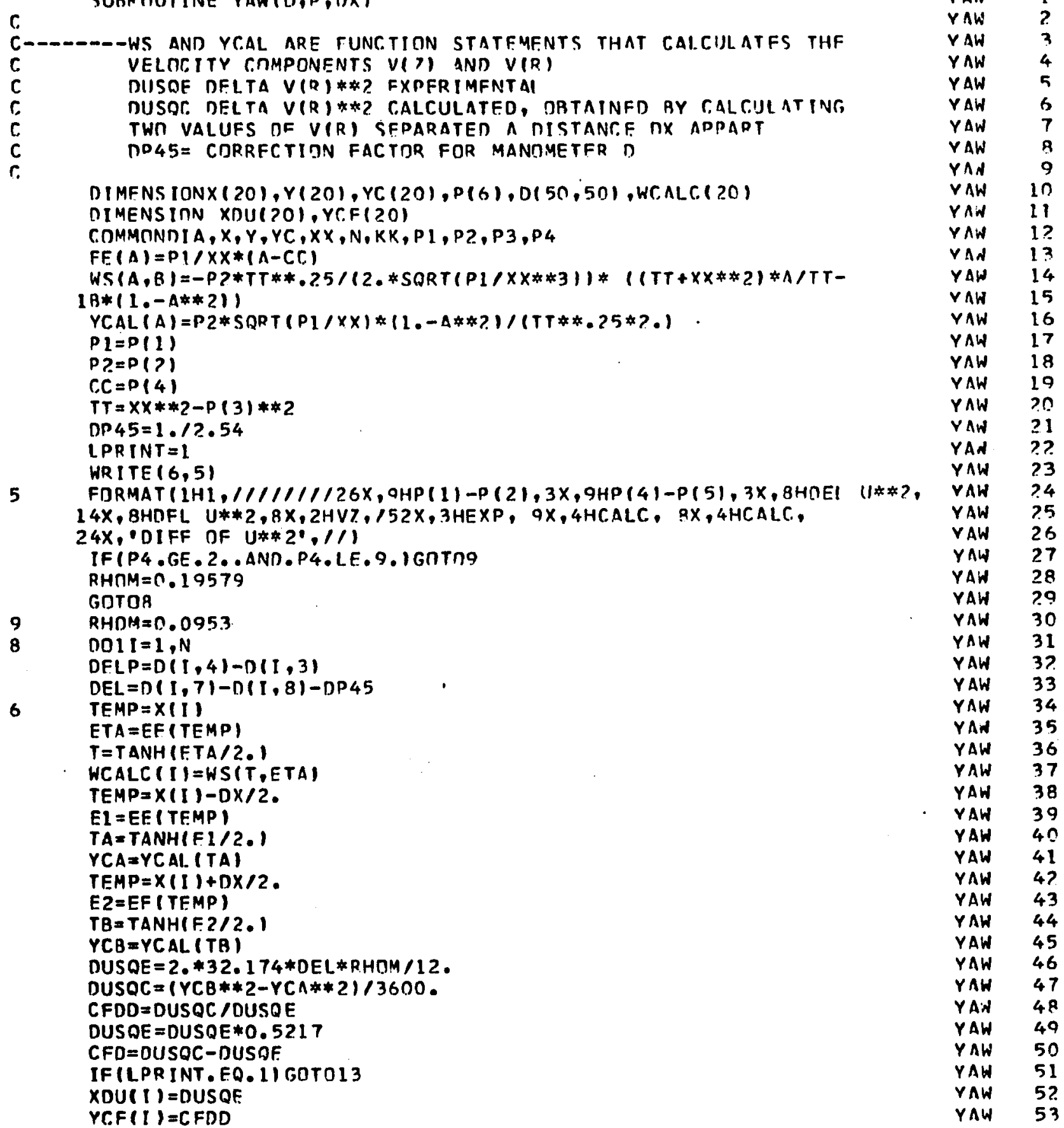



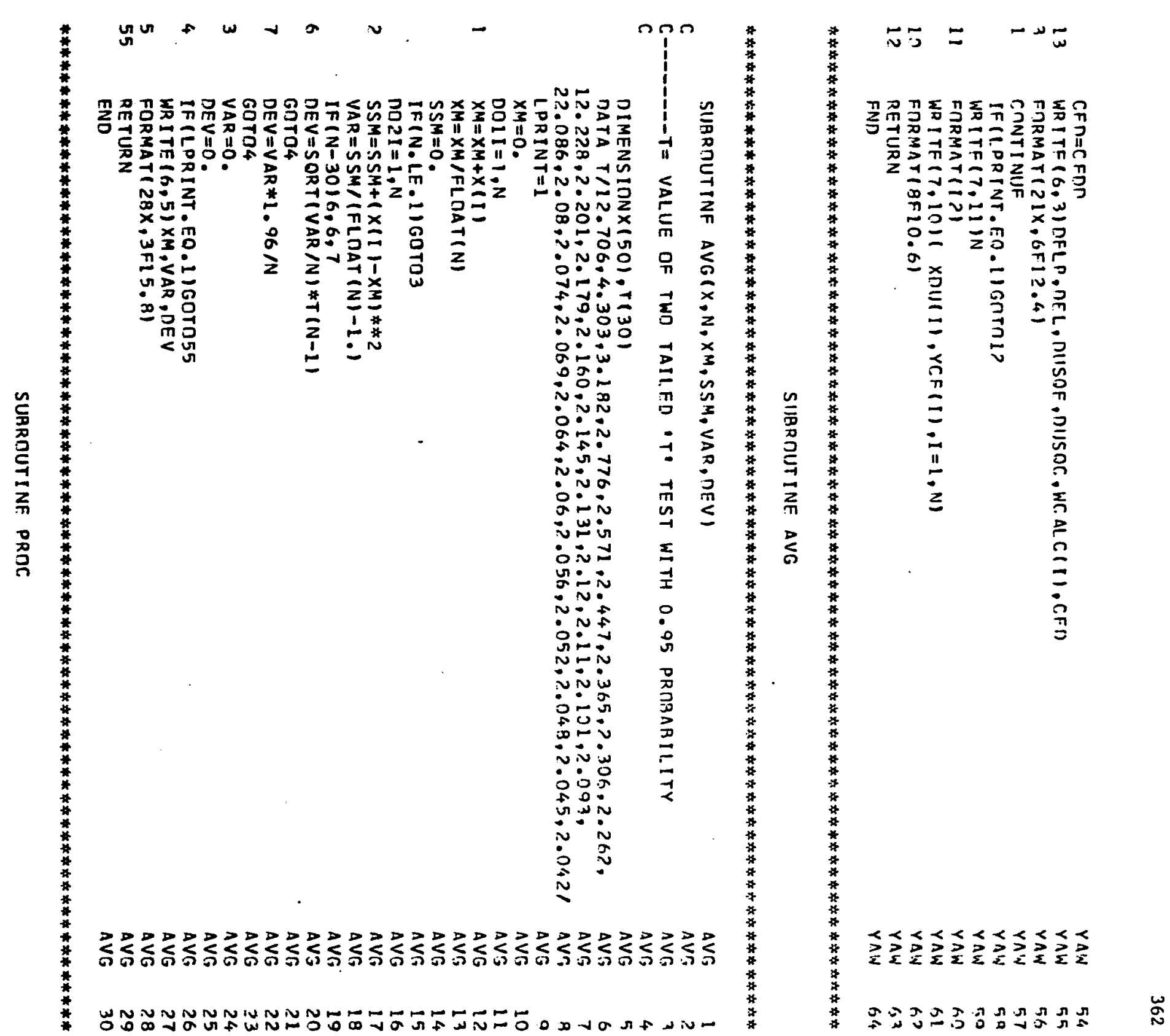


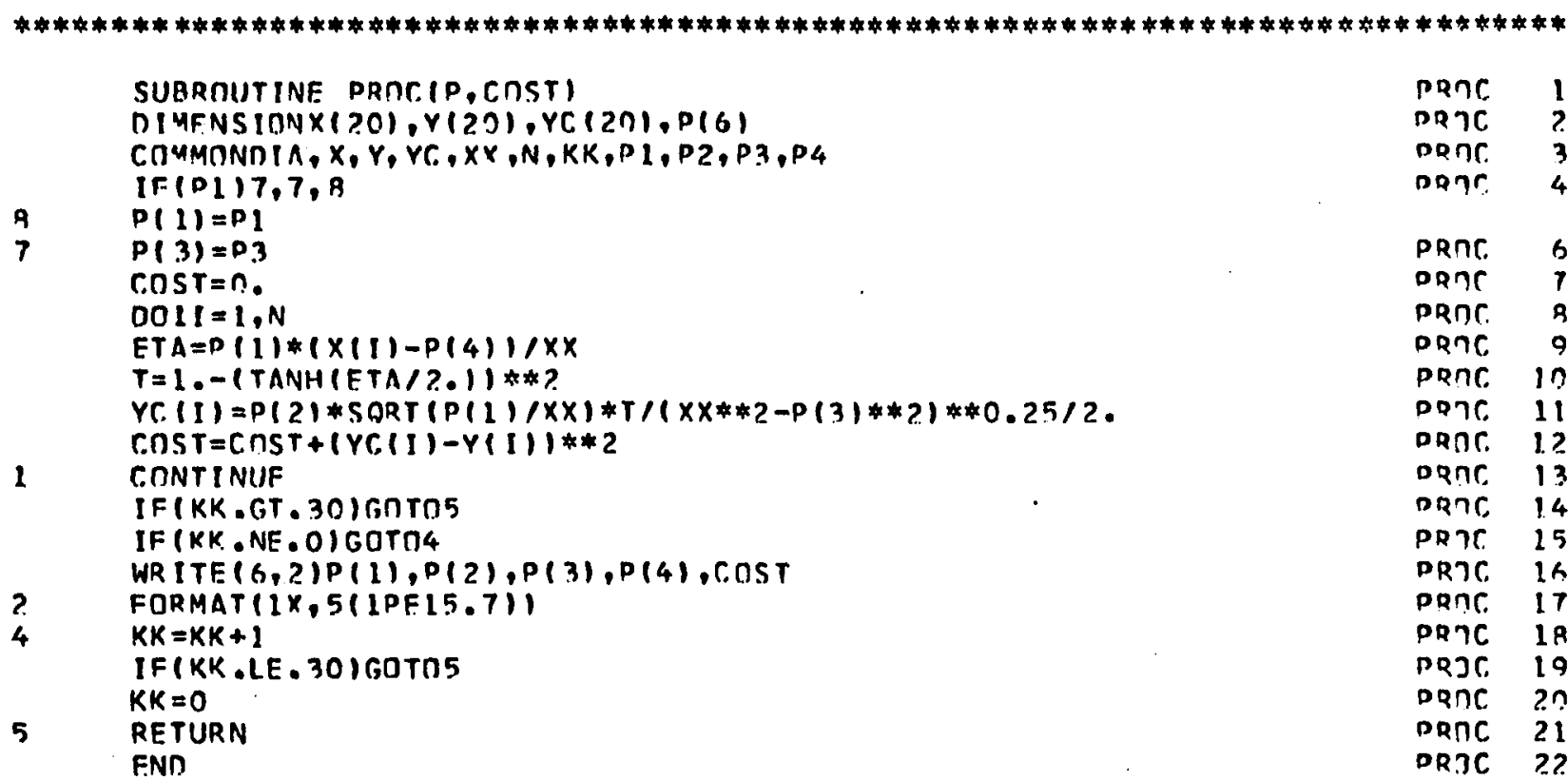

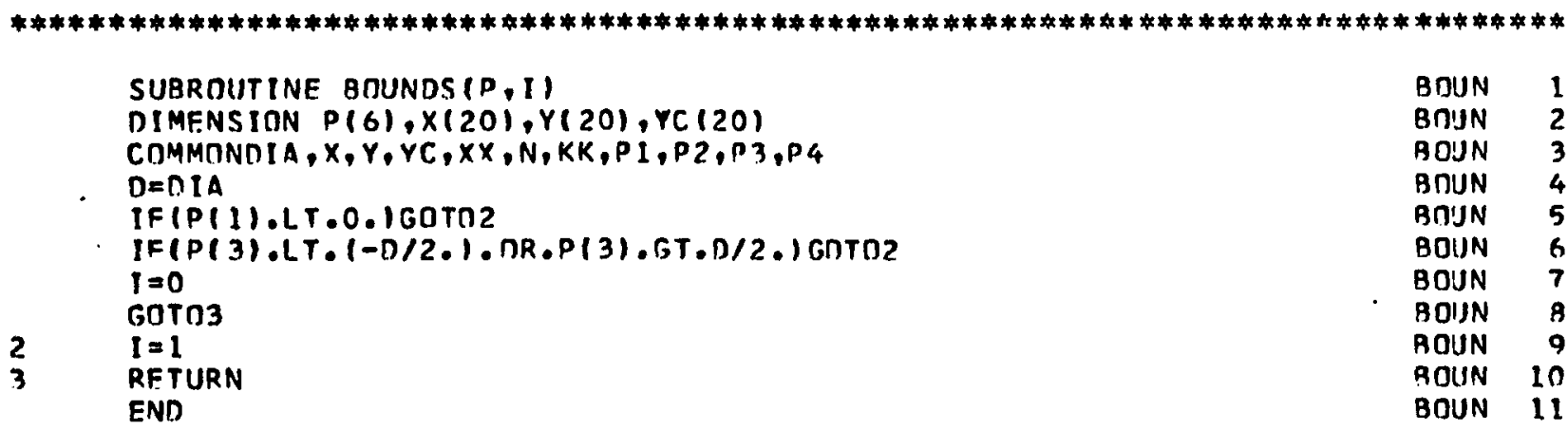

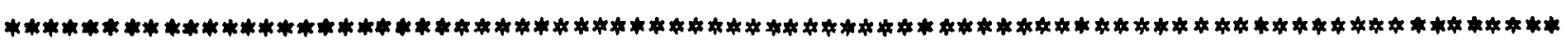
SUBROUTINE OMEGA

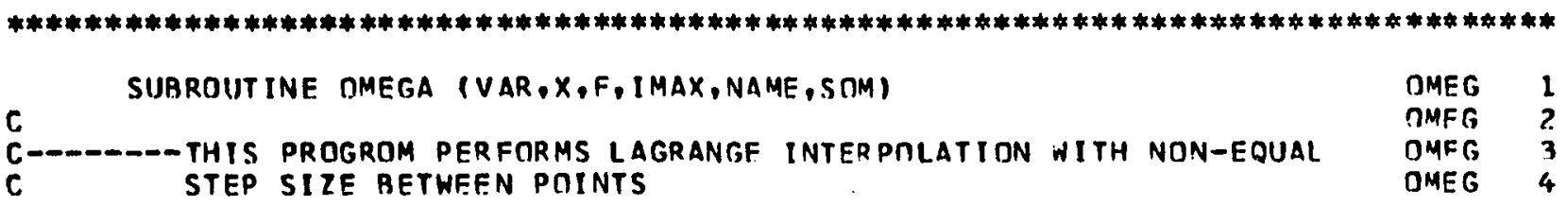




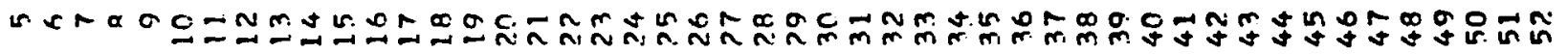

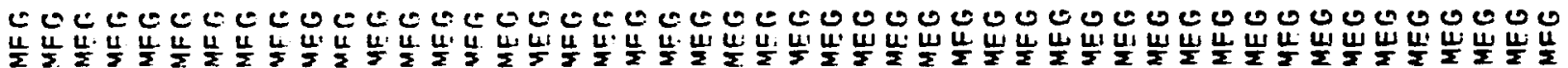

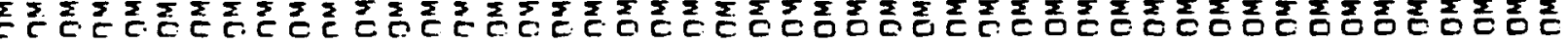

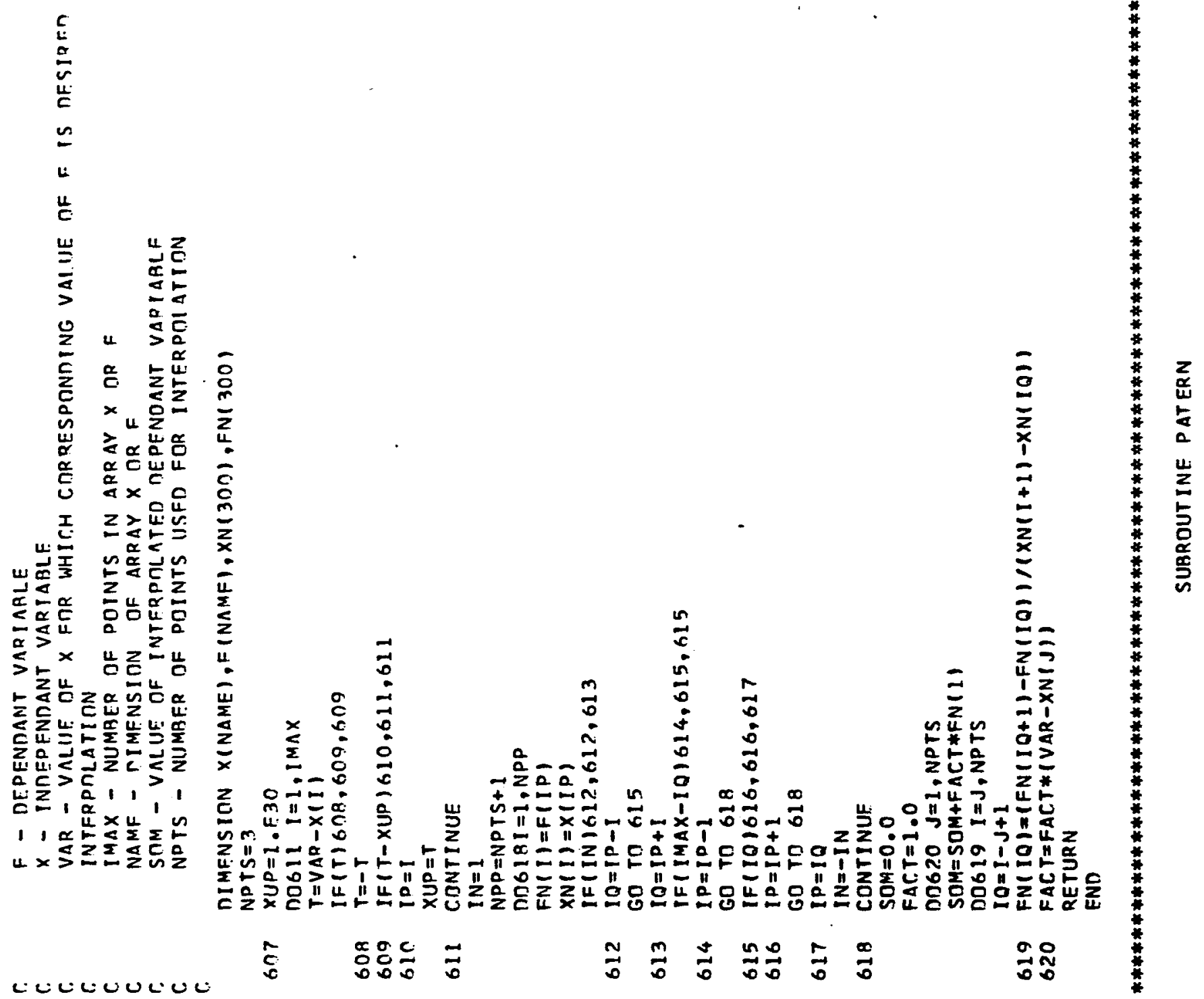




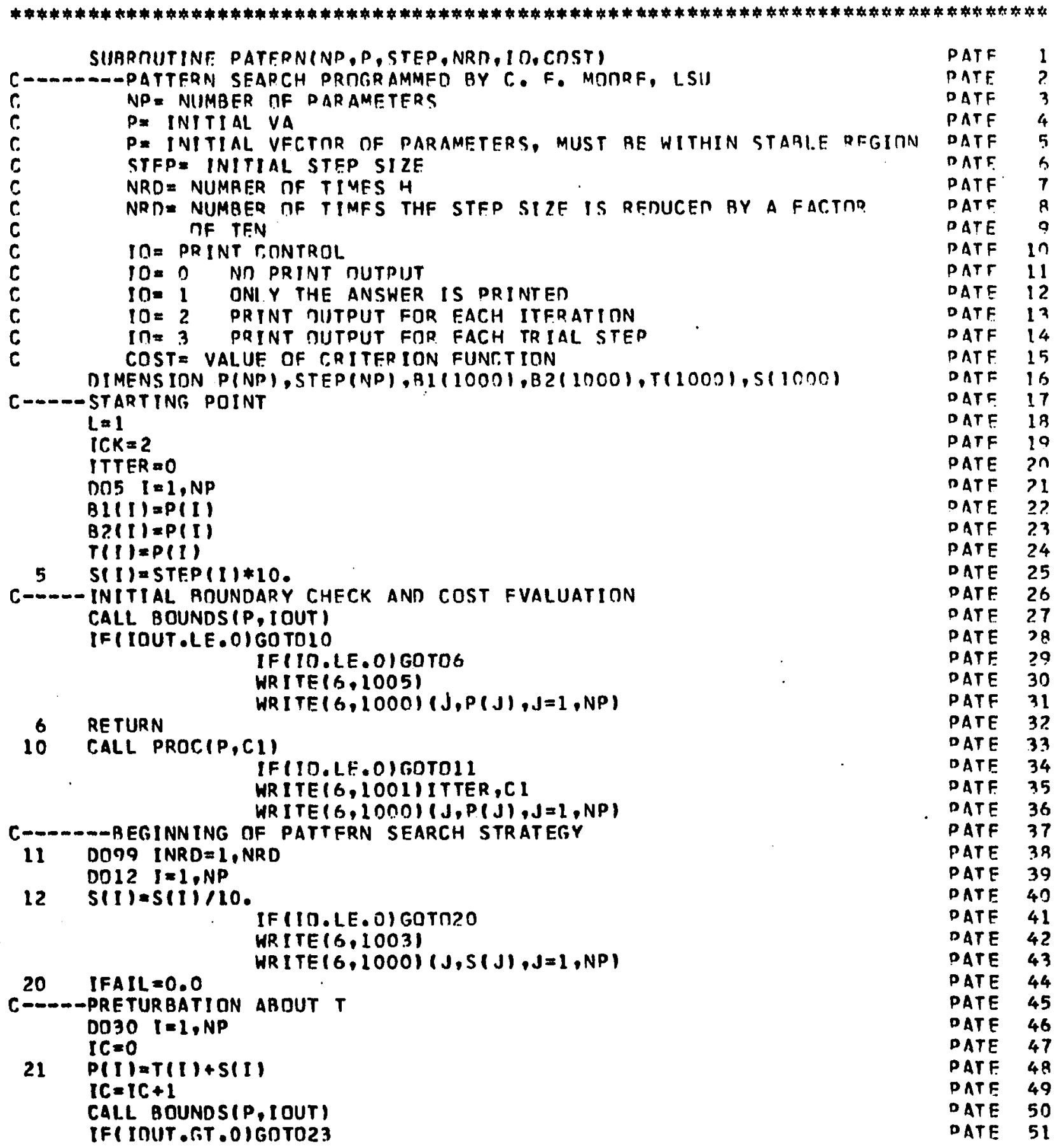




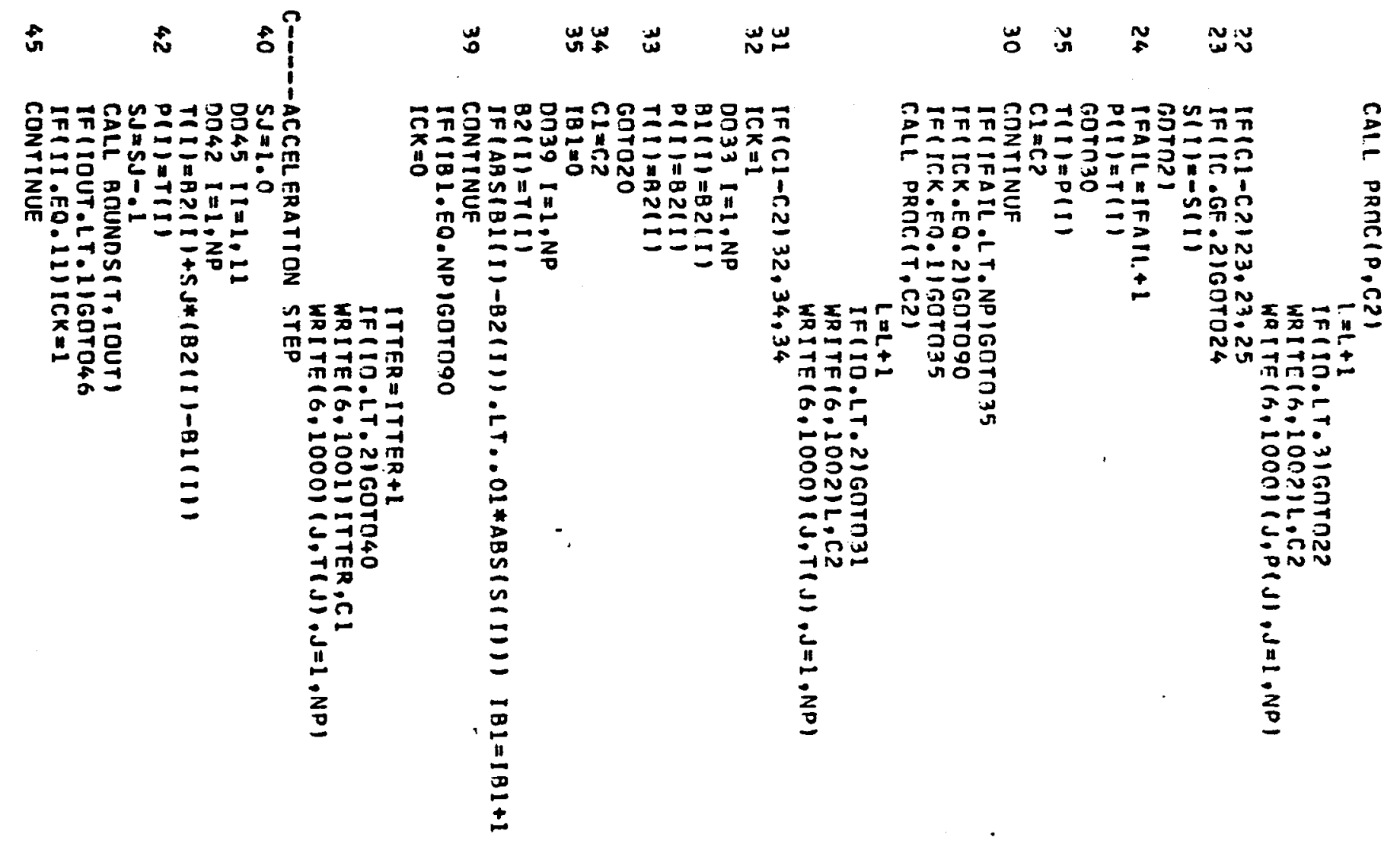

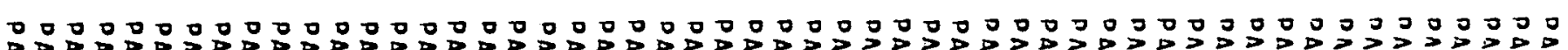

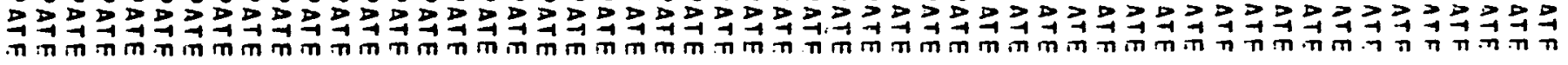

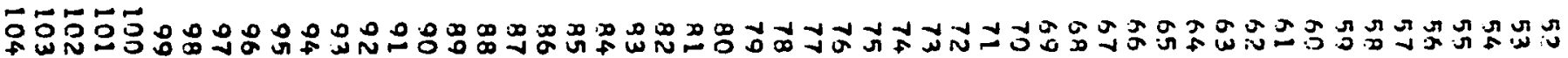




\begin{tabular}{|c|c|}
\hline $\begin{array}{l}46 \\
47\end{array}$ & $\begin{array}{l}D \cap 47 \quad I=1, N P \\
B I(I)=B 2(I) \\
\text { GNTO20 }\end{array}$ \\
\hline 9 & $D \cap 91 \quad I=1, N P$ \\
\hline & $\begin{array}{l}T(I)=R 2(I) \\
\text { CONTINUE: }\end{array}$ \\
\hline חפח & $\begin{array}{l}D \cap 100 \quad I=1, N P \\
P(I)=T(I) \\
C \cap S T=C I\end{array}$ \\
\hline
\end{tabular}

\section{RFTIIRN}

IF (IO.LE.OIRETURN

WR I TE $(6,1004) L, C I$

WRITE $(6,1,000)(J, P(J), J=L, N P)$

1000 FORMAT(10X,5(17,E13.6)/1)

1001 FORMATI/IIXI4HITTERATION NO. ,I5/5X,5HCOST=,E15.6,?DX, 1 INHPARAMFTFRSI

INO? FORMAT(1OX3HNO. I $14,8 \times 5 H C O S T=, E 15.6)$

1003

FTRMAT //IX?RHSTFP SIZE FOR EACH PARAMETER,

1004

FORMAT I IHI 13 3HANSWERS AFTER, I $3,2 X, 23$ HFUNCT IONAL
1 5X5HCOST=,E15,6, 20X, I BHOPTIMAL PARAMETERS I

1005 FORMATIIHI35HINITIAL PARAMETERS OUT OF BUUNDS END
PATF 105

DATE lng

DATF 107

DATE Ing

Data ino

Date llo

DATF 111

DATF 11?

PATF 113

DATE 114

PATF 115

DATE 116

DATF 117

PATF IIR

PATE 119

DATE 1 ?

PATE 1?:

PATF $1 ? 2$

I) PATE 123

DATE 124

onTE 125

PATF $1 \geq 6$

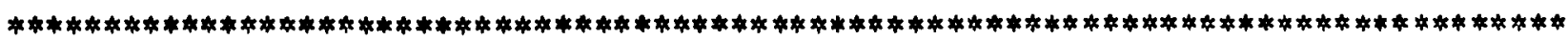
INPUT DATA

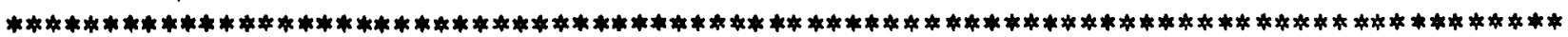

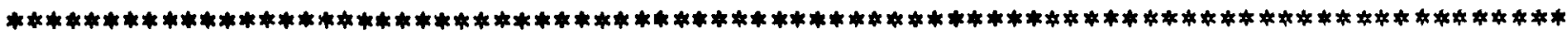

CONTROL PARAMETERS TO GBTAIN CONDFNSED TABLES AF APPENDIX G

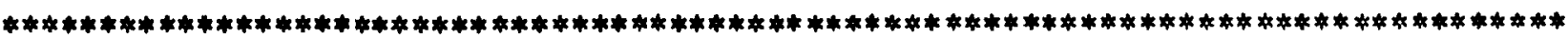
$\begin{array}{llll}140 & 1 & 0.1015 & 1\end{array}$
0.5
0.19579

11.121

0.5

0.5

$0.1147 \quad 0.01$

$0.001 \quad 0.0001$

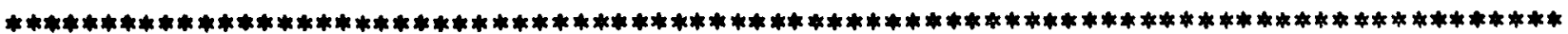

CONTROL PARAMETERS TO OBTAIN DETAIL PRINTOUT DF SECTION 4.4

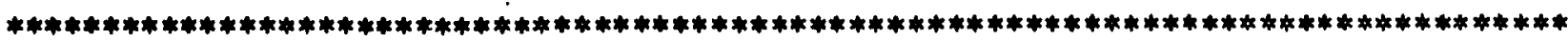
240
0.10150
0.5
0.19579 


$\begin{array}{ll}11.121 & 0.5 \\ 18.0 & 0.5 \\ 0.1147 & 0.01 \\ 0.001 & 0.0001\end{array}$

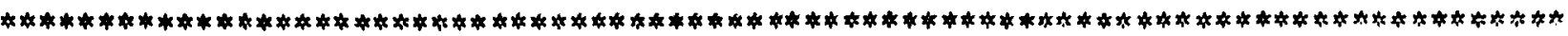

VELICITY PROFILES MFASURFD AT 2.43 RDM

TANK DIAMETFR $=12.25$ IN.

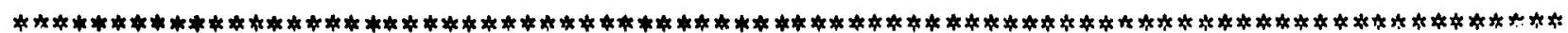

1

14

LINOV 22, 1969 1 .

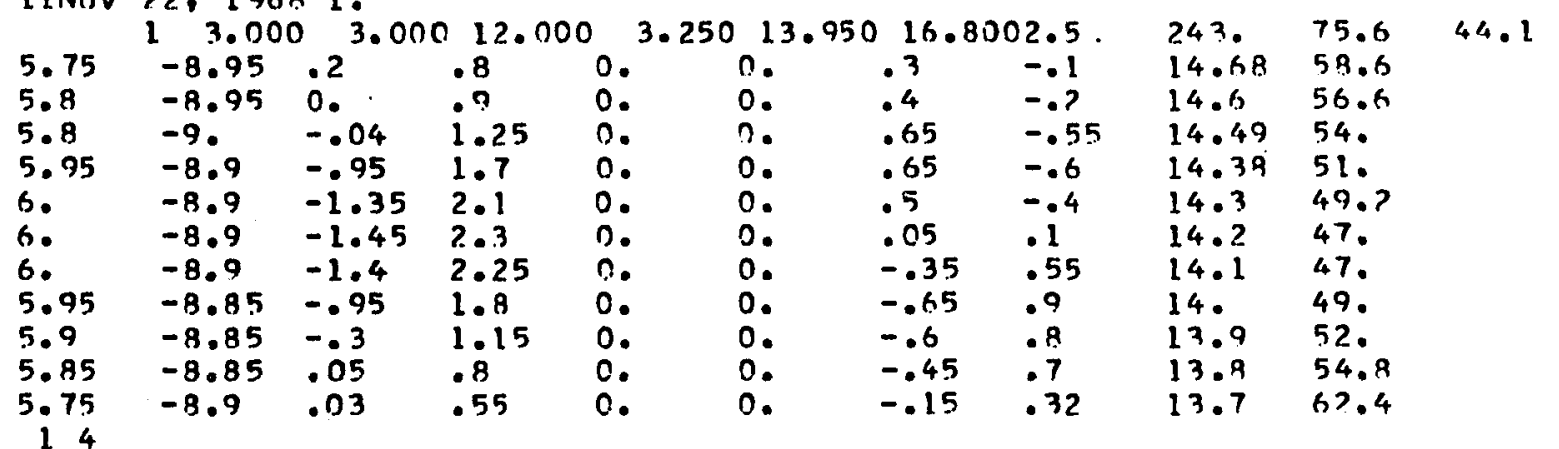

11DEC 9, 19681

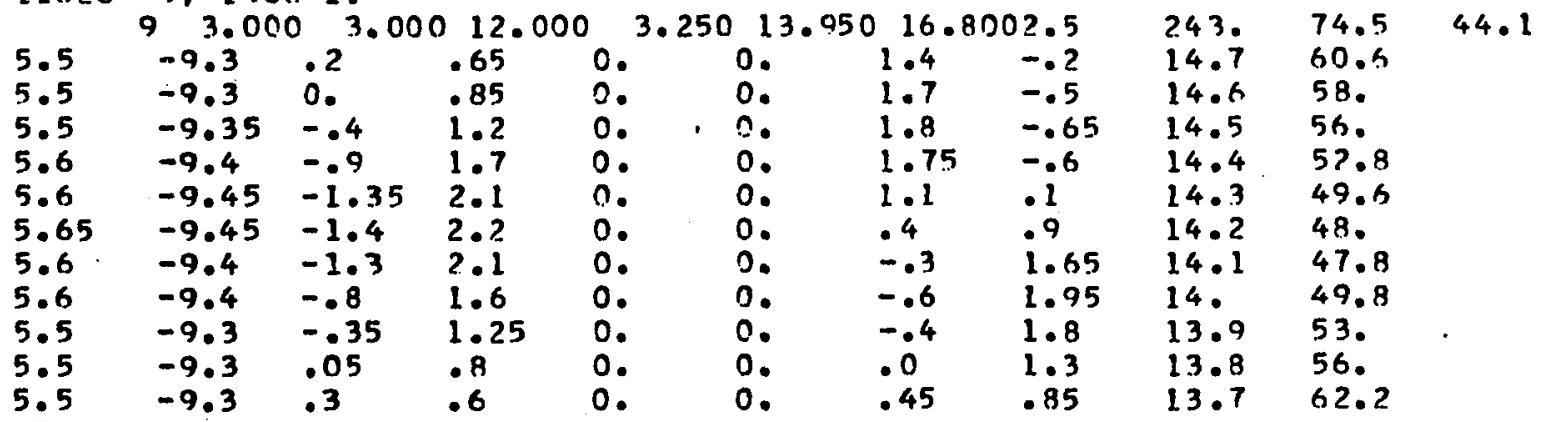

14

$12 \mathrm{NOV} 26,19681$

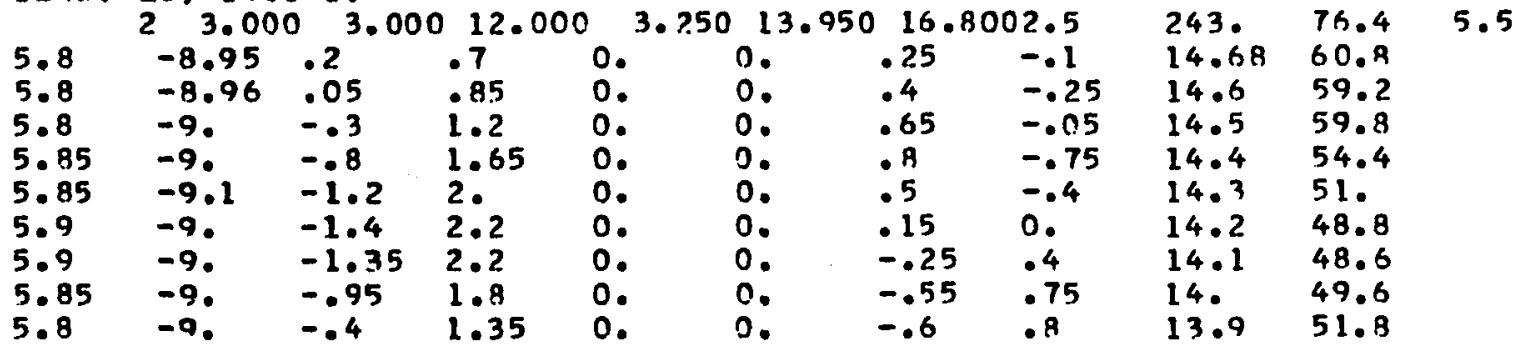


$\begin{array}{llllllllll}5.8 & -9 . & -.1 & .95 & 0 . & 0 . & -.5 & .7 & 13.8 & 55.8 \\ 5.75 & -9.35 & .2 & .7 & 0.7 & 0 . & -.25 & .45 & 13.7 & 60.1\end{array}$

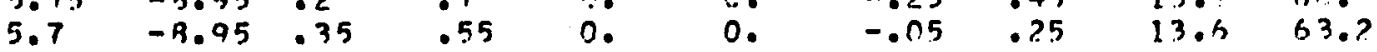

13 LFC 3.1969 1.

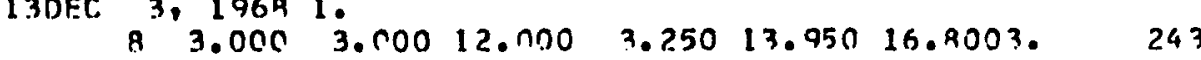

5.5

5.5

5.5

-9.25
-9.25

0.00

5.5 $-9.305$

n. 0.

1.25

$\begin{array}{ll}243 . \\ -.1 & 14.4 \\ -.1 & 14.7\end{array}$

$-9.25 \div 0$.

0.

$1.3-.1$

$1.4 \quad-.2 \quad 14.6$

5.55

$\begin{array}{llll}-9.3 & -.45 & 1.25 & 0 .\end{array}$

0.

$1.5 \quad-.3 \quad 14.5$

5.55

$-9.3 \quad-.55 \quad 1.35$

$-9.35$

$-.55$

1.4

0.

0.

$1.55-. .35$

$1.45 \quad-.25$

14.4

5. 5

$-9.35$

1.2

0 .

$14 \cdot 3$

$\begin{array}{llll}5.5 & -9.35 & -.525 & 1.3 \\ 5.55 & -9.35 & -.3 & 1.1\end{array}$

5.55

$-9.35$

$-9.3 \quad 01 \quad .9$

5.5

$-9.3$

$0.15 \quad .7$

o.

0.

- 8

.35

.25

.25

.5

$14 . ?$

1. 14.

1. 1.13 .9

$1.1 \quad 13.9$

.31 .13 .7

0.

0 .

.45

.9

13.6

14

27.19681.

$13 \mathrm{NOV} 27,19681$.

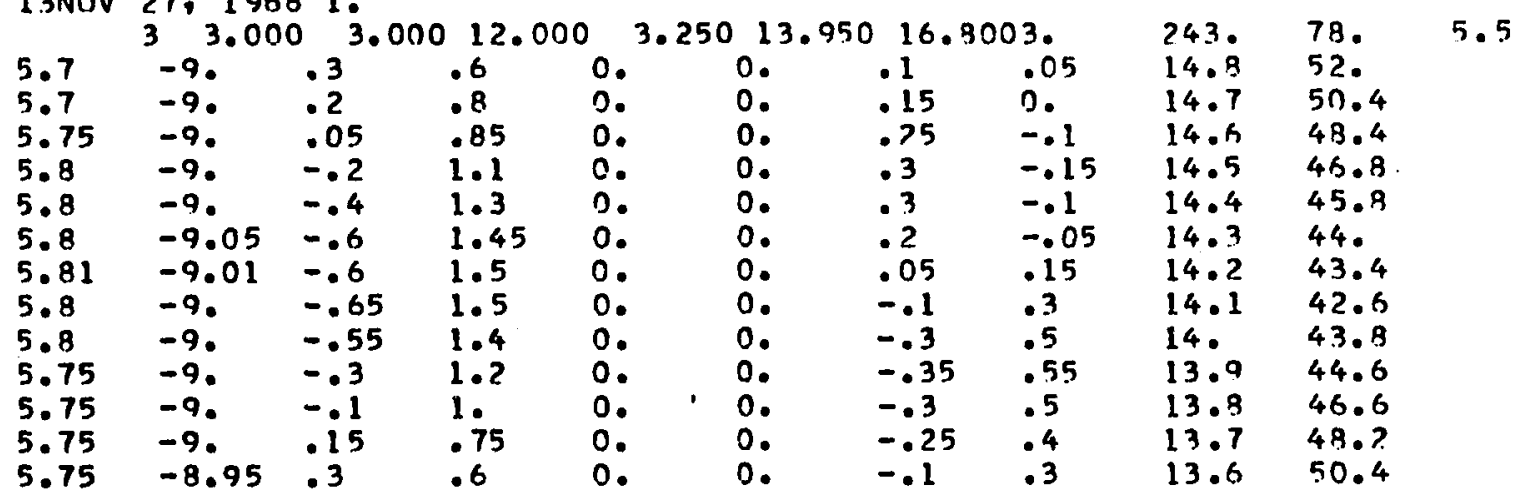

14

$-8.95 .3 \quad .6$

0.0 .

$-.1 \quad .3$

74.5

44.1

46.2

45. 9

45.2

44.

43.

43.

42.4

43.

44.4

46.

48. 8

50.

$13 D E C \quad 3,19681$.

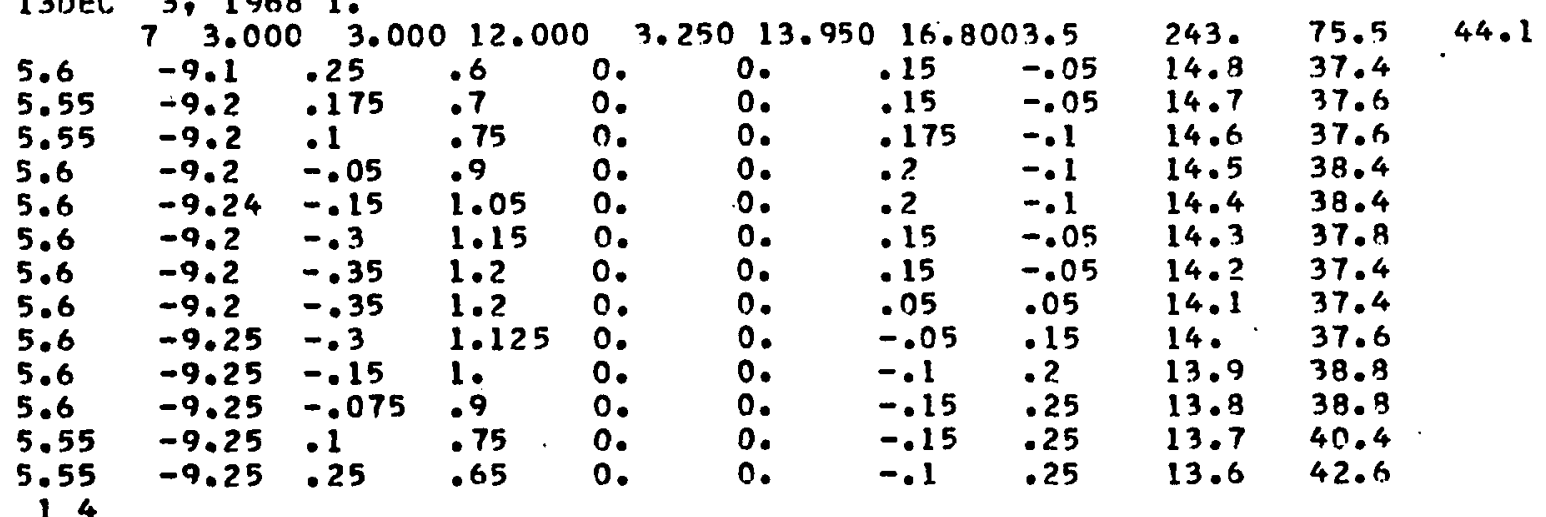

$14 N O V 28,19681$. 


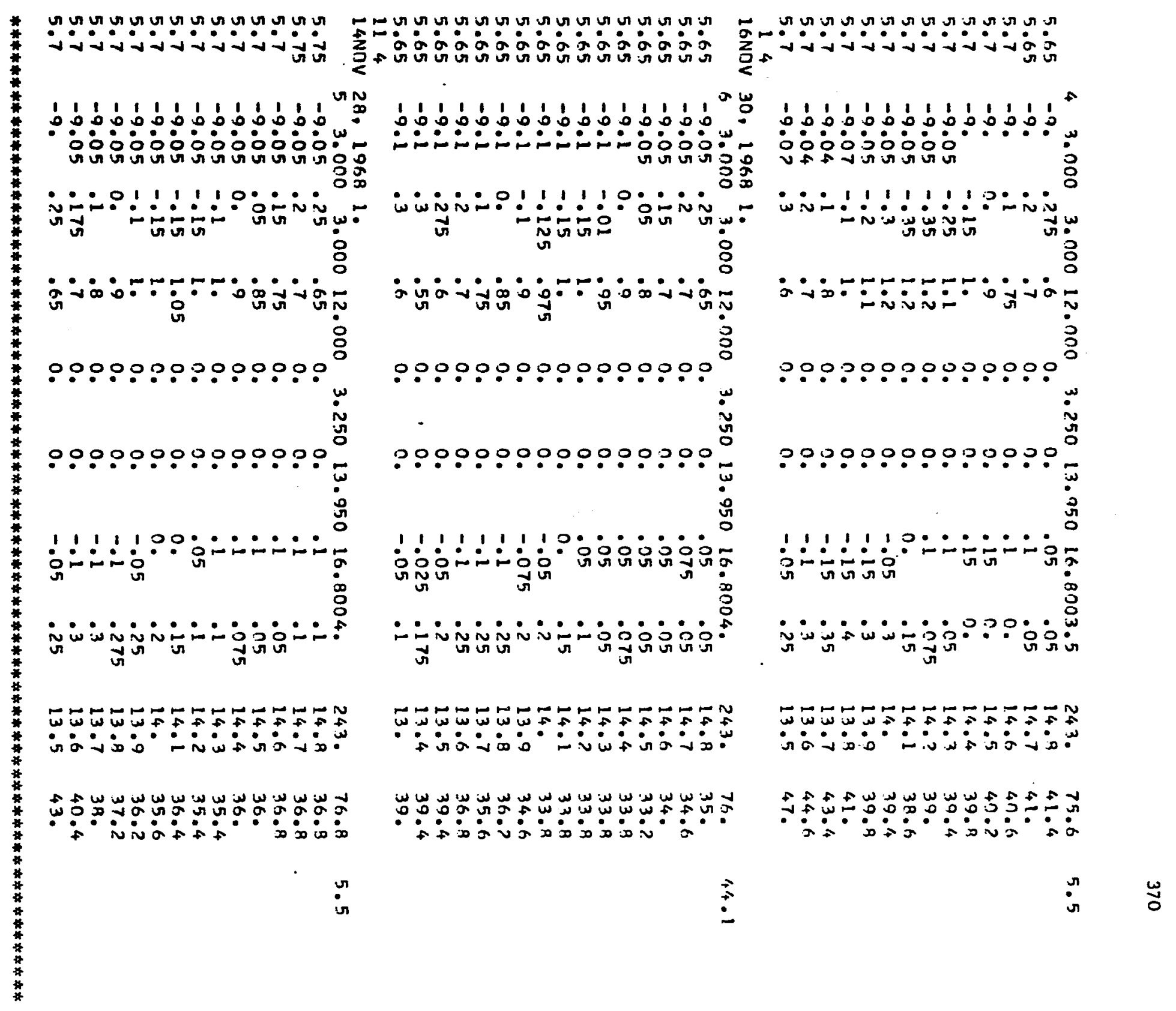


VFLOCITY PROFILES MEASURED AT 250 RPM

TANK DIAMETER $=1 ? .25$ I N.

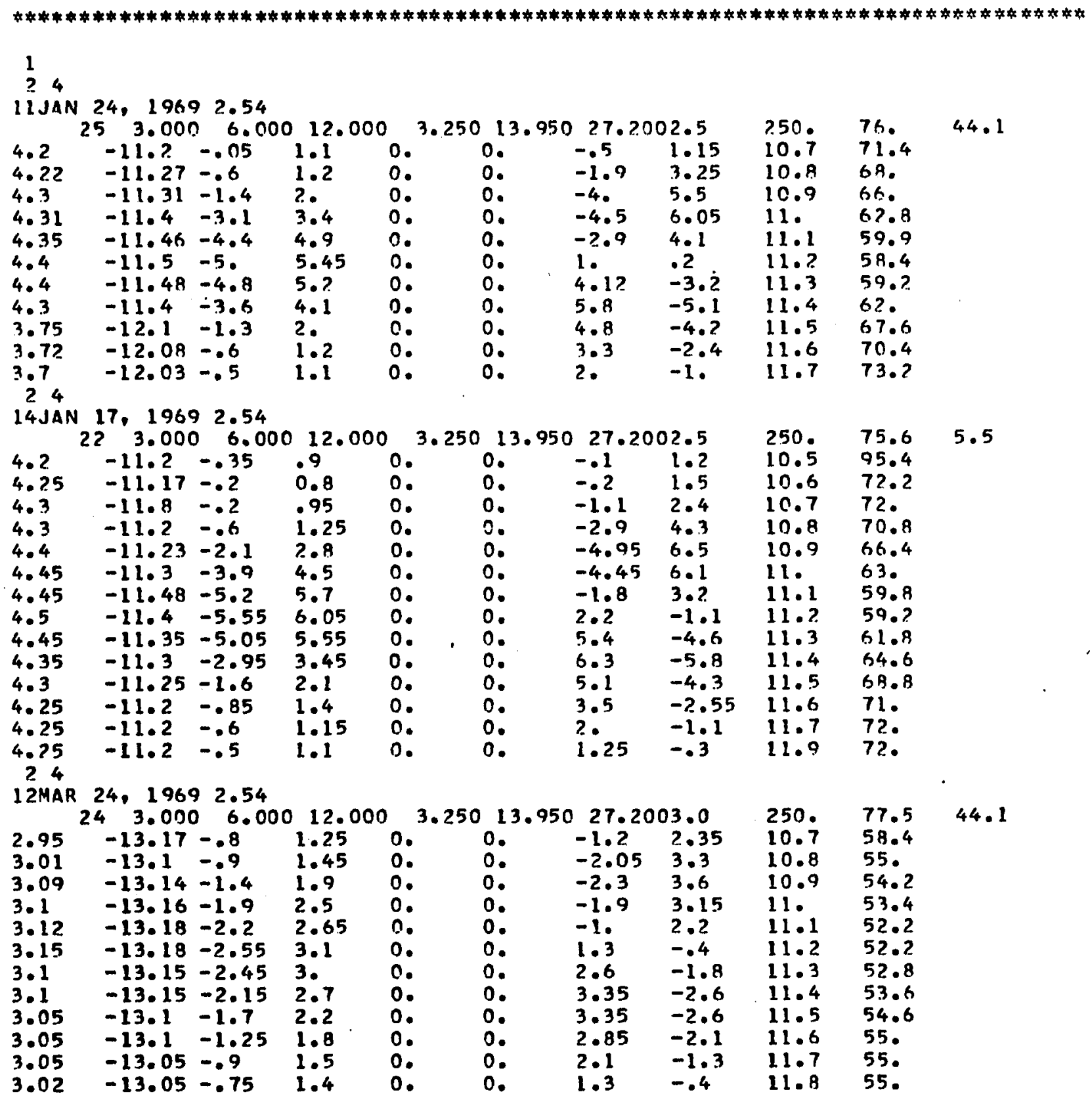


94

$13 J 4 N 22,19692.54$

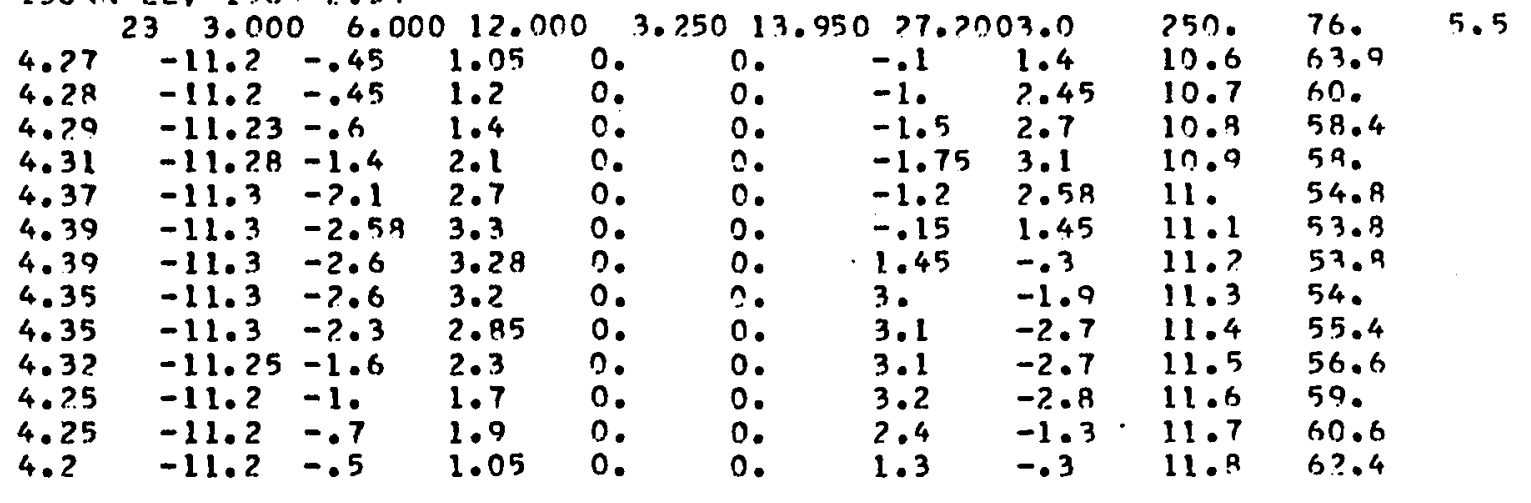

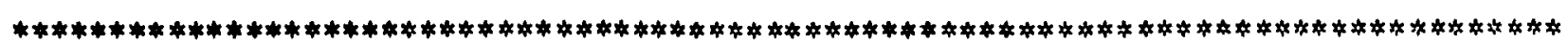

VELOCITY PROFILES MEASUREO AT $333.3 \mathrm{PPM}$

TANK DI AMETER $=12.25 \mathrm{IN}$.

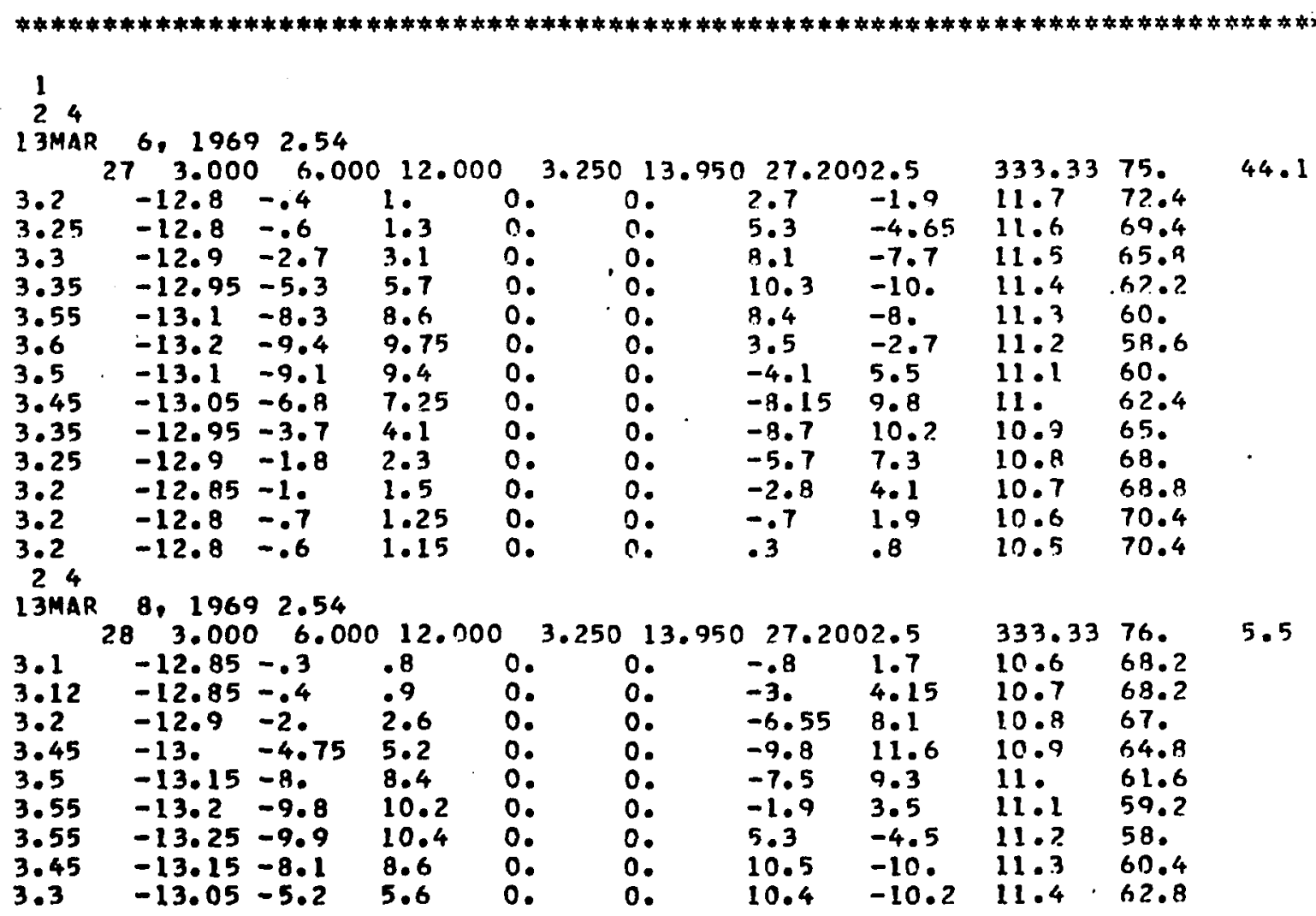




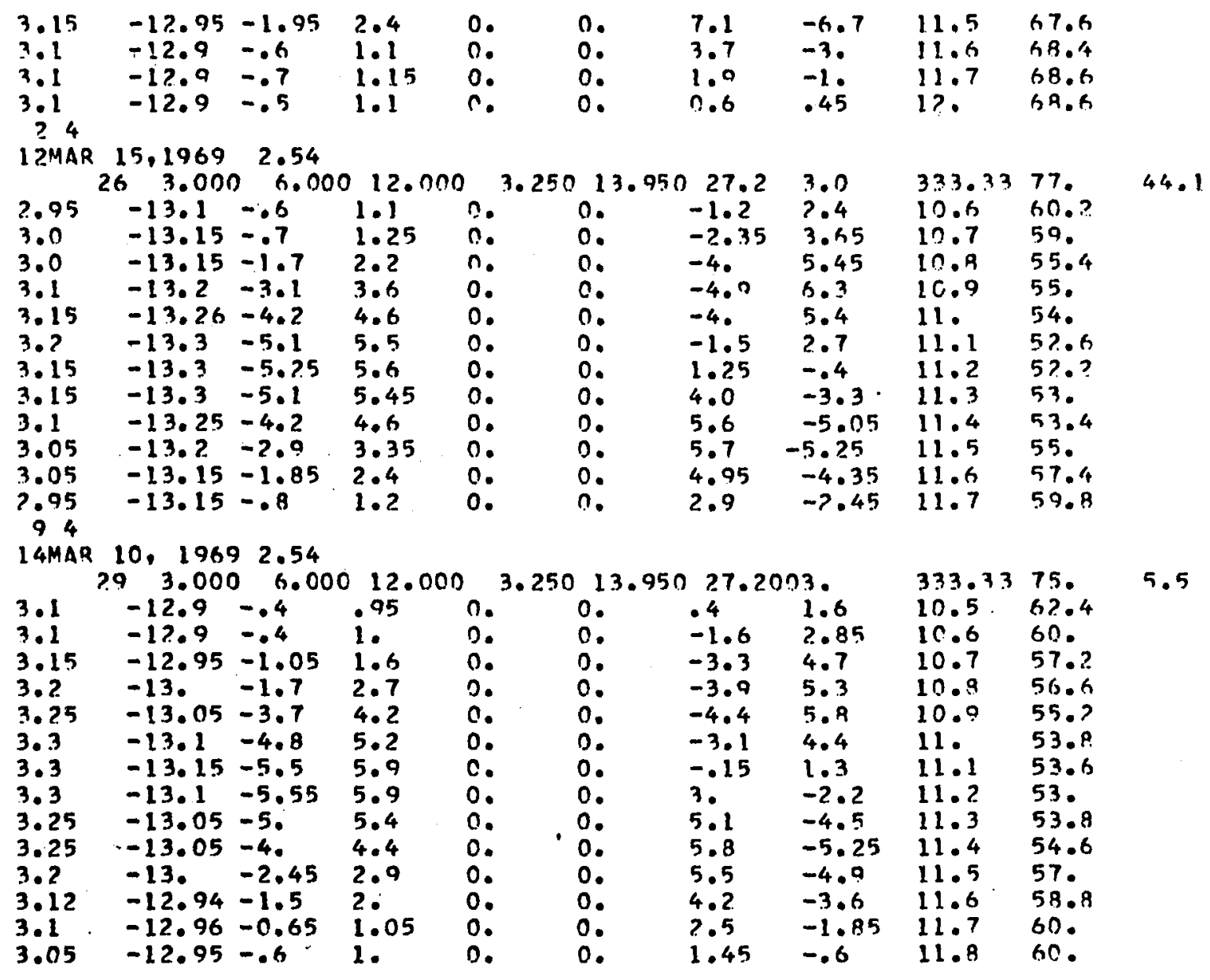

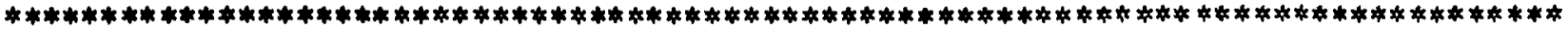

VELICITY PRDFILES MEASURED AT 400 RPM

TANK DIAMETFR $=12.25$ IN.

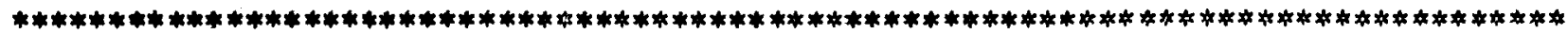

1

2. 4

L2MAR 12, 19692.54

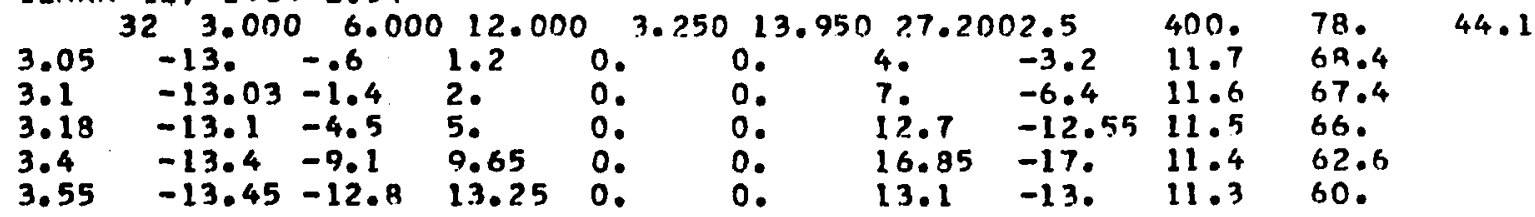




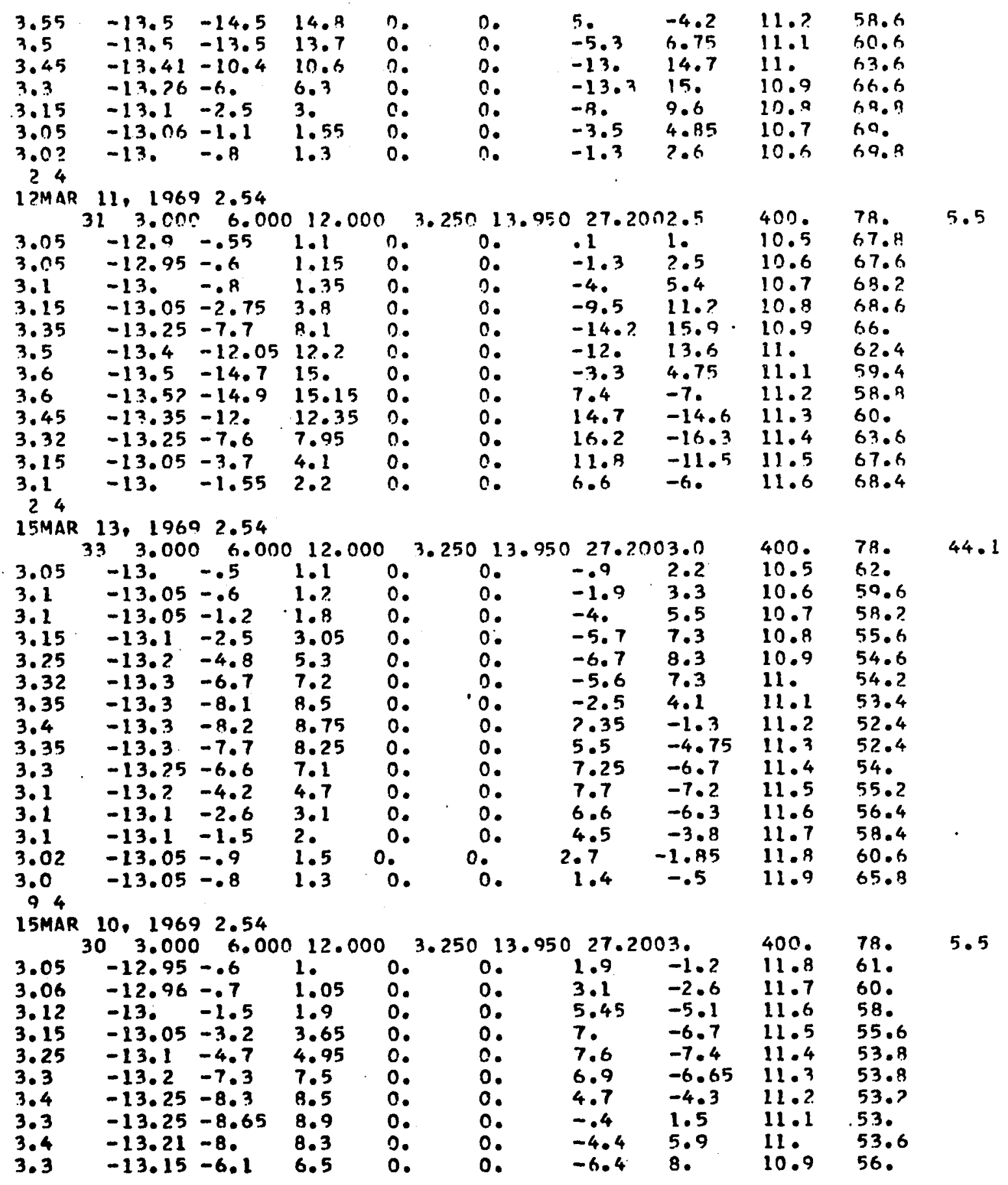




\begin{tabular}{|c|c|c|c|c|c|c|c|c|}
\hline $\begin{array}{l}3 . ? \\
3.15 \\
3.1 \\
3.1 \\
3.05\end{array}$ & $\begin{array}{l}-13.05 \\
-13 . \\
-12.95 \\
-12.95 \\
-12.95\end{array}$ & $\begin{array}{l}-4.25 \\
-2.5 \\
-1.2 \\
-.85 \\
-.7\end{array}$ & $\begin{array}{l}4.7 \\
3.05 \\
1.8 \\
1.5 \\
1.4\end{array}$ & $\begin{array}{l}0 . \\
0 . \\
0 . \\
0 . \\
0 .\end{array}$ & $\begin{array}{l}0 . \\
0 . \\
0 . \\
0 . \\
0 .\end{array}$ & $\begin{array}{l}-6.5 \\
-5.1 \\
-2.7 \\
-1 . \\
0 .\end{array}$ & $\begin{array}{l}8.15 \\
6.7 \\
4.2 \\
2.3 \\
1.3\end{array}$ & $\begin{array}{l}10.8 \\
10.7 \\
10.4 \\
10.5 \\
10.4\end{array}$ \\
\hline
\end{tabular}

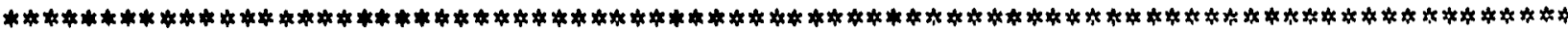

VELOCITY PROFILES MFASURED AT 500 ROM

TANK DIAMETFR= 12.25 IN.

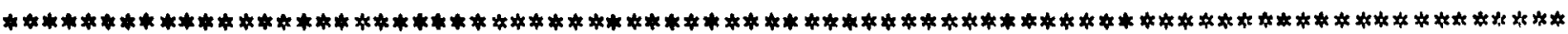

1

24

ISDFr 5,19681 .

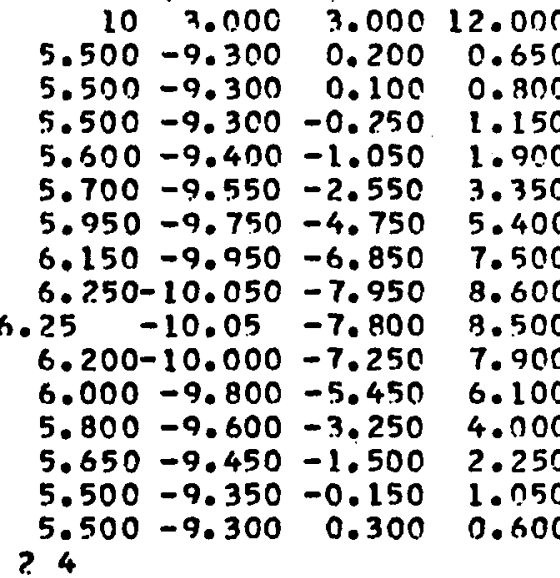

14DEC 18, 1968 1.

$\begin{array}{rrrr}.17 & 3.000 & 3.000 & 12.000 \\ 5.150 & -9.800 & -0.050 & 0.500 \\ 5.200 & -9.900 & -0.200 & 0.650 \\ 5.200 & -9.900 & -0.500 & 0.900 \\ 5.250-9.950 & -1.100 & 1.500 \\ 5.450-10.000 & -2.600 & 2.950 \\ 5.600-10.200 & -4.500 & 4.800 \\ 5.850-10.400 & -6.750 & 7.000 \\ 5.900-10.600 & -7.900 & 8.150 \\ 5.900-10.600 & -7.750 & 8.000 \\ 5.700-10.400 & -5.950 & 6.200 \\ 5.550-10.200 & -3.950 & 4.250 \\ 5.300-9.950 & -1.850 & 2.200 \\ 5.200-9.850 & -0.500 & 1.000 \\ 5.100-9.900 & 0.000 & 0.400 \\ 24 & & & \end{array}$

12DEC 22, 19682.54

$\begin{array}{llll}18 & 3.000 & 6.000 & 12.000\end{array}$

$\begin{array}{llll}5.050 & -9.900 & 0.254 & 1.143\end{array}$
$3.250 \quad 13.950 \quad 16.800$

0.000

0.000

0.000

0.000

0.000

0.000

0.000

0.000

0.000

0.000

0.000

0.000

0.000

0.000

0.000

0.000

16.800

$2.530500 .000 \quad 76.50044 .1$

0.000

0.000

0.000

1.250

0.32014 .900

58.300

0.000

$2.250-1.000 \quad 14.700 \quad 57.600$

0.000

$4.200-3.20014060057 .200$

$6.450-5.60014 .50056 .400$

0.000

7.450

$\begin{array}{rr}0.000 & 1.850 \\ 0.000 & -0.400\end{array}$

$6.300-5.500 \quad 14.300 \quad 50.000$

$0.000-3.200$

$0.000-6.800$

$0.000-7.100$

$0.000-5.150$

$0.000 \quad 1.500$

0.000

0.450

$-0.70014 .20048 .000$

$1.80514 .150 \quad 47.800$

$4.750 \quad 14.100 \quad 47.800$

$8.50014 .000 \quad 49.4 n ?$

$8.75013 .900 \quad 52.200$

$6.800 \quad 13.900 \quad 56.000$

$2.05013 .700 \quad 61.000$

$1.9 .00 \quad 13.600 \quad 69.200$

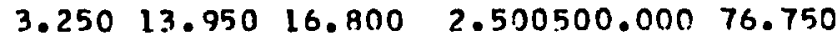 \\ $\begin{array}{lllllll}0.000 & 0.000 & 0.800 & 0.500 & 14.950 & 73.400\end{array}$ \\ 5.500}

$0.000 \quad 0.000 \quad 1.600-0.400 \quad 14.800 \quad 65.000$

$\begin{array}{llllll}0.000 & 0.000 & 2.300 & -1.200 & 14.700 & 62.000 .\end{array}$

$0.000 \quad 0.000 \quad 3.950-3.000 \quad 14.600 \quad 61.400$

$0.000 \quad 0.000 \quad 6.700 \quad-5.850 \quad 14.500 \quad 58.600$

$0.000 \quad 0.000 \quad 8.100 \quad-7.300 \quad 14.400 \quad 55.200$

$0.000 \quad 0.000 \quad 6.950-6.150 \quad 14.300 \quad 51.600$

$0.000 \quad 0.000 \quad 3.100-2.050 \quad 14.200 \quad 48.600$

$\begin{array}{llllllll}0.000 & 0.000 & -2.200 & 3.700 & 14.100 & 45.600\end{array}$

$\begin{array}{lllllll}0.000 & 0.000 & -6.650 & 8.350 & 14.000 & 49.600\end{array}$

$0.000 \quad 0.000-7.200 \quad 8.900 \quad 13.900 \quad 52.400$

$\begin{array}{lllllll}0.000 & 0.000 & -5.000 & 6.650 & 13.800 & 56.600\end{array}$

$0.000 \quad 0.000-2.150 \quad 3.700 \quad 13.700 \quad 61.600$

$0.000 \quad 0.000-0.050 \quad 1.200 \quad 13.600 \quad 65.800$

$3.25013 .5016 .8002 .5 \quad 500.76 .6 \quad 44.1$

$0.000 \quad 0.000 \quad 4.445-1.39711 .80060 .000$ 


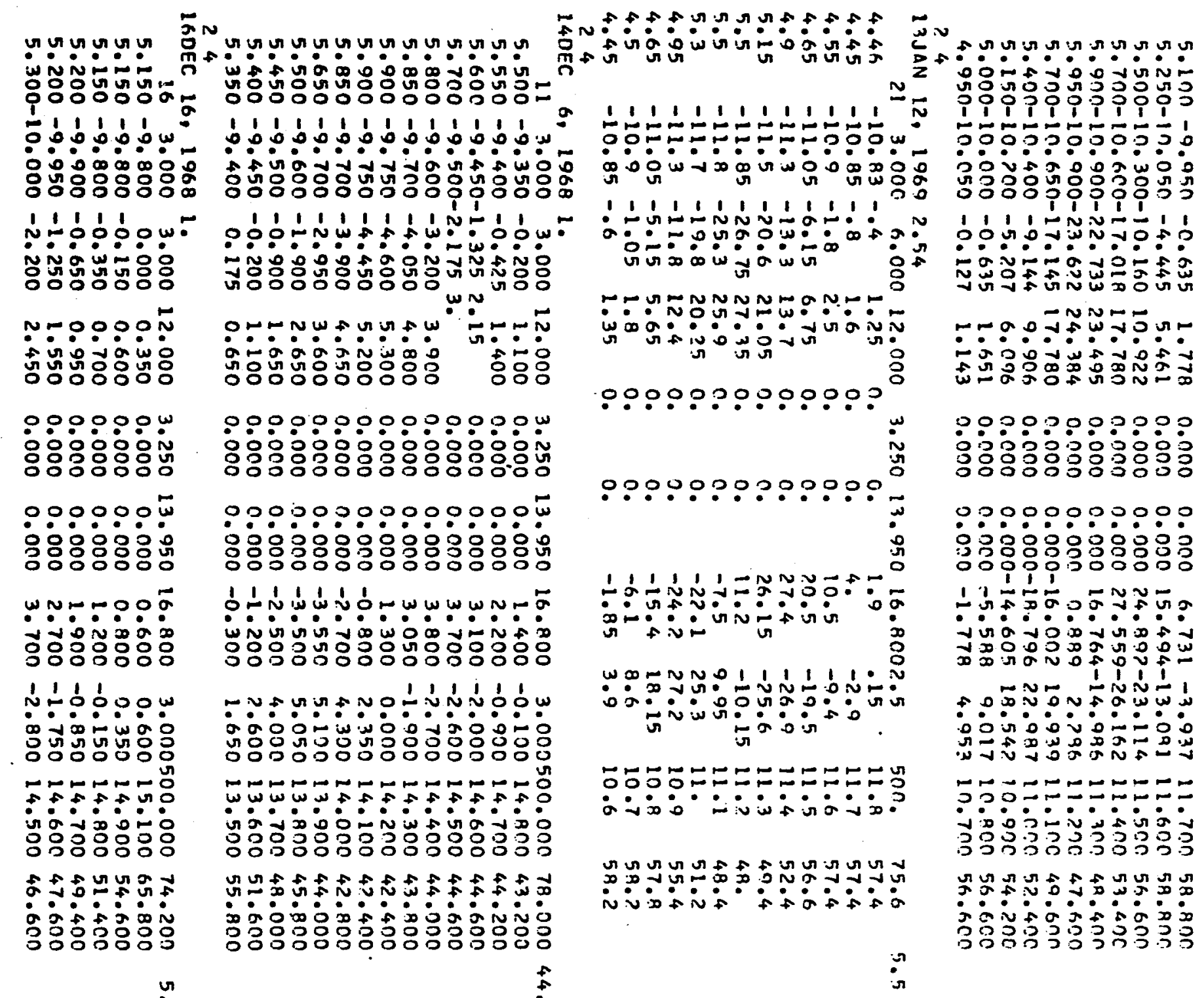




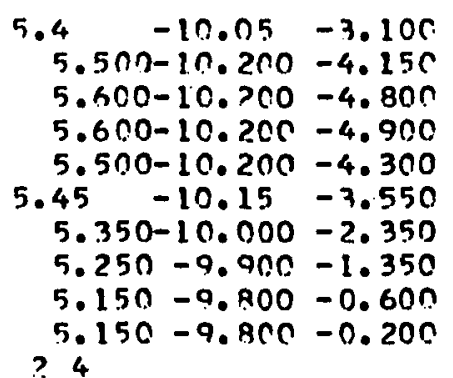

$\begin{array}{ll}3.350 & 0.000 \\ 4.300 & 0.000 \\ 5.000 & 0.000 \\ 5.100 & 0.000 \\ 4.500 & 0.000 \\ 3.700 & 0.000 \\ 2.050 & 0.000 \\ 1.700 & 0.000 \\ 0.950 & 0.000 \\ 0.550 & 0.000\end{array}$

0.000

0.000

0.000

0.000

0.000

0.000

0.000

$0.000-2: 700$

$0.000-1.600$

$0.000-0.450$
$3.90 n-3.00 n 14.4 n C 45.400$

$1.900-0.80$ r 14.23043 .700

1.47014 .10242 .600

$3.92014 .0 n \pi$ 43.0กn

$5.050 \quad 13.9 n 0 \quad 44.000$

$5.00013 .920 \quad 45.600$

4.13013 .70048 .40 r

$3.0 n c 13.60051 .000$

1.70013 .50057 .000

17 DEC $30,19682.54$

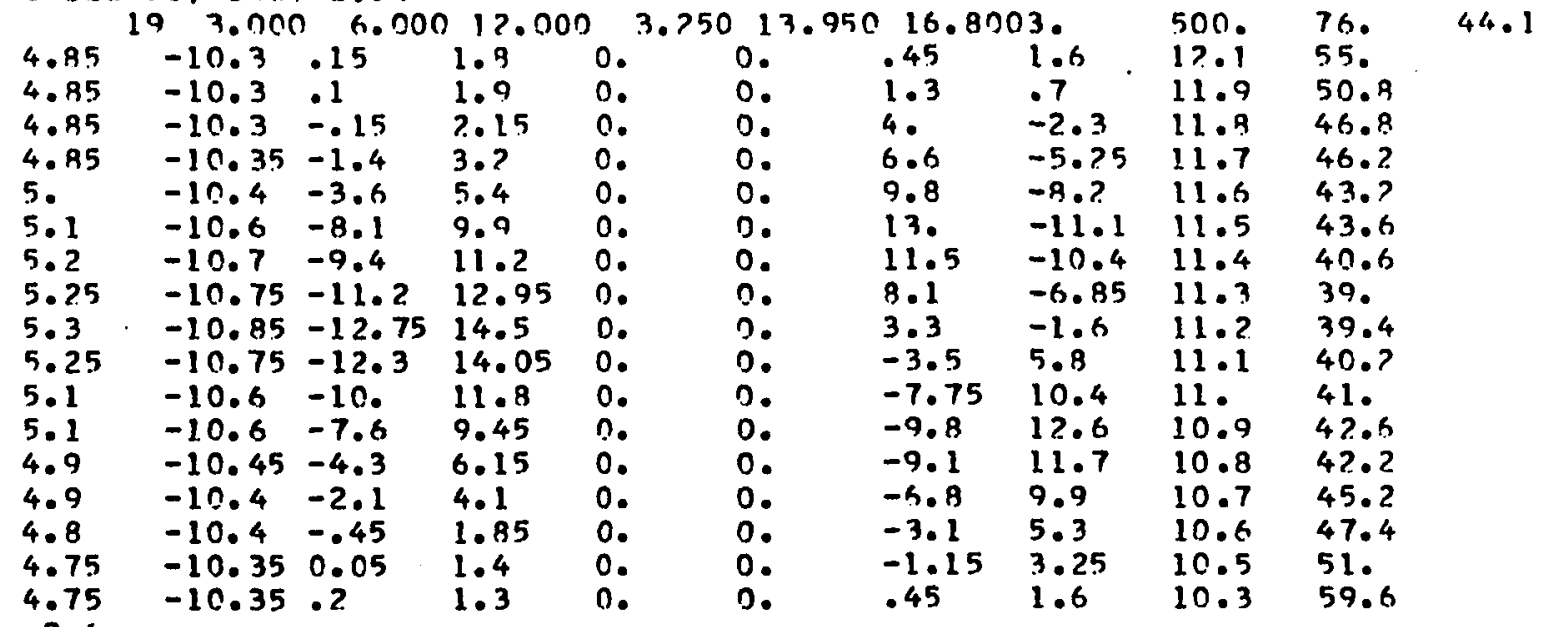

24

I6JAN 7, 19692.54

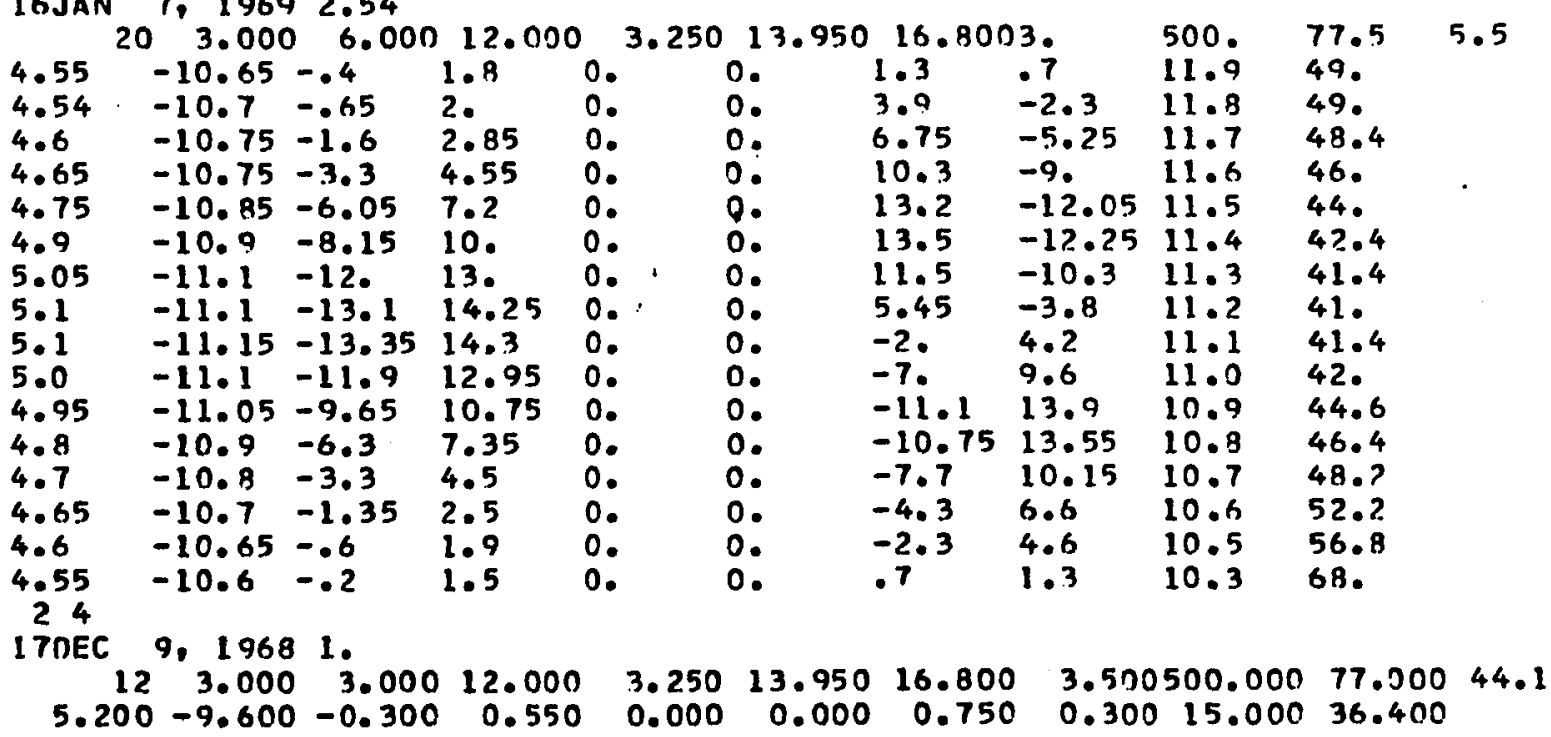


$\stackrel{\infty}{n}$

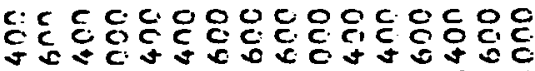

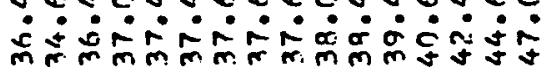

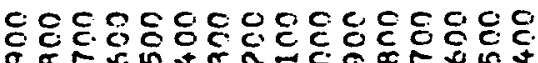

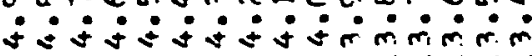

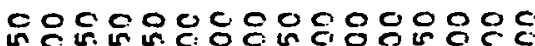

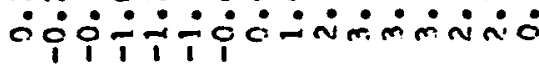

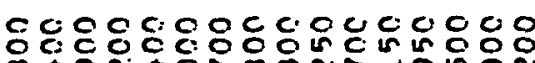

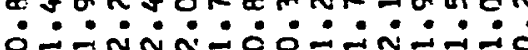

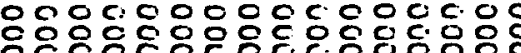
idicididididididi

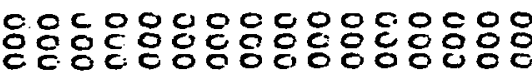
वंidiódididididid

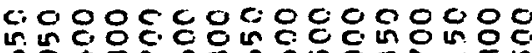
过

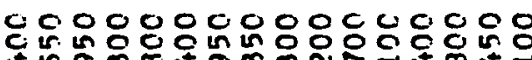

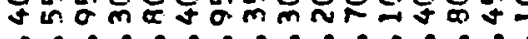
ioำnmmiñio

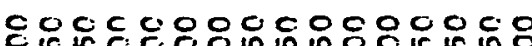
our uncomatarkroun un

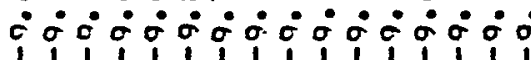

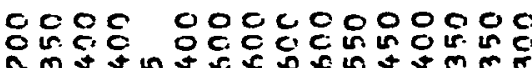

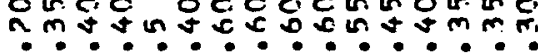

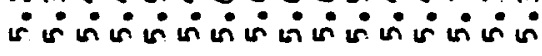

点

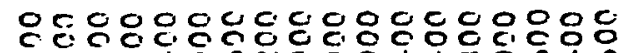

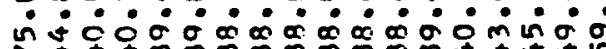

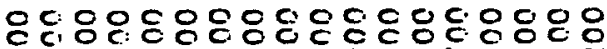

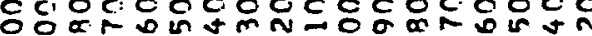

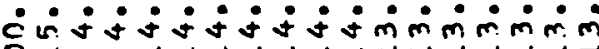

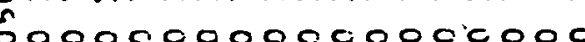

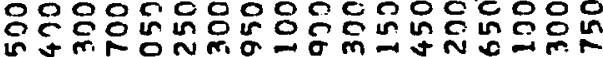

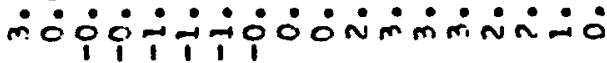

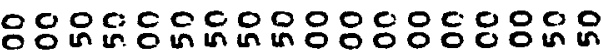

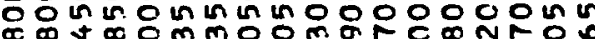

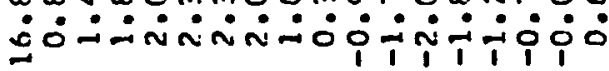

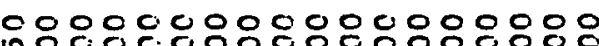

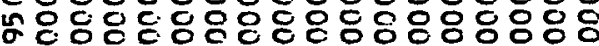

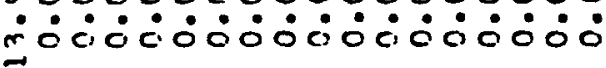

00000000000000000

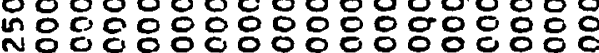

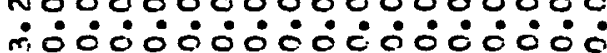

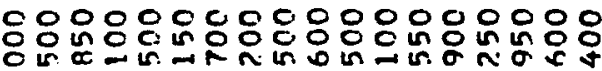

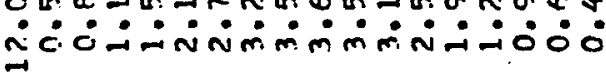

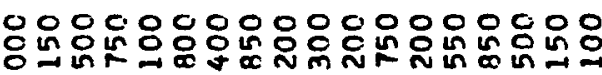

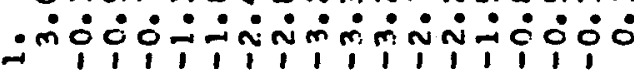

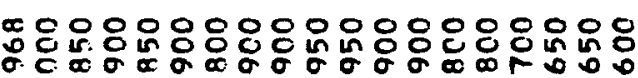

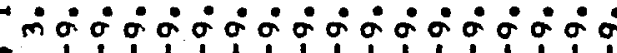
noco00000000000000

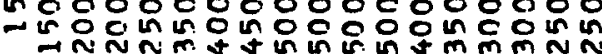
ง it

영영영영영영영영

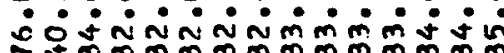
응응응영영응응등응 $\dot{0} \dot{1} \dot{j} \dot{j} \dot{j} \dot{j} \dot{j} \dot{j} \dot{m}$

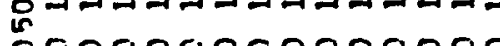

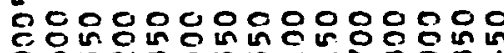
-ocóóióóñn

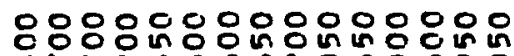
a.?

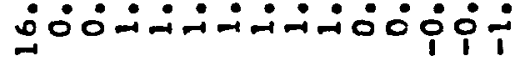

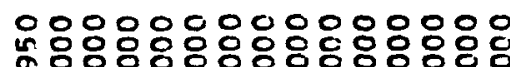
mó0ல் $\dot{n} \dot{0} \dot{0} \dot{0} \dot{0} \dot{0} \dot{0}$

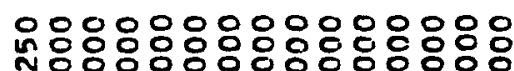

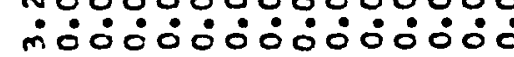

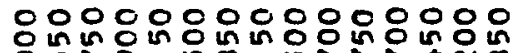
noojaminininan.

000000000000000

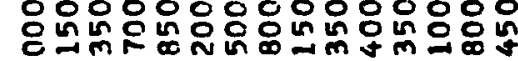

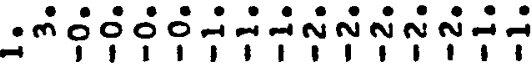

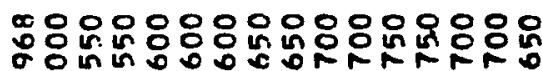

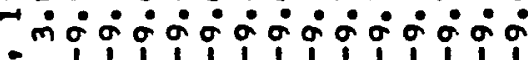
Ino0000000000

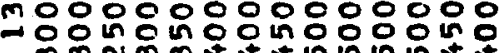
mmnmmst. 


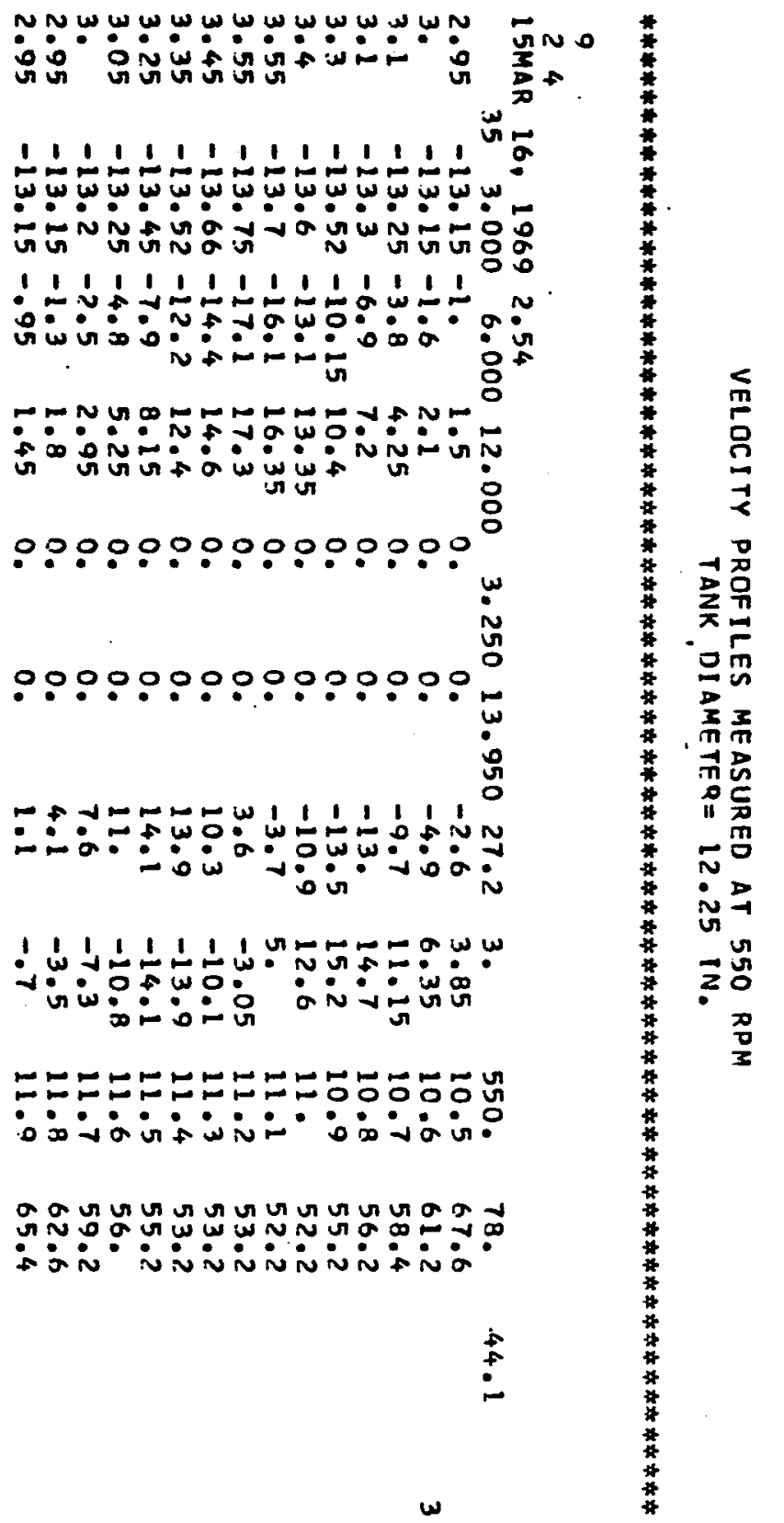

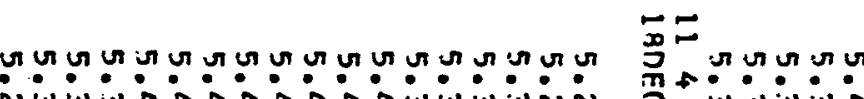

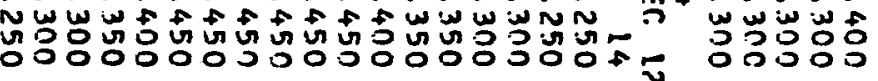

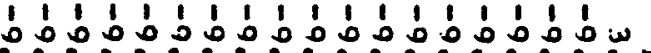

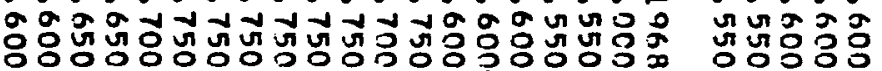

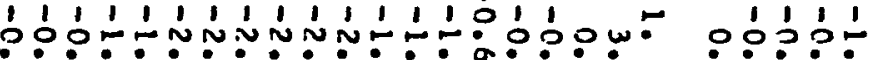

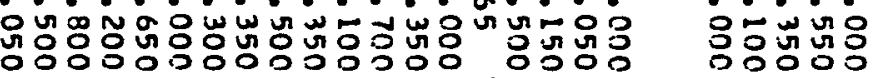

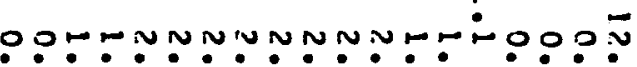

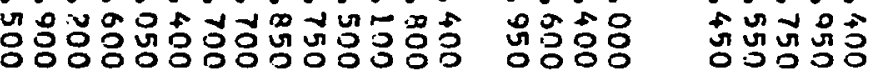

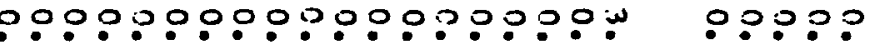

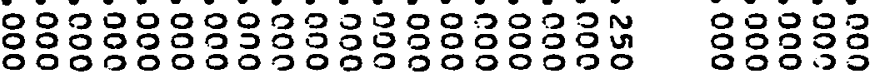
000000000000000000w 00000

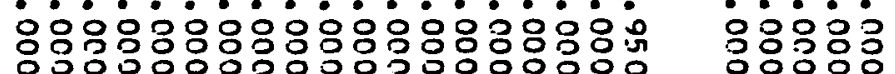

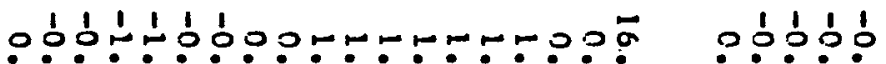

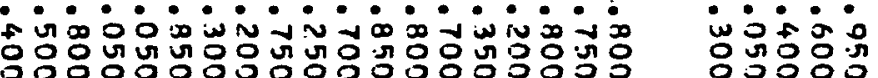

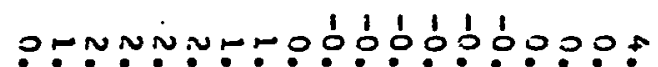

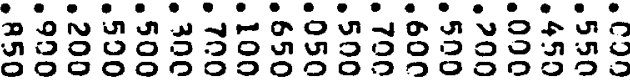

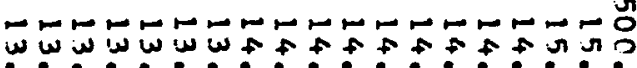

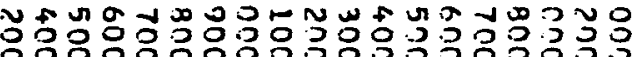

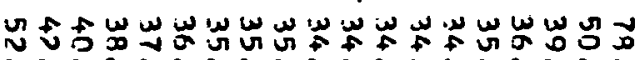

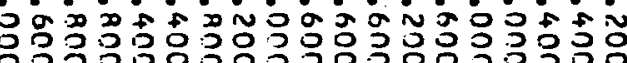

:.:.:.

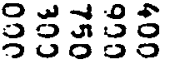

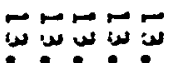

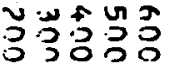
華岂岂

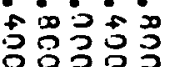

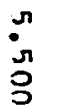


94

14MAP $17,1969 ? .54$

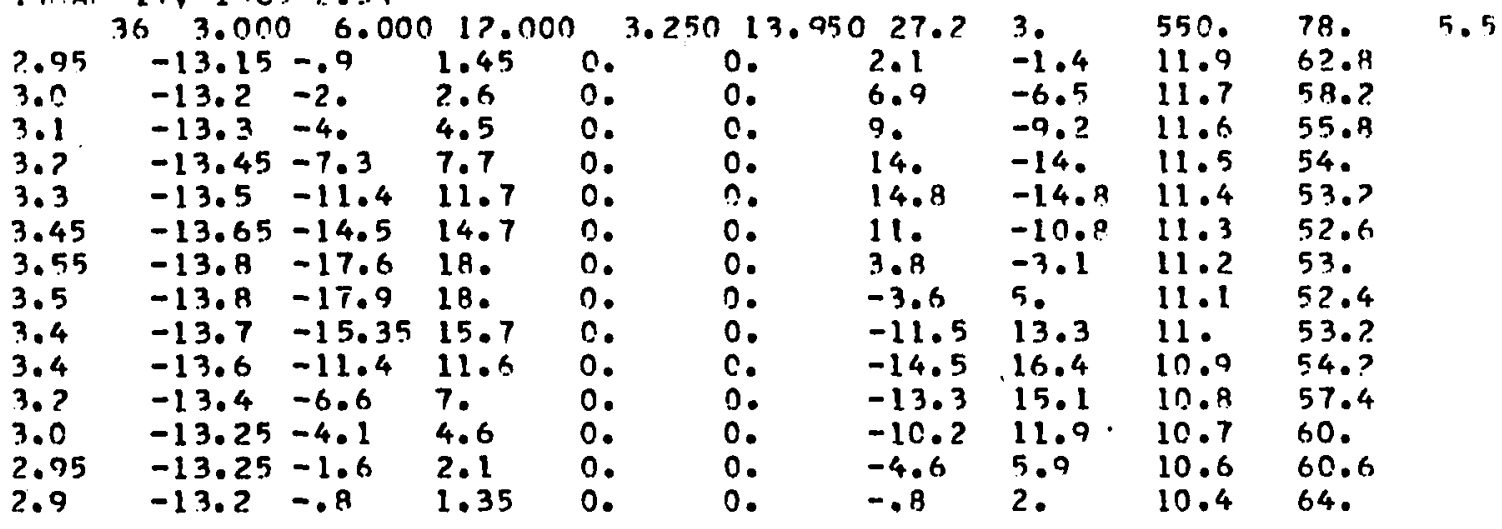

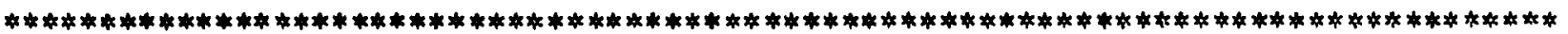

\section{VFLDCITY PRDFILES MEASURED AT 333.3 RPM} TANK DIAMETER $=11.5$ IN.

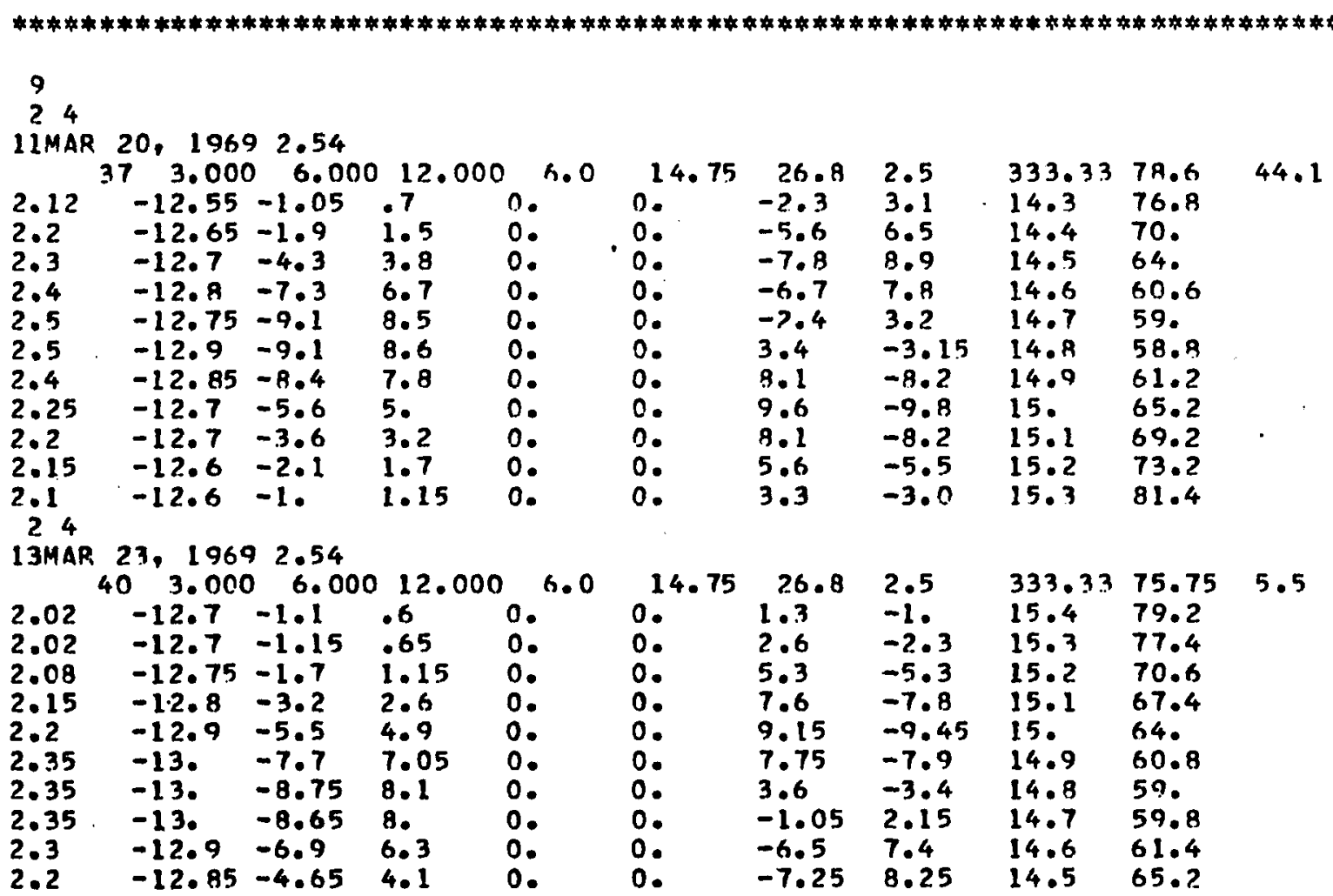




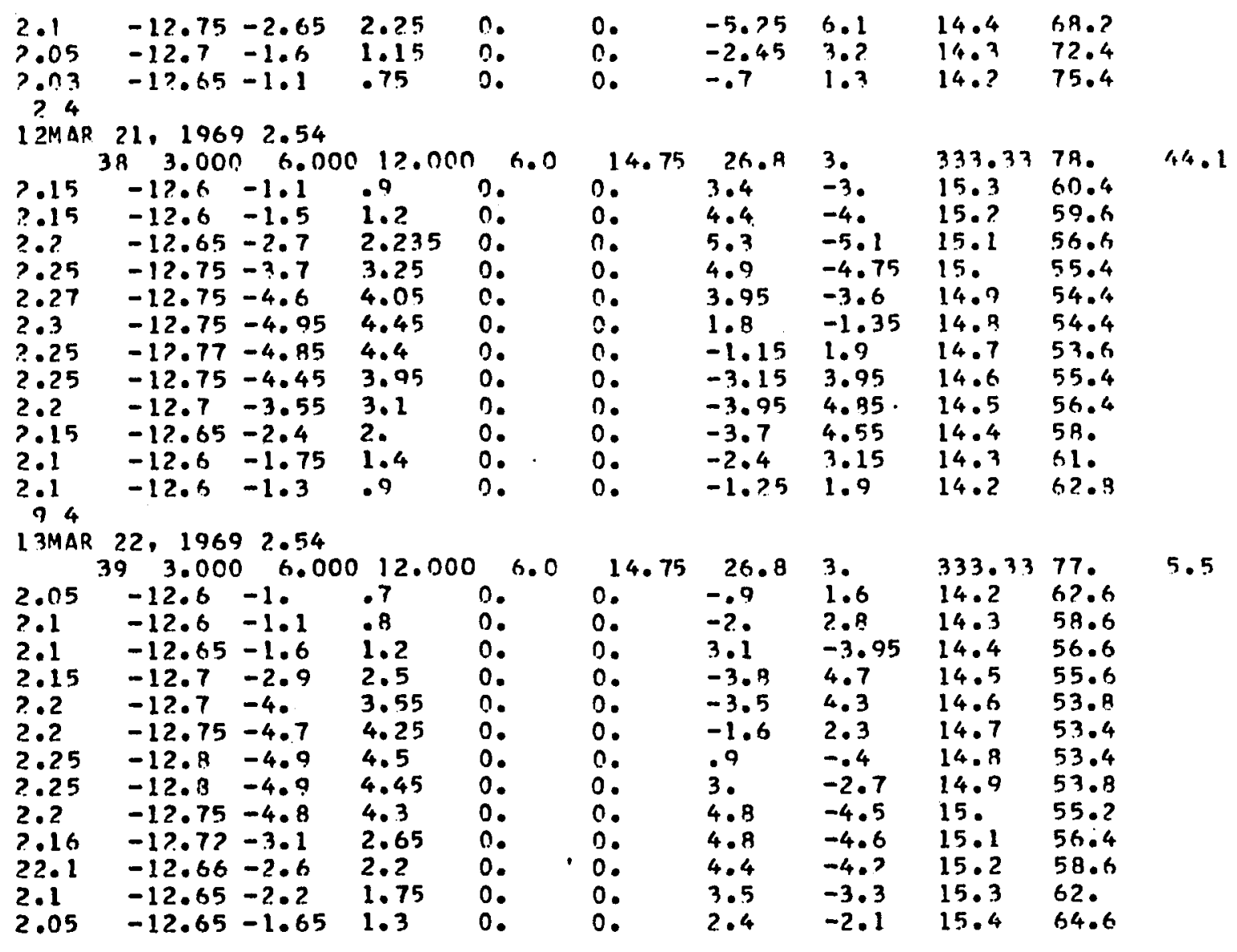


SAMPLE OUTPUT FOR RUN NUMBER 29

PAW nATA

MAR 10,1969

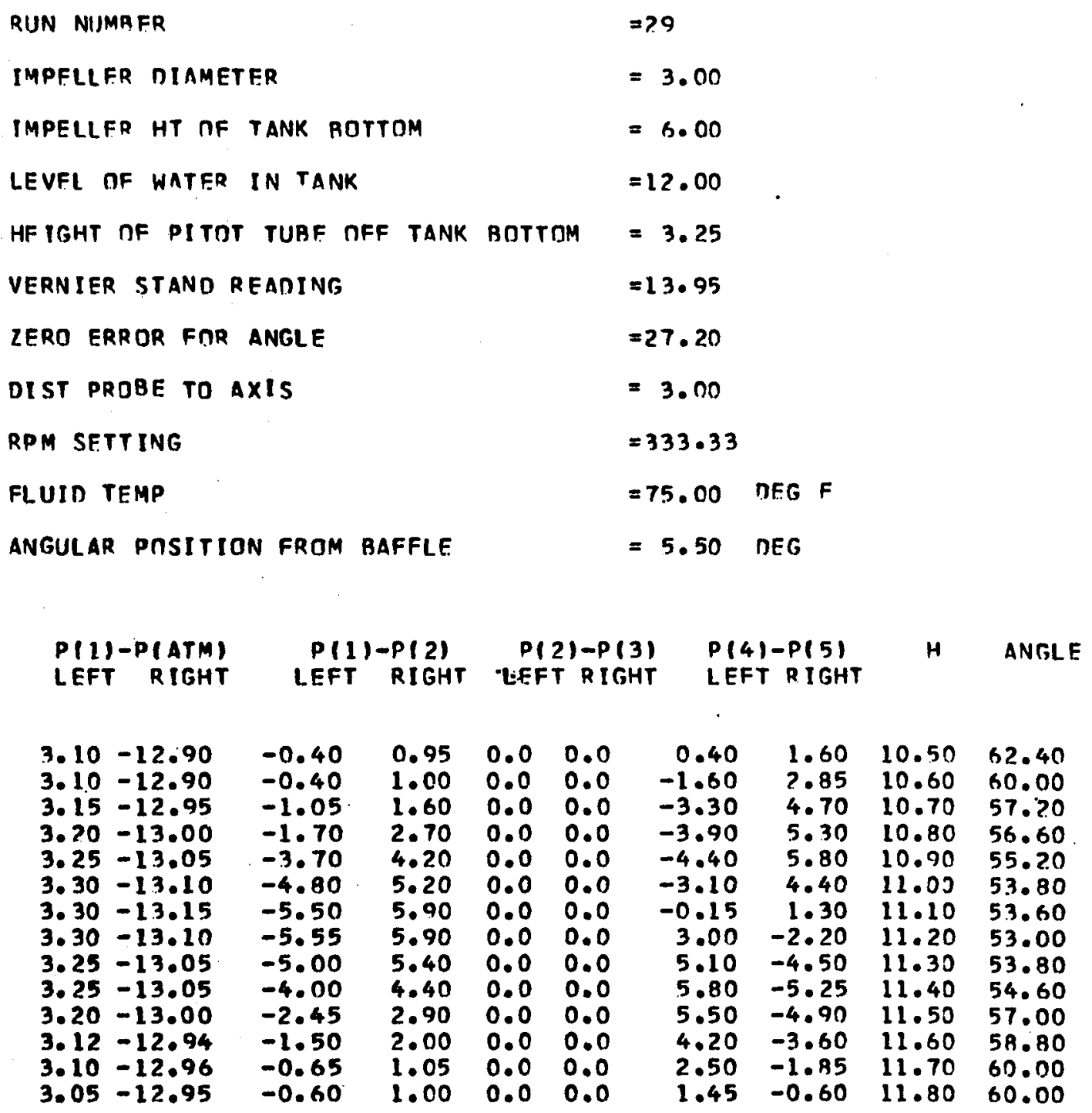


$P(1) \quad P(2) \quad P(3) \quad P(4) \quad$ SIM SQ RF

\begin{tabular}{|c|c|c|c|c|c|c|}
\hline $\begin{array}{l}9 F \\
2 F \\
1 F \\
9 F \\
9 F \\
9 F\end{array}$ & & $\begin{array}{l}\text { DOOOODF } \\
500000 F \\
500000 F \\
499954 F \\
549973 E \\
544972 E\end{array}$ & $\begin{array}{l}01 \\
01 \\
01 \\
01 \\
01 \\
01\end{array}$ & $\begin{array}{l}87989 F-01 \\
87989 F-01 \\
87989 E-01 \\
87989 F-01 \\
87989 E-01 \\
87989 F-01\end{array}$ & $\begin{array}{l}9999903 \mathrm{~F}-04 \\
9999065 \mathrm{~F}-03 \\
7999969 \mathrm{~F}-03 \\
7499963 \mathrm{~F}-03 \\
7499963 \mathrm{~F}-03 \\
7429950 \mathrm{~F}-03\end{array}$ & $\begin{array}{l}0601 F \\
071 \mathrm{AF} \\
7212 \mathrm{~F} \\
8941 \mathrm{~F} \\
4842 \mathrm{~F} \\
3470 \mathrm{~F}\end{array}$ \\
\hline
\end{tabular}

The above is the value of the parameters $\sigma, A, a$, and $z_{0}$ at every 30 th evaluation of SS as defined by Equation $\mathrm{C}-2$. 

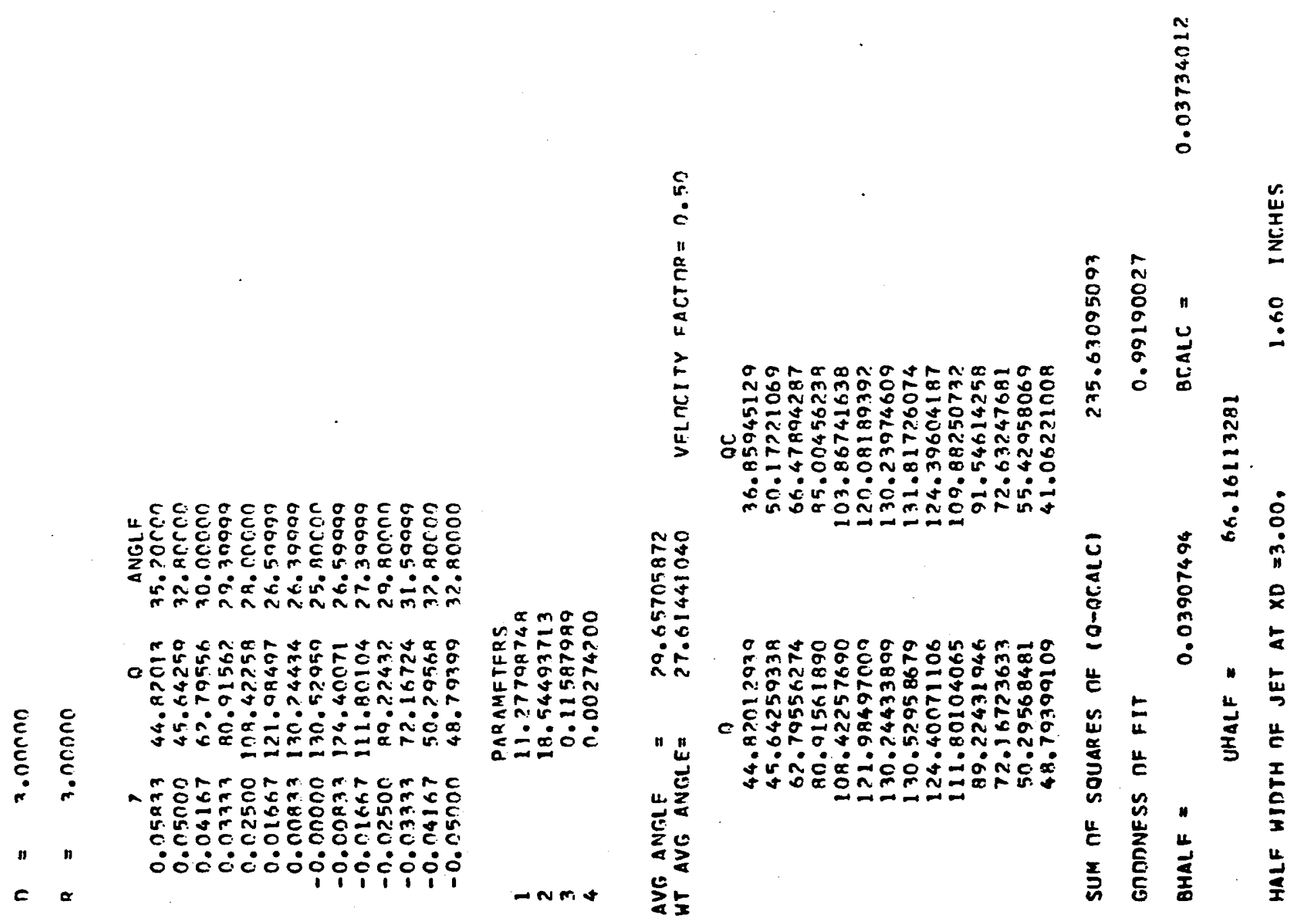


\begin{tabular}{|c|c|c|c|c|c|}
\hline$p(1)-p(2)$ & $P(4)-P(5)$ & 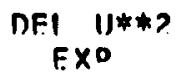 & $\begin{array}{l}\text { DFI U* } \% \text { ? } \\
\text { C.ALC. }\end{array}$ & $\begin{array}{r}V Z \\
\text { C.ALr. }\end{array}$ & nIfr חF $J * *$ ? \\
\hline $\begin{array}{l}0.5315 \\
0.5512 \\
1.0433 \\
1.7723 \\
3.1102 \\
3.9370 \\
4.4982 \\
4.5079 \\
4.0945 \\
3.3071 \\
2.1063 \\
1.3780 \\
0.6693 \\
0.6799\end{array}$ & $\begin{array}{l}-0.9661 \\
-2.1457 \\
-3.5433 \\
-4.0157 \\
-4.4094 \\
-3.3465 \\
-0.9646 \\
1.6535 \\
3.3858 \\
3.9567 \\
3.7008 \\
2.6772 \\
1.3189 \\
0.4134\end{array}$ & $\begin{array}{l}-0.7309 \\
-0.5770 \\
-0.9447 \\
-1.0706 \\
-1.1756 \\
-0.1927 \\
-0.2572 \\
0.4408 \\
0.9027 \\
1.0549 \\
0.9866 \\
0.7137 \\
0.3516 \\
0.1102\end{array}$ & $\begin{array}{l}-0.2475 \\
-0.4274 \\
-0.6617 \\
-0.9114 \\
-1.0494 \\
-0.9140 \\
-0.4419 \\
0.2228 \\
0.7891 \\
1.0399 \\
0.9791 \\
0.7505 \\
0.4990 \\
0.2998\end{array}$ & $\begin{array}{l}-0.2037 \\
-0.1577 \\
-0.1172 \\
-0.0768 \\
-0.0432 \\
-0.0197 \\
-0.0060 \\
0.0029 \\
0.0141 \\
0.0340 \\
0.0644 \\
0.1029 \\
0.1452 \\
0.1869\end{array}$ & $\begin{array}{r}-0.0165 \\
0.1487 \\
0.2929 \\
0.1592 \\
0.1771 \\
-0.0218 \\
-0.1849 \\
-0.2190 \\
-0.1145 \\
-0.0159 \\
-0.0076 \\
0.0368 \\
0.1474 \\
0.1896\end{array}$ \\
\hline
\end{tabular}


QATIAL VFLTCITY ANALYSIS
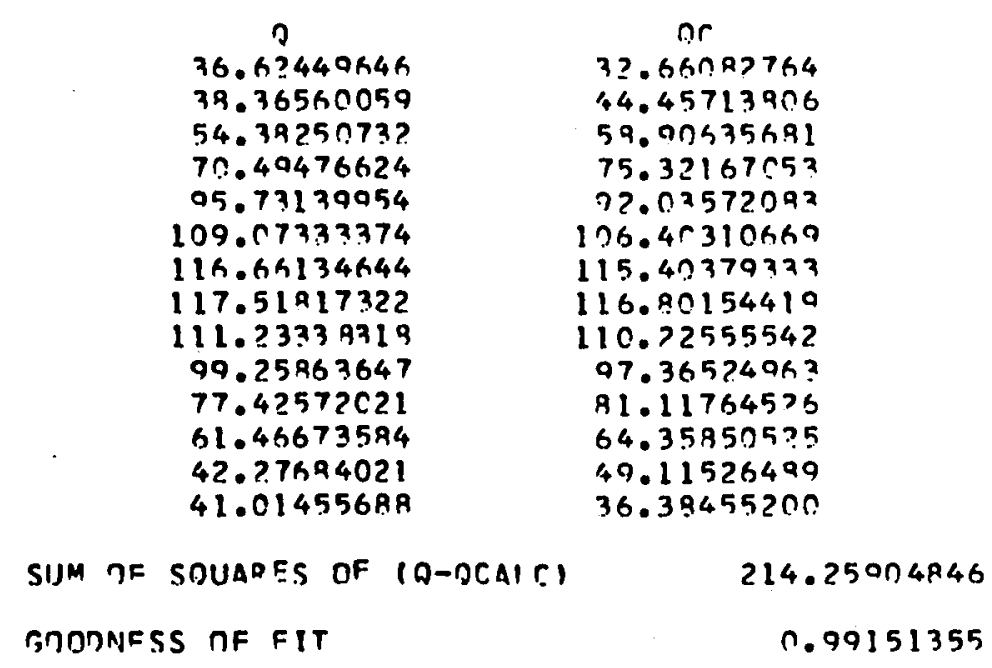
LIST F-2

FORTRAN LISTING FOR PROGRAM VELPRO, SUPPORTING SUBSTANCES ARE POLY AND CURVEF.

ALSO GIVEN ARE THE NECESSARY INPUT DATA AND SAMPLE OUTPUT.

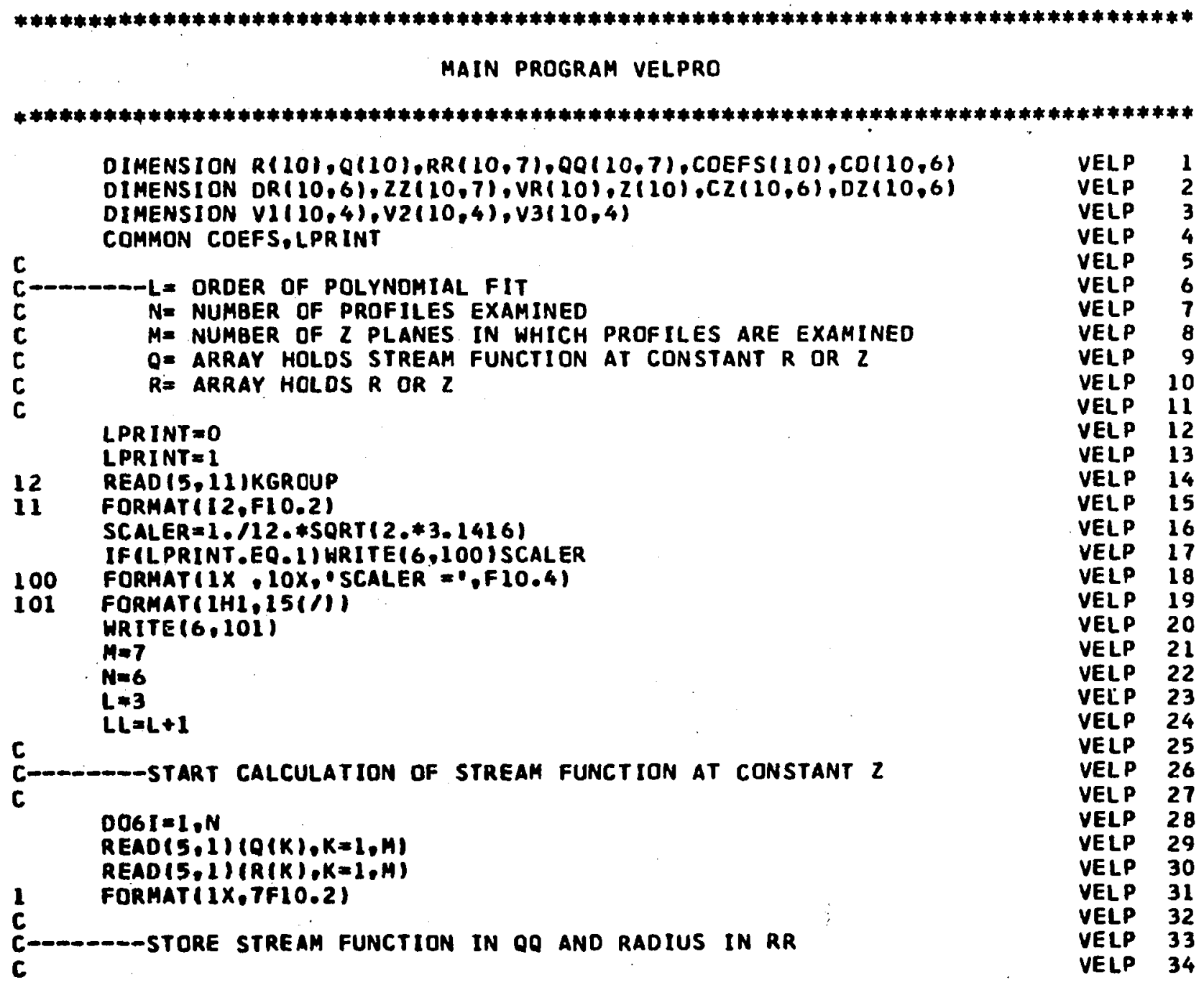




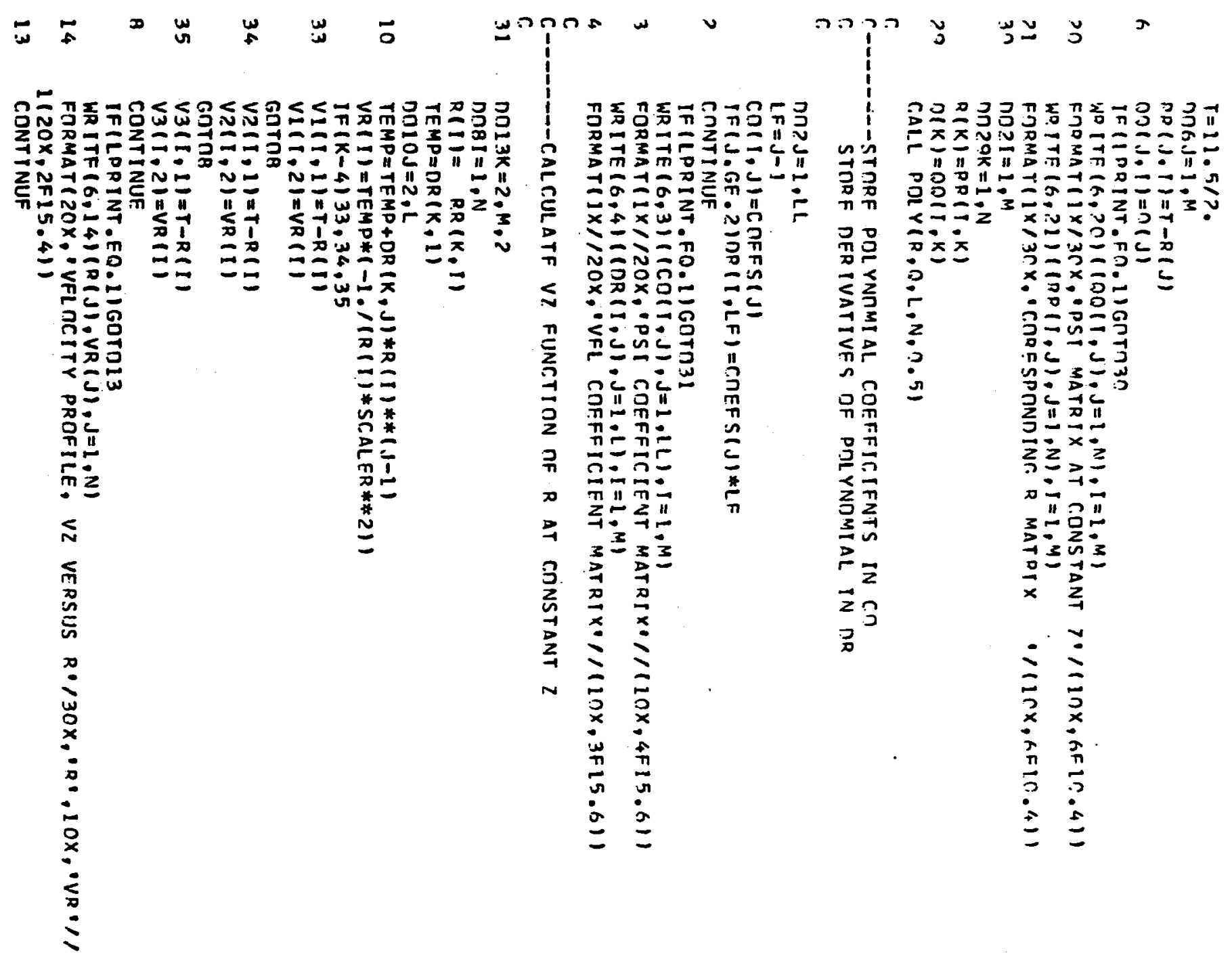

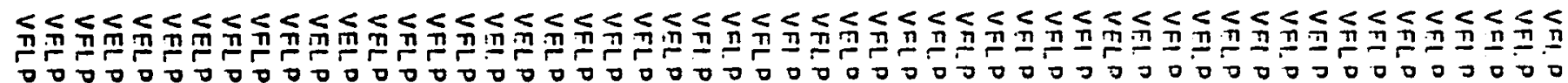

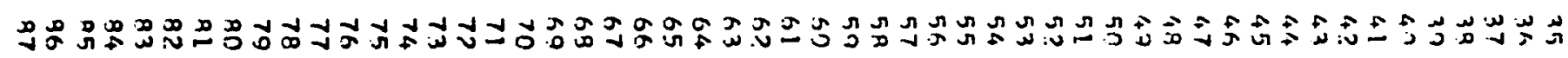


$r$

r.----OSTAPT CALCIJLATINA OF STPEAM FUNCTIINN AT CONSTAVT

r. K. SFLFTTS ALTFRNATF DNW

r. I. COUNTS CIMLIIMNS IN SFI.FCTFO ROW

I. FVALIIATFS NFW ELFMFNTS DF 7 AND STPFAM FIJNC.TINN PST
$r$.
$r$

c. STROE OFR.IVATIVES RF OMI.YMIMIAL AT CODNSTANT? IN $n$

$r$

DO1 $5 K=2, M, ?$

$D \cap 9 J=L, N$

$R T E M=R R(K, J)$

$? 7(M+1, J)=R$ TFM

TEMP $=1$.

$\cap \cap 5 I=I, M$

QTEM=Cח I, I)

$n \cap T K K=1, L$.

$7 \quad$ OTEM=QTFM+CN(I,KK+1)*RTFM*KK

$D([)=0$ TFM

$T F M P=T E M P+0.5$

$R(I)=T F M P$

$5 \quad$ LI I, J)=R(I)

CALL PMY $(R, O, L, M, 2,5)$

DNOKK $=1,1 . L$

$\mathrm{LF}=\mathrm{KK}-1$

C $?(J, K K)=C \cap F F S(K K)$

9 CONTINUF

IFILPR INT . FO. IIGOTN3?

WRITE $(6,2$ ? $)((Q O)(I, J), J=1, N), I=2, M)$

22. FORMATIIX/3NX, PSI MATRIX AT CONSTANT RI/(10X,6F10.41)

WRITE $(6,23)((2 Z(I, J), J=1, N), I=1, M)$

23 FORMATI $1 X / 30 X$. CORESPONDING $Z$ MATRIX $/(10 X, 6 F 10.2) 1$

HR ITF $(6,24)(27,(M+1, J), J=1, N)$

24 FIRMATIIX/30X, 'CORESPONIING VALUFS OF R, WHFN CONSTANT',

$1110 \times, 7 F 10.41)$

WRITE $(6,3)(\mid C Z(I, J), J=1, I L), I=1, N)$

WRITE $(6,4)((D 2) I, J), J=1, L), I=1, N)$

\section{C}

C-D---CALCULATE VR FUNCTION OF 7 . AT CONSTANT R

C KL SELFCTS POINTS IN DESIRED ROW

C I EVALUATES STREAM FUNCTION, FUNCTION DF 7 AT DESIRFD PIINT

$32 \quad$ T.TEM $=Z Z(K, 1)$

DO16KL $=1, N$

$R(K L)=22(M+1, K L)$

TEMP $=D Z(K L, 1)$

$D O 18 \mathrm{~J}=2, \mathrm{~L}$

$18 T$ TFMP $=$ TEMP $+D Z(K L, J) * Z T E M * *(J-1)$

VR $(K L)=T E M P /(R(K L) * S C A L E R * * 2)$

IF $(K-4) 37,38,39$

$37 \quad V_{1}(K L, 3)=V R(K L)$

GoTn 16

VFI $\cap \quad 99$

VFI $\triangle 97$

VFI.D an

VFin ?

VEI. ? ?

VFLD OR

VFLP $9 /$

VFI.P 25

VFID $D A$

VFI. 97

VEI $P \quad 79$

Vriln on

vrip inn

Vㄷ․ P 1n!

VELO IC?

VFI 1.3

VEI. 104

VFI.D 125

VELD IOS

VFI D 107

VFI $P$ 109

Vri.P 109

VFLP 110

VELP 111

VFL.P 112

VFLP 117

VFLP 114

VELP 115

VELP 116

VFLP 117

VFI. D 119

VFI $P 119$

VFL 120

VELP 121

VFLP 12?

VFLP 123

VFLP $1 ? .4$

- VFLP $1 ? 5$

VFLP 126

VELP 127

VEL.P 1?

VFLP 129

VFI P 130

VEI.P 131

VFLP 132

VFLP 133

VFI.P 134

VELP 135

VELP 1.36

VFLP 137

VFLP 138

VFLP 1.39

VELP 140 


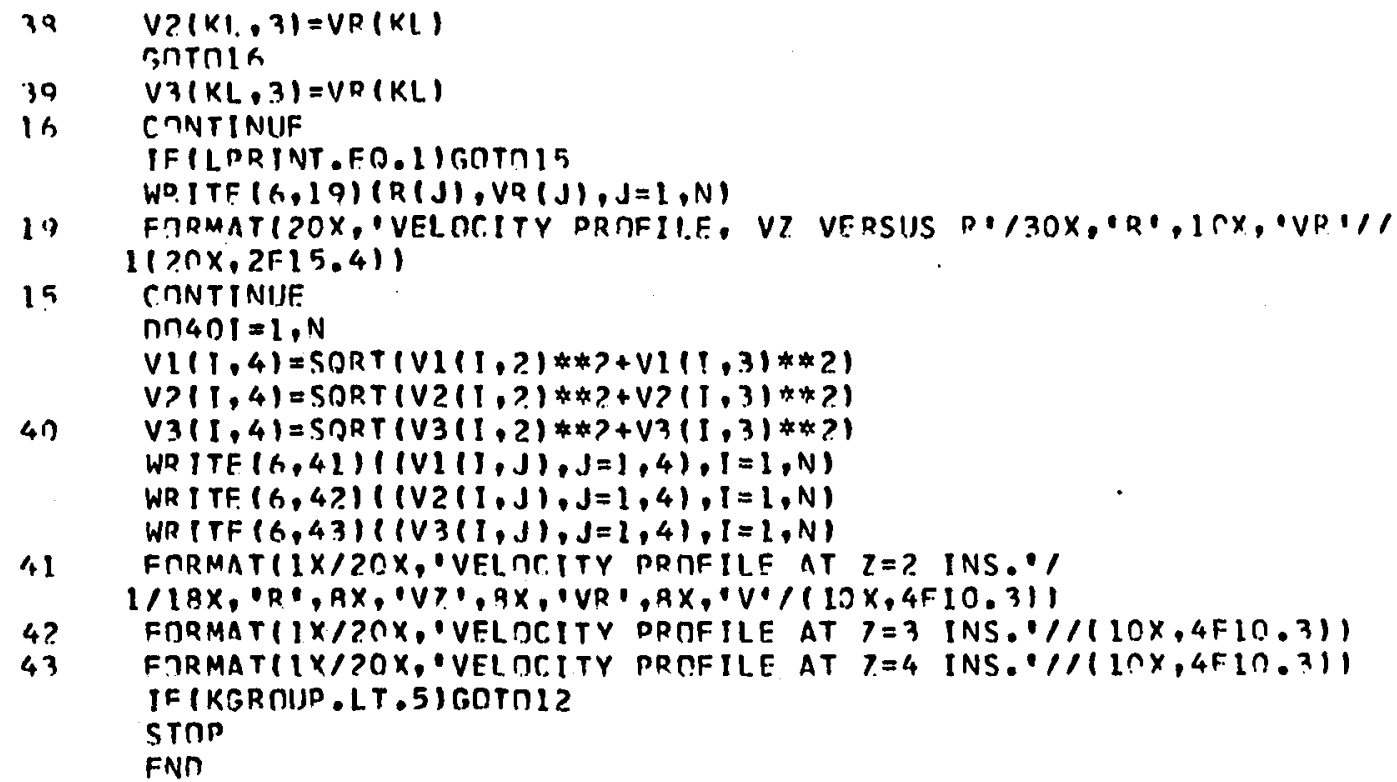

VII. $1+1$

VI:I.P 14 ?

$V=1 \cap 1 \% 3$

VILO L144

VFLD 1145

VELO 11\%

$V=1 P 147$

$V=10 \quad 149$

VFI 149

VFID 150

VFID 151

VELD 15?

VIID 153

VFLP 154

VFLO 155

VII. 156

VFL 157

VFI.D 159

Vㄷ․ 157

VEL IGO

VFLD 141

Vㄷ․ P 16?

VFID 163

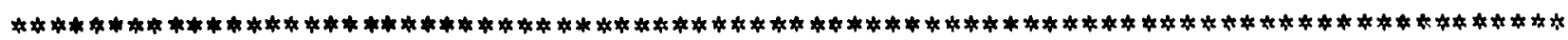
SURROUTINE PRLY

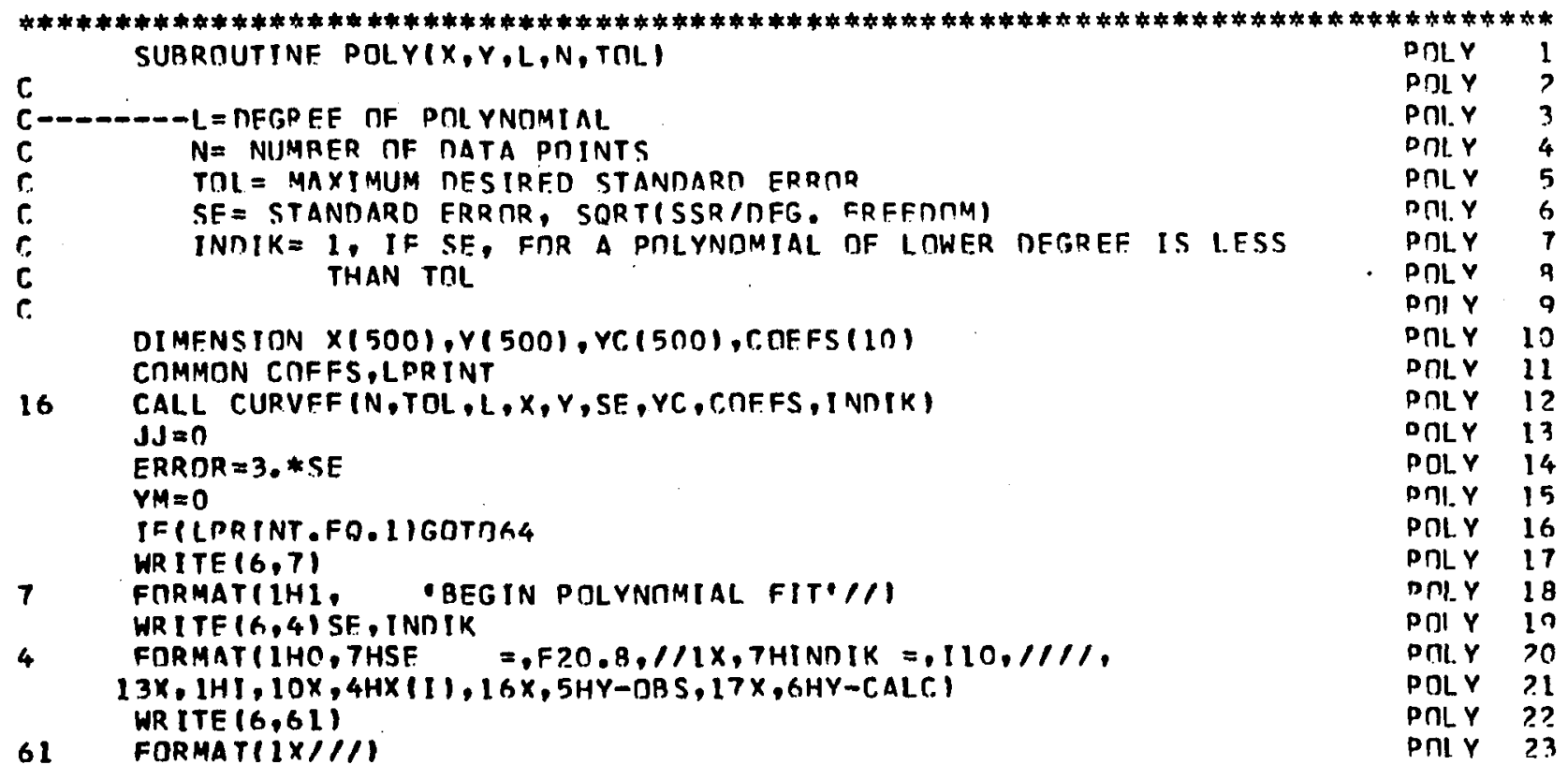




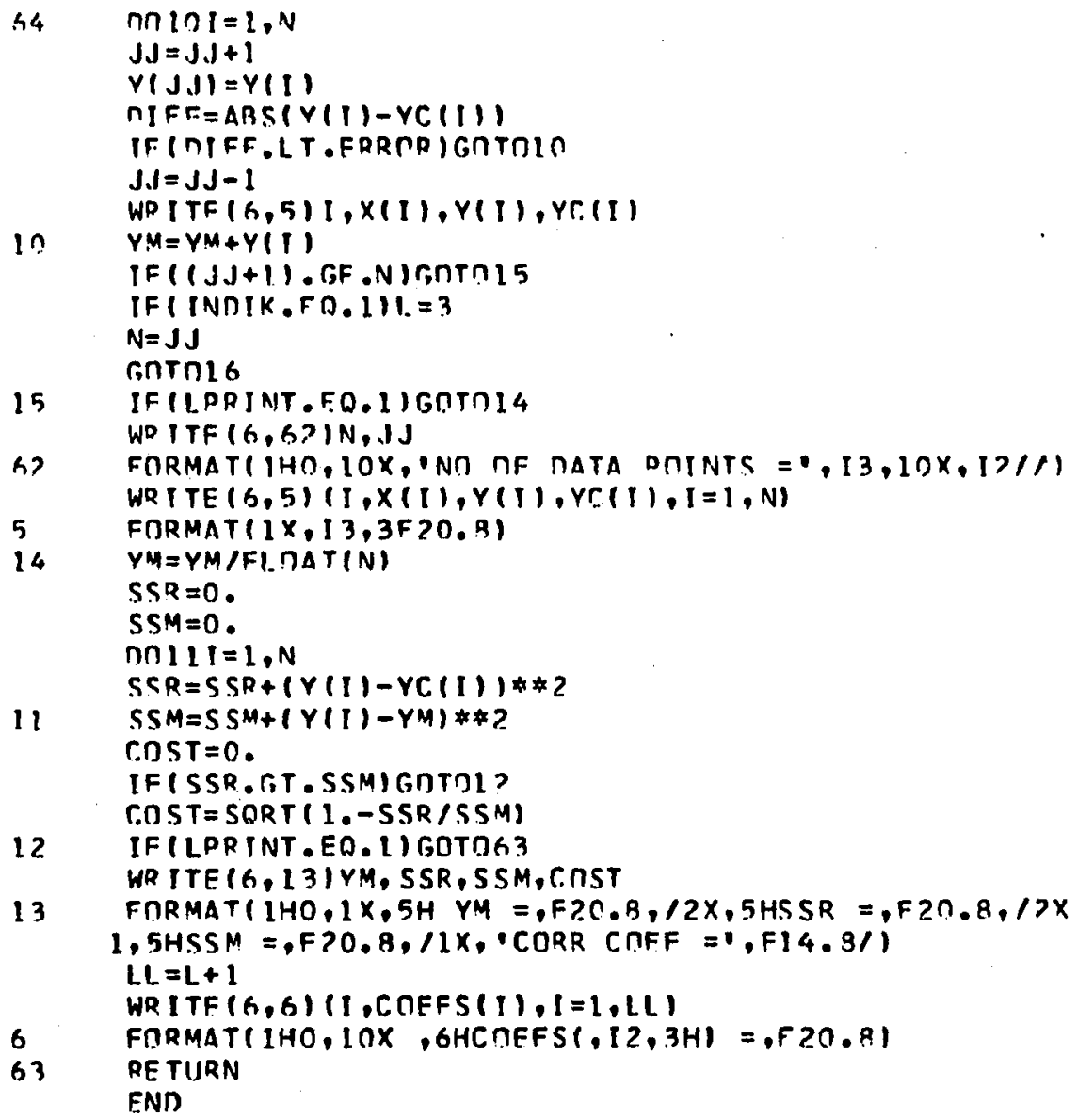

Dा $Y \quad 34$ $P \cap L Y$ ? $P$ PחI $Y$ ?h DחL $P 7$ nחi $Y$ in nกוY ?a PחL $Y$ 3n DOL $\quad 31$ pกI $Y$ is Bก $Y 33$ D) $Y$ 3/4 DกI $Y 35$ PII Y 36 PกI. $Y \quad 37$ DIL $Y 3 R$ nחi $Y$ 3n PกL $Y 40$ PחLY 41 PחI.Y 4 ? PחLY 43 PกL 144 PחI. 45 PחI. 45 DII.Y 47 PחL $Y$ 4R PII Y 49 POI.Y 50 PחL Y 51 PDL $Y 52$ PDLY 53 PחLV 54 PnI Y 55 POLY 56 nILY 57 PDLY 59

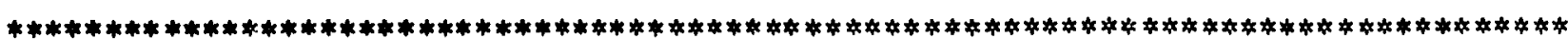
SURROUT INE CURVFF THIS SUBROUTINE IS A PART DF LSU SHARE LIBPARY

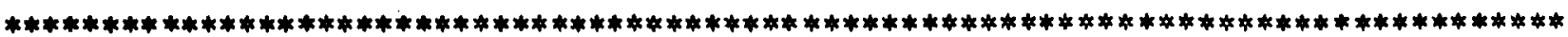

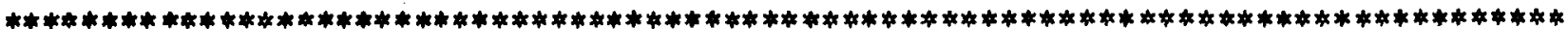
INPUT DATA PUNC.HFD DUTPUT FROM SUBRIUTINF SPRINT OF PRMGRAM FIDWANL FIR LISTING SEE LIST F-1 


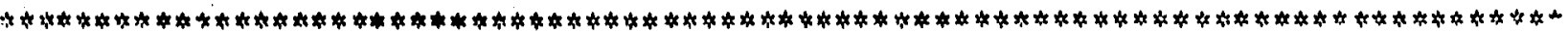

STDFAMFUNCTINN PSI VERSIJS Q FOP MATPIX OF POINTS |FIGIJRF IV-2) BFI. NW IMPFI.L.FR CENTERL INA

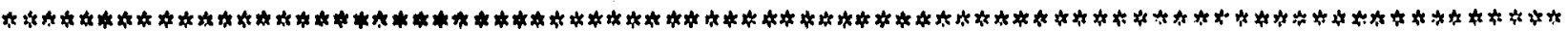

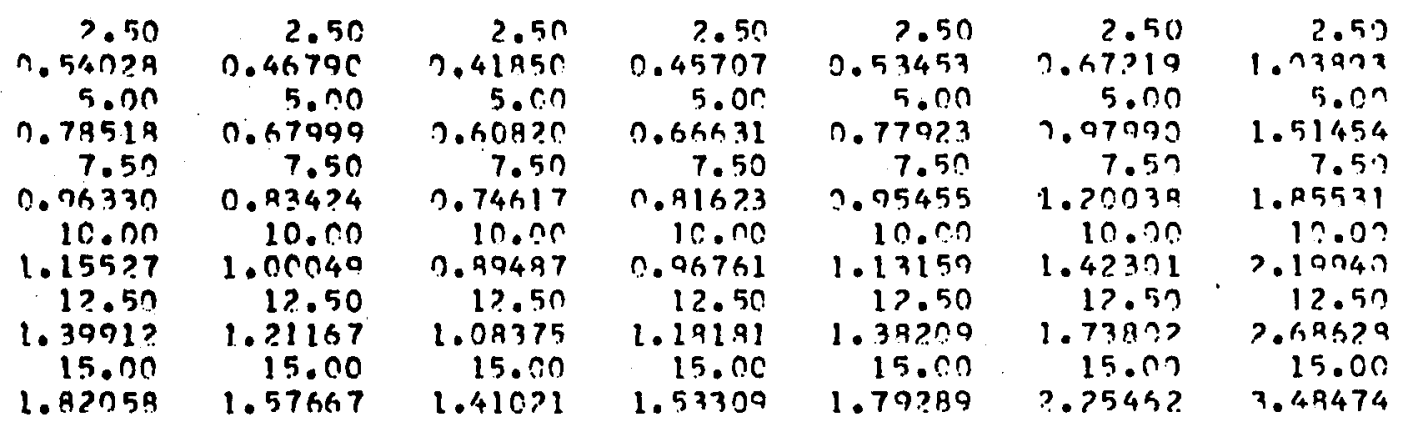

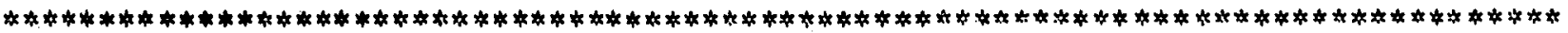

STRFAMFUNCTION OSI VFPSUS R FOR MATPIX TF PMINTS (FIGUPF IV-? ARNVF IMPFLLFR CENTFRLINE

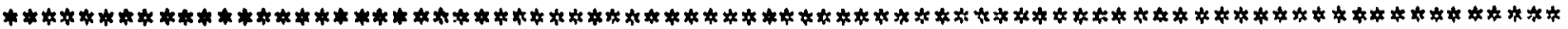

\begin{tabular}{|c|c|c|c|c|c|c|}
\hline $\begin{array}{r}2.50 \\
0.54101 \\
5.00 \\
0.78674 \\
7.50 \\
0.96459 \\
10.00 \\
1.15683 \\
12.50 \\
1.40100 \\
15.00 \\
1.82302\end{array}$ & $\begin{array}{r}2.50 \\
0.46853 \\
5.00 \\
0.68090 \\
7.50 \\
0.83536 \\
10.00 \\
1.00184 \\
12.50 \\
1.21330 \\
15.00 \\
1.57879\end{array}$ & $\begin{array}{r}2.50 \\
0.41906 \\
5.00 \\
0.60902 \\
7.50 \\
0.74717 \\
10.00 \\
0.89607 \\
12.50 \\
1.08521 \\
15.00 \\
1.41211\end{array}$ & $\begin{array}{r}2.50 \\
0.38 ? 55 \\
5.00 \\
0.55595 \\
7.50 \\
0.68207 \\
10.00 \\
0.81800 \\
12.50 \\
0.99066 \\
15.00 \\
1.7 .9907\end{array}$ & $\begin{array}{r}2.50 \\
0.38820 \\
5.00 \\
0.56574 \\
7.50 \\
0.69314 \\
10.70 \\
0.82260 \\
12.50 \\
1.00399 \\
15.00 \\
1.30266\end{array}$ & $\begin{array}{r}2.50 \\
0.43402 \\
5.00 \\
0.63252 \\
7.50 \\
0.77495 \\
10.00 \\
0.91960 \\
12.50 \\
1.12239 \\
15.90 \\
1.45642\end{array}$ & $\begin{array}{r}2.50 \\
0.52116 \\
5.00 \\
0.73037 \\
7.50 \\
0.89483 \\
10.00 \\
1.96197 \\
12.59 \\
1.79501 \\
1.5 .00 \\
1.68172\end{array}$ \\
\hline
\end{tabular}


SAMPLE OUTPUT FOR PROGRAM VELPRO

\begin{tabular}{cccc}
\multicolumn{5}{c}{ VFLOCITY PROFILF $A T ~ Z=2$} & INS. \\
$R$ & $V Z$ & VR & $V$ \\
0.5 & 74.3 & .8 .3 & 74.7 \\
0.7 & 67.2 & 10.3 & 68.3 \\
0.8 & $67 . ?$ & 11.7 & 63.3 \\
1.0 & 56.8 & 12.9 & 58.3 \\
1.2 & 50.2 & 14.0 & 52.1 \\
1.6 & 39.1 & 14.4 & 41.7
\end{tabular}

VELDCITY PRDF ILF AT $7=3$ INS.

$\begin{array}{llll}0.5 & 76.2 & -10.5 & 76.9 \\ 0.7 & 69.0 & -10.8 & 69.3 \\ 0.8 & 63.9 & -11.0 & 64.8 \\ 1.0 & 58.7 & -11.3 & 59.8 \\ 1.2 & 51.4 & -11.7 & 52.7 \\ 1.5 & 39.5 & -11.8 & 41.7\end{array}$

VEI. OCITY PROFILF AT $Z=4$ INS.

$\begin{array}{llll}0.7 & 59.1 & -14.9 & 61.0 \\ 1.0 & 50.5 & -29.2 & 58.3 \\ 1.2 & 45.5 & -36.7 & 58.5 \\ 1.4 & 41.6 & -47.2 & 59.3 \\ 1.7 & 38.4 & -46.7 & 60.5 \\ 2.3 & 41.0 & -47.7 & 62.9\end{array}$


VEL RCITY PROFILF AT $7=$ ? INS.

$\begin{array}{cccc}R & V I & \text { VR } & V \\ 0.5 & 73.9 & 9.7 & 74.5 \\ 0.7 & 67.1 & 12.5 & 69.3 \\ 0.8 & 67.2 & 14 . ? & 63.9 \\ 1.0 & 57.0 & 15.6 & 59.7 \\ 1 . ? & 50.2 & 16.7 & 52.9 \\ 1.6 & 38.5 & 16.5 & 41.9 \\ \text { VELOCITY PRAFILE AT } 7=3 \text { INS. }\end{array}$

$\begin{array}{llll}0.4 & 98.9 & 1.9 & 88.9 \\ 0.6 & 80.2 & 7.7 & 80 . ? \\ 0.7 & 73.9 & 3.1 & 73.9 \\ 0.8 & 67.1 & 3.5 & 67 . ? \\ 1.0 & 58.7 & 3.8 & 58.9 \\ 1.3 & 44.3 & 3.9 & 44.5 \\ \text { VELOCITY PROFILE } & \text { AT } 7=4 \text { INS. }\end{array}$

$\begin{array}{llrl}0.4 & 79.6 & -1.7 & 80.9 \\ 0.6 & 72.1 & -11.3 & 73.0 \\ 0.8 & 66.7 & -12.8 & 68.0 \\ 0.9 & 61.2 & -14.0 & 62.8 \\ 1.1 & 53.5 & -15.0 & 55.5 \\ 1.5 & 40.4 & -14.9 & 43.1\end{array}$


LIST $\mathbf{R - 3}$

FORTRAN LISTING FOR PROGRAM TANRANL WITH SUPPORTING SUBROUTINES OMEGA. ALSO GIVEN ARE THE

RAW DATA AND A SAMPLE OUTPUT FOR PORT 1.

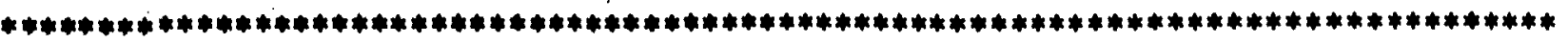

MAIN PROGRAM TANKANL

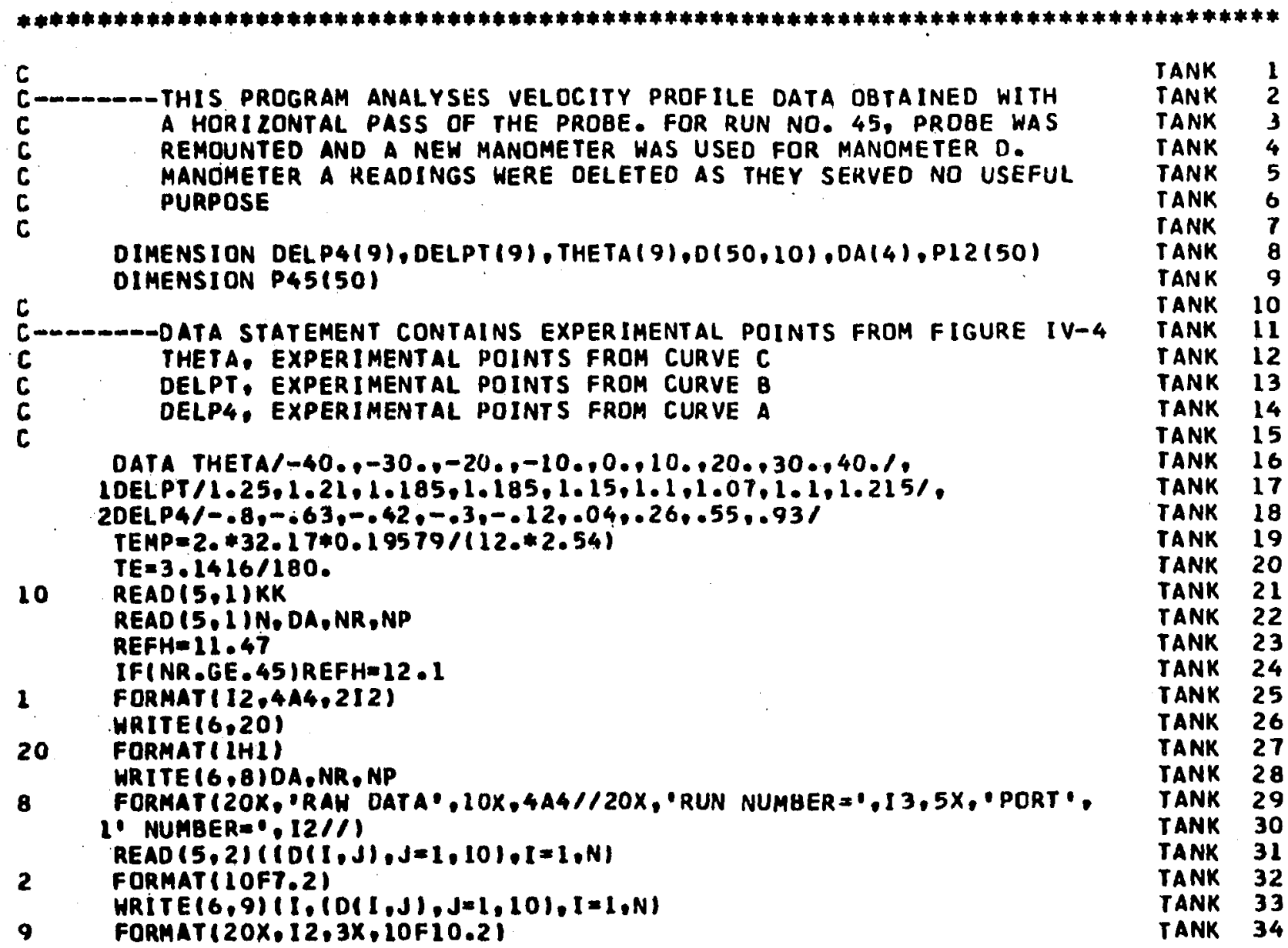




$$
c
$$

TAVK 75

TAVK 30

TAYK 37

TAYK 38

TAVK 37

TAVK 40

TANK 41

TAVK 4?

TAVK 43

TAVP 44

TAVK 45

TAVK. 46

TAVK 47

TAVK 48

TAVK 49

TAVK 50

TANK 51

TANK 5?

TAVK 5.3

TAVK 54

TAYK 55

TAVK 56

TAVK 57

TANK 58

TANK 59

TAVK 6?

TANK 61

TAVK 6?

TANK 63

TAVK 64

TANK 65

TAVK 66

TANK 67

TANK 68

TAVK 69

TANK 70

TAVK 71

TANK 72

TANK 73

TAVK 74

TAVK 75

TAVK 76

TAVK $7 T$

TAVK 78

TANK 79

TANK RO

TAVK 81

TANK B?

TANK 93 
FOR LISTIVG SFF I. IST F-I

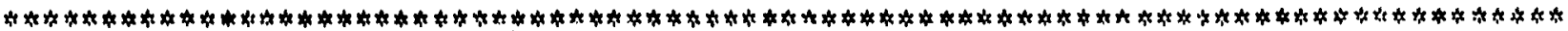

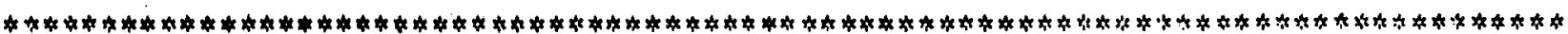
INPIIT INTA

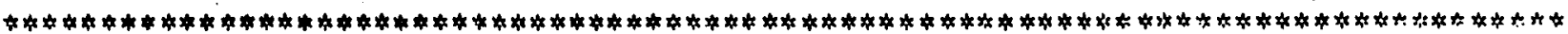

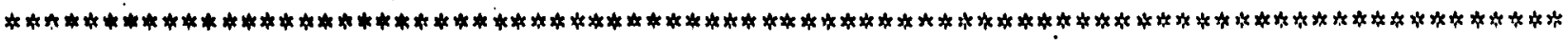
DIRT NO. 1

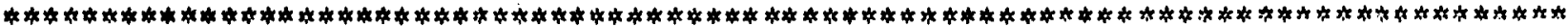

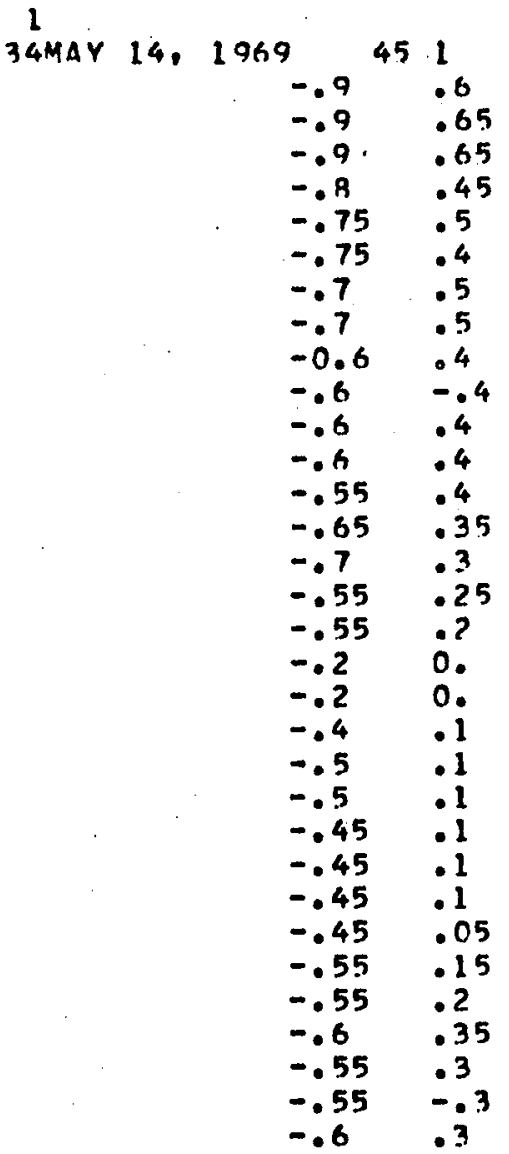

$\begin{array}{llll}-7.08 & -5.97 & 12 . ? & 146 . \\ -6.85 & -6.1 & 12.3 & 146 . \\ -6.61 & -6.31 & 12.4 & 146 . \\ -6.56 & -6.4 & 12.5 & 158.8 \\ -6.5 & -6.46 & 12.55 & 157.6 \\ -6.6 & -6.49 & 12.6 & 157.6 \\ -6.39 & -6.56 & 12.7 & 151.2 \\ -6.32 & -6.6 & 12.9 & 144.7 \\ -6.35 & -6.55 & 17.9 & 144.9 \\ -6.35 & -6.55 & 13.9 & 138.7 \\ -6.4 & -6.5 & 13.1 & 132.9 \\ -6.45 & -6.45 & 13.7 & 124 . \\ -6.56 & -6.35 & 13.3 & 106 . \\ -6.65 & -6.3 & 13.4 & 105.4 \\ -6.71 & -6.24 & 13.5 & 99 . \\ -6.8 & -6.15 & 13.6 & 94 . \\ -6.78 & -6.11 & 13.7 & 89.4 \\ -6.75 & -6.15 & 13.9 & 2.9 \\ -6.78 & -6.15 & 14.1 & 2.8 \\ -6.8 & -6.12 & 14.3 & 23 . \\ -6.82 & -6.11 & 14.5 & 7.2 \\ -6.85 & -6.1 & 14.7 & 12.8 \\ -6.85 & -6.1 & 14.9 & 17.4 \\ -6.8 .5 & -6.1 & 15.1 & 17.4 \\ -6.82 & -6.1 & 15.3 & 26.7 \\ -6.85 & -6.1 & 15.5 & 21.6 \\ -6.9 & -6.05 & 15.7 & 31.9 \\ -6.9 & -6.05 & 15.9 & 31.9 \\ -7.6 & -5.3 & 16.1 & 31.4 \\ -7.5 & -5.4 & 16.3 & 33.8 \\ -7.4 & -5.5 & 16.5 & 39 . \\ -7.39 & -5.51 & 16.7 & 39 . \\ & & & \end{array}$




$$
\begin{array}{llllll}
-0.55 & 0.3 & -7.4 & -5.5 & 1.9 .9 & 4.3 . ? \\
-0.55 & 0.3 & -7.4 & -5.5 & 17.1 & 43.2
\end{array}
$$

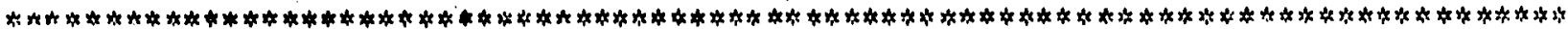

PNRT NO. ?

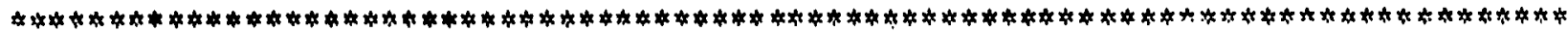

1

72ADQIL 14,1969 4? 2

$6.1 \quad-6.45 \quad-1.75 \quad 1.4$

$6.1-6.45 \quad-1.9 \quad 1.4$

$\begin{array}{llll}6.1 & -6.45 & -1.75 & 1.4\end{array}$

$6.1 \quad-6.45 \quad-1.75 \quad 1.35$

$6.1-6.45-1.75 \quad 1.35$

$\begin{array}{llll}6.08 & -6.45 & -1.8 & 1.4\end{array}$

$\begin{array}{llll}6.05 & -6.4 ? & -1.9 & 1.4\end{array}$

$6.05 \quad-6.4 ?-1.45 \quad 1.05$

$\begin{array}{llll}6.05 & -6.4 & -1.55 & 1.15\end{array}$

6.05 $-6.4 \quad-1.5 \quad 1.15$

$6.04 \quad-6.4 \quad-1.3 \quad 1$.

$6.05 \quad-6.4 \quad-1.3 \quad 1$.

$\begin{array}{llll}5.95 & -6.5 & -1.1 & .1\end{array}$

5.95. $-6.5 \quad-1.1 \quad .8$

$5.95 \quad-6.5 \quad-1.1 \quad .8$

$\begin{array}{llll}5.95 & -6.5 & -1.1 & .75\end{array}$

$5.95 \quad-6.55-1.15 \quad .8$

$5.95 \quad-6.53 \quad-1.15 \quad .8$

$5.05-6.55-1 . ? \quad .85$

$5.95-6.55-1.2 \quad .8$

$\begin{array}{llll}5.05 & -6.55 & -1.2 & .8 \\ 6.0 & -6.52 & -1.4 & 1.1\end{array}$

6. $\quad-6.5 \quad-1.2 \quad .95$

6. $\quad-6.51-1.15 \quad .9$

6. $-6.52-1.15 \quad .85$

6. $-6.55-1.15 \quad .85$

6. $-6.55-1.15 \quad .85$

6. $-6.55-1.150 .85$

6. $-6.55-1.55 \quad .85$

6. $-6.55-1.2 \quad .9$

6. $-6.55-1.2 \quad: 9$

\begin{tabular}{|c|c|c|c|}
\hline $\begin{array}{l}-18 . \\
-17.9 \\
-17.65 \\
-17.5 \\
-17.4 \\
-17.4 \\
-17.4 \\
-18.95 \\
-18.95 \\
-19 . \\
-19.6 \\
-19.6 \\
-24.9 \\
-25 . \\
-25.1 \\
-25 . \\
-25.9 \\
-24.9 \\
-24.9 \\
-24.8 \\
-24.8 \\
-24.3 \\
-24.3 \\
-24.25 \\
-24.25 \\
-24.2 .5 \\
-24.75 \\
-24.25 \\
-24.25 \\
-24.2 \\
-24.2 \\
-20.2\end{array}$ & $\begin{array}{l}-18.3 \\
-18.5 \\
-18.9 \\
-19.15 \\
-19.5 \\
-19.9 \\
-19.7 \\
-21 . \\
-21.2 \\
-21.15 \\
-21.2 \\
-21.25 \\
-25.3 \\
-25.2 \\
-25.1 \\
-25 . \\
-25 . \\
-24.9 \\
-25 . \\
-25 . \\
-25 . \\
-24.4 \\
-24.4 \\
-24.35 \\
-24.35 \\
-24.35 \\
-24.35 \\
-24.35 \\
-24.35 \\
-24.35 \\
-24.3 \\
-21.1\end{array}$ & $\begin{array}{l}11.55 \\
11.65 \\
11.7 \\
11.75 \\
11.9 \\
11.7 \\
12.9 \\
12.1 \\
12.2 \\
12.3 \\
12.4 \\
12.5 \\
12.6 \\
12.7 \\
12.9 \\
13.9 \\
13.2 \\
13.4 \\
13.6 \\
13.9 \\
14.9 \\
14.2 \\
14.4 \\
14.6 \\
14.9 \\
15.9 \\
15.3 \\
15.6 \\
15.8 \\
16.9 \\
16.9 \\
16.4\end{array}$ & $\begin{array}{l}160 . \\
160 . \\
160 . \\
159.6 \\
155 . \\
149.4 \\
144.6 \\
144.6 \\
135 . \\
130 . \\
111 . \\
111 . \\
82 . \\
89.9 \\
48 . \\
23.4 \\
18.8 \\
17.8 \\
10.6 \\
6.6 \\
6.6 \\
8.6 \\
8.6 \\
8.6 \\
12.8 \\
15.6 \\
27.2 \\
22.2 \\
21.6 \\
24.2 \\
26 . \\
26 .\end{array}$ \\
\hline
\end{tabular}

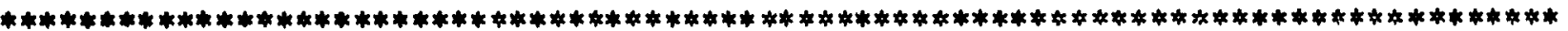
PAP.T Nח. 3

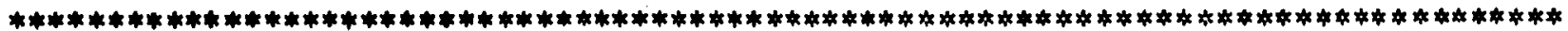

9

28APRIL 14, 1969433

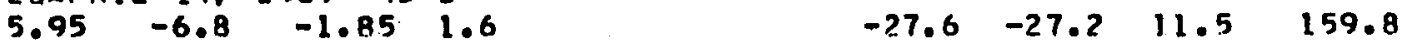




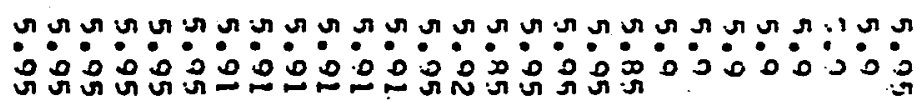

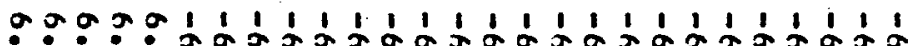

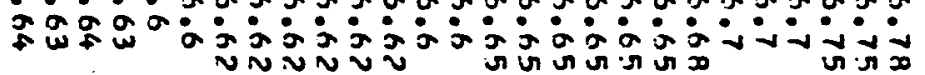

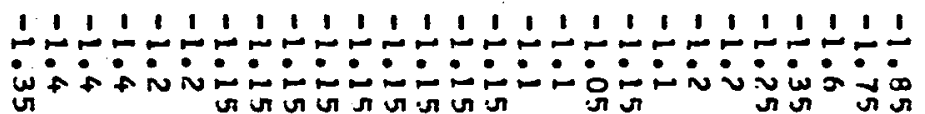

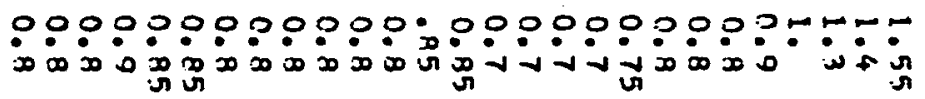

:००?:००:?:?? ?: ?:?

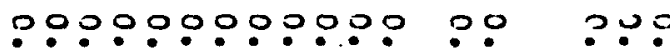

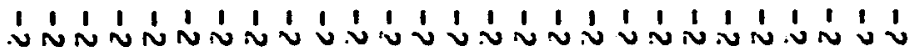

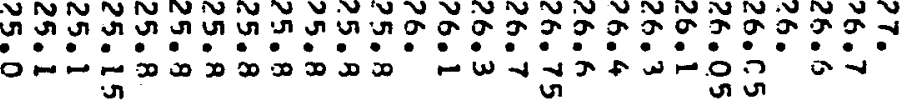

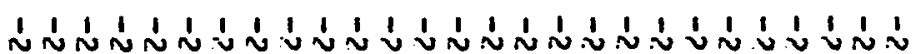

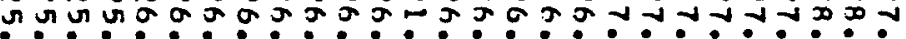

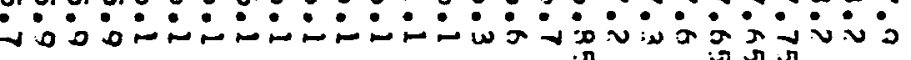

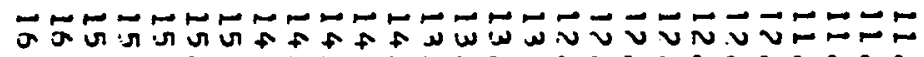

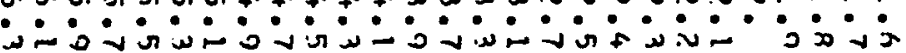

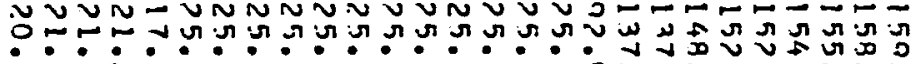




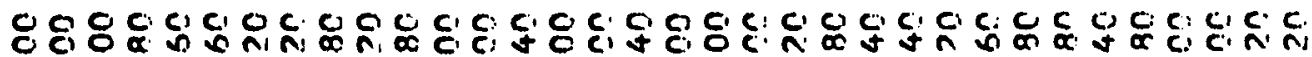
-

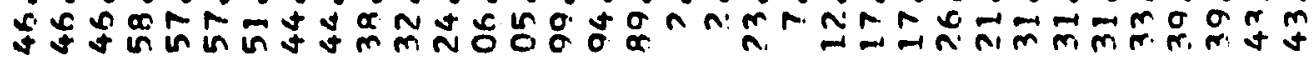

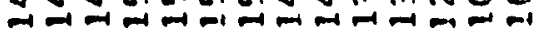

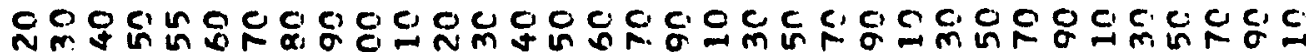

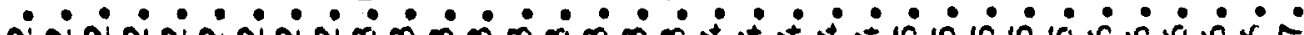

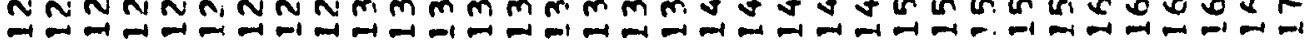

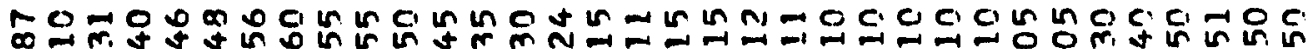

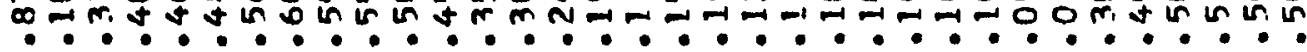

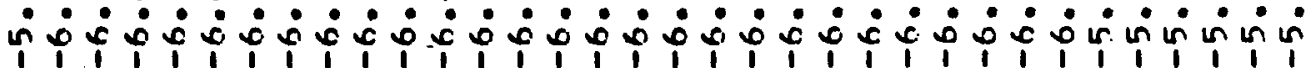

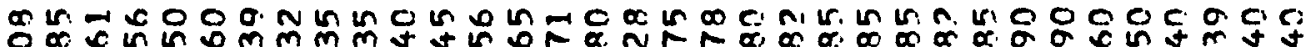
†ि

000000000000000000000000000000000

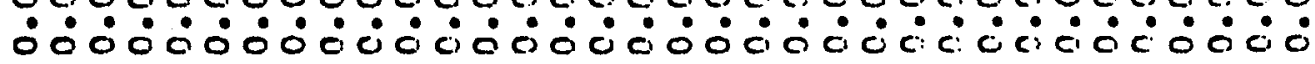

0000000000000000000000000000000000

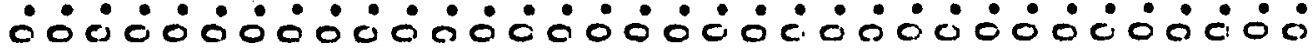

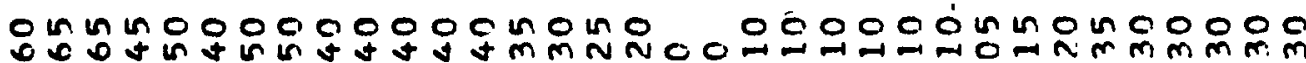

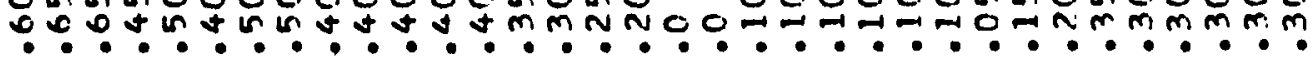

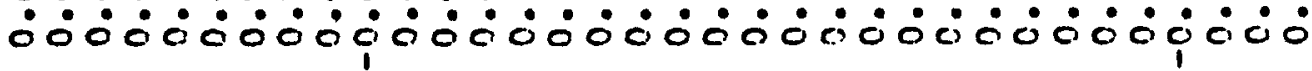

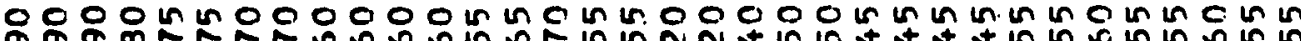

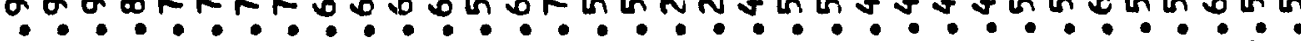

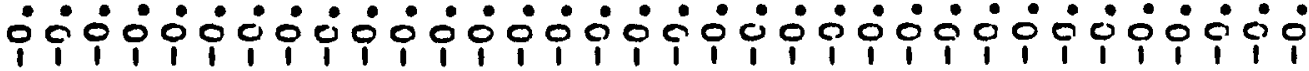

0000000000000000000000000000000000

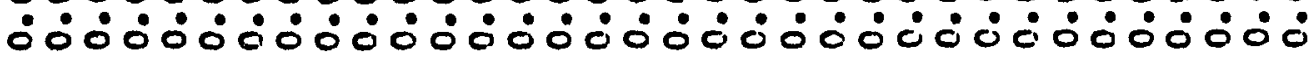

0000000000000000000000000000000000

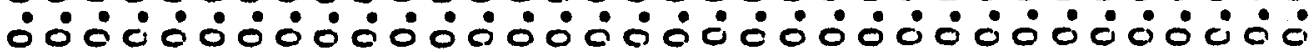




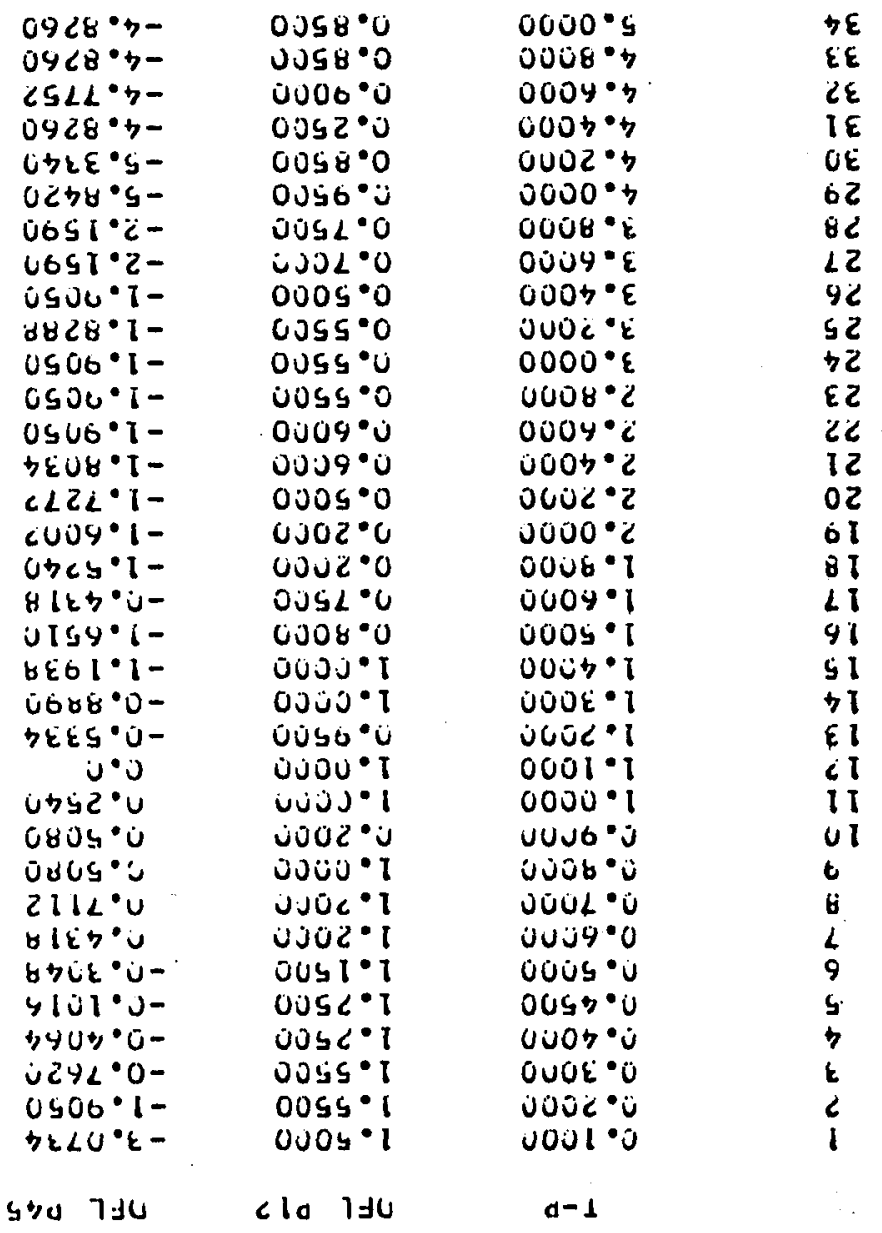




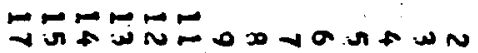

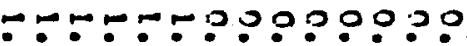

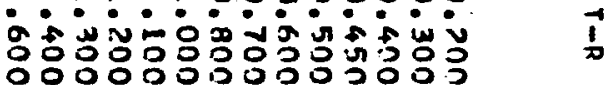

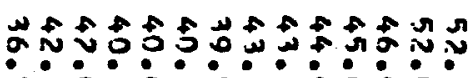

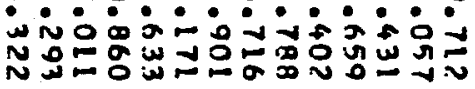

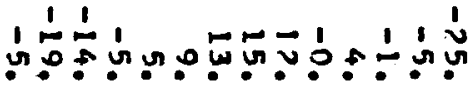

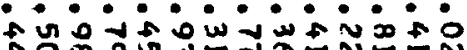

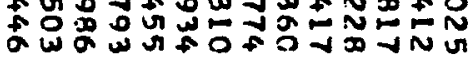

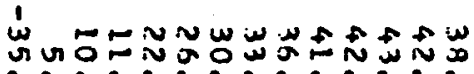

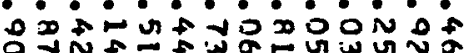

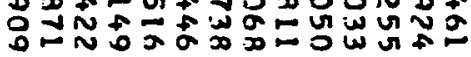
눈

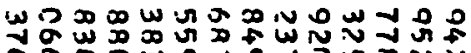

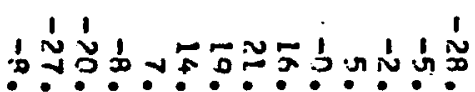
o N

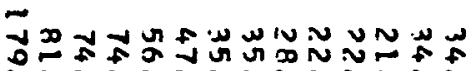

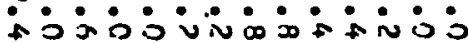

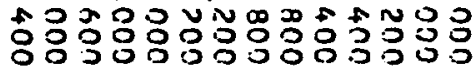

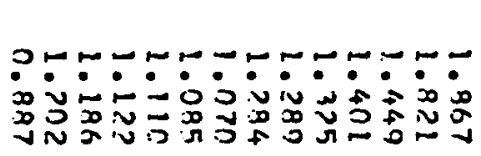


LIST F-4

FORTRAN LISTING FOR PROGRAM YAWANL WITH SUPPORTING SUBROUTINE OMEGA AND ILLUSTRATED WITH SAMPLE OUTPUT FOR EXPERTMENTAL DATA FROM RUN NUMBER 29.

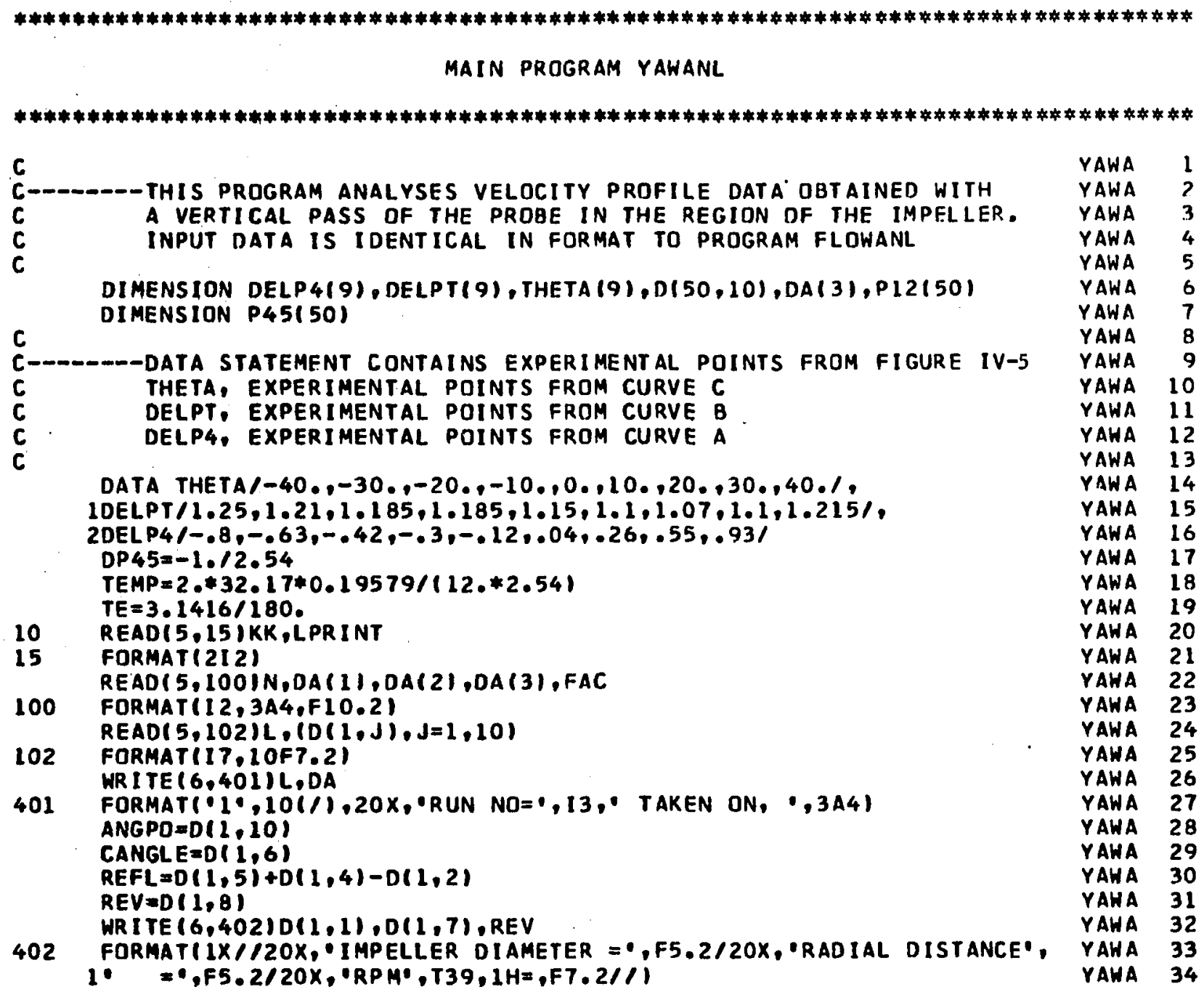


READ $(5,104)(C D(I, J), J=L, 10), I=L, N) \quad$ YAWA 35

104 FORMAT(1OF 7.2)

IF (LPRINT, EQ.4) GOTOI6

WRITE $(6,105)(10(1, J), J=1,10), I=1, N)$

105 FORMAT $(24 X, 1$ IHP(1)-P(ATM), $5 X, 9 H P(1)-P(2), 4 X, 9 H P(2)-P(3), 3 X$,

I9HP(4)-P(5),5X,1HH, 4X,5HANGLE, $24 X, 1$ IHLEFT RIGHT, $4 X$,

21 LHLET RIGHT $2 X, 10 H L E F T$ RIGHT, 3X, LOHLEFT RIGHT, $/ 1$

$3(20 x, 2 F 7,2, F B .2, F 7,2,1 X, 2 F 5.2,4 F 7.2))$

C------TRANSFORM RAH DATA INTO PRESSURE OROPS VERSUS DISTANCE FROM

C TANK HALL

$1600106 I=1, N$

$D(I, 9)=($ REFL-D $(1,9))$

$D(1,10)=D(I, 10)-C A N G L E$

IF IFAC . NE . I. IGOTOL.07

$D(I, 3)=D(1,3) * 2.54$

$D(I, 4)=0(1,4) * 2.54$

$D(I, 7)=0(1,7) * 2.54$

$D(I, 8)=D(1,8) * 2.54$

$107 \quad P 12(1)=0(1.4)-0(1.3)$

$P 45(1)=0(1,7)-D(1,8)-D P 45$

106 CONTINUE

WRITE $(6,4)(I, D(I, 9), P 12(I), P 45(I), I=I, N)$

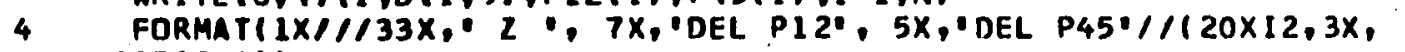
$13 F 12.411$

WR ITE 16.61

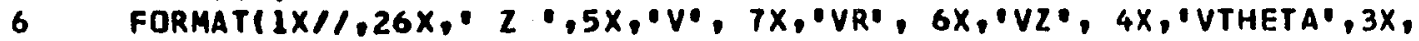

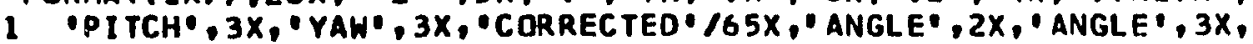
2 ODEL P12०/11

C

C-- - - CALCULATE VELOCITY PROFILE USING EQUATIONS IV-27(a) TO IV-27(c)

C

$005 I=1, N$

RAT IO=P45(1)*0.09537/ (P12(1)*0.19579)

C

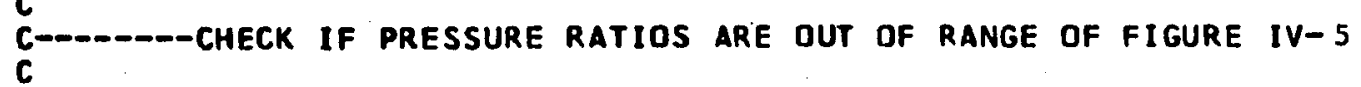

C

IFIRATIO.LT.I-.8).OR.RATIO.GT.I.) GOTO5

C-D-DINTERPOLATE TO OBTAIN TRUE KINETIC HEAD, P(T)-P(S).

C EQUIVALENT TO LOOKING UP FIGURE IV-4

CALL DMEgAIRATIO,DELP4,THETA,9,9, ANGLE)

$T P=A N G L E * T E$

$T Y=0(1,10) * T E$

CALL OHEGA (ANGLE, THETA,DELPT $, 9,9$, DELTA)

DELTA=DELTA*P12(I)

$V=S O R T(T E M P * D E L T A I * 60$.

$V Z=V * S I N(T P)$

VTE $=V * \operatorname{COS}(T P)$

$V R=V T E * \operatorname{COS}(T Y)$

VTHETA=VTE*SIN(TY)

URITE $(6,7) I, D(1,9), V, V R, V Z, V T H E T A, A N G L E, D(I, 10), D E L T A$

$\begin{array}{ll}\text { YAWA } & 35 \\ \text { YAWA } & 36 \\ \text { YAWA } & 37 \\ \text { YAWA } & 38 \\ \text { YAWA } & 39 \\ \text { YAWA } & 40 \\ \text { YAWA } & 41 \\ \text { YAWA } & 42 \\ \text { YAWA } & 43 \\ \text { YAWA } & 44 \\ \text { YAWA } & 45 \\ \text { YAWA } & 46 \\ \text { YAWA } & 47 \\ \text { YAWA } & 48 \\ \text { YAWA } & 49 \\ \text { YAWA } & 50 \\ \text { YAWA } & 51 \\ \text { YAWA } & 52 \\ \text { YAWA } & 53 \\ \text { YAWA } & 54 \\ \text { YAWA } & 55 \\ \text { YAWA } & 56 \\ \text { YAWA } & 57 \\ \text { YAWA } & 58 \\ \text { YAWA } & 59 \\ \text { YAWA } & 60 \\ \text { YAWA } & 61 \\ \text { YAWA } & 62 \\ \text { YAWA } & 63 \\ \text { YAWA } & 64 \\ \text { YAWA } & 65 \\ \text { YAWA } & 66 \\ \text { YAHA } & 67 \\ \text { YAWA } & 68 \\ \text { YAWA } & 69 \\ \text { YAWA } & 70 \\ \text { YAHA } & 71 \\ \text { YAHA } & 72 \\ \text { YAWA } & 73 \\ \text { YAWA } & 74 \\ \text { YAHA } & 75 \\ \text { YAWA } & 76 \\ \text { YAWA } & 77 \\ \text { YAWA } & 78 \\ \text { YAWA } & 79 \\ \text { YAHA } & 80 \\ \text { YAWA } \\ \text { YAHA } & 86 \\ \text { YAHA } & 81 \\ \text { YAHA } & 87 \\ \text { YAHA } & 88 \\ \text { YAHA } & 83 \\ \text { YA } & \end{array}$ 
7 FORMAT(20X,12,8FB,3)

YAWA 89

CONTINUE

YAWA 90

IFIKK.LT. 5 )GOTO10

YAWA 91

99

STOP

YAWA 92

END

YAWA 93

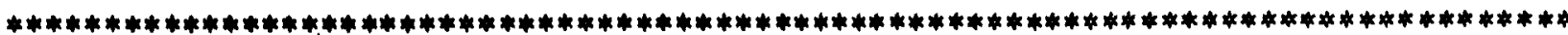
SUBROUT INE OMEGA

FOR LISTING SEE LIST F-I

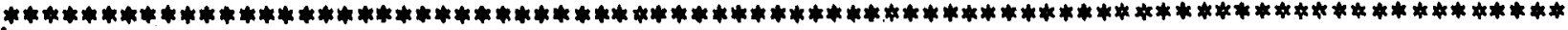

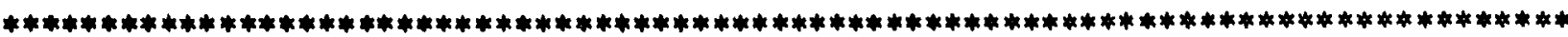

INPUT DATA

SAME AS FOR PROGRAM FLOHANL, SEE LIST F-l
\end{abstract}

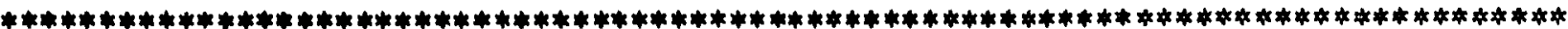


SAMPLE OUTPUT

RUN NU= 29 TAKEN DN, MAR 10, 1969

$\begin{array}{ll}\text { IMPELLER DIAME TER } & =3.00 \\ \text { RADIAL DISTANCE } & =3.00 \\ \text { RPM } & =333.33\end{array}$

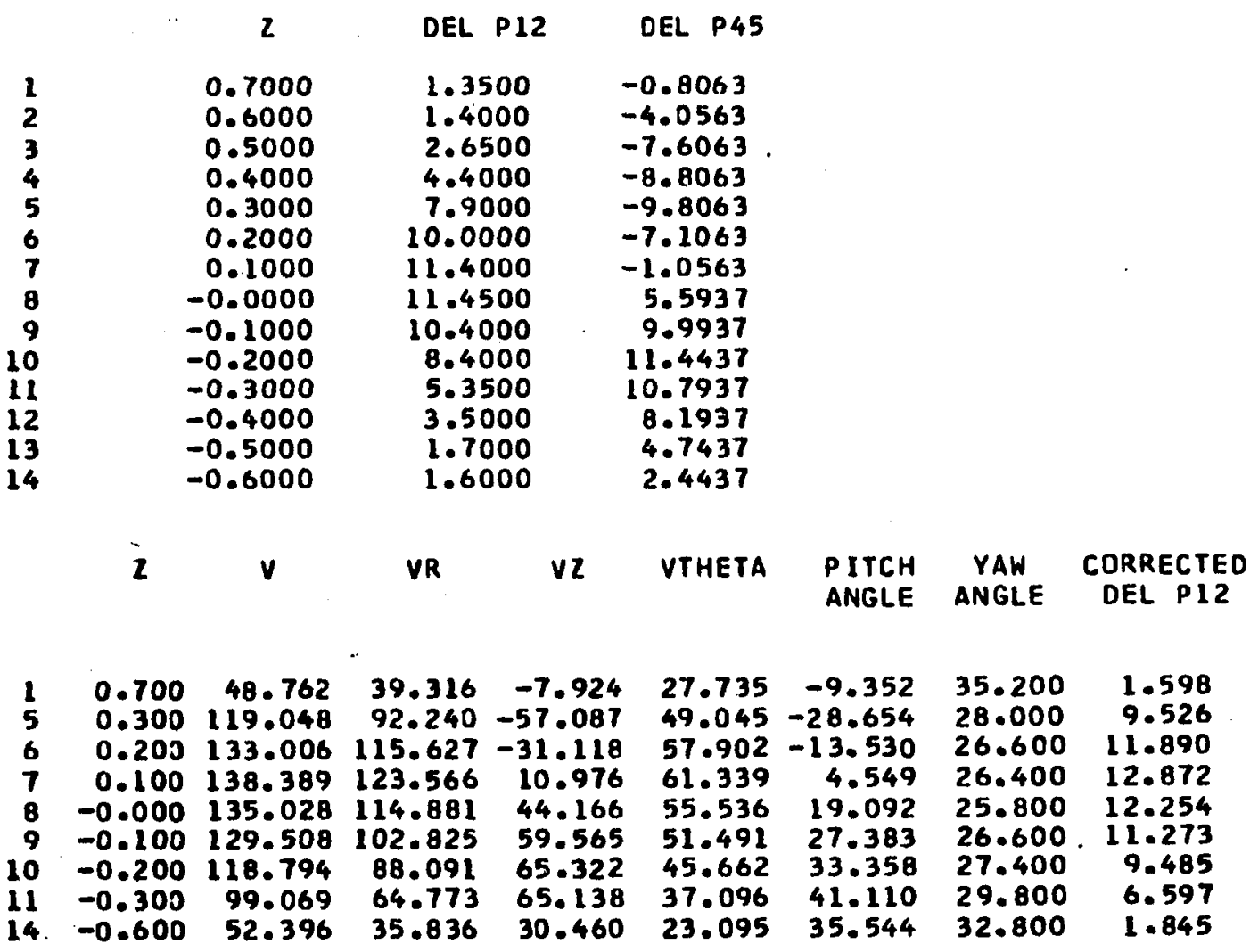




\section{LIST Ph-5}

FORTRAN LISTING FOR PROGRAM COOPER WITH SUPPORTING SUBROUTINES PATERN, PROC, BOUNDS, AVG AND OMEGA. ALSO GIVEN IS COOPER'S (8) VELOCITY PROFILE DATA.

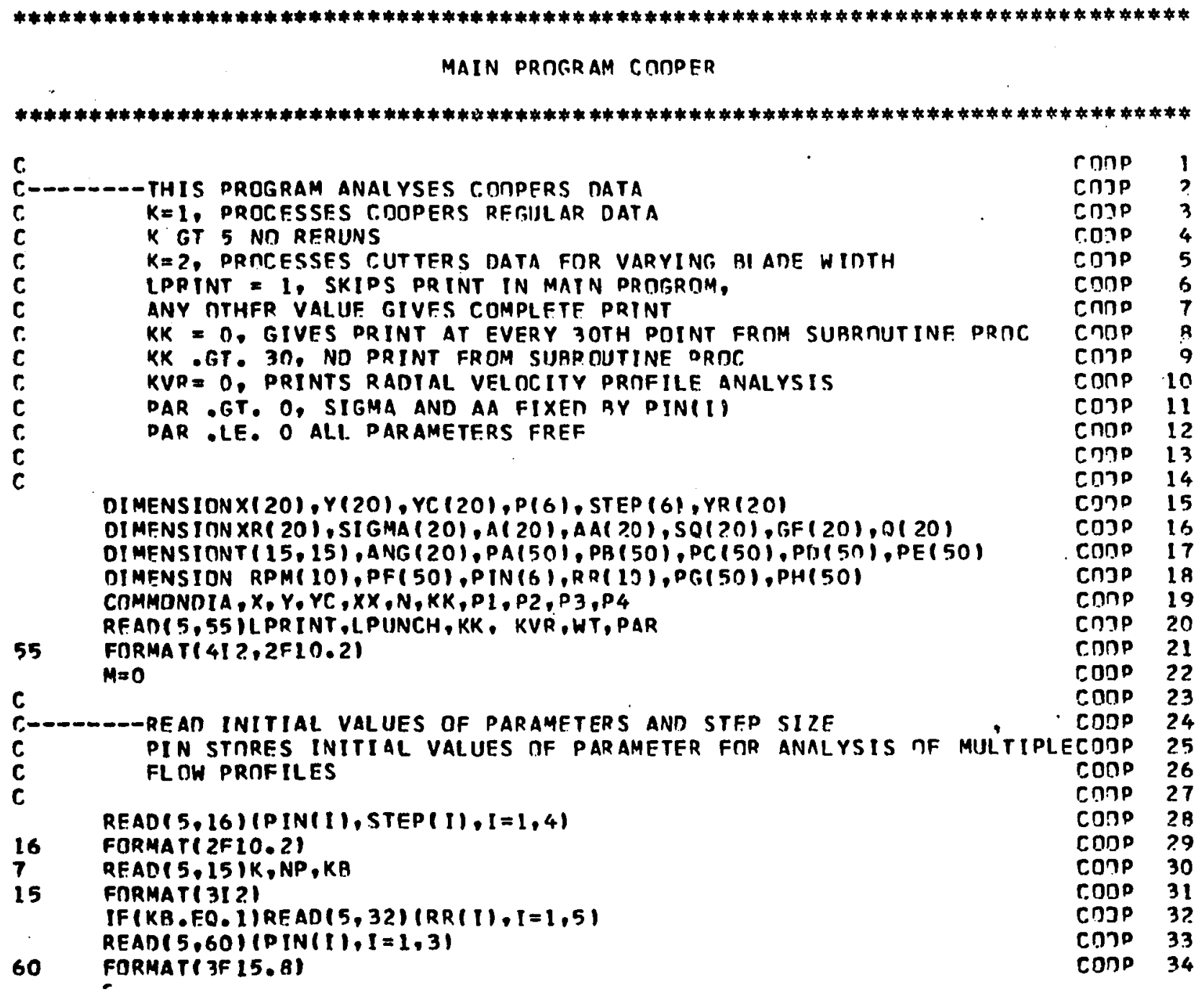




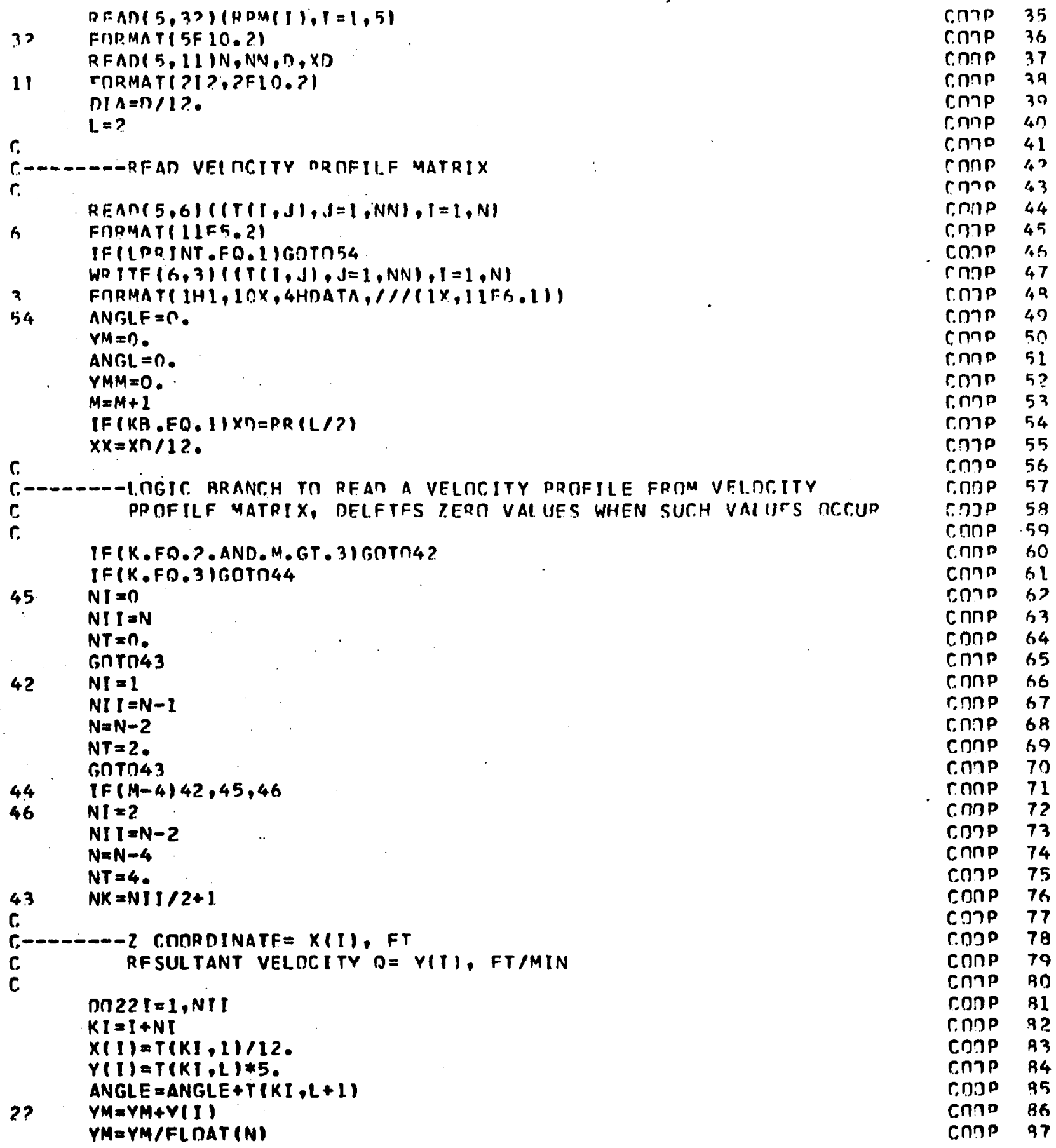




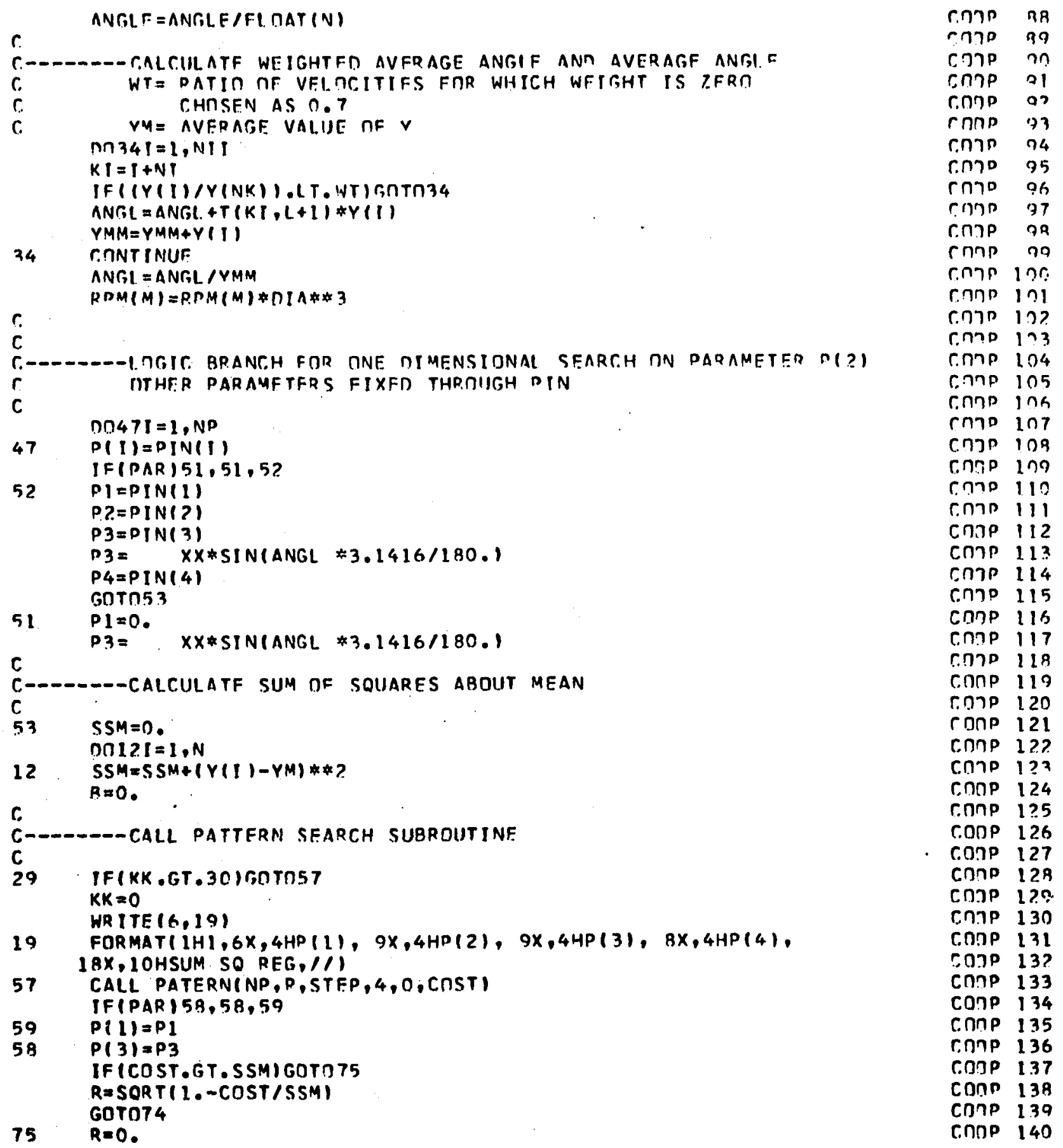




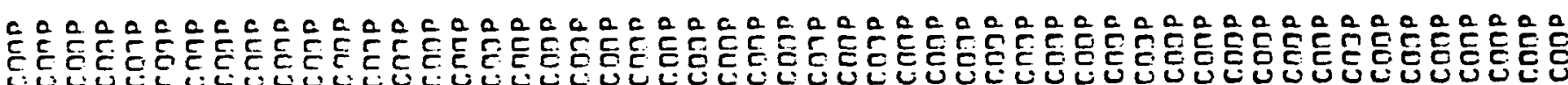

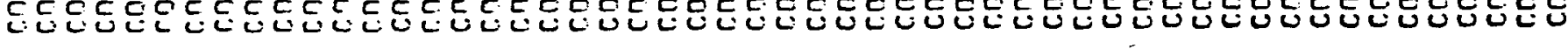

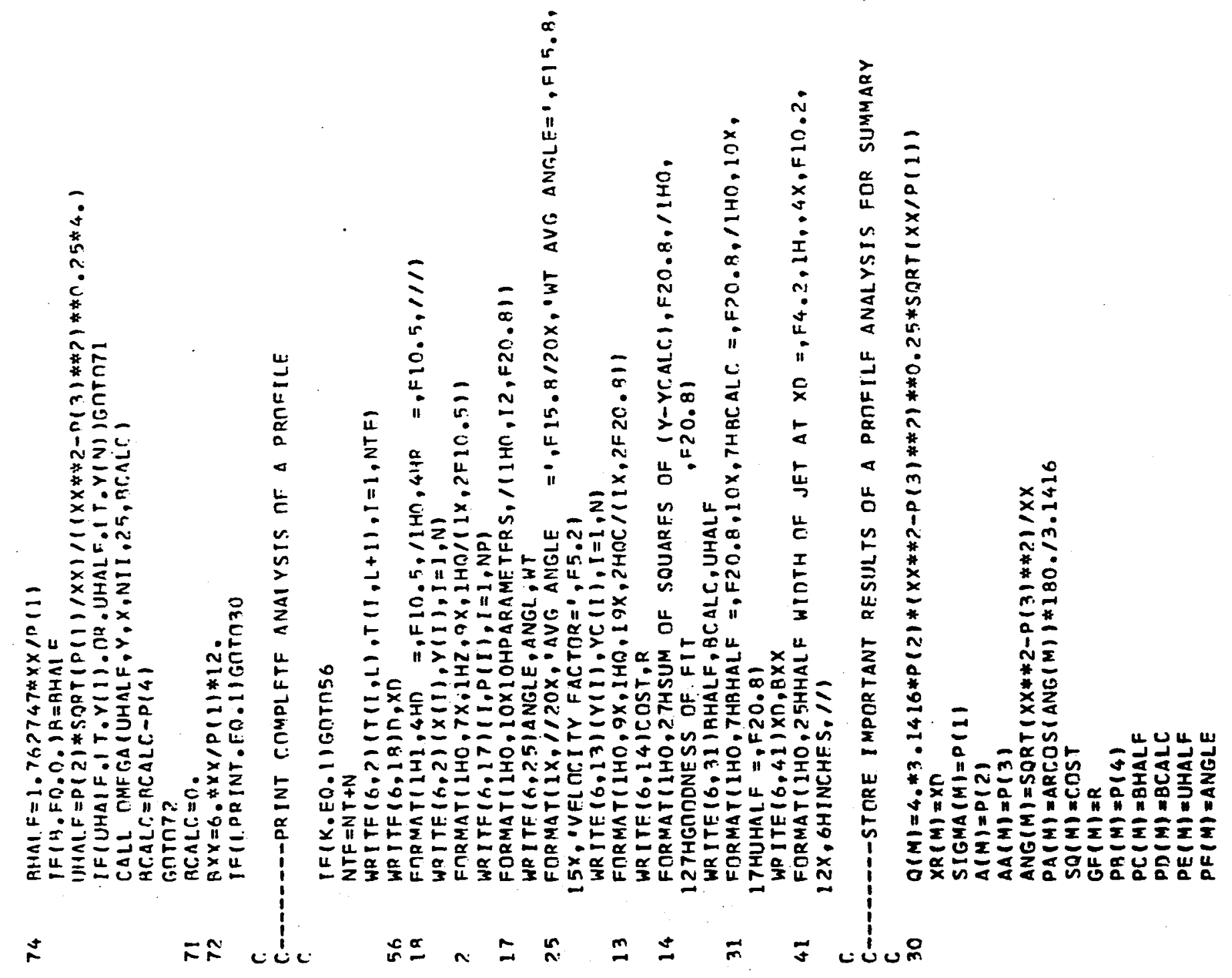




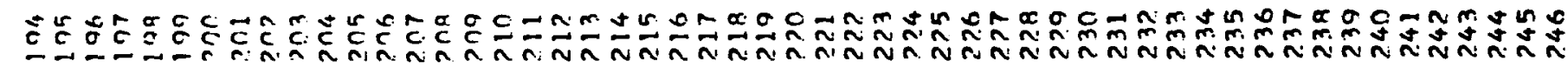

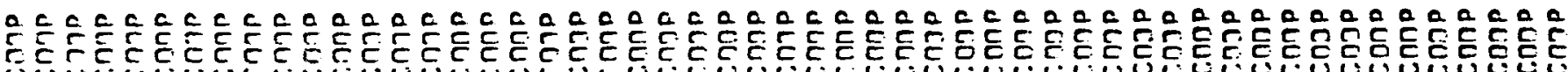

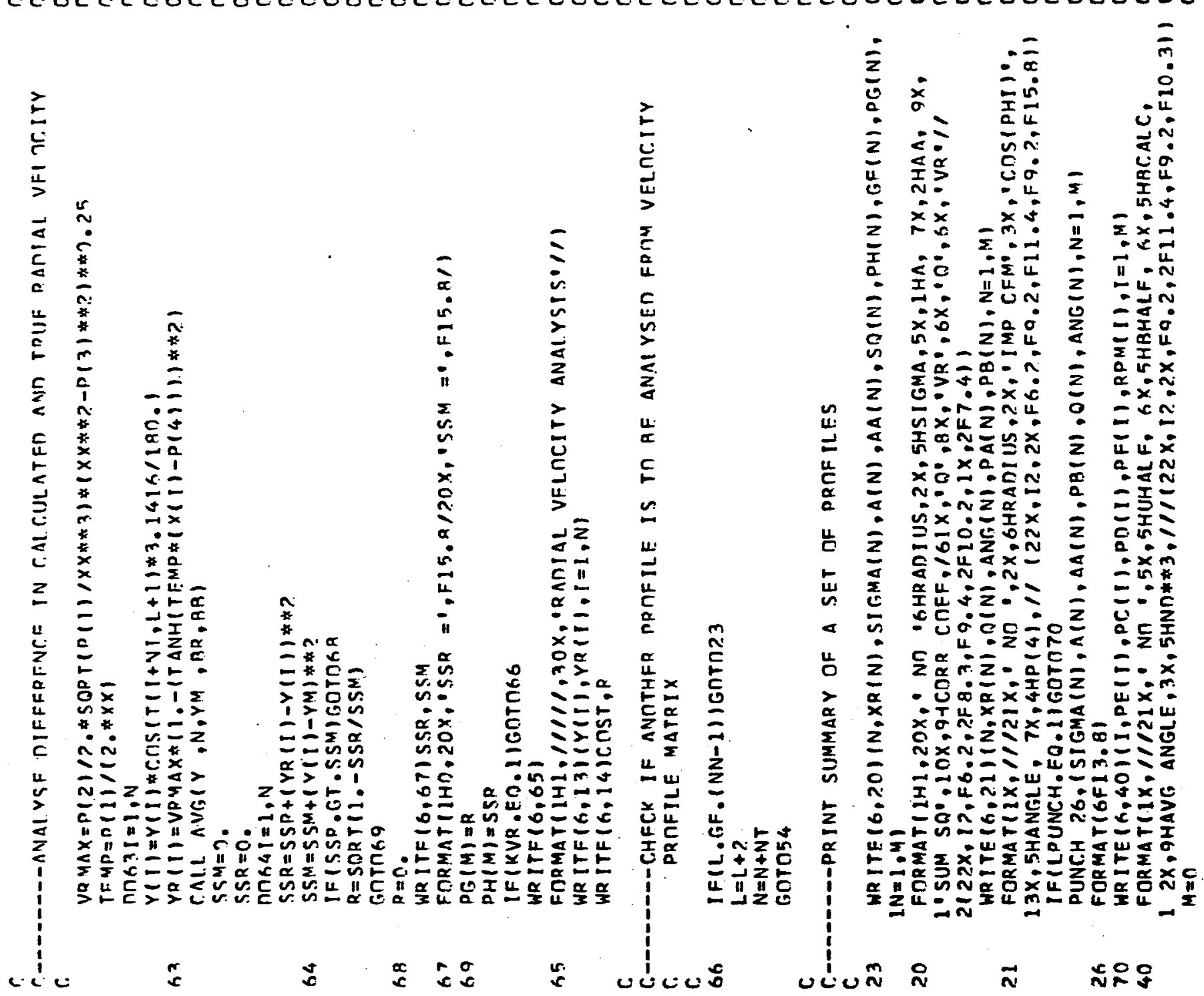




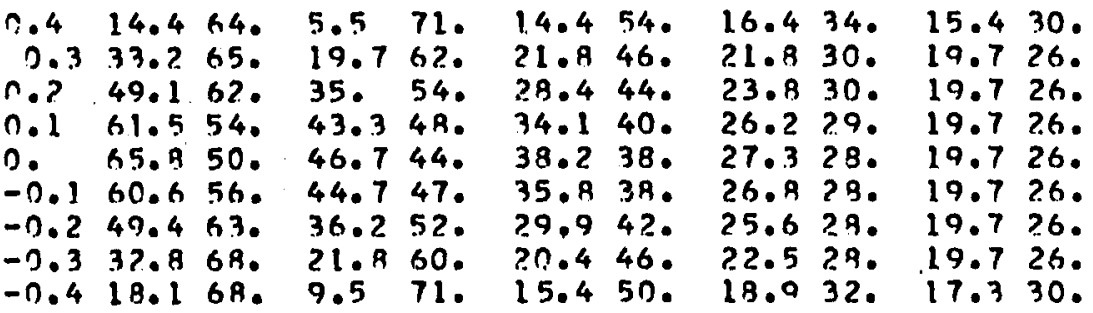

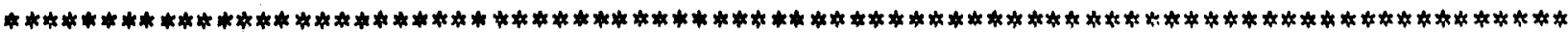

VELOCITY PPITFILE DATA, 4 INCH TURBINE AT 100 RPM, VARYING QLADF. WINTHS

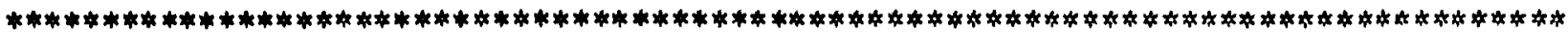

$$
24
$$

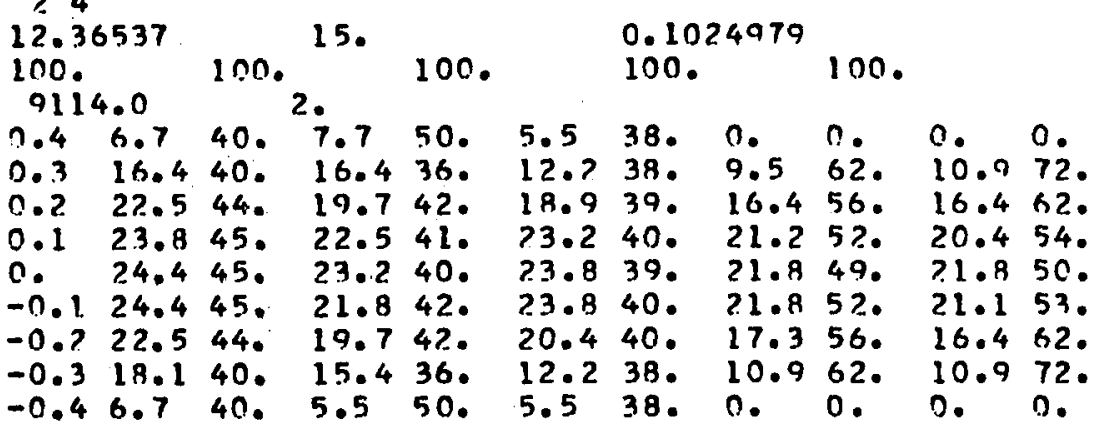

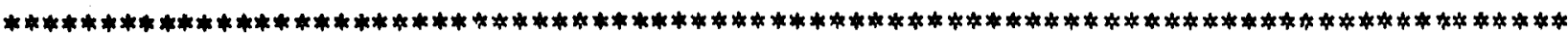

VELOCITY PROFILE DATA, 4 INCH TURBINE AT 200 RPM, VARYINS RLADE WIDTHS

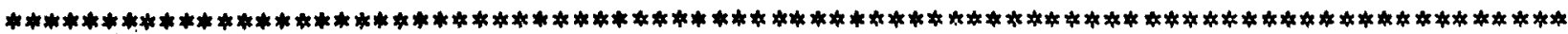

34

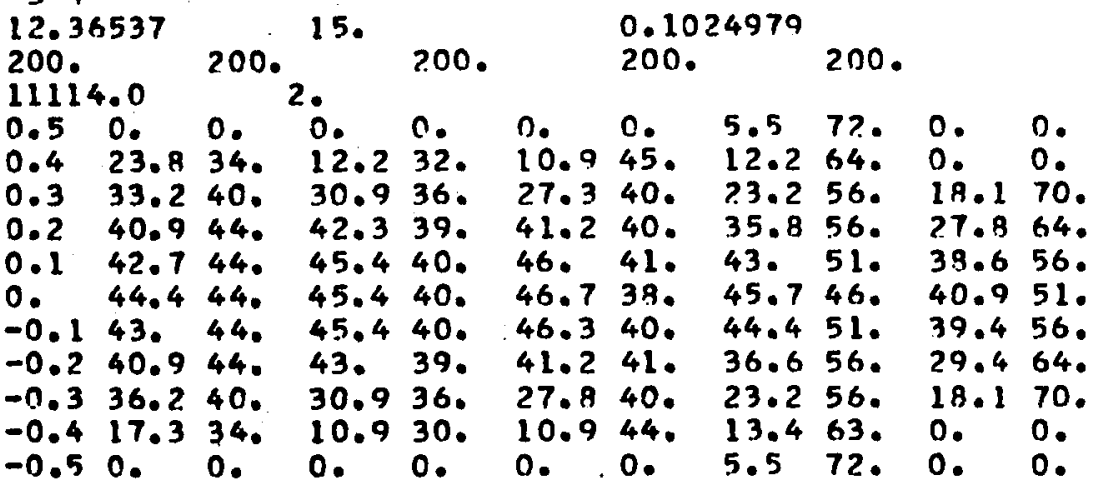




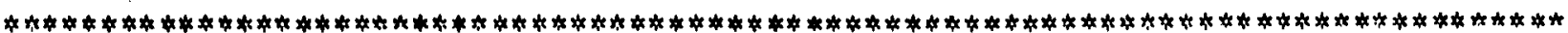
VFLTCITY PROFII.F MATA 3 INCH TIIPBINF IN WATEP

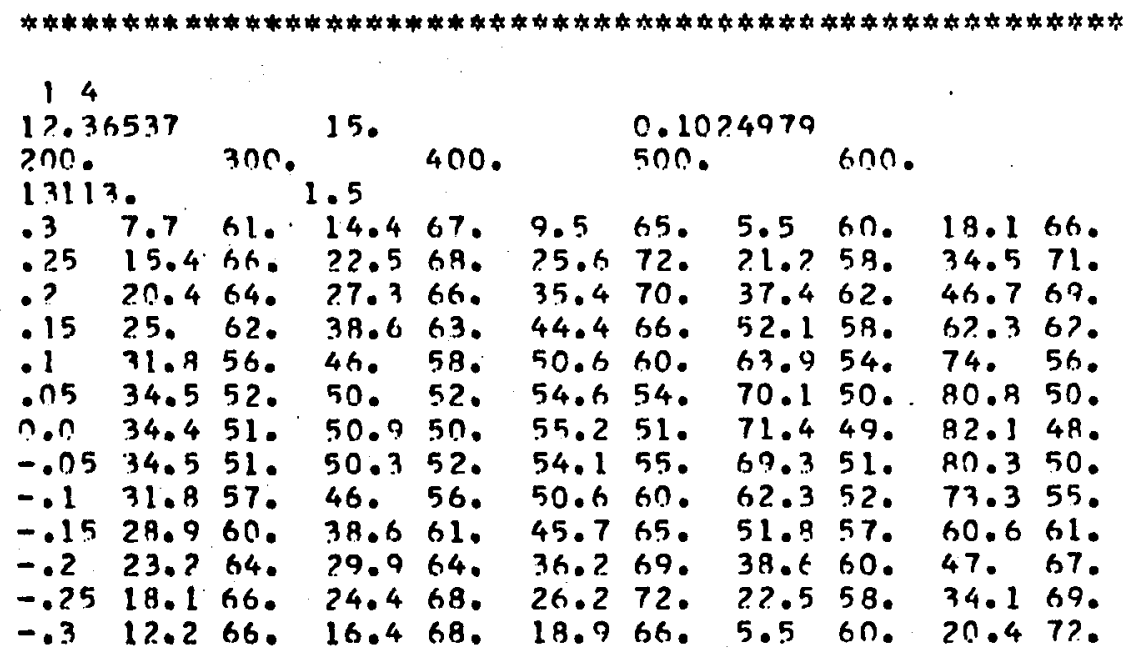

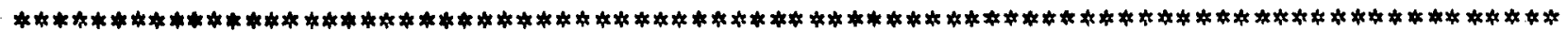
VELOCITY PROFILE TATA 4 INCH TURRINE IN WATFR

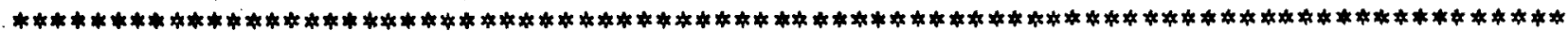

14

\begin{tabular}{|c|c|c|c|c|c|c|c|c|c|c|}
\hline $\begin{array}{r}120 \\
100 \\
91\end{array}$ & $\$ 03$ & 150. & 15. & 200 & & $\begin{array}{l}0.1 \\
250\end{array}$ & 107 & 300 & & \\
\hline $\begin{array}{l}.4 \\
0.3 \\
.2 \\
0.1 \\
0 .\end{array}$ & $\begin{array}{l}3.9 \\
10.2 \\
14.4 \\
21.2 \\
23.2 \\
21.5 \\
16.4 \\
9.5 \\
3.9\end{array}$ & $\begin{array}{l}67 . \\
61 . \\
61 . \\
58 \\
51 . \\
55 . \\
61 . \\
64 . \\
68 .\end{array}$ & $\begin{array}{l}9.5 \\
16.4 \\
23.8 \\
31.4 \\
34.1 \\
31.4 \\
25.6 \\
18.1 \\
9.5\end{array}$ & $\begin{array}{l}62 . \\
67 . \\
65 . \\
58 . \\
52 . \\
54 . \\
62 . \\
66 . \\
64 .\end{array}$ & $\begin{array}{l}9.5 \\
20.8 \\
33.2 \\
45.7 \\
48.8 \\
47 . \\
37.4 \\
20.4 \\
10.9\end{array}$ & $\begin{array}{l}62 . \\
66 . \\
65 . \\
56 . \\
51 . \\
55 . \\
63 . \\
68 . \\
68 .\end{array}$ & $\begin{array}{l}18.9 \\
32.3 \\
46.7 \\
56 . \\
57.5 \\
53.5 \\
45.4 \\
28.9 \\
19.7\end{array}$ & $\begin{array}{l}66 . \\
67 . \\
63 . \\
56 . \\
52 . \\
60 . \\
66 . \\
69 . \\
69 .\end{array}$ & $\begin{array}{l}21.2 \\
33.7 \\
49.1 \\
61.1 \\
66.7 \\
63.2 \\
50.9 \\
34.5 \\
20.4\end{array}$ & $\begin{array}{l}62 . \\
66 . \\
65 . \\
58 . \\
53 . \\
58 . \\
66 . \\
68 . \\
68 .\end{array}$ \\
\hline
\end{tabular}

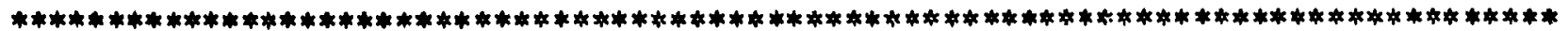

VELOCITY PROFILF DATA 5 INCH TURBINE IN WATER

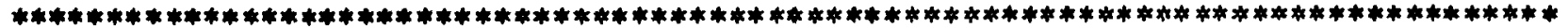

14

$12.66316 \quad 25$. 


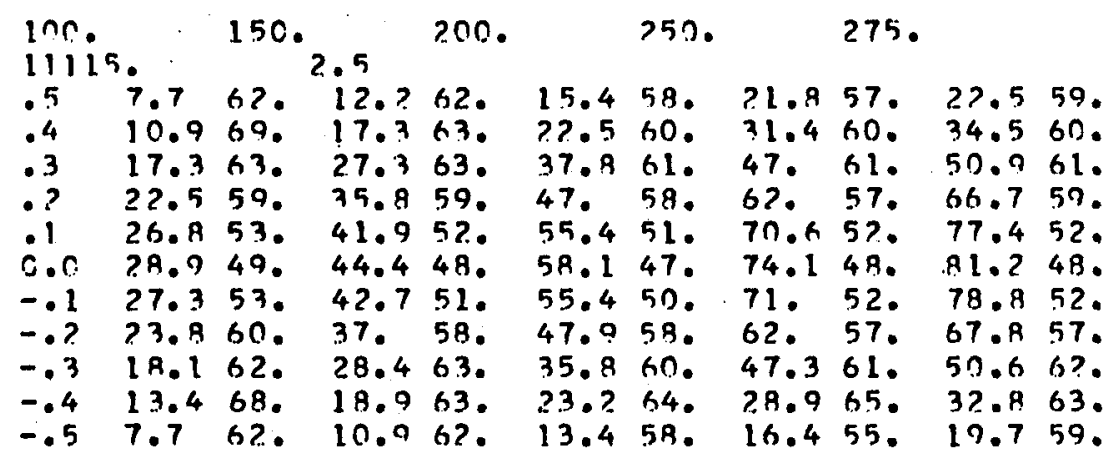

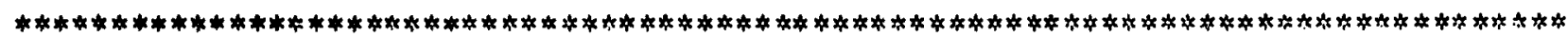

VEI. OCITY PROFILF DATA G INCH TUPBINF IN WATER.

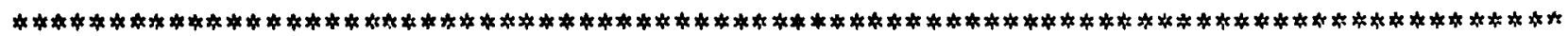

14

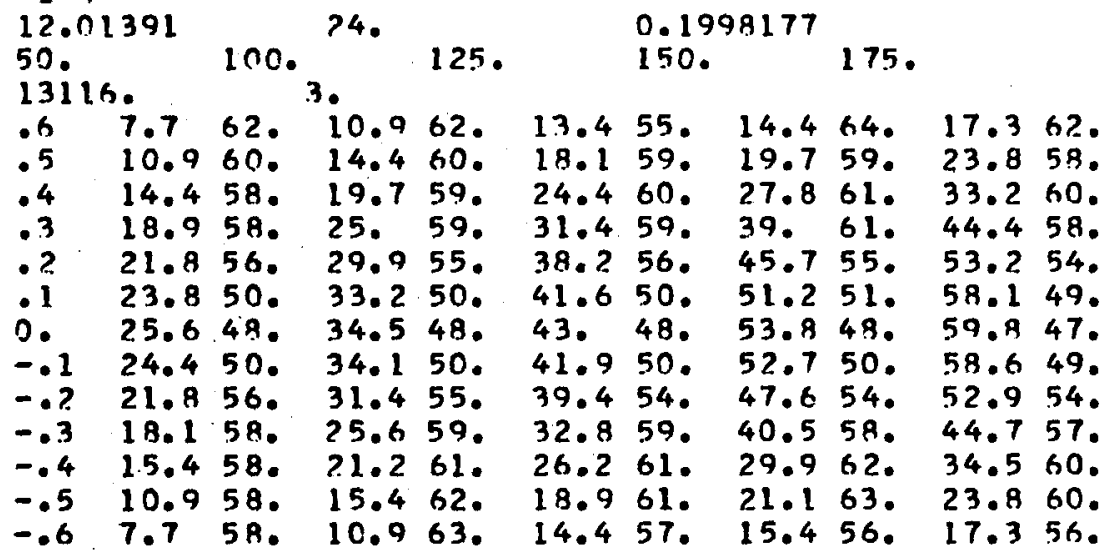

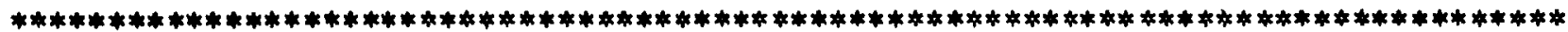

VEL NCITY PROFILE DATA 4 INCH TURAINE IN AIR

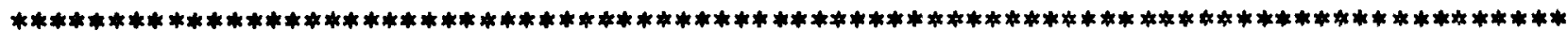

14

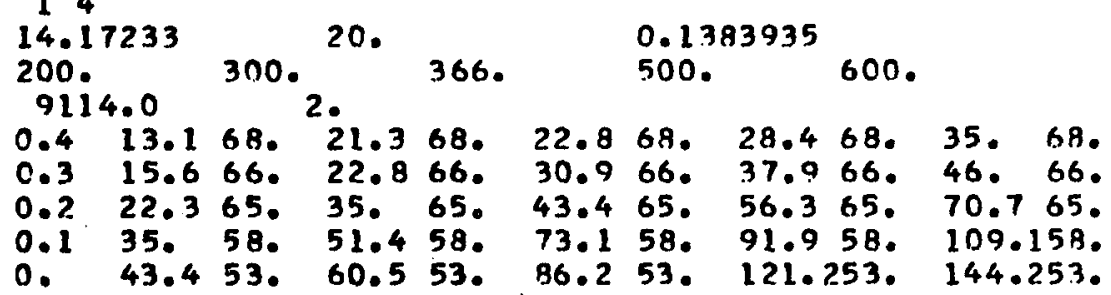




$\begin{array}{llllllllll}-0.1 & 37.9 & 58 . & 53.3 & 58 . & 75.6 & 58 . & 104.259 . & 130.459 . \\ -0.2 & 74.9 & 66 . & 75 . & 66 . & 48.6 & 66 . & 64.966 . & 86.266 . \\ -0.3 & 16.4 & 68 . & 22.8 & 68 . & 30.968 . & 40.2 & 68 . & 53.368 . \\ -0.4 & 12.4 & 68 . & 19 . & 68 . & 2.2 .868 . & 29.6 & 68 . & 35.768 .\end{array}$

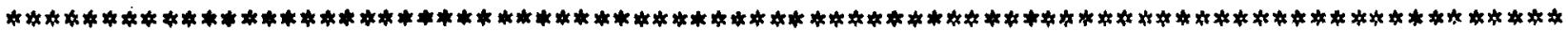

VELICITY PROFILF DATA 5 INCH TURBINE IN AIR

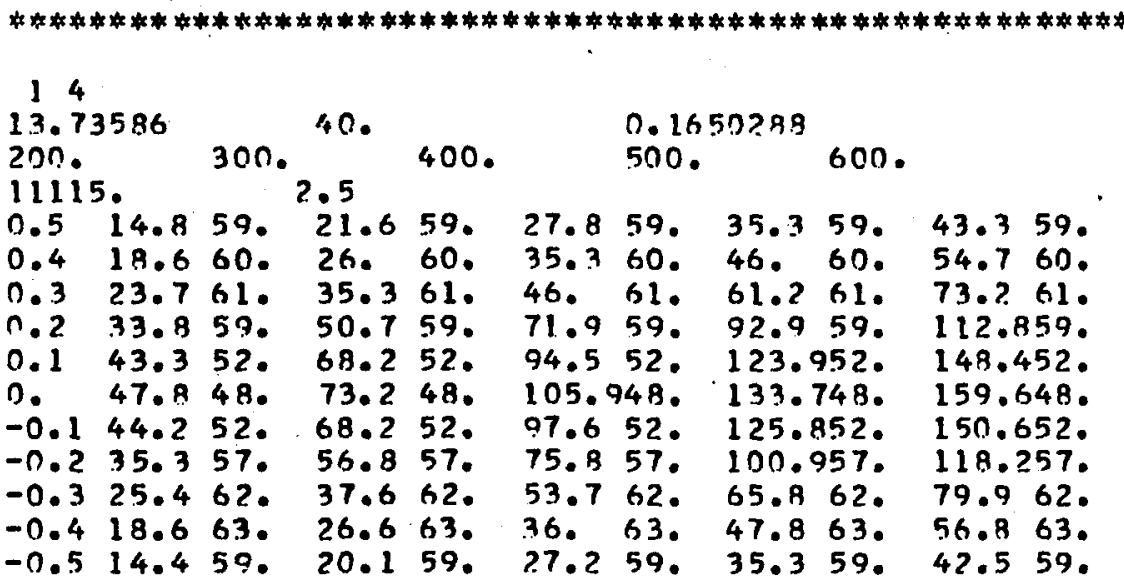

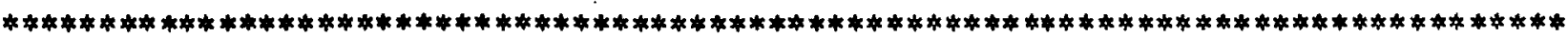

VELOCITY PROFILE DATA 6 INCH TURRINE IN AIR

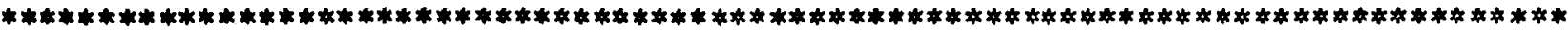

94

\begin{tabular}{|c|c|c|c|c|c|c|c|c|c|c|}
\hline $\begin{array}{l}17.9 \\
100 . \\
1311\end{array}$ & 968 & 200. & 40. & 300 & & $\begin{array}{l}0.10 \\
500\end{array}$ & 1546 & 600. & & \\
\hline $\begin{array}{l}.6 \\
.5 \\
.4 \\
.3 \\
.2 \\
.1\end{array}$ & $\begin{array}{l}11.8 \\
14.5 \\
16.5 \\
21.3 \\
25.5 \\
30.9 \\
34.3 \\
32.9 \\
27.8 \\
23.9 \\
18.1 \\
14.1 \\
11.8\end{array}$ & $\begin{array}{l}62 . \\
60 . \\
59 . \\
59 . \\
55 . \\
50 . \\
48 . \\
50 . \\
55 . \\
59 . \\
61 . \\
62 .\end{array}$ & $\begin{array}{l}21.5 \\
25.6 \\
30 . \\
38.4 \\
50.4 \\
61.1 \\
65.2 \\
61.1 \\
51.7 \\
41.2 \\
31.2 \\
25.6 \\
21.3\end{array}$ & $\begin{array}{l}62 . \\
60 . \\
59 . \\
59 . \\
55 . \\
50 . \\
48 . \\
50 . \\
55 . \\
59 . \\
61 . \\
62 . \\
63 .\end{array}$ & $\begin{array}{l}28.9 \\
33 . \\
39.8 \\
54.4 \\
71.6 \\
83.5 \\
90 . \\
83.5 \\
71.6 \\
56.3 \\
41.2 \\
33.2 \\
28.9\end{array}$ & $\begin{array}{l}62 . \\
60 . \\
59 . \\
59 . \\
55 . \\
50 . \\
48 . \\
50 . \\
55 . \\
59 . \\
61 . \\
62 . \\
63 .\end{array}$ & $\begin{array}{l}45.9 \\
53.5 \\
64.1 \\
90 . \\
123 . \\
148 . \\
160 . \\
152 . \\
129 . \\
101 . \\
68.3 \\
56.3 \\
46.7\end{array}$ & $\begin{array}{r}62 . \\
60 . \\
590^{\circ} \\
59 . \\
550^{\circ} \\
250 . \\
248 . \\
150 . \\
855 . \\
159 . \\
61 . \\
62 . \\
63 .\end{array}$ & $\begin{array}{l}53.5 \\
63.1 \\
77.4 \\
108.5 \\
140 . \\
172 . \\
186 . \\
177 . \\
150 . \\
116 . \\
83.5 \\
66.2 \\
53.5\end{array}$ & $\begin{array}{l}62 . \\
60 \\
59 \\
59 . \\
55\end{array}$ \\
\hline
\end{tabular}


LIST $F-6$

FORTRAN LISTING FOR PROGRAM NIELCUT WITH SUPPORTING SUBROUTINES, PATERN, BOUNDS AND OMEGA.

ALSO GIVEN ARE VELOCITY PROFILE DATA OF NIELSON (21) AND CUTTER (9).

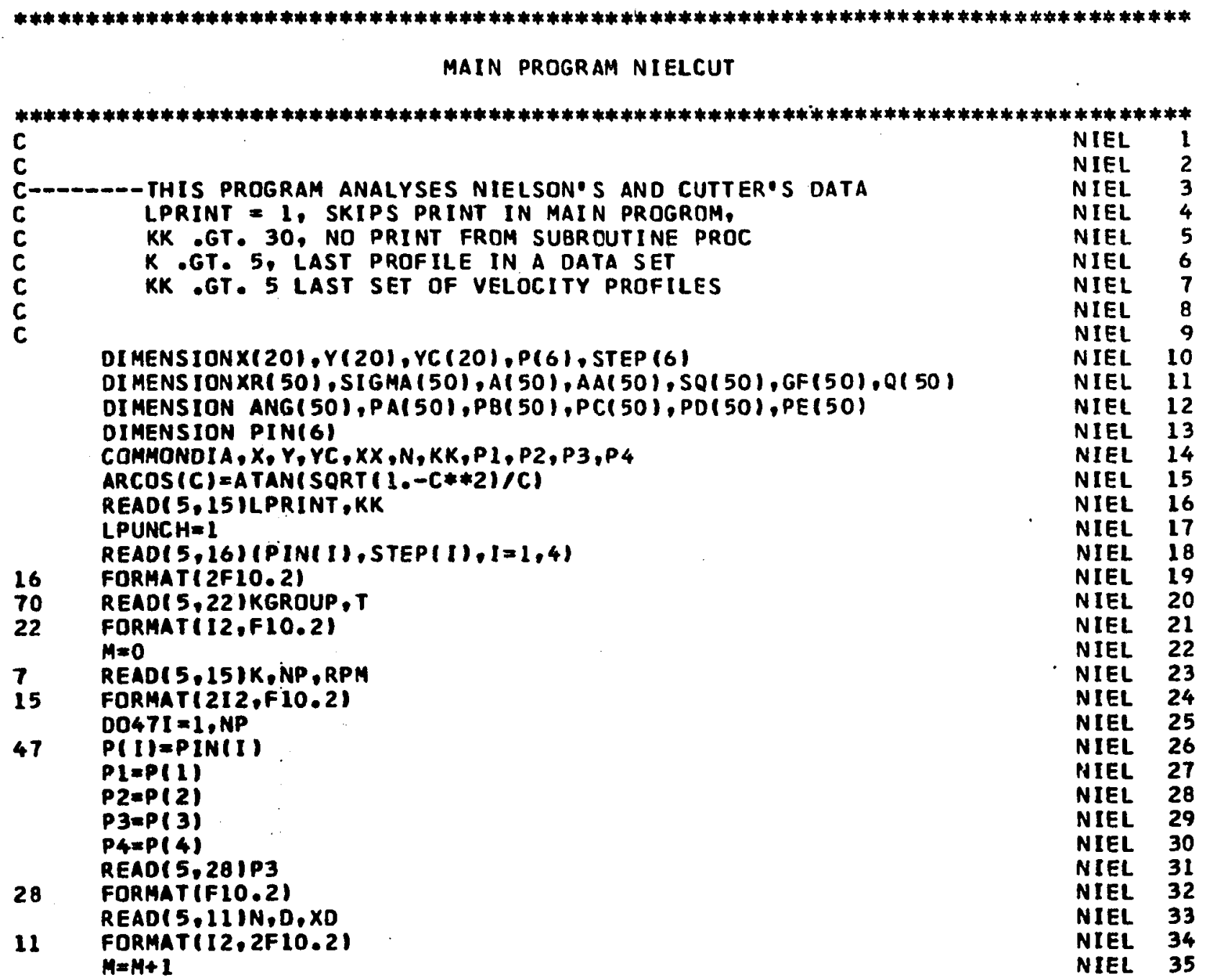




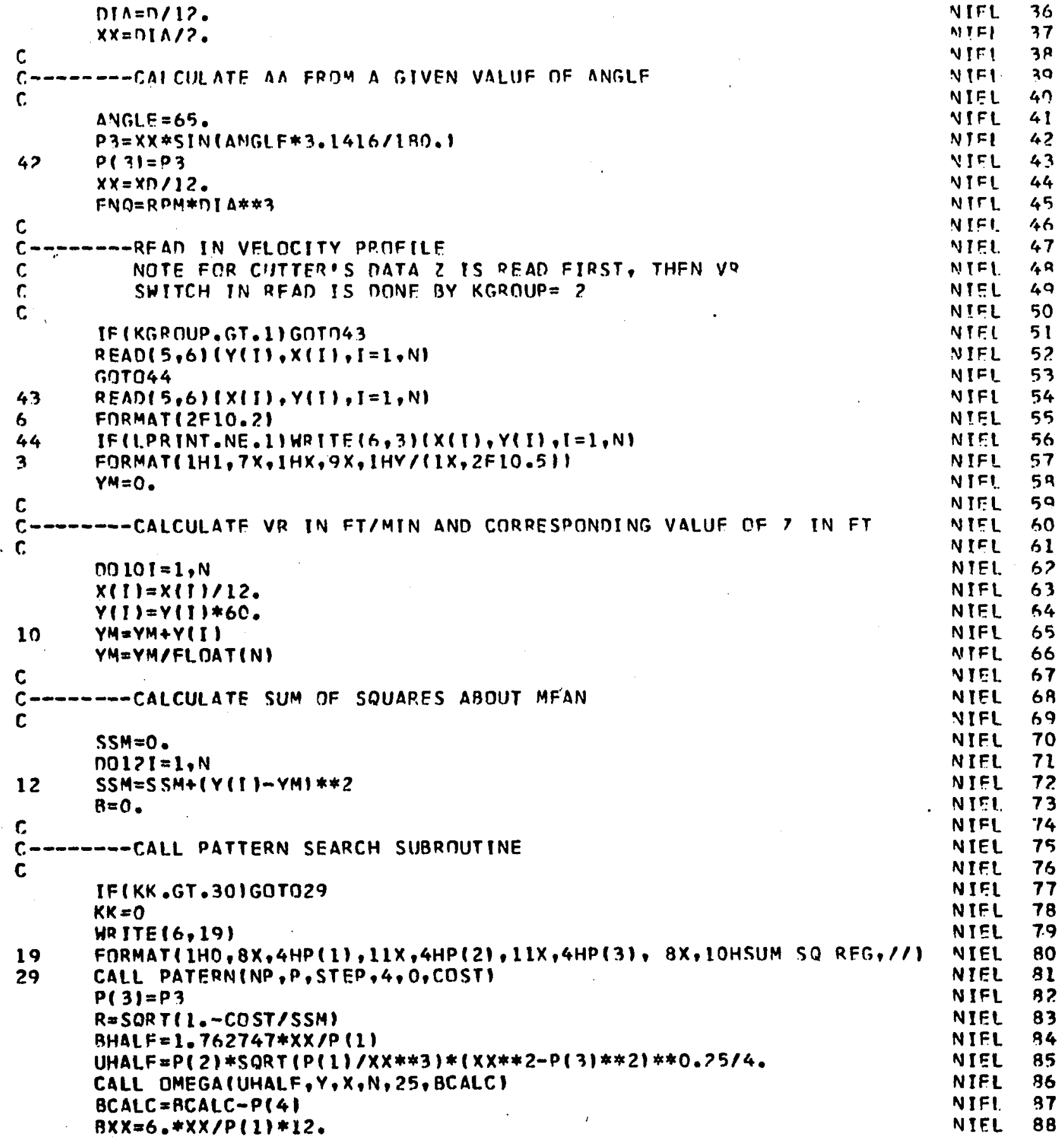

NIFL 36

NIFI 37

NIFI 38

NIFI 30

NIEL 4 ?

VIFL 41

NTFI 4?

VIFL 43

NIFL 44

NITL 45

NIFI. 46

NIFL. 47

NIFI. 49

NITL 49

NIFL 50

NIFI 5

NIFL 5?

NIFL 53

NIFI 54

NIFL 55

NIFL 56

NIFI 57

NIFI 59

NIFL 50

NIEL 50

NIFL $6 I$

NIEL. 6?

NIFL 63

NIEL $\quad 64$

NIFL 65

NIFL 66

NIEL 67

NIEL 68

VIFL 69

NIFL 70

NIFL 71

NIFL 7?

NIEL 73

NIFL 74

NIEL 75

NIFL 76

NIFI 77

NIFL 78

NIEL 7.9

NIEL 80

NIEL 81

NIFL B?

NIEL 83

NIFL 94

NIEL 85

NIFL 86

NIFI. $\quad 97$

NIEL 88 
r.

C-D--DPRINT TMMPLETE ANALYSIS DF A PRTFILF

NIFL 99

r.

IFILPR INT - FO. IIGOTOZO

WP. ITF $(6,18) \cap, \times D$

19 FIRMAT $1 \mathrm{H} 1,4 \mathrm{HO}=, \mathrm{F} 10.5,11 \mathrm{HO}, 4 \mathrm{H} \times \mathrm{H}=, \mathrm{F} 10.5,1 / 11$

WR ITF $(6,2)(X \mid I), Y(I), I=1, N)$

? FORMAT $(1 H O, 7 X, 1 H X, 2 X, 1 H Y(1,1 X, 2 F 10.5))$

WR ITF $(6,17)(1, n(1), 1=1, N P)$

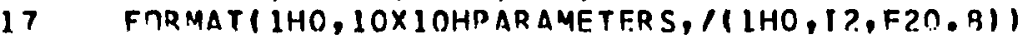

WR ITF $(6,13)(Y(I), Y C(I), I=1, N)$

13 FDRMAT(1HO,9X,1HY, $19 X, 2 H Y C /(1 X, ? F 20.81)$

WR ITF $(6,14)$ C.NST, $R$

14 FIRMAT(IHO,?7HSUM TF SOIJARES DF (Y-YCALC),F20,8,11HO, 12 THGONONFSS NF FIT .F20.81

WR ITF 16,31 I RHALF, BCALC, UHALF

31 FORMATI IHO, 7HBHAL.F =,F? $0,8,10 X, 7 \mathrm{HBC}, \mathrm{LLC}=, F ? 0,8,11 \mathrm{HO}, 10 \mathrm{X}$,

1 THIIHALF $=, F ? 0$. Q $)$

WR ITF $16,411 \times 0, B \times X$

41 FRRMAT(1HO,20X,25HHALF WIDTH OF JET AT $X \cap=, F 4,2,1 H,, 4 \times, F 10,2$, $12 \times, 6 H I N C H F S, 1 / 1$

$3 n \quad D(M)=4 . * 3.1416 * P(2) *(X X * 2-P(3) * 2) * 0.25 * S Q R T(X X) P(1))$

$X R(M)=X D$

$\operatorname{SIGMA}(M)=P(1)$

$A(M)=P(2)$

$A A(M)=P(3)$

ANG $(M)=\operatorname{SART}(X X * * 2-P(3) * * 2) / X X$

$P A(M)=A R C O S(A N G(M)) * 180.13 .1416$

$S Q(M)=C O S T$

$G F(M)=R$

$P B(M)=P(4)$

$P C(M)=$ RHALF

$P \cap(M)=B C A L C$

C

PF $(M)=$ IJHALF

C-D---CHFCK IF ANDTHER PROFILE IS TO BE ANALYSFD

c

IFIK.LT. 5 IGOTnT

c

C-D---DRINT SUMMARY OF A SET OF PROFILES

c

23 WRITE $(6,20)(N, X R(N), S I G M A(N), A(N), A A(N), S D(N), G F(N), N=1, M)$

20. FORMATIIHI, 22X,'ND', $1 X, 6 H R A D I U S, 2 X, 5 H S I G M A, 6 X, 1 H A, 7 X, ' A A^{\prime}, 5 X$,

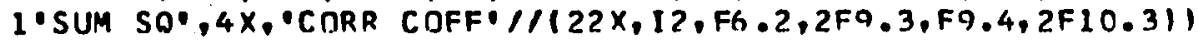

WR ITF $(6,2)(N, X R(N), D(N), A N G(N), P A(N), P B(N), N=1, M)$

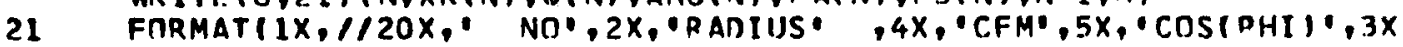

1.ANGLE', $10 X, P P(4),, / /(22 x, 1 ?, F 6,2,3 F 10,4, F(5,8) 1$

IF (LPUNCH.FQ.1IGOTOTI

PUNCH 26, $(S I G M A(N), A(N), A A(N), P B(N), Q(N), A N G(N), N=1, M)$

26 FORMAT (6F 12.4$)$

71 WR I TE $(6,40)(1, P E(I), P C(I), P D(I), I=1, M)$

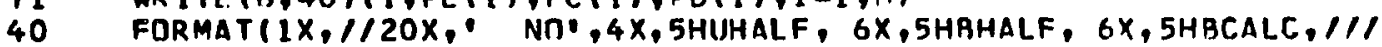
$1(22 x, 12, F 9,2,2 F 11.41)$

NIFI on

NIET. QI

NIFL 9?

NIFI 97

NIFL 94

NIEL 95

NIEL 96

NIFE. 97

NIFL 98

NIEL 99

NIFL 192

NIFL IOI

NIEL 19?

NIFL 103

NTEL 104

NIEL 105

NICL 106

NIEI 107

NIEl Ing

NIEL 109

NIFI. 110

NIFL 111

NIFL 112

NIFL 113

NIFL 114

NIEL 115

NIFL 116

NIFL 117

NIEL 119

NIEL 119

NIFL $12 ?$

NIEL 121

NIEL 122

NIFI 123

NIEL 124

NIFL 125

NIFL 126

NIEL 127

NIEL 128

NIFL 129

NIFL 130

NIEL 131

NIEL 132

NIFL 133

NIEL 134

NIFL 135

NIFL 136

NIFL 137

NIEL 138

NIFL 139

NIEL 140

NIFL 141 


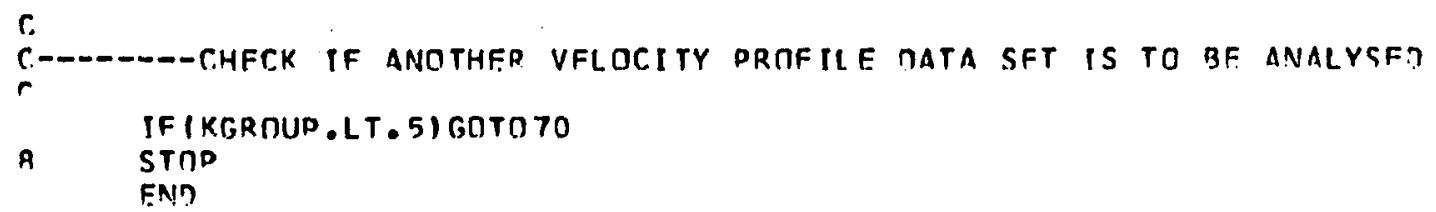

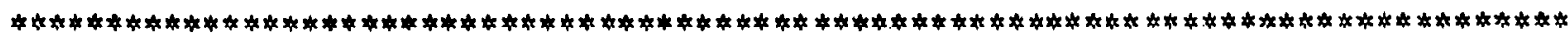
SURROUT INE PRDC.

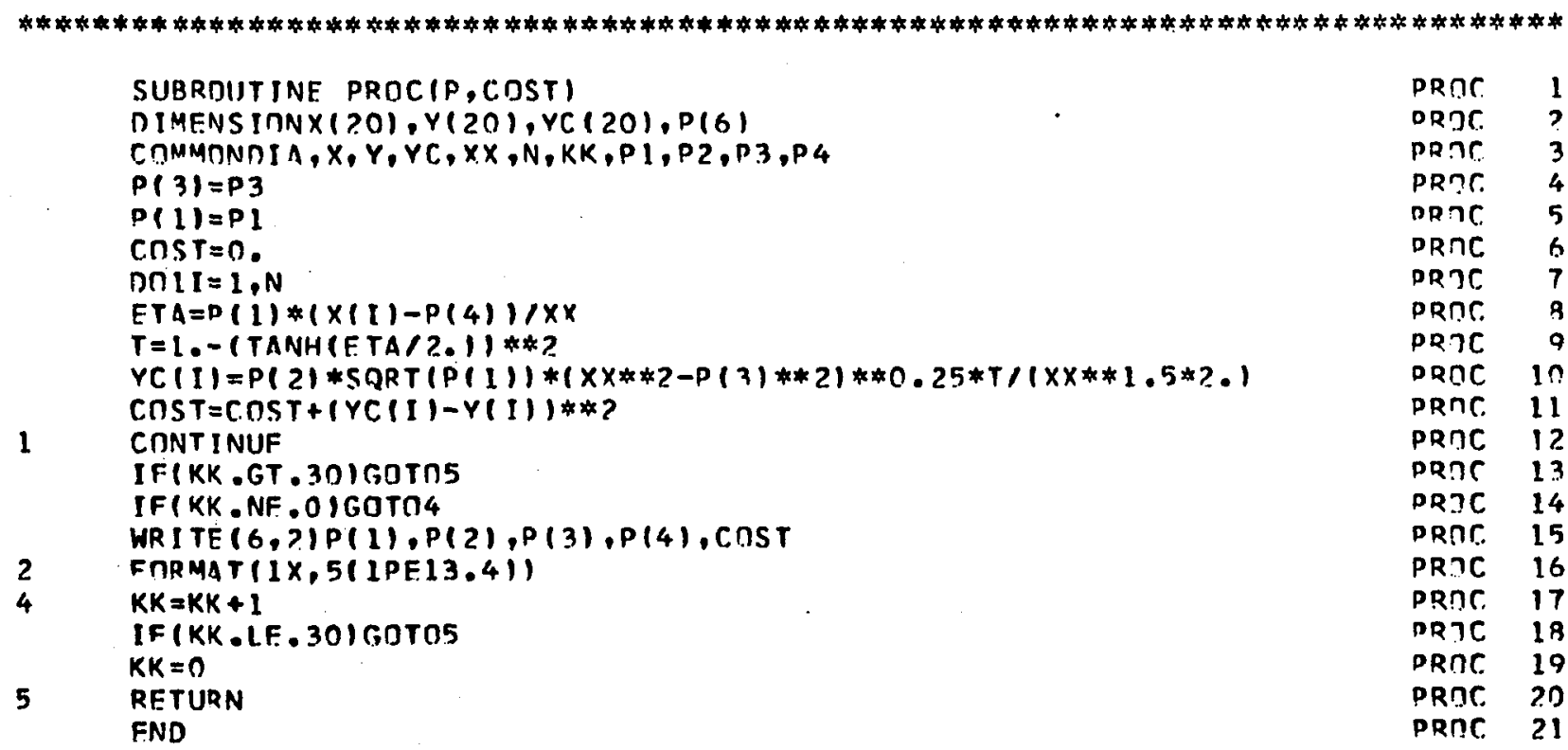

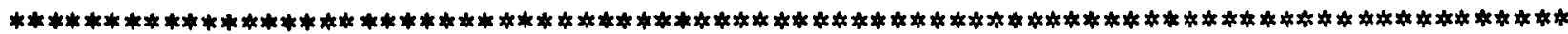

THE FOLLOWING SUPPRRTING SUBRDUTINE ARE USED

IN COMMDN WITH FLOWANL, FOR LISTING SFE LIST F-1

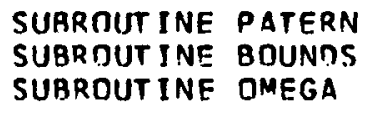

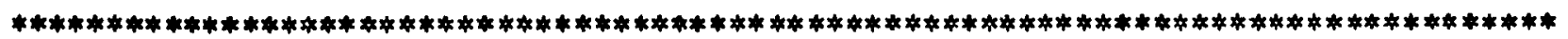




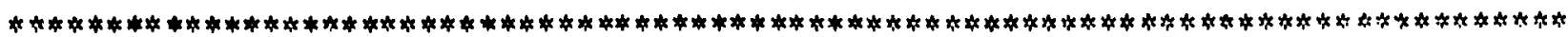

PRFLIMINARY TATA, CONTROLS MINF OF RRFRATION FOP INSTRUCTIONS SFE COMMENT CAROS AT THE RFGINING, DF THF: PROGRAM

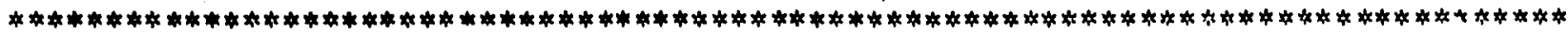

140

17.621

18.

.01

0.1165

0.5

ก. .001$$
\text { 0.001 }
$$

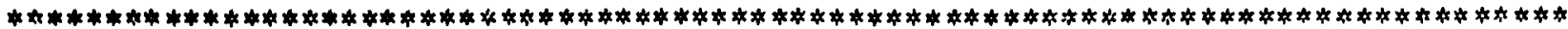

NIELSON'S DATA

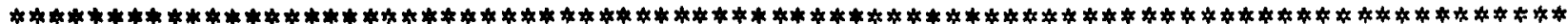

111.25

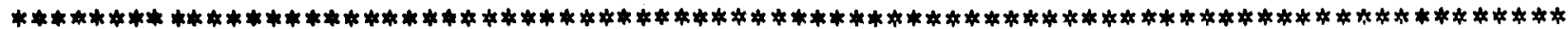

VFLDCITY PROFILF DATA, 2 INCH TURRINE AT 600 RPM AND

VARYING RADIAL DISTANCE R

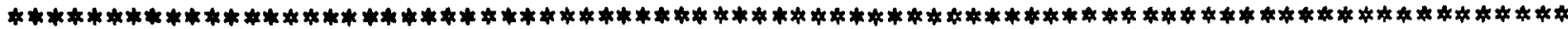

14600 .

\begin{tabular}{ll}
72.0 & \multicolumn{1}{l}{1.0} \\
0.38 & 0.166667 \\
1.32 & 0.1 \\
2.41 & 0.03333333 \\
3.47 & 0.0 \\
1.67 & -0.033333 \\
1.01 & -0.1 \\
0.34 & -0.166667 \\
14600. & \\
72.0 & \multicolumn{1}{l}{3.0} \\
0.54 & .5571429 \\
0.89 & -3714286 \\
1.15 & -1857143 \\
1.21 & 0.0 \\
0.81 & -.1857143 \\
0.54 & -.3714286 \\
0.37 & -.05571429 \\
144600. &
\end{tabular}




$\begin{array}{ll}72.0 & 4.0 \\ 0.55 & .5541426 \\ 0.71 & .3594284 \\ 0.81 & .1847142 \\ 0.9 & .0 \\ 0.78 & -.1847147 \\ 0.65 & -.3694784 \\ 0.56 & -.5541426\end{array}$

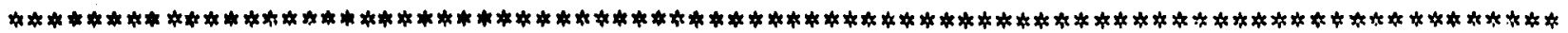

VFLCCITY PRDFILE DATA, 4 INCH TURBINE AT 200 RPM IN WATFP PADIAL. DISTANCE R=? INCH

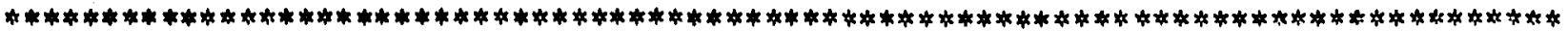
14200.

$\begin{array}{ll}74.0 & 2.0 \\ 0.54 & .355555556 \\ 1.01 & .266666667 \\ 1.53 & .177777778 \\ 2.07 & .089898889 \\ 2.22 & .0 \\ 1.60 & -.08888889 \\ 0.83 & -.17777778 \\ 0.81 & -.2666667 \\ 0.42 & -.35555556\end{array}$

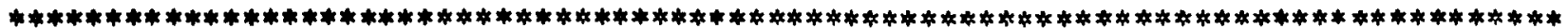

VELOCITY PROFILE DATA, 4 INCH TURBINE AT 100 RPM IN C.ORN SYRUP. RAMTAL DISTANCE R= 2 INCH

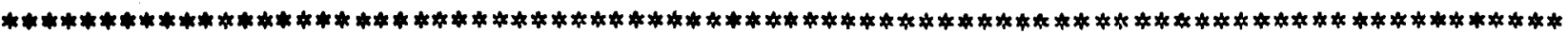
14100.

$\begin{array}{ll}94.0 & 2.0 \\ 0.22 & : 355555556 \\ 0.33 & .266666667 \\ 0.74 & .177777778 \\ 0.88 & .088888889 \\ 1.14 & .0 \\ 1.02 & -.08888889 \\ 0.68 & -.17777778 \\ 0.34 & -.2666667 \\ 0.12 & -.35555556\end{array}$

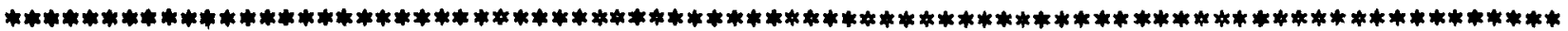

VELOCITY PROFILE DATA, 4 INCH TURRINE AT 200 RPM IN WATER RADIAL DI STANCE $R=4$ 


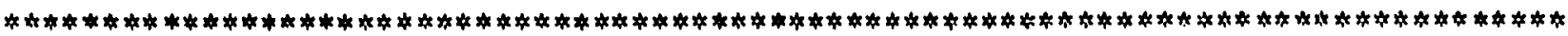

14300.

$\begin{array}{ll}11.4 .0 & 4.0 \\ 0.31 & .3409096 .909 \\ 0.45 & .7527272777 \\ 0.75 & .5445454545 \\ 1.14 & .3763636364 \\ 1.27 & .1891818191 \\ 1.26 & -0 \\ 1.06 & -.188181818 \\ 0.7 & -.376363636 \\ 0.47 & -.564545454 \\ 0.29 & -.752727273 \\ 0.13 & -.940909091\end{array}$

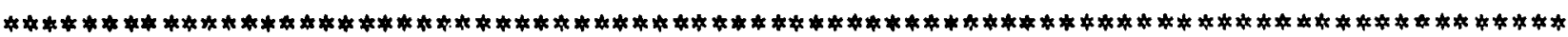

VELDCITY PROFILE DATA, 7 INCH TURBINE IN WATFR。

RANIAL DISTANCE R $=3.5$ INCH

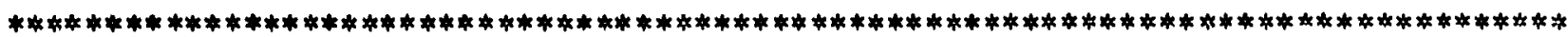

$$
9475 .
$$

$77.0 \quad 3.5$

$\begin{array}{ll}0.61 & .6 \\ 0.74 & .4 \\ 1.37 & .2 \\ 1.5 & .0 \\ 1.33 & -.2 \\ 1.0 & -.4 \\ 0.75 & -.6\end{array}$

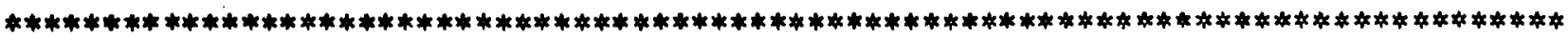
CUTTER'S OATA

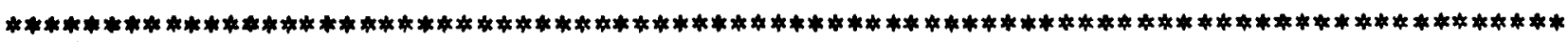

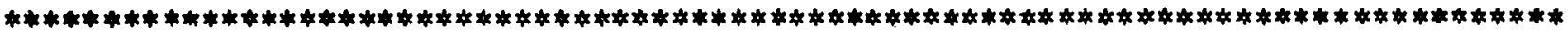

VELOCITY PROFILE DATA, 4 INCH TURBINF AT 200 RPM IN HATER

AT VARYING RADIAL DISTANCE.

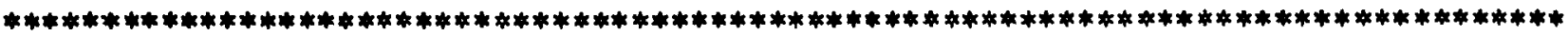

211.5

14200.

0.13101295 


\begin{tabular}{|c|c|}
\hline 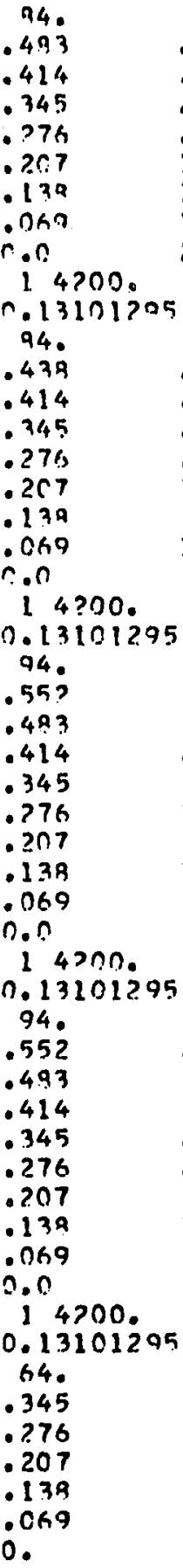 & $\begin{array}{l}4.13 \\
.72 \\
.78 \\
.59 \\
.79 \\
.89 \\
.87 \\
1.03 \\
1.08 \\
1.09\end{array}$ \\
\hline
\end{tabular}


94700 .

0.13101295

$\begin{array}{ll}64 . & 5.13 \\ .345 & : 08 \\ .276 & 1.09 \\ .207 & : 79 \\ .139 & 1.03 \\ .069 & 1.04 \\ n .0 & 1.33\end{array}$

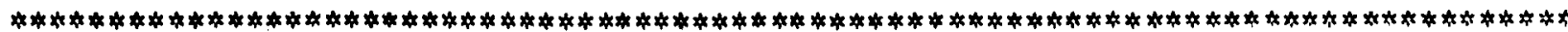

VEI OCITY PROFIIE MATA, 4 INCH TIJRATNF AT 400 PDM IN WATFR AT VARYING RAMIAL DISTANCE

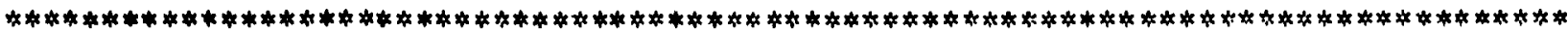

$? 11.5$

14400.

0.17355394

74.

$.414 \quad .62$

$.345 \quad 1.26$

$.276 \quad 2.70$

$.207 \quad 3.65$

$.138 \quad 4.68$

$.069 \quad 4.47$

$0.0 \quad 4.84$

14400 .

0.12355394

64.2 .81

$.345 \quad 1.87$

$.276 \quad 2.50$

$.207 \quad 2.70$

$.138 \quad 3.04$

$.069 \quad 3.54$

$0.0 \quad 3.94$

14400.

0.12355394

64.2 .86

$.345 \quad 2.14$

$.276 \cdot 2.21$

.2072 .81

$.138 \quad 3.32$

$.069 \quad 3.97$

$0.0 \quad 4.11$

14400.

0.12355394

74.

.414

.345

.276

.207

2.13

1.98

2.56 


\begin{tabular}{|c|c|}
\hline 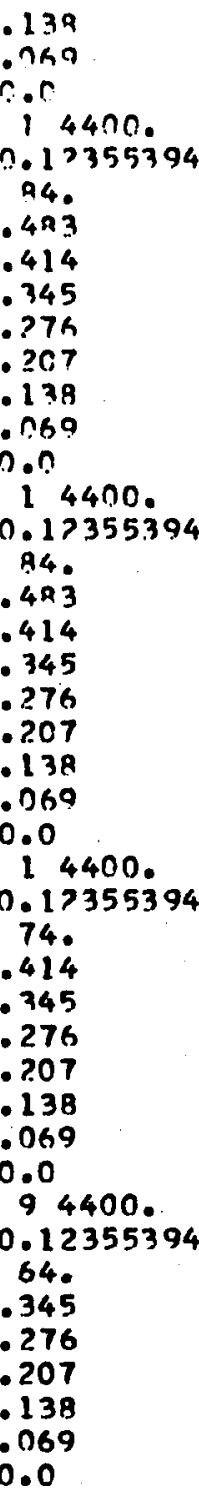 & $\begin{array}{l} \\
1.3 .56 \\
1.29 \\
1.30 \\
1.71 \\
1.74 \\
2.76 \\
2.46 \\
2.46 \\
2.62 \\
4 \\
\\
\\
1.4 .19 \\
2.85 \\
2.3 \\
2.36 \\
2.36 \\
2.39 \\
2.53 \\
2.53 \\
? .58\end{array}$ \\
\hline
\end{tabular}

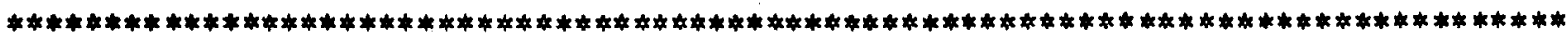

VFL BCITY PROFILE DATA, 4 INCH TURAINF AT 600 RPM IN WATER AT VARYING RADIAL DISTANCF

911.5 
14 4ก0.

C. 121 8ก9?5

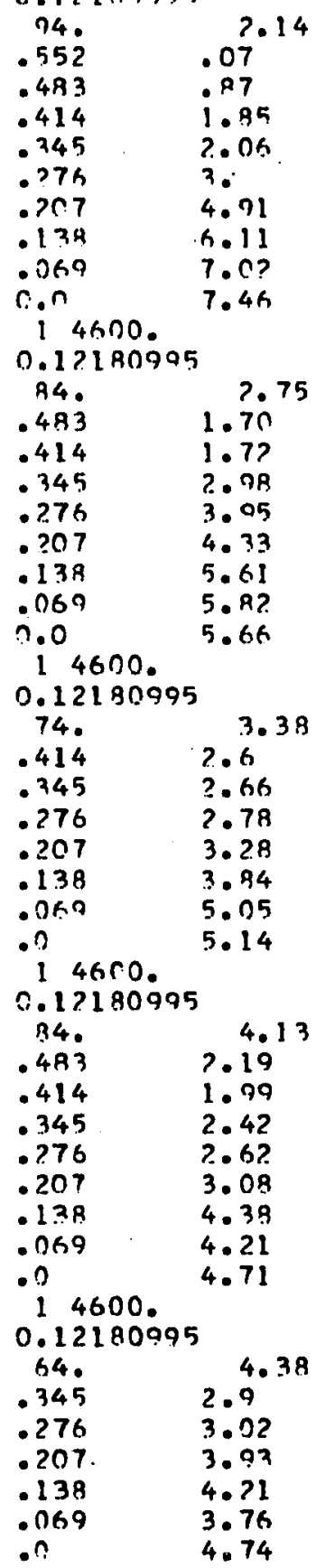


$\cap 4600$.

ก.12180705

64.5 .13

$.345 \quad 1.97$

$.276 \quad 2.04$

$.207 \quad 2.13$

$.138 \quad ? .48$

- D69 ?.65

$.0 \quad 2.34$ 
LIST $F-7$

FORTRAN LISTING FOR GRAPH WITH SUPPORTING SUBROUTINE SOLVE AND SAMPLE INPUT. TYPICAL

OUTPUT FROM PROGRAM IS ALSOO GIVEN

MAIN PROGRAM GRAPH

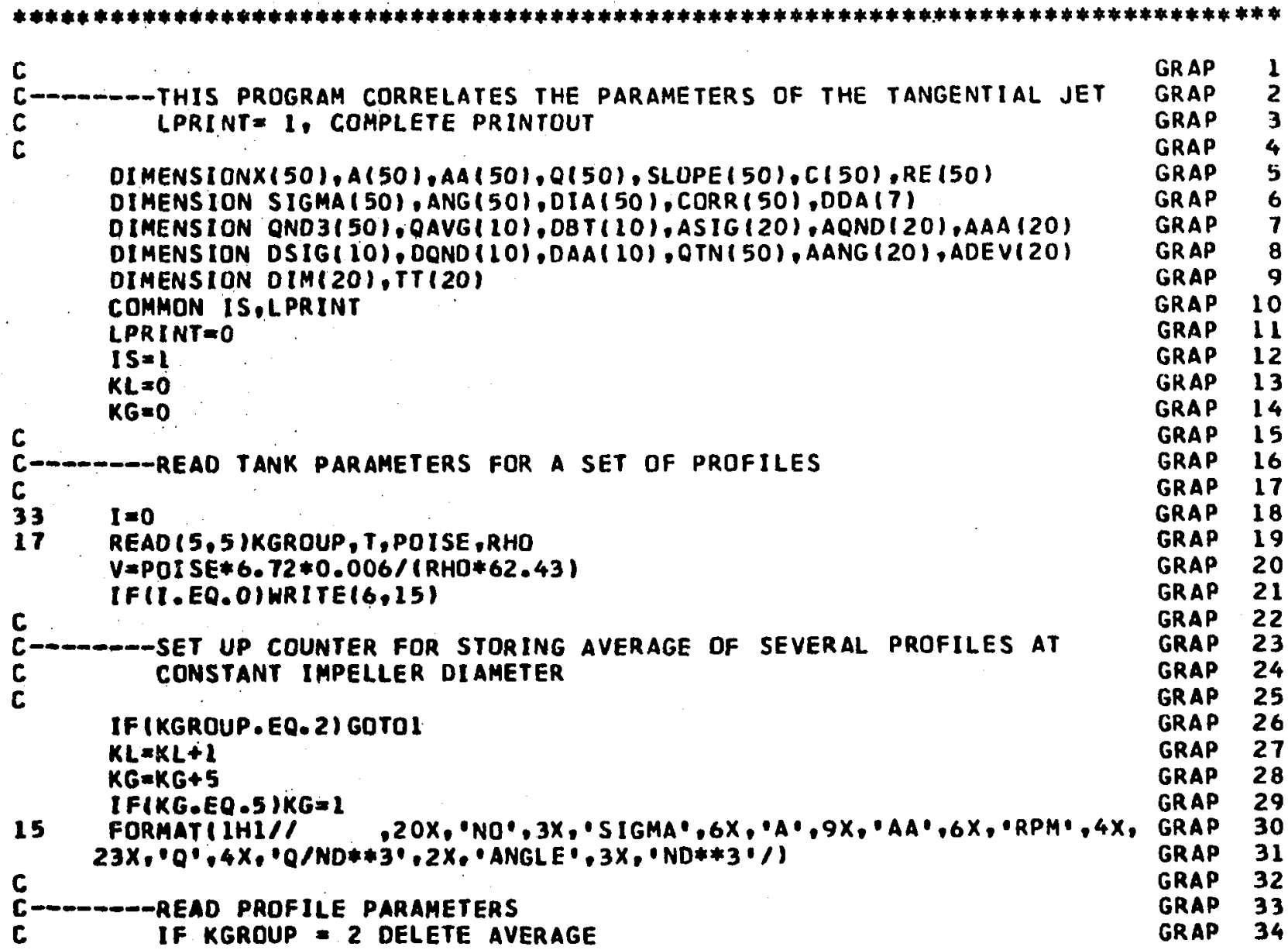




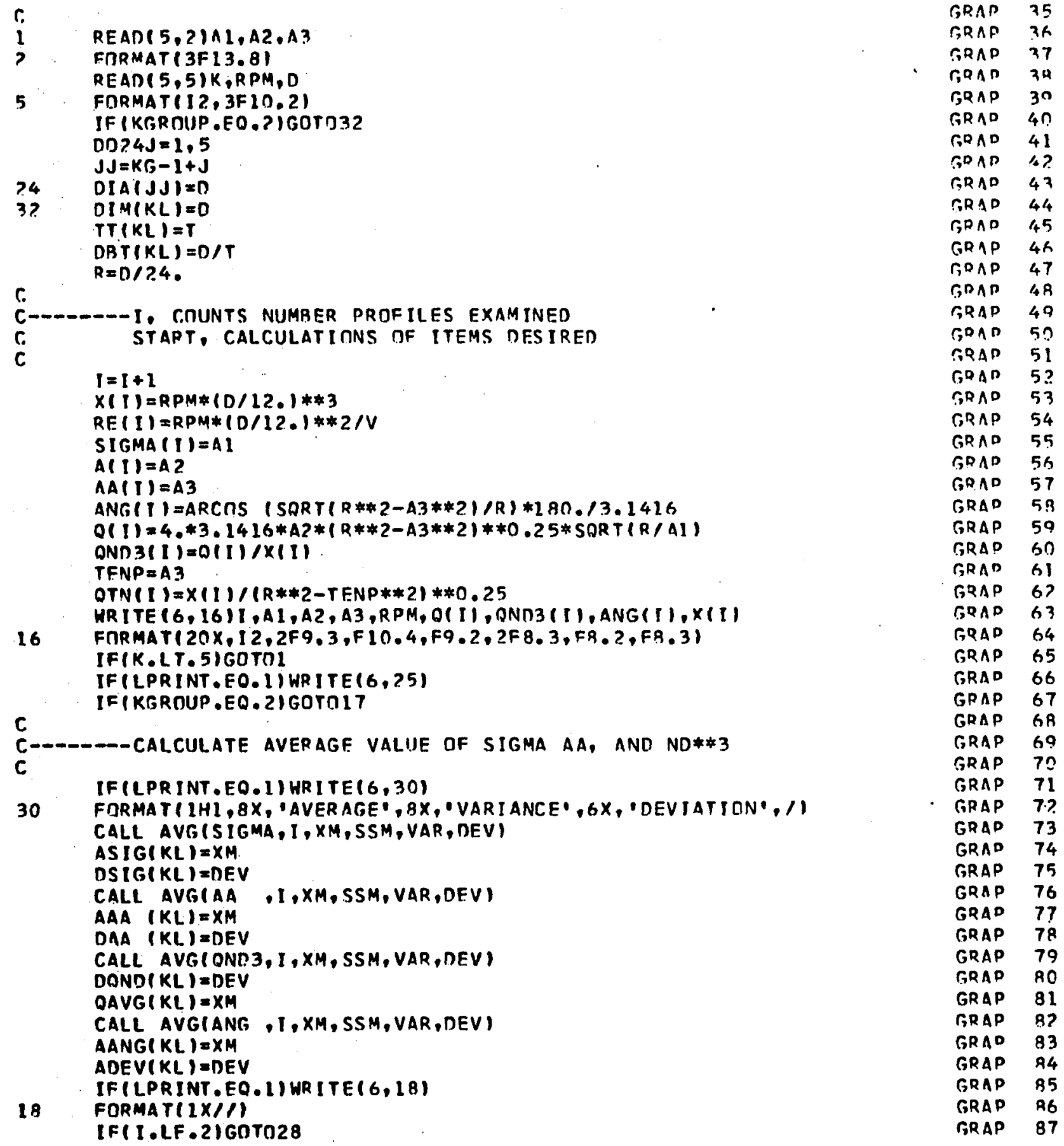




$$
c
$$
C-D.--OFINO EQUATIINN FOR NO**3 VERSUS A

C. IF (LPRINT, EN.1)WR ITF $(6,7)$

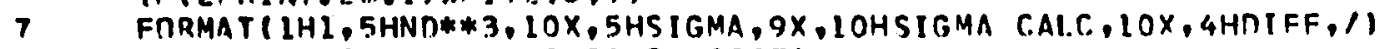
CALL SRLVFIX,A, I,CL,C.2,COSTI

SLOPF IKG $I=C L$.

CIKG $\quad I=C 2$

CORR (KG I I COST

IF (L.PRINT E EQ.1)WRITE (G, A)

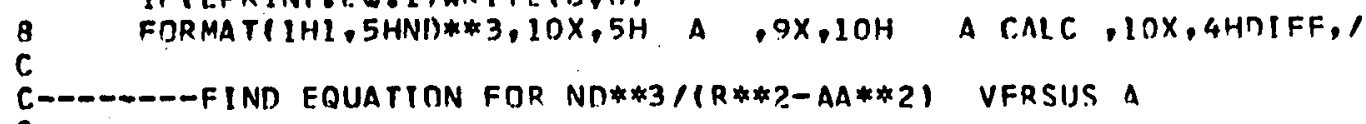

c

CALL SOLVE IOTN,A ,I,Cl,C2,COST)

SLNPE $(K G+1)=C 1$

$C(K G+1)=C 2$

CARR $(K G+1)=$ COST

\section{C}

C-----OFIND EQUATION FOR RF VERSUS A

IF(LPR INT , EQ. I) WR I TE $(6,12)$

12 FRRMATIIHI,5HRE NO, $10 \times, 5 \mathrm{H}$ A $9 \times, 10 \mathrm{H}$

CALL SOLVE (RE,A , I,CL,C2,COST)

A CALC, 10X,4HOIFF, 11

SLOPE $(K G+2)=C 1$

$C(K G+2)=C 2$

$\operatorname{CORR}(K G+2)=\operatorname{COS} T$

C

C-DONIND FOUATION FOR ND**3 VERSUS 0

10 IFILPRINT,EQ,1)WRITF(6,10)

CALL SOLVE $(X, Q \quad, I, C 1, C 2, C O S T)$

$S L \cap P F(K G+3)=C I$

$C(K G+3)=C 2$

CORR $(K G+3)=C O S T$

C

C IFILPRINT EQ E IIWRITE 16,13$)$

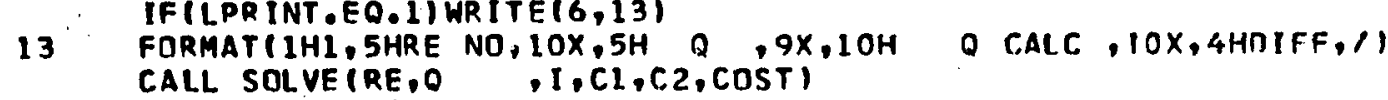

SLOPE $(K G+4)=C I$

$C(K G+4)=C 2$

CORR $(K G+4)=\operatorname{COST}$

GOT029

$28 \quad K G=K G-5$

29 IF (KGROUP.LT.5)GOT033

IFIKL .LT. 3 )GOTOZ6

IFILPR INT. EQ.I I WR I TE $(6,25)$

C

C

QNNOH 
CALL SOI VEICRT, OAVG,KL,C1,C.2,COSTI

IFILPRINT. EO. I IWR ITE 16,27 I I, I, C?, COST

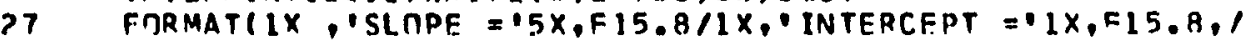

$1 \mid X_{0} \cdot$ CORR CDEF $=1,\left|X_{0}, F 15.8\right| 1$

is $s=0$

C.

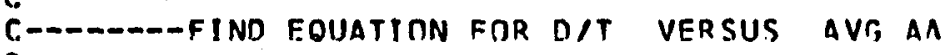

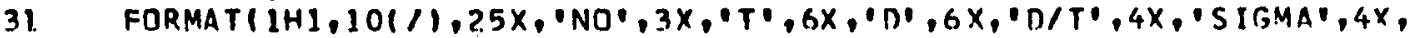

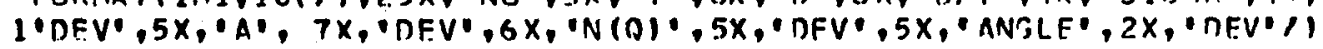
$0036 I=1, K I$.

36 WRITF (6,37)I,TTIII,DIMII),DATI I), ASIRII),DSIG(I), AAA II, DAAII), IDAVG (I), DQND (I), AANG (I), ADEVII)

37 FORMAT(25X,12,2F6.?,FB.4,F9,3,F7,3,F9,4,F9,5,2F9,4,F8,2,F6,2) STOP END

GRAP 141

TDND 14?

T.RAn 142

SRAP 144

FRAD 145

CRAD 146

GDAD 147

GRAD 14.9

FPAD 149

GONP 150

GRAn 151

GRAD 15?

GRAD 153

C.DAD 154

GRAR 155

GRAD 156

GRAD 157

CDAD 15R

GRAD 153

GOAP ISO

TPAD 161

GRAD 16?

SPAO 153

GRAD 164

GRAP 1.65

GRAD 166

GRAD 167

CQAD 168

GRAP 169

GRAP 17R

GRAP 171

GRAP 172

GRAP 173

GRAD 174

GRAD 175

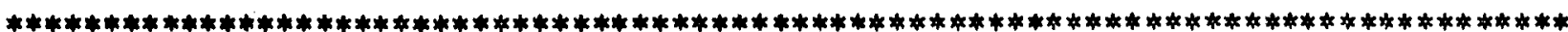
SURRDUT INE SOLVF

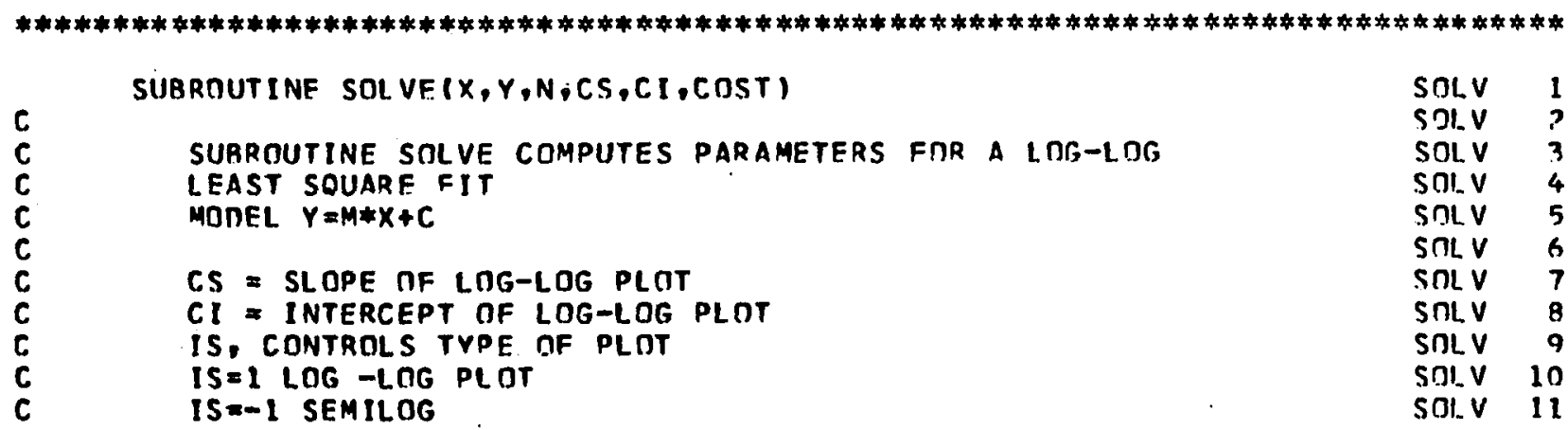




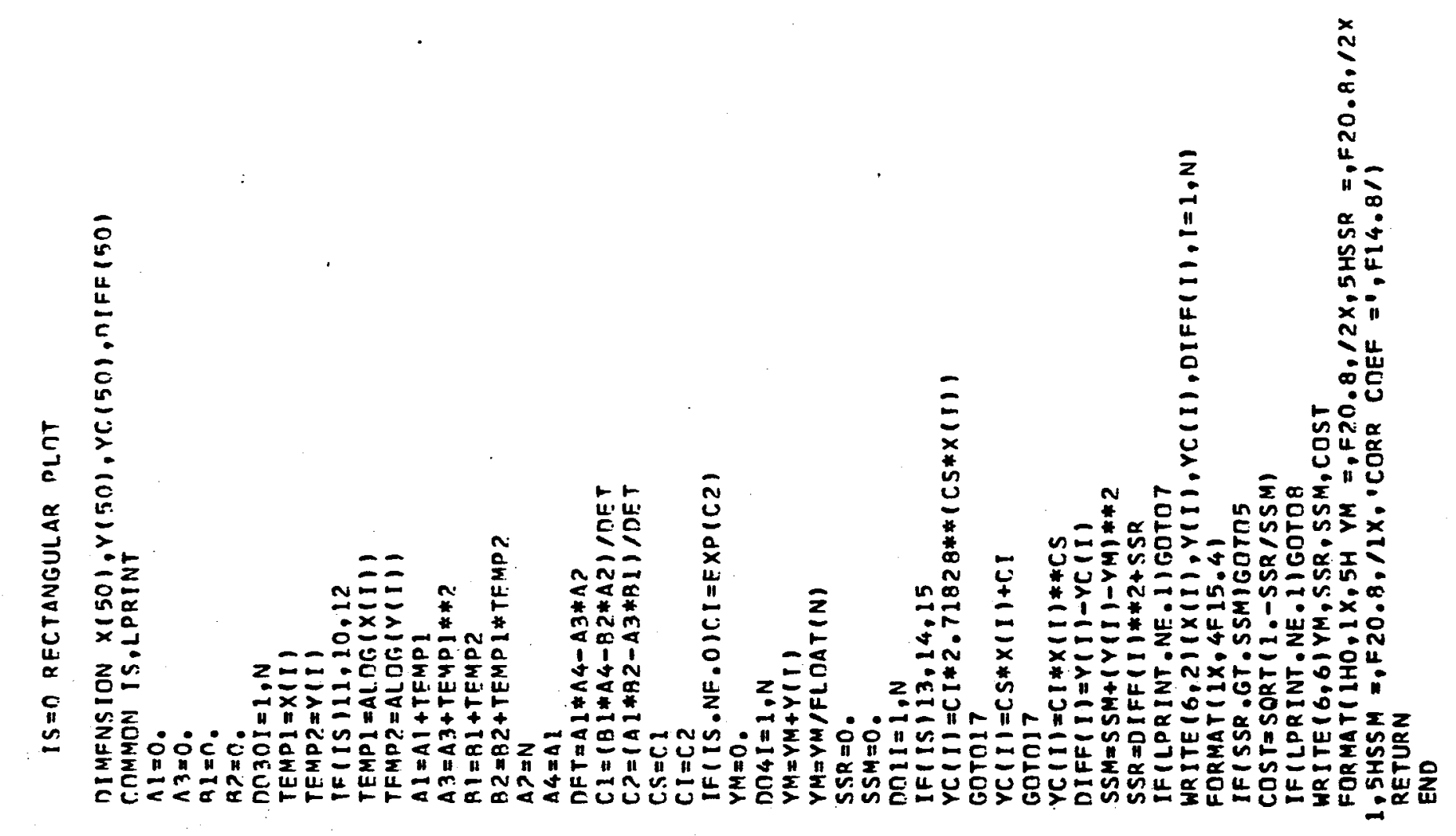

$\sim c$

$\cong ニ$

요

$\checkmark$

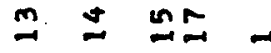

nit in $e x$. 


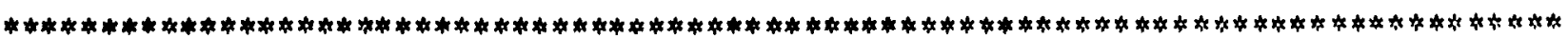

SURROUT INF AVG,

FIR LISTING SEE LIST F-1

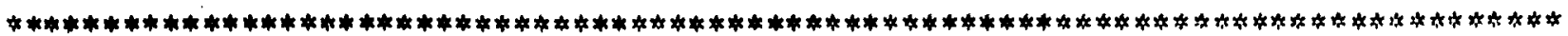

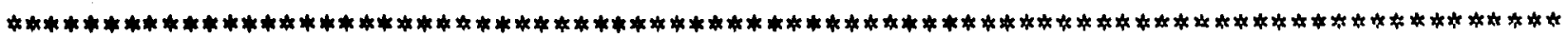
INPIJT DATA

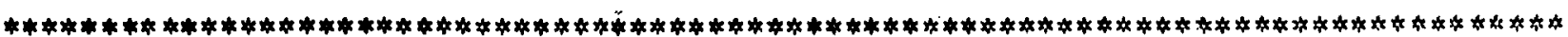

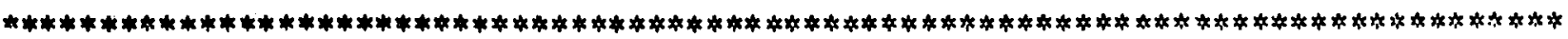

TANGENTIAL JET PARAMFTERS TRTAINED IN A 12.25 INCH DIAMETER TANK

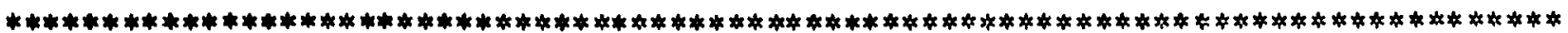

\begin{tabular}{|c|c|c|}
\hline $\begin{array}{c}112.25 \\
10.5175 \\
1243 . \\
10.0544 \\
1250 . \\
11.7746 \\
1333.33 \\
11.8492 \\
1400 \\
11.3673 \\
1500 . \\
11.5885 \\
9550 .\end{array}$ & $\begin{array}{l}0.8937 \\
13.4772 \\
3 . \\
14.285 \\
3 . i 8.557 \\
18.5 \\
22.7386 \\
3 . \\
28.412 \\
3 . \\
31.6168 \\
3 .\end{array}$ & $\begin{array}{c}0.99708 \\
0.114 \\
0.119\end{array}$ \\
\hline
\end{tabular}


(a) Results of Analysis for Data Sets Given In Table IV-20.

\begin{tabular}{|c|c|c|c|c|}
\hline & $N * D * * 3$ & VERSUS & A & \\
\hline \multirow[t]{2}{*}{$\begin{array}{l}D 1 A \\
3.00 \\
3.00 \\
4.00 \\
5.00 \\
6.00 \\
3.00 \\
3.00 \\
4.00 \\
5.00\end{array}$} & $\begin{array}{l}\text { SLOPF } \\
0.7252 \\
0.6796 \\
1.0313 \\
1.0516 \\
0.6966 \\
1.0239 \\
1.0931 \\
1.0731 \\
1.1035\end{array}$ & & $\begin{array}{c}\text { INTFRCEPT } \\
4.3155 \\
0.0038 \\
0.8706 \\
0.0003 \\
1.9184 \\
3.4737 \\
0.0001 \\
0.6218 \\
0.0000\end{array}$ & $\begin{array}{l}\text { CORR CHEF } \\
0.9851 \\
0.9729 \\
0.9901 \\
0.9996 \\
0.9717 \\
0.9996 \\
0.9897 \\
0.9997 \\
0.9992\end{array}$ \\
\hline & \multicolumn{4}{|c|}{$N D * * 3 /(R * * 2-A A * * 2)$ VERSUS $A$} \\
\hline \multirow[t]{2}{*}{$\begin{array}{l}014 \\
3.00 \\
4.00 \\
4.00 \\
5.00 \\
6.00 \\
3.00 \\
4.00 \\
4.00 \\
5.00\end{array}$} & $\begin{array}{l}\text { SL NPF } \\
0.7308 \\
1.0040 \\
1.0308 \\
1.0638 \\
0.6967 \\
1.1558 \\
1.1085 \\
1.0724 \\
1.0708\end{array}$ & & $\begin{array}{l}\text { INTFRR.FPT } \\
1.6014 \\
2.1553 \\
0.0011 \\
0.8673 \\
0.0128 \\
0.4820 \\
1.408 ? \\
0.0000 \\
0.6449\end{array}$ & $\begin{array}{l}\text { CORP COEF } \\
0.9728 \\
0.9913 \\
0.9902 \\
0.9995 \\
0.9742 \\
0.9878 \\
0.9978 \\
0.9986 \\
0.9986\end{array}$ \\
\hline & RE & VFRSUS & $A$ & \\
\hline \multirow[t]{2}{*}{$\begin{array}{l}014 \\
3.00 \\
4.00 \\
5.00 \\
5.00 \\
6.00 \\
3.00 \\
4.00 \\
5.00 \\
5.00\end{array}$} & $\begin{array}{l}\text { SLOPE } \\
0.7248 \\
0.9488 \\
1.0523 \\
1.0630 \\
0.6768 \\
1.0238 \\
1.1254 \\
1.1042 \\
1.0702\end{array}$ & & $\begin{array}{l}\text { INTERCEPT } \\
0.0071 \\
0.7498 \\
1.6058 \\
0.0001 \\
2.7376 \\
0.0004 \\
0.3414 \\
1.0644 \\
0.0000\end{array}$ & $\begin{array}{c}\text { CORR COEF } \\
0.9851 \\
0.9881 \\
0.9996 \\
0.9995 \\
0.9731 \\
0.9996 \\
0.9978 \\
0.9992 \\
0.9986\end{array}$ \\
\hline & $N * D * * 3$ & VERSUS & 0 & \\
\hline $\begin{array}{l}D 1 A \\
3.00 \\
4.00 \\
5.00 \\
6.00 \\
6.00 \\
3.00 \\
4.00 \\
5.00 \\
6.00\end{array}$ & $\begin{array}{l}\text { SLOPE } \\
0.6790 \\
1.0034 \\
1.0705 \\
0.6968 \\
0.6766 \\
1.0934 \\
1.1079 \\
1.1094 \\
0.9301\end{array}$ & & $\begin{array}{l}\text { INTERCEPT } \\
1.5467 \\
0.0004 \\
0.4907 \\
3.7437 \\
0.0111 \\
0.9031 \\
0.0000 \\
0.3271 \\
1.6988\end{array}$ & $\begin{array}{c}\text { CORR COEF } \\
0.9728 \\
0.9914 \\
0.9994 \\
0.9742 \\
0.9731 \\
0.9897 \\
0.9977 \\
0.9985 \\
0.9984\end{array}$ \\
\hline
\end{tabular}




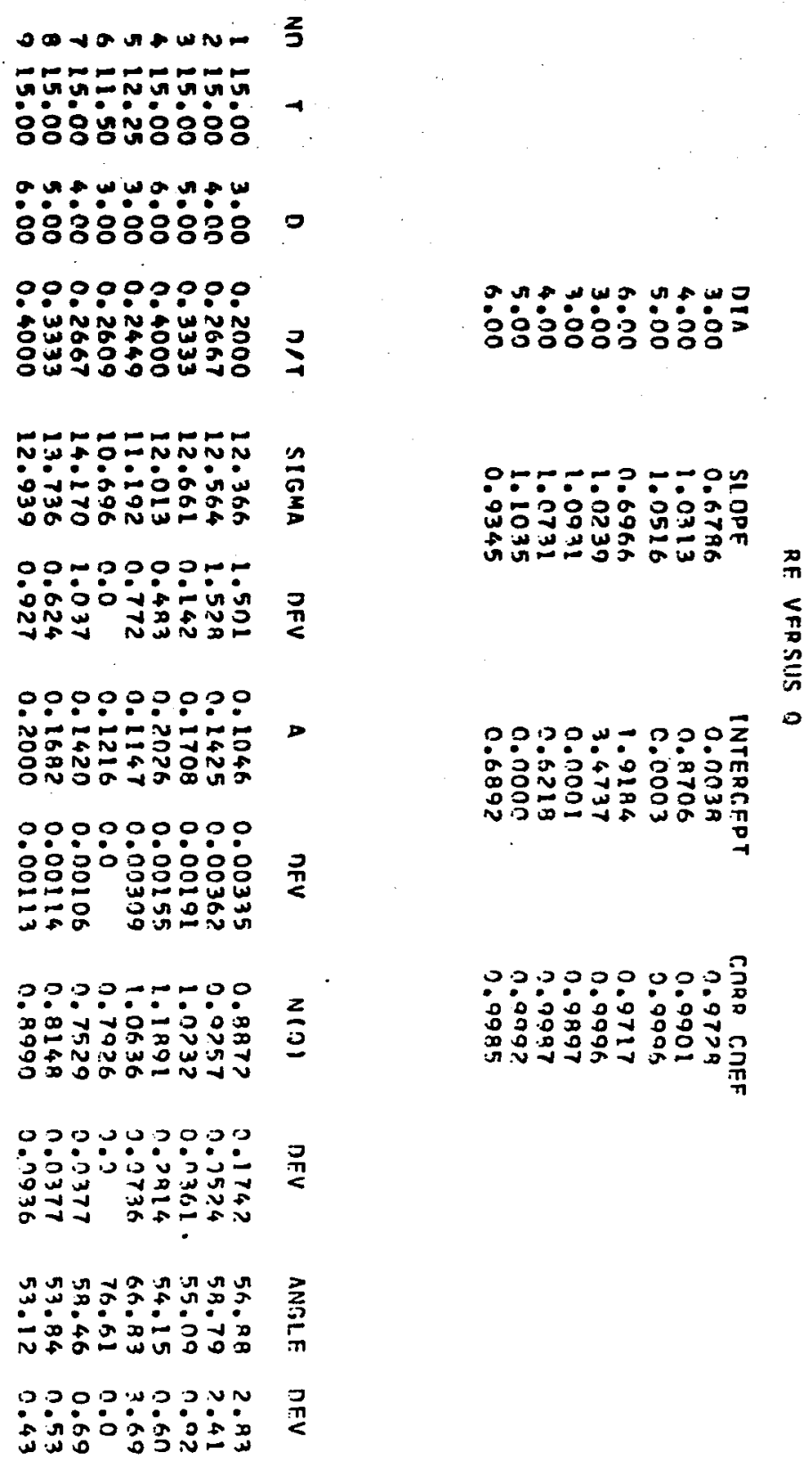

$0000 \mathrm{popoz} 0$

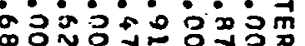

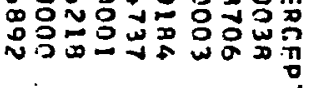

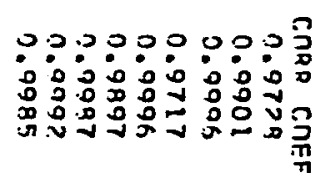

\section{是}


(b) Results of Analysis of (1) Tangential Jet Parameters from Table IV-20 and (2) Tangential Jet Parameters Obtained by Keeping $\sigma$ Constant.

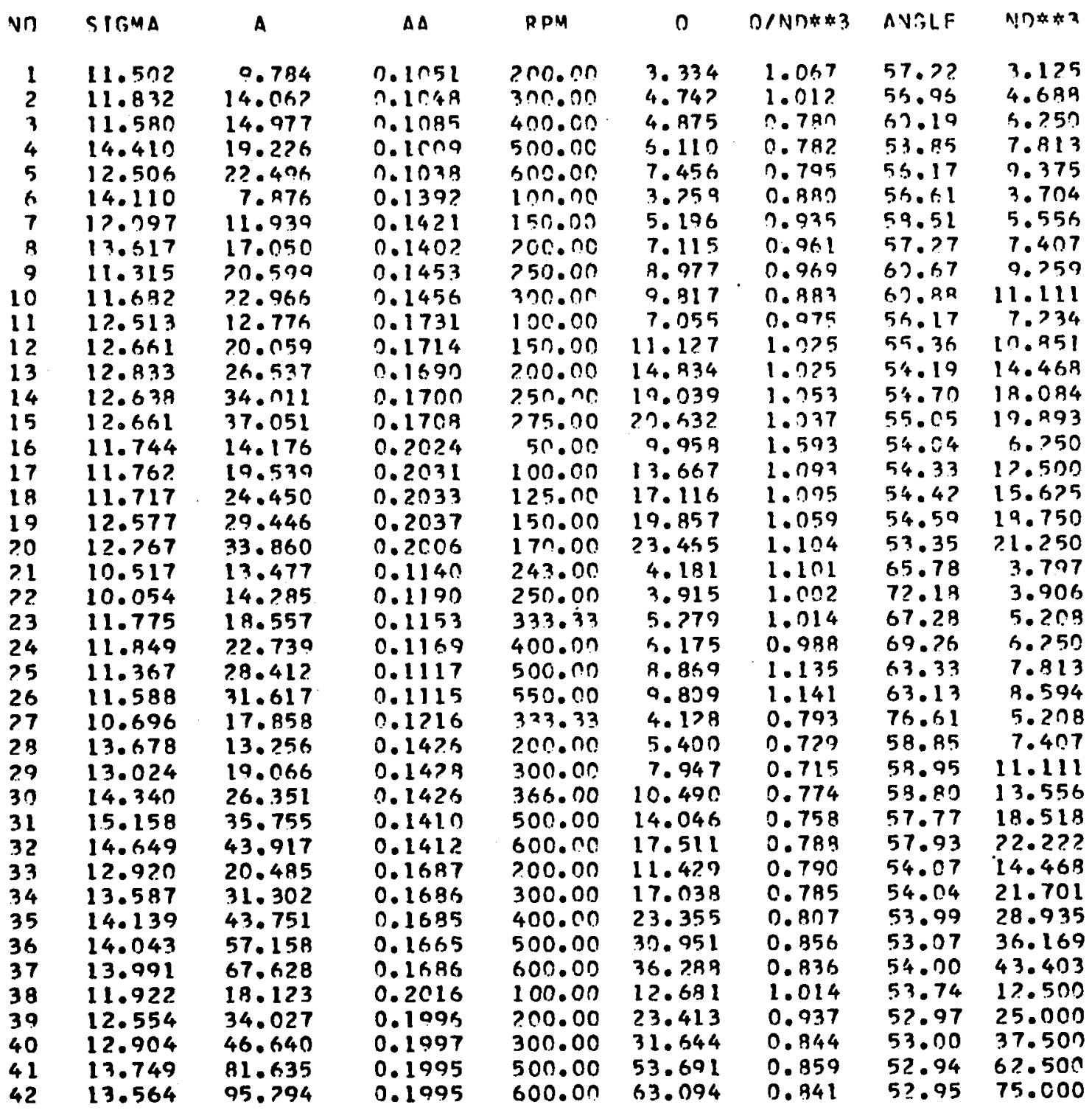




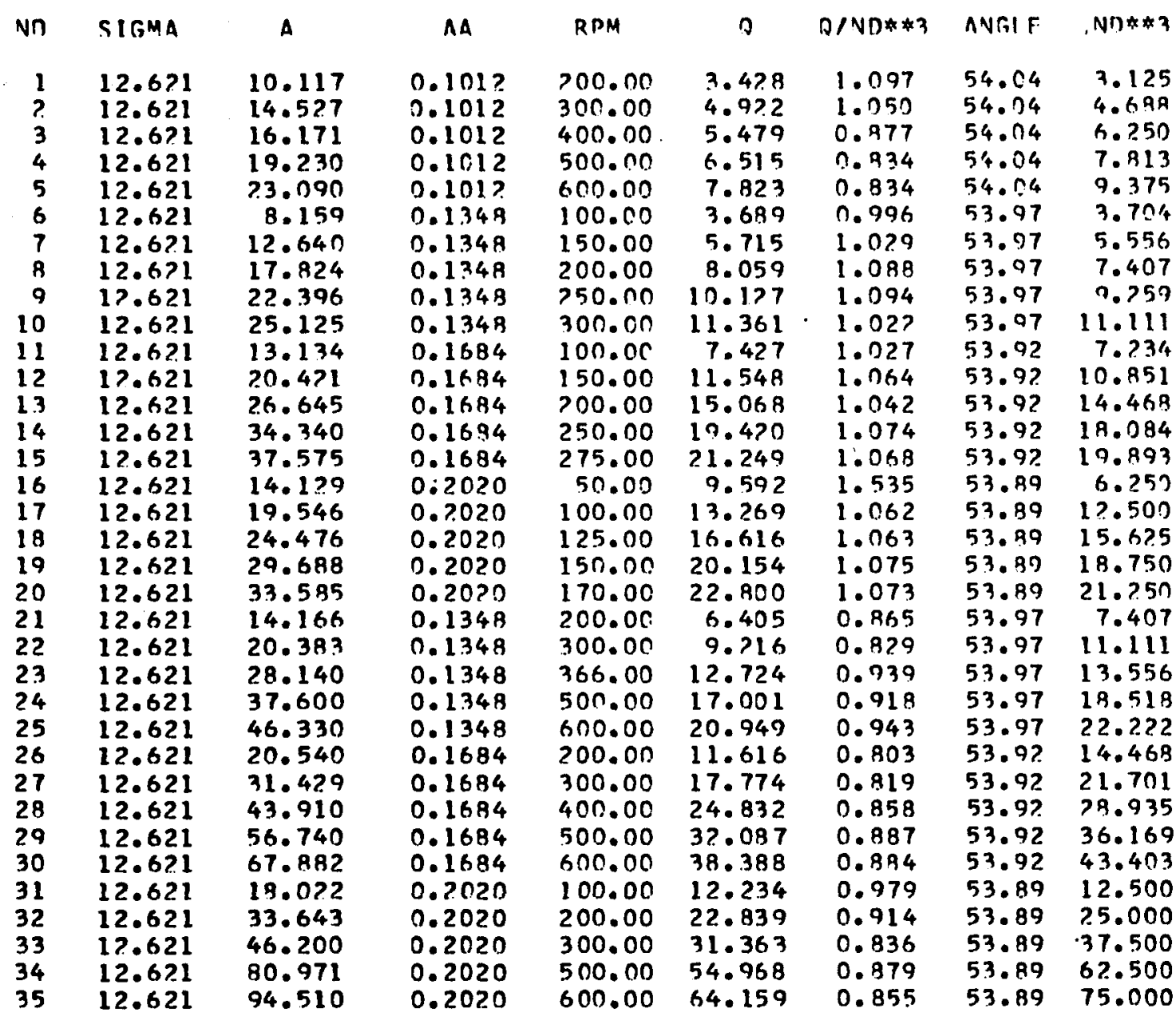

$N * n * * 3$ VFRSUS $A$

$\begin{array}{lccc}\text { DIA } & \text { SLOPE } & \text { INTERCEPT } & \text { CORR COFF } \\ 6.00 & 0.6625 & 4.7008 & 0.9583 \\ 6.00 & 0.7316 & 3.7712 & 0.9771 \\ & \text { RE VERSUS A } & \\ \text { DIA } & \text { SLOPE } & \text { INTERCEPT } & \text { CORR COEF } \\ 6.00 & 0.2397 & 1.2031 & 0.7098 \\ 6.00 & 0.2424 & 1.3471 & 0.7564 \\ & \text { N*D**3 VFRSUS } & & \\ & & & \\ \text { DIA } & \text { SLOPE } & \text { INTERCEPT } & \text { CORR COEF }\end{array}$




\begin{tabular}{|c|c|c|c|}
\hline \multirow[t]{2}{*}{$\begin{array}{l}6.00 \\
6.00\end{array}$} & $\begin{array}{l}0.9421 \\
0.9293\end{array}$ & $\begin{array}{l}1.0745 \\
1.1665\end{array}$ & $\begin{array}{l}0.9904 \\
n .9941\end{array}$ \\
\hline & RE VERSUS & 0 & \\
\hline $\begin{array}{l}014 \\
6.00 \\
6.00\end{array}$ & $\begin{array}{l}\text { SL חPF } \\
0.382 ? \\
0.2 .961\end{array}$ & $\begin{array}{c}\text { INTEP.CFPT } \\
0.0921 \\
0.4114\end{array}$ & $\begin{array}{c}\text { CORP COFF } \\
0.7926 \\
0.7108\end{array}$ \\
\hline AVG & DEVIATION & & \\
\hline $\begin{array}{c}12.621 \\
0.1539 \\
0.944\end{array}$ & $\begin{array}{l}0.066 \\
0.00006 \\
0.001 ?\end{array}$ & & \\
\hline AVr & NEVIATION & & \\
\hline $\begin{array}{l}12.621 \\
0.1588 \\
0.977\end{array}$ & $\begin{array}{l}0.000 \\
0.00007 \\
0.0011\end{array}$ & & \\
\hline
\end{tabular}


LIST $\mathbf{F}-8$

FORTRAN LISTING FOR MAIN PROGRAM RESULT WITH SUPPORTING SUBROUTINE VARI AND SAMPLE OUTPUT.

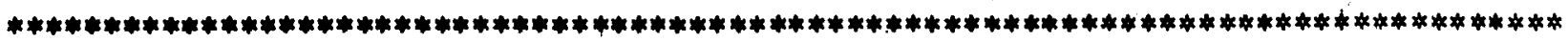

MAIN PROGRAM RESULT

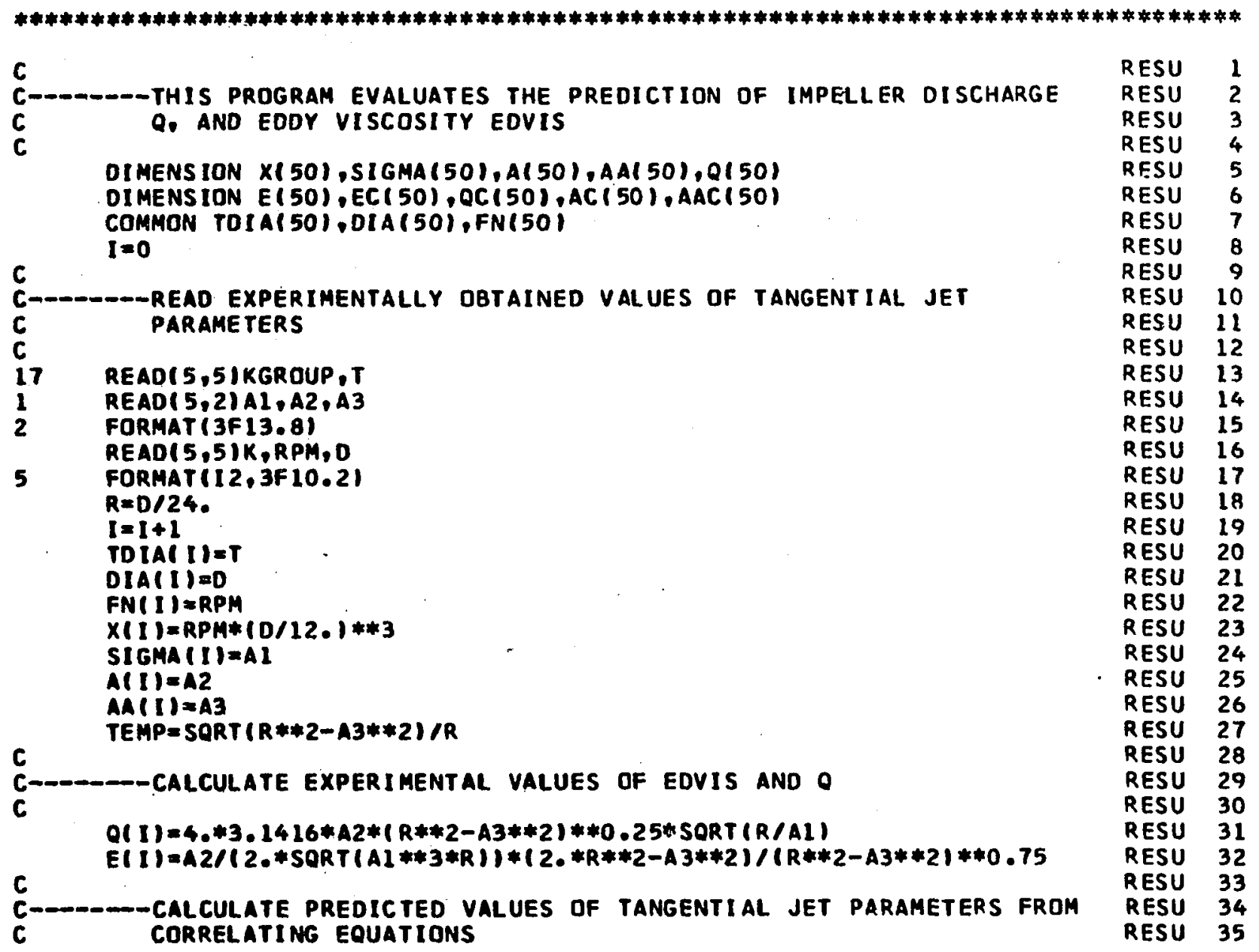


C

$A 1=12.621$

DA $T=(T-D) / T$

$T A=O B T$

IFI DB T $-0.73516,6,7$

$7 \quad A 3=0.06924 * T A * *(-1.837)$

GOTOB

$6 \quad A 3=\quad 0.08354 * T A * *(-1.7281)$

8 TEMP $=x(1) /(R * 2-A 3 * * 2) * 0.25$

$A 2=1.1436 * T E M P * 0.8337$

TEMP $=S Q R T(R * * 2-A 3 * * 2) / R$

C

C-- - - CALCULATE PREDICTED VALUES OF 0 AND EOVIS

C

$Q C(I)=4 . * 3.1416 * A 2 *(R * * 2-A .3 * * 2) * 0.25 * S Q R T(R / A L)$

$E C(I)=A 2 /(2 . * S Q R T(A) * 3 * R) / *(2 . * R * * 2-A 3 * * 2) /(R * 2-A 3 * 2) * 0.75$

$A C(I)=A 2$

$A A C(I)=A 3$

IFIK.LT.5)GOTOL

C

IFIKGROUP.EQ.1IGOTOLI

C------CALCULATE difference Between Calculated and PREOICTED

C VALUE AND PRINT RESULTS

WR ITE $(6,20)$

20 FORMATIIHI, $28 X$, ANALYSIS FOR IMPELLER DISCHARGE $0^{\circ} / 1$

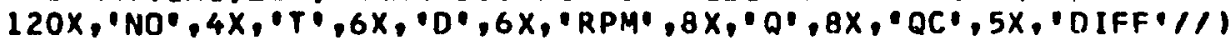

CALL VAR I (Q,QC, I)

WR ITE $(6,21)$

21 FRRMATIIHI, 20X, 'ANALYSIS FOR VOLUMETRIC FLOW, $A, / 11$

CALL VARI $(A, A C, I)$

HR I TE $(6,22)$

22 FORMATIIHI, 20X, 'ANALYSIS FOR RADIUS OF SOURCE AA', $/ 1$

CALL VARI ( AA, AAC, I)

WR ITE $(6,23)$

23 FORMATIIHI, 28X, ANALYSIS FOR EDDY VISCOSITY,,/I

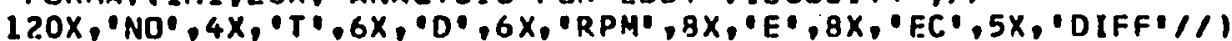

CALL VARIIE, EC, I)

STOP

END

RFSU 36

RESU 37

RESU 38

RESU 39

RESU 40

RESU 41

RESU 42

RESU 43

RESU 44

RFSU 45

RESU 40

RESU 47

RESU 48

RESU 49

RESU 50

RESU 51

RESU 5?

RESU 53

RESU 54

RFSU 55

RESU 56

RESU 57

RESU 5R

RESU 59

RESU 60

RESU 61

RESU 62

RESU 63

RESU 64

RESU 65

RESU 66

RESU 67

RESU 68

RFSU 69

RESU 70

RESU 71

RESU 72

RESU 73

RESU 74

RESU 75

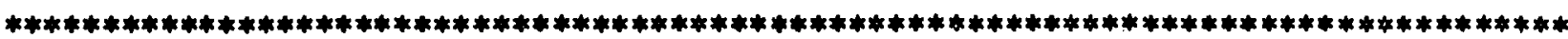

SUBROUT INE VARI

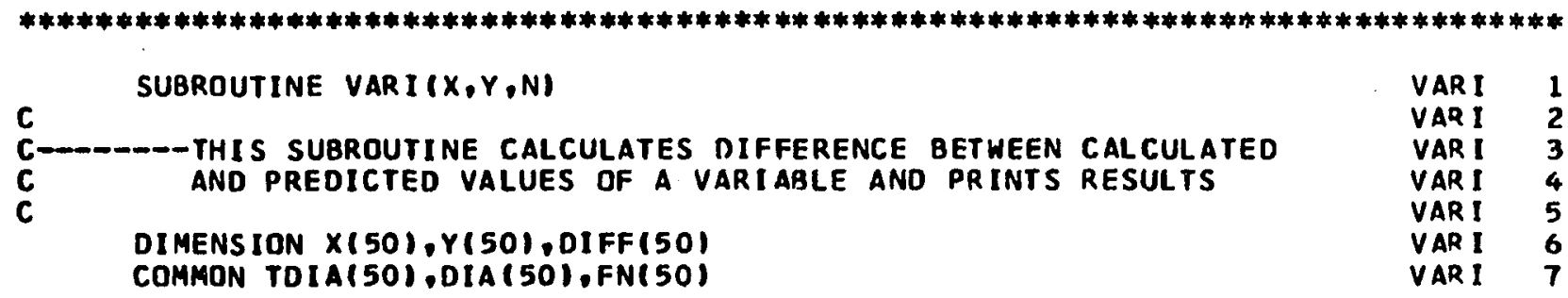




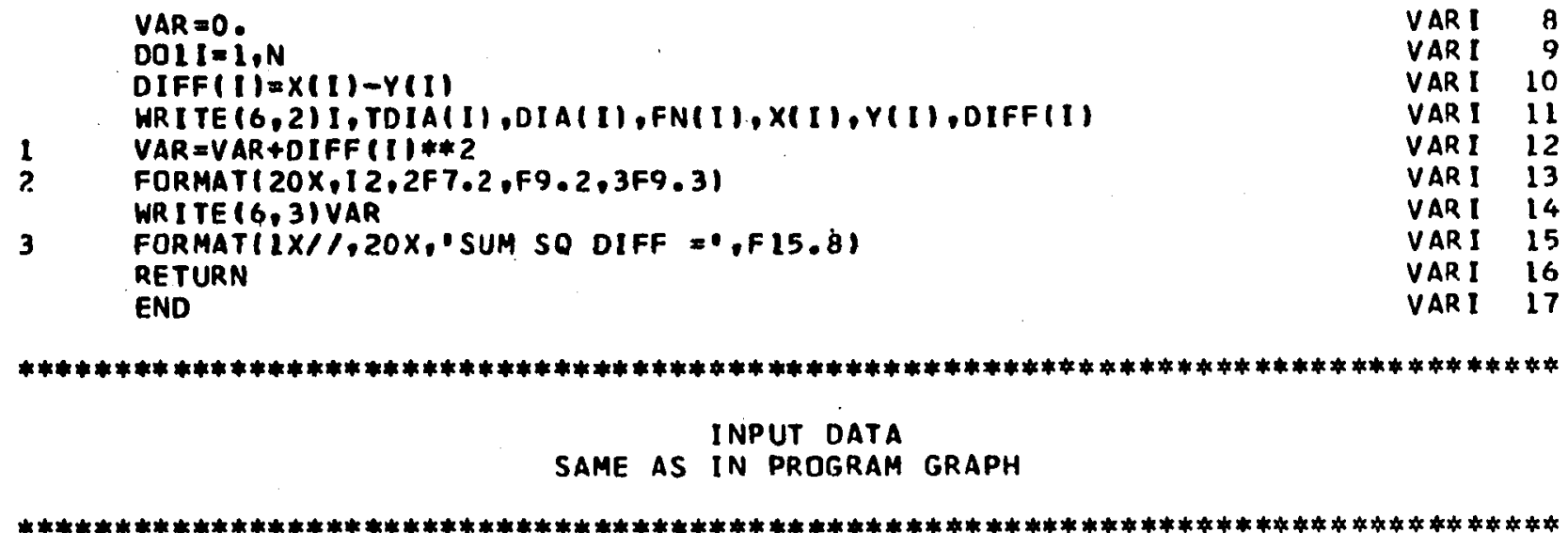


RESULTS OF ANALYSIS BY PROGRAM RESULT

ANALYSIS FOR EDDY VISCUSITY

$\begin{array}{rrrrrrr}N O & T & 0 & R P M & F & F C & 0 I F F \\ & & & & & & \\ 1 & 15.00 & 3.00 & 200.00 & 0.407 & 0.321 & 0.016 \\ 2 & 15.00 & 3.00 & 300.00 & 0.557 & 0.450 & 0.107 \\ 3 & 15.00 & 3.00 & 400.00 & 0.676 & 0.57 ? & 0.105 \\ 4 & 15.00 & 3.00 & 500.00 & 0.523 & 0.688 & -2.165 \\ 5 & 15.00 & 3.00 & 600.00 & 0.802 & 0.801 & 0.011 \\ 6 & 15.00 & 4.00 & 100.00 & 0.237 & 0.361 & -0.124 \\ 7 & 15.00 & 4.00 & 150.00 & 0.478 & 0.506 & -0.028 \\ 8 & 15.00 & 4.00 & 200.00 & 0.552 & 0.643 & -C .092 \\ 9 & 15.00 & 4.00 & 250.00 & 0.979 & 0.775 & 0.214 \\ 10 & 15.00 & 4.00 & 300.00 & 1.048 & 0.902 & 0.146 \\ 11 & 15.00 & 5.00 & 100.00 & 0.455 & 0.474 & -0.019 \\ 12 & 15.00 & 5.00 & 150.00 & 0.687 & 0.665 & 0.072 \\ 13 & 15.00 & 5.00 & 200.00 & 0.866 & 0.845 & 0.021 \\ 14 & 15.00 & 5.00 & 250.00 & 1.149 & 1.018 & 0.132 \\ 15 & 15.00 & 5.00 & 275.00 & 1.260 & 1.102 & 0.158 \\ 16 & 15.00 & 5.00 & 50.00 & 0.526 & 0.339 & 0.139 \\ 17 & 15.00 & 6.00 & 100.00 & 0.729 & 0.693 & 0.036 \\ 18 & 15.00 & 6.00 & 125.00 & 0.919 & 0.335 & 0.085 \\ 19 & 15.00 & 6.00 & 150.00 & 1.000 & 0.97 ? & 0.028 \\ 20 & 15.00 & 6.00 & 170.00 & 1.159 & 1.078 & 0.081 \\ 21 & 12.25 & 3.00 & 243.00 & 0.879 & 0.697 & 0.181 \\ 22 & 12.25 & 3.00 & 250.00 & 1.447 & 0.714 & 0.733 \\ 23 & 12.25 & 3.00 & 333.33 & 1.099 & 0.908 & 0.192 \\ 24 & 12.25 & 3.00 & 400.00 & 1.489 & 1.057 & 0.432 \\ 25 & 12.25 & 3.00 & 500.00 & 1.481 & 1.273 & 0.208 \\ 26 & 12.25 & 3.00 & 550.00 & 1.588 & 1.373 & 0.210 \\ 27 & 11.50 & 3.00 & 333.33 & 2.412 & 1.677 & 0.735 \\ 28 & 15.00 & 4.00 & 200.00 & 0.446 & 0.643 & -0.197 \\ 29 & 15.00 & 4.00 & 300.00 & 0.693 & 0.902 & -0.209 \\ 30 & 15.00 & 4.00 & 366.00 & 0.825 & 1.065 & -0.240 \\ 31 & 15.00 & 4.00 & 500.00 & 0.999 & 1.381 & -0.382 \\ 32 & 15.00 & 4.00 & 600.00 & 1.298 & 1.608 & -0.310 \\ 33 & 15.00 & 5.00 & 200.00 & 0.660 & 0.845 & -0.185 \\ 34 & 15.00 & 5.00 & 300.00 & 0.934 & 1.185 & -0.251 \\ 35 & 15.00 & 5.00 & 400.00 & 1.228 & 1.506 & -0.278 \\ 36 & 15.00 & 5.00 & 500.00 & 1.587 & 1.814 & -0.227 \\ 37 & 15.00 & 5.00 & 600.00 & 1.929 & 2.112 & -0.183 \\ 38 & 15.00 & 6.00 & 100.00 & 0.653 & 0.693 & -0.040 \\ 39 & 15.00 & 6.00 & 200.00 & 1.115 & 1.235 & -0.120 \\ 40 & 15.00 & 6.00 & 300.00 & 1.468 & 1.732 & -0.264 \\ 41 & 15.00 & 6.00 & 500.00 & 2.333 & 2.651 & -0.318 \\ 42 & 15.00 & 6.00 & 600.00 & 2.780 & 3.086 & -0.306\end{array}$




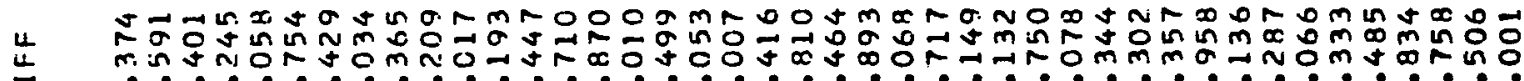

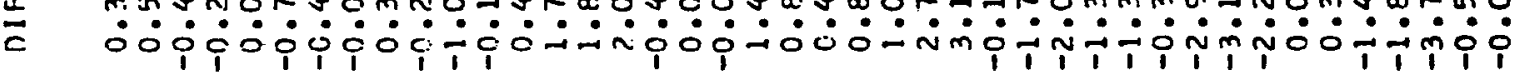

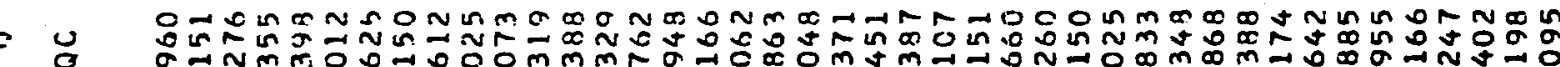

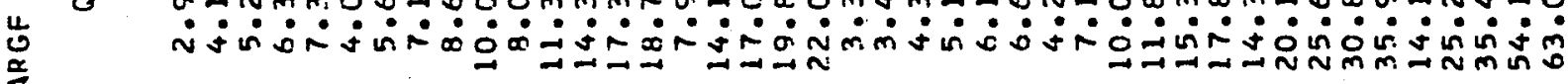

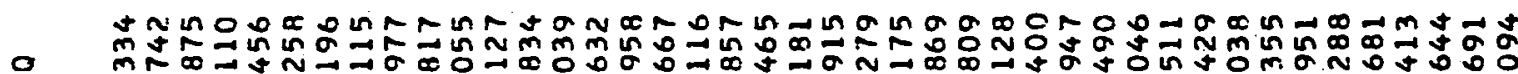

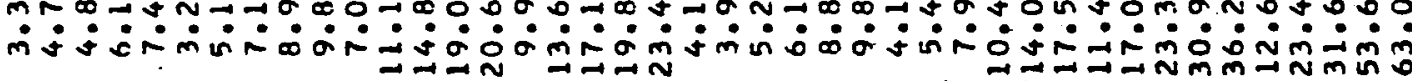

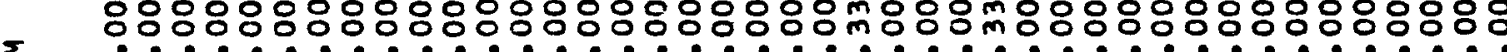
施

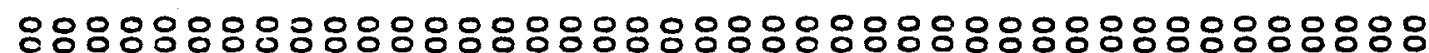

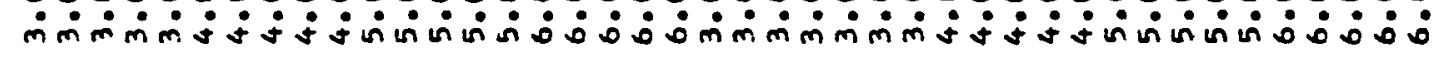

은.

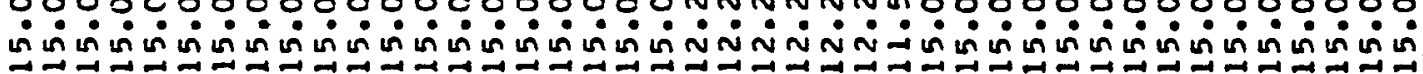


ANALYSIS FOR RADIUS OF SOURCE AA

$\begin{array}{rrrrrrr}1 & 15.00 & 3.00 & 200.00 & 0.105 & 0.104 & 0.001 \\ 2 & 15.00 & 3.00 & 300.00 & 0.101 & 0.104 & 0.000 \\ 3 & 15.00 & 3.00 & 400.00 & 0.108 & 0.104 & 0.004 \\ 4 & 15.00 & 3.00 & 500.00 & 0.101 & 0.104 & -0.003 \\ 5 & 15.00 & 3.00 & 600.00 & 0.104 & 0.104 & -0.010 \\ 6 & 15.00 & 4.00 & 100.00 & 0.139 & 0.143 & -1.004 \\ 7 & 15.00 & 4.00 & 150.00 & 0.142 & 0.143 & -0.001 \\ 8 & 15.00 & 4.00 & 200.00 & 0.140 & 0.143 & -0.003 \\ 9 & 15.00 & 4.00 & 250.00 & 0.145 & 0.143 & 0.003 \\ 10 & 15.00 & 4.00 & 360.00 & 0.146 & 0.143 & 0.003 \\ 11 & 15.00 & 5.00 & 100.00 & 0.173 & 0.169 & 0.005 \\ 12 & 15.00 & 5.00 & 150.00 & 0.171 & 0.164 & 0.003 \\ 13 & 15.00 & 5.00 & 200.00 & 0.169 & 1.168 & 0.001 \\ 14 & 15.00 & 5.00 & 250.00 & 0.170 & 0.168 & 0.00 ? \\ 15 & 15.00 & 5.00 & 275.00 & 0.171 & 0.163 & 0.002 \\ 16 & 15.00 & 6.00 & 50.00 & 0.202 & 0.202 & 0.000 \\ 17 & 15.00 & 6.00 & 100.00 & 0.203 & 0.202 & 0.001 \\ 18 & 15.00 & 6.00 & 125.00 & 0.203 & 0.202 & 0.001 \\ 19 & 15.00 & 6.00 & 150.00 & 0.204 & 0.202 & 0.00 ? \\ 20 & 15.00 & 6.00 & 170.00 & 0.201 & 0.202 & -0.001 \\ 21 & 12.25 & 3.00 & 243.00 & 0.114 & 0.116 & -1.002 \\ 22 & 12.25 & 3.00 & 250.00 & 0.119 & 0.116 & 0.003 \\ 23 & 12.25 & 3.00 & 333.33 & 0.115 & 0.116 & -0.001 \\ 24 & 12.25 & 3.00 & 400.00 & 0.117 & 0.116 & 0.001 \\ 25 & 12.25 & 3.00 & 500.00 & 0.112 & 0.116 & -0.004 \\ 26 & 12.25 & 3.00 & 550.00 & 0.111 & 0.116 & -0.005 \\ 27 & 11.50 & 3.00 & 333.33 & 0.122 & 0.121 & 0.001 \\ 28 & 15.00 & 4.00 & 200.00 & 0.143 & 0.143 & -0.000 \\ 29 & 15.00 & 4.00 & 300.00 & 0.143 & 0.143 & 0.000 \\ 30 & 15.00 & 4.00 & 366.00 & 0.143 & 0.143 & -0.000 \\ 31 & 15.00 & 4.00 & 500.00 & 0.141 & 0.143 & -0.002 \\ 32 & 15.00 & 4.00 & 600.00 & 0.141 & 0.143 & -0.002 \\ 33 & 15.00 & 5.00 & 200.00 & 0.169 & 0.168 & 0.000 \\ 34 & 15.00 & 5.00 & 300.00 & 0.169 & 0.168 & 0.000 \\ 35 & 15.00 & 5.00 & 400.00 & 0.169 & 0.168 & 0.000 \\ 36 & 15.00 & 5.00 & 500.00 & 0.167 & 0.168 & -0.002 \\ 37 & 15.00 & 5.00 & 600.00 & 0.169 & 0.168 & 0.000 \\ 38 & 15.00 & 6.00 & 100.00 & 0.202 & 0.202 & -0.000 \\ 39 & 15.00 & 6.00 & 200.00 & 0.200 & 0.202 & -0.002 \\ 40 & 15.00 & 6.00 & 300.00 & 0.200 & 0.202 & -0.002 \\ 41 & 15.00 & 6.00 & 500.00 & 0.200 & 0.202 & -0.002 \\ 42 & 15.00 & 6.00 & 600.00 & 0.200 & 0.202 & -0.002\end{array}$

SUM SB DIFF $=0.00019938$ 
ANALYSIS FOR VOLUMETRIC FLOW, A

$\begin{array}{rlllllr}1 & 15.00 & 3.00 & 200.00 & 9.784 & 9.020 & 0.764 \\ 2 & 15.00 & 3.00 & 300.00 & 14.062 & 12.648 & 1.413 \\ 3 & 15.00 & 3.00 & 400.00 & 14.977 & 16.077 & -1.100 \\ 4 & 15.00 & 3.00 & 500.00 & 19.226 & 19.364 & -0.137 \\ 5 & 15.00 & 3.00 & 600.00 & 22.496 & 22.542 & -0.046 \\ 6 & 15.00 & 4.00 & 100.00 & 7.876 & 9.474 & -1.599 \\ 7 & 15.00 & 4.00 & 150.00 & 11.939 & 13.285 & -1.346 \\ 8 & 15.00 & 4.00 & 200.00 & 17.050 & 16.886 & 0.164 \\ 9 & 15.00 & 4.00 & 250.00 & 20.599 & 20.338 & 0.261 \\ 10 & 15.00 & 4.00 & 300.00 & 22.966 & 23.677 & -0.711 \\ 11 & 15.00 & 5.00 & 100.00 & 12.776 & 14.272 & -1.496 \\ 12 & 15.00 & 5.00 & 150.00 & 20.059 & 20.013 & 0.046 \\ 13 & 15.00 & 5.00 & 200.00 & 26.537 & 25.437 & 1.100 \\ 14 & 15.00 & 5.00 & 250.00 & 34.011 & 30.638 & 3.373 \\ 15 & 15.00 & 5.00 & 275.00 & 37.051 & 33.172 & 3.880 \\ 16 & 15.00 & 6.00 & 50.00 & 14.176 & 11.708 & 2.469 \\ 17 & 15.00 & 6.00 & 100.00 & 19.539 & 20.866 & -1.328 \\ 18 & 15.00 & 6.00 & 125.00 & 24.450 & 25.133 & -0.683 \\ 19 & 15.00 & 6.00 & 150.00 & 29.446 & 29.254 & 0.187 \\ 20 & 15.00 & 6.00 & 170.00 & 33.860 & 32.477 & 1.383 \\ 21 & 12.25 & 3.00 & 243.00 & 13.477 & 12.477 & 0.988 \\ 22 & 12.25 & 3.00 & 250.00 & 14.285 & 12.789 & 1.496 \\ 23 & 12.25 & 3.00 & 333.33 & 18.557 & 16.255 & 2.302 \\ 24 & 12.25 & 3.00 & 400.00 & 22.739 & 18.923 & 3.815 \\ 25 & 12.25 & 3.00 & 500.00 & 28.412 & 22.792 & 5.620 \\ 26 & 12.25 & 3.00 & 550.00 & 31.617 & 24.677 & 6.939 \\ 27 & 11.50 & 3.00 & 333.33 & 17.358 & 18.836 & -0.978 \\ 28 & 15.00 & 4.00 & 200.00 & 13.256 & 16.886 & -3.629 \\ 29 & 15.00 & 4.00 & 300.00 & 19.066 & 23.677 & -4.611 \\ 30 & 15.00 & 4.00 & 366.00 & 26.351 & 27.946 & -1.595 \\ 31 & 15.00 & 4.00 & 500.00 & 35.755 & 36.248 & -0.493 \\ 32 & 15.00 & 4.00 & 600.00 & 43.917 & 42.199 & 1.719 \\ 33 & 15.00 & 5.00 & 200.00 & 20.485 & 25.437 & -4.952 \\ 34 & 15.00 & 5.00 & 300.00 & 31.302 & 35.667 & -4.365 \\ 35 & 15.00 & 5.00 & 400.00 & 43.751 & 45.335 & -1.584 \\ 36 & 15.00 & 5.00 & 500.00 & 57.158 & 54.604 & 7.5 .54 \\ 37 & 15.00 & 5.00 & 600.00 & 67.628 & 63.568 & 4.060 \\ 38 & 15.00 & 6.00 & 100.00 & 18.123 & 20.866 & -2.743 \\ 39 & 15.00 & 6.00 & 200.00 & 34.027 & 37.189 & -3.162 \\ 40 & 15.00 & 6.00 & 300.00 & 46.640 & 52.146 & -5.506 \\ 41 & 15.00 & 6.00 & 500.00 & 81.635 & 79.832 & 1.803 \\ 42 & 15.00 & 6.00 & 600.00 & 95.294 & 92.937 & 2.357\end{array}$


ANALYSIS FOR IMPELLER DISCHARGF 0

$\begin{array}{rrrrrrr}N D & T & 0 & R P M & 0 & n C & \text { DIFF } \\ & & & & & & \\ 1 & 15.00 & 3.00 & 200.00 & 3.334 & 2.960 & 0.374 \\ 2 & 15.00 & 3.00 & 300.00 & 4.742 & 4.151 & 0.591 \\ 3 & 15.00 & 3.00 & 400.00 & 4.875 & 5.276 & -0.401 \\ 4 & 15.00 & 3.00 & 500.00 & 6.110 & 6.355 & -0.245 \\ 5 & 15.00 & 3.00 & 600.00 & 7.456 & 7.398 & 0.058 \\ 6 & 15.00 & 4.00 & 100.00 & 3.258 & 4.012 & -0.754 \\ 7 & 15.00 & 4.00 & 150.00 & 5.196 & 5.625 & -0.429 \\ 8 & 15.00 & 4.00 & 200.00 & 7.115 & 7.150 & -0.034 \\ 9 & 15.00 & 4.00 & 250.00 & 8.977 & 8.612 & 0.365 \\ 10 & 15.00 & 4.00 & 300.00 & 9.817 & 10.025 & -0.209 \\ 11 & 15.00 & 5.00 & 100.00 & 7.055 & 1.073 & -1.017 \\ 12 & 15.00 & 5.00 & 150.00 & 11.127 & 11.319 & -0.193 \\ 13 & 15.00 & 5.00 & 200.00 & 14.834 & 14.389 & 0.447 \\ 14 & 15.00 & 5.00 & 250.00 & 19.039 & 17.329 & 1.710 \\ 15 & 15.00 & 5.00 & 275.00 & 20.632 & 18.762 & 1.870 \\ 16 & 15.00 & 6.00 & 50.00 & 9.958 & 7.948 & 2.010 \\ 17 & 15.00 & 6.00 & 100.00 & 13.667 & 14.166 & -0.499 \\ 18 & 15.00 & 6.00 & 125.00 & 17.116 & 17.062 & 0.053 \\ 19 & 15.00 & 6.00 & 150.00 & 19.857 & 19.863 & -0.007 \\ 20 & 15.00 & 6.00 & 170.00 & 23.465 & 27.048 & 1.416 \\ 21 & 12.25 & 3.00 & 243.00 & 4.181 & 3.371 & 0.810 \\ 22 & 12.25 & 3.00 & 250.00 & 3.915 & 3.451 & 0.464 \\ 23 & 12.25 & 3.00 & 333.33 & 5.279 & 4.387 & 0.893 \\ 24 & 12.25 & 3.00 & 400.00 & 6.175 & 5.107 & 1.068 \\ 25 & 12.25 & 3.00 & 500.00 & 8.869 & 6.151 & 2.717 \\ 26 & 12.25 & 3.00 & 550.00 & 9.809 & 6.660 & 3.149 \\ 27 & 11.50 & 3.00 & 333.33 & 4.128 & 4.260 & -0.132 \\ 28 & 15.00 & 4.00 & 200.00 & 5.400 & 7.150 & -1.750 \\ 27 & 15.00 & 4.00 & 300.00 & 7.947 & 10.025 & -2.078 \\ 30 & 15.00 & 4.00 & 366.00 & 10.490 & 11.833 & -1.344 \\ 31 & 15.00 & 4.00 & 500.00 & 14.046 & 15.348 & -1.302 \\ 32 & 15.00 & 4.00 & 600.00 & 17.511 & 17.868 & -0.357 \\ 33 & 15.00 & 5.00 & 200.00 & 11.429 & 14.388 & -2.958 \\ 34 & 15.00 & 5.00 & 300.00 & 17.038 & 20.174 & -3.136 \\ 35 & 15.00 & 5.00 & 400.00 & 23.355 & 25.642 & -2.297 \\ 36 & 15.00 & 5.00 & 500.00 & 30.951 & 30.885 & 0.066 \\ 37 & 15.00 & 5.00 & 600.00 & 36.288 & 35.955 & 0.333 \\ 38 & 15.00 & 6.00 & 100.00 & 12.681 & 14.166 & -1.485 \\ 39 & 15.00 & 6.00 & 200.00 & 23.413 & 25.247 & -1.834 \\ 40 & 15.00 & 6.00 & 300.00 & 31.644 & 35.402 & -3.758 \\ 41 & 15.00 & 6.00 & 500.00 & 53.691 & 54.198 & -0.506 \\ 42 & 15.00 & 6.00 & 600.00 & 63.094 & 63.095 & -0.001\end{array}$




\section{APPENDIX G \\ SUMMARY OF TANGENTIAL JET ANALYSIS}

This appendix gives the summary of results obtained from velocity profile data analyzed by the program FLOWANL. The listing of the program and the input data were given in Appendix F. The velocity profile summary is presented in Tables which are used in Chapter IV:

Section 4.1 of Chapter IV draws important conclusions and comments on the material given in the tables of this section. Section $4.4,4.5$ and Appendix $H$ form the basis of the tables in this section. Appendix. $H$ presents the analysis of a single velocity profile in four tables. A typical set of tables for a velocity profile are Tables $\mathrm{H}-1, \mathrm{H}-2$, $\mathrm{H}-3$, and $\mathrm{H}-4$. Seventeen items from these tables are considered to be of interest and form a single line of a table in this appendix.

In Section 4.2 it was shown that the average values of $\sigma$ the jet width parameter and a the radius of source parameter are reasonable estimates of these parameters, independent of impeller speed. The profile analysis was thus repeated with $\sigma$ and a constants, their values being given by Equations IV-9, and IV-10. The results of their analysis are presented back to back with corresponding tables where these parameters were originally free. This point will be made clear when the tables are presented. 
In Table G-1 is given a list of abbreviations that form the column headings of the tables presented in this section. These abbreviations were needed because of use of upper case letters by the $\infty$ mputer and the need to keep these headings as brief as possible. The abbreviations are in most cases the variables names used in the program FLOWANL. Each pair of tables that follow are for a constart impeller speed. The first table in the pair is with $\sigma$, and a (AA) obtained by a least square fit. The second table of the pair is with $\sigma$ and $a$ (AA) fixed by Equations $I V-9$ and $I V-10$ as noted above. Thus for example, Table G-2 and G-3 is for impeller speed at $243 \mathrm{RPM}$, and $h=3.0$ inches, $G-4$ and $G-5$ at $250 \mathrm{RPM}$ and $\mathrm{h}=6.0$ inches, etc. Tables G-2 to G-13 are for velocity profiles taken in 12.25 inch. diameter tank while Tables G-14 and G-15, the data was taken in 11.5 inch diameter tank.

\section{Summary of Cooper!s: Data}

In Tables G-16 to G-33 are summarized the result of velocity profile analysis obtained from the program COOPER. The program and input data are given in Appendix F. Tables G-16 to G-23 are for velocity profiles obtained in water, Tables G-23 to G-29 are for profiles obtained in air, and Tables G-30 to G-33 are for varying radial distance and impeller blade width. Profile measurements in air are made with a hot wire anemometer.

In Section 4.7 of Chapter IV, Figure IV-13 suggests that $\sigma$ is a constant independent of impeller diameter, impeller speed, tank diameter and fluid in the tank. The constant value of $\sigma$ was estimated as 
$12.621 \pm 0.066$. Hence the analysis was repeated with $\sigma$ kept constant at this value for Cooper's data. The result of this analysis is presented back to back with the case for $\sigma$ obtained by a least square fit. This facilitates comparison between the two different cases. Thus for example, pairs of Tables G-16 and G-17, G-18 and G-19, etc. are results of analysis of the same data. The first table in the pair G-16 is for $\sigma$ free while the second table is with $\sigma$ constant. In some of these tables a zero value is recorded for BALC and for the correlation coefficient. This occurs for example in Table G-30, Profile Number 5. This means that both these quantities coule not be calculated and were set equal to zero.

\section{Summary of Nielson's and Cutter's Data}

The summary of Nielson's data is given in Tables G-34 and G-35, while that of Cutter's data is given in Tables G-36 through G-41. In the analysis of the data the radius of source a, was selected by choosing a value for $\bar{\theta}_{y}$. The value of $\bar{\theta}_{y}$ is given under the heading ANGLE and is calculated from A-14. It is a function of $r$ and it will be noticed that it decreases with increasing $r$. The value of $\bar{\theta}_{y}$ selected for evaluating a can be found in these tables by looking up $\bar{\theta}_{\mathrm{y}}$ corresponding to requal to $\mathrm{D} / 2$. 


\section{TABLE G-1 \\ LIST OF ABBREVIATIONS USED FOR COLUMN HEADINGS \\ IN TABLES OF APPENDIX G}

NO

RADIAL ANGLE

SIGMA

A

AA

SUM SQ

Q

VR

CORR COEF

CFM IMP

CFM

$\cos (\mathrm{PH} 1)$
$=$ Profile Number

$=$ Angular position of radial plane in which velocity profile measurements are made. The angle is measured with respect to a baffle as shown in Figure III-1. (degrees)

$=\sigma$

$=A,\left(f t^{3} / \mathrm{min}\right)$

$=a,(f t$,

$=$ Sum of Squares defined by Equation IV-1

$=\vec{q}$

$=\bar{v}_{r}$

$=$ Correlation Coefficient $R$, defined by Equation IV-

= Volumetric flow at impeller periphery from Equation $\mathrm{IV}-7\left(\mathrm{ft}^{3} / \mathrm{min}\right)$

= Volumetric flow at radial distance $r$ from Equatio $\mathrm{iB}-31\left(\mathrm{ft}^{3} / \mathrm{min}\right)$

$=\cos \theta_{y}$ 


\section{TABLE G-1 CONTINUED}

ANGLE

$\mathrm{P}(4)$

UHALD

BHALF

BCALC

AVG ANGLE

NAD**3 $=\bar{\theta}_{\mathrm{y}}$ obtained from Equation A-14, (degrees)

$=z_{0},(f t)$

$=\frac{1}{2} \bar{q}_{\max },\left(f t^{3} / \mathrm{min}\right)$

$=z$, corresponding to $\bar{q}=\frac{\bar{z}}{\varepsilon} \bar{q}_{\max }$ frim Equation $\mathrm{H}-1$, (ft)

$=$ BHALF obtained by direct interpolation of the data ( $f t$ )

$=$ Arithmetic average angle of angle profile $\theta_{y}$ (degrees)

$=\mathrm{ND}^{3}(\mathrm{ft} / \mathrm{min})$

At the bottom of the tables the following headings are used:

AVERAGE

VARIANCE

DEVIATION
$=$ Arithmetic Average

$=$ Variance $s_{X}^{2}$, defined by Equation IV-

$=0.95$ confidence 1imit from t-test from 
TABLE G-2

SUMMARY OF VELOCITY PROFILE ANALYSIS AT 243 RPM, IN

A 12.25 INCH DIAMETER TANK

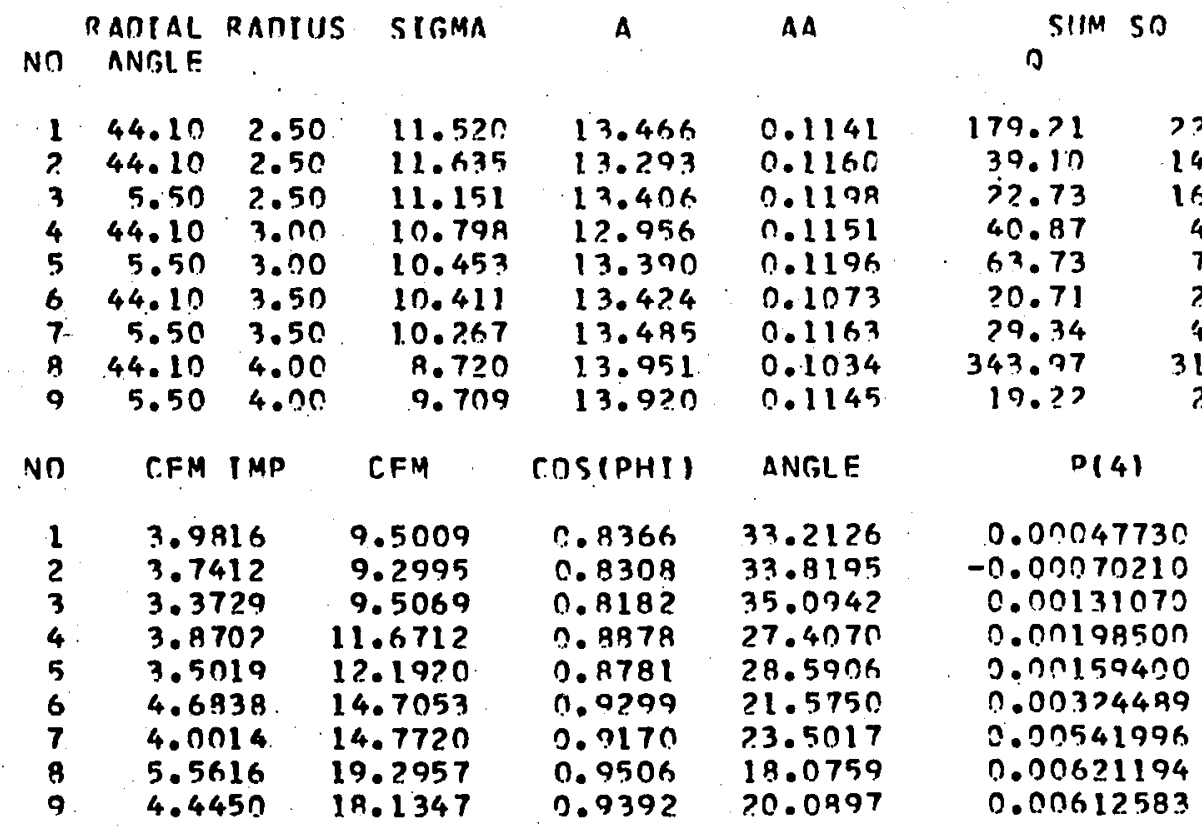

CARR CIFF

n VD

$0.9895 \quad 0.9851$

$0.9079 \cap .2025$

ก. 9989 n. nan?

0.09610 .974 ?

0.99380 .7920

0.99590 .994 ?

0.9952 n. $97 ? ?$

$0.01710 .921 \%$

$9.9932 \quad 2.9003$ 
TABLE G-2 CONTINUED

\begin{tabular}{|c|c|c|c|c|c|}
\hline NO & UHALF & BHALF & ACALC & AVG ANGLE & ND**3 \\
\hline $\begin{array}{l}1 \\
2 \\
3 \\
4 \\
5 \\
6 \\
7 \\
8 \\
9\end{array}$ & $\begin{array}{l}59.96 \\
59.69 \\
59.39 \\
45.19 \\
45.20 \\
38.50 \\
38.68 \\
31.69 \\
33.57\end{array}$ & $\begin{array}{l}0.0319 \\
0.0316 \\
0.0329 \\
0.0408 \\
0.0422 \\
0.0494 \\
0.0501 \\
0.0674 \\
0.0605\end{array}$ & $\begin{array}{r}-0.0326 \\
-0.031 ? \\
0.0336 \\
0.0399 \\
-0.0446 \\
0.0471 \\
-0.0523 \\
0.0895 \\
-0.0646\end{array}$ & $\begin{array}{l}36.07 \\
37.19 \\
38.45 \\
29.52 \\
29.89 \\
21.68 \\
24.79 \\
18.62 \\
70.34\end{array}$ & $\begin{array}{l}3.797 \\
3.797 \\
3.797 \\
3.797 \\
3.797 \\
3.797 \\
3.797 \\
3.797 \\
3.797\end{array}$ \\
\hline & & AVERAGE & \multicolumn{2}{|c|}{ VAR I ANCF } & NEVIATION \\
\hline & & $\begin{array}{r}13.4770 \\
10.518 ? \\
0.1140\end{array}$ & \multicolumn{2}{|c|}{$\begin{array}{r}0.0977 \\
0.8770 \\
0.00502014\end{array}$} & $\begin{array}{l}0.234 \\
0.703 \\
0.00415\end{array}$ \\
\hline & & FACTO & \multicolumn{2}{|l|}{0.50} & \\
\hline
\end{tabular}


TABLE G-3

SUMMARY OF VELOCITY PROFILE ANALYSTS AT 243 RPM $\sigma$ AND A CONSTANT,

IN A 12.25 INCH DIAMETER TANK

\begin{tabular}{|c|c|c|c|c|c|c|c|c|c|}
\hline NO & $\begin{array}{l}\text { PANIAL. } \\
\text { ANFI E }\end{array}$ & PADIUS & SIRMA & A & AA & $0^{S 1 / M}$ & VP. & $\begin{array}{c}\text { CORP } \\
n\end{array}$ & VR \\
\hline $\begin{array}{l}1 \\
? \\
3 \\
4 \\
5 \\
6 \\
7 \\
8 \\
9\end{array}$ & $\begin{array}{r}44.10 \\
44.10 \\
5.50 \\
44.10 \\
5.50 \\
44.10 \\
5.50 \\
44.10 \\
5.50\end{array}$ & $\begin{array}{l}2.50 \\
2.50 \\
2.50 \\
3.00 \\
3.00 \\
3.50 \\
3.50 \\
4.00 \\
4.00\end{array}$ & $\begin{array}{l}11.192 \\
11.19 ? \\
11.192 \\
11.192 \\
11.192 \\
11.19 ? \\
11.19 ? \\
11.197 \\
11.192\end{array}$ & $\begin{array}{l}13.483 \\
13.361 \\
13.539 \\
12.919 \\
13.377 \\
13.204 \\
13.355 \\
13.275 \\
13.560\end{array}$ & $\begin{array}{l}0.1147 \\
0.1147 \\
0.1147 \\
0.1147 \\
0.1147 \\
0.1147 \\
0.1147 \\
0.11147 \\
0.11147\end{array}$ & $\begin{array}{r}194.14 \\
67.51 \\
23.03 \\
57.12 \\
124.94 \\
57.65 \\
94.10 \\
652.03 \\
119.73\end{array}$ & $\begin{array}{r}299.07 \\
259.28 \\
194.73 \\
35.73 \\
51.96 \\
53.55 \\
50.31 \\
551.76 \\
95.44\end{array}$ & $\begin{array}{l}0.9896 \\
n .9963 \\
0.9989 \\
0.99411 \\
0.9970 \\
0.9893 \\
0.9946 \\
0.8359 \\
0.9569\end{array}$ & $\begin{array}{l}0.9470 \\
0.9931 \\
0.9997 \\
7.9941 \\
0.9937 \\
0.9979 \\
0.9973 \\
0.9591 \\
0.9693\end{array}$ \\
\hline NO & CFM & IMD & C.FM & Cns(PHI) & ANBLE & \multicolumn{2}{|c|}{$D(4)$} & & \\
\hline $\begin{array}{l}1 \\
2 \\
3 \\
4 \\
5 \\
6 \\
7 \\
8 \\
9\end{array}$ & $\begin{array}{l}3.99 \\
3.95 \\
4.00 \\
3.97 \\
3.96 \\
3.91 \\
3.95 \\
3.97 \\
4.01\end{array}$ & $\begin{array}{l}915 \\
554 \\
080 \\
244 \\
600 \\
088 \\
5.35 \\
? 98 \\
143\end{array}$ & $\begin{array}{l}9.6407 \\
9.5535 \\
9.6804 \\
1.4357 \\
1.8411 \\
3.8710 \\
14.0296 \\
16.1061 \\
16.4524\end{array}$ & $\begin{array}{l}0.8348 \\
0.8348 \\
0.8348 \\
0.9985 \\
0.8985 \\
0.9194 \\
0.9194 \\
0.9389 \\
0.9389\end{array}$ & $\begin{array}{l}33.4053 \\
33.4053 \\
33.4053 \\
27.3096 \\
27.3096 \\
23.1573 \\
23.1573 \\
20.1769 \\
20.1269\end{array}$ & \multicolumn{2}{|c|}{$\begin{array}{r}0.0 n n 46300 \\
-0.0 n n 73120 \\
0.00131400 \\
0.0 n 195000 \\
0.0015700 n \\
0.00309000 \\
0.0 n 540906 \\
0.00549493 \\
0.0 n 596895\end{array}$} & & \\
\hline
\end{tabular}


TABLE G-3 CONTINUED

\begin{tabular}{|c|c|c|c|c|c|}
\hline No & IJHALF & BHALF & AC.Al. C. & AVG ANSLLF & $N \cap * * 3$ \\
\hline $\begin{array}{l}1 \\
2 \\
3 \\
4 \\
5 \\
6 \\
7 \\
8 \\
9\end{array}$ & $\begin{array}{l}50.24 \\
59.71 \\
59.49 \\
45.85 \\
47.49 \\
39.49 \\
39.94 \\
34.37 \\
35.11\end{array}$ & $\begin{array}{l}0.0328 \\
0.0379 \\
0.0328 \\
0.0394 \\
0.0394 \\
0.0459 \\
0.0459 \\
0.0525 \\
0.0525\end{array}$ & $\begin{array}{r}-0.0331 \\
-0.0316 \\
0.0336 \\
0.0396 \\
-0.0434 \\
0.0464 \\
-0.0510 \\
0.0528 \\
-0.0621\end{array}$ & $\begin{array}{l}36.07 \\
37.18 \\
38.45 \\
28.57 \\
29.80 \\
21.69 \\
24.20 \\
18.63 \\
20.34\end{array}$ & $\begin{array}{l}3.707 \\
3.797 \\
3.797 \\
3.707 \\
3.797 \\
3.797 \\
3.797 \\
3.797 \\
3.797\end{array}$ \\
\hline & & AVFRAGF & \multicolumn{2}{|c|}{ VAR I ANICF } & DFVIATINI \\
\hline 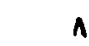 & & 13.3416 & \multicolumn{2}{|c|}{0.0389} & $n .15 ?$ \\
\hline
\end{tabular}


TABLE G-4

SUMMARY OF VELOCITY PROFILE ANALYSIS AT 250 RPM IN A 12.25 INCH DIAMETER TANK

\begin{tabular}{|c|c|c|c|c|c|c|c|c|c|}
\hline NO & $\begin{array}{l}\text { RADIAL } \\
\text { ANGLF }\end{array}$ & RADIL & SIGMA & A & AA & $0^{\text {SUM }}$ & VR & $\begin{array}{c}\text { CORRR } \\
n\end{array}$ & IFF \\
\hline $\begin{array}{l}1 \\
? \\
3 \\
4\end{array}$ & $\begin{array}{r}44.10 \\
5.50 \\
44.10 \\
5.50\end{array}$ & $\begin{array}{l}2.5 \\
2.5 \\
3.0 \\
3.0\end{array}$ & $\begin{array}{r}12.133 \\
10.525 \\
9.312 \\
9.251\end{array}$ & $\begin{array}{l}13.639 \\
14.600 \\
14.590 \\
14.310\end{array}$ & $\begin{array}{l}0.1177 \\
0.1 ? 1 ? \\
0.1120 \\
0.124 ?\end{array}$ & $\begin{array}{r}783.65 \\
1684.47 \\
7 ? .46 \\
17 ? .94\end{array}$ & $\begin{array}{r}347.16 \\
137.71 \\
71.78 \\
137.0 ?\end{array}$ & $\begin{array}{l}0.9863 \\
0.9455 \\
0.9814 \\
0.0773\end{array}$ & $\begin{array}{l}0.2904 \\
0.0524 \\
0.0790 \\
0.0772\end{array}$ \\
\hline NO & C.FM & IMP & CFM & COS(PHI) & ANGLE & \multicolumn{2}{|c|}{$P(4)$} & & \\
\hline $\begin{array}{l}1 \\
2 \\
3 \\
4\end{array}$ & $\begin{array}{l}3.5 \\
3.50 \\
5.28 \\
2.4\end{array}$ & $\begin{array}{l}377 \\
963 \\
075 \\
513\end{array}$ & $\begin{array}{r}9.3114 \\
10.6265 \\
15.0174 \\
13.7689\end{array}$ & $\begin{array}{l}0.8251 \\
0.8135 \\
0.8923 \\
0.8678\end{array}$ & $\begin{array}{l}34.4734 \\
35.5601 \\
26.84 ? ? \\
29.7993\end{array}$ & \multicolumn{2}{|c|}{$\begin{array}{r}-0.00076299 \\
0.00054890 \\
-1.00362798 \\
-0.9 n>43599\end{array}$} & & \\
\hline NO & UHAI & & AHALF & BCALC. & AVG ANGLE & \multicolumn{2}{|l|}{ ND**3 } & & \\
\hline \multirow[t]{2}{*}{$\begin{array}{l}1 \\
2 \\
3 \\
4\end{array}$} & $\begin{array}{l}67 . \\
63.1 \\
44.9 \\
46 .\end{array}$ & & $\begin{array}{l}0.0303 \\
0.0349 \\
0.0530 \\
0.0476\end{array}$ & $\begin{array}{r}-0.0277 \\
-0.0295 \\
0.0826 \\
0.0547\end{array}$ & $\begin{array}{l}39.15 \\
42.01 \\
27.08 \\
30.55\end{array}$ & $\begin{array}{l}3.906 \\
3.906 \\
3.906 \\
3.906\end{array}$ & & & \\
\hline & & & AVERAGE & \multicolumn{2}{|c|}{ VARIANCE } & \multicolumn{2}{|c|}{ REVIATION } & & \\
\hline \multicolumn{3}{|c|}{$\begin{array}{l}\text { A } \\
\text { SIGMA } \\
\text { AA }\end{array}$} & $\begin{array}{r}14.2847 \\
10.0553 \\
0.1190\end{array}$ & \multicolumn{2}{|c|}{$\begin{array}{r}0.2034 \\
2.7412 \\
0.00002373\end{array}$} & $\begin{array}{l}0.718 \\
2.634 \\
0.00775\end{array}$ & & & \\
\hline & & $L \pi$ & & & & & & & \\
\hline
\end{tabular}


TABLE G-5

SUMMARY OF VELOCITY PROFILE ANALYSIS AT 250 RPM $\sigma$ AND A CONSTANT

\begin{tabular}{|c|c|c|c|c|c|c|c|c|c|}
\hline NO & $\begin{array}{l}\text { RANIAL } \\
\text { ANGLF }\end{array}$ & RADIII & SIGMA & A & An & $0^{511 M}$ & VP & $\begin{array}{c}\text { CORR CO } \\
a\end{array}$ & IFF \\
\hline $\begin{array}{l}1 \\
2 \\
? \\
4\end{array}$ & $\begin{array}{r}44.10 \\
5.50 \\
44.10 \\
5.50\end{array}$ & $\begin{array}{l}2.50 \\
3.50 \\
3.00 \\
3.00\end{array}$ & $\begin{array}{l}11.192 \\
11.19 ? \\
11.19 ? \\
11.19 ?\end{array}$ & $\begin{array}{l}13.795 \\
14.744 \\
13.919 \\
14.126\end{array}$ & $\begin{array}{l}0.1147 \\
0.1147 \\
0.11147 \\
0.11147\end{array}$ & $\begin{array}{r}410.55 \\
177 ? .99 \\
873.17 \\
611.03\end{array}$ & $\begin{array}{r}694.31 \\
1147.34 \\
545.45 \\
77 ? .49\end{array}$ & $\begin{array}{l}0.9801 \\
n .94 ? 6 \\
0.7661 \\
0.0191\end{array}$ & $\begin{array}{l}0.9611 \\
0.9583 \\
0.9354 \\
0.9525\end{array}$ \\
\hline No & CFM. & IMP & C. FM & COSIPHI) & ANGI.E & \multicolumn{2}{|c|}{$P(4)$} & & \\
\hline $\begin{array}{l}1 \\
? \\
3 \\
4\end{array}$ & $\begin{array}{l}4.01 \\
4.34 \\
4.09 \\
4.18\end{array}$ & $\begin{array}{l}308 \\
649 \\
09 \\
117\end{array}$ & $\begin{array}{r}0.8563 \\
10.5423 \\
12.2324 \\
17.5047\end{array}$ & $\begin{array}{l}0.9348 \\
0.8349 \\
0.9985 \\
0.9985\end{array}$ & $\begin{array}{l}3.3 .40 .53 \\
33.4053 \\
27.3096 \\
77.3096\end{array}$ & \multicolumn{2}{|c|}{$\begin{array}{r}-0.00078600 \\
0.00 \cap 75880 \\
-0.0036421 ? \\
-0.002107 ? 0\end{array}$} & & \\
\hline ND & UHAL. & & BHALF & RC, ALC & AVG ANGLE & Nก**3 & & & $\because$ \\
\hline $\begin{array}{l}1 \\
2 \\
3 \\
4\end{array}$ & $\begin{array}{l}60.5 \\
64.7 \\
49.1 \\
50.1\end{array}$ & & $\begin{array}{l}0.0328 \\
0.0328 \\
0.0394 \\
0.0394\end{array}$ & $\begin{array}{r}0.0792 \\
-0.0286 \\
0.0647 \\
0.0421\end{array}$ & $\begin{array}{l}38.15 \\
42.01 \\
27.09 \\
30.55\end{array}$ & $\begin{array}{l}3.906 \\
3.906 \\
3.906 \\
3.906\end{array}$ & & & \\
\hline & & & AVFRAGE & VAF & I ANC.E & nQFVIATIR & & & \\
\hline & $A$ & & $14.11 \mathrm{BA}$ & & 1976 & 0.707 & & & \\
\hline
\end{tabular}


TABLEE G-6

SUMAARY OF VELOCITY PRORILE ANALYSIS AT 333.3 RPM IN A 12.25 INCH DIAMETER TANK

RADIAL RADIUS SIGMA
NO ANGLF


TABLE G-7

SUMAARY OF VELOCITY PROFILE ANALYSIS AT 333.3 RPM $\sigma$ AND A CONSTANT

\begin{tabular}{|c|c|c|c|c|c|c|c|c|c|}
\hline vo & $\begin{array}{l}\text { RADIAL } \\
\text { ANSLLE }\end{array}$ & PADI & S IGMA & $A$ & AA & $0^{\text {stun }}$ & VP & $\begin{array}{r}\text { CARR } \\
0\end{array}$ & ${ }_{\text {VR }}$ \\
\hline $\begin{array}{l}1 \\
? \\
3 \\
4\end{array}$ & $\begin{array}{r}44.10 \\
5.50 \\
44.10 \\
5.50\end{array}$ & $\begin{array}{l}2.5 \\
2.5 \\
3.0 \\
3.0\end{array}$ & $\begin{array}{l}11.192 \\
11.192 \\
11.19 ? \\
11.19 ?\end{array}$ & $\begin{array}{l}19.671 \\
19.960 \\
19.247 \\
19.590\end{array}$ & $\begin{array}{l}0.1147 \\
0.1147 \\
0.1147 \\
0.1147\end{array}$ & $\begin{array}{r}1778.12 \\
2853.65 \\
125.96 \\
237.39\end{array}$ & $\begin{array}{r}1491.56 \\
2579.7 ? \\
146.19 \\
237.09\end{array}$ & $\begin{array}{l}0.7761 \\
0.9559 \\
0.9034 \\
0.9918\end{array}$ & $\begin{array}{l}0.9671 \\
n .7424 \\
i .0711 \\
0.0071\end{array}$ \\
\hline No & CFM & IMP & CFM & CDS(PHI) & ANGLE & \multicolumn{2}{|c|}{$P(4)$} & & \\
\hline $\begin{array}{l}1 \\
2 \\
3 \\
4\end{array}$ & $\begin{array}{l}5.52 \\
5.61 \\
5.41 \\
5.51\end{array}$ & $\begin{array}{l}273 \\
128 \\
217 \\
004\end{array}$ & $\begin{array}{l}13.3500 \\
13.5564 \\
16.1519 \\
16.4471\end{array}$ & $\begin{array}{l}0.8349 \\
0.8348 \\
0.8885 \\
0.8885\end{array}$ & $\begin{array}{l}33.4053 \\
33.4053 \\
27.3006 \\
27.3096\end{array}$ & \multicolumn{2}{|c|}{$\begin{array}{l}0.0 \cap 371520 \\
0.00379900 \\
0.00019410 \\
0.00273499\end{array}$} & & \\
\hline Nח & IHHAC & & BHALF & ACALC. & AVG ANRIF & \multicolumn{2}{|l|}{$N D * * 3$} & & \\
\hline $\begin{array}{l}1 \\
2 \\
3 \\
4\end{array}$ & $\begin{array}{l}82.0 \\
83.3 \\
64.7 \\
65.9\end{array}$ & & $\begin{array}{l}0.0328 \\
0.0329 \\
0.0394 \\
0.0394\end{array}$ & $\begin{array}{r}0.0286 \\
0.0299 \\
-0.039 n \\
0.0374\end{array}$ & $\begin{array}{l}38.45 \\
37.68 \\
28.38 \\
29.68\end{array}$ & \multicolumn{2}{|l|}{$\begin{array}{l}5.208 \\
5.209 \\
5.209 \\
5.2 \cap 8\end{array}$} & & \\
\hline . & & & AVERAGE & \multicolumn{2}{|c|}{ VAR I ANC.F } & \multicolumn{2}{|c|}{ DFVIATIDN } & & \\
\hline & A & & 18.6146 & & 386.3 & 0.467 & & & $\because$ \\
\hline
\end{tabular}


TABLE G-8

SUMARAR OR VELOCITY PROFILE ANALYSIS AT 400 RPM IN A 12.25 INCH DIAMETER TANK

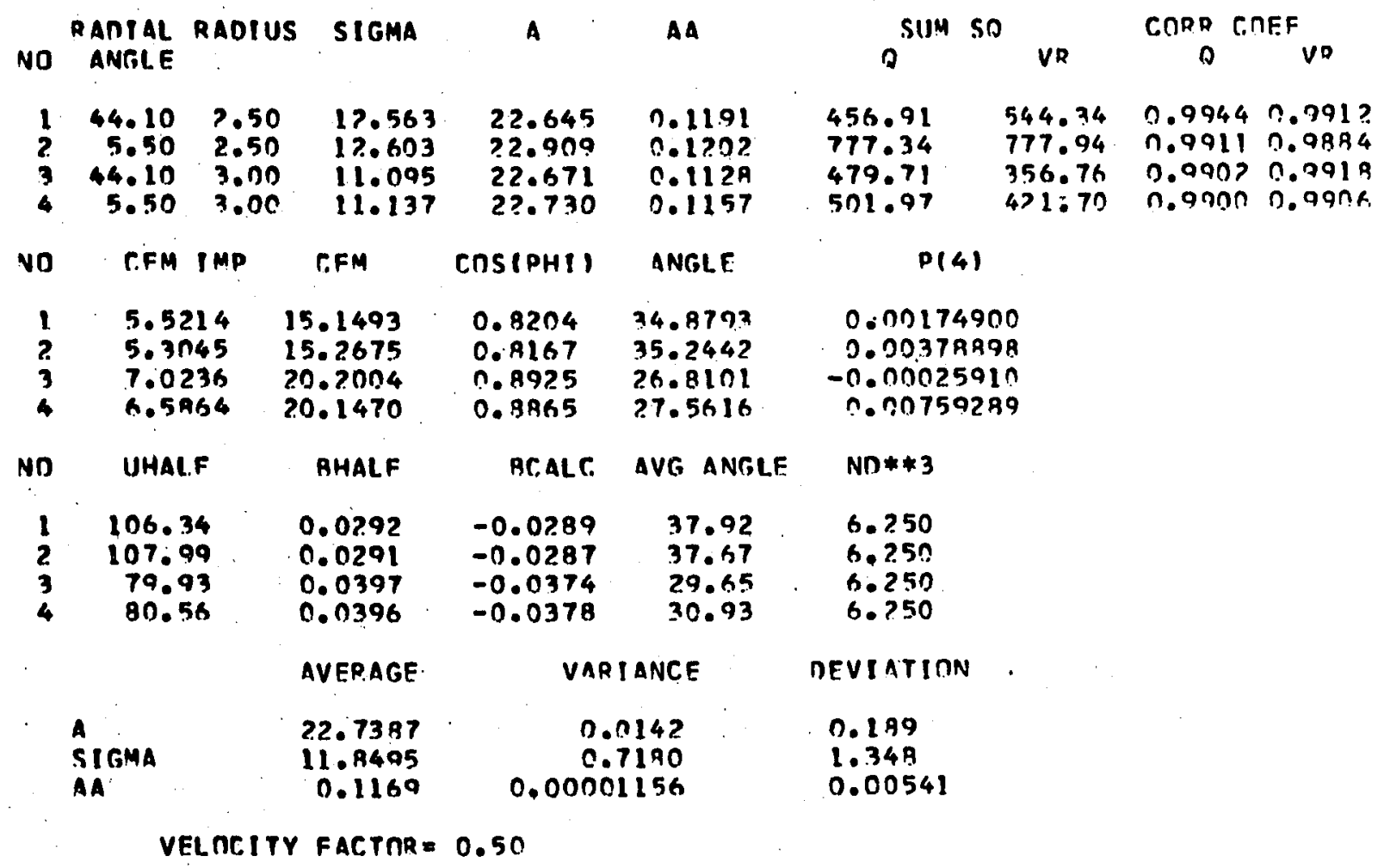


TABLE G-9

SUMAMARY OR VELOCITY PRORILB ANALYSIS AT 400 RPM $\sigma$ AND A CONSTANT

\begin{tabular}{|c|c|c|c|c|c|c|c|c|}
\hline in & $\begin{array}{l}\text { RANIAL } \\
\text { ANGI.F }\end{array}$ & RAnII & signa & A & $A n$ & $a^{\text {sum }}$ & vo & $\begin{array}{r}\text { R.OPR C.רFF } \\
D\end{array}$ \\
\hline $\begin{array}{l}1 \\
2 \\
3 \\
4\end{array}$ & $\begin{array}{r}44.11 \\
5.50 \\
4.10 \\
5.50\end{array}$ & $\begin{array}{l}2.50 \\
2.50 \\
3.00 \\
3.00\end{array}$ & $\begin{array}{l}11.197 \\
11.192 \\
11.192 \\
11.177\end{array}$ & $\begin{array}{l}22.924 \\
23.285 \\
2 ? .610 \\
22.750\end{array}$ & $\begin{array}{l}0.1147 \\
0.1147 \\
0.1147 . \\
0.11147\end{array}$ & $\begin{array}{r}1347.7 ? \\
1644.98 \\
493.41 \\
503.17\end{array}$ & $\begin{array}{l}1944.56 \\
2177.71 \\
374.84 \\
425.32\end{array}$ & 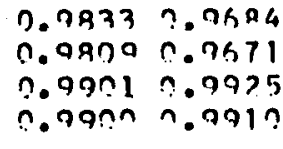 \\
\hline & C.FM & I MP & C.FM & CחS(PHI) & ANGLE & \multicolumn{2}{|c|}{$0(4)$} & \\
\hline $\begin{array}{l}1 \\
? \\
3 \\
4\end{array}$ & $\begin{array}{l}6.7 \\
6.7 \\
6.6 \\
6.7\end{array}$ & $\begin{array}{l}864 \\
931 \\
933 \\
347\end{array}$ & $\begin{array}{l}16.3909 \\
16.6487 \\
20.0140 \\
20.1380\end{array}$ & $\begin{array}{l}0.8348 \\
0.9348 \\
0.8895 \\
0.8895\end{array}$ & $\begin{array}{l}33.4053 \\
33.4053 \\
27.3096 \\
27.3096\end{array}$ & \multicolumn{2}{|c|}{$\begin{array}{r}0.00173600 \\
0.0 n 342990 \\
-0.00075000 \\
0.00759693\end{array}$} & \\
\hline 0 & IJHA & & BHALF & AC.AL.C. & AVG ANRILF & \multicolumn{2}{|l|}{$N \cap * * 3$} & \\
\hline $\begin{array}{l}1 \\
3 \\
3\end{array}$ & $\begin{array}{r}100 . \\
102 \\
80 . \\
90 .\end{array}$ & & $\begin{array}{l}0.0328 \\
0.0378 \\
0.0394 \\
0.0394\end{array}$ & $\begin{array}{r}0.0291 \\
0.0293 \\
-0.0373 \\
-0.0377\end{array}$ & $\begin{array}{l}37.92 \\
37.67 \\
39.65 \\
30.93\end{array}$ & \multicolumn{2}{|l|}{$\begin{array}{l}6.250 \\
6.250 \\
6.250 \\
6 . ? 50\end{array}$} & \\
\hline & & & AVERACEE & \multicolumn{2}{|c|}{ VARIANCT: } & \multicolumn{2}{|c|}{ OFVIATION } & \\
\hline & A & & 22.8923 & & & & & \\
\hline
\end{tabular}


TABLE G-10

SUMMARY OF VELOCITY PROFILE ANALYSIS AT 500 RPM IN A 12.25 INCH DIAMETER TANK

$\begin{array}{rrrr} & \begin{array}{r}\text { RADIAL RADIUS } \\ \text { ANGLE }\end{array} & \text { SIGMA } \\ \text { NO } & & \\ 1 & 44.10 & 2.50 & 12.187 \\ 2 & 5.50 & 2.50 & 11.939 \\ 3 & 44.10 & 2.50 & 14.318 \\ 4 & 5.50 & 2.50 & 13.460 \\ 5 & 44.10 & 3.00 & 11.165 \\ 6 & 5.50 & 3.00 & 10.846 \\ 7 & 44.10 & 3.00 & 11.265 \\ 8 & 5.50 & 3.00 & 10.940 \\ 9 & 44.10 & 3.50 & 10.270 \\ 10 & 5.50 & 3.50 & 10.486 \\ 11 & 44.10 & 4.00 & 9.646 \\ 12 & 5.50 & 4.00 & 9.844 \\ \text { NO } & \text { C.FM IMP } & \text { C.FM }\end{array}$

A

27.425
27.380
27.339
29.854
27.726
27.729
28.910
29.331
28.608
28.325
29.215
29.304

cns(PHI)
AA

$$
\begin{aligned}
& 0.1158 \\
& 0.1185 \\
& 0.1148 \\
& 0.1186 \\
& 0.1141 \\
& 0.1173 \\
& 0.1039 \\
& 0.1115 \\
& 0.1064 \\
& 0.1102 \\
& 0.0967 \\
& 0.1102
\end{aligned}
$$

$$
\text { SUM SD }
$$

م

654.33
912.57
730.47
759.04
311.34
$441.6 ?$
$5 ? 7.45$
603.73
377.70
$200.1 ?$
555.15
150.41

ANGLE

33.7755

34.6829

34.0977

34.6889

27.1446

27.9948

24.5491

26.4931

21.3866

2.2. 2064

16.8662 ?

19.2982

$$
\text { P } 4 \text { ) }
$$

$$
\begin{array}{r}
0.00070500 \\
0.01289900 \\
-0.07912266 \\
0.00371010 \\
0.00203899 \\
0.00432395 \\
0.00055100 \\
0.00434996 \\
0.00351099 \\
0.00593193 \\
0.00459896 \\
0.00967099
\end{array}
$$

CORP COFF

a VR
$0.99640 .79 ? ?$

$0.9940 \quad 0.9875$

$0.9955 \quad 0.0937$

ก.9751 ก. 0941

0.995 .70 .9226

0.99510 .7279

ก. 986a ก. จRव9

ก.9939 $0.79 ? 9$

$0.99240 .990 n$

0.99650 .794 ?

ก.9850 ก.9R6A

$0.996 ? 0.9940$ 
TABLE G-10 CONTINUED

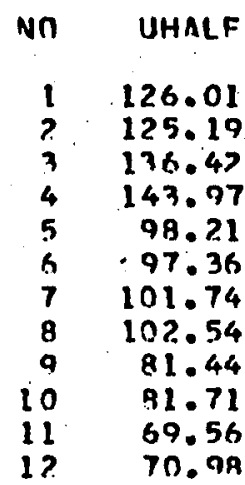

BHALF.

RCAI.C AVG, ANGLE ND**3

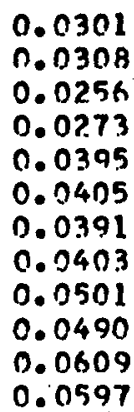

0.031
0.031

7. 913

0.0279

40.27

37.62

$-0.0775$

37.65

28.71

32.0 ?

28.7 ?

30.75

21.75

2.5 .04

19.77

?. 19

7. 913

7.813

7. 813

7. 813

7. 813

7.913

7.913

7.913

0.0485
-0.0488

$-0.0649$

7.91?

7.913

AVFRAGF.

VARI ANC. E

7. 913

\section{A \\ StGMa}

2.8.41?2

11.3672

0.7499

$1.983 C$

0.1117

0.00004487

DFVIATION

VFL RCITY FACTOR $=0.50$ 
TABLE G-11

SUMLARY OF VELOCITY PROFILE ANALYSIS AT. 500 RPM, $\sigma$ AND A CONSTANT

\begin{tabular}{|c|c|c|c|c|c|c|c|}
\hline No, & $\begin{array}{l}\text { RANIAL } \\
\text { ANGI E }\end{array}$ & RADIIIS & SIGMA & A & AA & $0^{\text {SUM }}$ & $\$ 0$ \\
\hline $\begin{array}{l}1 \\
7 \\
3 \\
4 \\
5 \\
6 \\
7 \\
9 \\
9 \\
10 \\
11 \\
1\end{array}$ & $\begin{array}{r}44.10 \\
5.50 \\
44.10 \\
5.50 \\
44.10 \\
5.50 \\
44.10 \\
5.50 \\
44.10 \\
5.50 \\
44.10 \\
5.50 .\end{array}$ & $\begin{array}{l}2.50 \\
2.50 \\
2.50 \\
2.50 \\
3.00 \\
3.00 \\
3.00 \\
3.00 \\
3.50 \\
3.50 \\
4.00 \\
4.00\end{array}$ & $\begin{array}{l}11.192 \\
11.192 \\
11.19 ? \\
11.19 ? \\
11.19 ? \\
11.19 ? \\
11.19 ? \\
11.192 \\
11.192 \\
11.19 ? \\
11.19 ? \\
11.19 ?\end{array}$ & $\begin{array}{l}77.669 \\
27.670 \\
27.428 \\
29.875 \\
27.700 \\
? 7.775 \\
? 8.580 \\
29.194 \\
28.240 \\
28.074 \\
78.470 \\
? 8.779\end{array}$ & $\begin{array}{l}0.11147 \\
0.11147 \\
0.1117 \\
0.11147 \\
0.1147 \\
0.11147 \\
0.1147 \\
0.1147 \\
0.11147 \\
0.11147 \\
0.1147 \\
0.11147\end{array}$ & $\begin{array}{r}1.569 .98 \\
1378.70 \\
7341.79 \\
5203.04 \\
311.73 \\
499.49 \\
1531.09 \\
547.89 \\
901.59 \\
443.68 \\
1518.9 ? \\
937.30\end{array}$ & $\begin{array}{r}279 \\
299 \\
741 \\
675 \\
41 \\
76 \\
129 \\
44 \\
75 \\
39 \\
177 \\
5 ?\end{array}$ \\
\hline in & CFM & IMP & CFM & r.NS(PHI) & ANGLE & $\mathrm{p} / \mathrm{l}$ & \\
\hline $\begin{array}{l}1 \\
2 \\
3 \\
4 \\
5 \\
5 \\
7 \\
8 \\
9 \\
10 \\
11 \\
12\end{array}$ & $\begin{array}{l}8.19 \\
9.17 \\
8.11 \\
8.84 \\
9.20 \\
9.2 ? \\
8.46 \\
8.64 \\
8.75 \\
9.31 \\
9.4 ? \\
8.51\end{array}$ & $\begin{array}{l}09 \\
614 \\
95 \\
41 \\
01 \\
73 \\
06 \\
75 \\
99 \\
09 \\
90 \\
95\end{array}$ & $\begin{array}{l}9.7832 \\
9.7482 \\
9.6109 \\
1.3609 \\
4.5196 \\
4.5860 \\
5.2985 \\
5.8425 \\
9.6664 \\
9.4921 \\
4.5413 \\
4.9162\end{array}$ & $\begin{array}{l}0.8348 \\
0.8348 \\
0.8348 \\
0.8348 \\
0.8885 \\
0.9895 \\
0.8385 \\
0.8985 \\
0.9194 \\
0.9194 \\
0.9389 \\
0.9389\end{array}$ & $\begin{array}{l}37.4053 \\
33.4053 \\
33.4 n 53 \\
33.4053 \\
27.3096 \\
27.3096 \\
27.3096 \\
77.3096 \\
23.1573 \\
23.1573 \\
20.1269 \\
70.1269\end{array}$ & $\begin{array}{r}0.00075 \\
0.00188 \\
-0.07906 \\
0.00376 \\
0.00205 \\
0.00435 \\
0.00054 \\
0.00434 \\
0.00354 \\
0.00607 \\
0.00545 \\
0.00956\end{array}$ & $\begin{array}{l}100 \\
100 \\
994 \\
309 \\
010 \\
? 97 \\
000 \\
007 \\
019 \\
395 \\
494 \\
598\end{array}$ \\
\hline
\end{tabular}

CIRA CTFF VP $D$ VD

$2794.750 .7917 \quad 0.98 \% ?$ $2994.59 \quad 0.09199 \quad 0.7756$ $7417.00 \quad 0.95390 .9373$ $6.055 .44 \quad 0.9727 \quad 0.7578$ $416.15 \quad 0.9953 \quad 0.0977$ $360.38 \quad 0.39452 .3754$ $1236.93 \quad 0.72690 .9296$ 4R6.R6 0.99330 .0749 $757.67 \quad 0.9939 \quad 0.7937$ $339.29 \quad 0.9972 \quad 0.99 \% 3$ $1777.50 \quad 0.96110 .7659$ 5?9.59 7.079R 0.0RG1 
TABLE G-11 CONTINUED

\begin{tabular}{|c|c|c|c|c|c|}
\hline NO & UHALF & BHALF & RCALC. & AVR ANRIF & $N \cap * * 3$ \\
\hline \multirow[t]{2}{*}{$\begin{array}{r}1 \\
? \\
2 \\
4 \\
5 \\
6 \\
7 \\
8 \\
9 \\
10 \\
11 \\
12\end{array}$} & $\begin{array}{r}121.57 \\
121.36 \\
120.51 \\
131.27 \\
98.31 \\
98.58 \\
101.43 \\
103.61 \\
84.45 \\
93.96 \\
73.72 \\
74.5 ?\end{array}$ & $\begin{array}{l}0.0328 \\
0.0328 \\
0.0328 \\
0.0328 \\
0.0394 \\
0.0394 \\
0.0394 \\
0.0394 \\
0.0459 \\
0.0459 \\
0.0525 \\
0.0525\end{array}$ & $\begin{array}{r}0.0322 \\
0.0319 \\
-0.0794 \\
0.0395 \\
0.0396 \\
-0.0390 \\
0.0397 \\
0.0394 \\
0.0471 \\
-0.0475 \\
0.0539 \\
-0.0590\end{array}$ & $\begin{array}{l}38.34 \\
49.97 \\
37.62 \\
37.65 \\
29.71 \\
3 ? .0 n \\
28.77 \\
30.75 \\
21.95 \\
25.04 \\
18.77 \\
21.39\end{array}$ & $\begin{array}{l}7.813 \\
7.819 \\
7.813 \\
7.813 \\
7.813 \\
7.913 \\
7.813 \\
7.913 \\
7.813 \\
7.913 \\
7.813 \\
7.813\end{array}$ \\
\hline & & AVERACF & \multicolumn{2}{|c|}{ VAP I ANCF } & DFVIATINN \\
\hline & & 28.2836 & \multicolumn{2}{|c|}{0.5398} & 0.466 \\
\hline
\end{tabular}


TABLE G-12

SUMMARY OF THE VBLOCITY PROFILE ANALYSIS AT 550 RPM IN A 12.25 INCH DIAMETER TANK

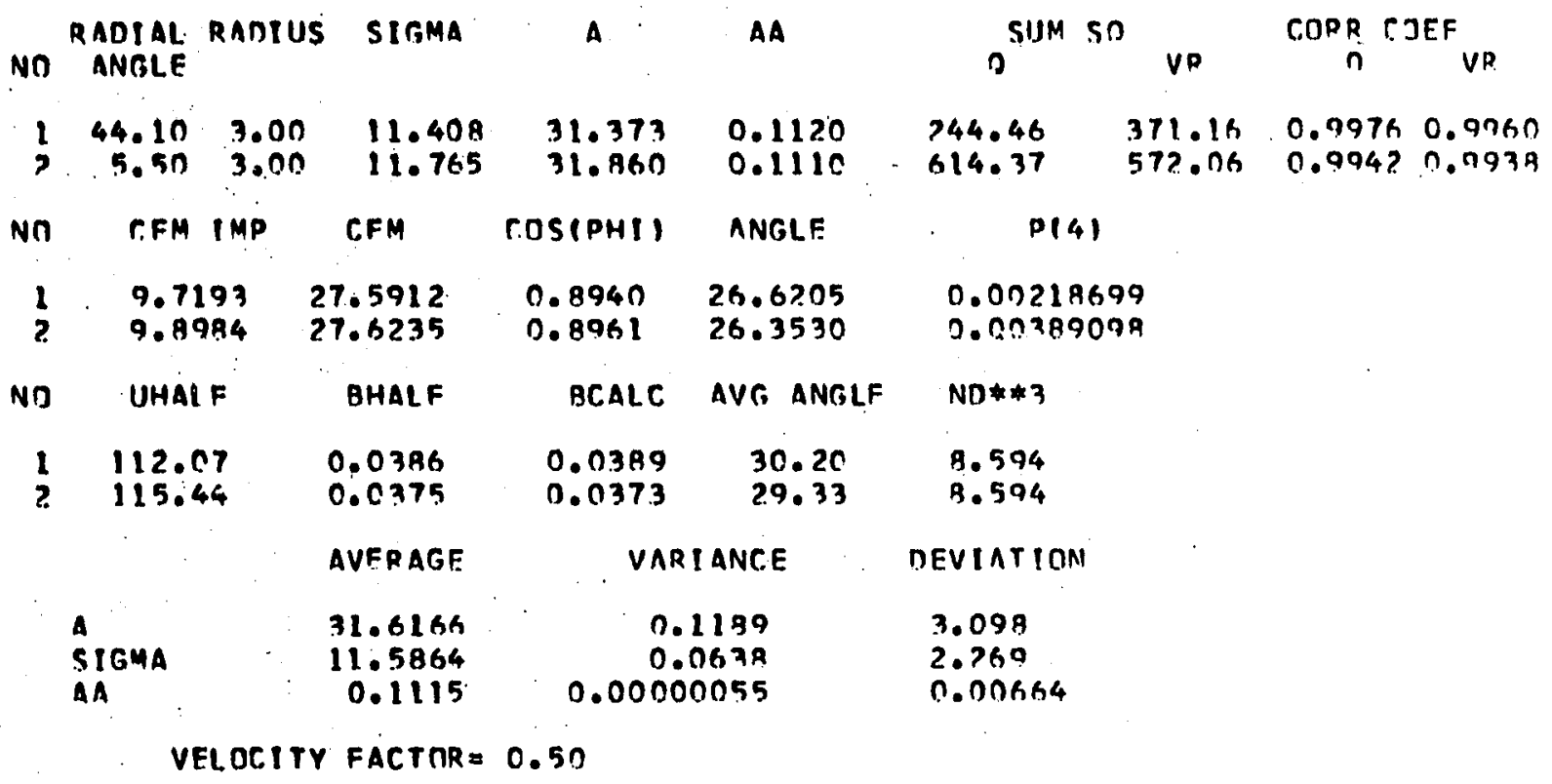


TABLE G-13

SUMMARY OF VBLOCITY PROFILE ANALYSIS AT 550 RPM, $\sigma$ AND A CONSTANT

RADIAL DANIIS SIGMA
NO ANFIF


TABLE G-14

SUMMARY OF VELOCITY PROFILE ANALYSIS AT 333.3 RPM IN A 11.5 INCH DIAMETER TANK

\begin{tabular}{|c|c|c|c|c|c|c|c|c|c|}
\hline No & $\begin{array}{l}\text { RADIAL. } \\
\text { ANGLF }\end{array}$ & RADI & S I GMA & A & AA & $0^{\text {SUM S }}$ & VR & $\begin{array}{c}\text { CORP } C \\
0\end{array}$ & ${ }_{\text {UEF }}$ \\
\hline $\begin{array}{l}1 \\
? \\
3 \\
4\end{array}$ & $\begin{array}{r}44.10 \\
5.50 \\
44.10 \\
5.50\end{array}$ & $\begin{array}{l}2.5 \\
2.5 \\
3.5 \\
3.0\end{array}$ & $\begin{array}{r}11.823 \\
10.810 \\
10.476 \\
9.712\end{array}$ & $\begin{array}{l}18.210 \\
18.080 \\
17.278 \\
17.866\end{array}$ & $\begin{array}{l}0.1201 \\
0.1217 \\
0.1221 \\
0.1223\end{array}$ & $\begin{array}{r}225.89 \\
360.08 \\
61.63 \\
32.9 .94\end{array}$ & $\begin{array}{r}573.10 \\
596.46 \\
90.46 \\
299.59\end{array}$ & $\begin{array}{l}0.0937 \\
0.9915 \\
0.995 ? \\
0.9779\end{array}$ & $\begin{array}{l}0.9704 \\
0.0837 \\
0.7921 \\
0.7797\end{array}$ \\
\hline Nn & CFM & $I M P$ & $C F M$ & COS $(P H I)$ & ANGLE & \multicolumn{2}{|l|}{$P(4)$} & & \\
\hline $\begin{array}{l}1 \\
2 \\
3 \\
4\end{array}$ & $\begin{array}{l}4.37 \\
4.12 \\
3.87 \\
4.08\end{array}$ & $\begin{array}{l}724 \\
207 \\
776 \\
891\end{array}$ & $\begin{array}{l}12.5321 \\
12.9693 \\
15.7125 \\
16.8199\end{array}$ & $\begin{array}{l}0.8170 \\
0.8116 \\
0.9725 \\
0.8721\end{array}$ & $\begin{array}{l}35.2152 \\
35.7503 \\
29.2449 \\
29.2917\end{array}$ & \multicolumn{2}{|c|}{$\begin{array}{l}-0.00373699 \\
-0.09131700 \\
-0.00004790 \\
-0.01963706\end{array}$} & & \\
\hline NO & WHAL & & BHALF & BCALC & AVG ANGLE & ND* *3 & & & \\
\hline $\begin{array}{l}1 \\
2 \\
3 \\
4\end{array}$ & $\begin{array}{l}83.1 \\
79.1 \\
59.1 \\
59.1\end{array}$ & & $\begin{array}{l}0.0311 \\
0.0340 \\
0.0423 \\
0.0454\end{array}$ & $\begin{array}{r}-0.0313 \\
0.0327 \\
0.0440 \\
0.0411\end{array}$ & $\begin{array}{l}40.42 \\
40.95 \\
30.53 \\
30.48\end{array}$ & $\begin{array}{l}5.208 \\
5.203 \\
5.208 \\
5.208\end{array}$ & & & \\
\hline & & & AVERAGE & \multicolumn{2}{|c|}{ VAR I ANCE } & DEVIATION & . & & \\
\hline$:$ & $\begin{array}{l}\text { SIGMA } \\
\text { SA }\end{array}$ & & $\begin{array}{r}17.8584 \\
10.6927 \\
0.1216\end{array}$ & $\begin{array}{r}0 \\
0.000\end{array}$ & $\begin{array}{l}6999 \\
750 \\
1098\end{array}$ & $\begin{array}{l}0.656 \\
1.401 \\
0.00159\end{array}$ & & & \\
\hline
\end{tabular}


TABLE G-15

SUMMARY OF VELOCITY PROFILE ANALYSIS AT 333.3 RPM, $\sigma$ AND A CONSTANT IN A 11.5 INCH DIAMETER TANK

\begin{tabular}{|c|c|c|c|c|c|c|c|c|c|}
\hline NO & RADIAL & RADIUS & SIGMA & A & $A A$ & SUN & So & \multicolumn{2}{|c|}{$\begin{array}{rr}\text { CARR } & \text { CIEF } \\
0 & \text { VQ }\end{array}$} \\
\hline $\begin{array}{l}1 \\
2 \\
3 \\
4\end{array}$ & $\begin{array}{r}44.10 \\
5.50 \\
44.10 \\
5.50\end{array}$ & $\begin{array}{l}2.5 \\
2.5 \\
3.0 \\
3.0\end{array}$ & $\begin{array}{l}11.192 \\
11.192 \\
11.192 \\
11.19 ?\end{array}$ & $\begin{array}{l}18.220 \\
18.053 \\
17.137 \\
17.576\end{array}$ & $\begin{array}{l}0.1216 \\
0.1216 \\
0.1216 \\
0.1216\end{array}$ & $\begin{array}{l}334.2 .4 \\
409.49 \\
150.27 \\
731.47\end{array}$ & $\begin{array}{r}1090.77 \\
397.88 \\
51.68 \\
301.44\end{array}$ & $\begin{array}{l}0.9907 \\
0.9903 \\
0.9992 \\
0.9500\end{array}$ & $\begin{array}{l}0.9663 \\
0.9995 \\
0.9955 \\
0.9779\end{array}$ \\
\hline No & C.FM & IMP & CFM & $\cos (\mathrm{PHI})$ & ANGLE & \multicolumn{2}{|c|}{$P(4)$} & & \\
\hline $\begin{array}{l}1 \\
? \\
3 \\
4\end{array}$ & \multicolumn{2}{|c|}{$\begin{array}{l}4.1174 \\
4.0798 \\
3.8728 \\
3.9720\end{array}$} & $\begin{array}{l}12.8480 \\
12.7306 \\
15.0429 \\
15.42 .83\end{array}$ & $\begin{array}{l}0.8120 \\
0.8120 \\
0.8737 \\
0.8737\end{array}$ & $\begin{array}{l}35.7097 \\
35.7097 \\
29.104 ? \\
29.1042\end{array}$ & \multicolumn{2}{|c|}{$\begin{array}{l}-0.00326300 \\
-0.00133920 \\
-0.0000756 n \\
-0.00813694\end{array}$} & & \\
\hline NO & \multicolumn{2}{|c|}{ IJHALF } & BHALF & BC.ALC & AVG ANGLE & \multicolumn{2}{|l|}{$N D * * 3$} & & \\
\hline $\begin{array}{l}1 \\
? \\
3 \\
4\end{array}$ & \multicolumn{2}{|c|}{$\begin{array}{l}81.17 \\
80.43 \\
61.34 \\
62.91\end{array}$} & $\begin{array}{l}0.0328 \\
0.0328 \\
0.0394 \\
0.0394\end{array}$ & $\begin{array}{r}-0.0320 \\
0.0323 \\
-0.0391 \\
0.0383\end{array}$ & $\begin{array}{l}40.47 \\
40.95 \\
30.53 \\
30.48\end{array}$ & $\begin{array}{l}5.208 \\
5.209 \\
5.208 \\
5.208\end{array}$ & & & \\
\hline & & & AVFRAGE & \multicolumn{2}{|c|}{ VAR I ANC.F } & \multicolumn{2}{|c|}{ DEVIATION } & & \\
\hline & $\mathbf{A}$ & & 7468 & & 239.4 & 0.778 & & & \\
\hline
\end{tabular}


TABLE G-16

ANALYSIS OF COOPER'S DATA IN WATER FOR A 3.0 INCH DIAMETER IMPELLER

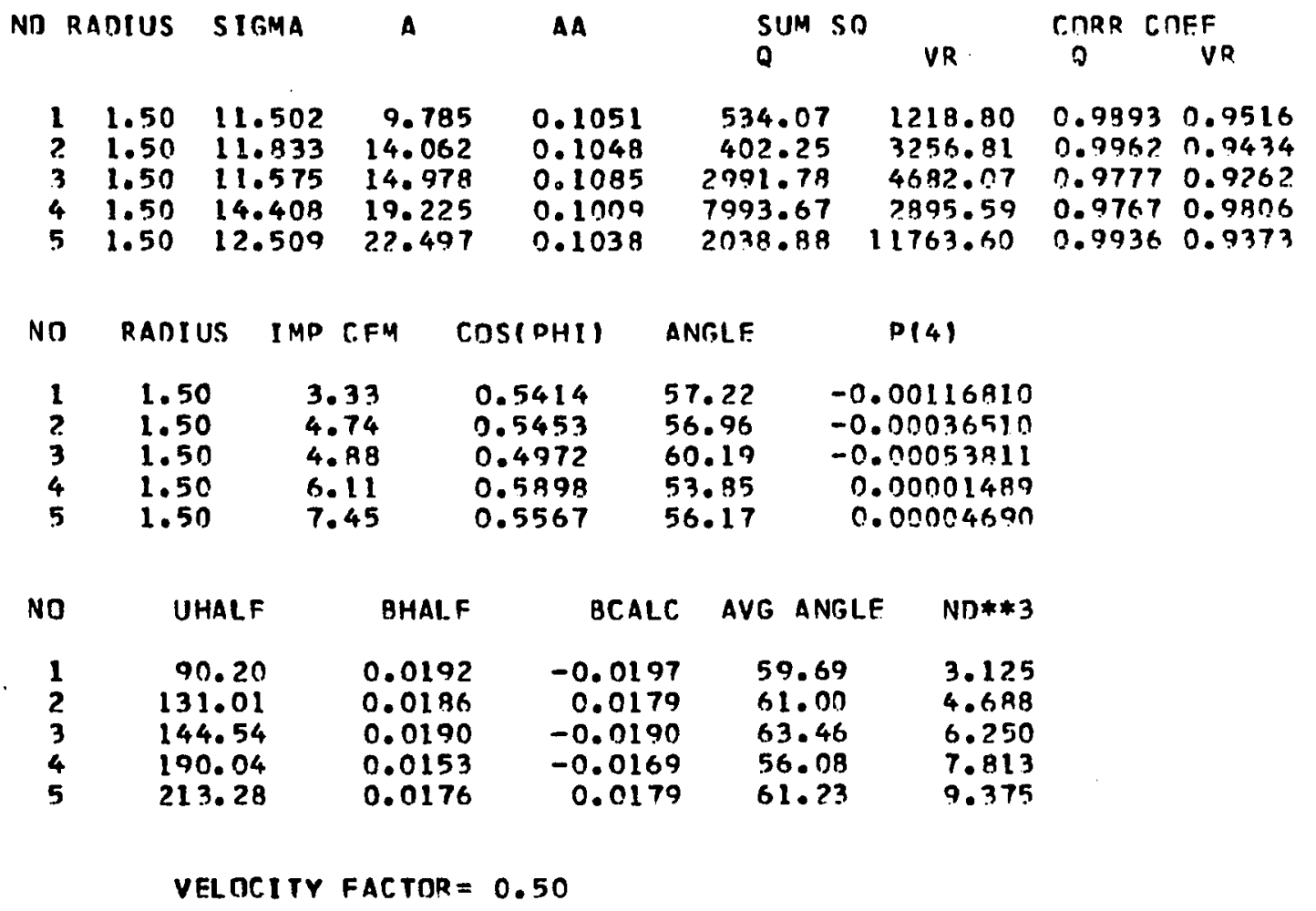


TABLE G-17

ANALYSIS OF COOPER'S DATA FOR A 3.0 INCH DIAMETER IMPELLER IN WATER, $\sigma$ CONSTANT

$\begin{array}{ccccc}\text { NO RADIUS } & \text { SIGMA } & \text { A } & \text { AA } \\ & & & & \\ 1 & 1.50 & 12.621 & 9.714 & 0.1051 \\ 2 & 1.50 & 12.621 & 13.999 & 0.1048 \\ 3 & 1.50 & 12.621 & 14.881 & 0.1085 \\ 4 & 1.50 & 12.621 & 19.272 & 0.1009 \\ 5 & 1.50 & 12.621 & 2.485 & 0.1038\end{array}$

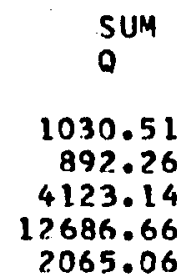

SUM SO

VR

CORRR COFF
0 $\quad$ VR

$\begin{array}{cccc}\text { NO } & \text { RADIUS } & \text { IMP CFM } & \text { C.OSIPHII } \\ 1 & 1.50 & 3.16 & 0.5414 \\ 2 & 1.50 & 4.57 & 0.5453 \\ 3 & 1.50 & 4.64 & 0.4972 \\ 4 & 1.50 & 6.54 & 0.5898 \\ 5 & 1.50 & 7.42 & 0.5567\end{array}$

ANGLE P(4)

$57.22 \quad-0.00109310$

$56.96 \quad-0.00034411$

$60.19 \quad-0.00047911$

$53.85-0.00000211$

$56.17 \quad 0.00004690$

$\begin{array}{rrrrrr}\text { NO } & \text { UHALF } & \text { BHALF } & \text { BCALC } & \text { AVG ANGLE } & \text { NO**3 } \\ & & & & & \\ 1 & 93.80 & 0.0175 & -0.0192 & 59.69 & 3.125 \\ 2 & 134.70 & 0.0175 & 0.0173 & 61.00 & 4.688 \\ 3 & 149.95 & 0.0175 & -0.0186 & 63.46 & 6.250 \\ 4 & 178.29 & 0.0175 & 0.0171 & 56.08 & 7.813 \\ 5 & 214.12 & 0.0175 & 0.0179 & 61.23 & 9.375\end{array}$

VEL OCITY FACTOR $=0.50$ 
TABLE G-18

ANALYSIS OF COOPER'S DATA IN WATER FOR A 4.0 INCH DIAMETER IMPELLER

\begin{tabular}{|c|c|c|c|c|c|c|c|c|c|c|c|}
\hline NO $R$ & ADIUS & SIGMA & \multicolumn{2}{|l|}{$A$} & \multicolumn{2}{|l|}{ AA } & \multicolumn{2}{|c|}{ SUM So } & VR & \multicolumn{2}{|c|}{$\begin{array}{cc}\text { CORR } & \text { COEF } \\
Q & \text { VR }\end{array}$} \\
\hline $\begin{array}{l}1 \\
2 \\
3 \\
4 \\
5\end{array}$ & $\begin{array}{l}2.00 \\
2.00 \\
2.00 \\
2.00 \\
2.00\end{array}$ & $\begin{array}{l}14.106 \\
12.095 \\
13.611 \\
11.317 \\
11.680\end{array}$ & \multicolumn{2}{|c|}{$\begin{array}{r}7.876 \\
11.040 \\
17.052 \\
20.599 \\
22.967\end{array}$} & \multicolumn{2}{|c|}{$\begin{array}{l}0.1392 \\
0.1421 \\
0.140 ? \\
0.1453 \\
0.1456\end{array}$} & \multicolumn{2}{|c|}{$\begin{array}{r}153.27 \\
90.20 \\
621.11 \\
514.96 \\
209.50\end{array}$} & $\begin{array}{r}212.73 \\
705.60 \\
1507.36 \\
2643.61 \\
7.969 .09\end{array}$ & \multirow[t]{2}{*}{$\begin{array}{l}0.9930 \\
0.9974 \\
0.9935 \\
0.9944 \\
0.9983\end{array}$} & \multirow[t]{2}{*}{$\begin{array}{l}0.9757 \\
0.9538 \\
0.9631 \\
0.9374 \\
0.9443\end{array}$} \\
\hline NO & RANIUS & IMP & CFM & $\mathrm{cn}$ & (PHI) & & GLE & & $P(4)$ & & \\
\hline $\begin{array}{l}1 \\
2 \\
3 \\
4 \\
5\end{array}$ & $\begin{array}{l}2.00 \\
2.00 \\
2.00 \\
2.00 \\
2.00\end{array}$ & & $\begin{array}{l}26 \\
20 \\
12 \\
98 \\
82\end{array}$ & $\begin{array}{l}0 \\
0 \\
0 \\
0 \\
0\end{array}$ & $\begin{array}{l}5503 \\
5224 \\
5407 \\
4898 \\
4866\end{array}$ & & $\begin{array}{l}61 \\
51 \\
27 \\
67 \\
88\end{array}$ & $\begin{array}{l}-0 . \\
-0 . \\
-0 . \\
0 . \\
-0 .\end{array}$ & $\begin{array}{l}00044112 \\
00064011 \\
00070011 \\
00063988 \\
00033811\end{array}$ & & \\
\hline NO & UHAL & & BHA & LF & BC & & AVG & ANGLE & $N D * * 3$ & & \\
\hline $\begin{array}{l}1 \\
2 \\
3 \\
4 \\
5\end{array}$ & $\begin{array}{r}59.8 \\
86.1 \\
128.3 \\
148.5 \\
168.7\end{array}$ & & $\begin{array}{l}0.02 \\
0.02 \\
0.02 \\
0.02 \\
0.02\end{array}$ & $\begin{array}{l}08 \\
43 \\
16 \\
60 \\
52\end{array}$ & $\begin{array}{r}0.0 \\
0.0 \\
0.0 \\
-0.0 \\
0.0\end{array}$ & $\begin{array}{l}22 \\
47 \\
23 \\
50 \\
53\end{array}$ & $\begin{array}{l}60 \\
61 \\
61 \\
63 \\
62\end{array}$ & $\begin{array}{l}.67 \\
11 \\
.56 \\
.11 \\
.67\end{array}$ & $\begin{array}{r}3.704 \\
5.556 \\
7.407 \\
9.259 \\
11.111\end{array}$ & & \\
\hline
\end{tabular}


TABLE G-19

ANALYSIS OF COOPER'S DATA FOR A 4.0 INCH DIAMETER IMPELLER IN WATER, $\sigma$ CONSTANT

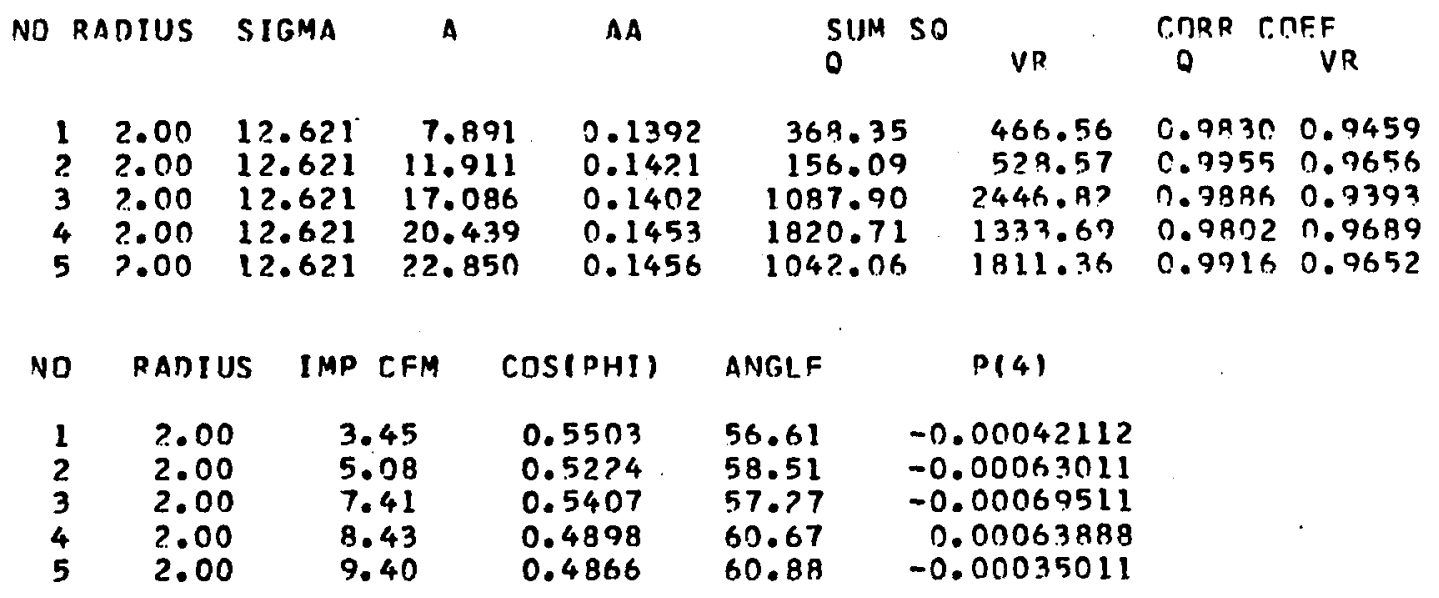

$\begin{array}{rrrrrr}\text { NO } & \text { UHALF } & \text { BHALF } & \text { BCALC } & \text { AVG ANGLF } & \text { N } \# \# 3 \\ & & & & & \\ 1 & 56.68 & 0.0233 & 0.0234 & 60.67 & 3.704 \\ 2 & 87.82 & 0.0233 & -0.0249 & 61.11 & 5.556 \\ 3 & 123.83 & 0.0233 & 0.0229 & 61.56 & 7.407 \\ 4 & 155.62 & 0.0233 & 0.0251 & 63.11 & 9.259 \\ 5 & 174.56 & 0.0233 & -0.0244 & 62.67 & 11.111\end{array}$

VELOCITY FACTDR $=0.50$ 
TABLE $\mathbf{G - 2 0}$

ANALYSIS OF COOPER'S DATA IN WATER FOR A 5.0 INCH DIAMETER IMPELLER

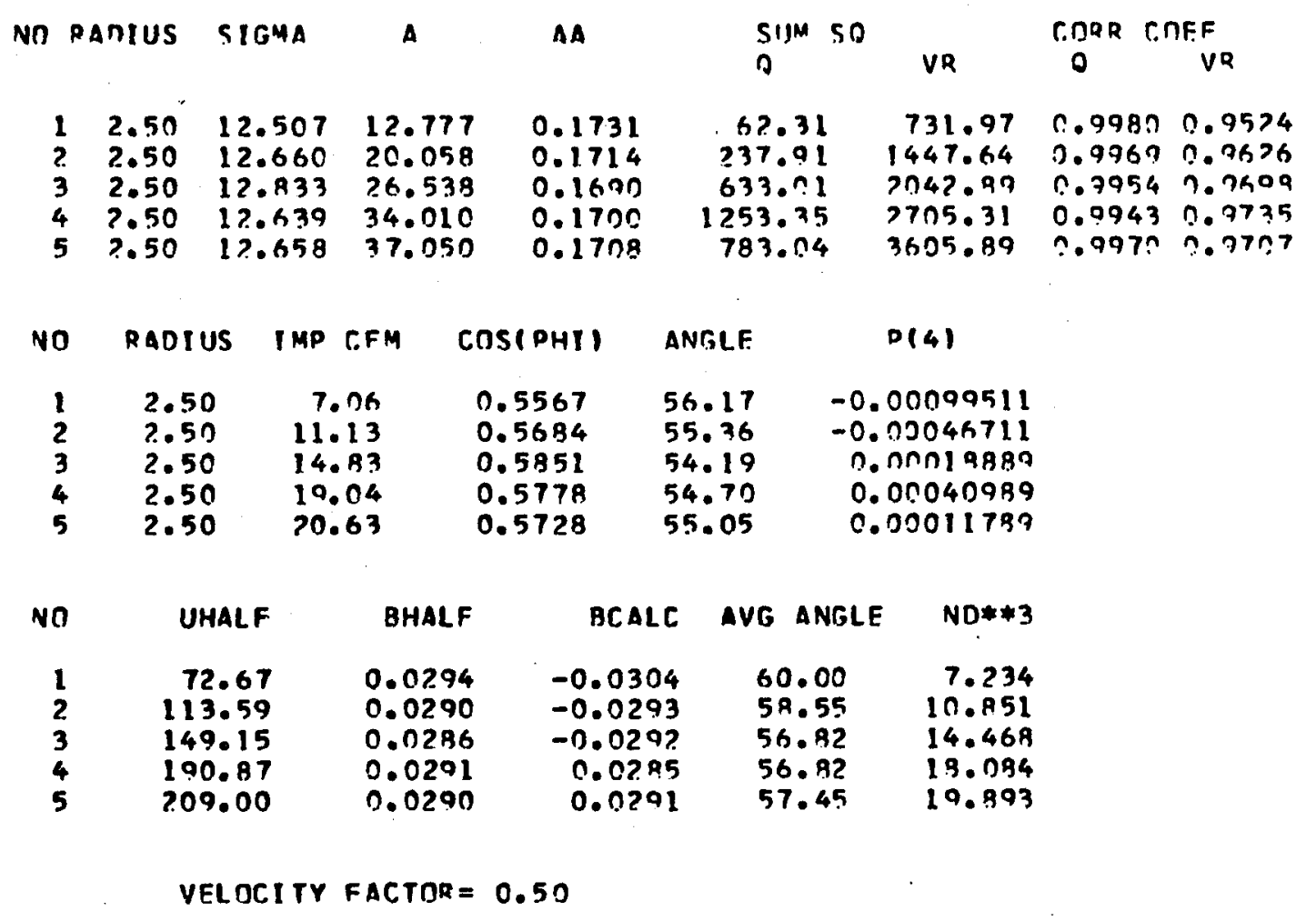


TABLE G-21

ANALYSIS OF COOPER'S DATA FOR A 5.0 INCH DIAMETER IMPELLER IN WATER, $\sigma$ CONSTANT

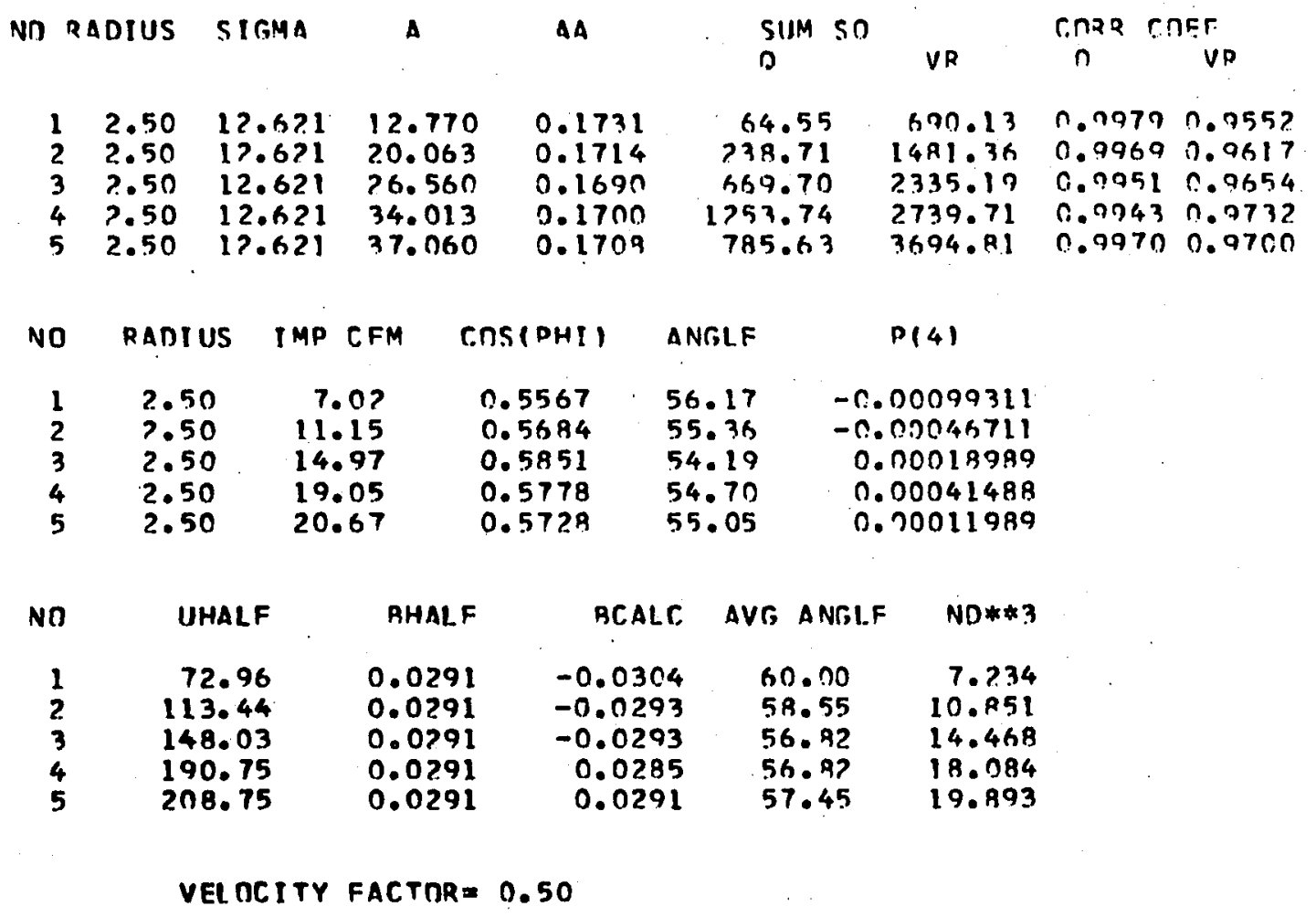


TABLE G-22

ANALYSIS OF COOPER'S DATA IN WATER FOR A 6.0 INCH DIAMETER IMPELLER

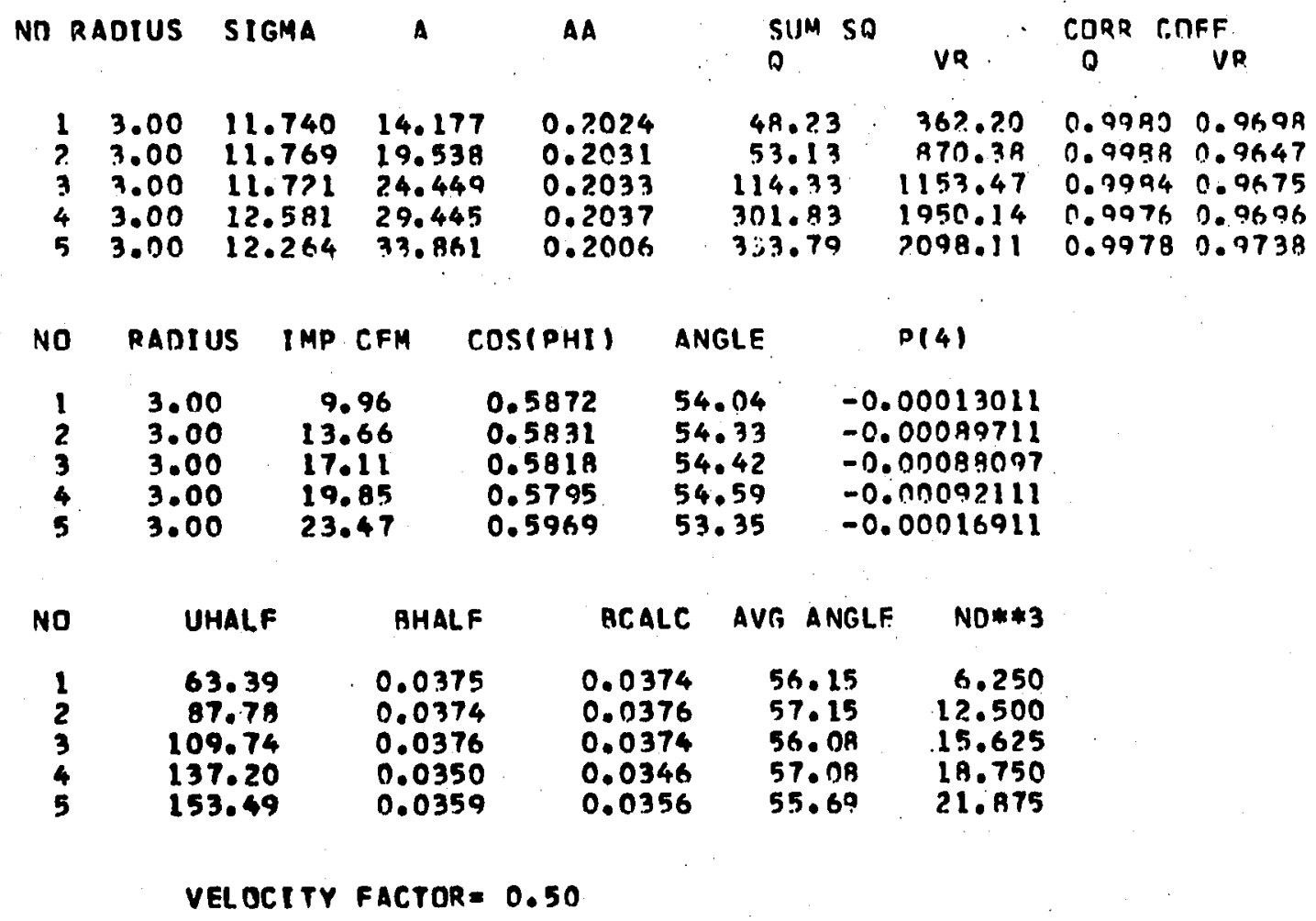


TABLE G-23

ANALYSIS OF COOPER'S DATA FOR A 6.0 DIAMETER IMPELLER IN WATER, $\sigma$ CONSTANT

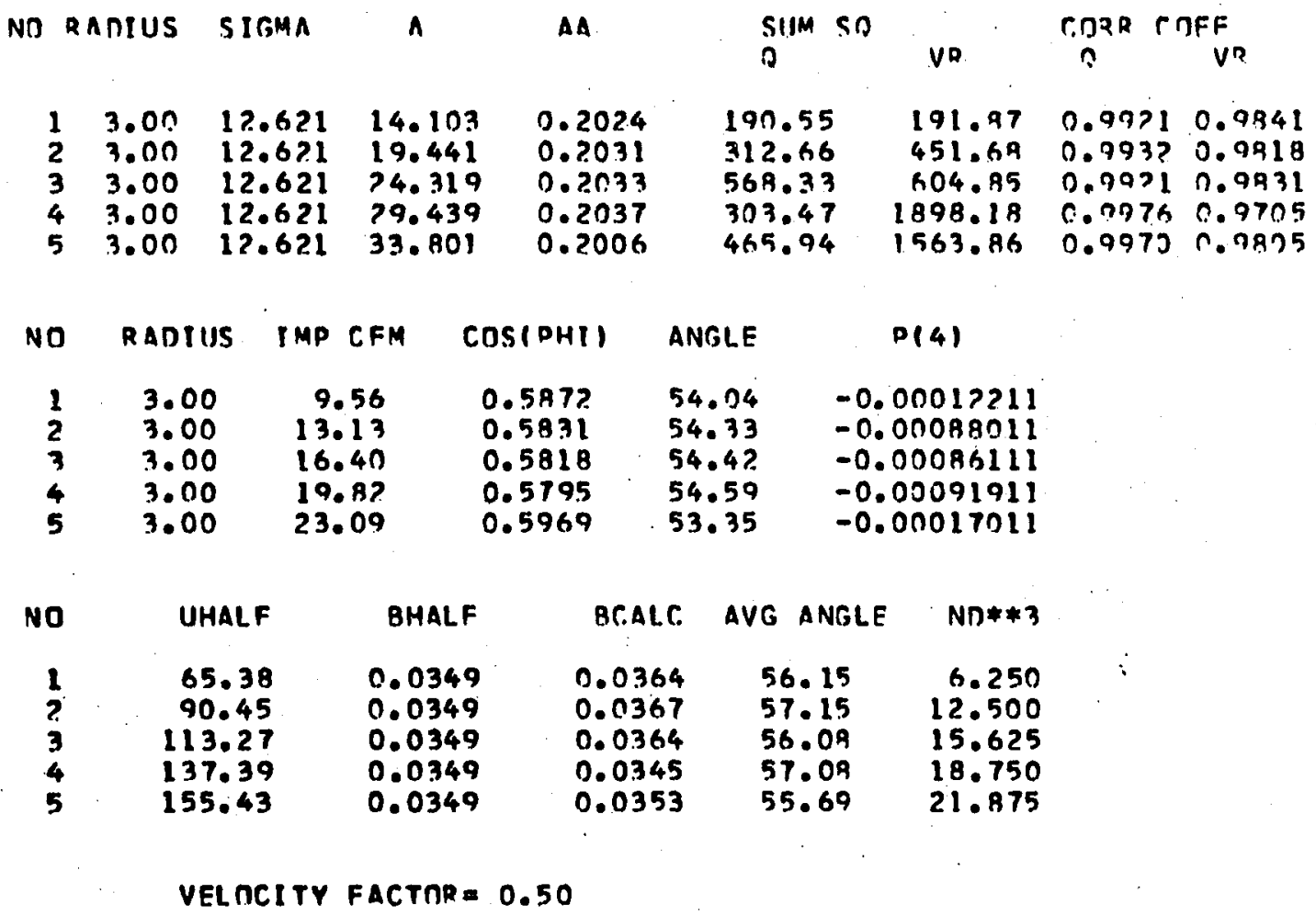


TABLE G-24

ANALYSIS OF COOPER'S DATA IN AIR FOR A 4.0 DIAMETER IMPELLER

\begin{tabular}{|c|c|c|c|c|c|c|c|c|c|c|c|}
\hline Nn & AnIIIS & GMA & $A$ & & AA & & $\begin{array}{l}\text { SIJ } \\
0\end{array}$ & J 5 & VR & $\begin{array}{c}\text { r.nop r } \\
r\end{array}$ & $\begin{array}{c}\text { PR } \\
\text { VR }\end{array}$ \\
\hline $\begin{array}{l}1 \\
? \\
3 \\
4 \\
5\end{array}$ & $\begin{array}{ll}2.00 & 1 \\
2.00 & 1 \\
? .00 & 1 \\
? .00 & 1 \\
? .00 & 1\end{array}$ & $\begin{array}{l}.679 \\
.024 \\
.345 \\
.165 \\
.649\end{array}$ & $\begin{array}{l}13 . \\
19 . \\
2.60 \\
35 . \\
43 .\end{array}$ & $\begin{array}{l}55 \\
66 \\
50 \\
60 \\
? 0\end{array}$ & $\begin{array}{l}0.1426 \\
0.1429 \\
0.1426 \\
0.1417 \\
0.141\end{array}$ & & $\begin{array}{l}1232 . \\
7776 . \\
3796 . \\
9793 . \\
9319 .\end{array}$ & $\begin{array}{l}42 \\
08 \\
01 \\
19 \\
10\end{array}$ & $\begin{array}{l}697.41 \\
497.60 \\
793.01 \\
595.16 \\
939.76\end{array}$ & $\begin{array}{l}0.7769 \\
\cap .7772 \\
n .7839 \\
C .7972 \\
0.9876\end{array}$ & $\begin{array}{l}0.9781 \\
0.9 ? 1 ? \\
0.9410 \\
0.9417 \\
0.0474\end{array}$ \\
\hline vn & RADIUS & IMP & CFM & & (PHI) & & IGLE & & 141 & & \\
\hline $\begin{array}{l}1 \\
? \\
? \\
4 \\
5\end{array}$ & $\begin{array}{l}2.00 \\
? .0 n \\
2.00 \\
2.00 \\
2.0 n\end{array}$ & $\begin{array}{r}5 \\
7 \\
10 \\
14 \\
17\end{array}$ & $\begin{array}{l}40 \\
95 \\
49 \\
04 \\
51\end{array}$ & & $\begin{array}{l}5173 \\
5158 \\
5180 \\
5333 \\
5309\end{array}$ & & $\begin{array}{l}.85 \\
.95 \\
.89 \\
.77 \\
.93\end{array}$ & $\begin{array}{l}-0 . \\
-0 . \\
-0 . \\
-0 . \\
-0 .\end{array}$ & $\begin{array}{l}0077011 \\
0001911 \\
0053011 \\
0109011 \\
0169011\end{array}$ & & \\
\hline No & UHAL & & $\mathrm{AH}$ & & BC: A & & AVE & ANGLE & $N D * * 3$ & & \\
\hline $\begin{array}{l}1 \\
2 \\
3 \\
4 \\
5\end{array}$ & $\begin{array}{l}102 . \\
143 . \\
207.0 \\
296.0 \\
346.0\end{array}$ & & $\begin{array}{l}0.0 \\
0.0 \\
0.0 \\
0.0 \\
0.0\end{array}$ & $\begin{array}{l}15 \\
76 \\
05 \\
94 \\
01\end{array}$ & $\begin{array}{l}0.01 \\
0.00 \\
0.01 \\
0.01 \\
0.01\end{array}$ & & $\begin{array}{l}63 \\
67 \\
63 \\
63 \\
63\end{array}$ & $\begin{array}{r}.33 \\
.33 \\
.33 \\
.33 \\
.33\end{array}$ & $\begin{array}{r}7.407 \\
11.111 \\
13.556 \\
19.518 \\
2 ? .72 ?\end{array}$ & & \\
\hline
\end{tabular}


TABLE G-25

ANALYSIS OF COOPER'S DATA FOR A 4.0. INCH DIAMETER IMPELLER IN AIR, O CONSTANT

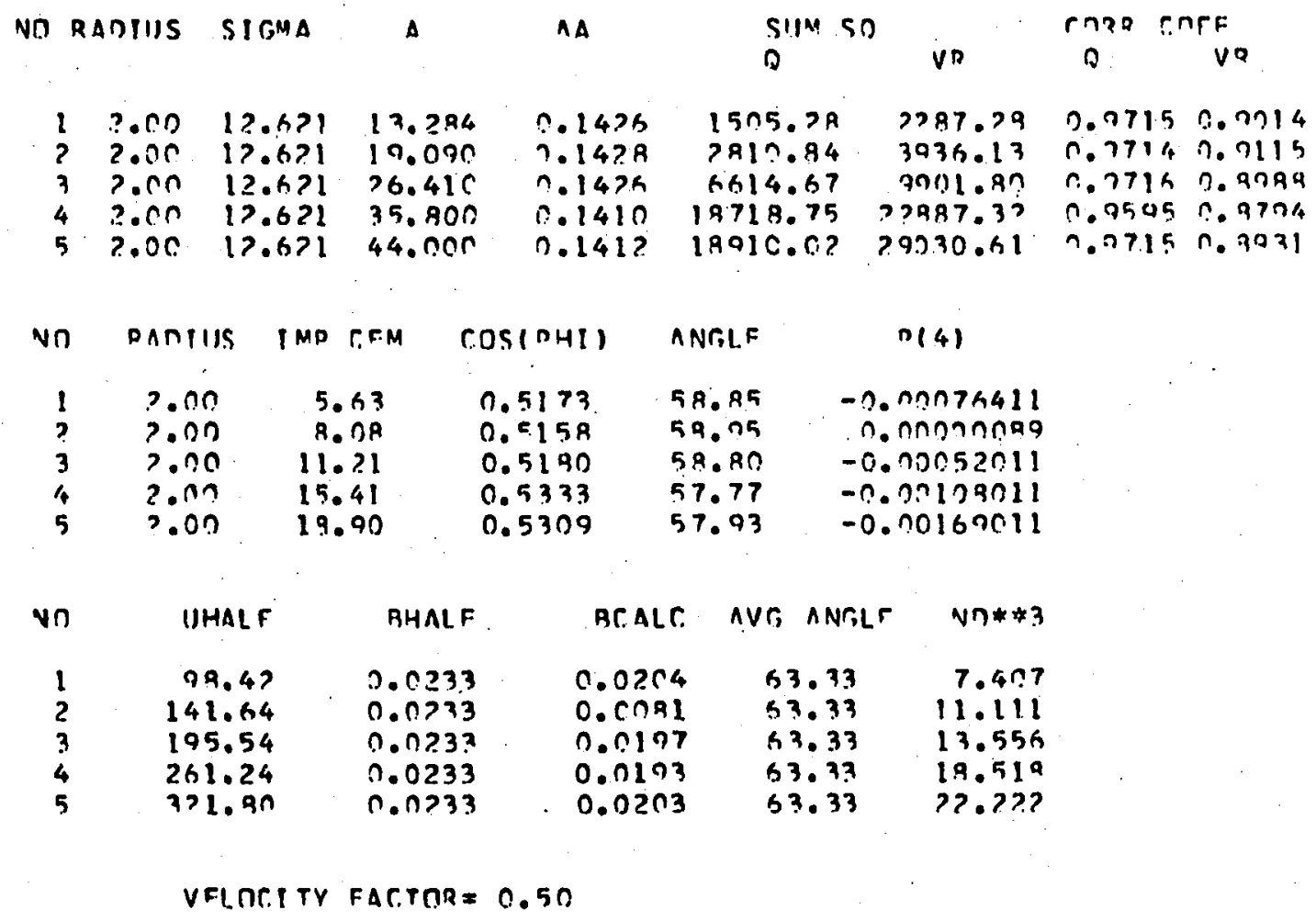


TABLE G-26

ANALYSIS OF COOPER'S DATA IN AIR FOR A 5.0 INCH DIAMETER IMPELLER

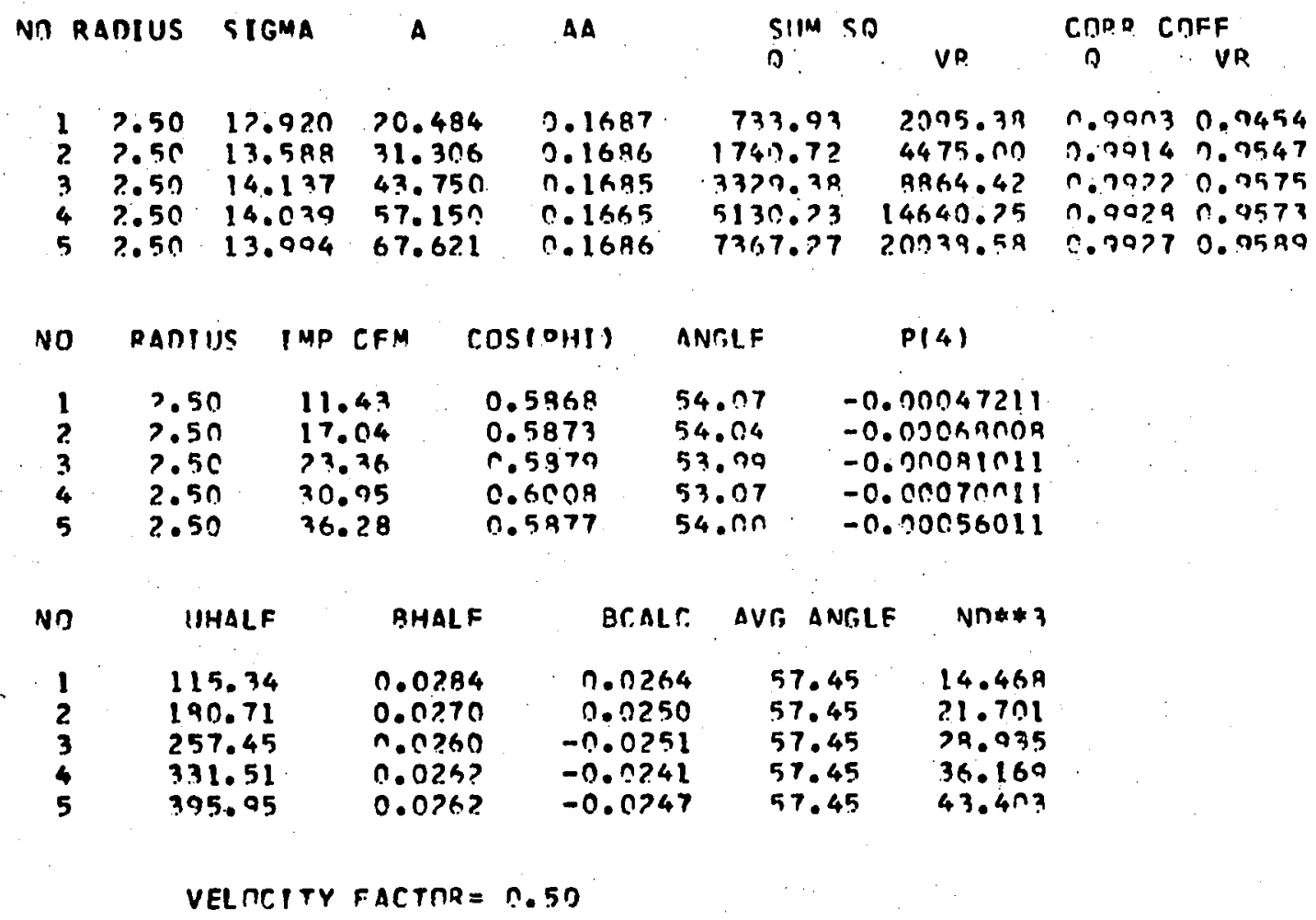


TABLE G-27

ANALYSIS OF COOPER'S DATA FOR A 5.0 DIAMETER IMPELLER IN AIR, - CONSTANT

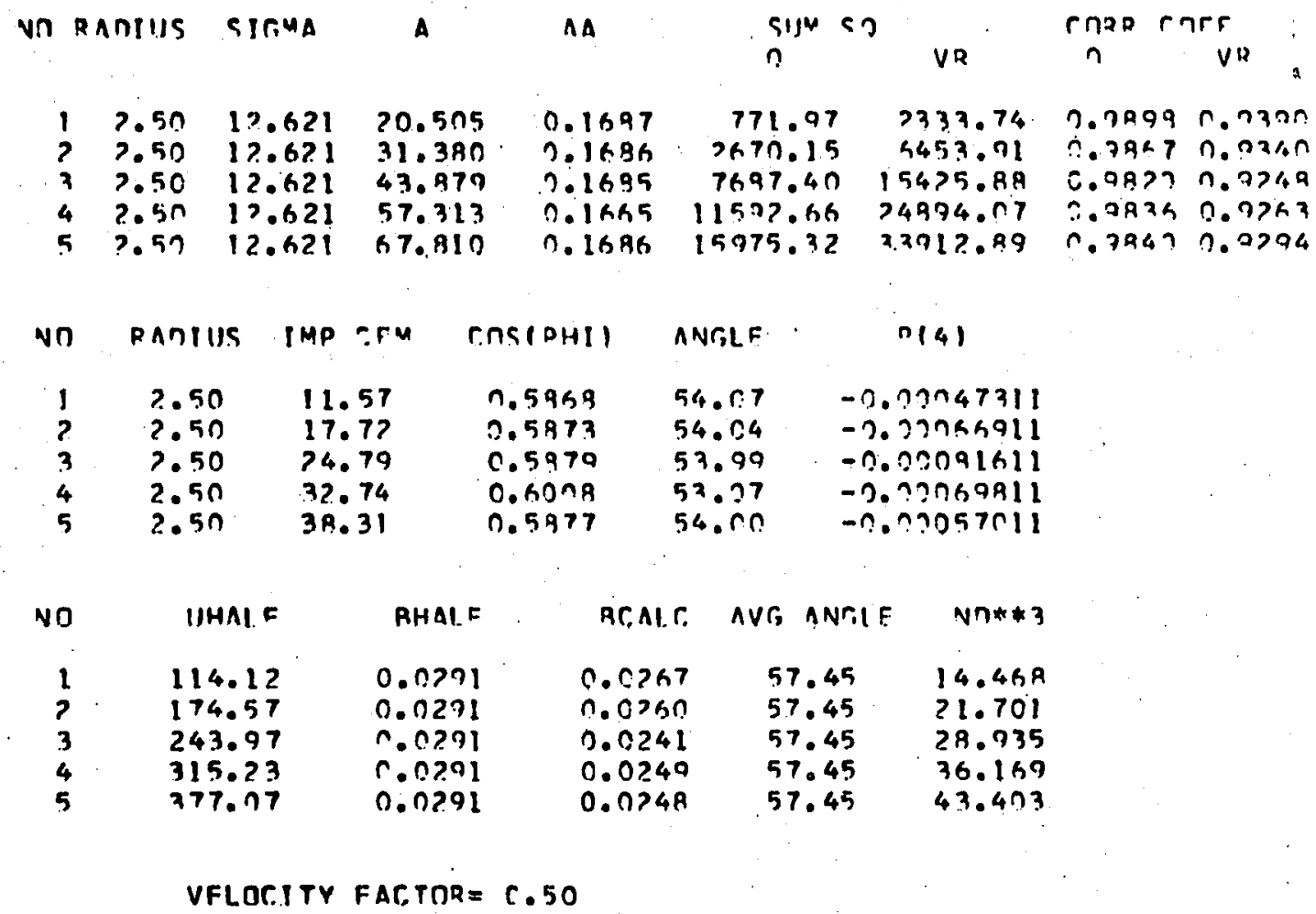


TABLE G-28

ANALYSIS OF COOPER'S DATA IN AIR FOR A 6.0 INCH DIAMETER IMPELLER

\begin{tabular}{|c|c|c|c|c|c|c|c|c|c|c|c|}
\hline NO & intus & SMA & A & & $\Delta \mathbf{\Delta}$ & & $\begin{array}{l}S U M \\
0\end{array}$ & $S Q$ & VR & $\begin{array}{l}C O P R \\
D\end{array}$ & $\begin{array}{l}\text { IFF } \\
\text { VR }\end{array}$ \\
\hline $\begin{array}{l}1 \\
2 \\
3 \\
4 \\
5\end{array}$ & $\begin{array}{l}3.00 \\
3.00 \\
3.00 \\
3.00 \\
3.00\end{array}$ & $\begin{array}{l}976 \\
559 \\
905 \\
747 \\
563\end{array}$ & $\begin{array}{l}18 . \\
34 . \\
46 . \\
81 . \\
95 .\end{array}$ & $\begin{array}{l}24 \\
20 \\
40 \\
30 \\
97\end{array}$ & $\begin{array}{l}0.2016 \\
0.1996 \\
0.1997 \\
0.1995 \\
0.1995\end{array}$ & & $\begin{array}{r}537.92 \\
268.21 \\
379.09 \\
066.95 \\
704.00\end{array}$ & $\begin{array}{l}2 \\
1 \\
9 \\
5 \\
0\end{array}$ & $\begin{array}{l}57.60 \\
46.09 \\
797.27 \\
783.27 \\
668.21\end{array}$ & $\begin{array}{l}C .9860 \\
0.9850 \\
0.9878 \\
0.7890 \\
0.7012\end{array}$ & $\begin{array}{l}0.7474 \\
0.9449 \\
c .0521 \\
0.9603 \\
0.9598\end{array}$ \\
\hline ח & RADIUS & I MP & C.FM & C.D & OHII & $A N$ & iLE & & 141 & & \\
\hline $\begin{array}{l}1 \\
2 \\
3 \\
4 \\
5\end{array}$ & $\begin{array}{l}3.00 \\
3.00 \\
3.00 \\
3.00 \\
3.00\end{array}$ & $\begin{array}{l}12 \\
23 \\
31 \\
53 \\
63\end{array}$ & $\begin{array}{l}68 \\
40 \\
64 \\
69 \\
10\end{array}$ & $\begin{array}{l}0 \\
0 \\
0 \\
0\end{array}$ & $\begin{array}{l}5914 \\
5023 \\
5018 \\
5026 \\
5075\end{array}$ & $\begin{array}{l}53 \\
57 \\
53 \\
5 ? \\
52\end{array}$ & $\begin{array}{l}74 \\
97 \\
00 \\
94 \\
95\end{array}$ & $\begin{array}{l}-0.0 \\
-0.0 \\
-0.1 \\
-0.1 \\
-0.1\end{array}$ & $\begin{array}{l}149011 \\
055310 \\
024010 \\
104009 \\
0094009\end{array}$ & & \\
\hline NO & IIHAI & & $\mathrm{BH}$ & & BCAI & & $\triangle V G \triangle N$ & NGLE & $N \cap * * 3$ & & \\
\hline $\begin{array}{l}1 \\
2 \\
3 \\
4 \\
5\end{array}$ & $\begin{array}{l}91 . \\
155 . \\
215 . \\
389 . \\
452 .\end{array}$ & & $\begin{array}{l}0.0 \\
0.0 \\
0.0 \\
0.0 \\
0.0\end{array}$ & $\begin{array}{l}70 \\
51 \\
41 \\
21 \\
25\end{array}$ & $\begin{array}{r}0.03 \\
-0.03 \\
-0.03 \\
-0.02 \\
-0.02\end{array}$ & & $\begin{array}{l}57.1 \\
57.1 \\
57.1 \\
57.1 \\
57.1\end{array}$ & $\begin{array}{l}15 \\
15 \\
15 \\
15 \\
15\end{array}$ & $\begin{array}{l}12.500 \\
25.000 \\
37.500 \\
62.500 \\
75.000\end{array}$ & & \\
\hline
\end{tabular}


TABLE G-29

ANALYSIS OF COOPER'S DATA FOR A 6.0 INCH DIAMETER IMPELLER IN AIR, $\sigma$ CONSTANT

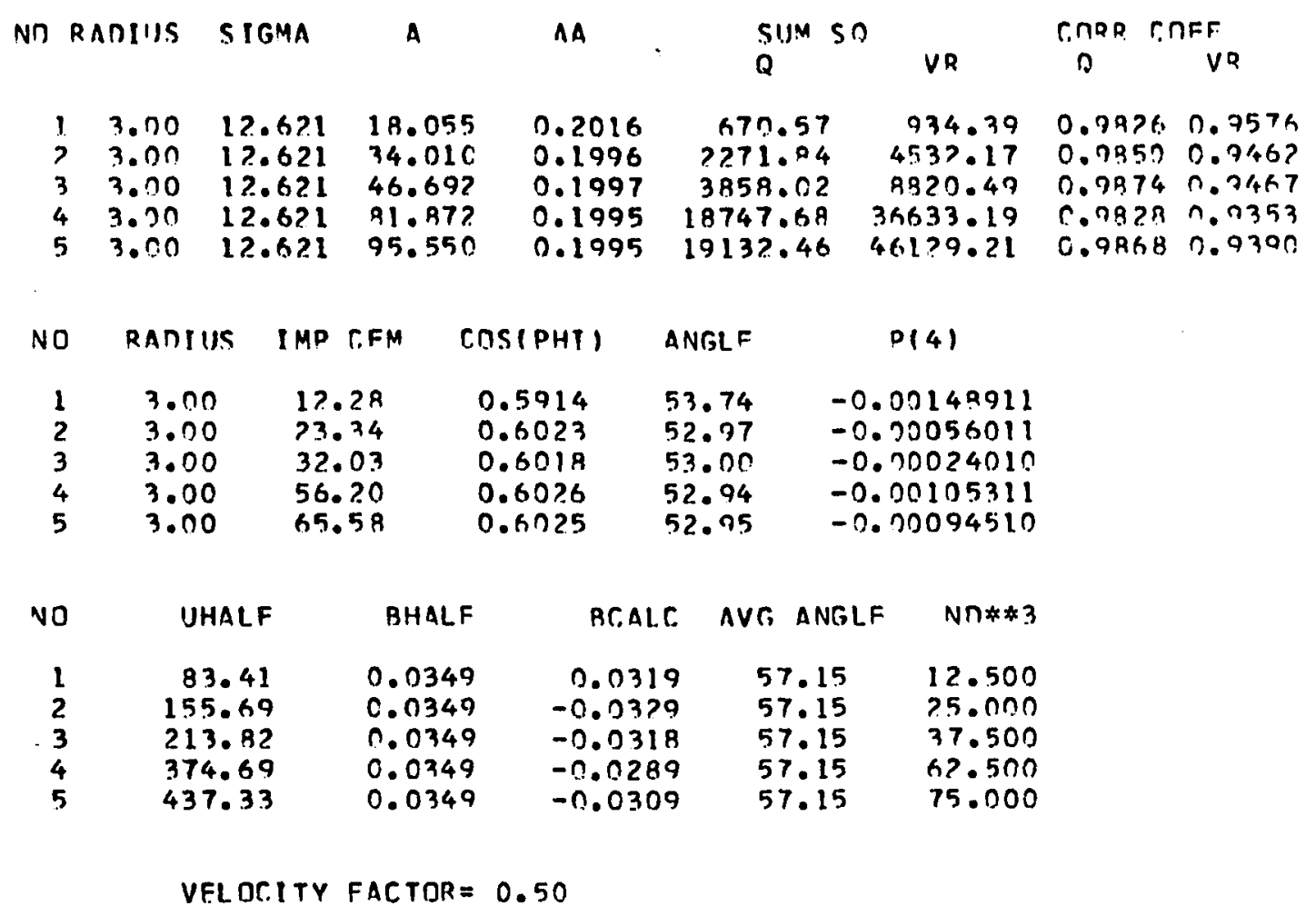


TABLE G-30

ANALYSIS OF COOPER'S DATA FOR A 4.0 INCH IMPELLER AT 280 RPM AND VARYING RADIAL DISTANCE

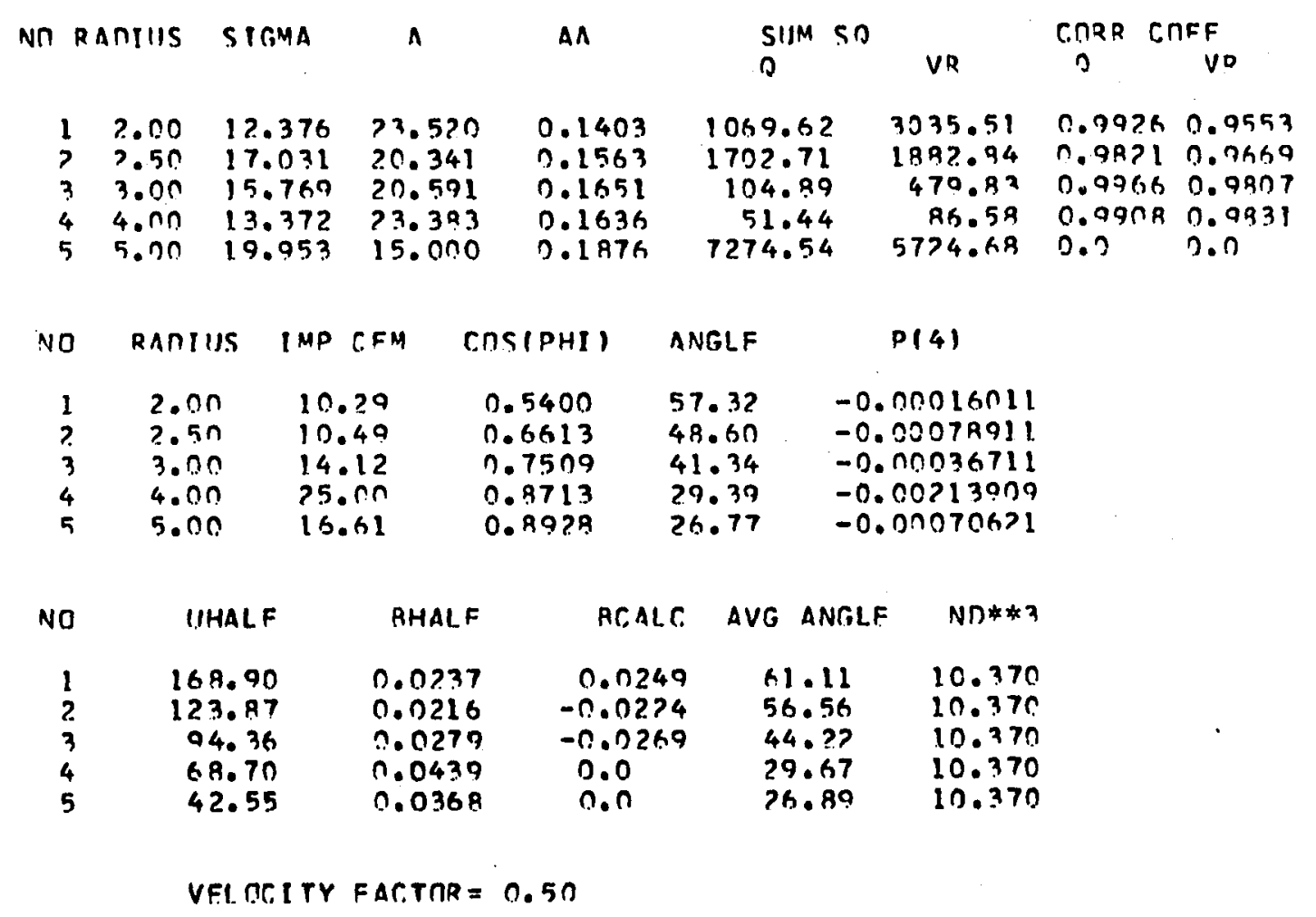


TABLE G-31

ANALYSIS OF COOPER'S DATA FOR A 4.0 INCH IMPELLER AT 280 RPM AND VARYING DISTANCE, $\sigma$ CONSTANT AND a EVALUATED FROM EQUATION IV-38

\begin{tabular}{|c|c|c|c|c|c|c|c|c|c|c|c|}
\hline Nn & An?lus & SIrima & \multicolumn{2}{|c|}{ A } & AA & \multicolumn{3}{|c|}{$\begin{array}{l}\text { SIJM SO } \\
0\end{array}$} & VR & $\begin{array}{l}\text { CORR C. } \\
n\end{array}$ & $\begin{array}{l}\text { TEF } \\
\text { VR }\end{array}$ \\
\hline $\begin{array}{l}1 \\
2 \\
3 \\
4 \\
5\end{array}$ & $\begin{array}{ll}2.00 & 1 \\
2.50 & 1 \\
3.00 & 1 \\
4.00 & 1 \\
5.00 & 1\end{array}$ & $\begin{array}{l}12.621 \\
12.671 \\
12.671 \\
12.621 \\
12.621\end{array}$ & \multicolumn{2}{|c|}{$\begin{array}{l}2 ? .961 \\
? 1.478 \\
? ? .040 \\
74.163 \\
23.753\end{array}$} & \multicolumn{2}{|c|}{$\begin{array}{l}0.1479 \\
0.1429 \\
0.1479 \\
0.1429 \\
0.1478\end{array}$} & \multicolumn{2}{|c|}{$\begin{array}{r}1124.54 \\
7625.15 \\
1521.79 \\
78.7 ? \\
215.76\end{array}$} & $\begin{array}{r}2007.59 \\
7657.66 \\
3383.99 \\
263.99 \\
356.83\end{array}$ & \multirow[t]{5}{*}{$\begin{array}{l}n .7922 \\
0.7161 \\
n .7501 \\
n .7959 \\
n .7420\end{array}$} & \multirow[t]{2}{*}{$\begin{array}{l}0.9572 \\
0.9160 \\
n .9545 \\
0.9477 \\
0.5637\end{array}$} \\
\hline NO & RADIUS & I AP & C.FM & & (PHI) & & IriLE & & $D(4)$ & & \\
\hline $\begin{array}{l}1 \\
? \\
3 \\
4 \\
5\end{array}$ & $\begin{array}{l}2.00 \\
2.50 \\
3.00 \\
4.00 \\
5.00\end{array}$ & $\begin{array}{l}9 \\
13 \\
17 \\
27 \\
33\end{array}$ & $\begin{array}{l}7 ? \\
51 \\
66 \\
08 \\
93\end{array}$ & & $\begin{array}{l}5156 \\
7281 \\
\text { A2 ก } \\
9 \cap 36 \\
9304\end{array}$ & & $\begin{array}{l}.96 \\
.27 \\
.87 \\
.37 \\
.04\end{array}$ & $\begin{array}{l}-0 . \\
-0 . \\
-0 . \\
-0 . \\
-0 .\end{array}$ & $\begin{array}{l}n 0015211 \\
0010 \times 71 ! \\
n n 39011 \\
00230110 \\
00190110\end{array}$ & & \\
\hline NO & UHAI & & BH & & & & AV $r_{i}$ & ANGLF & Nก**3 & & \\
\hline $\begin{array}{l}1 \\
2 \\
3 \\
4 \\
5\end{array}$ & $\begin{array}{r}170 . \\
107 . \\
36 . \\
67 . \\
57 .\end{array}$ & & $\begin{array}{l}0.0 \\
0.0 \\
0.0 \\
0.0 \\
0.0\end{array}$ & & $\begin{array}{r}0.0 \\
-0.0 \\
-0.0 \\
0.0 \\
0.0\end{array}$ & & $\begin{array}{l}6 \\
5 \\
4 \\
? \\
?\end{array}$ & $\begin{array}{l}1.11 \\
6.56 \\
4.2 ? \\
9.67 \\
6.89\end{array}$ & $\begin{array}{l}10.370 \\
10.370 \\
10.370 \\
10.370 \\
10.370\end{array}$ & & \\
\hline
\end{tabular}


TABLE G-32

ANALYSIS OF COOPER'S DATA FOR A 4.0 INCH IMPELLER AT 100 RPM WITH VARYING BLADE WIDTHS

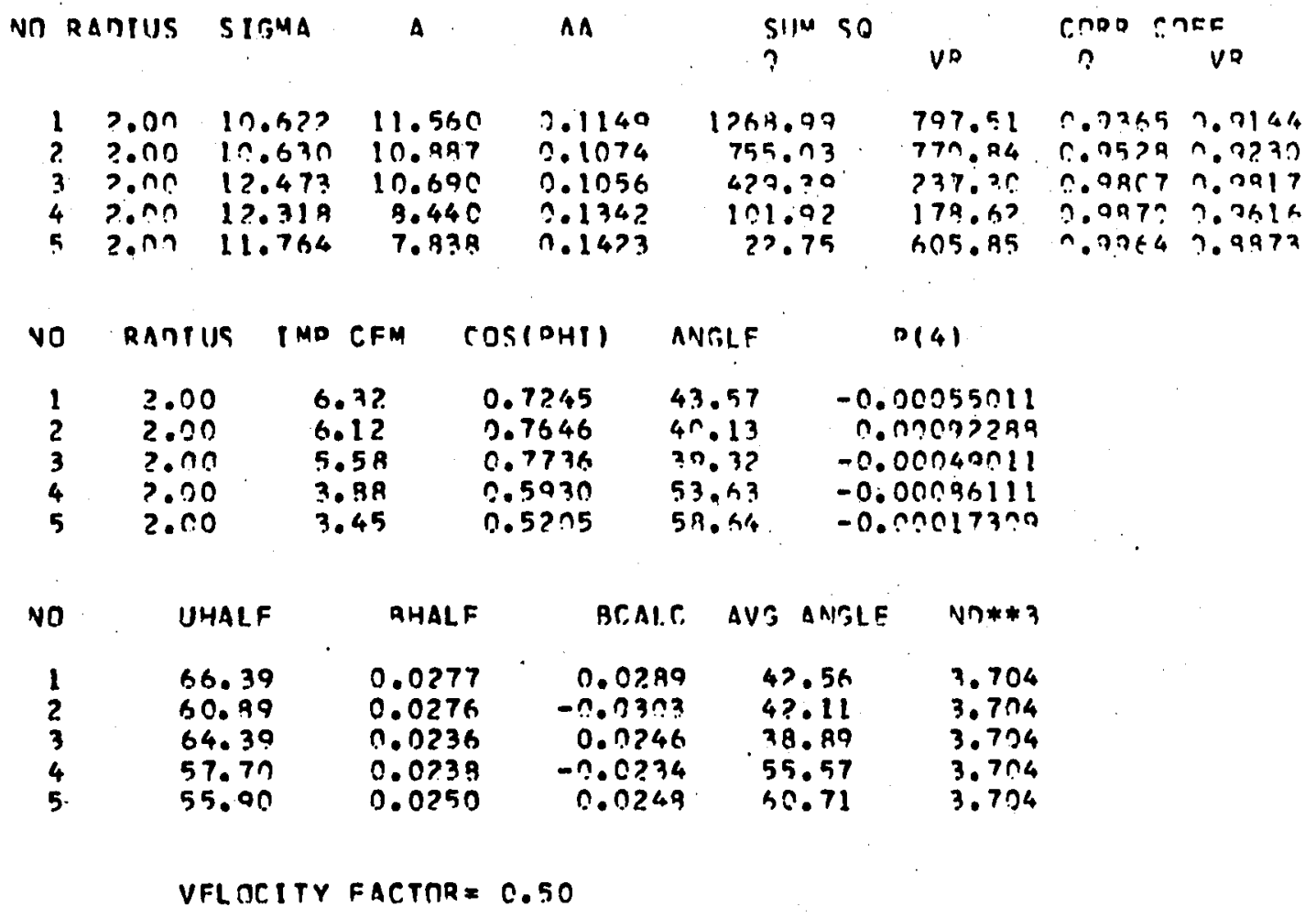


TABLE G-33

ANALYSIS OF COOPER'S DATA FOR A 4.0 INCH IMPELLER AT 200 RPM WITH VARYING BLADE WIDTHS

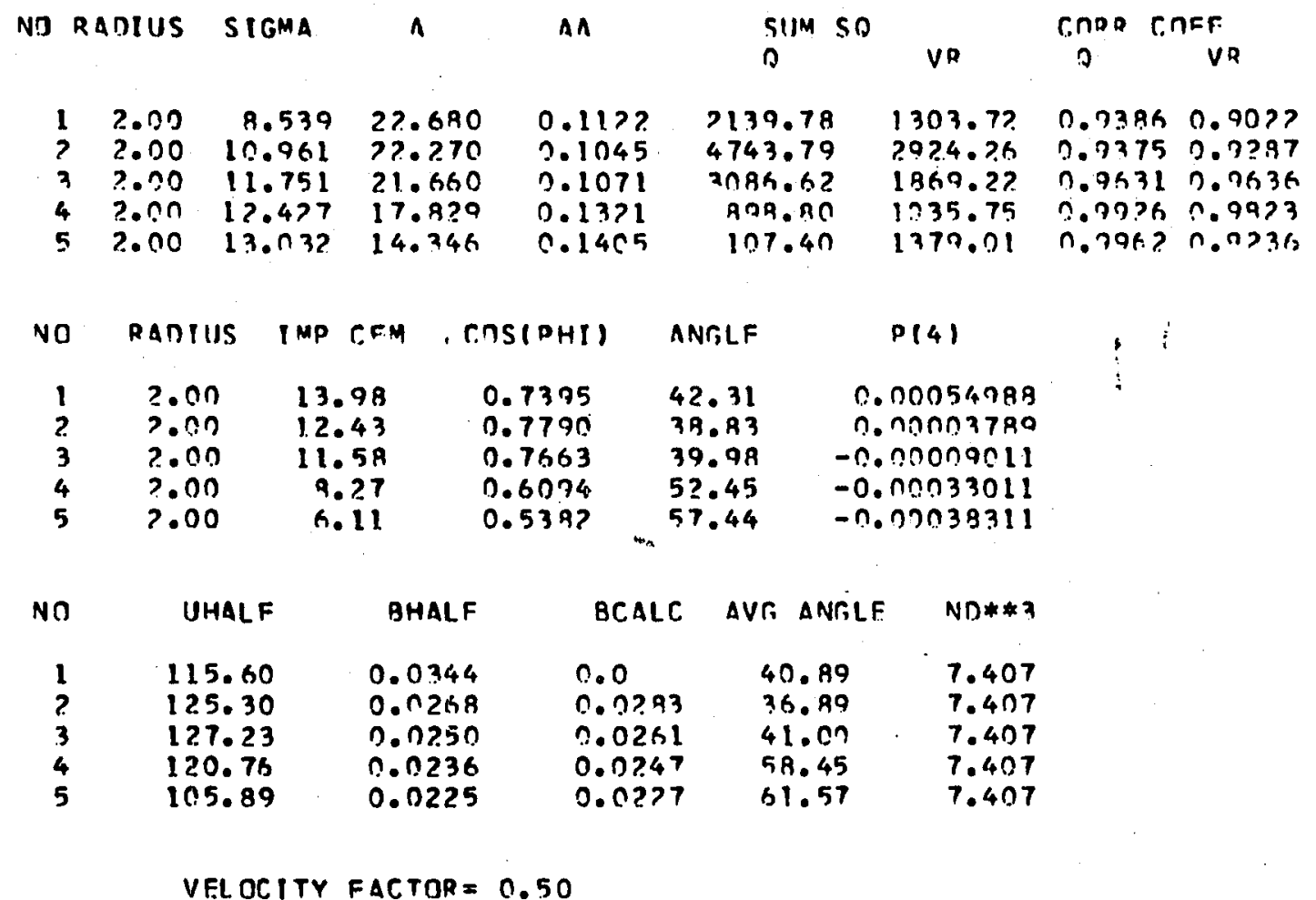


TABLE G-34

ANALYSIS OF NIBLSON'S DATA AT VARYING IMPELLER DIAMETER, IMPELLER SPEED. PROFILE 5 IS MEASURED IN CORN SYRUP AND a WAS CALCULATED FROM $\bar{\theta}_{y}$

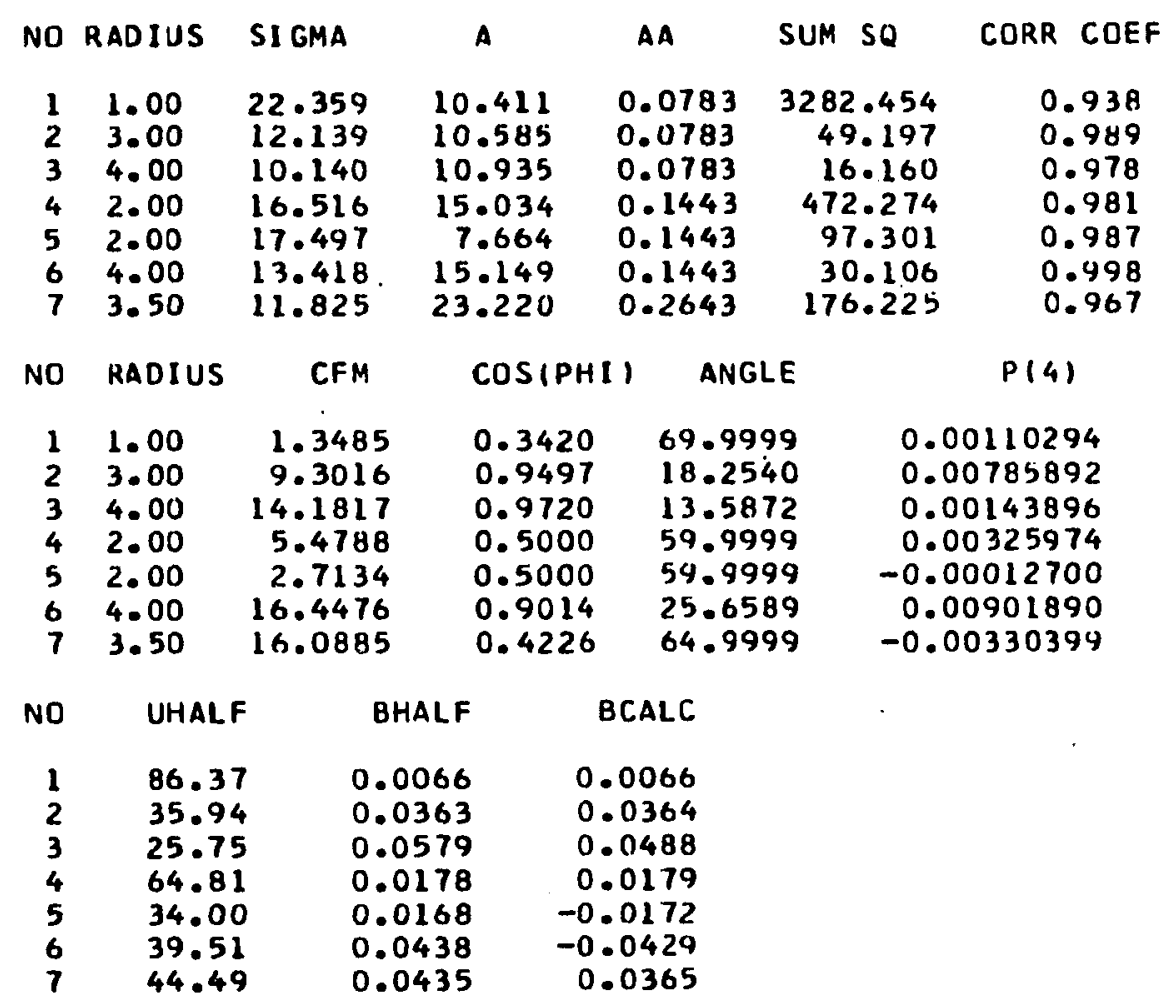


TABLE G-35

ANALYSIS OF NIELSON'S DATA AT VARYING IMPELLER DIAMETER IMPELLER SPEED. PROFILE 5 IS MEASURED CORN SYRUP, a WAS CALCULATED FROM $\overline{\boldsymbol{\theta}}_{y}$ AND $\sigma$ IS CONSTANT

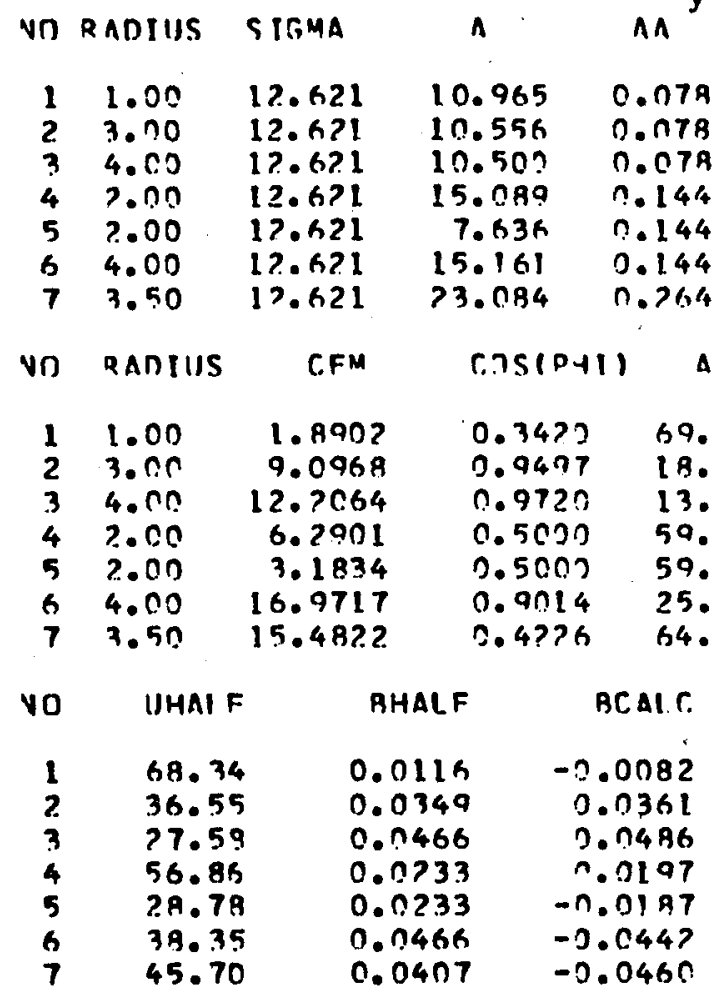


TABLE G-36

ANALYSIS OF CUTTER'S DATA WITH A 4.0 INCH DIAMETER IMPELLER AT VARYING RADIAL DISTANCE $r$. IMPELLER SPEED IS 200 RPM

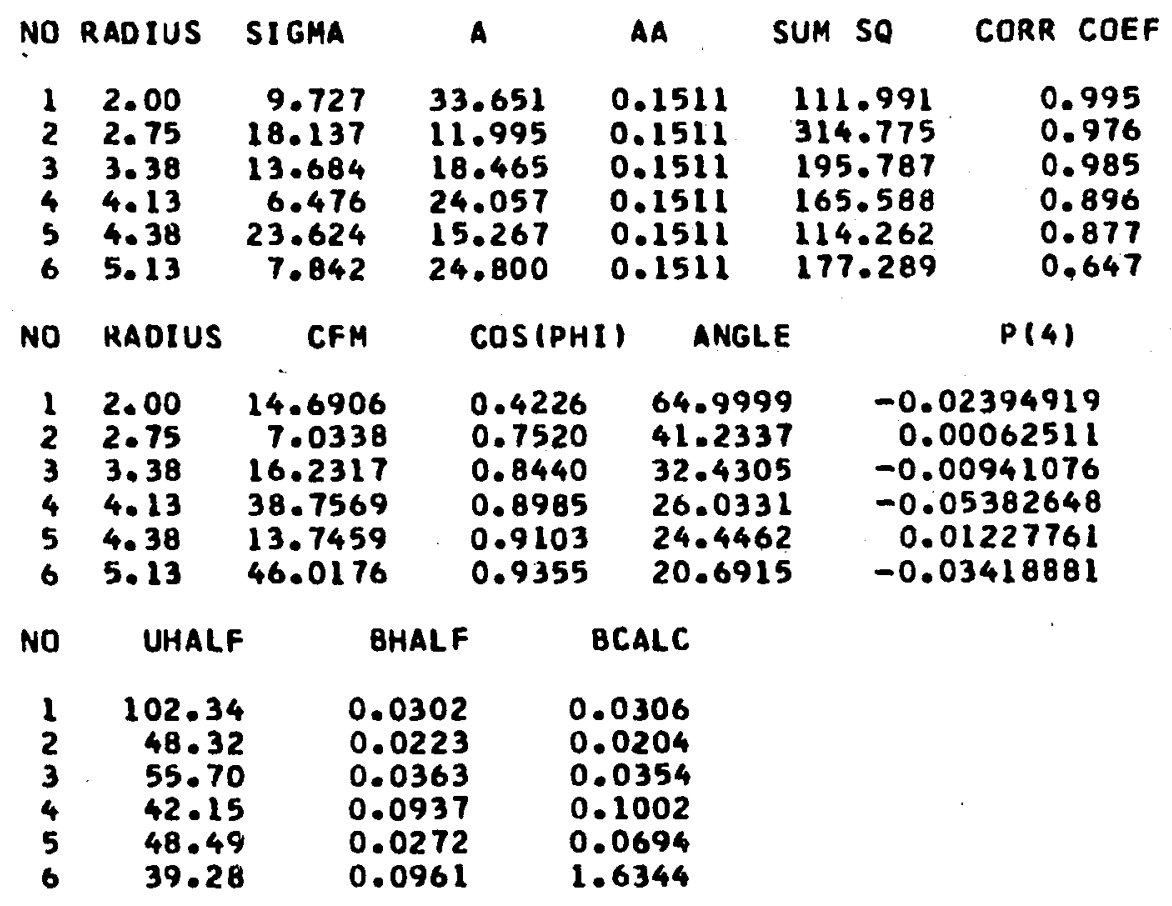


TABLE G-37

ANALYSIS OF CUTTER'S DATA WITH A 4.0 INCH DIAMETER IMPELLER AT VARYING RADIAL DISTANCE $x$. TMPELLER SPEED

IS 200 RPM AND $\sigma$ IS CONSTANT

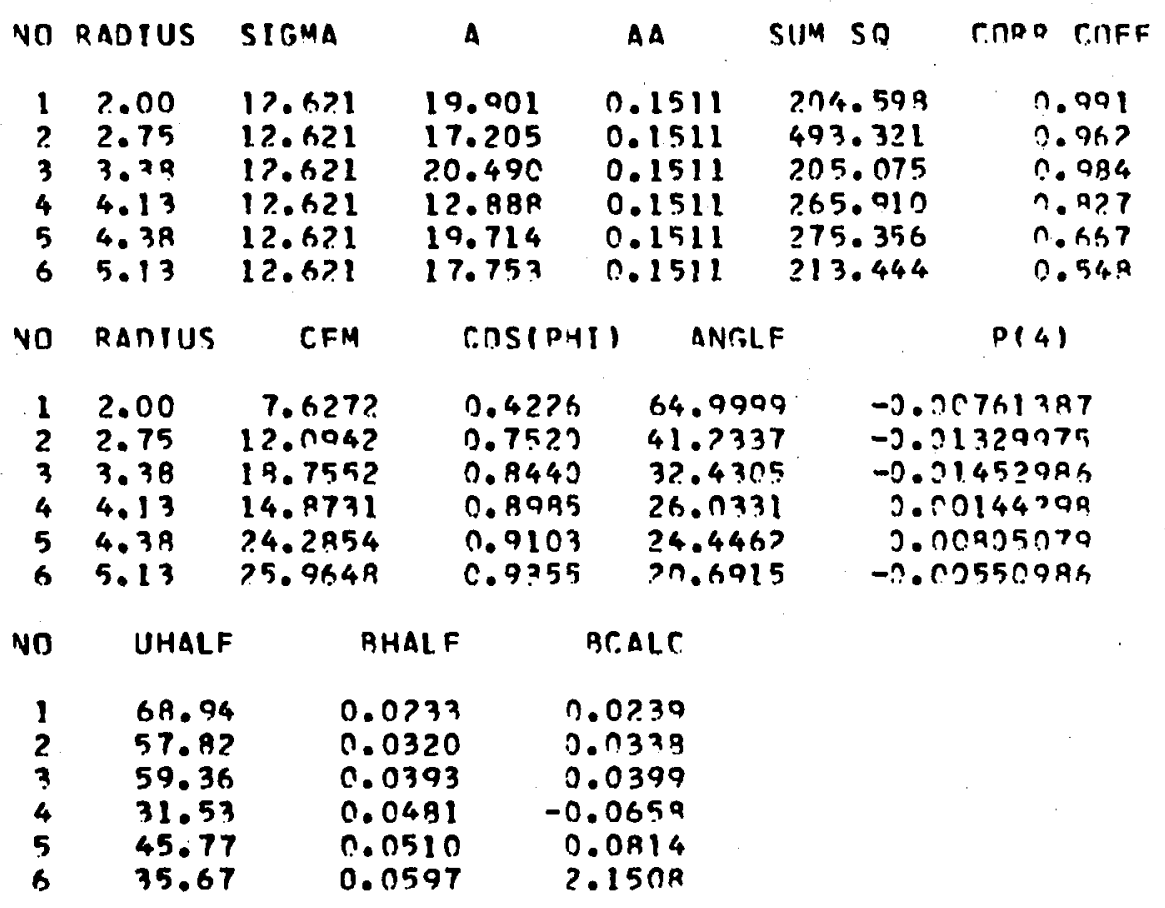


TABLE G-38

ANALYSIS OF CUTTER'S DATA AT 400 RPM, IMPELLER DIAMETER $=4.0$

INCH AND VARYING RADIAL DISTANCE $r$

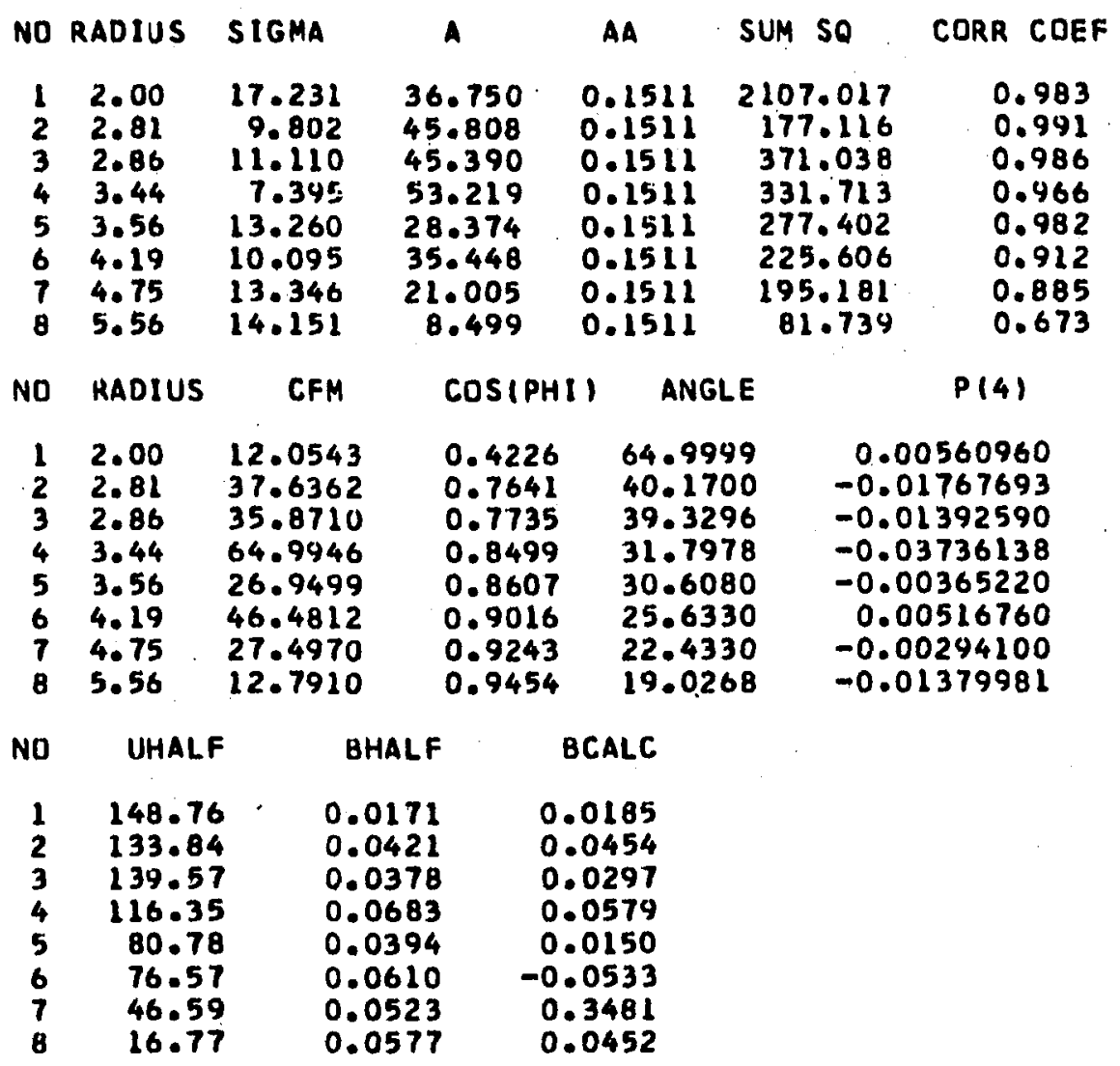


TABLE G-39

ANALYSIS OF CUT'TER'S DATA AT 400 RPM, 4.0 INCH DIAMETER IMPELLER, WITH VARYING RADIAL DISTANCE AND $\sigma$ CONSTANT

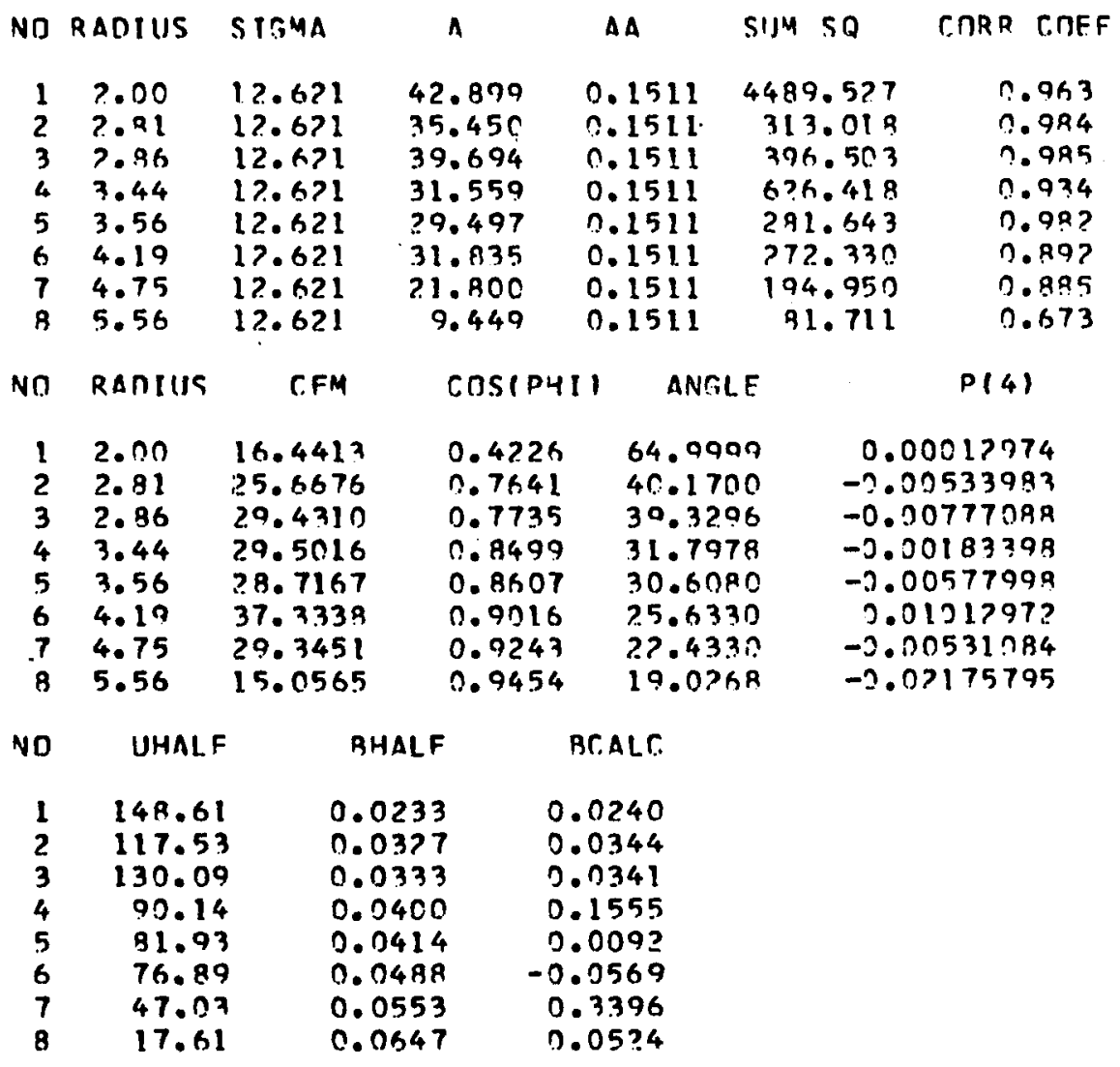


TABLE $G=40$

ANALYSIS OF CUTTER'S DATA AT 600 RPM, 4.0 INCH DIAMETER

IMPELLER, WITH VARYING RADIAL DISTANCE

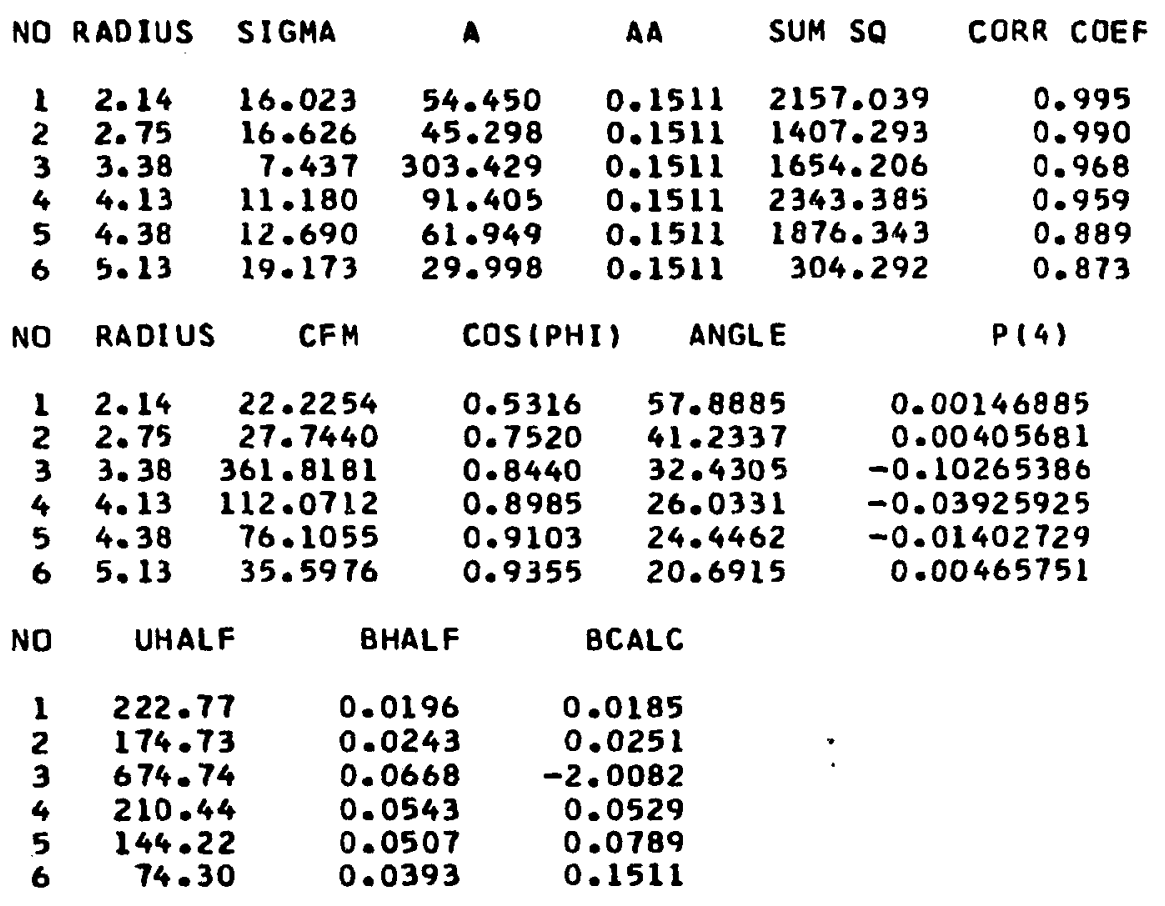


TABLE G-41

ANALYSIS OF CUTTER'S DATA AT 600 RPM, 4.0 INCH DIAMETER IMPELLER, WITH VARYING RADIAL DISTANCE AND $\sigma$ CONSTANT

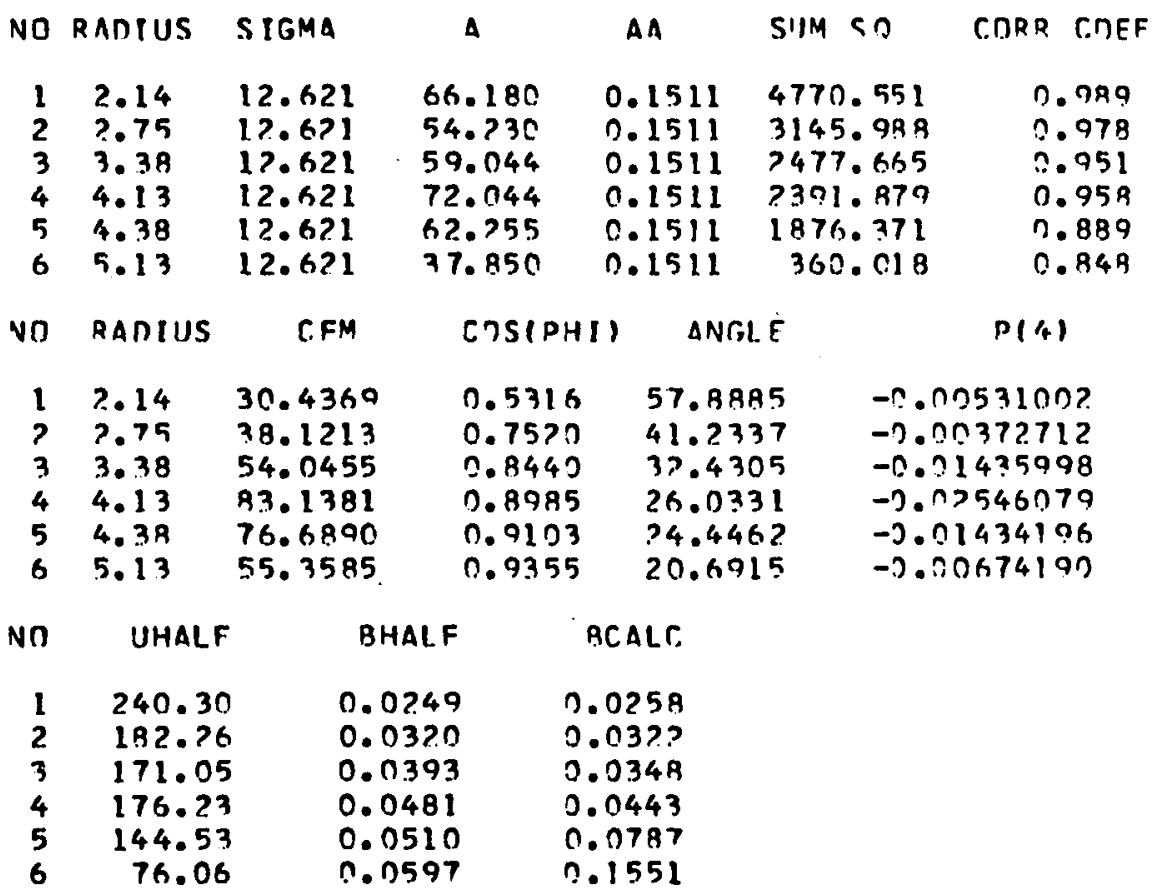




\section{APPENDIX H \\ DETAILED ANALYSIS OF A VELOCITY PROFILE \\ IN THE NEIGHBORHOOD OF THE IMPELLER}

In this Appentix a velocity profile analysis will be presented in detail. The results of this analysis has been treated in section 4.1 and 4.4. The analysis was performed on an IBM 360/ 65 computer. The program written for this analysis is called FLOWANL and is described in Appendix F. The raw data are punched on cards and these are also given in Appendix $F$ at the end of List,F-1. The data is presented in sets; each set is for a constant impeller speed. In Table H-1 is shown a typical printout of the raw data by the program FLOWANL. The item height of pitot tube off tank bottom given in the list in Table H-1 needs explanation. It is the height of the pressure tap $P_{1}$ on the probe when the "VERNIER STAND READING" $=13.95$. This serves to locate the position of the pressure tap $P_{1}$ in the tank. A simple calculation will show that the vernier stand will read 14.10 when $P_{1}$ is in the plane of the impeller centerline. This point is considered as $z=0$ for analysing the flow profile. Also given in the list is the relative location of $\theta_{y}=0$ and is called the zero error for the yaw angle, this corresponds to the reading from the vernier protractor when pressure tap $P_{1}$ is aligned with the center of the impeller. It is used to correct the item ANGLE to obtain the true direction of the velocity vector $\vec{q}$, 
Table H-1. Raw Data for Run No. 29.

\begin{tabular}{|c|c|c|c|c|c|c|c|c|}
\hline RUN NUMBER & & & & $=7$ & $? 7$ & & & \\
\hline IMPEI LER DIAMFTF & & & & $=$ & 3.00 & & & \\
\hline IMPELLFP HT OF $T$ & ANK BOTY & 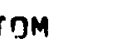 & & $=$ & 6.00 & & & \\
\hline LFVFL OF WATER & N TANK & & & & $1 ? .0 n$ & & & \\
\hline HF IGHT DF PITOT & TUAF NFF & $=$ TANK & AnTt & $n$ & 3.2 .5 & & & \\
\hline VERNIFR STAND RE & ADING & & & & 13.95 & & & \\
\hline ZERO ERRกR FחR A & NrgLF & & & & $27.2 \mathrm{C}$ & & & \\
\hline DIST PRMBE IN $A X$ & 15 & & & & 3.00 & & & \\
\hline RPM SETTING & & & & & 333.33 & & & \\
\hline FLUIN TEMP & & & & & 75.00 & DES $F$ & & \\
\hline ANGULAR POSITION & FRDM BA & AFFLF & & $=$ & 5.50 & DES & & \\
\hline $\begin{array}{l}\text { P(II-P(ATM) } \\
\text { LEFT RIGHT }\end{array}$ & $\begin{array}{l}\text { P(1)- } \\
\text { I.FFT }\end{array}$ & $\begin{array}{l}\text { P } P \text { ? } \\
\text { RIGHT }\end{array}$ & $\begin{array}{l}P P \\
I E F\end{array}$ & $\begin{array}{l}2)-P(3) \\
T \text { RIGH }\end{array}$ & $\begin{array}{l}\text { P } 14 \\
\text { LEF }\end{array}$ & $\begin{array}{l}\text { I-P(5) } \\
\text { T RITHT }\end{array}$ & $H$ & $\triangle N R L F$ \\
\hline $\begin{array}{l}3.10-12.90 \\
3.10-12.90 \\
3.15-12.95 \\
3.20-13.00 \\
3.25-13.05 \\
3.30-13.10 \\
3.30-13.15 \\
3.30-13.10 \\
3.25-13.05 \\
3.25-13.05 \\
3.20-13.00 \\
3.12-12.94 \\
3.10-12.96 \\
3.05-12.95\end{array}$ & $\begin{array}{l}-0.40 \\
-0.40 \\
-1.05 \\
-1.70 \\
-3.70 \\
-4.90 \\
-5.50 \\
-5.55 \\
-5.00 \\
-4.00 \\
-2.45 \\
-1.50 \\
-0.65 \\
-0.60\end{array}$ & $\begin{array}{l}0.95 \\
1.00 \\
1.60 \\
2.70 \\
4.20 \\
5.20 \\
5.90 \\
5.90 \\
5.40 \\
4.40 \\
2.90 \\
2.00 \\
1.05 \\
1.00\end{array}$ & $\begin{array}{l}0.0 \\
0.0 \\
0.0 \\
0.0 \\
0.0 \\
0.0 \\
0.0 \\
0.0 \\
0.0 \\
0.0 \\
0.0 \\
0.0 \\
0.0 \\
0.0\end{array}$ & $\begin{array}{l}0.0 \\
0.0 \\
0.0 \\
0.0 \\
0.0 \\
0.0 \\
0.0 \\
0.0 \\
0.0 \\
0.0 \\
0.0 \\
0.0 \\
0.0 \\
0.0\end{array}$ & $\begin{array}{r}0.40 \\
-1.60 \\
-3.30 \\
-3.90 \\
-4.40 \\
-3.10 \\
-0.15 \\
3.00 \\
5.10 \\
5.80 \\
5.50 \\
4.20 \\
2.50 \\
1.45\end{array}$ & $\begin{array}{l}1.60 \\
2.85 \\
4.70 \\
5.30 \\
5.90 \\
4.40 \\
1.30 \\
-7.20 \\
-4.50 \\
-5.25 \\
-4.70 \\
-3.60 \\
-1.85 \\
-0.60\end{array}$ & $\begin{array}{l}10.50 \\
10.60 \\
10.70 \\
10.80 \\
10.90 \\
11.05 \\
11.10 \\
11.20 \\
11.30 \\
11.40 \\
11.50 \\
11.60 \\
11.70 \\
11.80\end{array}$ & $\begin{array}{l}52.40 \\
60.00 \\
57.20 \\
56.60 \\
55.20 \\
53.80 \\
53.60 \\
53.00 \\
53.80 \\
54.60 \\
57.00 \\
58.80 \\
60.00 \\
60.00\end{array}$ \\
\hline
\end{tabular}


The headings of table H-2 will now be explained. The column headings $P(1)-P(A T M), P(1)-P(2)-P(3)$ and $P(4)-P(5)$ are readings taken on manometers $A, B, C$, and $D$ of Figure III-7. The subscripts on $\mathrm{P}$ refer to the pressure tap of the three dimensional probe. LEFT and RIGHT signifies the left and right arms of the manometer. The heading $\mathrm{H}$ refers to the vernier stand reading. Since the vernier stand reading for $z=0$ is known, the $z$ coordinate or the location of pressure tap $P_{1}$ can thus be calculated. The ANGLE refers to the reading on the vernier protractor and together with the value of the zero error gives the yaw angle $\theta_{y}$ of the velocity vector $\vec{q}$. The program FLOWANL consists of a MAIN program and several subroutines. A brief description of FLOWANL and its subroutines follow.

FLOWANL: Main program, reads in raw data and other necessary information. It calculates the $\mathrm{z}$ coordinates and corresponding values of $\vec{q}$. It calls the various subroutines and prints output in desired format.

PATERN: Subprograms called by FLOWANL. It performs Patters Search and returns to FLOWANL the values of parameters obtained for a least square fit on the data. The theoretical basis of this program is described in Appendix $C$.

PROC: Subprogram called by PATERN. It calculates the least square criteria which is stored as COST.

BOUNDS: Subprogram called by PATERN. This program checks parameters for violation of constraints. 
Table H-2, Anslysis of Velocity Profile $\vec{q}$, for Run No. 29.

$$
\begin{aligned}
& D=3.00000 \\
& R=3.00000
\end{aligned}
$$

\begin{tabular}{|c|c|c|}
\hline $\begin{array}{c}7 \\
0.05833 \\
0.05000 \\
0.04167 \\
0.03333 \\
0.02500 \\
0.01667 \\
0.00833 \\
0.00000 \\
0.00833 \\
0.01667 \\
0.02500 \\
0.03333 \\
0.04167 \\
0.05000\end{array}$ & $\begin{array}{c}n \\
44.82013 \\
45.64259 \\
62.79556 \\
90.91562 \\
108.47758 \\
171.98497 \\
130.74434 \\
130.52959 \\
124.40071 \\
111.801144 \\
89.27432 \\
72.16724 \\
50.29568 \\
48.79399\end{array}$ & $\begin{array}{c}\text { ANGLF } \\
35.20000 \\
32.80000 \\
30.00000 \\
29.39999 \\
28.00000 \\
26.59999 \\
26.39999 \\
25.80000 \\
26.59999 \\
27.39999 \\
29.80000 \\
31.59999 \\
32.80000 \\
32.80000\end{array}$ \\
\hline & $\begin{array}{r}\text { PAR AME TERS } \\
11.2779874 \\
18.544937 \\
0.1158798 \\
0.0027420\end{array}$ & \\
\hline
\end{tabular}

AVG ANGLF $=29.65705872$ WT AVG ANGLF $=\quad 27.61441040$

VELOCITY FACT DR $=0.50$

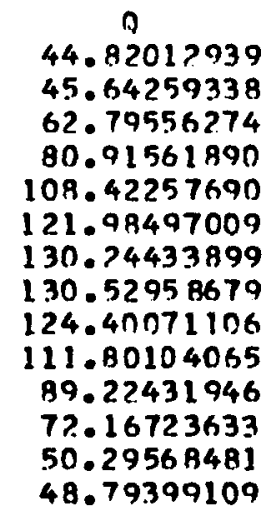

QC.

36. B59451?9

50.17221069

66.47894287

R5. 00456238

103.86741638

120.08189392

130.23974609

131.81726074

124.39604187

109.89250732

91.54614258

72.63247681

55.47 .958069

41.06221008

SUM MF SQUARES DF (O-DCALC)

235.63095093

GOODNFSS OF FIT

0.99190027

BHALF =

0.03907494

BCALC, =

0.03734012

HALF HIDTH OF JFT AT XO $=3.00$.

1.60 INCHFS 
OMEGA: Subprogram called by FLOWANL. This program performs a three point Lagrange interpolation and is used to obtain the value of $z$ at which $\bar{q}=1 / 2 \bar{q}_{\max }$.

YAW: Subporgram called by FLOWANL. This program analyses the readings obtained from manometer $D$.

AVG: Subporgram called by FLOWANL. It calculates an average value of a data set and also its variance and a confidence limit or deviation based on a "t-test" with a 0.95 confidence level.

In Table H-2 is shown a sample printout of the output obtained from the program FLOWANL. The symbols in Table $\mathrm{H}-2$ are:

$\mathrm{D}=$ diameter of impeller, inches

$R=$ distance of prove form impeller axis, inches

$z=$ vertical distance at which velocity $\bar{q}$ is measured, $z=0$ is at impeller centerline, ft. $Q=\vec{q}$, ft./min., calculated from equation ANGLE $=$ yaw angle $\theta_{y}$ in degrees which $\vec{q}$ makes with the radius vector

$\mathrm{QC}=\overrightarrow{\mathrm{q}}$ calculated from equation II-13, ft./min.

$\vec{q}$ is experimental calculated using equation III-3

PARAMETERS, are the parameters obtained from Pattern Search subroutine and are as follows.

$$
\begin{aligned}
& 1=\sigma \\
& 2=A \\
& 3=a \\
& 4=z_{0}
\end{aligned}
$$


Table H-3. Radial Velocity Profile Analysis for Run No. 29.

RADIAL VFLOCITY ANALYSIS

0

$36.6244964 h$

38.36560059

54.38750732

70.49476624

95.73139954

109.07337374

116.66134644

117.51817372

$111 . ? 3338318$

99.25963647

77. $47.572 \cap 21$

61.46677584

42.?76940?1

41.01455688
$D C$

32. 660 OR2764

44.45713806

58.90635681

75.32167053

97.0357? 093

106.40310669

115.4037933 ?

116. 80154419

120.2255554 ?

97.36524963

81.11764526

64.35850525

49.11526489

$36.3845520 n$

SUM TF SQUARFS TF (D-DCALC)

714.25904946

GOONNFSS OF FIT

0.99151355 
The value of $\bar{q}$ theoretical or sometimes referred to as calculated, is obtained farm equation II-10 which is reproduced below

$$
\bar{q}_{c a l c}=\frac{A}{2}\left(\frac{\sigma}{r}\right)^{\frac{t}{2}} \frac{1}{\left(r^{2}-a^{2}\right)^{\frac{T}{4}}}: 1-\tan h^{2}(\eta / 2) \bar{J}
$$

II- 10

Three of the parameters $\sigma, A$ and $z_{0}$ are obtained by a least square fit of the data. The parameter a; is obtained from a weighted average value of $\theta_{y}$, the yaw angle, as explained in Section 4.1. Both average value of $\theta_{y}$ and the weighted average value of $\theta_{y}$ are next reported in Table $\mathrm{H}-2$. Also given is the velocity factor that modifies the weighted.average angle.

QC. is the predicated value of $\vec{q}$ and is seen to be close to the experimental value. The goodness of fit is the value of the correlation coefficient $R$ defined by Equation 4-1. The sum of squares $(Q-Q C)$ is the value of the sum squares about regressions, given by Equation $\mathrm{C}-2$.

The quantity BHALF in Table $\mathrm{H}-2$ should not be confused with $b_{\frac{1}{2}} \cdot$ It is the value of $z$ for which $\bar{q}$ is $1 / 2 \bar{q}_{\max }$. From a similar reasoning as that used in Appendix B to obtain the half width of jet, Equation B-33, it can be shown that

$$
\text { BHALF }=1.763 \underline{\underline{r}}
$$

The factor of 1.763 is the value of $\eta / 2$ which makes $\tanh ^{2}(\eta / 2)=0.5$ 
UHALF is equal to $1 / 2 \bar{q}_{\max }$ and is obtained by calculating $\bar{q}_{\max }$ from equation II-10 putting $\eta=0$. UHALF is used to interpolate $\bar{q}$ experimental to obtain BCALC. The interpolation is done using a three point Lagrange interpolation formula. The interpolated value is corrected for shift in origin by subtracting $z_{0}$, and the result reported as BCALC. It is secondary check on the goodness of fit. The closeness between BHALF and BCALC in Table $\mathrm{H}-2$ indicates a very good fit between the experimental and calculated values of $\vec{q}$. BCALC is sometimes reported as a negative value because the interpolation program OMEGA chooses three values of $\vec{q}$ closest to UHALF which in this case corresponds with negative values of $z$. As the velocity profile is symmetirc about the impeller centerline the sign of $z$ is hence of no significance.

The last item in Table H-2 is the half width of the jet at the point of measurement. It is calculated from Equation B-34 and is the value of $z$ at which the velocity is less than $1 \%$ of $\bar{q}_{\max }$. Thus at $r=3$ the width of the tangential jet is 3.34 inches. These facts are shown in Figure IV-11 which gives both the experimental and theoretical value of $\bar{q}$ versus $z$ for Run Number 29 . The theoretical line is obtained from Equation II-10 using the value of the parameters as given in Table $\mathrm{H}-2$. The fit by the model is seen to be very good except at the profile ends where the probe response is poor. This is because for a velocity of $30 \mathrm{ft} / \mathrm{min}$, the $\Delta \mathrm{H}_{21}$ recorded by a manometer using a fluid of sp. $\mathrm{gr}$. of 0.8 is 0.66 inch (calculated from Equation III-3). At this low value of $\Delta \mathrm{H}_{21}$ the manometer 
response falls tending to take an extremely long time to reach equilibrium. Thus velocity measurements are not feasible below this limiting velocity. Hence with the present probe the profile cannot be checked very far beyond BHALF as shown in Figure. IV-11, which shows a plot of the experimental and thearetical velocity profilesi.

Also shown in Figure IV-11 is the jet displacement $z_{0}$ and the half width of the jet $b_{\frac{1}{2}}$. It is seen that for $z= \pm\left(b_{\frac{1}{2}}+z_{0}\right)$ the velocity reaches less than $1 \%$ of $\bar{q}_{\max }$. Figure IV-11 also demonstrates the goodness of fit as measured by the correlation coefficient. The correlation coefficient for this particular profile from Table H-2 is 0.99 and the agreement between experiment and theory is excellent as shown in Figure IV-11. In general the fit by the model for the velocity profiles is excellent in a majority of cases and can be verified by examining the correlation coefficient in Appendix G.

In Table H-3 is shown the result of the analysis of the radial velocity profile for Run Number 29. Q is the exprrimentally determined value of $\overline{\mathrm{v}}_{\mathrm{r}}$ and is obtained from Equation B-22. QC is the calculated value of $\bar{v}_{r}$ obtained from the tangential jet model using Equation B-22 with the parameters $\sigma, A$, $a$ and $z_{0}$ obtained for the least square fit on $\vec{q}$ and given in Table $\mathrm{H}-2$.

The goodness of fit has the same meaning as in Table H-2 and is obtained from Equation IV-1 written for $\bar{v}_{\mathbf{r}}$. Similarly, sum of squares is obtained from Equation $C-2$ written for $\bar{v}_{\mathbf{r}}$. The correlation coefficient is 0.99 indicating that the prediction for $\bar{v}_{r}$ is 
very good. In Figure IV-17 is plotted the experimental and calculated values of $\bar{v}_{r}$. This figure also illustrates the relationship between $\bar{v}_{r}$ and the pumping capacity $Q$.

In Table $\mathrm{H}-4$ is shown the results obtained from Subroutine YAW. $P(1)$ - $P(2)$ and $P(4)$ - $P(5)$ are the pressure drops recorded by manometers $B$ and $D$ in inches of manometer fluid. DEL U**2 EXP and DEL $U * * 2$ CALC are the experimental and calculated values of $\Delta q^{2}$ obtained from Equations IV-28 (b) and IV-31 respectively. Next VZ CALC is $\bar{v}_{z}$ obtained from Equation B-23. Finally the last column gives the difference between the calcula ted and experimental values of $\Delta q^{2}$. 
Table H-4: Results of Analysing Manometer D for Run No. 29

\begin{tabular}{|c|c|c|c|c|c|}
\hline$P(1)-P(2)$ & $P(4)-P(5)$ & $\underset{F \times P}{D F * 2}$ & $\begin{array}{l}\text { DEL U**?? } \\
\text { CALC }\end{array}$ & $\begin{array}{r}v 7 \\
\text { CAL. }\end{array}$ & DIFF DF U**? \\
\hline $\begin{array}{l}0.5315 \\
0.5512 \\
1.0433 \\
1.7323 \\
3.1102 \\
3.9370 \\
4.4882 \\
4.5079 \\
4.0945 \\
3.3071 \\
2.1063 \\
1.3780 \\
0.6693 \\
0.6299\end{array}$ & $\begin{array}{l}-0.8661 \\
-7.1457 \\
-3.5433 \\
-4.0157 \\
-4.4094 \\
-3.3465 \\
-0.9646 \\
1.6535 \\
3.3858 \\
3.9567 \\
3.7008 \\
2.6772 \\
1.3189 \\
0.4134\end{array}$ & $\begin{array}{l}-0.23199 \\
-0.5720 \\
-0.9447 \\
-1.0706 \\
-1.1756 \\
-0.8922 \\
-0.2572 \\
0.4408 \\
0.9027 \\
1.0549 \\
0.9866 \\
0.7137 \\
0.3516 \\
0.1102\end{array}$ & $\begin{array}{l}-0.2475 \\
-0.4234 \\
-0.6617 \\
-0.9114 \\
-1.0484 \\
-0.9140 \\
-0.4419 \\
0.2278 \\
0.7881 \\
1.0399 \\
0.9791 \\
0.7505 \\
0.4990 \\
0.2998\end{array}$ & $\begin{array}{l}-0.2002 \\
-0.1597 \\
-0.11172 \\
-0.0768 \\
-0.0437 \\
-0.0197 \\
-0.0060 \\
0.0028 \\
0.0141 \\
0.0340 \\
0.0644 \\
0.1029 \\
0.1452 \\
0.1868\end{array}$ & $\begin{array}{r}-0.0165 \\
0.1487 \\
0.28 ? 9 \\
0.153 ? \\
0.1271 \\
-0.0718 \\
-0.1848 \\
-0.2180 \\
-0.1145 \\
-0.0150 \\
-0.0076 \\
0.0368 \\
0.1474 \\
0.1896\end{array}$ \\
\hline
\end{tabular}




\section{APPENDIX J \\ RESULTS OF VELOCITY PROFILES ANALYSIS FROM PROGRAM TANKANL}

The analysis of the manometer readings obtained from the pitot tube, in making a horizontal pass through Ports 1,2 , and 3 is present in this appendix. The theoretical basis of this analysis is discussed in Section 4.3 .

In Table $\mathrm{J}-1$ is given a list of the abbreviations used as column headings in this appendix. In Table $\mathrm{J}-2$ is given the raw data converted to pressure drops in inches of manometer fluid from manometers $B$ and $D$ for Port 1. The results of the analysis for Ports 1,2 , and 3 are given in Tables $\mathrm{J}-3, \mathrm{~J}-4$, and $\mathrm{J}-5$ respectively. In Table $\mathrm{J}-2$ it is seen that only fourteen of the readings could be analyzed out of thirty-four reported in Table $\mathrm{J}-2$. This is because twenty of these readings gave a $\theta_{p}$ larger than $40^{\circ}$ and thus was outside the range of probe response. 


\section{TABLE J-1}

\section{LIST OF ABBREVIATIONS USED FOR COLUMN HEADINGS}

IN THE TABLES OF APPENDIX $J$

NO

$\mathbf{T}-\mathbf{R}$

DEL P12

DEL P45

V

VR

VZ

VTHETA

PITCH ANGLE

YAW ANGLE

CORRECTED DEL P12
$=$ Item Number

$=T-r$, distance from tank wall, in.

$=\Delta \mathrm{P}_{12}$, pressure drop reading from manometer $B$, inches of manometer fluid

$=\Delta \mathrm{P}_{45}$, pressure drop reading from manometer $D$, onches of manometer fluid

$=\vec{v}$, resultant velocity (ft/min)

$=\bar{v}_{r},(f t / m i n)$

$=\overline{\mathrm{v}}_{\mathrm{z}},(\mathrm{ft} / \mathrm{min})$

$=\overline{\mathrm{v}}_{\theta},(\mathrm{ft} / \mathrm{min})$

$=\theta_{\mathrm{p}}$, (degrees)

$=\theta_{y}$, (degrees)

$=\Delta \mathrm{P}_{12}$, corrected pressure drop from probe manufacturers calibration chart, Figure ' IV-15 
TABLE $\mathrm{J}-2$

RAW DATA CONVERTED TO PRESSURE DROPS

ACROSS MANOMETERS B AND D FOR PORI 1

$$
T-R
$$

0.1000

0.2000

0.3000

0.4000

0.4500

0.5000

0.6000

0.7000

0.8000

0.9000

1.0000

1.1000

1.2 .000

1.3000

1.4000

1. 5000

1.6000

1. 8000

2. 0000

2. 2000

2. 4000

2. 6100

2. 8000

3.000 C

3.2000

3.4000

3. 5000

3. 8000

4. 0000

4. $? 000$

4.4000

4.6000

4. 8000

5.0000
DFL PI2

1.5000

1.5500

1.5500

$1.25 \mathrm{cn}$

1.2500

1.1500

1.2000

1. 2000

1. 0000

0.2000

1. 000 .

1.0000

0.9500

1.0000

1. 00ro

0.8000

$0.75 n 0$

0.2 .000

n. $? 000$

0.5000

0.6000

$0.60 \mathrm{CO}$

0. $55 \mathrm{cn}$

C. 5500

$0.55 \mathrm{CO}$

$0.506 ?$

0.7000

0.7500

$0.95 \mathrm{co}$

0.8500

$0.250 n$

0.9000

$0.85 \mathrm{CO}$

0.8500
DEL $\$ 45$

$-3.0734$

$-1.9050$

$-0.76 ? 0$

$-0.4064$

$-0.1016$

$-n .3048$

0.4318

ก.711?

$0.50 A 0$

0.50 an

0.2540

n. n

$-0.5334$

- C. 8R9ก

$-1.1939$

$-1.6510$

$-0.4318$

$-1.5240$

-1.600 ?

$-1.727 ?$

$-1.8034$

$-1.9050$

$-1.9050$

$-1.9050$

-1.828 B

$-1.9050$

$-2.1590$

$-2.1590$

-5. $94 ? 0$

$-5.3340$

-4.826 ?

-4.775 ?

$-4.8260$

$-4.9760$ 
TABLE $\mathbf{J}-3$

RESULTS OF VELOCITY PROFILE ANALYSIS FOR PORT 1

$T-R \quad V$ VR VZ VTHFTA PITCH VAW CORRFCTFN
ANGLE ANGLF DFL DI?

$\begin{array}{rrrr}0.200 & 52.71 ? & -25.025 & 38.461 \\ 0.300 & 52.057 & -5.412 & 42.924 \\ 0.400 & 46.431 & -1.817 & 43.255 \\ 0.450 & 45.659 & 4.278 & 42.033 \\ 0.500 & 44.407 & -0.417 & 41.050 \\ 0.300 & 43.788 & 12.360 & 36.811 \\ 0.700 & 43.716 & 15.774 & 33.069 \\ 0.800 & 39.901 & 13.310 & 30.738 \\ 1.000 & 40.171 & 9.934 & 26.446 \\ 1.100 & 40.633 & 5.455 & 22.516 \\ 1.200 & 40.860 & -5.793 & 11.149 \\ 1.300 & 42.011 & -14.986 & 10.472 \\ 1.400 & 42.293 & -19.503 & 5.871 \\ 1.600 & 36.372 & -5.446 & -35.909\end{array}$

$25.942-28.343 \cdot 34.00 r$

I. RAT

$28.952-5.967 \cdot 34.000$

I. R P I

$16.77 A \quad-7.247 \quad 71.700$

1.449

$17.325 \quad 5.313 \quad 22.402$

1.401

$16.920 \quad-0.539 \quad 2.2 .400$

1.325

$20.237 \quad 16.395 \quad 2 \mathrm{A.ROC}$

$23.849 \quad 21.151 \quad 35.900$

1.299

$21.693 \quad 19.495 \quad 35.200$

1. 294

1.076

$28.559 \quad 14.317 \quad 47.20$ ? 1. กคร

$33.391 \quad 7.715 \quad 56.000$

1.112

$38.880-8.151 \quad 74.000$

1. 127

$37.839-20.899 \quad 74.600$

$1.1 A B$

$37.066-27.460 \quad 81.70 n$

1. $2 n ?$

$0.376-8.6 ? 3179.4 n ? \quad$ ก.997 
TABLE J -4

RESULTS OF VELOCITY PROFILE ANALYSIS FOR PORT 2

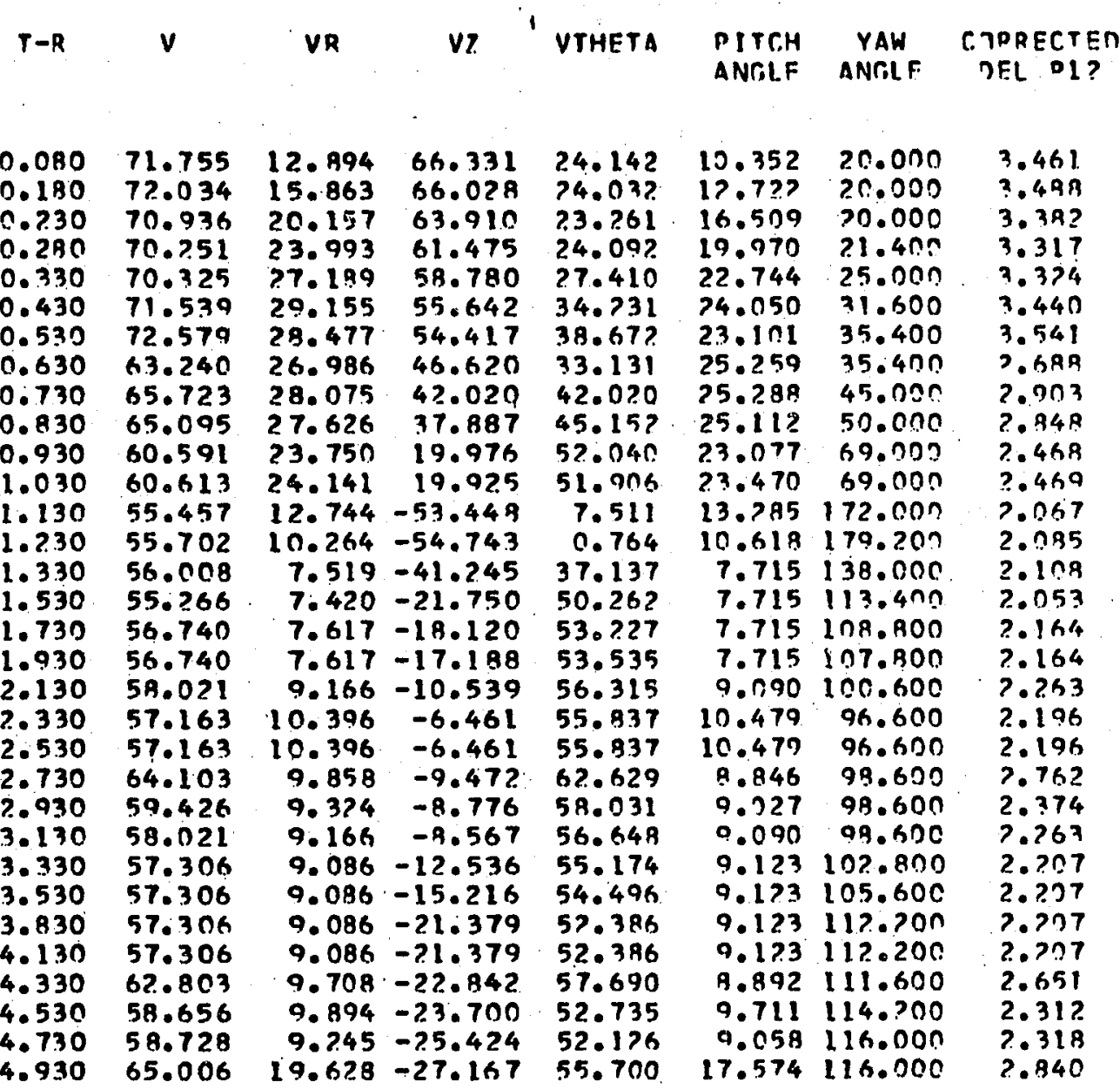


TABLE J-5

RUSULTS OF VELOCITY PROFILE ANALYSIS FOR PORT 3

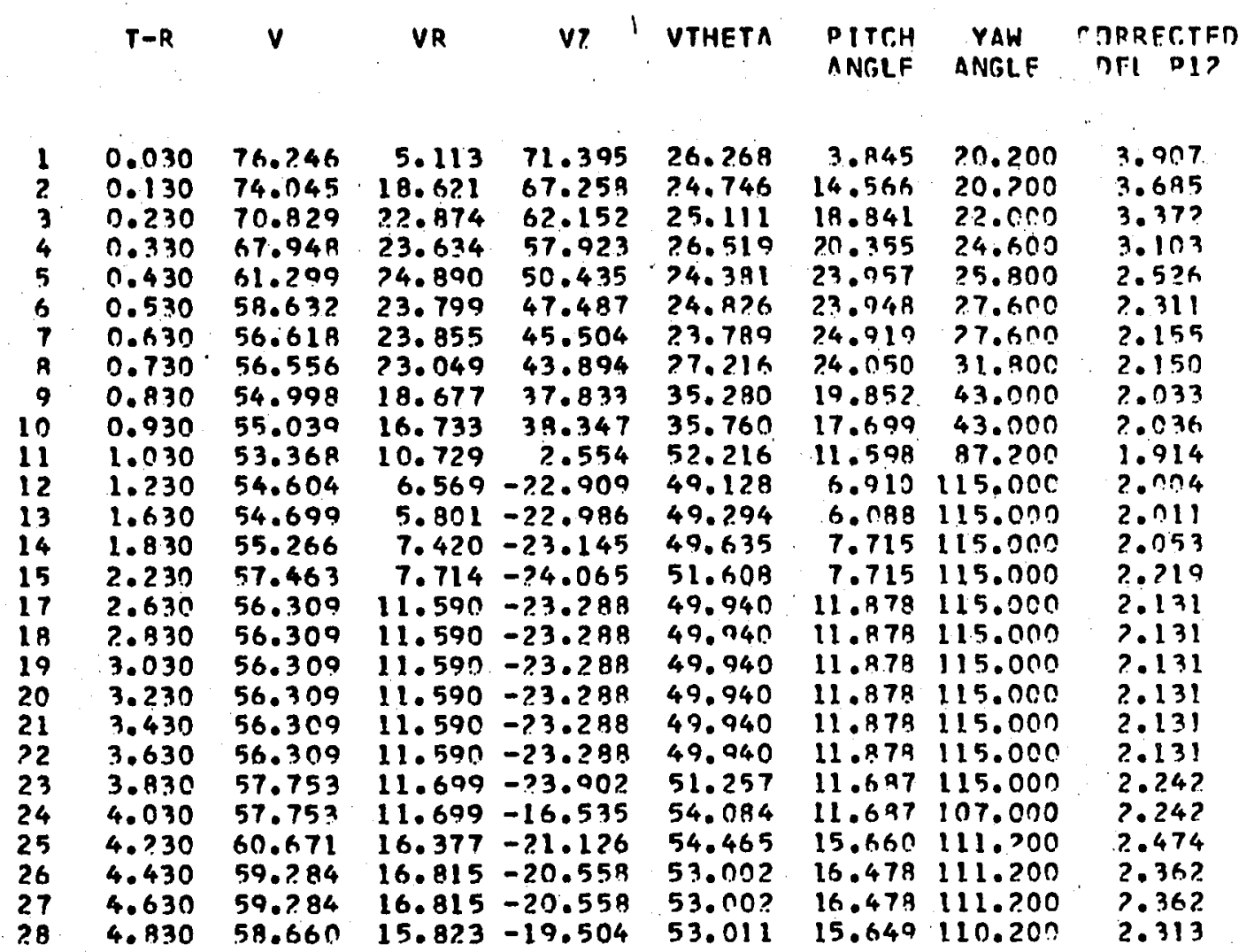




\section{VITA}

Abel DeSouza was born on November 1, 1936 in Ratlain, Madhya, - Pradesh, India. He completed his secondary education at St. Mary's High School, Bombay. He graduated from the Department of Chemical Technology, University of Bombay, Bombay, in March 1959, obtaining a Bachelor of Chemical Engineering degree. After graduation he was employed by the Government of India, Atomic Energy Establishment, Trombay,: Bombay, with their Chemical Engineering Division.

Five years later in September 1964, he joined the Graduate School of the Louisia na State University. In August 1966, he received the Master of Science degree in Chemical Engineering and is present1y working towards a Doctor of Philosophy degree in the same curriculum. He has accepted a position with the She11 Development Company, Emeryville, California. 
EXAMINATION AND THESIS REPORT

Candidate: Abel DeSouza

Major Field: Chemical Engineering

Title of Thesis: Fluid Dynamics and Flow Patterns in Stirred Tanks With a Turbine Impeller

Approved:

Papen w. Pike

Major Professor and Chairman

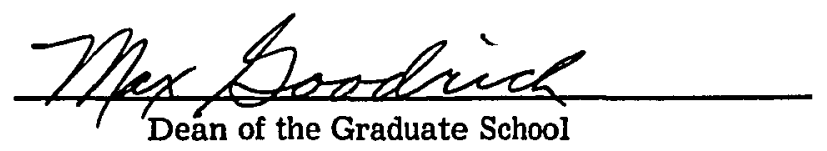

EXAMINING COMMITTEE:
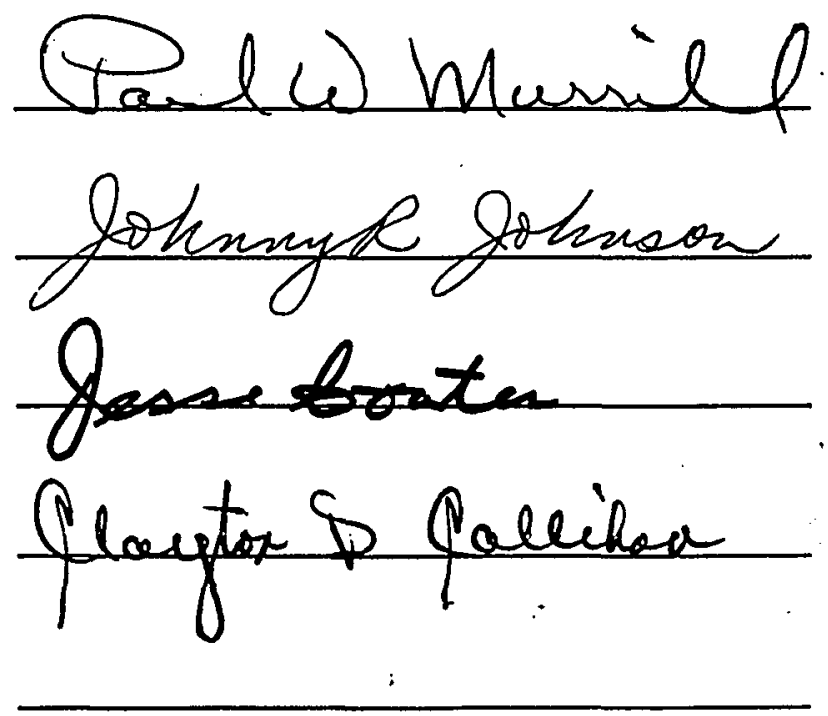

Date of Examination:

July 10,1969 